\title{
Origin of the Anisotropy and Structure of Ellipsoidal Polyfluorene Nanoparticles
}

\author{
Dissertation zur Erlangung des \\ akademischen Grades eines Doktors der Naturwissenschaften \\ (Dr. rer. nat.) \\ vorgelegt von \\ Annika Sickinger \\ aus Hechingen
}

an der

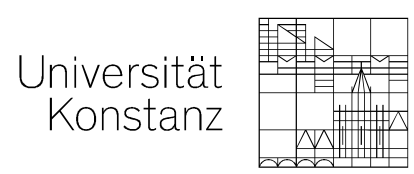

Mathematisch-Naturwissenschaftliche Sektion

Fachbereich Chemie 

Tag der mündlichen Prüfung:

1. Referent:

2. Referent:

Prüfungsvorsitz:
03.12 .2021

Prof. Dr. Stefan Mecking

Prof. Dr. Malte Drescher

Prof. Dr. Helmut Cölfen 

„Bei unvorhergesehenen Ereignissen muss man sich den Gegebenheiten anpassen."

- Loriot 
Die vorliegende Arbeit entstand im Zeitraum von Februar 2018 bis August 2021 unter der Leitung von Herrn Prof. Dr. Stefan Mecking am Fachbereich Chemie der Universität Konstanz. 


\section{DANKSAGUNG - ACKNOWLEDGEMENT}

An erster Stelle gilt mein Dank Prof. Dr. Stefan Mecking für die Aufnahme in die Arbeitsgruppe und die hervorragende Betreuung dieser Arbeit. Er vertraute mir ein extrem vielseitiges, interessantes und herausforderndes Thema an und zeigte stets Interesse an meiner Forschung. Für seine jahrelange Unterstützung, viele hilfreichen Gespräche und die mir gewährten Freiheiten möchte ich mich herzlich bedanken.

Weiterhin danke ich Prof. Dr. Malte Drescher für die Übernahme der Zweitbetreuung und des Zweitgutachtens genauso wie für die gute Zusammenarbeit im Rahmen des Kooperationsprojektes. Für die Übernahme des Prüfungsvorsitzes möchte ich Prof. Dr. Helmut Cölfen ganz herzlich danken.

Ein besonders großer Dank gilt Dr. Julian Ruiz Perez für die umfangreiche und geduldige Einführung in die Thematik der Polyfluoren-Nanopartikel, einschließlich zahlreicher Synthese- und Analytikmethoden. Ebenso danke ich inm, Dr. Steffen Huber, Florian Wimmer, Dr. Tobias Morgen und Dr. Manuel Schnitte für viele hilfreiche Diskussionen auf dem Gebiet der konjugierten Polymere und der Nanopartikeldispersionen. Weiterhin danke ich Dennis Bücker aus der AG Drescher für die gewissenhafte Durchführung und Auswertung von DEER-Experimenten beim gemeinsamen Bearbeiten des A9Kooperationsprojektes.

Dr. Marina Krumova danke ich herzlich für ihre ständige Hilfsbereitschaft und Unterstützung im Zusammenhang mit Mikroskopiemessungen jeder Art. Bei Lars Bolk bedanke ich mich für die Hilfestellungen bei GPC-Messungen und IT-Problematiken. Mein großer Dank gilt ebenso Anke Friemel und Ulrich Haunz für die technische Ermöglichung und die Durchführung verschiedener NMRExperimente. Silke Müller danke ich für die Aufnahme von zahllosen MALDI-TOF Massenspektren. Außerdem möchte ich mich bei Dr. Inaqui Göttker gen. Schnetmann für diverse konstruktive Betrachtungen rätselhafter NMR-Spektren und Anregungen bei chemischen Fragen bedanken. Robin Kirsten danke ich außerdem für die Hilfe in allen technischen Fragen und Gisela Berner in allen organisatorischen und bürokratischen Belangen.

Ich danke dem Sonderfoschungsbereich 1214 „Anisotropic Particles as Building Blocks: Tailoring Shape, Interactions and Structures" für die finanzielle Unterstützung während der Promotion. Den Mitgliedern der IRTG und vor allem meinen AG-internen Mitstreitern danke ich für den hilfreichen wissenschaftlichen Austausch bei allen gemeinsamen Veranstaltungen.

Ganz besonders danke ich auch Anja Rehse, Jonathan Angst, Frederic Hallmann, Magdalena Müller und Janine Scholliers, die mit ihrer hervorragenden Arbeit in ihren Mitarbeiterpraktika und Tätigkeiten als wissenschaftliche Hilfskräfte einen erheblichen Beitrag zu dieser Arbeit geleistet haben. Frederic Hallmann danke ich zudem für sein Engagement, Interesse und die großartige Labordynamik im Rahmen seiner Bachelorarbeit.

Ich danke außerdem von ganzem Herzen der gesamten AG Mecking mit allen gegenwärtigen und ehemaligen Mitgliedern, mit denen ich eine famose Promotionszeit erlebt habe. Danke für die allgemeine Hilfsbereitschaft, die gute Arbeitsatmosphäre und vor allem auch für so viele gelungene 
Aktivitäten inner- und außerhalb der Universität. Dabei sei besonders meinem fantastischen, 'präpandemischen' Büro L722 für stetiges Heben der Laune in allen Situationen gedankt, ebenso Dr. Manuel Schnitte für die gemeinsame Bewältigung des Alltags in guter Bürotischnachbarschaft und allen Laborkolleginnen und Laborkollegen, mit denen ich über die Jahre verschiedene Labore bewohnt habe. Des Weiteren möchte ich mich bei Dr. Tobias Morgen, Anne Staiger, Florian Wimmer, Andreas Scherer, Lukas Odenwald und Dr. Manuel Schnitte für das gründliche und konstruktive Korrekturlesen dieser Arbeit bedanken.

Ein großer Dank gilt außerdem meinen Freunden in Konstanz, der Gruppe „Flucht um 6“, den Mitgliedern des Superserienabends, meiner Inselgassen-WG und alle anderen, die zu vielen unvergesslichen Momenten während des Studiums und der Promotion beigetragen haben.

Zu guter Letzt gilt mein wichtigster Dank denjenigen, die mich in allen guten und nicht so guten Momenten der letzten Jahre stets unterstützt und ertragen haben: meiner wunderbaren Familie, langjährigen Freunden, Jules und ganz besonders meinen Eltern. Danke, dass ihr immer an mich geglaubt habt! 


\section{PUBLICATIONS \& COMMUNICATIONS}

Parts of this thesis have been published.

\section{JOURNAL PUBLICATIONS}

- Sickinger, A.; Mecking, S. Macromolecules 2021, 54, 5267-5277.

"Origin of the Anisotropy and Structure of Ellipsoidal Poly(fluorene) Nanoparticles".

- Bücker, D.; Sickinger, A.; Ruiz Perez, J. D.; Oestringer, M.; Mecking, S.; Drescher, M. J. Am. Chem. Soc. 2020, 142, 1952-1956.

"Direct Observation of Chain Lengths and Conformations in Oligofluorene Distributions from Controlled Polymerization by Double Electron-Electron Resonance".

\section{POSTER PUBLICATIONS}

- Sickinger, A.; Ruiz Perez, J. D.; Mecking, S.

at the $14^{\text {th }}$ European Congress on Catalysis, EuropaCat 2019 "Catalysis without Borders" Aachen, Germany, August 2019.

"Catalytic Synthesis of Anisotropic Conjugated Polymer Nanoparticles".

- $\quad$ Sickinger, A.; Ruiz Perez, J. D.; Schlötter, M.; Maret, G.; Mecking, S. at the SFB 1214 Symposium “Anisotropic Particles”, Konstanz, Germany, September 2018. "Charged Anisotropic Conjugated Polymer Nanoparticles". 
Parts of this thesis have been reproduced and reprinted with permission.

Bücker, D.; Sickinger, A.; Ruiz Perez, J. D.; Oestringer, M.; Mecking, S.; Drescher, M. Direct Observation of Chain Lengths and Conformations in Oligofluorene Distributions from Controlled Polymerization by Double Electron-Electron Resonance. J. Am. Chem. Soc. 2020, 142, 1952-1956. (DOI: 10.1021/jacs.9b11404) Copyright 2021 American Chemical Society. (Chapter 3)

Sickinger, A.; Mecking, S. Origin of the Anisotropy and Structure of Ellipsoidal Poly(fluorene) Nanoparticles. Macromolecules 2021, 54, 5267-5277. (DOI: 10.1021/acs.macromol.1c00597) Copyright 2021 American Chemical Society. (Chapter 4) 


\section{ZUSAMMENFASSUNG - ABSTRACT}

Die Eigenschaften von Nanopartikeln sind in hohem Maße abhängig von ihrer inneren Struktur. Ein detailliertes Verständnis der Prozesse und Mechanismen, die im Inneren von Partikel während deren Entstehung stattfinden, ermöglicht letztendlich die Herstellung von Materialien mit maßgeschneiderten Eigenschaften. Besonders deutlich wird dieser Zusammenhang bei Nanopartikeln mit anisotroper, also nicht-sphärischer Form. Während die Herstellung anisotroper Partikel aus anorganischen Materialien bereits in großem Umfang erforscht wurde, sind für organische Polymere als Ausgangsstoff nur wenige Beispiele bekannt, die nahezu alle auf der Verarbeitung zuvor synthetisierter Polymere basieren. Erst kürzlich wurde ein Verfahren entwickelt, um ellipsoide Nanopartikel aus Poly(9,9'-dioctylfluoren) (PF8) mit einer durchschnittlichen Partikellänge von ca. 120 nm und einem Aspektverhältnis von 3.5 direkt im Zuge der Polymersynthese herzustellen (Abbildung 1). Es handelt sich dabei um eine Palladiumkatalysierte Suzuki-Miyaura Polykondensation, die als Emulsionspolymerisation durchgeführt wird. In der Materialklasse der konjugierten Polymere stellt PF8 einen Sonderfall dar, da die Polymerketten verschiedene Konformationen annehmen und sich abhängig davon zu Strukturen mit unterschiedlich ausgeprägter Ordnung organisieren können. Im Fokus wissenschaftlichen Interesses ist hierbei vor allem die sogenannte $\beta$-Konformation, die durch eine Planarisierung des Polymerrückgrads zur Bildung von Bereichen hoher Kettenordnung im Material führt, welche wiederum außergewöhnliche optische Eigenschaften zur Folge haben. Obwohl von einem Zusammenhang zwischen der intermolekularen Anordnung der Polymerketten und der einzigartigen Form der blau fluoreszierenden Partikel ausgegangen wurde, waren der genaue Ursprung der Anisotropie und die zugrundeliegenden Mechanismen noch nicht geklärt.

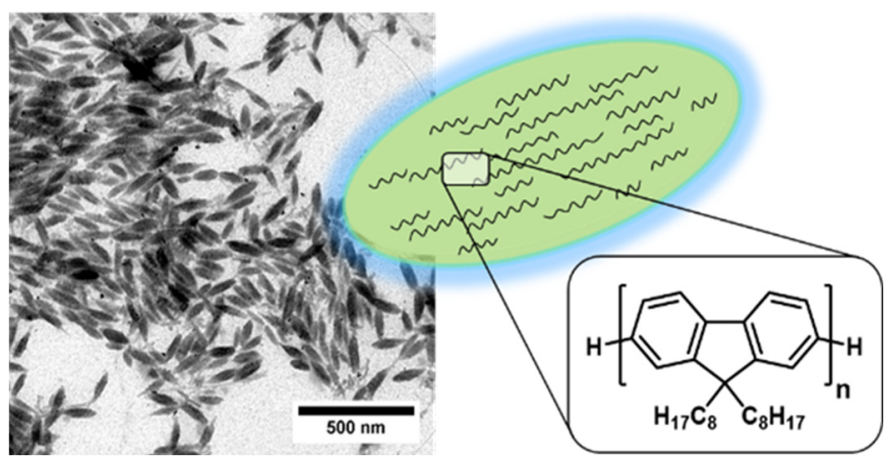

Abbildung 1: Transmissionselektronenmikroskopische Aufnahme einer Dispersion ellipsoider PF8-Nanopartikel mit schematischer Darstellung der inneren Partikelstruktur und chemischer Struktur von PF8.

In einem ersten Ansatz wurde die molekularer Anordnung in PF8 Nanopartikeln anhand von Elektronenspinresonanz untersucht. Dazu wurden Oligo(fluorene) definierter Länge mit je zwei paramagnetischen Spinsonden an beiden Kettenenden synthetisiert um anschließend ihre Konformation in Lösung zu charakterisieren. Aus den gemessenen Abständen zwischen den beiden Spinsonden konnten für das vorliegende Ensemble von einzelnen Oligomeren unterschiedlicher Kettenlänge die Kontur- und die Persistenzlängen errechnet werden. Während die Konturlänge die Länge eines Makromoleküls in vollständig ausgestrecktem Zustand wiedergibt und daher für jede 
Oligomer-Spezies einzeln bestimmt wurde, repräsentiert die Persistenzlänge als Maß für die Flexibilität eines Gesamtsystems eine globale Größe. Im vorliegenden Fall wurde eine Persistenzlänge von $L_{p}=14 \mathrm{~nm}$ ermittelt, was einem semi-flexiblen Charakter der Ketten entspricht. Mit dem erhaltenen Satz von Referenzdaten wurden weiterhin Messungen an realen Mischungen von Oligomeren durchgeführt. Diese wurden durch kontrollierte Suzuki-Miyaura Polymerisation erhalten und zeigten eine PoissonKettenlängenverteilung. Die durch Elektronenspinresonanz ermittelten Spin-Spin Abstände der Oligomere stimmten mit der erwarteten Form der Verteilung überein, was die Anwendbarkeit der Messmethode auf komplexe Polymermischungen bewies. Neben Experimenten in Lösung wurde das Verhalten der Oligomere in Partikeln, also umgeben von fester Materie, untersucht, um zusätzlich Aufschluss über den Anwendungsbereich der Messmethode zu erhalten. Die entsprechenden, kurzkettigen Oligomere wurden in einem Post-Polymerisationsverfahren in PF8-Nanopartikel eingebettet und zeigten in den anschließenden Messungen eine ungestörte Flexibilität. Zusammenfassend stellte Elektronenspinresonanz eine gewinnbringende Analytikmethode dar, um die Konformation und Flexibilität konjugierter Oligomere sowohl in Lösung als auch im Feststoff zu untersuchen. Allerdings repräsentierte der auf Oligo(fluorene) mit maximal 7 Wiederholeinheiten limitierte Messbereich einen entscheidenden Nachteil der Methode, da von Studien an längeren Oligomeren und Polymeren in Nanopartikeln weitere Informationen über die Prozesse der Partikelbildung erwartet wurden.

Dies wurde im Folgenden durch eine Kombination synthetischer und analytischer Methoden realisiert, die tiefgehende Erkenntnisse über die interne Anordnung von Polymerketten während der Herstellung ellipsoider PF8-Partikel ermöglichten. Zunächst wurden stufenweise, ausgehend von Trimeren als Untereinheiten, längere Oligo(9,9'-dioctylfluorene) hergestellt. Diese wurden durch Zugabe in die anfängliche Reaktionsmischung im Verlauf der etablierten Suzuki-Miyaura-Polykondensation unter Emulsionsbedingungen in die entstehenden Nanopartikel eingebaut. Durch mikroskopische und spektroskopische Betrachtung der Dispersionen konnte nachgewiesen werden, dass die Gegenwart von Oligomeren mit 3,6,9,12 oder 15 Wiederholeinheiten keinerlei Einfluss auf die resultierenden Partikel hatte, weder in Bezug auf die Partikelform noch auf die optischen Materialeigenschaften. Daher kann davon ausgegangen werden, dass unter der Bedingung eines geeigneten organischen Lösungsmittels selbst längere Oligomere die ausgeprägte Fähigkeit besitzen, sich in entstehenden Partikeln anzuordnen. Gleichermaßen konnte auch für Poly(9,9'-dioctylfluoren) eine ausreichende Mobilität nachgewiesen werden. Als Alternative zur oben erwähnten Suzuki-Miyaura-Polykondensation wurde eine Methode entwickelt, ellipsoide Nanopartikel in einem Post-Polymerisationsverfahren herzustellen. Dieses beinhaltete das Emulgieren einer Lösung des vorgefertigten Polymers in der wässrigen, kontinuierlichen Phase durch Anwendung von Ultraschall. Da die auf diese Art hergestellten Partikel eine vergleichbare Qualität wie das durch Emulsionspolymerisation gewonnene Referenzsystem aufwiesen, kann das Verfahren als nutzbringende Methode zur Herstellung anisotroper Partikel betrachtet werden. Durch Verwendung der vorgefertigten Oligomere im beschriebenen, optimierten Verfahren wurden ebenfalls Dispersionen definierter Partikel hergestellt und eine kritische Kettenlänge von mindestens 10 Wiederholeinheiten ermittelt, welche zur Ausbildung einer anisotropen Partikelform nötigt waren. Um weitere Einsicht in die Anordnung der Polymerketten im Laufe der SuzukiMiyaura Polykondensation zu gewinnen, wurde die laufende Reaktion mittels Messungen des 
Molekulargewichts und der Fluoreszenzemission verfolgt. Im Hinblick auf das Stufenwachstum der Polykondensationsreaktion wurde ein außergewöhnlich rasches Wachstum der Polymerkette festgestellt, wobei das endgültige Molekulargewicht von ca. $40 \mathrm{~kg} / \mathrm{mol}$ bereits nach wenigen Minuten der Reaktionszeit erreicht war. Der Anstieg des Molekulargewichts ging einher mit der Ausrichtung der Polymerketten zu höher geordneten Regionen von $\beta$-Phasenkonformation im Material, was durch parallele fluoreszenzspektroskopische Messungen ersichtlich wurde. Darüber hinaus wurde durch Studien an vernetztem PF8 die Erkenntnis gewonnen, dass die Prozesse der Kettenanordnung in PF8Partikeln linearem Polymer vorbehalten sind. Die Anwesenheit von Verzweigungen oder Vernetzungspunkten, die durch Zugabe multifunktionaler Monomere in der Suzuki-Miyaura Polykondensation generiert wurden, führte zu einem drastischen Verlust der hohen inneren Partikelordnung und der ellipsoiden Form zugunsten von sphärischen Partikeln mit amorpher Polymerstruktur. Gestützt auf die vorangegangenen Erkenntnisse wurde die Hypothese eines Bildungsmechanismus der Partikel im Laufe der Polymerisation erarbeitet, welche im Folgenden ausgeführt wird (Abbildung 2).

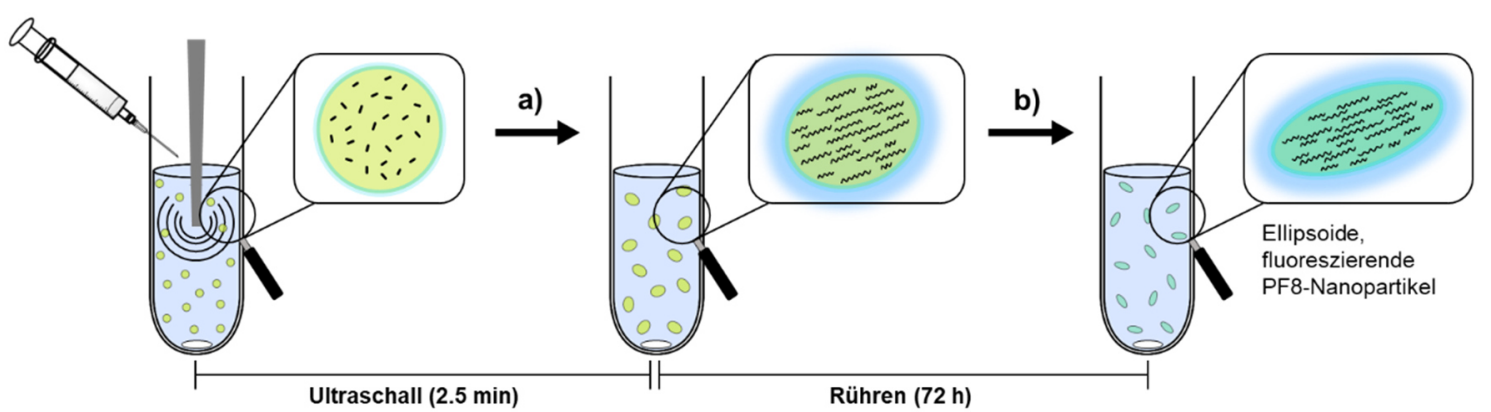

Abbildung 2: Angenommener Mechanismus der Bildung von ellipsoiden, fluoreszierenden Nanopartikeln aus Poly(9,9'-dioctylfluoren) im Laufe der Suzuki-Miyaura Polykondensation. Dieser umfasst (a) die Bildung von Oligomer- und Polymerketten und deren Anordnung in geordnete Phasen und (b) das Verdampfen des organischen Lösungsmittels.

Direkt mit dem Emulgieren der organischen Phase durch Ultraschall beginnt das Wachstum von Oligomer- und Polymerketten, welche sich gleichzeitig in diesem ersten Stadium der Reaktion zu Bereichen der $\beta$-Phasenkonformation anordnen (a). Dieser Vorgang wird zudem durch die hohe Polymerkonzentration in den Lösungsmitteltröpfchen, welche durch das schnelle Kettenwachstum vorliegt, begünstigt. Nach Beendigung des Ultraschalls ist das finale Molekulargewicht des Polymers bereits erreicht; dennoch ist die verbleibende Reaktionszeit von großer Relevanz für das Verdampfen des organischen Lösungsmittels aus den noch gequollenen Partikeln (b). Schlussendlich werden blau fluoreszierende Nanopartikel in einer charakteristischen, ellipsoiden Form erhalten. Die obigen Ergebnisse geben einen detaillierten Einblick in die Bildung von Nanopartikeln konjugierter Polymere mit einer anisotropen Form sowie in die involvierten Prozesse. Dabei wurde ein signifikanter Zusammenhang zwischen innerer Ordnung des Materials auf molekularer Ebene und Partikelform festgestellt, was die Erschließung neuartiger Materialeigenschaften in Partikeln, reguliert durch ihre innere Struktur, ermöglicht.

Neben Poly $\left(9,9^{\prime}\right.$-dioctylfluoren) als prominentestes Beispiel dieser Polymerklasse wurde auch für Poly(fluorene) mit zwei Aryl- statt Alkylsubstituenten an der verbrückenden C-9-Position ein ungewöhnliches, polymorphes Verhalten und das Auftreten von $\beta$-Phasenkonformation nachgewiesen. 
Aufgrund dieser Eigenschaften in Kombination mit der hohen Stabilität gegenüber Oxidation bietet dieses Polymer eine vielversprechende Alternative zu PF8 und wurde im Folgenden hinsichtlich seiner Fähigkeit zur Ausbildung anisotroper Partikelformen untersucht. Nach der Synthese der entsprechenden Monomere wurde erfolgreich Poly(5-octyloxy-9,9'-diphenylfluoren) über zwei Routen hergestellt: einerseits durch das etablierte Verfahren der Suzuki-Miyaura Polykondensation unter Emulsionsbedingungen und andererseits durch kontrollierte Suzuki-Miyaura Polymerisation in Lösung. Die aus der Emulsionspolymerisation hervorgegangenen Dispersionen wurden mittels Transmissionselektronenmikroskopie charakterisiert, welche das überwiegende Vorkommen von faserartigen, undefinierten Polymerstrukturen zeigten. Jedoch konnte die Bildung ellipsoider Nanopartikel als exklusive Spezies durch eine Anpassung verschiedener Reaktionsparameter erreicht werden, vor allem durch Erniedrigung der Monomerkonzentration in der anfänglichen Reaktionsmischung. Nanopartikel deutlich höherer Qualität in Bezug auf Anisotropie und Dispersität waren zudem in einem optimierten Post-Polymerisationsverfahren zugänglich (Abbildung 3).

a)

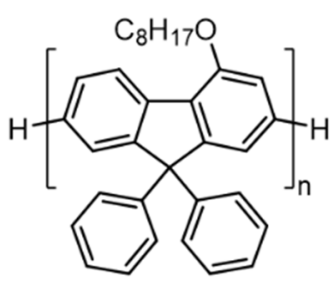

b)

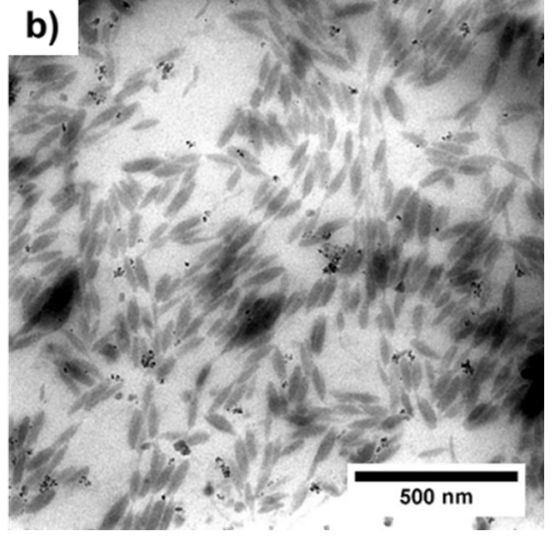

Abbildung 3: (a) Chemische Struktur von Poly(5-octyloxy-9,9'-diphenylfluoren) und (b) transmissionselektronenmikroskopische Aufnahme der durch ein Post-Polymerisationverfahren hergestellten Partikel (Länge $=104 \mathrm{~nm}$, Aspektverhältnis = 3.4).

Obwohl die grundsätzliche Fähigkeit des Materials zur Ausbildung von $\beta$-Phasen nachgewiesen wurde, wiesen die betrachteten Dispersionen ausschließlich Absorptions- und Fluoreszenzsignale amorpher Polymerstruktur auf, unabhängig von der Art ihrer Herstellung. Abschließend ist festzuhalten, dass durch Poly(5-octyloxy-9,9'-diphenylfluoren) ein Polymer gegeben ist, welches die Fähigkeiten zur Bildung anisotroper Partikelformen und zur Ausrichtung der Polymerketten in Regionen geordneter Zustände in sich vereint. Anders als im Fall von PF8 offenbarte sich basierend auf den durchgeführten Messungen der Fluoreszenzemission jedoch keine eindeutige Korrelation dieser beiden Phänomene. Im Zuge der oben ausgeführten Studien wurden hochwertige, ellipsoide Nanopartikel hergestellt und die Bandbreite der als anisotrope Partikel zugänglichen konjugierten Polymere erweitert.

Neben der Anwendung als Ausgangsmaterial für die Herstellung von Nanopartikeln stellen Poly(fluorene) und verwandte Polymere vielversprechende Komponenten in Block-Copolymeren dar. Durch Verknüpfung der hydrophoben, starren Polymerkette mit einem hydrophilen, flexiblen Segment können amphiphile Block-Copolymere erhalten werden, die aufgrund der Verschiedenheit der einzelnen Komponenten dazu in der Lage sind, sich im Zuge von Mikrophasenseparation zu komplexen Überstrukturen zu organisieren. Neben den zuvor betrachteten Poly(fluoren)-Nanopartikeln wurden in 
der vorliegenden Arbeit Block-Copolymere mit PF8-Segment synthetisiert und als alternatives System hinsichtlich der Organisation von Polymerketten auf molekularer Ebene untersucht. Als hydrophile, flexible Komponente wurden Poly(ethylenglykole) (PEGs) gewählt. Zur Synthese der gewünschten Copolymere wurden zunächst kommerzielle PEGs end-funktionalisiert mit einem Palladium-basierten Präkatalysator zu einer katalytisch aktiven Spezies umgesetzt. Die resultierenden Makroinitiatoren dienten anschließend im angepassten Protokoll einer kontrollierten Suzuki-Miyaura Polymerisation als Ausgangspunkt für die entstehende PF8-Kette (Abbildung 4).
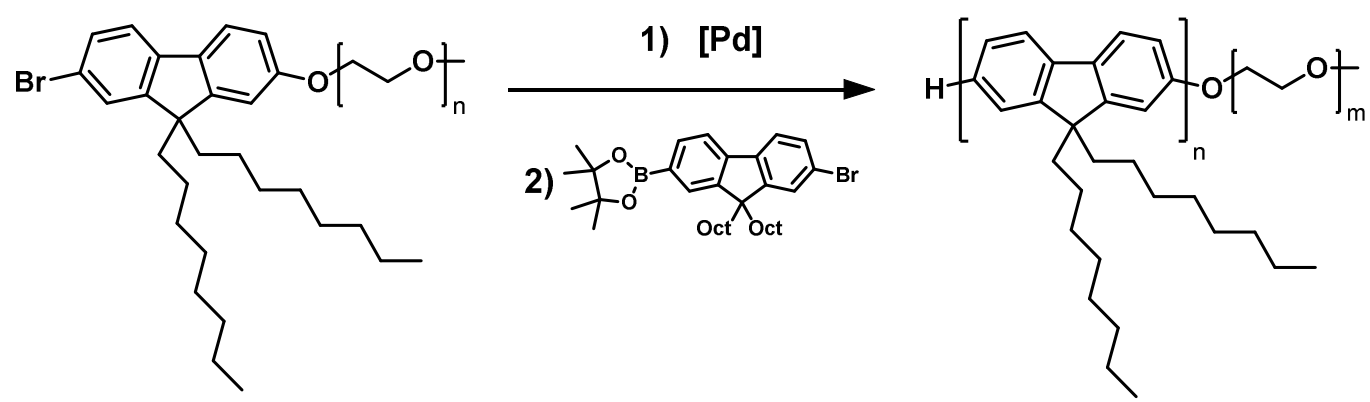

Abbildung 4: Schematische Darstellung der Synthese amphiphiler Block-copolymere durch Suzuki-MiyauraPolymerisation ausgehend von einem PEG-funktionalisierten Makromolekül.

Nach Bestätigung der erfolgreichen Verknüpfung der beiden Polymere mittels verschiedener Methoden wurden über den obigen Syntheseweg eine Reihe amphiphiler Block-Copolymere mit variierender Länge beider Segmente hergestellt. Neben PF8 als Referenzsystem wurde die Polymerisation mit alternativen konjugierten Monomeren durchgeführt, was die Möglichkeit zur Synthese amphiphiler Copolymere mit einstellbarer Emissionswellenlänge eröffnete. Erste Experimente bezüglich des Assemblierungsverhaltens der Copolymere zeigten, anhand mikroskopischer Studien, das Auftreten von gleichmäßigen, lamellaren Strukturen. Es wurde angenommen, dass die Bildung dieser Aggregate vor allem von der kovalenten Anbindung hydrophiler Segmente und den damit ermöglichten Prozessen der Mikrophasenseparation bedingt wurde. Zusammenfassend lässt sich sagen, dass die ausgearbeitete Syntheseroute eine nützliche Methode zur Herstellung amphiphiler Block-Copolymere bietet, die eine große Bandbreite von Copolymeren mit variabler Segmentlänge und unterschiedlichen Emissionseigenschaften zugänglich macht.

In ihrer Gesamtheit gewähren die dargelegten Ergebnisse am Beispiel von Poly(9,9'-dioctylfluoren) weitreichende Einsicht in die Zusammenhänge von anisotroper Partikelform und der Anordnung der Polymerketten auf molekularer Ebene. Durch verschiedene synthetische und analytische Ansätze wurde das Verhalten von Polymer- und Oligomerketten im Verlauf der Partikelentstehung verfolgt. Im Zuge dessen wurde ein Verfahren ausgearbeitet, um ellipsoide Nanopartikel durch Emulgieren von vorgefertigtem Polymer herzustellen. Die Bandbreite der als anisotrope Partikel zugänglichen konjugierten Polymere wurde durch Untersuchungen an Poly(5-octyloxy-9,9'-diphenylfluoren) erweitert. Die Steuerung der äußeren Dimensionen von Nanostrukturen durch intermolekulare Interaktionen der Polymerketten konnte weiterhin am Beispiel amphiphiler Block-Copolymere mit konjugiertem Segment demonstriert werden. Es wurde gezeigt, dass die Entstehung von Bereichen hoher molekularer Ordnung im betrachteten konjugierten Polymer in außerordentlichem Maße mit der Ausbildung anisotroper Partikelformen korreliert. 


\section{TABLE OF CONTENTS}

DANKSAGUNG - ACKNOWLEDGEMENT ..........................................................

PUBLICATIONS \& COMMUNICATIONS ........................................................... VI

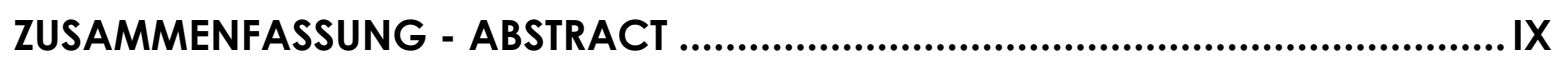

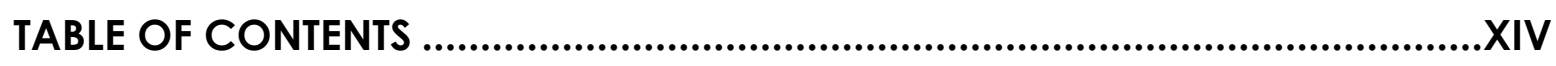

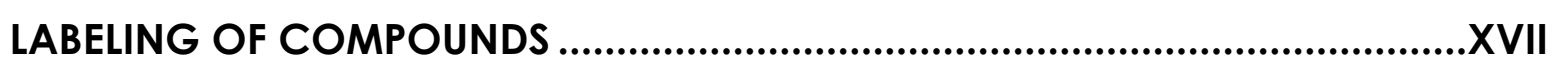

LIST OF ABBREVIATIONS ..............................................................................

1 GENERAL INTRODUCTION...................................................... 1

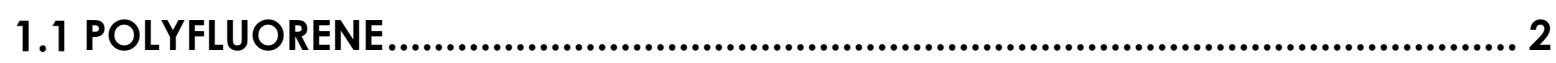

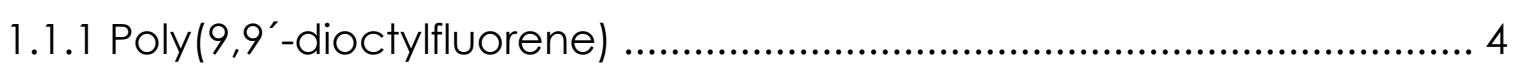

1.1.2 Poly $\left(9,9^{\prime}\right.$-diaryffluorene) ................................................................. 7

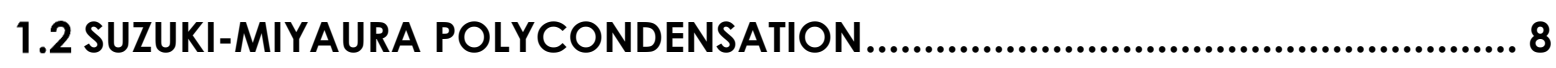

1.3 CONJUGATED POLYMER NANOPARTICLES ............................................. 10

1.3.1 Post-Polymerization Techniques ............................................................ 11

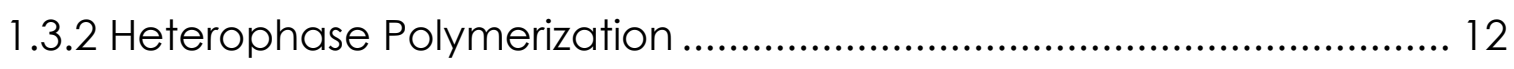

1.4 ANISOTROPIC POLYMER NANOPARTICLES ............................................. 14

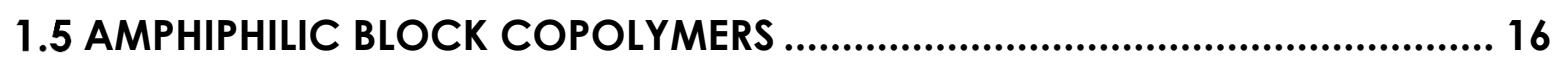

2 SCOPE OF THE THESIS .............................................................19

3 STUDIES ON OLIGO-FLUORENE CONFORMATION BY DOUBLE ELECTRON-ELECTRON RESONANCE..........................................220

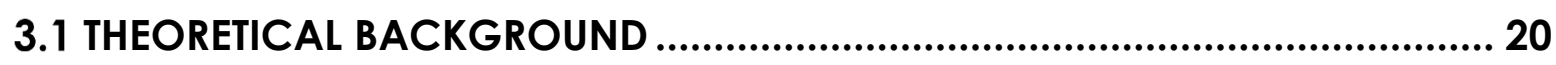

3.2 DEER MEASUREMENTS ON SPIN-LABELED OLIGOMERS ….......................... 22

3.2.1 Synthesis of Spin-Labeled Oligomers ................................................ 22

3.2.2 DEER Measurements on Individual Oligomer Species ......................... 24

3.3 DEER MEASUREMENTS ON A SPIN-LABELED OLIGOMER MIXTURE............... 27

3.4 EVALUATION OF DEER IN POLYFLUORENE PARTICLES …............................ 29

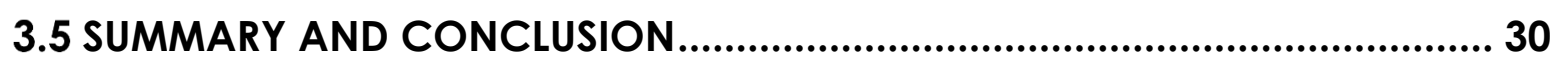

4 ORIGIN OF ANISOTROPY AND STRUCTURE OF ELLIPSOIDAL POLYFLUORENE NANOPARTICLES ............................................... 32

4.1 SUZUKI-MIYAURA POLYCONDENSATION WITH ADDED OLIGOFLUORENES 34 4.2 POST-POLYMERIZATION PARTICLE SYNTHESES........................................... 38

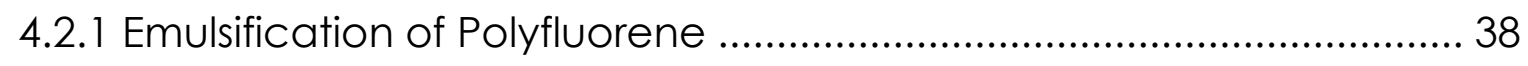

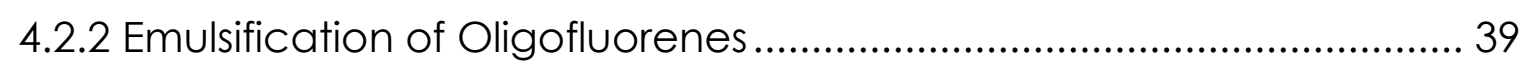


4.3 EVOLUTION OF MOLECULAR WEIGHT IN THE COURSE OF

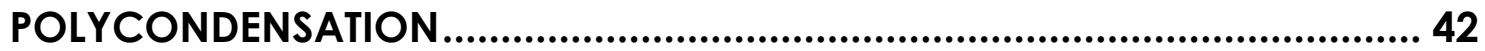

4.4 CHAIN-PACKING IN CROSS-LINKED POLYFLUORENE ................................. 44

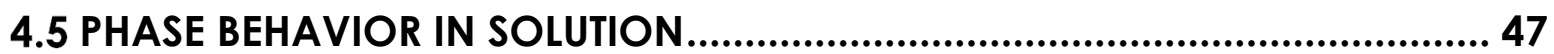

4.5.1 Concentration Dependent Solution Spectra of Polyfluorene ............ 47

4.5.2 Concentration Dependent Solution Spectra of Oligofluorenes......... 49

4.6 SELF-ASSEMBLY OF ELLIPSOIDAL POLYFLUORENE NANOPARTICLES ........... 51

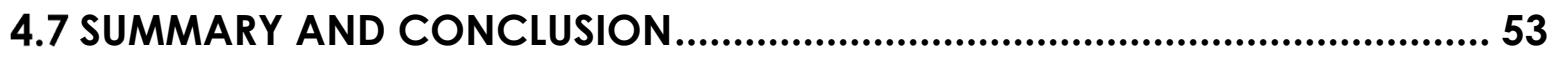

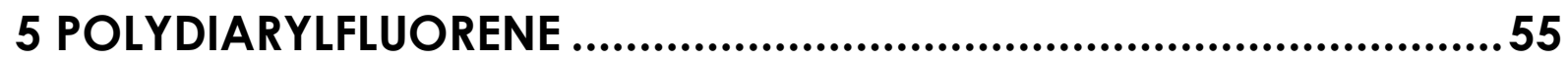

5.1 SYNTHESES OF DIARYLFLUORENE MONOMERS ........................................ 55

5.2 POLYDIARYLFLUORENE BY EMULSION POLYMERIZATION ............................ 57

5.3 CONCENTRATION DEPENDENT PHASE BEHAVIOR IN SOLUTION .................61

5.4 POST-POLYMERIZATION PARTICLE SYNTHESES................................................ 62

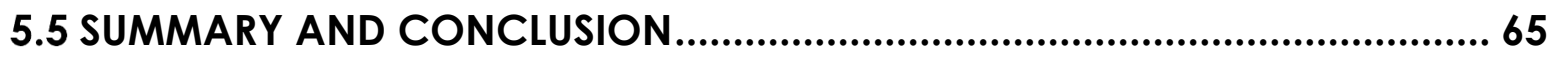

6 AMPHIPHILIC BLOCK COPOLYMERS ..........................................66

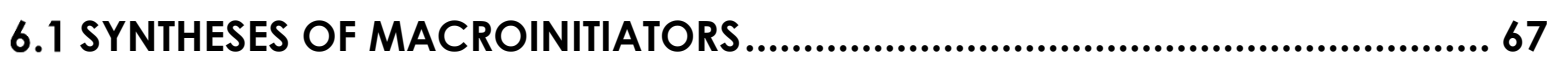

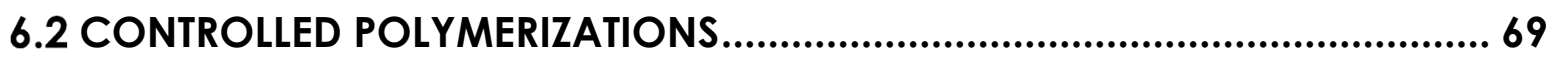

6.2.1 Copolymers with Polyfluorene Segment ............................................... 70

6.2.2 Copolymers with Other Conjugated Polymer Segments .................... 76

6.3 AGGREGATION BEHAVIOR OF AMPHIPHILIC COPOLYMERS ...................... 78

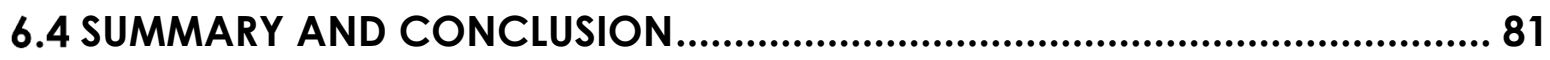

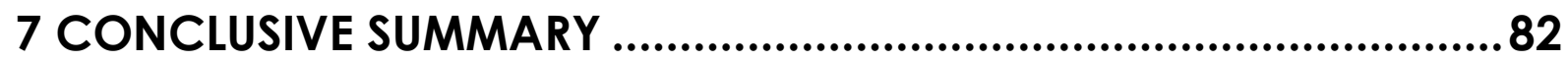

8 EXPERIMENTAL SECTION...........................................................88

8.1 MATERIALS AND GENERAL CONSIDERATIONS ............................................88

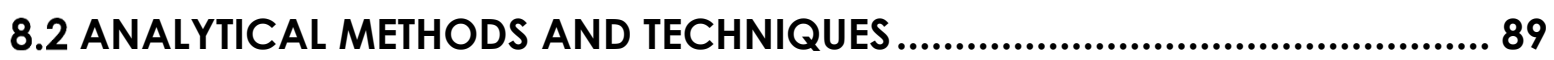

8.3 HETEROPHASE SUZUKI-MIYAURA POLYCONDENSATION ............................ 91

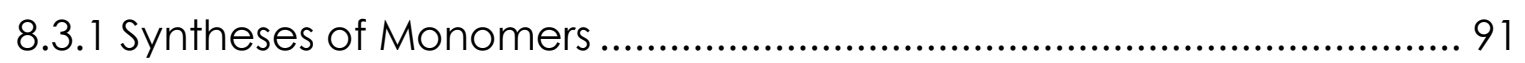

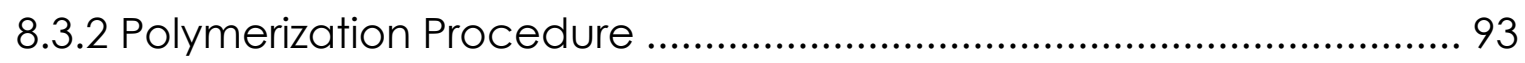

8.4 DOUBLE ELECTRON-ELECTRON RESONANCE MEASUREMENTS ON

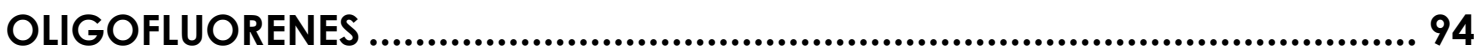

8.4.1 Spin-Labeled Oligomers by Controlled Suzuki-Miyaura Coupling

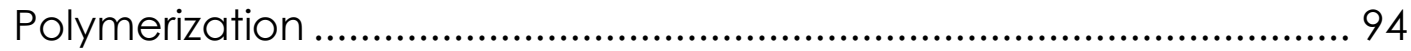

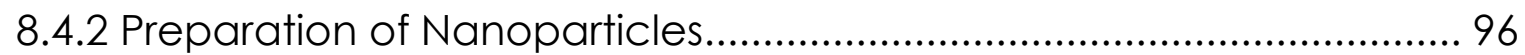

8.4.3 Electron Paramagnetic Resonance Measurements ............................ 96

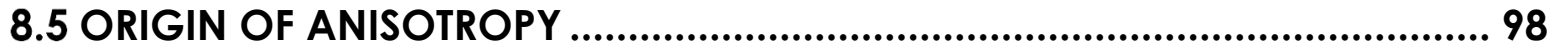

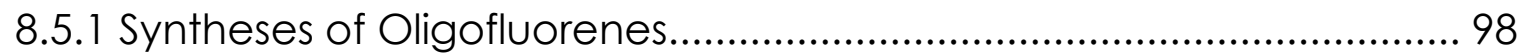


8.5.2 Suzuki-Miyaura Polycondensation with Added Oligomers 109

8.5.3 Post-Polymerization Particle Synthesis 109

8.5.4 Evolution of Molecular Weight in the Course of Polycondensation..110

8.5.5 Synthesis of Cross-Linked Polyfluorene. .111

8.5.6 Phase Behavior in Solution. .113

8.5.7 Vertical Deposition

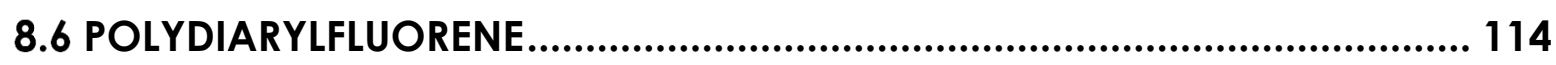

8.6.1 Syntheses of Diarylfluorene Monomers ...............................................114

8.6.2 Polydiarylfluorene by Emulsion Polymerization .....................................119

8.6.3 Polydiarylfluorene by Controlled Suzuki-Miyaura Coupling Polymerization

8.6.4 Post-Polymerization Synthesis of Polydiarylfluorene Particles ..............120

8.7 AMPHIPHILIC BLOCK COPOLYMERS ..................................................... 122

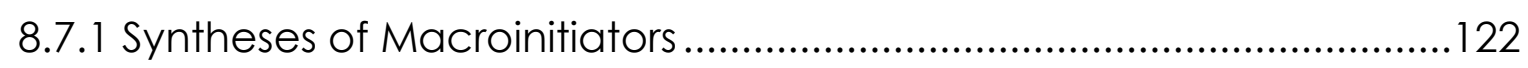

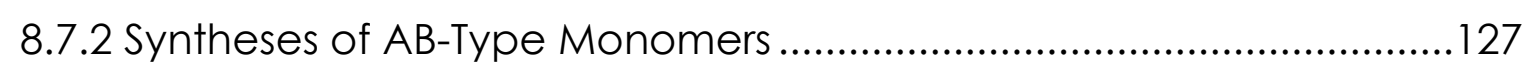

8.7.3 Controlled Suzuki-Miyaura Coupling Polymerization ...........................128

8.7.4 Aggregation Behavior of Amphiphilic Copolymers............................129

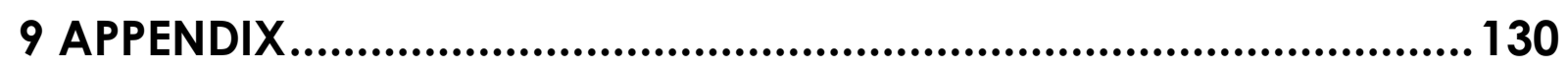

9.1 STUDIES ON OLIGOFLUORENE CONFORMATION BY DOUBLE ELECTRONELECTRON RESONANCE.

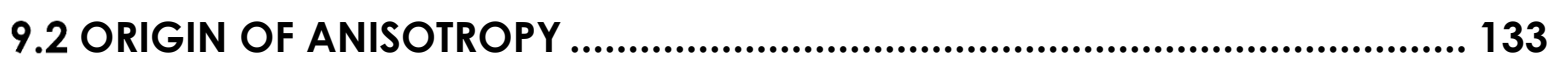

9.2.1 Syntheses of Oligofluorenes.............................................................133

9.2.2 Heterophase Suzuki-Miyaura Polycondensation with Alternative Solvents

9.2.3 Post-Polymerization Particle Syntheses ................................................134

9.2.4 Evolution of Molecular Weight in the Course of Polycondensation..135

9.2.5 Synthesis of Cross-Linked Polyfluorene ................................................138

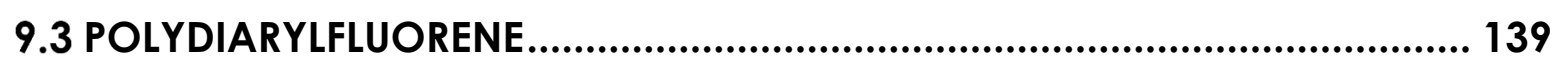

9.3.1 NMR-Spectra of Diarylfluorene Monomers ........................................139

9.3.2 Polydiarylfluorene by Emulsion Polymerization......................................143

9.3.3 Post-Polymerization Particle Syntheses ..............................................146

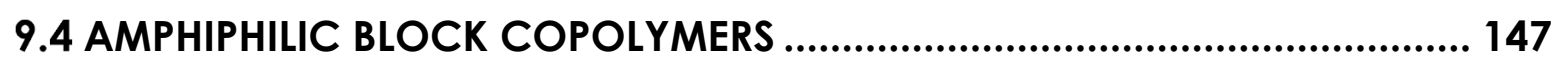

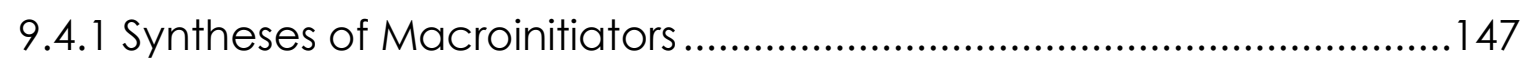

9.4.2 Controlled Suzuki-Miyaura Coupling Polymerization ...........................151

9.4.3 Aggregation Behavior of Amphiphilic Copolymers...........................154

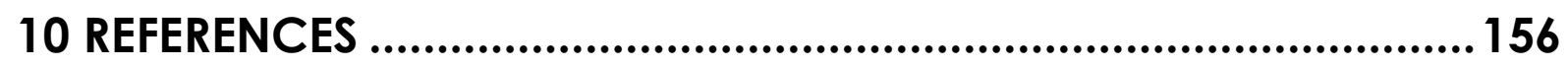




\section{LABELING OF COMPOUNDS}

\section{9,9'-DIOCTYLFLUORENE MONOMERS}<smiles>[13CH3]C1([14CH3])c2cc(Br)ccc2-c2ccc(Br)cc21</smiles>

$\mathrm{Br}-\mathrm{F}_{\mathbf{1}}-\mathrm{Br}$

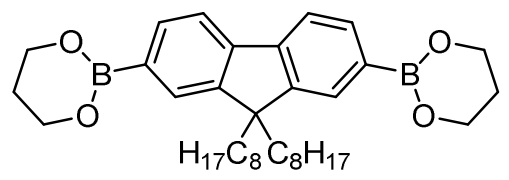

$\mathrm{PrO}_{2} \mathrm{~B}-\mathrm{F}_{1}-\mathrm{BO}_{2} \mathrm{Pr}$

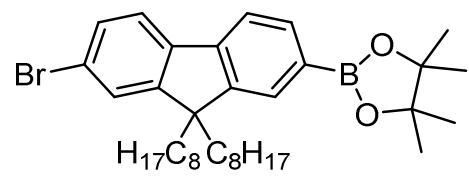

$\mathrm{Br}-\mathrm{F}_{\mathbf{1}}$-BPin

$9,9^{\prime}$-DIOCTYLFLUORENE OLIGOMERS<smiles>C=C(C)C1(CCCC)c2cc(C)ccc2-c2ccc(C)cc21</smiles>

$H-F_{n}-H$

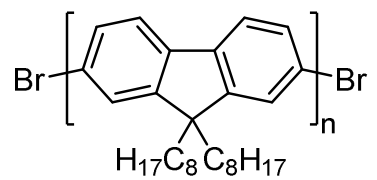

$\mathrm{Br}-\mathrm{F}_{\mathrm{n}}-\mathrm{Br}$

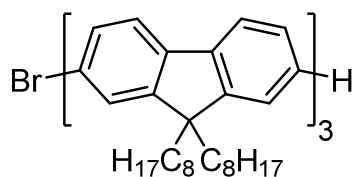

$\mathrm{Br}-\mathrm{F}_{3}-\mathrm{H}$

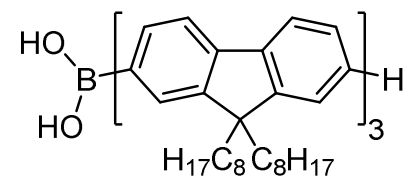

$(\mathrm{HO})_{2} \mathrm{~B}-\mathrm{F}_{3}-\mathrm{H}$

5-OCTYLOXY-9,9'-DIPHENYLFLUORENE MONOMERS<smiles>Brc1ccc2c(c1)C(c1ccccc1)(c1ccccc1)c1cc(Br)ccc1-2</smiles>

Br-ODPF-Br

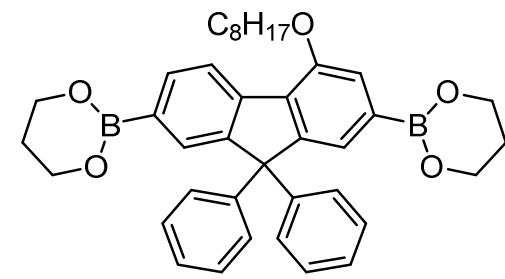

$\mathrm{PrO}_{2} \mathrm{~B}-\mathrm{ODPF}-\mathrm{BO}_{2} \mathrm{Pr}$

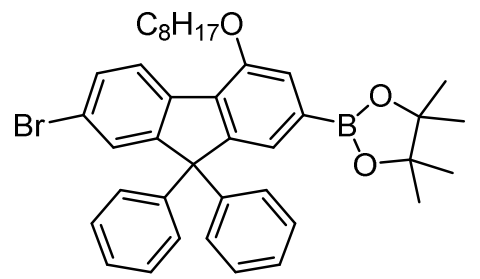

Br-ODPF-BPin

OTHER AB-TYPE MONOMERS<smiles>CC1(C)OB(c2ccc3c(c2)C2(CCCC2)c2cc(Br)ccc2-3)OC1(C)C</smiles>

Br-M3-BPin

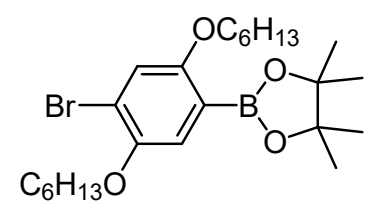

Br-M4-BPin<smiles>CC(C)(C)c1cc(B2OC(C)(C)C(C)(C)O2)sc1Br</smiles>

Br-M5-BPin 


\section{LIST OF ABBREVIATIONS}

a.i.

ar

ATRP

COSY

CPNs

$\delta$

DCM

DEER

DLS

DMF

DMSO

DOSY

$D P_{n}$

EPR

equiv.

GPC

HMBC

HOMO

${ }^{n} J_{X Y}$

$\lambda$

$\lambda_{\text {exc }}$

$\mathrm{L}_{c}$

$L_{p}$

LUMO

MALDI-TOF

$m$-CPBA

$M \mathrm{n}$

mPEG

$M$ w

$M_{\mathrm{w}} / M_{\mathrm{n}}$

$\mathrm{m} / \mathrm{z}$

naverage

NBS

NMR

OLED

PEG

PF

PF8 arbitrary intensity

aspect ratio

atom transfer radical polymerization

correlation spectroscopy

conjugated polymer nanoparticles

chemical shift [ppm]

dichloromethane

double electron-electron resonance

dynamic light scattering

$\mathrm{N}, \mathrm{N}$-dimethylformamide

dimethyl sulfoxide

diffusion ordered spectroscopy

number-average degree of polymerization

electron paramagnetic resonance

equivalents

gel permeation chromatography

heteronuclear multiple bond correlation

highest occupied molecular orbital

coupling constant $[\mathrm{Hz}]$ of atoms $X$ and $Y$ over $n$ bonds

expectation value

excitation wavelength

contour length

persistence length

lowest unoccupied molecular orbital

matrix-assisted laser desorption/ionization time-of-flight

meta-chloroperoxybenzoic acid

number-averaged molecular weight

poly(ethylene glycol) monomethylether

weight-averaged molecular weight

molecular weight distribution

mass-to-charge ratio

average number of PEG repeat units

$\mathrm{N}$-bromosuccinimide

nuclear magnetic resonance

organic light emitting diode

poly(ethylene glycol)

poly(fluorene)

poly $\left(9,9^{\prime}\right.$-dioctylfluorene $)$ 


$\begin{array}{ll}\text { PL } & \text { photoluminescence } \\ \text { PODPF } & \text { poly(5-octyloxy-9,9'-diphenylfluorene) } \\ \text { ppm } & \text { parts per million } \\ \text { PS } & \text { poly(styrene) } \\ \text { PTFE } & \text { poly(tetrafluoroethylene) } \\ \text { rpm } & \text { revolutions per minute } \\ \text { rt } & \text { room temperature } \\ \text { SDS } & \text { sodium dodecyl sulfate } \\ \text { SEM } & \text { scanning electron microscopy } \\ \text { SMPC } & \text { Suzuki-Miyaura polycondensation } \\ \text { cSMCP } & \text { controlled Suzuki-Miyaura coupling polymerization } \\ \text { TBAB } & \text { tetra- } n \text {-butylammonium bromide } \\ \text { TEM } & \text { transmission electron microscopy } \\ \text { TEMPO } & (2,2,6,6 \text {-tetramethylpiperidin-1-yl)oxyl } \\ \text { THF } & \text { tetrahydrofuran } \\ \text { TLC } & \text { thin layer chromatography } \\ \text { Triton X } & p \text {-(1,1,3,3-tetramethylbutyl)phenoxy-poly(ethylene glycol) } \\ \text { Ts } & \text { tosyl } \\ \text { US } & \text { ultrasonication } \\ \text { UV/Vis } & \text { ultraviolet/visible } \\ \text { vs. } & \text { versus } \\ \text { w } & \text { fraction [\%] } \\ \text { WLC } & \text { worm-like chain } \\ \text { wt\% } & \text { percentage by weight (solids content) } \\ \end{array}$




\section{1 general introduction}

In order to meet the increasing demand for photovoltaics and light-emitting devices, conjugated polymers have been much studied and proven themselves valuable due to their outstanding photo- and electroluminescent features. ${ }^{1,2}$ For the sake of facilitated processability, the utilization of conjugated polymers as nanoparticle dispersions provide an interesting alternative to the mere bulk material. Moreover, colloidal systems of conjugated polymer nanoparticles (CPNs) combine the optoelectronic features of conjugated polymers with the collective behavior of particles in dispersion, bearing high potential for the formation of films or related processes. ${ }^{3,4}$ In contrast to the broad range of wellestablished procedures towards inorganic nanostructures in various sizes and shapes, polymer nanoparticles are mostly limited to spherical morphologies or post-polymerization shaping methods. ${ }^{5-7}$ Recently, a protocol for the formation of ellipsoidal poly(9,9'-dioctylfluorene) nanoparticles by heterophase Suzuki-Miyaura polycondensation was established by Ruiz Perez et al., enabling the direct synthesis of anisotropic CPNs. ${ }^{8}$ The intersegmental packing of polymer chains typically plays a crucial role in the mechanism of particle formation; however, the origin of anisotropy remained elusive to date. Investigations on the optical properties of poly(fluorene) materials, for example by fluorescence measurements, gave insights on length and conformation of the conjugated backbone.9,10

As a detailed understanding of the processes involved in polymer chain arrangement enables an improved control over particle morphologies and optoelectrical material properties, the investigation of nanoscale materials based on poly(fluorene) is of high interest. 


\subsection{POLYFLUORENE}

Poly(acetylene), perhaps the most prominent and prototypical conjugated polymer, initially received only little interest since it was obtained as air-sensitive, infusible and insoluble black powder by any method of preparation. ${ }^{11}$ But in 1977, Shirakawa, MacDiarmid and Heeger discovered a conductivity in doped poly (acetylene) on par with metals, which opened up a completely new class of polymers. ${ }^{12}$ Since then, a great variety of different polymers were synthesized, all comprising a conjugated system of atomic bonds, resulting in the delocalization of $\pi$-molecular orbital electrons and their motion along the polymer backbone (Figure 1.1). ${ }^{13}$<smiles>[R]c1cc(C(C)(C)C)sc1C(C)(C)C</smiles>

poly(thiophene)<smiles>[R]/C(C)=C/C(C)(C)C</smiles>

poly(acetylene)

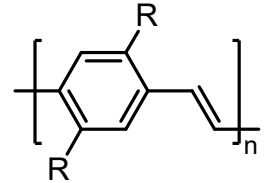

poly(phenylene vinylene)

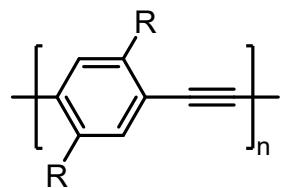

poly(phenylene ethynylene)

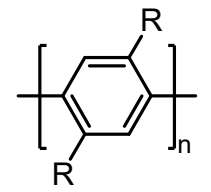

poly(phenylene)

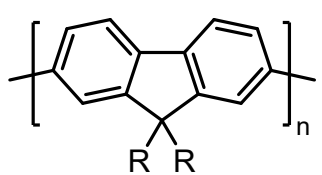

poly(fluorene)

Figure 1.1: Selected examples of conjugated homopolymers. $R$ represents proton, alkyl group or alkoxy group exemplarily located at the most common attachment points for functionalization. ${ }^{14,15}$

Since non-functionalized poly(acetylene) constitutes a rather poor luminophore, the interest in this polymer class was at first primarily based on the semiconducting properties, while the light-emitting qualities were largely neglected..$^{15}$ Nevertheless, high quantum yields for luminescence were revealed for other conjugated polymers such as poly( $p$-phenylenevinylene), which exhibits a larger band gap $\left(E_{\text {gap }} \approx 2.5 \mathrm{eV}\right.$, within the range of visible light) ${ }^{2}$ in contrast to poly(acetylene) $\left(E_{\text {gap }} \approx 1.4 \mathrm{eV}\right){ }^{16}$. This emission is induced by the radiative recombination of a singlet polaron exciton, more figuratively spoken, of an electron-hole pair formed by excitation..$^{1,2}$ Depending on the type of excitation, a distinction is drawn between photoluminescence and electroluminescence. In case of the former, an electron is excited from HOMO to LUMO state upon irradiation and subsequent relaxation to the energetic ground state leads to the emittance of light at a slightly longer wavelength compared to the absorbed light. In case of electroluminescence, electrons and holes are injected into the LUMO and the HOMO by means of connection of the material to an electric circuit. The emerging charges migrate within the conductive material and recombine under light emission (Scheme 1.1). ${ }^{14}$ 
a)

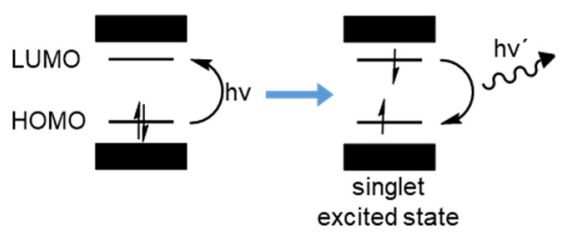

b)

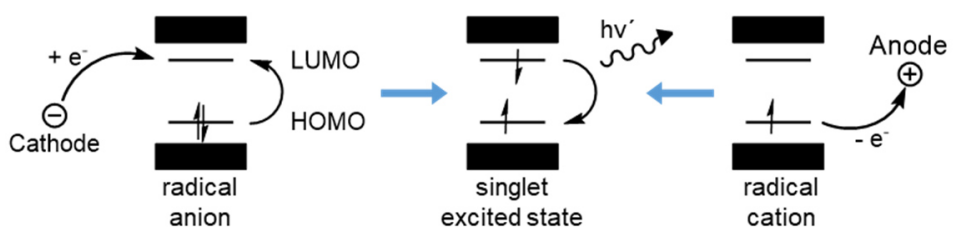

Scheme 1.1: Schematic illustration of (a) photoluminescence and (b) electroluminescence. ${ }^{14}$ Adapted with permission from Wiley-VCH Verlag GmbH \& Co. KGaA (Angew. Chem. Int. Ed. 1998, 37, 402-428; Copyright 2021).

A major benefit of conjugated polymers is the possibility to alter their chemical structure, for example by variation of the aromatic ring, the substituents or the regioregularity, which allows for precise tuning of the emission color. ${ }^{14,15}$ Beyond that, the functionalization of the polymer backbone is especially of interest as the majority of conjugated polymers feature a rigid molecular structure, resulting in poor solubility and challenging processing of materials. For instance, the attachment of alkyl or alkoxy groups in many cases has advantageous effects on both the processability and stability of the synthesized polymer. ${ }^{15}$

Due to the unusual range of ordered and disordered regions in conjugated polymers, the understanding of the interplay between microstructure and electrical properties is complex. Conjugated polymer chains are considered semi-flexible, with a rigid character at short length scales and flexibility at larger dimensions. ${ }^{17,18}$ The main proportion of electronic coupling within conjugated polymers was found to occur along the polymer backbone. But, in order to allow for macroscopic charge transport as necessary in applications, intermolecular aggregates, for example formed by m-stacking, are required. Additionally, the bridging of adjacent crystallite domains in the material by connecting chains provide for efficient charge transport between ordered regions. ${ }^{17}$ Upon certain conditions such as thermal treatment, the reorganization between various morphological states, driven by both intra- and interchain ordering, was observed. ${ }^{19}$ These findings indicated a complex interplay between the molecular design and the microstructure of semiconducting polymers affecting the overall electrical properties. ${ }^{17}$ Their ability to emit light made conjugated polymers attractive materials for low-cost, yet high-performing materials, as they exhibit both, the conducting and optical features of inorganic semiconductors and the properties of organic polymers. Hence, they found diverse applications in photovoltaics, chemical sensoring and especially in light emitting devices such as organic light-emitting diodes (OLEDs).,20 Due to the relevance of these technologies, the elaboration of new synthetic routes to conjugated polymers as well as the investigation of their photo- and electroluminescent properties are subject to ongoing studies. ${ }^{11,15}$

With the great variety of available conjugated polymers and their different wavelengths of emission, almost the entire spectrum of visible light is covered. For the emission of high-energetic blue light, poly(fluorene) (PF) homo- and copolymers emerged as popular materials. They combine excellent efficiencies in charge-transport and luminescence with a remarkable chemical and thermal 
stability. ${ }^{15,21,22}$ The fluorene monomer consists of a rigid biphenyl unit bridged by a carbon atom at the C-9 position. This forces the two aromatic rings in one plane, which increases the orbital overlap and thereby the degree of conjugation, resulting in the efficient emission of light in the blue wavelength range (Figure 1.2). ${ }^{15,23,24}$ The protons of the methylene bridge are rather acidic $\left(\mathrm{p} K_{A}=22.9\right)$ due to the effective resonance stabilization of the generated fluorenyl anion. ${ }^{24}$ This makes the C-9 position readily accessible for chemical modification, for instance by attachment of alkyl or aryl groups, which can improve the solubility in organic solvents and influence the PF backbone conformation by steric interactions. ${ }^{15,23}$

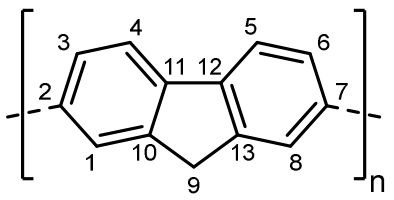

Figure 1.2: Chemical structure of poly(fluorene) with numbering of the carbon atoms. ${ }^{15}$

PF derivatives were originally prepared by metal catalyzed oxidative coupling and featured high degrees of branching or other defect sites, owed to the low specificity of the oxidation reaction. ${ }^{23,25}$ Nowadays, transition metal catalyzed cross-coupling reactions, for example the Suzuki-Miyaura polycondensation, are chosen for the synthesis of most PF homopolymers and copolymers, as described in more detail in the following (cf. Chapter 1.2). ${ }^{15,26-31}$ Due to the possible access to structural variety, high stability and simultaneously efficient luminescence, poly(fluorenes) became valuable materials for light emitting diodes or devices with polarized electroluminescence. ${ }^{15,21}$

\subsubsection{POLY(9,9'-DIOCTYLFLUORENE)}

By far the most investigated poly(fluorenes) are derivatives with alkyl substituents attached at the C-9 position. Among this polymer subclass, particularly attractive physical properties were found for poly $\left(9,9^{\prime}\right.$-dioctylfluorene) (PF8), which exhibited a high degree of intramolecular orientation and the ability to crystallize, with melting points at approximately $170{ }^{\circ} \mathrm{C} .{ }^{32}$ It showed an exceptional conformation-induced polymorphic behavior, involving crystalline structures as well as completely amorphous, nematic, isotropic or semiordered phases. ${ }^{22,32}$ Due to the outstanding conformationdependent photophysical properties, PF8 has emerged as a prototype for PFs.

Generally, PF8 adopts a planar zigzag-type conformation (Figure 1.3), with alkyl chains oriented perpendicular to the aromatic backbone, allowing a close packing of the polymer chains ${ }^{33-35}$ Due to the bridging C-9 carbon atom, the dioctylfluorene repeat units in PF8 are not oriented in a straight line. Instead, the connecting C-C bonds between adjacent monomers (C-2/C-7) form an angle of approximately $160^{\circ} .{ }^{34}$ Additionally, coplanarity of adjacent fluorene units is hindered by the steric repulsion induced by hydrogen atoms in ortho positions to C-2/C-7.34 Based on the resulting torsion angles, three conformational isomers can be distinguished: an $\alpha$-phase $\left(\theta=135^{\circ}\right)$, a more flattened $\beta$-phase conformation $\left(\theta=165^{\circ}\right)$ and a $\gamma$-phase $\left(\theta=155^{\circ}\right)$ with a backbone conformation intermittent 
to the $\alpha$ - and $\beta$-phase. ${ }^{15,36}$ As the $\beta$-phase comprises the most planarized backbone of all phases, this conformation exhibits the largest conjugation length, with tremendous impact on the optical properties of the polymer. ${ }^{37-39}$ The emergence of a $\beta$-phase was found to be inducible by thermal processing of PF8, ${ }^{32,33,40}$ treatment with solvent vapor ${ }^{33,41}$ or by changes in the surrounding environment such as the presence of poor solvents. ${ }^{15,42-44}$

a)

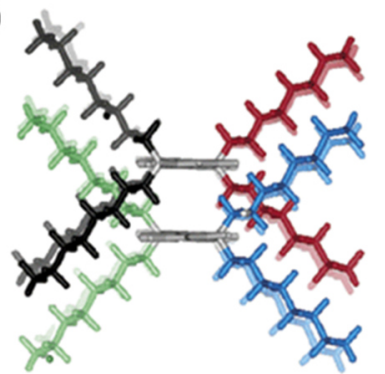

b)

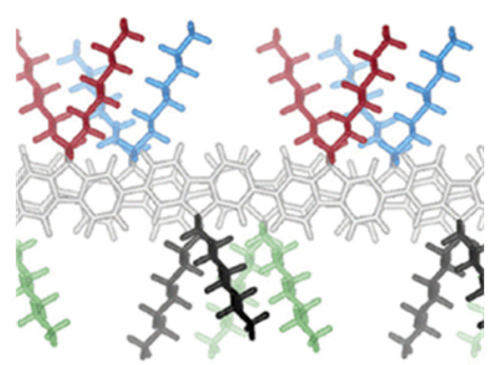

Figure 1.3: Molecular modeling of most stable packing of two poly $\left(9,9^{\prime}\right.$-dioctylfluorene) chains, adopting the 'planar zigzag' conformation, with polymer chains (a) perpendicular and (b) parallel to the view. ${ }^{35}$ Reprinted with permission from the American Chemical Society (Chem. Mater. 2004, 16, 994-1001; Copyright 2021).

PF8 of both ordered and amorphous morphology exhibit a broad $\pi-\pi^{*}$ transition absorption band around $380 \mathrm{~nm}$. Additionally, the extended conjugation length of $\beta$-phase regions causes a red-shift of the absorption wavelength as opposed to the amorphous phase, revealed by a sharp absorption peak at around $440 \mathrm{~nm}$ (Figure 1.4a). ${ }^{10,45}$ These optical properties are also visible in the fluorescence emission. Amorphous PF8 regions show a vibronically well-resolved pattern with peaks at $420 \mathrm{~nm}, 448 \mathrm{~nm}$ and $472 \mathrm{~nm}$, assignable to the 0-0, 0-1 and 0-2 intrachain singlet transitions, respectively. With increasing $\beta$-phase content, fluorescence signals at $441 \mathrm{~nm}, 468 \mathrm{~nm}$ and $498 \mathrm{~nm}$ emerge and the signals of lowordered PF phases decrease (Figure 1.4b). ${ }^{10,21,44}$ In addition to the described red-shift in absorption and emission, efficient energy transfer from $\alpha$ - to $\beta$-phase domains in PF8 is enabled, observable by the almost complete quenching of the emission at $420 \mathrm{~nm} .{ }^{44}$ The $\beta$-phase regions act as efficient `energy trap' inclusions in the less-ordered 'host' matrix, forming a self-dopant system. ${ }^{39}$ This leads to a strong domination of the material's optical properties by the presence of $\beta$-phase, even if its percentage is small. Considering photochemical stability and charge transport, the $\beta$-phase conformation can be regarded as superior to other morphologies in PF8. ${ }^{15,46}$
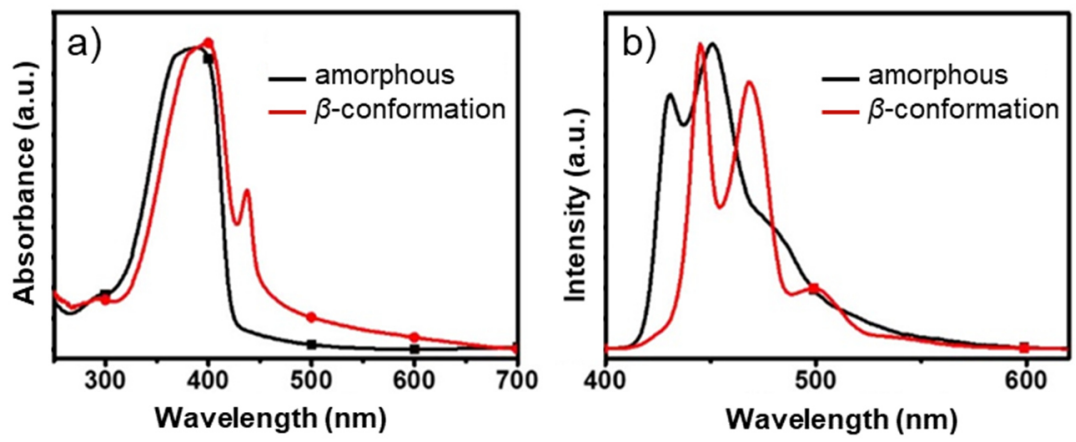

Figure 1.4: (a) UV/Vis absorption and (b) fluorescence emission spectra of poly (9,9'-dioctylfluorene) in amorphous state (black line) and in $\beta$-phase conformation (red line). ${ }^{39}$ Adapted with permission from the American Chemical Society (J. Phys. Chem. Lett. 2018, 9, 364-372, Copyright 2021). 
These unique optoelectronic features led to intensive studies on the formation of $\beta$-phase in PF8, including various scattering techniques, ${ }^{37,42}$ X-ray diffraction, ${ }^{33,40,41}$ nuclear magnetic resonance (NMR), ${ }^{47}$ photoluminescence and absorption measurements ${ }^{10,36,38}$ as well as transmission electron microscopic (TEM) studies. ${ }^{34}$ These investigations have been source of different interpretations and hypotheses regarding the structural chain packing in PF8, which remain subject of debate. The occurrence of $\beta$-phase was found to be dependent on many factors such as solvent nature and quality $43,48,49$ or temperature. ${ }^{45,47}$ Upon cooling dilute PF8 solutions or gels, the amount of $\beta$-phase conformation increases, as was observed from emerging absorption and emission signals. ${ }^{44,50,51}$ Additionally, the nature and concentration of surfactant in the synthesis of PF8 particles evidently influence the polymer chain separation from the solution and their condensation on the initially formed particle nuclei, thus effecting the resulting particle morphology. ${ }^{52}$ Apart from external factors, the amount of $\beta$-phase regions was proven to be strongly dependent on the nature of the C-9 substituents. It was shown that especially the attachment of $n$-octyl groups favors the formation of $\beta$-phase as opposed to other $n$-alkyl chains. ${ }^{42,45}$ This finding was attributed to the induction of van-der-Waals interactions which are strong enough to overcome the steric repulsion of the ortho-positioned protons to C-2/C-7 (vide supra). This leads to a planarization of the conjugated backbone, which can only be ensured by sufficient alkyl chain length. But, with an increasing size of longer or branched alkyl substituents, the steric demand outweighs the attractive forces and thereby hinders the formation of ordered morphologies. ${ }^{15,45}$ Regarding the influence of the molecular weight and the corresponding chain length, a minimum of 9 to 12 fluorene repeat units was revealed to be required for the appearance of the $\beta$-phase. .,10,50 $^{1}$ The correlation of intra- and interchain interactions upon $\beta$-phase formation are still discussed controversially. Based on the observation of thermodynamically stable, lamellar $\beta$-phase crystals with resolved lattice parameters, intermolecular interactions are considered crucial for $\beta$-phase formation in PF8 aggregates. ${ }^{40,42}$ This contradicts the theory of $\beta$-phase formation originating exclusively from backbone planarization. However, $\beta$-phase formation has been demonstrated in dilute polymer solutions, which suggests that the decisive interchain interactions result rather from the collapse of the individual polymer chains than from aggregation of multiple chains. ${ }^{33,47,50}$ An energy transfer from $\alpha$ - to $\beta$-phase regions was observed in dilute solutions, indicating the proximity of the phases or their location in the same polymer chain. ${ }^{44}$

From these different findings and interpretations a process of $\beta$-phase formation can be concluded, which originates, at least at early stages of formation, from intramolecular interactions and is then followed by the intermolecular aggregation of $\beta$-phase domains. ${ }^{44,50}$ The first, intramolecular step of conformational reorganization is thought to be the reversible planarization of the polymer chain, followed by aggregation to form sheet-like structures. The intermolecular aggregation step is considered irreversible. ${ }^{44}$ 


\subsubsection{POLY $\left(9,9^{\prime}-D I A R Y L F L U O R E N E\right)$}

The conformation-induced formation of highly ordered phases leaded to unique optical properties in poly(fluorenes) with alkyl chain substituents. However, a major challenge towards the establishment of poly(fluorene) based emitters was their limited stability to high operating voltages required for blue light emission, making these materials especially sensitive towards the formation of defects. ${ }^{24}$ PF8 and related polymers are prone to oxidation processes at the C-9 position at elevated temperatures in the presence of air, forming the corresponding fluorenone derivative. ${ }^{22,53}$ These on-chain keto defects are observable as red-shifted, low-energy emission components and deteriorate the luminescence color purity. 22,53 Hence, alternative poly(fluorenes) were searched for, capable of $\beta$-phase formation and with charge transport properties on par with poly(dialkylfluorenes). Poly(diarylfluorenes) as a materials class were found to meet these demands (Figure 1.5). ${ }^{39,54}$ The bulky aryl substituents at the C-9 position had a favorable impact on the chemical and thermal stability of the material, as compared to poly(dialkylfluorenes). ${ }^{35,39,55}$ The sensitivity towards oxidation was reduced and the blue-light emission showed improved stability. ${ }^{15,22}$

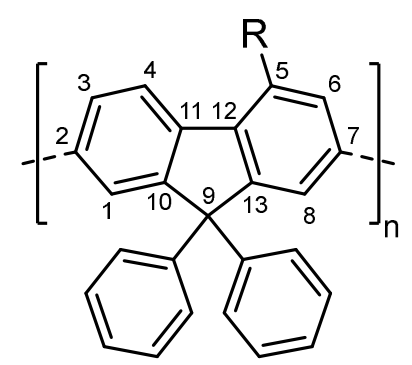

Figure 1.5: Chemical structure of poly(diarylfluorenes) with a possible functionalization site at C-5 position (denoted as $R$ ). The unsubstituted homopolymer is defined as $R=H^{22}$

The unsubstituted homopoly $\left(9,9^{\prime}\right.$-diphenylfluorene $)(R=H)$ was insoluble in common organic solvents and required alkyl or alkoxy moieties at the aryl substituents or at the aromatic backbone in order to provide processablity. ${ }^{22,56}$ The functionalization of the original polymer with alkoxy groups in C-5 position led to the induction of $\beta$-phase conformation, as observed by various analytical methods. ${ }^{54,55,57,58}$ The molecular arrangement was explained by the subtle interplay of steric repulsion of the bulky aryl groups and attractive van-der-Waals forces of the alkoxy substituents. ${ }^{55}$ This interplay enabled the planarization of the polymer backbone, comparable to the conformation found in PF8. ${ }^{57,58}$ The formation of $\beta$-phase in poly(alkoxy-9,9'-diarylfluorenes) was evident from additional shoulders or peaks in absorption and emission spectra upon preparation of solutions or gels or by film formation via spin-coating. ${ }^{54,55}$ Thermal post-processing of spin-coated films on glass substrates exhibited a significant increase in $\beta$-phase ratio (up to $45 \%$ at $240{ }^{\circ} \mathrm{C}$ ) upon annealing at elevated temperatures. ${ }^{57}$ Based on their molecular attractorrepulsor design, poly(alkoxy-9,9'-diarylfluorenes) constitute conjugated polymers of wide bandgap with high potential for extensive applications in optoelectronics. ${ }^{39,57}$ 


\subsection{SUZUKI-MIYAURA POLYCONDENSATION}

The synthetic access to conjugated polymers has been challenging for a long time, as the C-C bond formation between aromatic subunits is required. ${ }^{59}$ Furthermore, the material must be accessed in a sufficient pure form in order to control non-radiative decay of excited states based on defect sites. ${ }^{1}$ As the insolubility of many conjugated polymers limits their processability already during the synthesis, many preparative routes relied on solution-processable precursor polymers which were converted to the desired polymer in a second step, for instance by thermal treatment. ${ }^{1,14}$ However, only relatively short conjugated segments were accessible by this route and materials are often prone to structural defects such as cross-links. ${ }^{11,59}$ Alternative synthetic routes included electrochemical oxidation, cycloaddition reactions or organometallic coupling polymerizations. Especially with the latter a broad range of conjugated polymers were accessible. ${ }^{11,60,61}$ This became relevant for the first time when Yamamoto et al. successfully applied Ni-catalyzed cross-coupling reaction of aromatic Grignard reagents to the synthesis of poly(phenylenes) in $1978 .{ }^{62}$ With the discovery of the Suzuki-Miyaura cross-coupling reaction one year later, the connection of two $\mathrm{sp}^{2}$-hydridizised carbon atoms became possible under mild conditions, compatible with a broad range of functional groups. ${ }^{63,64}$ These findings laid the ground for the development of the nowadays well-established Suzuki-Miyaura polycondensation (SMPC). ${ }^{59}$ Depending on the type of monomer employed in the SMPC, two different mechanistic pathways are possible (Scheme 1.2). The AA/BB-type route requires two aryl monomers, each carrying either two boronic acid (ester) functionalities or two leaving groups such as halides or triflates. The resulting polymer backbone has an alternating structure with high structural versatility by variation of the aromatic repeat unit. ${ }^{15}$

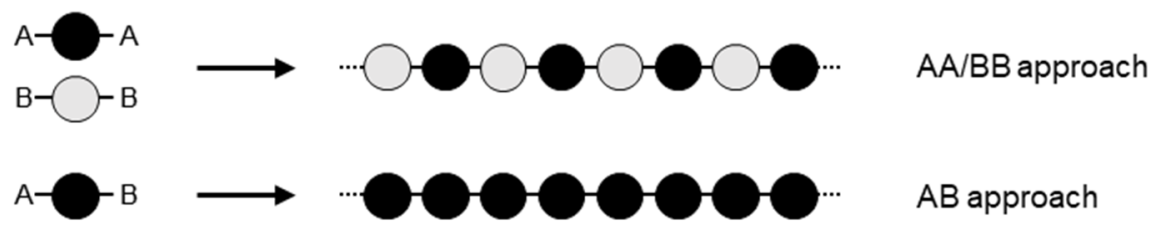

Scheme 1.2: Schematic illustration of the $A B$ - and $A A / B B$-type polycondensation. ${ }^{15}$

In contrast, the AB-approach implies only one aromatic species which is coupled in a head/tail fashion during the polymerization, providing access to polymers with regioregularity. However, the synthesis of the bifunctional monomer is more challenging compared to the symmetrically functionalized species. The major benefit of the $A B$-approach is the inherent stoichiometric balance between both functional groups involved in the cross-coupling, whereas the AA/BB-approach requires an equimolar ratio of the different monomers in order to achieve high molecular weights. Nevertheless, the majority of the performed SMPC reactions follow the AA/BB-principle, since the advantages outweigh the stoichiometry issue in most cases. . $^{15,28,65}$ The AA/BB-type SMPC proceeds in a step-growth mechanism, as concluded from the presence of short-chain oligomers at early stages, which are growing with ongoing reaction time..$^{15}$ The polymerization follows the general cross-coupling mechanism of two aromatic moieties, including oxidative addition, transmetalation and reductive elimination (Scheme 1.3). ${ }^{66}$ 


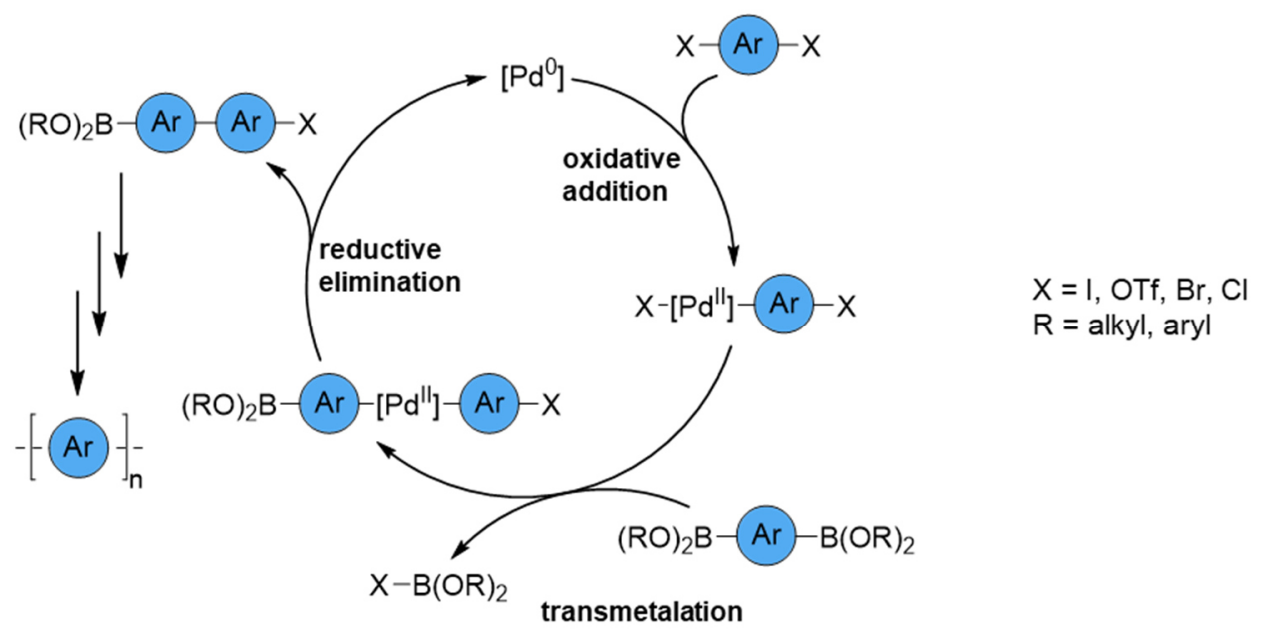

Scheme 1.3: Schematic representation of the Suzuki-Miyaura polycondensation of AA/BB-type monomers. ${ }^{66}$

The oxidative addition of the aryl halide to a $\left[\mathrm{Pd}^{0}\right]$ species yields a stable [Pd"] complex, followed by a transmetalation step with a second aromatic species bearing a boronic acid or ester functionality. Reductive elimination recovers the $\left[\mathrm{Pd}^{0}\right]$ complex and yields the dimeric coupling product; which undergoes further cross-coupling reactions towards oligo- and subsequently poly(arylene) segments. ${ }^{66}$ However, the step-growth character of the polymerization limits the control over molecular weight, polydispersity and end group identity. ${ }^{65}$ This can be overcome by a catalyst transfer polycondensation of AB-type monomers, initially reported by Yokozawa et al. and in the following referred to as controlled Suzuki-Miyaura coupling polymerization (CSMCP) (Scheme 1.4). ${ }^{30}$

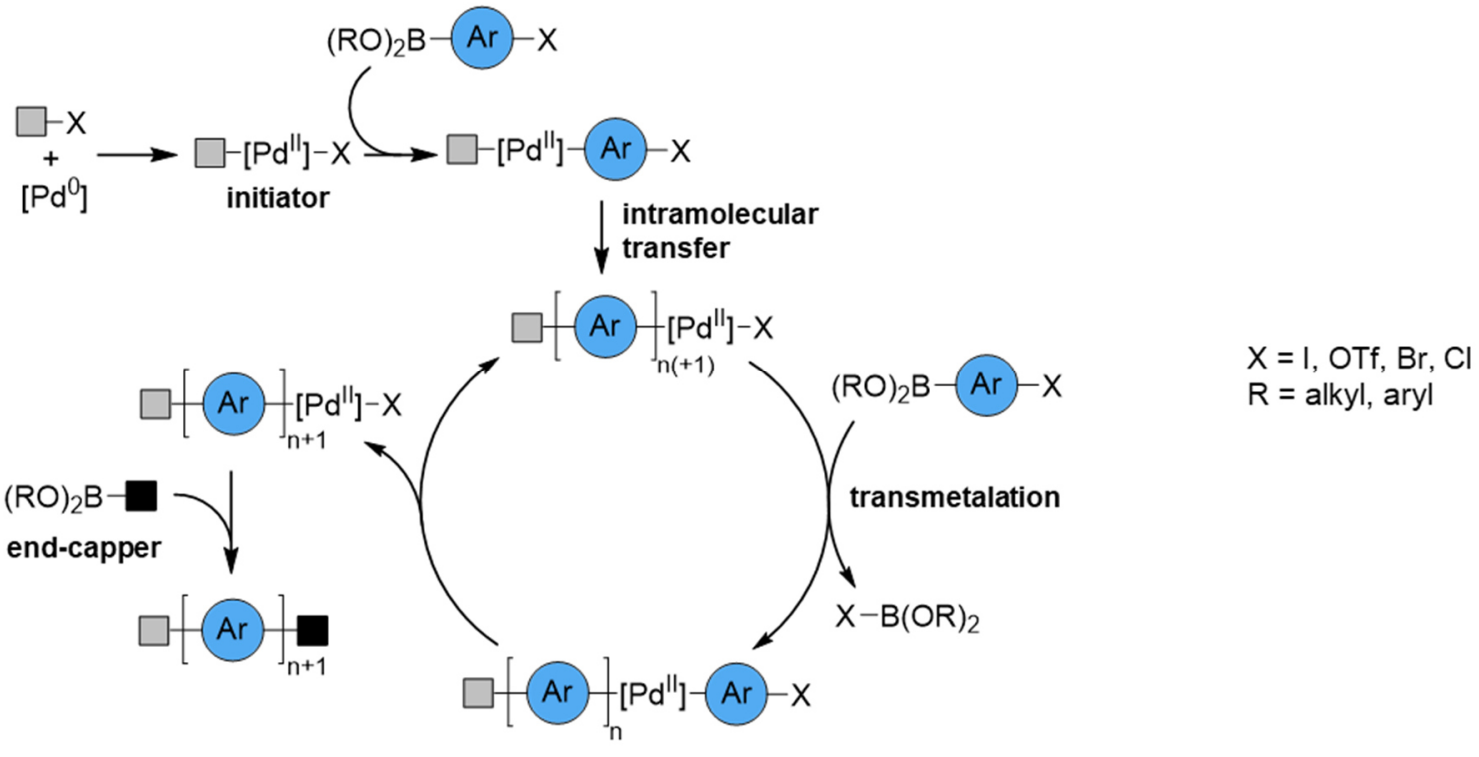

Scheme 1.4: Schematic representation of the controlled Suzuki-Miyaura coupling polymerization (CSMCP) of ABtype monomers. ${ }^{67}$

This approach requires the formation of an initiator by oxidative addition reaction of an aryl halide, chosen as initial unit of the polymer chain, to a palladium source. Subsequently, the polymerization is induced by a transmetalation reaction of the initiator species and the bifunctional AB-monomer. From this complex, chain propagation occurs by subsequent reductive elimination, intramolecular migration 
of the Pd-species to the terminal $\mathrm{C}-\mathrm{X}$ bond, followed by oxidative addition. Since the intramolecular oxidation is favored over intermolecular catalyst migration, the AB-type SMPC follows a chain-growth mechanism..$^{30,61,67}$ This type of polymerization enables control over molecular weight and polydispersity of conjugated polymers and has the additional benefit of a tunable initiator species, making targeted chain end functionalization feasible. ${ }^{30,68}$ Besides the introduction of an end group by the initiator complex, a second end group can be added by quenching the chain propagation with a boronic ester. This gives access to heterobis-functionalized conjugated polymers by an efficient, facile and controlled polymerization. ${ }^{69,70} \mathrm{~A}$ number of different palladium initiators, usually ligated by one or more phosphine species, have been synthesized and successfully used in chain growth type Suzuki-Miyaura polycondensation. ${ }^{71}$ The active $\mathrm{Pd}$-complex can also be formed in situ by conversion of a precatalyst in the presence of aryl halides, thereby omitting the additional step of catalyst isolation. ${ }^{71}$ Among various in situ systems, especially phosphine-coordinated 2-phenylaniline-based palladacycle complexes yielded excellent results in terms of catalyst activity. This class of catalysts was originally developed by Buchwald and co-workers for the Suzuki-Miyaura coupling reactions of sensitive boronic acid aryls and has since been established as a robust, yet reactive catalyst system for the CSMCP. ${ }^{72-74}$ Such initiators avoid the formation of less reactive 14-electron $\left[\mathrm{Pd}(0)\left(\mathrm{P}^{t} \mathrm{Bu}_{3}\right)_{2}\right]$ complexes during the initiator formation step, in contrast to other widely applied initiator systems with excessive use of phosphine ligands. Instead, 12-electron $\left[\mathrm{Pd}(0)\left(\mathrm{P}^{t} \mathrm{Bu}_{3}\right)\right]$ species are formed under basic conditions, which are prone to the oxidative addition of a broad spectrum of aryl bromides with different functional groups. ${ }^{72}$ Both polymerization mechanisms provide access to several classes of well-defined conjugated homo- and copolymers, including poly(phenylenes), poly(thiophenes) or poly(fluorenes), and are subject to ongoing research with continuing development and optimization of reaction components. ${ }^{65,67,75-78}$

\subsection{CONJUGATED POLYMER NANOPARTICLES}

Despite the numerous studies on conjugated polymers fueled by their desirable properties in terms of luminescence and variability by chemical modification, applications of this material class are limited by the low solubility and the resulting, challenging processing. This problem can be overcome by the use of conjugated polymers in the form of nanoparticles dispersed in aqueous media. Even at high polymer solids content dispersions of conjugated polymer nanoparticles (CPNs) retain low viscosity. ${ }^{3,79}$ They combine desirable processability features with the unique optoelectronic properties of conjugated polymers ${ }^{79}$ and the possibility of emission wavelength tuning by covalent incorporation of dyes or by employment of comonomers. ${ }^{8,80-83}$ Furthermore, colloidal dispersions of CPNs provide attractive building blocks for the formation of films by deposition onto solid substrates. ${ }^{84}$ Application fields for such uniform coverage over large areas are for instance photovoltaic devices ${ }^{85}$ or OLEDs. ${ }^{86} \mathrm{~A}$ further benefit of CPNs is the versatility of their surface chemistry by functionalization, which is especially relevant for biological applications. This advantage, in combination with their low cytotoxicity, make CPNs attractive materials for cell-labeling or bioimaging. ${ }^{81,82,87-90}$ 
The optoelectrical properties of CPNs show severe differences compared to the respective polymer in solution or bulk material, since the interactions between polymer chain segments are significantly altered within the particles. As a consequence, an overall red- or blue-shift of UV/Vis absorption and fluorescence emission can be observed: fluorescence spectra of CPNs display a general red-shift, as opposed to the polymers in solution. ${ }^{3,91}$ This was explained by enhanced intra- and interchain interactions between polymer chain segments such as aggregate formation by $\pi$-stacking, promoting radiationless decay and thereby favoring energy transfer from the excited state to low-energy-emitting sites. ${ }^{80,90,92}$ In contrast, a blue-shift in absorption is originating from the reduced conjugation length by bending and folding of the rigid polymer backbone, since the polymer chains are confined in the small, spherical particle volume.43,90,91 This aggregation-induced disorder occurs especially in case of nanoparticles generated rapidly, hence representing kinetically arrested structures. ${ }^{3,93,94}$ Particles prepared over extended time periods show the tendency to form more ordered structures of stretched, aligned polymer chains and consequently exhibit a red-shift in absorption. ${ }^{3}$ This was also observed for annealing or swelling of particles, indicating that distorted polymer chains can rearrange into a more ordered state. ${ }^{3,43}$

Two major techniques are distinguished to obtain dispersions of CPNs, as outlined more detailed in the following chapters. They can either by generated by emulsification or precipitation of the pre-synthesized polymer in a continuous, generally aqueous phase or by direct polymerization in a heterophase system, utilizing emulsion polymerization methods. . $^{3,7,83}$

\subsubsection{POST-POLYMERIZATION TECHNIQUES}

Post-polymerization techniques for particle formation, also referred to as secondary dispersion, are based on polymers dissolved in an organic solvent which are subsequently dispersed in a continuous medium. ${ }^{3}$ One possible procedure is given by the emulsion technique, meaning the generation of submicrometer scaled droplets of polymer solution upon the application of high shear, usually by ultrasonication. ${ }^{84,85,95}$ The resulting systems are generally miniemulsions, which have to be colloidally stable over a sufficient amount of time to allow for the solvent to evaporate. This can be ensured by adding surfactants like the commonly used sodium dodecyl sulfate (SDS), which are absorbed on the particle surface and prevent the droplets from coalescing. After complete solvent removal, stable aqueous dispersions of conjugated polymer particles are obtained. $3,84,85$ The size of the final particles, usually in the range of $30-500 \mathrm{~nm}$, can be tuned by adjusting the polymer or surfactant concentration. Hence, particles with smaller dimensions are obtained by decreasing the polymer concentration in the organic solvent or increasing the amount of added surfactant. ${ }^{83,84,95}$

In contrast, nanoparticle generation by nanoprecipitation involves the rapid addition of a dilute polymer solution, for example in tetrahydrofuran or acetonitrile, to an excessive amount of non-solvent, usually water, under vigorous mixing or ultrasonication. ${ }^{91,96}$ The sudden decrease of solvent quality upon addition induces precipitation, as the hydrophobic polymer chains tend to avoid contact with water and fold into spherical shapes to minimize exposure. ${ }^{83}$ The concentration of the initial polymer solution 
influences the dimensions of the obtained particles. ${ }^{88,90,94} \mathrm{~A}$ good miscibility of polymer solvent and the surrounding, continuous phase is a prerequisite for this technique. As for the abovementioned emulsion technique, the nascent particles are either stabilized by means of additional surfactants or intrinsically by incorporated steric or electrostatic moieties. ${ }^{43,88,91}$ Using nanoprecipitation, ultrasmall CPNs with diameters of a few nanometers can be generated by adjustment of polymer molecular weight and concentration. ${ }^{83,90,96}$ For very diluted polymer solutions, nanoparticles consisting of single conjugated polymer chains can be accessed. ${ }^{91}$

Both emulsion- and precipitation-based post-polymerization techniques are limited to some extent by the requirement of solution-processible polymers, which can be problematic due to the rigid polymer backbones and the resulting bad solubility of many non-substituted conjugated polymers. ${ }^{3}$

\subsubsection{HETEROPHASE POLYMERIZATION}

The restrictions of post-polymerization techniques associated with polymer solubility can be overcome by polymerizing in a heterophase system. With this approach, the polymer is directly generated in the form of nanoparticles from the respective monomers dispersed in a non-solvent regarding the polymer.3,97,98 The general concept of heterophase polymerization is based on the generation of small, homogeneous and stable droplets acting as independent nanoreactors. ${ }^{99,100}$ In addition to the low dispersion viscosity, the continuous phase facilitates the heat removal from the polymerization by providing an effective heat transfer medium, especially relevant in industrial free radical polymerizations. ${ }^{98}$ The heterophase polymerization approach allows the formation of any polymer particles insoluble in the surrounding medium, including conjugated polymers. ${ }^{99}$ The first reported heterophase synthesis of conjugated polymers was the preparation of submicrometer poly(acetylene) particles $(40-200 \mathrm{~nm})$ by polymerization of acetylene in tetrahydrofuran as continuous phase, containing the dissolved catalyst. The acetylene gas serving as monomer species was in this case bubbled through the organic solvent and the forming particles were stabilized by block-copolymers as surfactants. ${ }^{4}$

Within the class of heterophase polymerizations various types must be differentiated. Dispersion polymerizations start with a homogeneous reaction mixture of monomer, initiator and surfactant dissolved in the reaction medium. The solubility of the resulting polymer decreases with progressing polymerization and particle nucleation occurs upon a critical chain length. The particles are prevented from agglomeration by the presence of surfactants, either adsorbed or covalently attached to the particle surface. $3,7,52,101$ In contrast, the monomer used in emulsion polymerizations has only limited miscibility with the surrounding medium and forms a droplet phase. The droplets constantly coalesce and break by the exerted shear forces, for example by rigorous stirring. Chain growth is initiated by the catalyst or initiator dissolved in the continuous phase (typically water) and leads to the nucleation of hydrophobic particles at sufficient chain length. Further chain growth takes place within the forming particles, fed by the diffusion of monomers through the surrounding phase, which in turn requires a certain solubility of the monomer. ${ }^{3,93,99}$ By the addition of suitable surfactants providing either electrostatic or steric stabilization, the colloidal stability of the droplets is ensured. 
A variation of emulsion polymerization is the miniemulsion polymerization (Scheme 1.5). In miniemulsions, the small droplets narrowly distributed in a continuous phase can, once formed by high shear forces, remain stable over the time scale of the polymerization. ${ }^{98,102}$ Regarding thermodynamics, this represents a non-equilibrium state. However, the kinetics of the destabilization process are retarded by the presence of a hydrophobic agent in the dispersed phase, which is trapped inside the nanodroplets and prevents them from Ostwald ripening..$^{98,99}$ Unlike classical emulsion polymerization, in miniemulsion polymerization the (much smaller and more stable) droplets are the locus of particle nucleation, and ideally, one droplet results in the formation of one particle within a size range of $50-500 \mathrm{~nm} .{ }^{81,82,93} \mathrm{By}$ adjustment of the surfactant concentration, the particle size can be tuned. ${ }^{98,103}$
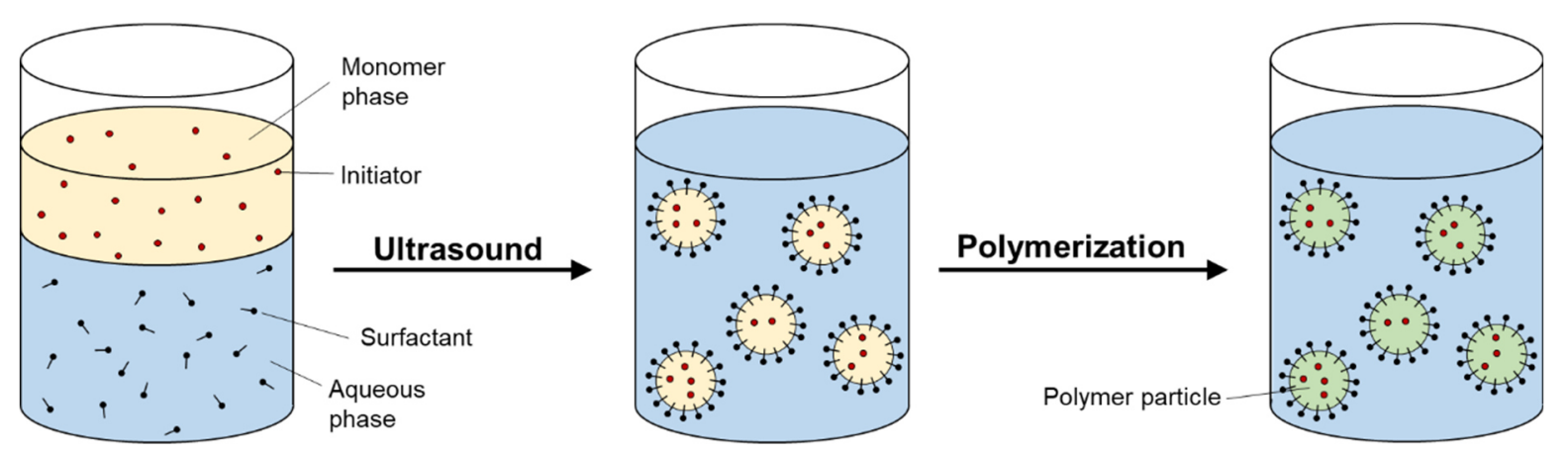

Scheme 1.5: Schematic illustration of the miniemulsion process, including ultrasonication of the two-phase system to yield stable nanodroplets dispersed in the continuous phase. The nanodroplets act as location for the polymerization, giving access to nanoparticle dispersions. ${ }^{99}$

In comparison, microemulsion polymerizations start with an emulsion of the same components (oil, water, surfactant and possibly a co-surfactant). The major difference to kinetically stable miniemulsions is that microemulsions are thermodynamically stable under certain conditions, depending on the nature, concentration and proportions of the reaction components. Usually, this requires large amounts of surfactants. ${ }^{3,104}$ Unlike miniemulsions, microemulsions are dynamic systems with the possibility of interdroplet material exchange. Although they can remain stable over an infinite time period, microemulsions are very sensitive towards environmental changes such as temperature or composition. ${ }^{105}$

The field of heterophase polymerization is traditionally known for free-radical-type polymerizations, with styrene or acrylates as typical monomers. ${ }^{93,99}$ Nevertheless, other types of polymerizations can be performed in emulsions, including anionic or cationic polymerizations, polycondensations and polyadditions, as well as metal-catalyzed polymerizations. ${ }^{99,102}$ For CPNs synthesized under emulsion condition, metal-catalyzed reactions such as Sonogashira-, 82 Heck-, 101 Glaser-80 or Suzuki-Miyauratype $52,81,106$ coupling polymerizations have emerged (cf. Chapter 1.2). Heterophase polymerizations are applicable to a great variety of conjugated homo- and copolymers and represent valuable routes towards high-performance materials. 


\subsection{ANISOTROPIC POLYMER NANOPARTICLES}

Nanoparticles of conjugated polymers predominantly render spherical shapes, regardless of the preparation method. ${ }^{79,81,84,90,101,103}$ Due to the high interfacial tension and the small particle radius, spheres have the lowest interfacial area for a given volume and are therefore favorable. ${ }^{104,107}$ Deviations from this energetic minimum and the development of anisotropic nanoscale shapes are still challenging for polymers and remain primarily a domain of inorganic materials. ${ }^{5,108-110}$ Nevertheless, anisotropy in particles as one or multiple asymmetries in their geometrical, chemical or physical nature fundamentally influence their properties independent of their chemical composition. ${ }^{109,111-113}$ The great potential and the growing variety of non-spherical nanoparticles and their optical, magnetic, electrical and biological properties are reflected by numerous studies on anisotropic particles and their synthesis. ${ }^{108-110,114}$ Anisotropic shapes and interactions are powerful tools for the construction of superstructures with unique, direction-dependent properties, for example optical birefringence, and a multitude of strategies to assemble anisotropic nanoparticles were conceived. ${ }^{111,112,115-117}$ In contrast to spherical particles generally assembling into close-packed symmetries, the mechanistic processes involved in the selfassembly of anisotropic building blocks are much more complex and enable a broad range of possible arrangements. ${ }^{6,109}$

For the organization of particles into ordered assemblies, two general principles are distinguished. Selfassembly of particles refers to the spontaneous self-organization of the building blocks, representing the minimum in the system's free energy, and is associated with a thermodynamic equilibrium state. ${ }^{118,119}$ This principle is relevant in the droplet evaporation, with attractive and repulsive forces controlling the assembling patterns. ${ }^{109,120}$ The second concept of particle organization is directed selfassembly, using external fields for the construction of nanomaterials with controlled alignment, location and spacing. This can be, for example, accomplished by the use of structure-guiding templates or by the application of electric or magnetic fields, flow fields or combination of these modes. ${ }^{115,118,121-124}$ In both cases, the particles' readiness to assemble depends on several parameters, above all on the potential interactions. ${ }^{117,120,125}$ The interplay of repulsive forces and attractive van-der-Waals forces between particles is quantified by the DLVO pair potential interaction ${ }^{126,127}$ and constitutes a major factor in the processes of structural organization. In addition, the selective modification of particle surfaces, for instance by ligating the particles or via surfactant variation, can also impact their assembling behavior. ${ }^{117,128-130}$

The versatile properties of anisotropic nanomaterials make them valuable for many applications including sensing methods, photovoltaics, photonics, for piezoelectric energy harvesting or biomedical applications. ${ }^{6,124}$ Despite the great potential of ordering the anisotropic particles into novel complex superstructures and materials, the full prediction and control of assembly remains challenging. ${ }^{116}$ This may originate from the requirement of narrow size and shape distributions of the colloidal particles. Aiming at the self-organization of building blocks to complex structures, strategies towards high-quality nanomaterials with anisotropic shapes are of great scientific interest. ${ }^{117}$ Although preparative approaches towards shapes beyond the thermodynamically favored spheres remain rather unexplored 
for organic polymers in general, especially anisotropic CPNs provide attractive building blocks for materials requiring directional properties, as they combine direction-dependent interactions with the unique optoelectronic features of $\pi$-conjugated polymers. For example, anisotropic CPNs could give access to crystals with geometry-controlled photonic band gaps. ${ }^{7,131}$

So far, strategies towards anisotropic polymer nanoparticles mostly rely on post-polymerization processing. ${ }^{132}$ Proven approaches are for example seeded polymerization methods, where the polymerization occurrs within monomer-swollen pre-synthesized particles, ${ }^{133,134}$ lithographic molding techniques ${ }^{135}$ or the deformation of spherical particles by electrospinning ${ }^{136}$ or stretching. ${ }^{7,137-139}$ Especially stretching methods, implying the embedding of the desired particles in a deformable matrix which is subsequently stretched under suitable temperatures, opened access to CPNs in various, anisotropic shapes.7,139 However, all abovementioned methods imply the deformation or shaping of polymer by external forces and are limited to micrometer-sized structures. In contrast, only few intrinsically driven processes towards anisotropic shapes are known. One possible approach was given by the synthesis of amphiphilic di- or triblock-copolymers with hydrophilic and hydrophobic segments. By crystallization-driven self-assembly in organic solvents, these copolymers readily formed anisotropic nanorods or platelets with a crystalline, conjugated polymer core, solubilized by a flexible, coronaforming segment. ${ }^{94,140-142}$ The assembly mechanism was assumed to be governed by the interplay of interchain interactions between the crystallizing core segments and the inherent tendency towards microphase separation (cf. Chapter 1.5). ${ }^{143-145}$ In addition, Yang et al. observed the formation of ellipsoidal CPNs with aspect ratios (ratio of an objects' width to its length) of up to 2.0 by emulsification of dissolved polymers. ${ }^{95}$ Even under annealing conditions exceeding $T_{\mathrm{g}}$ of PF8 $\left(78^{\circ} \mathrm{C}\right)$, the particles retained their ellipsoidal shape instead of recovering a spherical shape due to increased chain movement. These findings showed that anisotropic shapes are in a state of thermal equilibrium and result from the underlying liquid crystalline order in the polymer chain. ${ }^{95}$

In contrast to these post-polymerization techniques, the direct synthesis of polymers with anisotropic shapes gives access to monodisperse, nanometer-scaled particles in a scalable, single step method. The few direct approaches towards intrinsic non-spherical shapes on the nanoscale generally rely on internal molecular arrangement. ${ }^{146-149}$ This was formerly shown for poly(ethylene) nanocrystals, arising from growing polymer chains which are immediately deposited on the crystal growth front, yielding ideal lozenge-shaped nanocrystals of uniform size and shape distribution. ${ }^{148-150}$ Starting from the occasional observation of non-spherical CPNs in different protocols of heterophase polymerization, ${ }^{80,151}$ a protocol for the synthesis of anisotropic poly $\left(9,9^{\prime}\right.$-dioctylfluorene) nanoparticles via heterophase Suzuki-Miyaura polycondensation was evolved by Ruiz Perez et al. (Figure 1.6). ${ }^{8}$ The reaction parameters were examined and optimized for the Pd-catalyzed polymerization of AA/BB-type monomers to develop suitable conditions yielding well-defined ellipsoidal particles (length $\approx 140 \mathrm{~nm}$ ) with high aspect ratio (ar = 3.6) and narrow size and shape distribution. In order to red-shift the blue-colored fluorescence of the particles, the covalent incorporation of fluorescent dyes during polymerization was successfully demonstrated. 


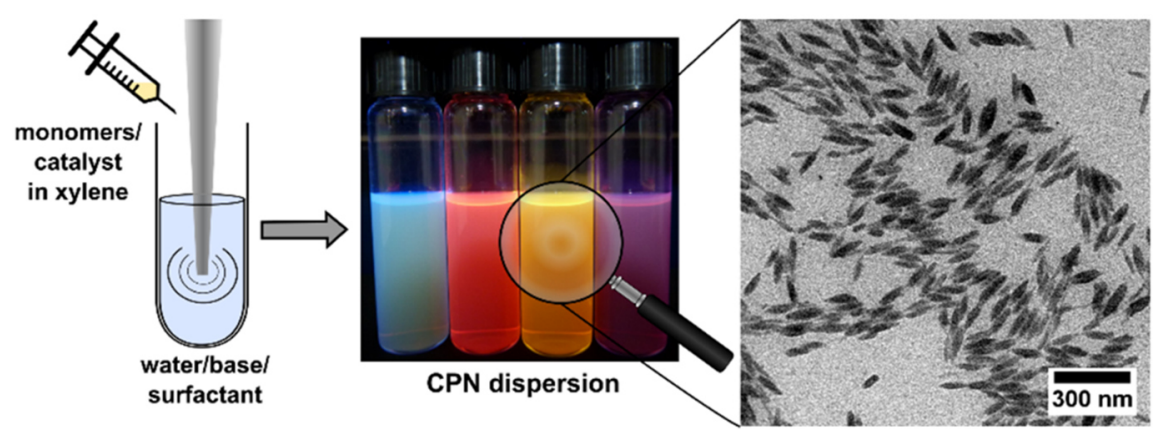

Figure 1.6: Schematic illustration of the preparation of ellipsoidal CPNs by heterophase polymerization (left), resulting in dispersions with varying emission wavelengths (center), investigated by TEM measurements (right). ${ }^{8}$ Reprinted with permission from Wiley-VCH Verlag GmbH \& Co. KGaA (Angew. Chem. Int. Ed. 2017, 56, 61476151; Copyright 2021).

By spectroscopic monitoring, a preliminary mechanism of particle formation was elaborated, showing an important contribution of chain conformation and intermolecular polymer packing. ${ }^{8}$ Several individual steps (namely chain growth, orientation, particle formation and diffusion of the organic phase) were suggested to occur in a concerted fashion. Although the detailed origin and formation of particle anisotropy remained elusive, a strong correlation between internal polymer chains arrangement and external particle dimension were considered. A major factor for the appearance of ellipsoidal shapes was stated to be the high persistence length of conjugated polymers, based on the backbones' rigid character. ${ }^{80}$ Also, the treatment of the polymerizing system with shear forces seemed to induce the deviation from spherical shapes. Ultrasonication was demonstrated to enable the sonocrystallization of dissolved conjugated polymers which subsequently solidified as nanofibers. ${ }^{152}$ In a fully dissolved state, the polymer chains were considered to be thermodynamically instable, but located in a local energetic minimum. However, the mechanical energy provided by the ultrasound-induced cavitation events was sufficient to overcome the energetic barrier, resulting in the formation of crystalline nuclei and the growth of larger nanofibers. ${ }^{152}$ As the intrinsic order in anisotropic particles is considered to be a decisive factor in overcoming the tendency to surface minimization, insight into the internal particle structure and its correlation with the external shape is of major interest to understand and control particle anisotropy.

\subsection{AMPHIPHILIC BLOCK COPOLYMERS}

Apart from homopolymer-based materials, conjugated polymers have been much studied as hydrophobic components of block copolymers. ${ }^{140,141,153,154}$ Their rigid chain character and their tendency to form agglomerates, facilitated by $\pi-\pi$ stacking effects, can be used as an advantage by the attachment of complementary, flexible groups with hydrophilic properties. ${ }^{155}$ The resulting amphiphilic rod-coil type copolymers can self-organize into supramolecular morphologies such as micellar, vesicular, cylindrical, lamellar or honeycomb-like structures, driven by microphase separation processes. ${ }^{15,93,142}$ As compared to 'classical' coil-coil block copolymers consisting of two flexible components, the choice of conjugated polymers with rigid character as hydrophobic part gives access 
to different superstructures, caused by the high disparity between the segments regarding flexibility and solubility. ${ }^{156,157}$

For the choice of rod and coil segments, numerous types and combinations of polymers were proven to be feasible. ${ }^{154,158}$ A broad range of conjugated polymers serving as hydrophobic moiety was reported, including poly(p-phenyleneethynylenes), ${ }^{94}$ poly(phenylenevinylenes), ${ }^{159,160}$ poly(fluorenes) ${ }^{140,156,161}$ or poly(thiophenes). ${ }^{141,162-164}$ The range of possible coil components includes the commonly used hydrophilic poly(ethylene glycols) (PEGs), ${ }^{93,140,141,156}$ poly(acrylates), ${ }^{15,163}$ poly(dimethylsiloxanes), ${ }^{165}$ poly(styrenes), ${ }^{163,164}$ polypeptides or protein-like polymers, which are of particular interest for biologic applications. ${ }^{142}$ Amphiphilic block copolymers are accessible by various synthetic methods (Scheme 1.6).

a)
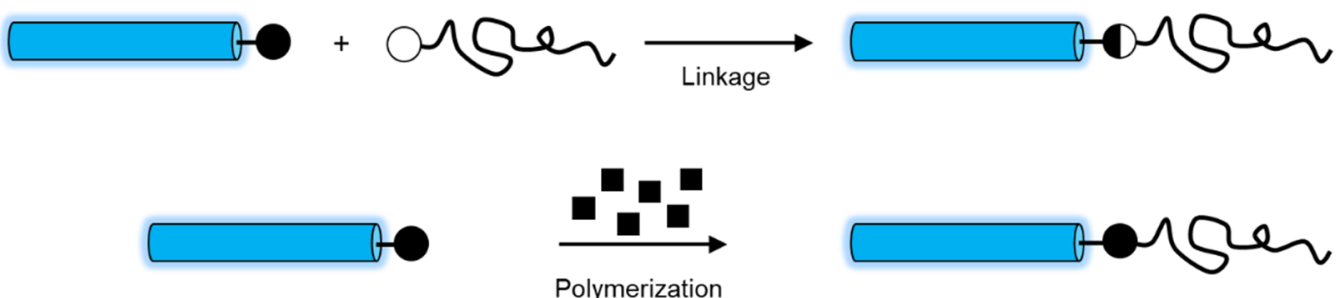

b)

Polymerization

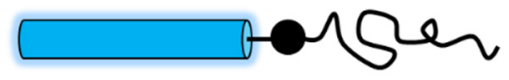

Polymerization
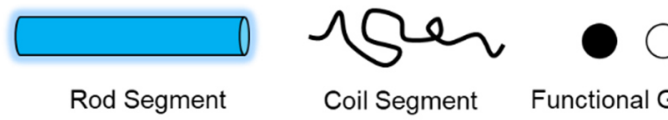

Functional Groups

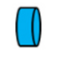

Monomers

Scheme 1.6: Synthetic approaches towards amphiphilic rod-coil block copolymers. (a) Linkage of hydrophobic rod and hydrophilic coil segment by functional chain ends. (b) Chain extension from one end-functionalized segment, serving as macroinitiator for the polymerization of the second segment.

The most straightforward approach (a) is the linkage of two independently synthesized segments, which are end-functionalized and subsequently connected, for example via copper-catalyzed click reactions ${ }^{140,162,164,166}$ or condensation-type reactions..$^{70,94,156,159,160,167}$ This approach potentially involves demanding purification procedures as well as low coupling efficiencies resulting from the low overall concentration of terminal functionalities. These issues can be overcome by consecutively operated polymerizations for each segment (b). To this end, one segment is end-functionalized and serves as macroinitiator, from which the second segment is grown in the course of the respective anionic polymerization, ${ }^{141,165}$ ATRP ${ }^{161,163}$ or other polymerization type. In this context, the development of Pdor Ni-catalyzed controlled coupling polymerizations provides a valuable tool to access the conjugated block (cf. Chapter 1.2).30,168 Whereas the low solubility of conjugated polymer macroinitiators can constitute a restriction for the following polymerization, this can be avoided by end-functionalization of the flexible coil segment, which simultaneously serves as solubilizing group. ${ }^{153}$

Dispersions of nanoparticles, intrinsically stabilized by the hydrophobic segment, were prepared from such amphiphilic block copolymers in a nanoprecipitation approach (cf. Chapter 1.3.1). A dilute copolymer solution in a water-miscible solvent was added to rigorously stirred water, resulting in the 
formation of core-shell structures..$^{70,169}$ As an alternative pathway towards well-defined superstructures, the slow mixing of various solvents with different quality regarding the solubilization of the individual copolymer segments effected homogeneous particle nucleation, followed by the self-organization of stable and fluorescent micelles. ${ }^{141,160,162}$ Induced by to the mutual repulsion of the different blocks and the packing constraints due to the connection of the blocks, microphase separation into hydrophilic and hydrophobic domains occurred. ${ }^{144,157,163}$ Depending on the lengths and thereby the relative volume fractions of rod and coil segments, these domains arranged periodically, yielding structures with variable shapes and dimensions. ${ }^{70,94,157,162}$ Theoretic studies on the behavior of rod-coil-type copolymers yielded various hierarchical superstructures, taking into account several factors such as the steric interaction between rigid segments or stretching of the flexible counterpart (Figure 1.7). ${ }^{170,171}$
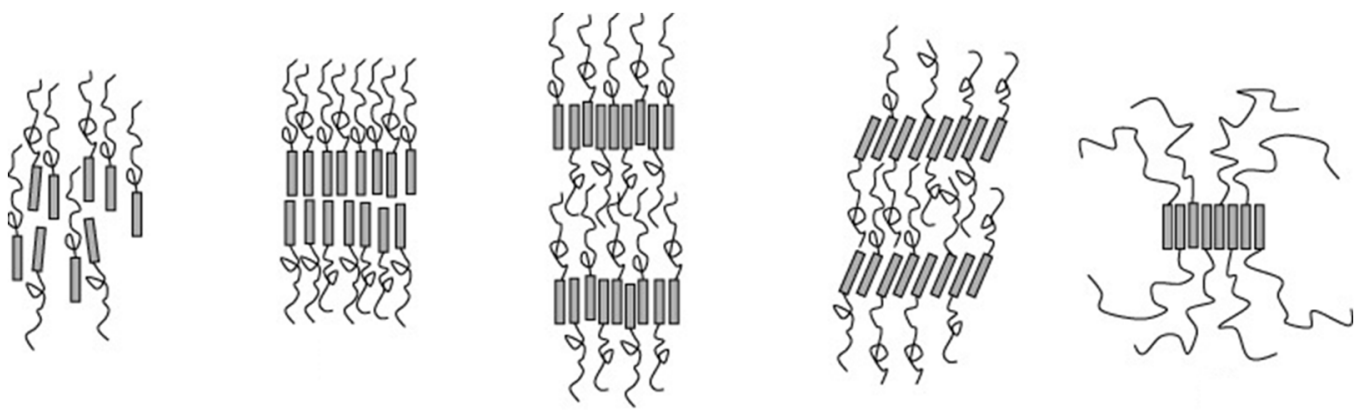

Figure 1.7: Schematic illustration of various superstructures, obtained from self-assembly of rod-coil-type copolymers. Reprinted with permission from John Wiley and Sons, Inc. (Polym. Int. 2010, 59, 1452-1476. Copyright 2021).

The obtained shapes, predominantly spherical micelles or nanofibres, were found to be further tunable by external stimuli such as concentration, temperature or choice of solvent. ${ }^{94,141,161,164}$ Simultaneously, the formation of supramolecular aggregates was assumed to be strongly affected by electrostatic $\pi-\pi$ stacking interactions between conjugated polymer chains, favored by the delocalized $\pi$-electrons and the planar structure of the rod segment. ${ }^{142,156}$ Even at low concentrations, di- or triblock copolymers with conjugated blocks exhibited remarkable capabilities in terms of molecular aggregation, which distinguished them significantly from rod-coil copolymers without conjugated segment. ${ }^{159}$ The formation of supramolecular structures dramatically effected the physical properties of the involved conjugated polymer and gave rise to unusual photophysical behavior in both solution and solid state. ${ }^{142,162}$ Their unique material properties and aggregation behavior make amphiphilic block copolymers with conjugated segments an interesting object of research to study on a molecular scale the organization of PF chains upon attachment of a hydrophilic moiety. 


\section{SCOPE OF THE THESIS}

The properties of polymer nanoparticles, including their shape and size, are known to strongly correlate with polymer chain alignment and the involved mechanisms on a molecular scale. A unique example for this relation is given by the material class of conjugated polymers. Directed via internal chain order, conjugated polymer nanoparticles of non-spherical shape are accessible directly in the process of polymerization. This constitutes a rare exception, as the preparation of such particle shapes is primarily a domain of inorganic materials. Furthermore, the few existing examples for organic polymers rely on post-polymerization techniques. Among the broad variety of conjugated polymers, poly $\left(9,9^{\prime}-\right.$ dioctylfluorene) exhibits an exceptional polymorphic behavior, which manifests in the coexistence of amorphous, disordered phases along with domains of high order, with a strong impact on the optoelectrical properties. The evolution of ordered phases in poly $\left(9,9^{\prime}\right.$-dioctylfluorene $)$ is supposed to be related to the unusual ellipsoidal nanoparticle shape. However, the detailed origin of anisotropy and the underlying mechanisms of chain arrangement remained elusive.

To reveal the internal molecular order within the particles, the conformation of oligomers with spin-labels attached to both chain termini was investigated by electron paramagnetic resonance spectroscopy (Chapter 3$)$. The origin of anisotropy in ellipsoidal poly(9,9'-dioctylfluorene) nanoparticles and its correlation with the evolution of ordered phases was elucidated by a combination of preparative approaches. These allowed insight into several decisive factors for the mechanism of anisotropic particle formation. Especially the molecular weight of the respective polymer chains and their arrangement within the nascent particles was examined (Chapter 4). In order to explore the scope of conjugated polymers forming nanoparticles of non-spherical shape, poly(fluorene) with aryl instead of alkyl substituents was studied (Chapter 5). To shed further light on the underlying processes of nanoparticle and shape formation, a synthetic route to block rod-coil type copolymers with a conjugated polymer segment was evolved and their intermolecular interactions in solution were studied (Chapter 6). 


\section{STUDIES ON OLIGO- FLUORENE CONFORMATION BY DOUBLE ELECTRON- ELECTRON RESONANCE}

\subsection{THEORETICAL BACKGROUND}

The external dimensions of nanoscale particles are strongly influenced by their internal morphology and the orientation of the components on a molecular level. The illumination and understanding of the inner structure of conjugated polymer nanoparticles and the mechanistic details of particle formation are considered to be crucial for the control over particle size and shape. ${ }^{7,101}$ Considering this objective, numerous analytical methods were developed and optimized, including double electron-electron resonance (DEER) spectroscopy.

DEER is an electron paramagnetic resonance (EPR) technique that allows the determination of the distance between two unpaired electrons or other paramagnetic centers. The electron spins present in the system under investigation are exposed to an external static magnetic field and excited by microwave radiation of a suitable frequency. Compared to the closely related technique of nuclear magnetic resonance (NMR) spectroscopy, using radio frequency pulses, EPR benefits from a larger Zeeman splitting and therefore significantly higher sensitivity. ${ }^{172-174}$ By incorporation of spin probes into the sample of interest by site-directed labeling, the otherwise only rare natural occurrence of paramagnetic species can be turned into an advantage. Nitroxide functionalities, such as $2,2,6,6,-$ tetramethylpiperidin-1-yloxyl (TEMPO), are widely used as spin-labels of good synthetic accessibility and high stability. ${ }^{175}$ DEER measurements can elucidate the distance between a pair of paramagnetic centers on an approximate scale of 1.8-8 nanometers, while longer ranges typically require deuterated samples. ${ }^{176,177}$ The most common technique for interspin distance determination is the four-pulse DEER experiment, which provides the possibility to measure the dipolar coupling between two unpaired electrons amongst all contributions to the EPR spectrum and to subsequently determine the respective distance distribution. The pump-probe type experiment is based on a principle comprising two channels of different microwave frequencies, which target different spins within the sample (Scheme 3.1). One four-pulse DEER sequence consists of a refocused primary Hahn echo sequence at the observer frequency with fixed interpulse delays ( $\mathrm{T}_{1}$ and $\mathrm{T}_{2}$ ). The observer sequence produces a refocused echo

Parts of Chapter 3 are published in J. Am. Chem. Soc. 2020, 142, 1952-1956. 
signal, which factors out inhomogeneous broadening of the observer spin's EPR line caused by effects such as the dipole-dipole interactions of observer spin $A$ to electron spins, which are not excited by the observer pulse. A fraction of the electron spins B, coupled to observer spin A, is inverted by a m-pulse at the pump frequency at a variable time referring to the first observer echo $(t)$. The inversion of the pump spins lead to a change of the effective magnetic field that is felt by the observer spins. Given the prerequisite that the spins excited by the two frequencies exert electron-electron coupling, the inversion of spin $B$ changes the frequency of spin $A$. This results in an intensity change of the refocused echo. When the pump pulse is shifted in time, the echo intensity oscillates with a frequency that is directly dependent on the dipolar coupling between the observer and pump spin. Hence, the respective modulation frequency of the echo amplitude correlates with the interspin distance. By subsequent processing of the experimental DEER data the distance distribution of the spins can be determined. The two applied frequencies are chosen based on their excitation of different ranges of the samples EPR spectrum. While the pump frequency is placed at the intensity maximum of the spectrum, the observer frequency is shifted to a separated intense frequency.

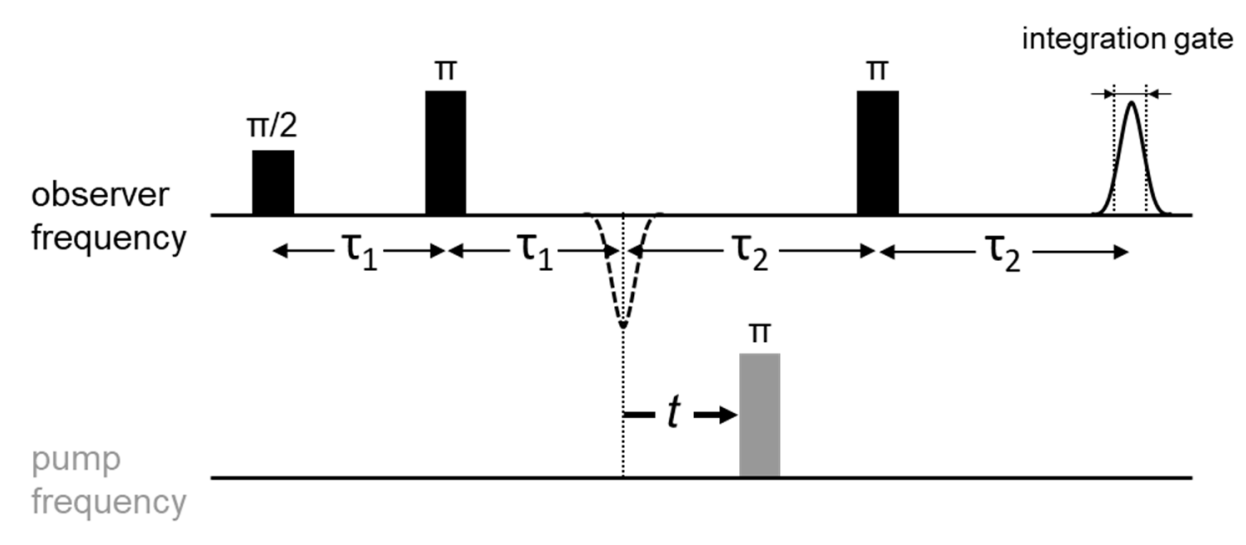

Scheme 3.1: Pulse sequence of the four-pulse DEER experiment. Delays $\tau_{1}$ and $\tau_{2}$ are kept fixed, while the delay $t$ between the unobserved first primary echo (dotted line) and the pump pulse is varied. The echo intensity integrated over the full width at half height is recorded. Adapted with permission from Royal Society of Chemistry (Phys. Chem. Chem. Phys. 2007, 9, 1895-1910; Copyright 2021).

In past studies, DEER measurements have successfully been employed to provide access to structural information in biomacromolecules, such as proteins. ${ }^{177-180}$ Moreover, valuable insight into the conformation and flexibility of defined monodisperse synthetic molecules in solution, including rod-like, conjugated oligomers, was enabled by determination of their contour lengths, persistence lengths and the respective correlation. ${ }^{181,182}$ The contour length $L_{c}$ of a polymer or oligomer represents the total length along its backbone in a fully extended state. In contrast, the persistence length $L_{p}$ is defined as the characteristic length designating the decay of tangent-tangent correlations. More figuratively, the distance between two segments of the same chain necessary for the canceling of their directiondependent correlation is described by $L_{p}$, which therefore provides a quantitative measure for the flexibility or stiffness of a chain. Considering flexible macromolecules, the contour length exceeds the persistence length by far $\left(L_{c}>L_{p}\right)$ and the system can be described as a self-avoiding, freely jointed chain. Meanwhile, a rigid, rod-like behavior is expected for molecules or chain segments which are considerably shorter than $L_{p}$. In the case of semi-flexible molecules, contour length and persistence length are in the same order of magnitude. ${ }^{183,184}$ 
Based on these perceptions, the flexibility of double spin-labeled oligo(p-phenyleneethynylenes) was investigated using acquired end-to-end distance distributions. ${ }^{181,185}$ However, respective DEER measurements were imprecise for longer molecules exceeding the elucidated persistence length of around $14 \mathrm{~nm}$. Apart from studies in solution, the DEER technique was successfully applied to illuminate the chain conformation of oligo( $p$-phenyleneethynylenes) in conjugated polymer nanoparticles prepared from nanoprecipitation. Revealed by a drastic decrease of interspin distance as compared to the values obtained in solution, the conjugated oligomers showed the ability to bend and to adopt the energetically favorable spherical particle shape. ${ }^{169}$ As the DEER technique combines spatial resolution for short range distances over several nanometers and virtual insensitivity to the chemical environment, it is a potent method to investigate the conformation of poly(fluorene) chains within anisotropic nanoparticles. Considering the DEER measurement range limited to interspin distances up to approximately $8 \mathrm{~nm}$, oligo(fluorenes) were initially chosen as samples to study the chain length distributions and conformational flexibility.

Please note, that the preparation of the spin-labeled oligo(fluorenes) under investigation were performed according to procedures elaborated by J. D. Ruiz Perez. Details on the synthesis and labeling of oligomers are more thoroughly elucidated in the corresponding doctoral thesis and in cited literature. ${ }^{18,186}$ DEER measurements and the processing of the acquired data were performed by $D$. Bücker from the group of Prof. Dr. M. Drescher. Extensive protocols and parameters of the DEER measurements can be found in published literature. ${ }^{18}$

\subsection{DEER MEASUREMENTS ON SPIN-LABELED OLIGOMERS}

\subsubsection{SYNTHESIS OF SPIN-LABELED OLIGOMERS}

For the synthesis of oligo(fluorenes) with spin-labels attached to both ends, two different pathways were of relevance. Owed to the limited DEER measurement range, the synthesis of comparatively short oligomers was envisaged. Oligomers of defined lengths were accessible in a stepwise approach, including repetitive cycles of Suzuki-Miyaura coupling reactions and subsequent brominations in an alternate fashion (Scheme 3.2). ${ }^{10,26,187}$ For oligomers with odd and even numbers of repeat units, two slightly different routes opened up, starting from a twofold brominated monomer or dimer, respectively. After achievement of the desired number of repeat units, the spin-label was introduced in a further Suzuki-Miyaura coupling reaction between the brominated oligomer and the corresponding aromatic boronic acid ester, previously connected to the TEMPO-label via esterification. ${ }^{169}$ For the following DEER studies, oligomers up to $n=5$ were synthesized according to this method, which yielded precise, monodisperse molecules. However, the synthetic effort of this approach increased considerably with the 
chain length. As well, threefold-brominated oligomers, forming in slight amounts as by-products in every halogenation step and potentially leading to the occurrence of undesired branched or threefold-labeled products, accumulated with growth of the oligomer chain.

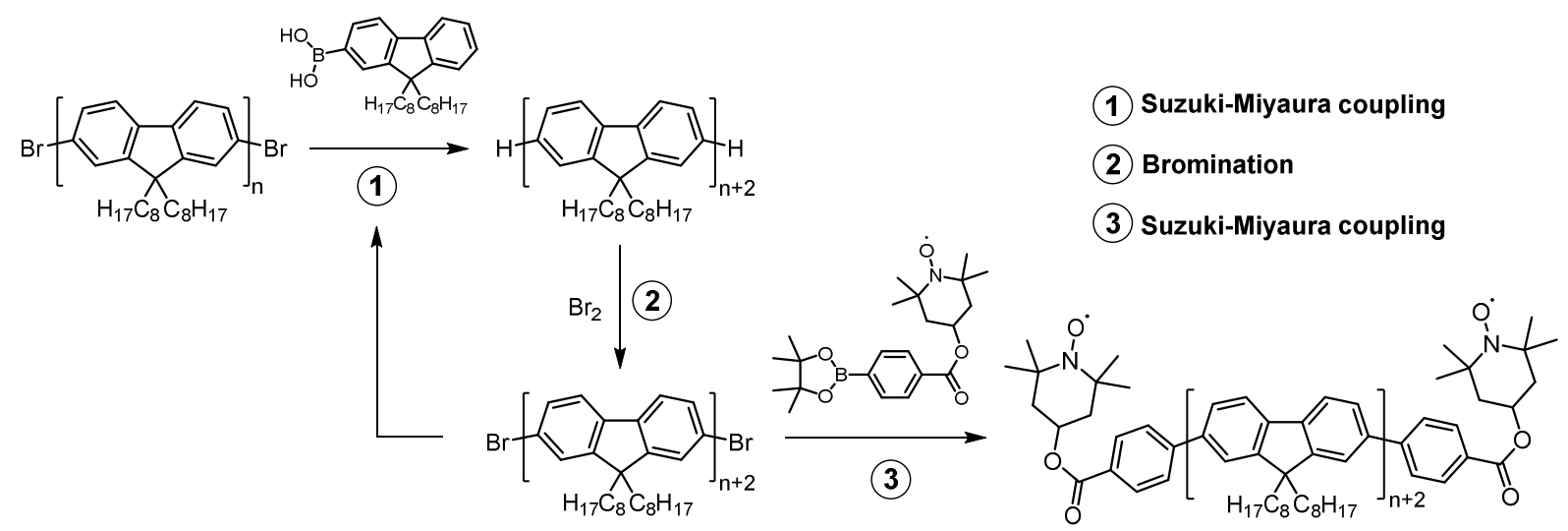

Scheme 3.2: Synthetic pathway to defined, doubly labeled oligo(fluorenes) by a stepwise approach, including sequences of extension of the oligomer chain by two units via Suzuki-Miyaura coupling reaction (1), followed by bromination (2). The TEMPO based spin-label is attached in a further Suzuki-Miyaura coupling step (3).

Hence, a second method to synthetically access labeled oligomers, especially the longer homologues, was elaborated. By controlled Suzuki-Miyaura coupling polymerization (CSMCP), the resulting polymer could be functionalized on both chain termini directly in the course of the reaction, by well-considered choice of initiator system and end-capping agent (cf. Chapter 1.2). Thereby, mixtures of oligo- and poly(fluorenes) with spin-labels on both ends became accessible (Scheme 3.3). The polymerization procedure used in this work is based on established protocols, ${ }^{69,70}$ which have been optimized with regard to the involved reagents by J. D. Ruiz Perez. ${ }^{186}$ The polymerization was initiated by an in situ formed catalyst system of chloro[(tri-tert-butylphosphine)-2-(amino-biphenyl)]palladium(II) as source of $\left[\mathrm{Pd}^{0}\right]$ and TEMPO-labeled 4-bromobenzoic acid which added oxidatively to the Pd-complex, serving as first unit of the growing chain. The polymerization reaction was subsequently quenched by the addition of TEMPO-labeled 4-carboxyphenyl-boronic acid pinacol ester, acting as end-capping agent. Owed to the living character of the polymerization reaction, the molecular weight distribution of the obtained oligoand polymers corresponds to a Poisson distribution with a maximum adjustable by the initiator to monomer ratio. For the intended EPR experiments, a distribution maximum of 5 repeat units was targeted, in accordance with the DEER measurement range. Although this approach is based on a synthetically straightforward method, the challenge emerged from the necessity to separate macromolecules with almost identical polarity, only differing in their chain length. Valuable techniques to this end are provided by chromatographic methods, as previously shown by the successful fractionation of oligo(fluorenes) and oligo(thiophenes). ${ }^{10,188}$ Size exclusion techniques rely on the molecular mass, more precisely on the hydrodynamic radii of the molecules. Hence, gel permeation chromatography (GPC) operated in a semi-preparative mode constituted a suitable approach for the separation of doubly spin-labeled oligo(fluorenes). ${ }^{10}$ For this purpose, the purified and dried oligomer mixture obtained from controlled Suzuki-Miyaura coupling polymerization was eluted from a semipreparative column while the outflow was automatically collected in various fractions. If necessary, individual fractions were further purified by consecutive cycles of fractionation, to obtain precise, 
monodisperse oligomers. The drawback of this method lied in the feasibility of only small sample loads ( $2.5 \mathrm{mg}$ per fractionation cycle) and the resulting, low amounts of purified product. This was, however, no severe limitation for EPR measurements, which required only very little amounts of sample.
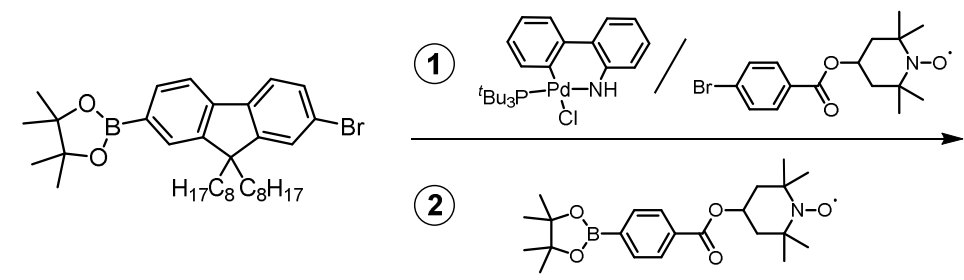

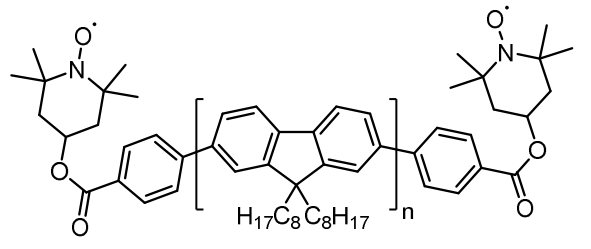

(1) Suzuki-Miyaura coupling polymerization

(2) Quenching

Scheme 3.3: Synthetic pathway to defined, doubly labeled oligo(fluorenes) by controlled Suzuki-Miyaura coupling polymerization.

Due to the paramagnetic character of the compounds, excluding their analysis by NMR spectroscopy, the labeled oligomers from both stepwise synthesis and CSMCP were characterized by MALDI-TOF measurements (cf. Figure 9.1). The successful incorporation of TEMPO-labels at both chain termini was confirmed, proving CSMCP and subsequent semi-preparative GPC fractionation to be a suitable method to obtain defined, labeled oligomers with efficient spin-labeling directly during polymerization. Especially for long or intermediate length oligomers $(n \geq 5)$, the considerably lower synthetic effort of this approach ruled out the advantage of higher product yields and scalability enabled by the stepwise synthetic approach.

\subsubsection{DEER MEASUREMENTS ON INDIVIDUAL OLIGOMER SPECIES}

With a series of doubly spin-labeled, precise oligo(fluorenes) in hand, DEER measurements were conducted for each individual oligomer chain length, in order to study their behavior in solution, with unrestricted mobility. Along with the increasing number of repeat units, increasing distance distributions were expected to be found. The results were intended to serve as reference data for the studies on oligomer mixtures and oligomers in particles. For this study, oligomers with $n=1-4$ repeat units were obtained from stepwise synthesis, whereas longer homologues with $n=5-6$ were prepared by cSMCP, followed by GPC fractionation. After flash-freezing of the respective oligomer solution in toluene-d8, DEER measurements were carried out in Q-band (34 GHz). Using the software DeerAnalysis, ${ }^{189,190}$ the experimental data acquired for the individual oligomers were fitted with the worm-like chain model (WLC), as recommended by previous literature (Figure 3.1a). ${ }^{183,185}$ The WLC model, introduced by Kratky and Porod for semi-flexible polymers $\left(L_{p} \approx L_{c}\right)$, presumes short-range interactions between individual monomer segments, which roughly have the same spatial orientation, and is considered wellsuited to describe the behavior of conjugated polymers. ${ }^{183}$ From processing of these data, distance distributions were obtained (Figure 3.1b). 


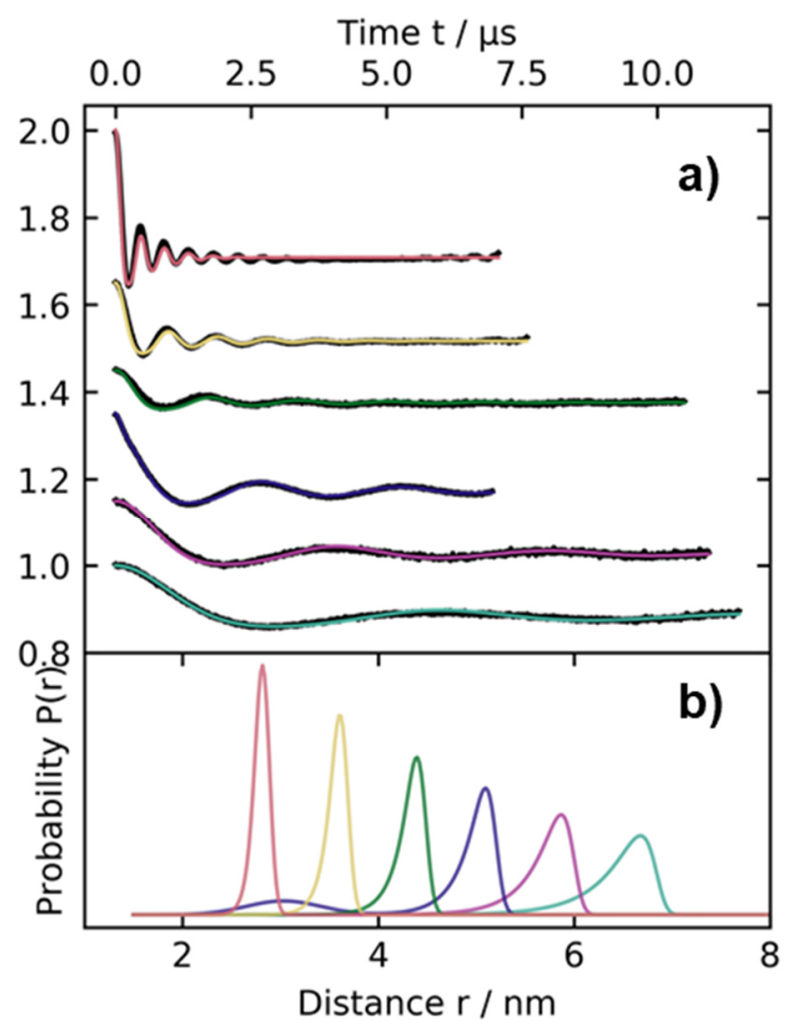

Figure 3.1: Distance determination by DEER spectroscopy for doubly spin-labeled, precise oligomers with repeat unit numbers from $n=1$ (top) to 6 (bottom) in toluene-d8. (a) Background-corrected form factor with model fit. (b) Distance distributions obtained by model fit, normalized to the integral over the distance probability.

For the doubly labeled monomer consisting of only one fluorene repeat unit, a completely stiff, inflexible character was assumed. Hence, the width of the respective distance distribution exclusively resulted from the flexibility of the TEMPO-based spin-labels. In accordance with this presumption, the distribution width for $n=1$ could be described by a Gaussian broadening with $\sigma=0.06 n m,{ }^{169}$ which was considered as spin-label contribution for the overall flexibility in all further experiments. Resulting from the stepwise character of the synthetic procedure, the occurrence of three-fold bromination and hence, the attachment of a third spin-label in the center of the oligomer chain was observable for the species with $n=4$ units $(21 \%$ of spin-labels were attached to three-fold labeled oligomers). The additional, broad distance contribution below the expected end-to-end distance was therefore described by a further Gaussian distribution for this oligomer species in the fitting process, which was confirmed by model free analysis. As outlined above, the probability of excessive bromination and, consequently, the introduction of additional spin-labels increased with the oligomer chain length in the course of the stepwise synthesis approach. Hence, the synthesis of TEMPO-labeled oligomers by CSMCP and subsequent fractionation into individual species was considered the preferable synthetic pathway, especially for reference compounds with higher chain lengths.

The contour and persistence lengths of the oligo(fluorenes) were obtained by the analysis of the DEER data. The contour length $L_{c}$ is the total length of a polymer or macromolecule along its backbone in a fully extended state and therefore requires individual fitting for each species investigated. In contrast, the persistence length $L_{p}$ is a global parameter, characterizing a specific material or, in this case, a set of oligomers which only differ in their length. As apparent from Figure 3.1b, the distance distributions 
become broader with greater chain lengths, which can be attributed to the increasingly flexible oligomer backbone. Beyond the contribution of the spin-label flexibility, this second contribution to the distribution broadening is characterized by the persistence length $L_{p}$. Regarding the set of oligo(fluorenes) under investigation, $L_{p}$ was calculated under exclusion of the doubly spin-labeled fluorene monomer $(n=1)$, due to its mainly monomeric character. Accordingly, a global persistence length of $L_{p}=14 \mathrm{~nm}$ was determined. The corresponding parameters of free persistence lengths and the contour lengths, which were calculated with either free or with fixed persistence length, are listed in Table 3.1.

Table 3.1: Summary of fitting parameters for global persistence length determination of spin-labeled, defined oligomers ( $L_{p}$, free and $L_{c}$, free) and parameters of model-based fit with WLC upon fixed persistence length $\left(L_{c}\right)$.

\begin{tabular}{c|c|c|c}
$\begin{array}{c}\text { oligomer length } \\
\text { [number } n \text { of } \\
\text { repeat units] }\end{array}$ & $\begin{array}{c}\mathrm{L}_{p, \text { free }}{ }^{a} \\
{[\mathbf{n m}]}\end{array}$ & $\begin{array}{c}\mathrm{L}_{\mathrm{c}, \text { free }}{ }^{a} \\
{[\mathrm{~nm}]}\end{array}$ & $\begin{array}{c}\mathrm{L}_{\mathrm{c}}{ }^{b} \\
{[\mathrm{~nm}]}\end{array}$ \\
\hline $\mathrm{n}=1$ & 50.42 & 2.84 & 2.90 \\
$\mathrm{n}=2$ & 19.77 & 3.69 & 3.73 \\
$\mathrm{n}=3$ & 15.06 & 4.55 & 4.56 \\
$\mathrm{n}=4^{c}$ & 14.26 & 5.30 & 5.31 \\
$\mathrm{n}=5$ & 12.27 & 6.11 & 6.14 \\
$\mathrm{n}=6$ & 11.00 & 7.14 & 7.02
\end{tabular}

${ }^{a}$ Free persistence length and $\sigma=0.06 \mathrm{~nm}$. ${ }^{b} \mathrm{Global}$ parameters: Persistence length $\mathrm{L}_{p}=14 \mathrm{~nm}$ and $\sigma=0.06 \mathrm{~nm}$. ${ }^{c}$ For $\mathrm{n}=4$ an additional Gaussian distribution was introduced ( $r=3.03 \mathrm{~nm}, \sigma=0.267 \mathrm{~nm}, 21 \%$ weighting factor).

As expected, both the distance distributions and the determined values for $L_{c}$ showed that the extension of the oligomer chain by one monomer unit resulted in the same increase in contour lengths for all oligomer species. Concerning this increase, a general correlation of $L_{c} \approx(2.1+n \times 0.8) n m$ was elaborated, including a length of $0.82 \mathrm{~nm}$ for each additional repeat unit. This result is in good agreement with the values found for a monomeric fluorene unit, as obtain by XRD measurements $(0.83 \mathrm{~nm})^{33}$ or for repeat units of poly $\left(9,9^{\prime}\right.$-bis(2-ethylhexyl)-fluorene-2,7-diyl) $(0.75 \mathrm{~nm}) \cdot{ }^{191}$ More details on the measurements of the individual oligomer species can be found in recent literature. ${ }^{18}$ As apparent from the contour lengths $L_{c}$, determined using either free or fixed persistence length, the two data sets only showed marginal differences, which indicated accessibility of the form factors using fixed persistence lengths. Conclusively, the model WLC_rigid_gauss, a Gaussian broadening of $\sigma=0.06 \mathrm{~nm}$ and a global persistence length of $L_{p}=14 \mathrm{~nm}$ were suitable conditions to obtain a good accordance of experimental data and fit. It was furthermore possible to access conformational data for precise, doubly spin-labeled oligo(fluorenes) of semi-flexible nature, ready to be used as valuable reference data for DEER measurements on oligomer mixtures or solid samples. 


\subsection{DEER MEASUREMENTS ON A SPIN-LABELED OLIGOMER MIXTURE}

After determination of the WLC parameters for each individual oligomer species, DEER measurements were performed on a mixture of doubly labeled oligomers, resulting from a cSMCP reaction with a fivefold amount of monomer relative to the initiator. Due to the occurrence of spin-spin relaxation, the dipolar evolution time of the experiment was limited to $10 \mu \mathrm{s}$. This means that an accurate determination of the mean and the width of the distance distribution was only possible to an upper limit of $7 \mathrm{~nm}$, whereas the shape of the distribution could be precisely elucidated up to $5 \mathrm{~nm} .{ }^{177}$ The obtained experimental data were described using a model comprising a superposition of different oligomers, according to a Poisson distribution with an expectation value $\lambda$. Each of the oligomer species in turn was described by the WLC parameters derived from the abovementioned reference measurements of isolated oligomers with chain lengths of $n=1-6$. Persistence length $\left(L_{p}=14 \mathrm{~nm}\right)$ and Gaussian broadening $(\sigma=0.06 \mathrm{~nm})$ were kept fixed (Figure 3.2). As a consequence of the post-polymerization work-up of the mixture, required for sample preparation, the fractions of the short oligomers with $n=1-3$ were underrepresented referred to a Poisson distribution. Considering this deviation, monomer and dimer were excluded in the fitting process of the distribution. For lack of respective reference data, the contour length of the oligomer with $\mathrm{n}=7$ was fitted as an additional parameter. For the model based fit including seven individual WLCs, the contour length $L_{c}$ for $n=1-6$ was fixed using the values previously determined for the individual oligomers, whereas the value for $n=7$ was varied in the fit. Although for oligomer lengths up to $n=7$ their respective contributions to the distance distribution could be separated from the background without disturbing the analysis, this was not possible for longer oligomers under the given experimental conditions. Consequently, oligo(fluorenes) with $n>7$ present in the polymerization mixture were not analyzable by DEER. In order to illustrate the amount of longer oligomers in the overall distribution, these were calculated according to the revealed Poisson distribution (Figure 3.2b, dashed line).

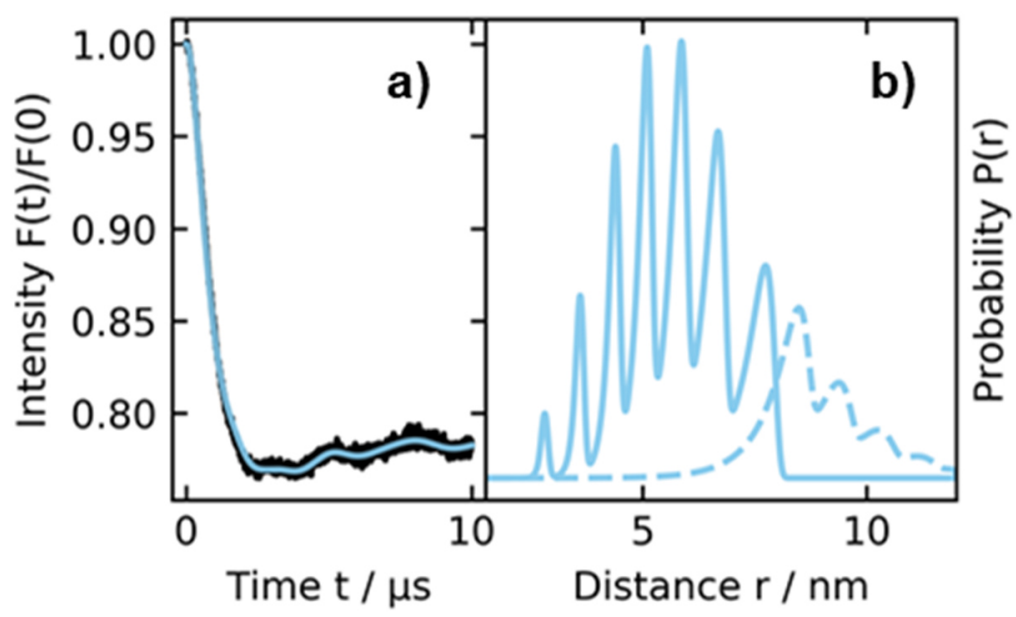

Figure 3.2: Distance measurement of an oligomer mixture, obtained from controlled Suzuki-Miyaura polymerization, in toluene-d8. (a) Background-corrected form factor with model fit (blue). (b) Distance distributions according to the fitted model, following a Poisson distribution, for $n=1-7$ (solid line) and for $n>7$ (dashed line) with an expectation value of $\lambda=5.4$ and a fixed global persistence length of $L_{p}=14 \mathrm{~nm}$. 
For each chain length, the experimentally obtained fractions for oligomers of $n=1-7$ were normalized to $100 \%$ and were compared to the probability values of a theoretical Poisson distribution as expected from an ideal living polymerization with fast and efficient initiation (Table 3.2). It can be concluded that the experimental DEER data is in very good conformity with a Poisson distribution of chain lengths around an expectation value of $\lambda=5.4$. Moreover, the results correspond excellently with a chain conformation described by a WLC with a persistence length of approximately $14 \mathrm{~nm}$.

Table 3.2: Contour lengths $L_{c}$ used for the fit of seven WLCs, experimentally found, normalized fractions $w$ of individual oligomer species and fractions expected from a theoretical Poisson distribution resulting from an ideal living polymerization.

\begin{tabular}{c|c|c|c}
$\begin{array}{c}\text { oligomer length } \\
\text { [number } n \text { of } \\
\text { repeat units] }\end{array}$ & $\begin{array}{c}\mathbf{L}_{\mathrm{c}} \\
{[\mathrm{nm}]}\end{array}$ & $\begin{array}{c}\text { fraction } \mathbf{w} \\
{[\%]}\end{array}$ & $\begin{array}{c}\text { Poisson } \\
\text { normalized } \\
\text { fraction [\%] }\end{array}$ \\
\hline $\mathrm{n}=1$ & 2.90 & 0.4 & 3.0 \\
$\mathrm{n}=2$ & 3.73 & 1.6 & 8.1 \\
$\mathrm{n}=3$ & 4.56 & 9.9 & 14.5 \\
$\mathrm{n}=4$ & 5.31 & 24.8 & 19.6 \\
$\mathrm{n}=5$ & 6.14 & 26.6 & 21.1 \\
$\mathrm{n}=6$ & 7.02 & 20.8 & 19.0 \\
$\mathrm{n}=7^{b}$ & 8.20 & 15.9 & 14.7
\end{tabular}

aProbability values for $n=1-7$ were taken from a theoretical Poisson distribution $(\lambda=5.4)$ and normalized to $100 \%$. ${ }^{b}$ Value of $L_{c}$ for $n=7$ were taken from fit.

In order to evaluate the robustness of the determined persistence length $L_{p}=14 \mathrm{~nm}$, the fit for the oligomer mixture under investigation was repeated with different fixed persistence lengths, but identical contour lengths, in accordance with the previously identified values (vide supra). The weight of the different oligomer fractions were allowed to vary freely. Although the widths of the distributions for the individual WLCs were changing upon a variation in $L_{p}$, the relative weight of the individual fractions was not significantly affected by a change in persistence length in a range of $12 \mathrm{~nm}<\mathrm{L}_{\mathrm{p}}<20 \mathrm{~nm}$ (cf. Figure 9.2). Hence, persistence lengths below $12 \mathrm{~nm}$ and beyond $20 \mathrm{~nm}$ could be excluded, which furthermore supported the validity of the determined parameters. For reasons of comparison, the evaluation of the oligomer mixture was, in addition to the model based approach described above, also performed with a model free system. Thereby, the fractions for each oligomer species were determined in a model free approach, yielding data which were still in reasonable agreement with a Poisson distribution. Hence, the determination of the persistence length was, although less accurate, also possible without previous measurements on monodisperse, doubly labeled reference oligomers. More detailed elucidation of model based and model free evaluation of DEER data on oligomer mixtures obtained from controlled Suzuki-Miyaura coupling polymerization can be found in the cited litertaure. ${ }^{18}$ In conclusion, the successful implementation of DEER measurements on a real-life synthetic polymer, containing a multitude of different chain length species, was demonstrated. The analysis technique 
allows for the quantification of individual oligomer species and the respective conformation and flexibility directly on mixtures.

\subsection{EVALUATION OF DEER IN POLYFLUORENE PARTICLES}

Apart from oligo(fluorenes) moving freely in solution, also DEER studies on TEMPO-labeled oligomers in conjugated polymer nanoparticles were envisaged, in order to estimate the method's applicability on spin systems in solid environment. As the chain conformation and flexibility of poly(fluorene) and related materials are strongly connected with particle shapes and morphologies, access to these information was of high interest. To this end, spherical poly(fluorene) nanoparticles, containing a predefined mixture of doubly spin-labeled oligomers, were prepared using the emulsification approach (cf. Chapter 1.3.1).84,85,95 The short oligomers of $n=1,2$ and 3 (in a 4:3:3 ratio) were blended with the bulk polymer by dissolving both in tetrahydrofuran, yielding an overall spin concentration of $0.25 \mathrm{mM}$. The organic mixture was emulsified by ultrasonication in an aqueous solution of sodium dodecyl sulfate (SDS), serving as stabilizing agent. Colloidally stable, fluorescent dispersions of particles with an average diameter of around $190 \mathrm{~nm}$ were yielded, as confirmed by TEM and DLS measurements (Figure 3.3a, Figure 9.3). In order to remove excessive surfactant, the dispersion was purified by dialysis prior to DEER measurements. Furthermore, it was freeze-dried to enhance the overall concentration of spin-labels in the measurement volume, while the particle structure remains unchanged. Owed to the in the non-deuterated environment of the nanoparticle, the phase memory time was reduced to $T_{m}=1.9 \mu \mathrm{s}$, as opposed to the previous studies in deuterated solution $\left(T_{m}=8.0 \mu \mathrm{s}\right)$. Hence, the nanoparticles were investigated by DEER with a dipolar evolution time of $3 \mu \mathrm{s}$, which constituted a significantly shorter time referred to the times of $8-12 \mu$ s used for the experiments in toluene-d8.
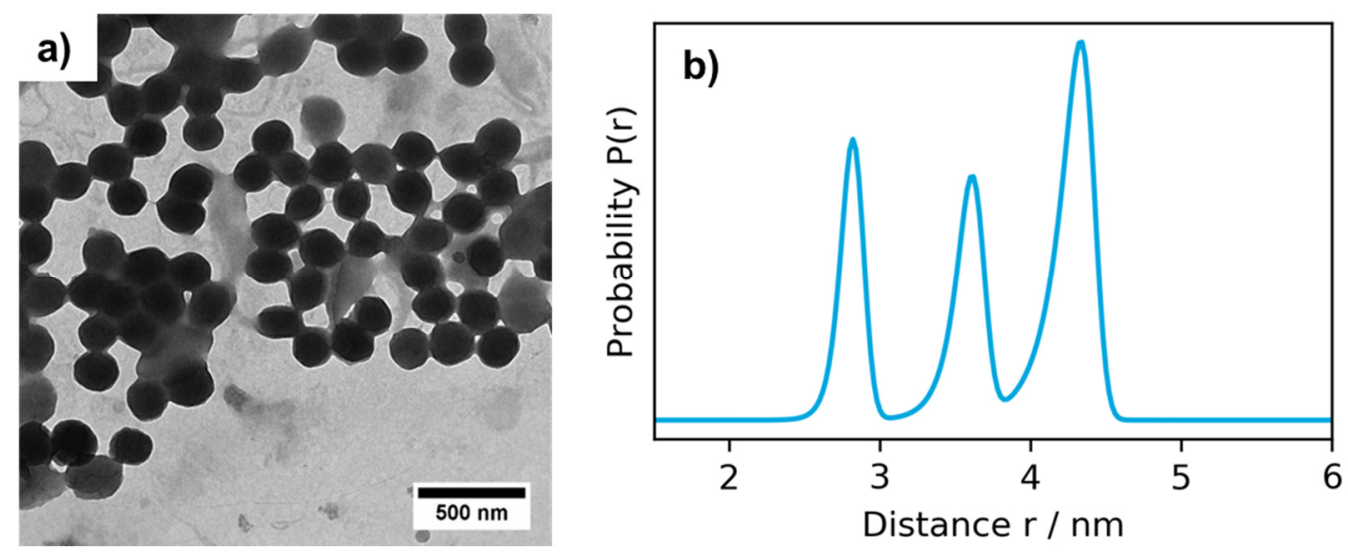

Figure 3.3: Poly(fluorene) nanoparticles with incorporated spin-labeled reference oligomers of $n=1,2$ and 3 . (a) TEM image of respective particles. (b) Distance distributions obtained by model fitting with three WLCs and $D E E R N e t$ background. The fit was performed with fixed persistence length $\left(L_{p}=14 \mathrm{~nm}\right)$ and fixed Gaussian broadening $(\sigma=0.06 \mathrm{~nm})$. Weights of the respective oligomers from fit are $w(1)=0.32, w(2)=0.28$ and $w(3)=0.40$. 
The acquired experimental data were processed using the values for contour length and broadening, induced by flexibility of spin-labels, obtained from the previous reference measurements. The relative weights of the oligomers as well as the global persistence length were fitted, to yield the corresponding distance distribution for spin-labeled oligomers, incorporated in nanoparticles, by a model-based fit (Figure 3.3b). The respective oligomer fractions of 32\% $(n=1), 28 \%(n=2)$ and $40 \%(n=3)$ were obtained by integration of the peaks in the distance distribution from a model free fit created by Tikhonov regularization (cf. Figure 9.5). The results agreed reasonably with the values envisaged by sample preparation (40\%, 30\% and $30 \%$ ), especially when taking into account the limited signal-to-noise ratio of distance measurements in solids. A persistence length of $L_{p} \approx 14 \mathrm{~nm}$ was derived, in consistency with the results found for dissolved oligomers. It can therefore be assumed, that the worm-like chain nature of the oligomers, including undisturbed flexibility, is retained also in solid environment, at least for the short chain homologues under investigation. As this result is not necessarily representative for longer polymer chains, ${ }^{169}$ which are, however, not accessible by DEER under the given conditions, further studies on this subject are required.

\subsection{SUMMARY AND CONCLUSION}

With the aim to access insight into the internal structure of anisotropic conjugated polymer nanoparticles, knowledge on the conformation of poly- and oligomer chains occurring as mixtures within solid environment is of great significance. Double electron-electron resonance (DEER) measurements were applied, monitoring the applicability of this technique to poly(fluorene) nanoparticles. The required oligomers were synthesized either by stepwise synthesis or by controlled Suzuki-Miyaura coupling polymerization and subsequent fractionation via gel permeation chromatography, yielding precise oligo(fluorenes) with 1-6 repeat units, bearing TEMPO spin-labels on both chain termini.

In order to acquire a set of reference data for following studies on mixtures or oligomers in solid material, DEER measurements in solution were conducted for each individual oligo(fluorene) species. From processing of the acquired data, the spin-spin distance distributions were obtained, which showed the expected consistent increase with every added monomer unit and an increasing broadening of the distribution, assignable to the enhanced flexibility of longer chains. From these results, a set of parameters for the system studied was calculated, described by the contour lengths $L_{c}$ of each species and the global persistence length $L_{p}$. With these parameters in hand, studies on doubly spin-labeled oligomer mixtures obtained from controlled Suzuki-Miyaura coupling polymerization were performed. The resulting distribution of spin-spin distance peaks, related to the different oligomer chain lengths, very well reflects the Poisson-type distribution expected from the controlled character of the polymerization. The accessibility of oligo(fluorene) mixtures by DEER was furthermore confirmed by processing of the data in a model free approach without using reference parameters of individual oligomer species, yielding highly consistent results. 
As oligo(fluorenes) are key intermediates in the formation of anisotropic poly(fluorene) nanoparticles, the fundamental feasibility of conformational analysis of oligomers within polymer particles by DEER was demonstrated. Despite the limited evaluability owed to the non-deuterated environment within the nanoparticles, the fractions of short chain oligomers found were in good agreement with values expected from sample preparation. Moreover, the chain flexibility of oligo(fluorene) was found to be unchanged as compared to measurements in solution, which hints at a worm-like chain nature with undisturbed flexibility to be retained in the nanoparticles. However, this can only be stated for the short chain oligomers under investigation and is not necessarily representative for longer oligo- or poly(fluorene) chains. Apparently, the measurement range of the analytic method with maximum determinable spinspin distances of around $7 \mathrm{~nm}$ constitutes a limitation and the incorporation of longer oligomers is expected to be more illuminating in view of the internal chain conformation of the polymer particles. Nevertheless, the general accessibility of chain conformation and flexibility of either individual oligo(fluorenes) in an isolated state or directly on mixtures by DEER measurements was demonstrated. It can therefore be considered as a valuable complementary technique to established standard methods for chain length distribution and conformation analysis. 


\section{ORIGIN OF ANISOTROPY AND STRUCTURE OF ELLIPSOIDAL POLYFLUORENE NANOPARTICLES}

Polymer nanoparticles with a non-spherical shape are difficult to access and therefore rare, which is also the case for conjugated polymer nanoparticles (CPNs). Compared to solutions of conjugated polymers, the intra- and interchain interactions between polymer chain segments are significantly altered by confinement in the small volume of nanoparticles. Overall red- or blue-shifts of UV/Vis absorption and fluorescence emission spectra are the result in many cases.,31,92 Hence, a strong correlation of internal chain arrangement, external particle morphology and optoelectrical properties has emerged as a frequently observed phenomenon for $\mathrm{CPNs} .{ }^{20} \mathrm{~A}$ reproducible protocol for the synthesis of anisotropic poly(9,9'-dioctylfluorene) (PF8) nanoparticles via heterophase Suzuki-Miyaura polycondensation was empirically developed by Ruiz Perez et al. ${ }^{8}$ In this Pd-catalyzed polycondensation of diboronic acid ester and dibromo-substituted fluorene monomers, the polymerization takes place within the emulsified organic solvent droplets, containing monomers and catalyst, and the final particle shape evolves during polymerization along with evaporation of the solvent (Scheme 4.1).

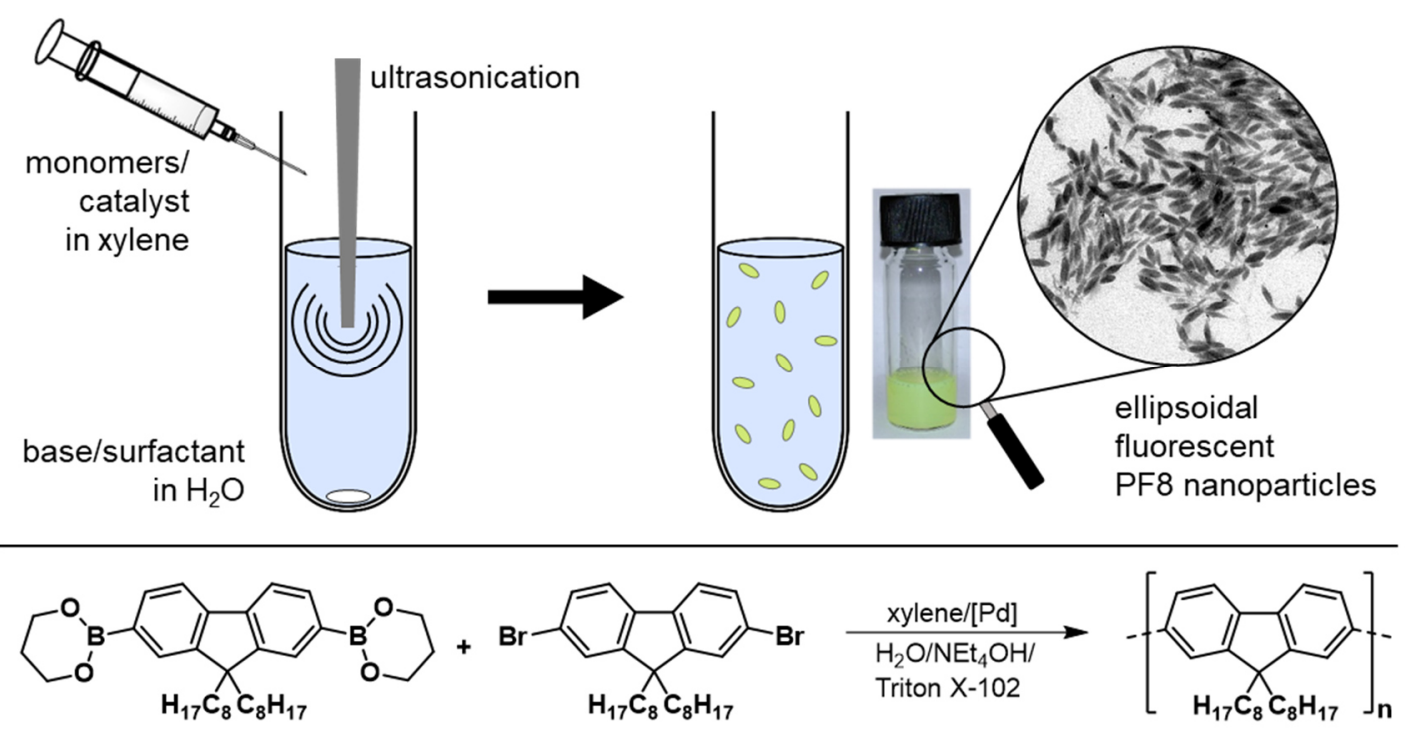

Scheme 4.1: Top: Schematic illustration of the preparation of ellipsoidal poly(fluorene) nanoparticles. Bottom: Reaction scheme of the applied heterophase Suzuki-Miyaura polymerization.

Parts of Chapter 4 are published in Macromolecules 2021, 54, 5267-5277. 
Well-defined ellipsoidal particles (length: $140 \mathrm{~nm}$, aspect ratio: 3.6) with narrow size and shape distribution and bright blue fluorescence are obtained. Although the origin of PF8 particle anisotropy was not yet sufficiently understood, a preliminary mechanism of particle formation was elaborated, suggesting the occurrence of several individual steps (polymerization chain growth, orientation, particle formation and diffusion of the organic phase) in a concerted fashion. The oligo(fluorenes) formed during step-growth are considered to be key intermediates which align within the emulsified droplets, due to their comparatively high mobility. As the arrangement of polymer chains is regarded as a decisive factor in the generation of particle anisotropy, the insight into the internal particle structure and its relation to the characteristic ellipsoidal shape is of major interest. In the context of polymers' chain order and the resulting bulk morphology, PF8 has emerged as an exemplary case for the field of poly(fluorene) homoand copolymers due to its exceptional polymorphic behavior, including the appearance of the ordered $\beta$-phase.22,32 Although the detailed mechanism of $\beta$-phase formation in PF8 still remains elusive, numerous investigations have been the source of differing interpretations. A dependence of $\beta$ conformation ratio on the number of monomer repeat units was ascertained, revealing a critical chain length necessary for the onset of $\beta$-phase evolution. 9,10,50,192 The numerous studies support the overall picture that the $\beta$-phase essentially originates - at least at early stages of formation - from intramolecular interactions, followed by the transition to intermolecular conformational order emerging from $\beta$-phase domains. ${ }^{44,50}$

The very few reported direct bottom-up approaches towards anisotropic, nanometer-scaled polymer particles generally rely on an internal molecular arrangement. ${ }^{147-150}$ For instance, this was elaborated in depth for nanoscale polyethylene single crystals, which arise from immediate deposition of the growing polymer chain on the crystal growth front, to yield single-chain anisotropic nanocrystals of uniform size and shape distribution. ${ }^{148-150}$ This suggests an understanding of internal particle morphology and how it affects the evolution of particle shape can be essential to exploit the scope of anisotropic polymer nanoparticles. By double electron-electron resonance experiments, the behavior of oligo(fluorenes) concerning their general flexibility in solution and within nanoparticles was approached (cf. Chapter 3). However, the measurement range of the analytic method is limited to short chain oligomers and the investigation of longer oligo- or poly(fluorene) chains is expected to be relevant for the internal chain conformation of the polymer particles. The following chapter reports insights on the origin of the shape of ellipsoidal PF8 nanoparticles and decisive factors for the mechanism of particle formation, revealed by a combination of experimental approaches. These studies comprise, apart from the evaluation of conditions required for the synthesis of anisotropic particles, the influence of internal factors like the chain length and the corresponding molecular weight of linear oligo(fluorenes). 


\subsection{SUZUKI-MIYAURA POLYCONDENSATION WITH ADDED OLIGOFLUORENES}

In order to shed light on the ability of PF8 segments for internal packing already in early stages of the polymerization and on its potential impact on particle dimensions, the reported Suzuki-Miyaura polycondensation (SMPC) was performed in the presence of defined, pre-synthesized oligomers of nonpolymerizable character (Figure 4.1), added to the initial polymerization mixture.

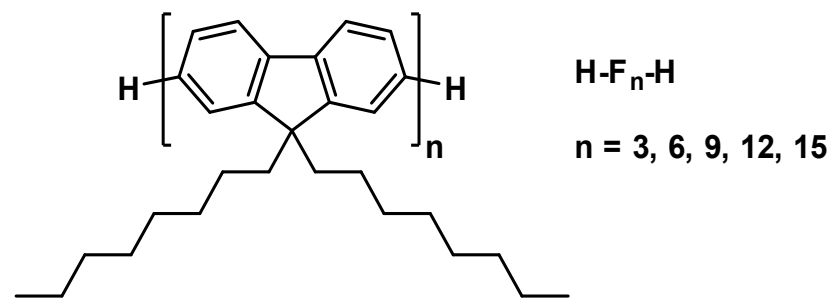

Figure 4.1: General structure of the non-polymerizable oligo(fluorenes) added to heterophase Suzuki-Miyaura polycondensation.

The n-mers with $n=3,6,9,12$ and 15 , respectively, were synthesized by consecutive cycles of bromination and Suzuki- or Yamamoto-type coupling steps (cf. Chapter 8.5.1). ${ }^{10,193}$ This construction of oligomers based on trimer blocks was chosen to reduce the overall number of coupling steps required, and to avoid the accumulation of undesired by-products. Measurement of UV/Vis absorption and fluorescence emission spectra of the oligo(fluorenes) in solution revealed the expectable red-shift with growing chain length. All oligomers with $n>3$ displayed emission spectra with a characteristic peak pattern identical with the spectrum of PF8 solutions (Figure 9.6). These pre-fabricated oligomers were added to the SMPC reaction mixture in amounts of $11 \mathrm{mg}(10 \mathrm{wt} \%$ referred to the weight of both monomers) by dissolution along with the standard AA/BB-type monomers and the catalyst (cf. Chapter 8.5.2). The data acquired for the obtained dispersions by various measurement techniques were compared to a reference PF8 dispersion synthesized under identical conditions, but without addition of pre-fabricated oligomers (Table 4.1). For the following transmission electron microscopy (TEM) measurements, the mean values of length and aspect ratio as well as of the corresponding coefficient of variation $v$ were calculated from the measurement of 50 nanoparticles in each case. In this context, the coefficient of variation $v$ as a measure for the particle dispersity is defined by the ratio of the standard deviation of either particle length or aspect ratio to the respective mean value.

Independent of the chain length, all oligomers under consideration were readily soluble along with the polycondensation monomers in the course of the preparation of the reaction mixture, which indicates high solubility in aromatic solvents like xylene (>11 mg/mL in each case). Although uninvolved in the step-growth reaction itself, the oligomers are expected to be located in the emulsified xylene droplets during the polycondensation and are embedded in the final particles obtained after polymerization and organic solvent evaporation. TEM analysis revealed that particle dimensions are largely unaffected by the presence of additional oligo(fluorenes) (Figure 4.2). The particles' lengths $(I \approx 120 \mathrm{~nm}$ ), their aspect ratios $(\operatorname{ar} \approx 3.3)$ and the respective coefficients of variation $v$ differ only little from the reference system. 
Table 4.1: Results of Suzuki-Miyaura polycondensation in the presence of pre-fabricated oligo(fluorenes). ${ }^{a}$

\begin{tabular}{c|c|c|c|c|c}
$\mathbf{n}\left(\mathbf{H}-\mathbf{F}_{\mathbf{n}}-\mathbf{H}\right)$ & $\begin{array}{c}\text { particle length } \\
{[\mathbf{n m}] \mathbf{v}^{\mathbf{b}}}\end{array}$ & $\begin{array}{c}\text { aspect } \\
\mathbf{r a t i o} / \boldsymbol{v}^{\mathbf{b}}\end{array}$ & $\begin{array}{c}\boldsymbol{M}_{\mathbf{n}}{ }^{\mathbf{c}} \\
{[\mathbf{k g} / \mathbf{m o l}]}\end{array}$ & $\begin{array}{c}\boldsymbol{\lambda}_{\mathrm{abs}, \max } \\
{[\mathbf{n m}]}\end{array}$ & $\begin{array}{c}\lambda_{\mathrm{em}, \max } \\
{[\mathbf{n m}]}\end{array}$ \\
\hline 3 & $126 / 0.11$ & $3.1 / 0.12$ & $36.8(18.4)$ & 410 & 440 \\
6 & $114 / 0.13$ & $3.2 / 0.09$ & $57.0(15.4)$ & 407 & 441 \\
9 & $114 / 0.13$ & $3.4 / 0.09$ & $63.8(21.9)$ & 407 & 441 \\
12 & $124 / 0.12$ & $3.4 / 0.08$ & $70.4(31.5)$ & 408 & 441 \\
15 & $117 / 0.10$ & $3.2 / 0.10$ & $63.8(25.8)$ & 408 & 441 \\
- & $111 / 0.11$ & $3.5 / 0.11$ & 42.5 & 406 & 441
\end{tabular}

${ }^{a}$ Conditions: Diboronic acid ester and dibromo-substituted fluorene monomers $\left(0.1 \mathrm{mmol}\right.$ each), $\left[\mathrm{Pd}\left(\mathrm{P}^{t} \mathrm{Bu}_{3}\right)_{2}\right]$ $(4 \mu \mathrm{mol})$ and respective oligo(fluorene) $(11.1 \mathrm{mg})$ dissolved in xylene $(1 \mathrm{~mL})$, addition to $5 \mathrm{wt} \%$ aqueous Triton $\mathrm{X}$ 102 solution $(25 \mathrm{~mL})$, simultaneous ultrasonication $\left(2.5 \mathrm{~min}, 60 \%\right.$ amplitude), $72 \mathrm{~h}$ of stirring. ${ }^{b}$ Mean values of lengths, aspect ratios and coefficient of variation $v$ in each case. TEM statistics were calculated from dispersions after purification by redispersion. 'Determined by GPC with RI detection vs. PS standards. $M_{\mathrm{n}}$ was calculated under exclusion of oligomer peak; values with included oligomer peak are given in brackets.
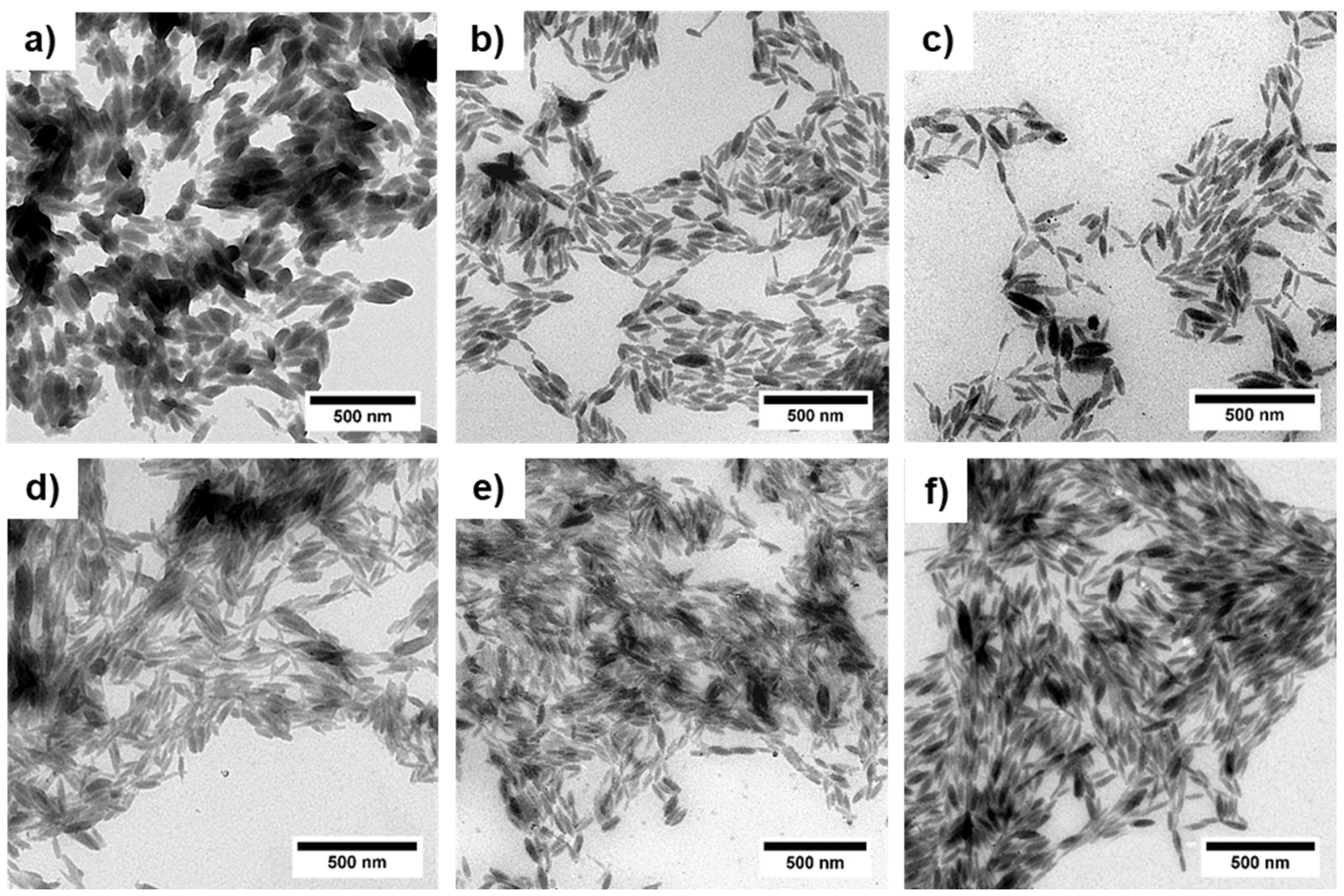

Figure 4.2: TEM images of PF8 nanoparticles obtained by polymerization in the presence of pre-fabricated (a) 3-mer, (b) 6-mer, (c) 9-mer, (d) 12-mer, (e) 15-mer, and of (f) reference particles generated without added oligomer. 
Likewise, no adverse effects on the optical properties of the particles were found. All samples showed the UV/Vis absorption band around $400 \mathrm{~nm}$ for amorphous PF8 and the sharp, characteristic $\beta$-phase peak at $440 \mathrm{~nm}$ as well as the typical fluorescence emission pattern of $\beta$-phase poly(fluorene) with peaks at $441 \mathrm{~nm}, 466 \mathrm{~nm}$ and $499 \mathrm{~nm}$, unaltered by the added pre-fabricated oligomers (Figure 4.3).
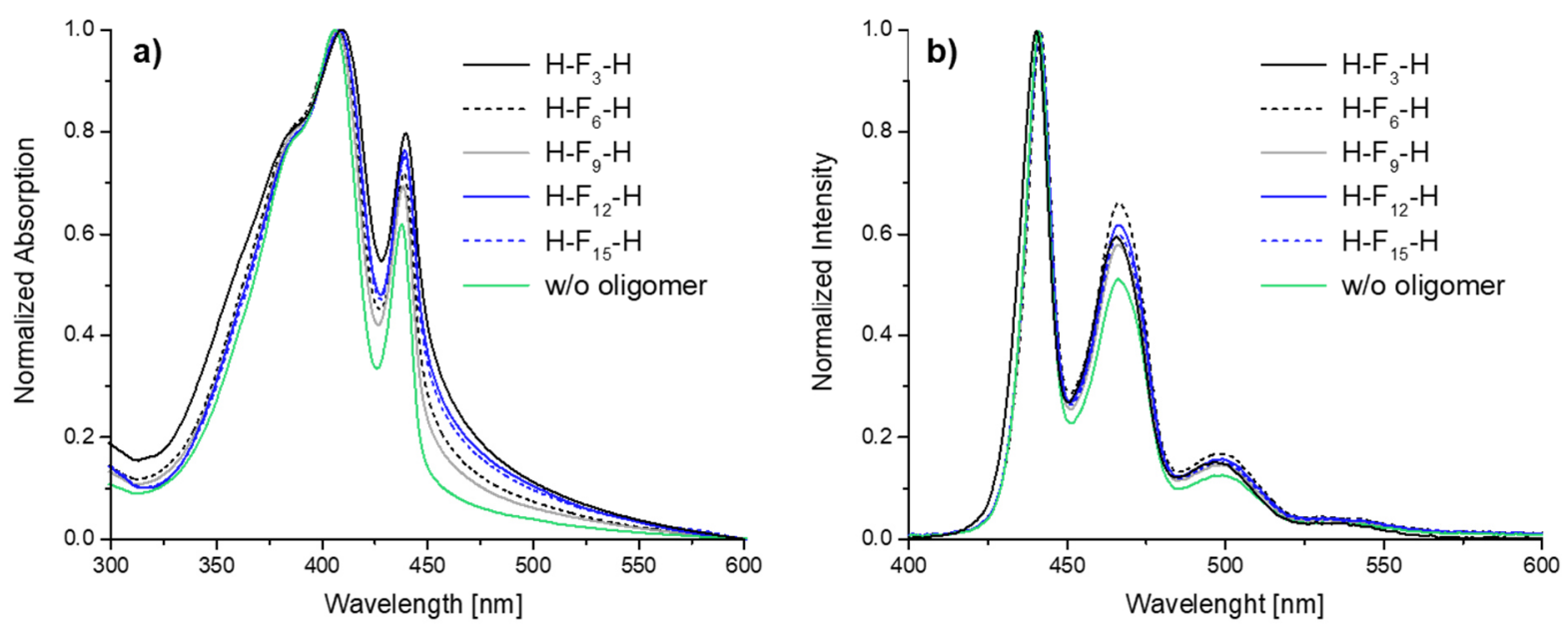

Figure 4.3: (a) UV/Vis absorption spectra and (b) fluorescence emission spectra ( $\lambda_{\text {exc }}=380 \mathrm{~nm}$ ) of PF8 nanoparticles with oligomers $H-F_{n}-H(n=3,6,9,12$ and 15) added to the reaction mixture, with spectra of the standard system for comparison.

These findings revealed that the incorporation of $\mathrm{H}-\mathrm{F}_{\mathrm{n}}-\mathrm{H}$ oligomers did not affect the ongoing polymerization, for example by promoting phase nucleation independently of the collapse of the growing polymer chain, or the properties of the resulting particles. In contrast, the oligomers were capable to align into the internal structure of the evolving particles. This was even the case for the higher homologues, which still seemed to be sufficiently mobile to be integrated into the solvent-swollen particles. Compared to the initial assumption of a concerted occurrence of both polymer step growth and chain assembly, the alignment of polymer or oligomer chains appears to be possible at any stage of the polymerization. This is especially noteworthy in connection with the fundamental impact of the aromatic solvent on the resulting dispersions. The impact of the nature of the aromatic solvent employed as the lipophilic phase in the SMPC was also explored. Therefore, heterophase Suzuki-Miyaura polycondensation was performed according to the reaction procedure described in Chapter 8.3.2, with the substitution of xylene by other aromatic solvents. (Table 4.2). 
Table 4.2: GPC data of poly(fluorene) dispersions prepared by Suzuki-Miyaura polycondensation with alternative aromatic solvents. ${ }^{a}$

\begin{tabular}{c|c|c|c|c} 
solvent & $\begin{array}{c}\boldsymbol{M}_{\mathbf{n}}{ }^{b} \\
{[\mathbf{k g} / \mathbf{m o l}]}\end{array}$ & $\boldsymbol{M}_{\mathbf{w}} \boldsymbol{M}_{\mathbf{n}}{ }^{b}$ & $\begin{array}{c}\boldsymbol{\lambda}_{\mathrm{abs}, \max } \\
{[\mathbf{n m}]}\end{array}$ & $\begin{array}{c}\boldsymbol{\lambda}_{\mathrm{em}, \max } \\
{[\mathbf{n m}]}\end{array}$ \\
\hline benzene & 18.6 & 2.6 & 408 & 441 \\
toluene & 22.3 & 2.7 & 402 & 439 \\
xylene & $42 . d$ & 2.7 & 406 & 441 \\
mesitylene & 27.9 & 3.0 & 408 & 440
\end{tabular}

aConditions: Diboronic acid ester and dibromo-substituted fluorene monomers $\left(0.1 \mathrm{mmol}\right.$ each) and $\left[\mathrm{Pd}\left(\mathrm{P}^{\mathrm{t} B \mathrm{Bu}_{3}}\right)_{2}\right](4 \mu \mathrm{mol})$ dissolved in respective solvent $(1 \mathrm{~mL})$, addition to $5 \mathrm{wt} \%$ aqueous Triton X-102 solution $(25 \mathrm{~mL})$, simultaneous ultrasonication ( $2.5 \mathrm{~min}, 60 \%$ amplitude), $72 \mathrm{~h}$ of stirring. ${ }^{b}$ Determined by GPC with RI detection vs. PS standards. "Mixed isomers. ${ }^{d}$ Data of standard dispersion for comparison.

Xylene was found the most appropriate solvent when it comes to the dimensions of the particles as well as the polymer properties, while reduced molecular weights were observed resulting from polymerizations with alternative solvents. Although ellipsoidal nanoparticles were as well obtained by protocols employing the lower boiling solvents benzene or toluene, undefined, fibrous structures represented the major component of the resulting dispersions (Figure 4.4). In contrast, dispersions with quality comparable to the reference system were obtained using mesitylene, although slightly reduced particle lengths and aspect ratios were apparent from TEM images (length $=104 \mathrm{~nm}(v=0.09)$, aspect ratio $=3.1(v=0.10))$. With regard to these findings, the internal chain packing presumably occurs independently of the chain length but more interrelated to the nature of the organic solvent.
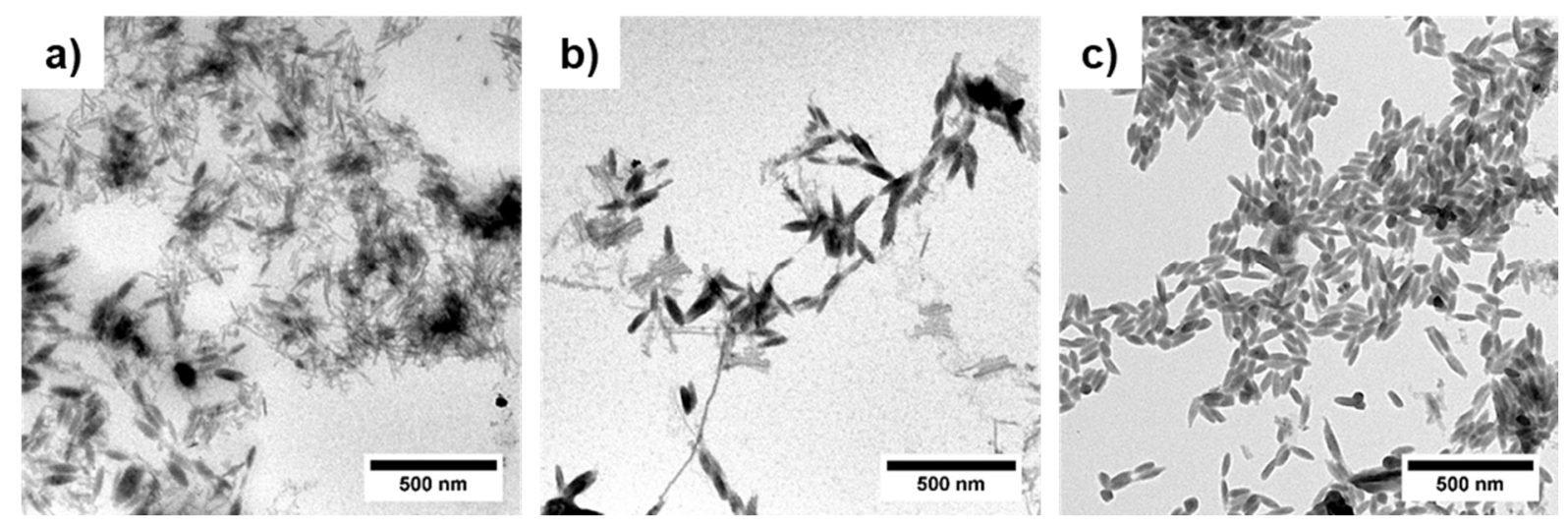

Figure 4.4: TEM images of particles prepared by heterophase Suzuki-Miyaura polycondensation with alternate organic solvents: (a) benzene, (b) toluene and (c) mesitylene. 


\subsection{POST-POLYMERIZATION PARTICLE SYNTHESES}

\subsubsection{EMULSIFICATION OF POLYFLUORENE}

In view of these findings, the generation of nanoparticles by post-polymerization dispersion ${ }^{3,84}$ of prefabricated high molecular weight PF8 under appropriate conditions was explored. Pursuant to adjustment of several parameters, best results were obtained by emulsification of PF8 solutions in xylene $(1 \mathrm{mg} / \mathrm{mL})$ in water as dispersing medium, using Triton X-102 as surfactant and ultrasonication as origin of shear force (cf. Chapter 8.5.3). PF8 $\left(M_{\mathrm{n}}=33 \mathrm{~kg} / \mathrm{mol}\right)$, synthesized by SMPC under heterophase conditions as outlined above, was purified to remove residual amounts of surfactant or other impurities prior to utilization in post-polymerization particle synthesis. At the aforementioned suitable conditions this emulsification yielded stable dispersions of anisotropic PF8 nanoparticles with lengths around $100 \mathrm{~nm}(v=0.12)$ (Figure 4.5). Although the mean aspect ratio of $2.9(v=0.10)$ calculated from TEM statistics did not entirely reach the values typically obtained for PF8 particles prepared by SMPC $(I=111 \mathrm{~nm}$, ar = 3.5), the post-polymerization technique provided dispersions with largely comparable dimensions and dispersity. Both, the absorption peak around $440 \mathrm{~nm}$, assignable to $\beta$-phase-type poly(fluorene), and the corresponding, characteristic fluorescence emission pattern were clearly observed for these particles.
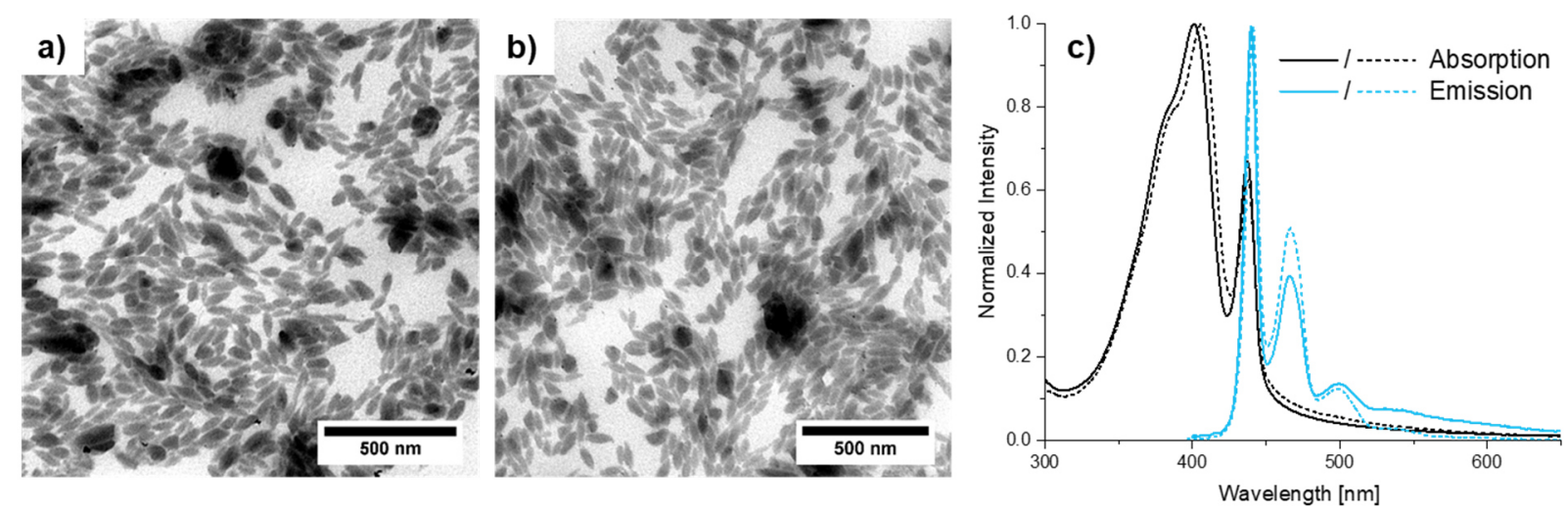

Figure 4.5: (a,b) TEM images of poly(fluorene) particle dispersions prepared by post-polymerization emulsification of PF8 $(I=100 \mathrm{~nm}(v=0.12)$, ar $=2.9(v=0.10))$ and $(c)$ the corresponding UV/Vis absorption (black) and fluorescence emission (blue) spectra (solid lines), compared to a dispersion prepared by heterophase SuzukiMiyaura polycondensation (dashed lines).

The post-polymerization emulsification procedure showed sensitivity towards changes in conditions in a qualitatively similar manner as the heterophase SMPC procedure. Variations of the organic solvent, the duration of ultrasonication or the surfactant applied, for instance, resulted in drastic changes of the particle dimensions, yielding dispersions with spherical particles or fibrous structures as main components or inhomogeneous mixtures with ellipsoidal particles (cf. Table 9.1, Figure 9.9). This further underlines the crucial role of the presence and especially the nature of the (ultimately evaporating) aromatic solvent for the mobility of the polymer chain. To exclude the possibility that any specific trace residues or any particular details of molecular weight distribution originating from the SMPC procedure 
are responsible for the ellipsoidal particle shape, the identical protocol was applied to commercial PF8 $\left(M_{n}=27 \mathrm{~kg} / \mathrm{mol}\right)$ purchased from Sigma-Aldrich, with analogous results (Figure 4.6). Upon implementation of the developed procedure, stable dispersions of uniform, ellipsoidal nanoparticles with an average length of $100 \mathrm{~nm}(v=0.10)$ and an aspect ratio of $3.0(v=0.09)$ were obtained. Similar to the abovementioned post-polymerization of PF8 fabricated by heterophase polycondensation, the respective UV/Vis absorption and emission spectra displayed the signals characteristic for PF8 with a significant proportion of $\beta$-phase conformation.
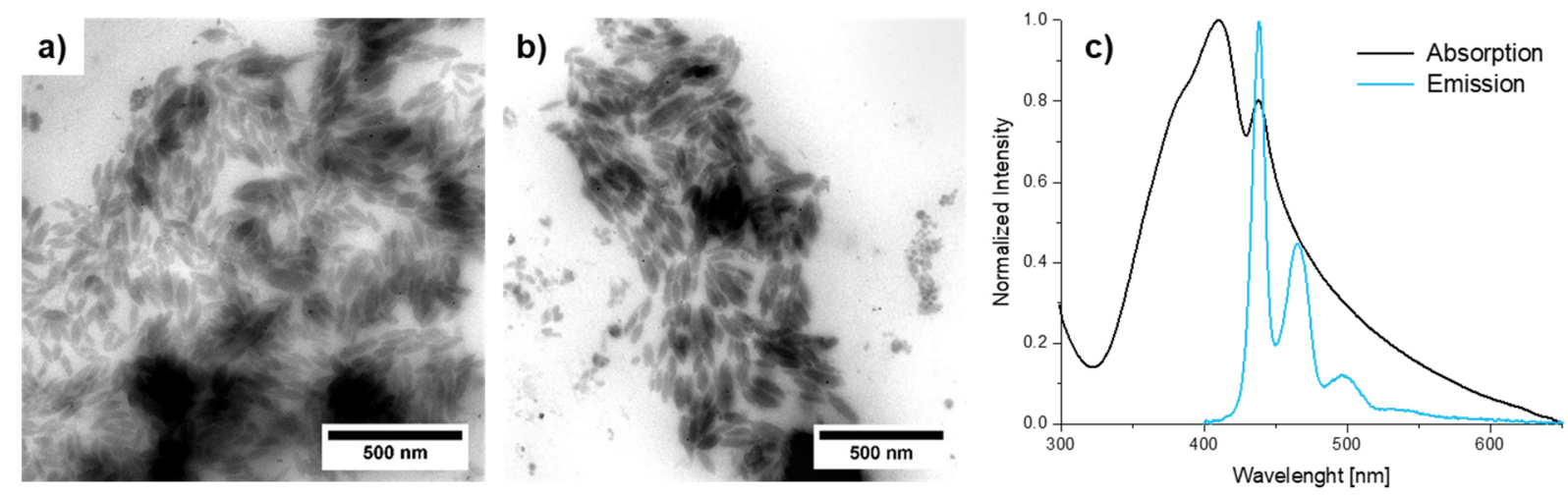

Figure 4.6: $(a, b)$ TEM images of poly(fluorene) particle dispersions prepared by post-polymerization emulsification of PF8 acquired from Sigma-Aldrich $(I=100 \mathrm{~nm}(v=0.10)$, ar $=3.0(v=0.09))$ and $(c)$ the corresponding UV/Vis absorption (black) and fluorescence emission (blue) spectra.

This implies that under appropriate conditions, even long poly(fluorene) chains possess the required mobility to rearrange within emulsified solvent droplets, to eventually form nanoparticles with a high anisotropy. The process of alignment is evidently accompanied by the formation of regions of $\beta$-phase conformation, which once more underlines the close correlation of internal particle order and anisotropic external dimensions.

\subsubsection{EMULSIFICATION OF OLIGOFLUORENES}

The successful incorporation of pre-fabricated oligomers in developing PF8 nanoparticles demonstrated the capability of short polymer fragments to align and adopt the $\beta$-phase conformation of the surrounding solid. In order to estimate the minimum number of fluorene repeat units necessary for the arrangement in $\beta$-phase conformation, oligomers with repeat unit numbers of $n=3,6,9,12$ and 15 , respectively, were studied as the sole component of post-polymerization generated particles (cf. Chapter 8.5.3). For all oligomer lengths, stable, fluorescent dispersions resulted (Figure 4.7). Both UV/Vis absorption and emission spectra of the obtained dispersions featured red-shifted maxima with increasing numbers of fluorene repeat units and the consequential extended conjugation length (Figure 4.8). 

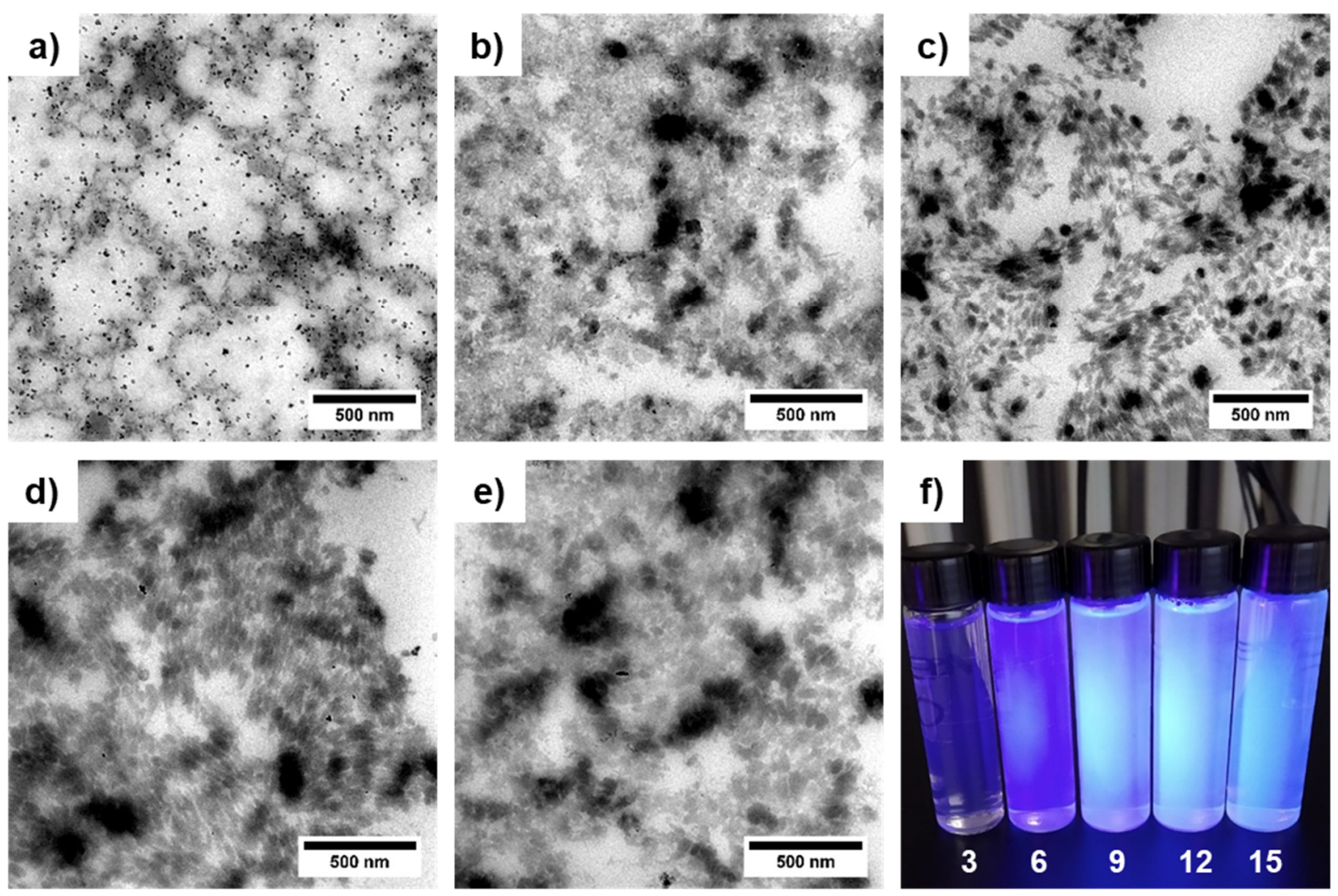

Figure 4.7: TEM images of dispersions prepared by post-polymerization emulsification of prefabricated oligo(fluorenes) $H-F_{n}-H$ with (a) $n=3$, (b) $n=6$, (c) $n=9$, (d) $n=12$ and (e) $n=15$. (f) Photograph of dispersions illuminated by UVNis light, value of $n$ indicated below.
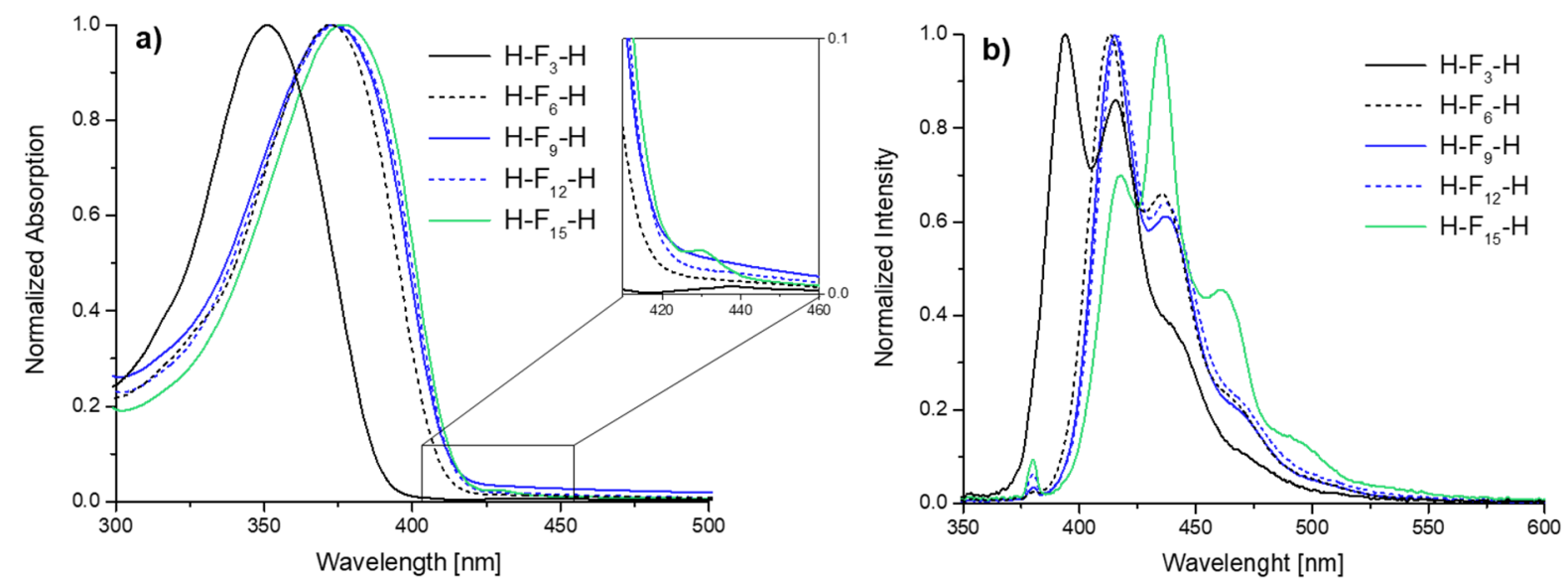

Figure 4.8: (a) UV/Vis absorption spectra of dispersions prepared by post-polymerization emulsification of prefabricated oligo(fluorenes) with enlarged region of $\beta$-phase absorption and (b) the respective fluorescence emission spectra $\left(\lambda_{\text {exc }}=380 \mathrm{~nm}\right)$.

Generally, dispersions of conjugated polymer nanoparticles frequently display overall red- or blue shifts of absorption and emission spectra relative to the spectra observed for organic solutions of the respective polymer. ${ }^{194}$ For nanoparticles prepared by emulsion techniques like the system under investigation, a general blue-shift in absorption would be expected, as the overall conjugation length is reduced by bending and kinking of the rigid polymer chain resulting from the confinement in the small particle volume..$^{91,97}$ In contrast, emission spectra of CPNs are typically red-shifted due to the increased 
occurrence of inter- and intrachain interactions; an effect which intensifies with growing particles size, as the system approaches bulk material properties. ${ }^{92}$ Comparing the optical properties of our postpolymerization generated oligomer particles with spectra of the corresponding oligomers dissolved in tetrahydrofuran (Table 4.3, cf. Figure 9.6), no significant shift of the absorption or emission maxima are apparent for the oligomers with $\mathrm{n} \leq 12$. Therefore, a comparable degree of polymer chain folding and order in dissolved state and in the particles can be assumed, which seems consistent with the data acquired by double electron-electron resonance spectroscopy on shorts-chain oligomers (cf. Chapter 3). The samples exhibited the broad UV/Vis absorption band corresponding to amorphous PF8 as well as the characteristic amorphous phase emission peaks at $415 \mathrm{~nm}, 438 \mathrm{~nm}$ and $468 \mathrm{~nm}$. In contrast, the particle dispersion prepared from the pre-fabricated 15-mer considerably differed from the respective oligomer solution and from the shorter homologues in their optical properties. In the absorption spectrum, a slight, yet assignable signal emerged in the typical region of $\beta$-phase absorption (enlarged section). At the same time, the presence of $\beta$-phase conformation in the 15 -mer particles was even more evident in the emission spectrum. In addition to the diminished amorphous phase peak at $417 \mathrm{~nm}$, a characteristic peak pattern with signals at $435 \mathrm{~nm}, 461 \mathrm{~nm}$ and $495 \mathrm{~nm}$ became observable, which compares well to the fluorescence data of the ellipsoidal particles obtained from standard SuzukiMiyaura polycondensation. As apparent from TEM images of the dispersions prepared, the higher oligo(fluorenes) $(n \geq 9)$ also featured the ability to form defined non-spherical particles, whereas only undefined structures were obtained for the short chain oligomers $(n<9)$ (cf. Figure 4.7).

Table 4.3: UV/Vis absorption and fluorescence emission ( $\lambda_{e x c}=380 \mathrm{~nm}$ ) peak maxima of $\mathrm{H}-\mathrm{F}_{\mathrm{n}}-\mathrm{H}$ oligomers dissolved in THF and of the particles prepared by post-polymerization emulsification of the respective oligomer. ${ }^{a}$

\begin{tabular}{c|c|c|c|c|c}
\multirow{n}{n}{$\begin{array}{c}\mathbf{n} \\
\left(\mathbf{H}-\mathbf{F}_{\mathbf{n}}-\mathbf{H}\right)\end{array}$} & $\begin{array}{c}\boldsymbol{M} \\
{[\mathbf{g} / \mathbf{m o l}]}\end{array}$ & \multicolumn{2}{|c|}{ oligomer solutions } & \multicolumn{2}{c}{ particle dispersions } \\
\cline { 3 - 6 } & $\begin{array}{c}\boldsymbol{\lambda}_{\text {abs, max }} \\
{[\mathbf{n m}]}\end{array}$ & $\begin{array}{c}\boldsymbol{\lambda}_{\text {em, max }} \\
{[\mathbf{n m}]}\end{array}$ & $\begin{array}{c}\boldsymbol{\lambda}_{\text {abs, max }} \\
{[\mathbf{n m}]}\end{array}$ & $\begin{array}{c}\boldsymbol{\lambda}_{\text {em, max }} \\
{[\mathbf{n m}]}\end{array}$ \\
\hline 3 & 1167.93 & 353 & 394 & 351 & 394 \\
6 & 2333.85 & 375 & 415 & 372 & 413 \\
9 & 3499.77 & 377 & 416 & 373 & 415 \\
12 & 4665.68 & 378 & 417 & 374 & 416 \\
15 & 5831.60 & 382 & 416 & 377 & 435
\end{tabular}

${ }^{a}$ Conditions: Dissolution of respective oligo(fluorene) $(5 \mathrm{mg})$, in xylene $(1 \mathrm{~mL})$, rapid injection into the aqueous solution $(10 \mathrm{~mL})$ of Triton $\mathrm{X}-102(5 \mathrm{wt} \%)$, ultrasonication (1 $\mathrm{min}, 60 \%$ amplitude), stirring for at least $24 \mathrm{~h}$.

In conclusion, these findings support the assumption of a critical minimum oligo(fluorene) chain length required for formation of defined, anisotropic particles, with a number of repeat units around $n \approx 10$. Although this critical number of fluorene units is not necessarily exactly the same for the formation of $\beta$ phase and the formation of ellipsoidal particles, respectively, a correlation of anisotropic particle dimensions and internal order of oligo(fluorene) chain packing is indicated by these observations. 


\subsection{EVOLUTION OF MOLECULAR WEIGHT IN THE COURSE OF POLYCONDENSATION}

The previous results showed that the formation of defined, anisotropic particles does not imperatively require a fully developed polymer with $M_{\mathrm{n}}$ values of several $10 \mathrm{~kg} / \mathrm{mol}$, as already fluorene oligomers can serve as the basic material for particle synthesis. Triggered by this finding, the increase of molecular weight in the course of Suzuki-Miyaura polycondensation was monitored by GPC measurements (Table 4.4). To this end, samples were drawn at various stages of polymerization followed by immediate precipitation of the formed polymer in methanol, thereby preventing further polycondensation steps (for details of sample preparation cf. Chapter 8.5.4). Surprisingly in view of the step-growth nature of the polymerization, the molecular weight of the nascent poly(fluorene) showed a rapid increase after the onset of polymerization and the final $M_{\mathrm{n}}$ of around $40 \mathrm{~kg} / \mathrm{mol}$ was virtually reached already at an early stage (2.5 min, that is during the ultrasonication step).

Table 4.4: GPC data of the poly(fluorene) samples drawn after different times of polycondensation reaction.

\begin{tabular}{l|c|c} 
reaction time & $\boldsymbol{M}_{\mathbf{n}}{ }^{a}[\mathbf{k g} / \mathbf{m o l}]$ & $\boldsymbol{M}_{\mathbf{w}} \boldsymbol{M}_{\mathbf{n}}{ }^{a}$ \\
\hline $0 \min ^{b}$ & 0.5 & 1.1 \\
$0.5 \min ^{c}$ & 8.5 & 1.8 \\
$1 \min ^{c}$ & 11.9 & 2.3 \\
$2 \min ^{c}$ & 17.6 & 2.1 \\
$2.5 \min ^{c}$ & 39.6 & 2.8 \\
$1 \mathrm{~h}$ & 37.5 & 2.9 \\
$2 \mathrm{~h}$ & 40.0 & 2.8 \\
$4 \mathrm{~h}$ & 39.8 & 2.7 \\
$6 \mathrm{~h}$ & 43.0 & 2.7 \\
$24 \mathrm{~h}$ & 40.0 & 2.8 \\
$48 \mathrm{~h}$ & 40.6 & 2.7 \\
$72 \mathrm{~h}$ & 41.2 & 2.7
\end{tabular}

aDetermined by GPC with RI detection vs. PS standards. ${ }^{b}$ Mixture of catalyst and AA/BB-type polycondensation monomers prepared according to the procedure outlined in section 8.3.2. 'Precipitation of complete reaction mixture.

Recorded GPC traces indicated a separation of the low molecular weight fraction in distinct signals for each oligomer; over time, these merged into a high molecular weight distribution (Figure 9.11Figure 9.13). After the ultrasonication process, the molecular weight only showed minor fluctuations, 
which are in the order of the accuracy of GPC analyses. Regarding the corresponding fluorescence spectra of the poly(fluorene) samples in solution, no change in the characteristic emission pattern with signals at $417 \mathrm{~nm}, 440 \mathrm{~nm}$ and $471 \mathrm{~nm}$ over time were evident (Figure 9.10). The development of the fluorescence in the early stages of the polymerization experiments was furthermore monitored for aqueous samples. Aliquots drawn were diluted in distilled water and fluorescence emission spectra were recorded immediately (cf. Chapter 8.5.4). They exhibited the emergence of peaks at $440 \mathrm{~nm}, 467 \mathrm{~nm}$ and $498 \mathrm{~nm}$, assignable to $\beta$-phase conformation, already at very short reaction times (1 min) (Figure 4.9).

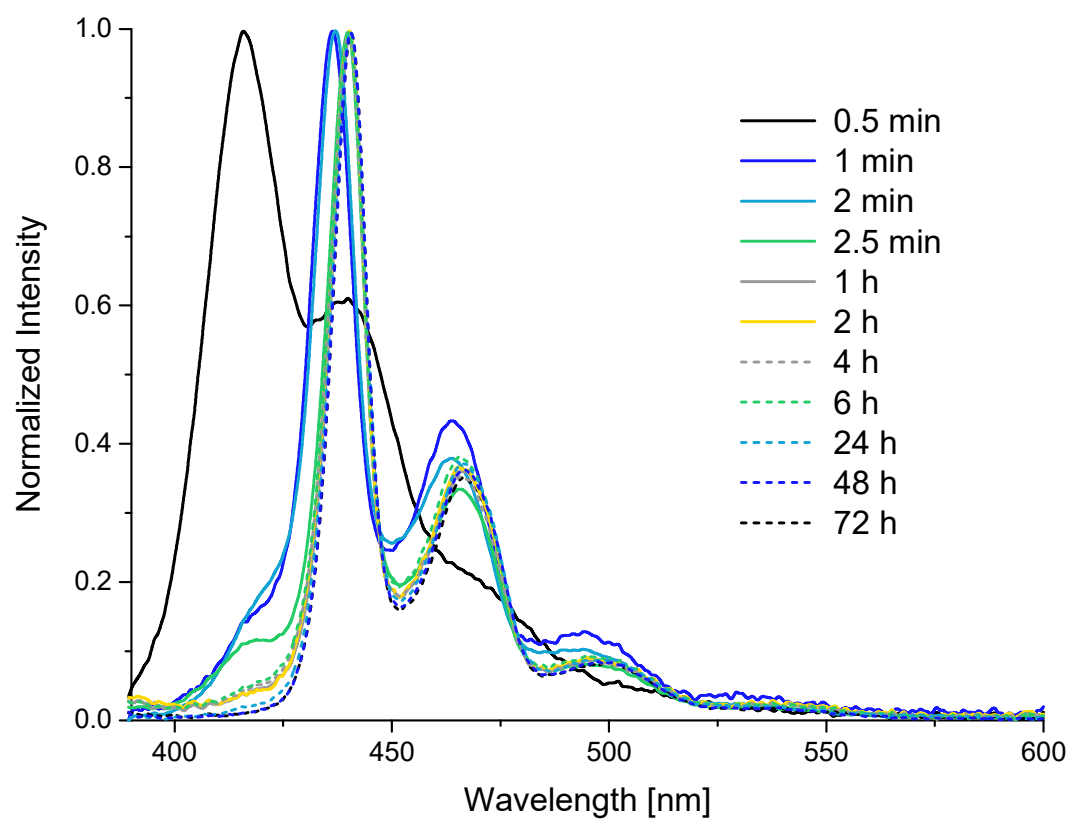

Figure 4.9: Evolution of fluorescence emission properties $\left(\lambda_{\mathrm{exc}}=380 \mathrm{~nm}\right.$ ) over time during Suzuki-Miyaura polycondensation, determined on aqueous aliquots drawn from an ongoing polymerization experiment.

In contrast, signals of mere amorphous poly(fluorene) $(416 \mathrm{~nm}$ and $439 \mathrm{~nm})$ were only observable beforehand and diminished drastically, to be completely absent after one hour of reaction time. The peak maxima of the emission spectra exhibited a red-shift with ongoing polymerization, which stagnated after only a few minutes of reaction time. In agreement with the GPC results, fluorescence spectra acquired at later stages of the polycondensation did not show any significant change. TEM images recorded on aliquots drawn at various stages of the polymerization agreed with these observations, showing the formation of defined particles already in early phases (Figure 9.14). Although keeping in mind that the optical properties of poly(fluorene) are to a large extent dominated by $\beta$-phase regions already at low ratios, both the onset of $\beta$-phase generation and the formation of particles were consequently evident at very early stages of the polymerization reaction.

These findings demonstrate that the heterophase Suzuki-Miyaura polycondensation with step-growth character evidently proceeds in a rapid manner with a large part of the coupling steps occurring within a few minutes. Simultaneously, the growing poly(fluorene) chains form regions of $\beta$-phase conformation already during the first minutes of polymerization within the emulsified droplets of organic solvent. Over the remaining reaction time, the organic solvent slowly evaporates, leaving solid particles with high amounts of ordered phases. 


\subsection{CHAIN-PACKING IN CROSS-LINKED POLYFLUORENE}

To further elucidate the role of chain-packing in the determination of particle shape, the unobstructed alignment of poly(fluorene) chains was disturbed by cross-linking of the forming polymer. To this end, the heterophase AA/BB-type Suzuki-Miyaura polycondensation was performed in the presence of additional tri- and tetrafunctional monomers $\left(\mathrm{B}_{3}\right.$ and $\left.\mathrm{B}_{4}\right){ }^{195}$ Tris(4-bromophenyl)amine, on account of its electron-donating properties a popular branching agent for conjugated polymers, ${ }^{196,197}$ and tetrakis(4bromophenyl)methane, respectively, were employed (Figure 4.10). The presence of the cross-linkers and the resulting occurrence of branched poly(fluorene) chains was expected to favor the formation of spherical particles with low internal chain order over the established non-spherical shape (c, d). The cross-linkers were added to the standard protocol of heterophase Suzuki-Miyaura polycondensation in various ratios $(0.5 \mathrm{~mol} \%$ to $5 \mathrm{~mol} \%)$, replacing the corresponding amount of the dibromo-substituted bifunctional fluorene monomer to retain the overall amount of monomers with both bromo- and boronic acid ester functionalities $(0.2 \mathrm{mmol}$ ) (for details cf. Chapter 8.5.5).
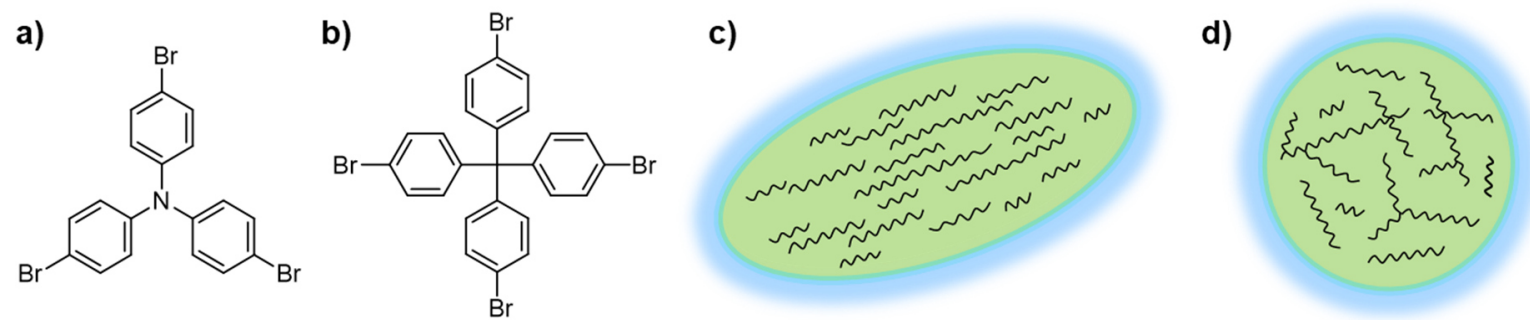

Figure 4.10: Structures of cross-linkers employed in Suzuki-Miyaura polycondensation: (a) tris(4-bromophenyl)amine and (b) tetrakis(4-bromophenyl)methane. Proposed arrangements of (c) linear and (d) cross-linked poly(fluorene) chains, resulting in either ellipsoidal or spherical particles.

GPC analyses (Table 4.5, Figure 9.15 and Figure 9.16) of the polymers formed confirmed that crosslinking indeed occurred, as evident from the emergence of species with extremely high molecular weights which increasingly dominate the molecular weight distribution (except for the case of low amounts of trifunctional cross-linker. Note that GPC only captures the sol fraction of cross-linked samples). At the same time, TEM images of the respective dispersions (Figure 4.11) revealed a strong impact on particle shape and size. Whereas at low amounts of cross-linkers the ellipsoidal shape and homogeneity of the nanoparticles was retained, incorporation of higher ratios $(1.25 \mathrm{~mol} \%$ and $2.5 \mathrm{~mol} \%)$ evidently favored the formation of smaller, less monodisperse particles (coefficients of variation $v$ for both particle lengths and aspect ratios around 0.20-0.40) with a diminished anisotropy. For the maximum amount of $5 \mathrm{~mol} \%$ for both types of cross-linker, monodisperse particles with diameters around $30 \mathrm{~nm}$ $(v=0.08-0.10)$ and virtually spherical dimensions (aspect ratios of $1.4(v=0.12)$ and $1.3(v=0.10))$ were observed exclusively. 
Table 4.5: Molecular weights of cross-linked poly(fluorene), synthesized by Suzuki-Miyaura polycondensation with varying amounts of tris(4-bromophenyl)amine and tetrakis(4-bromophenyl)methane as cross-linking additives, calculated particle dimensions of the respective dispersions and UV/Vis absorption and fluorescence emission $\left(\lambda_{e x c}=380 \mathrm{~nm}\right)$ peak maxima. Data of non-crosslinked standard dispersions are included as reference (last entry). ${ }^{a}$

\begin{tabular}{|c|c|c|c|c|c|c|c|}
\hline \multirow{2}{*}{$\begin{array}{c}\text { multi- } \\
\text { functional } \\
\text { monomer }\end{array}$} & \multirow{2}{*}{$\begin{array}{c}\text { amount }^{b} \\
\text { [mol\%] }\end{array}$} & \multicolumn{2}{|c|}{ peak $M_{\mathrm{n}}^{c}[\mathrm{~kg} / \mathrm{mol}]$} & \multirow{2}{*}{$\begin{array}{l}\text { length } \\
{[\mathrm{nm}] / v^{d}}\end{array}$} & \multirow{2}{*}{$\begin{array}{l}\text { aspect } \\
\text { ratio/ } v^{d}\end{array}$} & \multirow{2}{*}{$\begin{array}{c}\lambda_{\text {abs, } \max } \\
{[\mathrm{nm}]}\end{array}$} & \multirow{2}{*}{$\begin{array}{c}\lambda_{\text {em, max }} \\
{[\mathrm{nm}]}\end{array}$} \\
\hline & & low $M_{\mathrm{n}}$ & high $M_{\mathrm{n}}$ & & & & \\
\hline \multirow{4}{*}{$\mathrm{N}\left(\mathrm{C}_{6} \mathrm{H}_{4} \mathrm{Br}\right)_{3}$} & 0.5 & 35.5 & - & $107 / 0.11$ & $2.9 / 0.09$ & 397 & 440 \\
\hline & 1.25 & 33.3 & - & $56 / 0.27$ & $2.0 / 0.23$ & 397 & 440 \\
\hline & 2.5 & 26.7 & 3308 & $55 / 0.38$ & $1.6 / 0.40$ & 382 & 438 \\
\hline & 5 & 8.9 & 328 & $25 / 0.10$ & $1.4 / 0.12$ & 379 & 436 \\
\hline \multirow{4}{*}{$\mathrm{C}\left(\mathrm{C}_{6} \mathrm{H}_{4} \mathrm{Br}\right)_{4}$} & 0.5 & 30.6 & 2988 & $73 / 0.14$ & $2.4 / 0.11$ & 401 & 442 \\
\hline & 1.25 & 19.7 & 2189 & $53 / 0.24$ & $1.9 / 0.20$ & 380 & 440 \\
\hline & 2.5 & 16.6 & 817 & $34 / 0.14$ & $1.5 / 0.11$ & 378 & 440 \\
\hline & 5 & 4.8 & 643 & $30 / 0.08$ & $1.3 / 0.10$ & 372 & 437 \\
\hline- & - & 42.5 & - & $111 / 0.11$ & $3.5 / 0.11$ & 406 & 441 \\
\hline
\end{tabular}

aConditions: Diboronic acid ester $(0.1 \mathrm{mmol})$ and dibromo-substituted fluorene monomers $(0.1-\mathrm{x} \mathrm{mmol})$, multifunctional monomer $(\mathrm{x} \mathrm{mmol})$ and $\left[\mathrm{Pd}\left(\mathrm{P}^{t} \mathrm{Bu}_{3}\right)_{2}\right](4 \mu \mathrm{mol})$ dissolved in xylene $(1 \mathrm{~mL})$, addition to 5 wt $\%$ aqueous Triton X-102 solution ( $25 \mathrm{~mL}$ ), simultaneous ultrasonication ( $2.5 \mathrm{~min}, 60 \%$ amplitude), $72 \mathrm{~h}$ of stirring. ${ }^{b}$ Referred to the total amount of both AA/BB-type monomers $(0.2 \mathrm{mmol})$. ${ }^{\circ}$ Determined by GPC with RI detection vs. PS standards. ${ }^{d}$ Mean values of lengths, aspect ratio and coefficient of variation $v$ in each case. TEM statistics were calculated from dispersions after purification by redispersion.
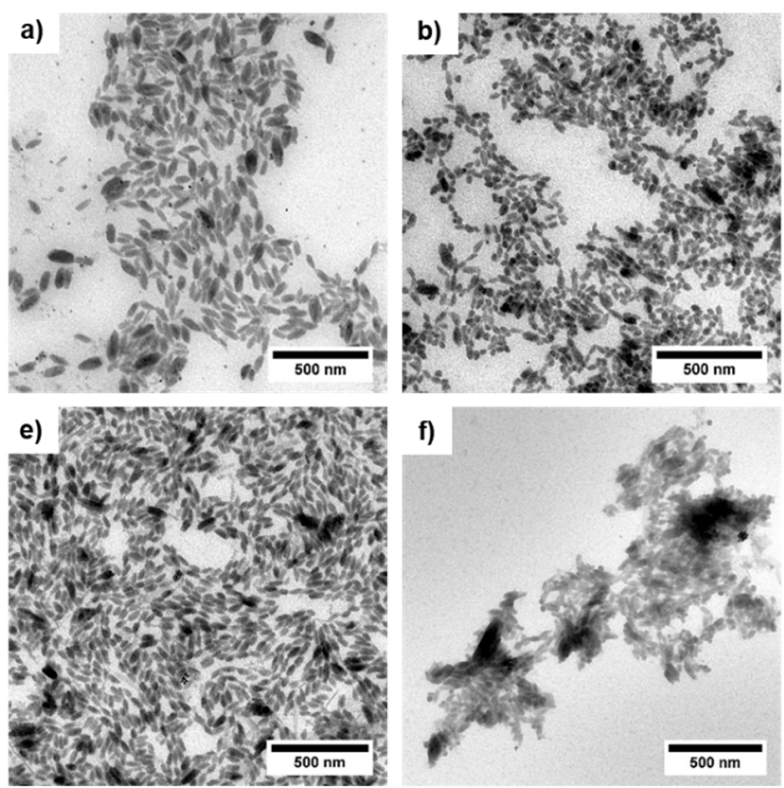
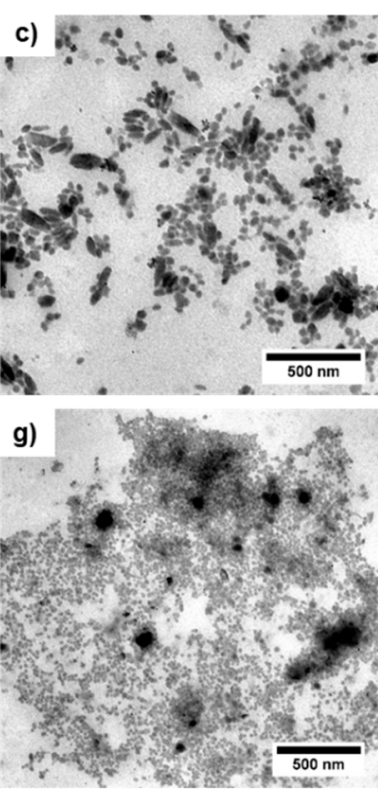
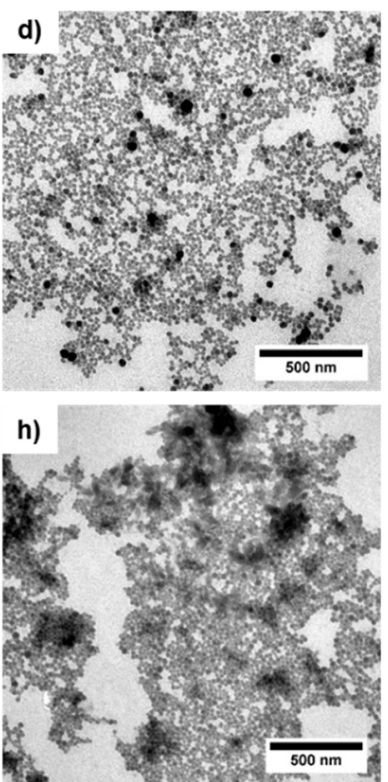

Figure 4.11: TEM images of cross-linked poly(fluorene) dispersions, synthesized by Suzuki-Miyaura polycondensation with variable amounts of cross-linkers. (a) $0.5 \mathrm{~mol} \%$, (b) $1.25 \mathrm{~mol} \%$, (c) $2.5 \mathrm{~mol} \%$ and (d) $5 \mathrm{~mol} \%$ of tris(4-bromophenyl)amine, and (e) $0.5 \mathrm{~mol} \%$, (f) $1.25 \mathrm{~mol} \%$, (g) $2.5 \mathrm{~mol} \%$ and (h) $5 \mathrm{~mol} \%$ of tetrakis(4bromophenyl)methane. 
In parallel to the growing portion of spherical particles with increased cross-linking, significant changes of the polymer morphology also became apparent from UV/Vis absorption spectra of the dispersions (Figure 4.12). With both cross-linkers studied, the characteristic absorption peak at around $440 \mathrm{~nm}$, assigned to $\beta$-phase conformation of poly(fluorene), diminished rapidly with increasing concentrations of cross-linker. The presence of cross-linking points evidently inhibits the planarization of the poly(fluorene) backbone as well as the intermolecular interactions required for the occurrence of $\beta$-phase regions. ${ }^{42}$ At the same time, the drastic increase of molecular weight restricts the mobility of polymer chains within the aromatic solvent droplets.
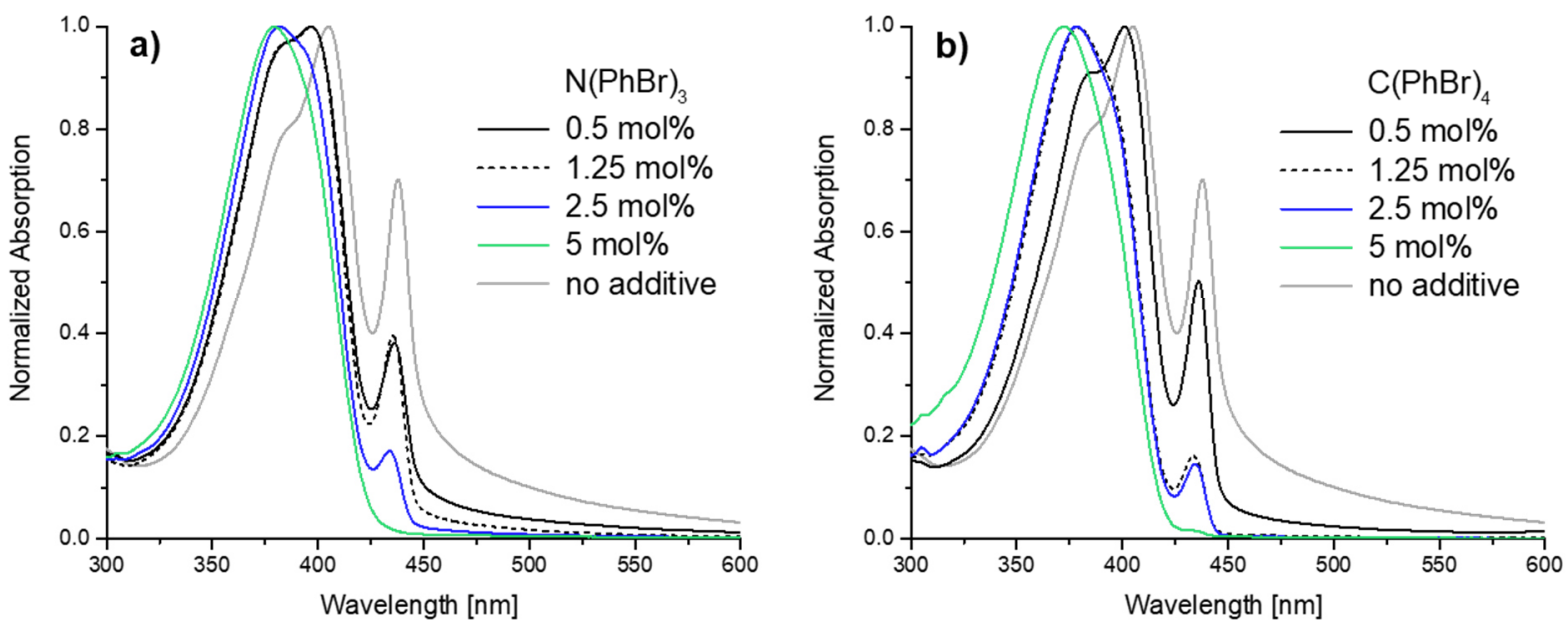

Figure 4.12: UV/Vis absorption spectra of poly(fluorene) dispersions synthesized by polymerization with variable amounts of (a) tris(4-bromophenyl)amine and (b) tetrakis(4-bromophenyl)methane as cross-linkers, with spectra of non-crosslinked standard dispersion as reference (grey lines).
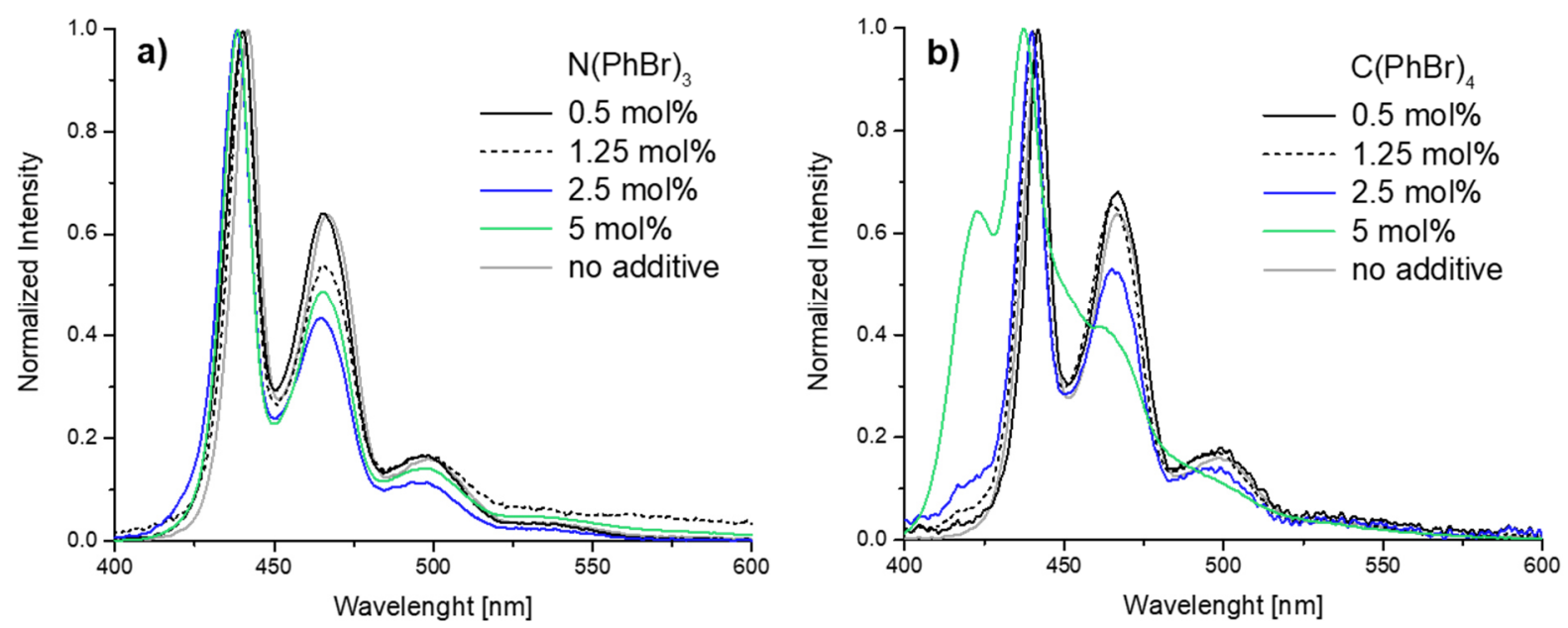

Figure 4.13: Fluorescence emission spectra $\left(\lambda_{e x c}=380 \mathrm{~nm}\right.$ ) of poly(fluorene) dispersions synthesized by polymerization with variable amounts of (a) tris(4-bromophenyl)amine and (b) tetrakis(4-bromophenyl)methane as cross-linkers, with spectra of non-crosslinked standard dispersion as reference (grey lines).

Apart from the suppression of $\beta$-phase formation, a significant blue-shift of the main absorption bands from $405 \mathrm{~nm}$ for non-crosslinked reference particles to $379 \mathrm{~nm}$ for $5 \mathrm{~mol} \%$ of trifunctional cross-linker or $372 \mathrm{~nm}$ for $5 \mathrm{~mol} \%$ of tetrafunctional cross-linker, respectively, was evident. This can be traced to an overall reduction of the conjugation length of the polymer and the decrease of intra- and interchain 
interactions by introduction of cross-links. ${ }^{3}$ The shift of peak maxima to lower wavelengths to a certain extent was also observed for emission spectra, especially for the case of relatively high amounts of tetrakis(4-bromphenyl)methane cross-linker, displaying the emergence of glassy-phase poly(fluorene) signals (Figure 4.13).

In conclusion, cross-linking resulted in a loss of the particle shape anisotropy and a concurrent reduction of $\beta$-phase domains, as the ability of intramolecular alignment was limited. These findings highlight the strong correlation of $\beta$-phase formation and the particles' anisotropy in poly(fluorene) nanoparticles.

\subsection{PHASE BEHAVIOR IN SOLUTION}

By a combination of experimental approaches, the previous chapters have revealed decisive factors of the formation mechanism of anisotropic particles such as the chain length of linear oligo(fluorenes). The evolution of a non-spherical ellipsoidal particle shape was shown to strongly correlate with the evolution of ordered $\beta$-phase regimes. Although the detailed mechanism of $\beta$-phase formation in PF8 remains elusive, numerous studies have been addressing this issue and revealed a dependence of $\beta$ conformation ratio on various factors such as the polymer chain length.9,50,192 These investigations have been source of the overall picture that the $\beta$-phase originates from intramolecular interactions at early stages of formation, followed by the occurrence of intermolecular conformational order emerging from $\beta$-phase domains. ${ }^{44,50}$ In the following, the genesis of $\beta$-phase conformation of poly- and oligo(fluorene) in solution, induced by change in concentration, was investigated in order to give insight into the role of intermolecular processes involved. To this end, solutions of varying concentration were examined by fluorescence spectroscopy, monitoring the presence of $\beta$-phase regions in comparison to disordered PF8 in a fully dissolved state.

\subsubsection{CONCENTRATION DEPENDENT SOLUTION SPECTRA OF POLYFLUORENE}

Prior to the recording of concentration-dependent fluorescence spectra, the measurement parameters of the used instrument were adjusted to the high polymer concentrations. The acquisition time of each measurement was reduced, while the overall number of measurement cycles, over which an average should be found, was increased to enhance the signal to noise ratio (for details cf. Chapter 8.5.6). Polymer concentrations between $0.005 \mathrm{mg} / \mathrm{mL}$ and $2 \mathrm{mg} / \mathrm{mL}$ were investigated. Following the optimized conditions elucidated for the heterophase Suzuki-Miyaura polycondensation (cf. Chapter 8.3.2), xylene was chosen as solvent for the following experiments. Commercial PF8 $\left(M_{n}=27 \mathrm{~kg} / \mathrm{mol}\right)$ served as a substrate. 
For low PF8 concentrations, the emission peak pattern characteristic for polymer in an amorphous or dissolved state was observed, evident from maxima at $416 \mathrm{~nm}$ and $438 \mathrm{~nm}$ and a shoulder at $465 \mathrm{~nm}$, respectively (Figure 4.14). With a gradual increase at generally still low concentration, the peak at approximately $440 \mathrm{~nm}$ became more pronounced. This observation was accompanied by a slight redshift of the entire spectrum, assumedly originating from the enhanced interchain interactions between polymer chain segments such as aggregate formation by $\pi$-stacking. ${ }^{90,92}$ For very concentrated solutions ( $2 \mathrm{mg} / \mathrm{mL})$, the $\beta$-phase conformation constituted the only present morphological species, as revealed by an emission spectrum with peaks at $440 \mathrm{~nm}, 468 \mathrm{~nm}$ and a less pronounced signal at approximately $500 \mathrm{~nm}$. Although the signals were not as sharply defined as for aqueous nanoparticle dispersions (cf. for example Figure 4.3), the respective wavelengths were in good agreement and were unambiguously assignable to PF8 exhibiting $\beta$-phase conformation.

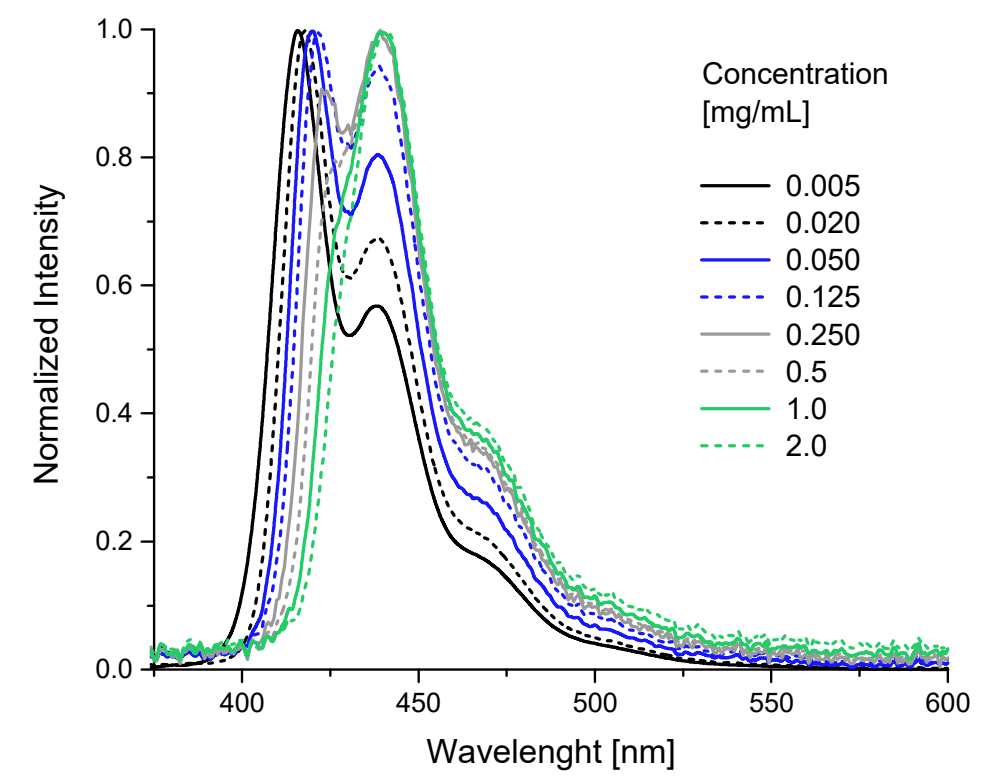

Figure 4.14: Fluorescence emission spectra $\left(\lambda_{e x c}=380 \mathrm{~nm}\right)$ recorded from a concentration series of commercial PF8 solutions in xylene.

As the increased appearance of $\beta$-phase PF8 and the concurrent decrease of disordered, amorphous PF8 presumably occurred in a gradual manner, the identification of a definite concentration inducing the onset of $\beta$-phase formation remained difficult. However, a dominance of $\beta$-phase over other PF8 conformation was observed at $0.25 \mathrm{mg} / \mathrm{mL}$ and higher concentrations, while it constituted the only species to be captured in the emission spectra at the highest concentration investigated $(2 \mathrm{mg} / \mathrm{mL})$.

These findings show a significant dependence of $\beta$-phase appearance on the PF8 concentration. This led to the assumption that the mechanism of $\beta$-phase formation relies to a great extent on intermolecular interactions of polymer chains rather than intramolecular arrangements, whose impacts on the PF8 morphology ought to be observable even at low concentrations. The results presented agree well with former studies on the dynamic behavior of PF8 solutions of varying concentration in toluene and on the formation of gels, induced by cooling and aging of samples. ${ }^{51}$ By means of dynamic light scattering (DLS), a decreased chain mobility was evidenced for less diluted solutions, which was associated with 
the onset of polymer chain aggregation. The corresponding formation of a gel-like material was found to be accompanied by enhancement of $\beta$-phase emission.

With regard to anisotropic PF8 nanoparticles, these intermolecular processes are supposed to be of great relevance in the mechanisms of particle formation. As shown by the experiments concerning the evolution of molecular weight in the course of heterophase Suzuki-Miyaura polycondensation (cf. Chapter 4.3), the final $M_{\mathrm{n}}$ of around $40 \mathrm{~kg} / \mathrm{mol}$ is already reached at early stages of polymerization. The hydrophobic PF8 is dissolved in the organic xylene phase $(1 \mathrm{~mL})$, dispersed as droplets in the continuous aqueous phase $(25 \mathrm{~mL})$, which results in a particularly high polymer concentration within the droplets (approximately $70 \mathrm{mg} / \mathrm{mL}$ ). This value by far exceeds the concentrations found above to be required for a predominant appearance of $\beta$-phase and hence, the polymer chain alignment within the forming particles is strongly favored. This effect is enhanced by the constant evaporation of the remaining xylene during the particle synthesis, which further increases the polymer concentration until the solvent has entirely vanished. The complex correlation of internal particle formation processes, external dimensions and the chosen reaction conditions is highlighted by these findings. In conclusion, apart from the chain length of linear oligo- and poly(fluorenes), the polymer concentration within the dispersed xylene droplets constitutes a decisive factor for the evolution of ordered $\beta$-phase regimes and hence for the mechanism of particle formation.

\subsubsection{CONCENTRATION DEPENDENT SOLUTION SPECTRA OF OLIGOFLUORENES}

From the previous studies on heterophase Suzuki-Miyaura polycondensation with added, presynthesized oligo(fluorenes) of varying chain length (cf. Chapter 4.1) it was concluded that the alignment of polymer or oligomer chains is possible at any stage of the polymerization. Post-polymerization experiments revealed the general ability of oligo(fluorenes) to form stable particle dispersions, similarly to PF8. In this context, a critical oligomer chain length of $\mathrm{DP}_{\mathrm{n}} \geq \mathrm{ca}$. 10 was determined to be required for the formation of anisotropic instead of spherical particles (cf. Chapter 4.2.2). However, the general ability of oligo(fluorenes) to form regions of $\beta$-conformation under appropriate conditions remains unclear.

Following the investigations on phase behavior of PF8 in solution, analogous fluorescence measurements were conducted for the respective oligomers. Solutions of varying concentration were prepared by dissolving pre-synthesized oligo(fluorenes) of different chain length in xylene, followed by the recording of fluorescence emission spectra (Figure 4.15). 


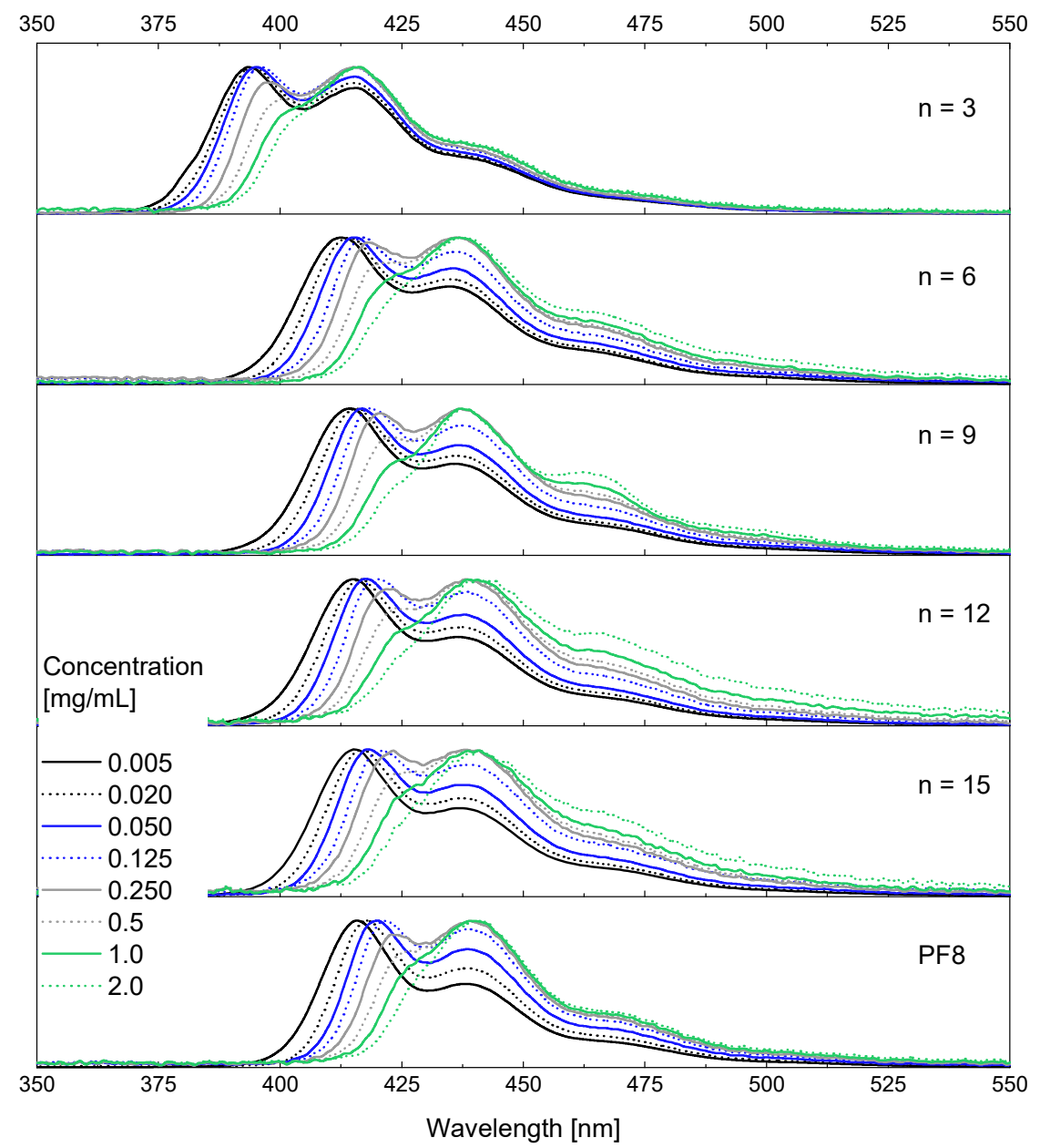

Figure 4.15: Fluorescence emission spectra recorded from a concentration series of solutions of oligo(fluorenes) with increasing number of repeat units $n$ (top to bottom) in xylene, with spectra of commercial PF8 as reference.

Comparing the results with the concentration series of PF8, a strong similarity could be observed for oligomers of $n \geq 6$. All these spectra showed at low concentrations the characteristic emission signals of amorphous or dissolved oligo- or poly(fluorene). Gradually, the peaks at around $440 \mathrm{~nm}, 470 \mathrm{~nm}$ and $500 \mathrm{~nm}$ gained in intensity for higher concentrated solutions, until the signals of $\beta$-phase emission dominated the spectrum for concentrations of $2 \mathrm{mg} / \mathrm{mL}$. The measurements of the pre-synthesized 3 -mer represented an exception as even for the highest concentration investigated no $\beta$-phase induced emission became evident. Instead, the entire set of recorded spectra seemed to be blue-shifted by approximately $25 \mathrm{~nm}$ with regard to the other concentration series, presumably owed to the relatively short conjugation length. Concluding these results, a critical chain length of $\mathrm{DP}_{n}>3$ necessary for oligo(fluorenes) to form regions of $\beta$-phase in solution could be elucidated. Long or medium-length oligomers exceeding this critical value demonstrated a concentration-dependent onset of $\beta$-phase formation, which shows no more correlation with the oligomer chain length. In summary, a similar behavior of poly(fluorene) and oligo(fluorenes) of a certain length was found, concerning the emergence of $\beta$-phase conformation at sufficient concentrations, starting from a state of low chain order. 


\subsection{SELF-ASSEMBLY OF ELLIPSOIDAL POLYFLUORENE NANOPARTICLES}

Beyond the properties of individual nanoparticles, colloidal systems exhibit unique collective behavior, which is especially the case for particles with anisotropic shapes, as they enable direction-dependent interactions. ${ }^{6,111,116}$ Since anisotropic conjugated polymer nanoparticles (CPNs) combine these features with their electroluminescence, their assembly by a suitable method can give access to complex superstructures with extraordinary properties. However, the formation of ordered structures from the ellipsoidal PF8 particles under investigation turned out to be challenging and only small regions of ordered assembly could be evidenced in most cases. Among the broad variety of methods, especially self-assembly approaches driven by evaporation but without further external forces were investigated, including drop-casting, spin-coating, dip-coating and vertical deposition. These techniques have in common that the particles have a distinct tendency to align along the receding front of the evaporating solvent under appropriate conditions, thereby forming small domains of orientation.

Most promising results in terms of particle order were achieved by the approach of vertical deposition, based on the controlled evaporation of dispersions in a vessel containing the desired substrate for particle deposition (Figure 4.16a). ${ }^{198,199}$ Over time, the capillary flow occurring to replenish the evaporated solvent carries the particles towards the contact line of the substrate-solvent-air interphase where they are deposited on the substrate surface. The deposition process and the properties of the resulting film are strongly influenced by various factors such as particle concentration, evaporation speed or the hydrophobicity of the substrate. In the vertical deposition experiments outlined in the following, PF8 particle dispersions obtained from heterophase Suzuki-Miyaura polycondensation according to the abovementioned protocol (cf. Chapter 8.3.2) were used (for details cf. Chapter 8.5.7). Prior to the experiments, the silicon wafers were purified by immersion in an aqueous solution of nascent peroxysulfuric acid, which simultaneously favors the formation of $\mathrm{SiO}_{2}$ groups on the substrate surface, thereby increasing the hydrophilicity.

a)

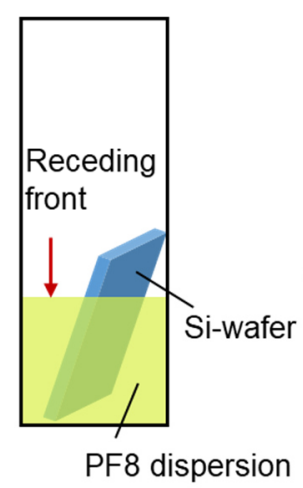

b)

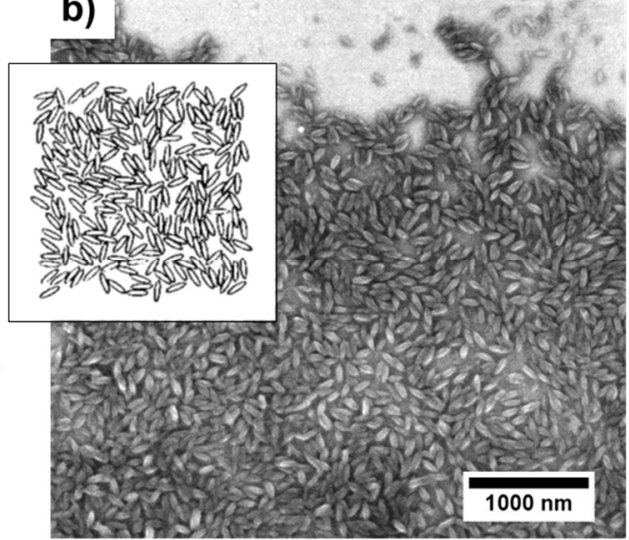

c)

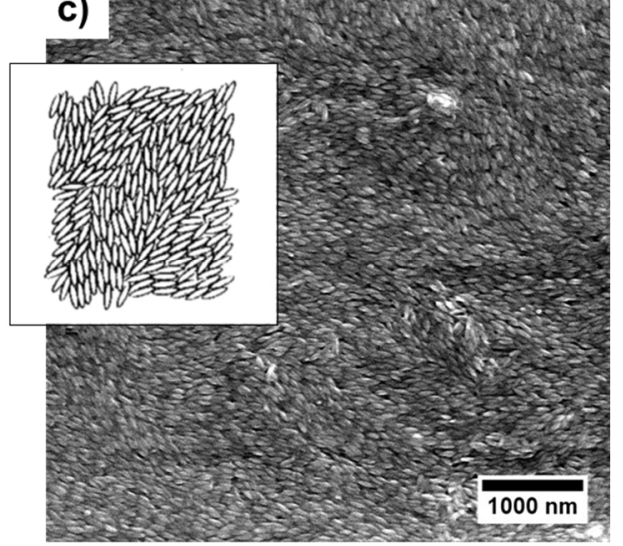

Figure 4.16: (a) Schematic illustration of the vertical deposition technique, including the immersion of a silicon wafer in an evaporating particle dispersion. (b,c) SEM images of the obtained particle films with inserted sections of Monte-Carlo simulations of isotropic phase $(\eta=0.440, b)$ and nematic phase $(\eta=0.785, c)$ of hard prolate ellipsoids of revolution with ar $=4.200$ 
The deposition samples were investigated by scanning electron microscopy (SEM) (Figure 4.16), which revealed both areas of low particle order (b) and regions with a significant degree of interparticle alignment (c). The assembly behavior of uniaxial ellipsoids has been intensively studied by Monte-Carlo simulations of two- and three-dimensional colloidal systems, providing detailed phase diagrams which consider the particle packing density $\eta$ and the particles' aspect ratio. ${ }^{200,201}$ As the simulated hard prolate ellipsoids with an aspect ratio of 4 agreed reasonably with the shape of the synthesized nanoparticles $(\mathrm{ar} \approx 3.5$ ), the corresponding results obtained for two-dimensional systems were compared with the produced particle films under investigation. ${ }^{200}$ The more ordered parts of the specimen surface showed close resemblance with the nematic phase of these simulations, defined by a packing fraction of $\eta=0.785$ (c). Both, the simulated phase and the recorded SEM images clearly showed the appearance of film sections, where nearly all particles are aligned along the same directional axis. The particle order within the nematic phase regions was visualized by performance of an illustrated orientation distribution. Using the software ImageJ, the particles were colored depending on their spatial orientation, which emphasized the partial alignment of the particles relative to each other (Figure 4.17).
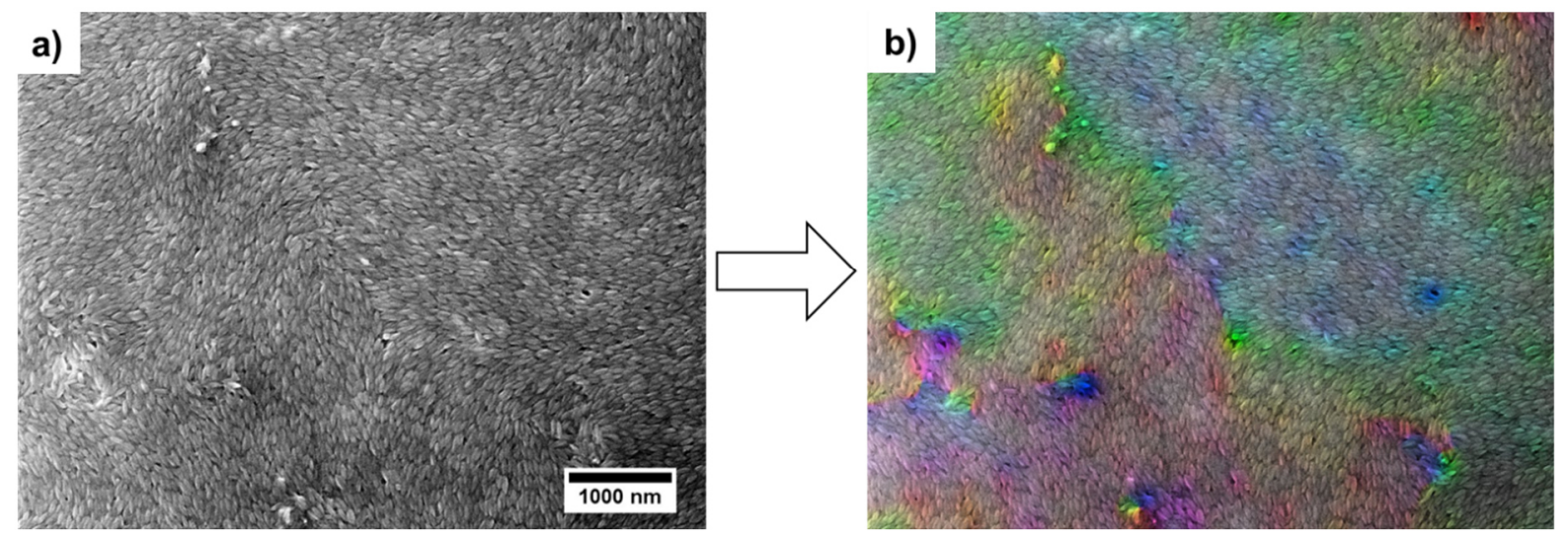

Figure 4.17: (a) SEM image of substrate prepared by vertical deposition of a PF8 particle dispersion and (b) illustrated orientation of the particles.

However, the alignment did not extend over the entire substrate, but was restricted to local order, as the greater proportion of the produced particle film showed similarity to less ordered, isotropic phases with $\eta=0.440$ (Figure 4.16b). A possible reason for this and hence a general drawback of the vertical depositing method might be given by the unsteady thickness of the deposited particle film, which arouses as the particle concentration increases with ongoing evaporation. ${ }^{198}$ As a result, the film thickness is higher in lower regions of the silicon wafer and the optimum concentration required for the onset of nematic phase formation is only ensured in a limited time period of the drying process and hence on a limited area of the substrate.

Conclusively, the abovementioned findings indicate the general possibility to organize ellipsoidal PF8 particles into assemblies of high order, on par with the degree of orientation found in simulated systems with ideal particle uniformity. This constitutes promising preliminary findings for the development of complex superstructures with unique electroluminescent properties. 


\subsection{SUMMARY AND CONCLUSION}

Ellipsoidal poly $\left(9,9^{\prime}\right.$-dioctylfluorene) (PF8) nanoparticles provide promising building blocks for the assembly of complex superstructures, as they are able to form regions of high alignment by appropriate deposition methods. The origin of the anisotropic shape of PF8 nanoparticles synthesized via SuzukiMiyaura-type emulsion polymerization and the mechanisms of their formation were elucidated by a combination of different preparative approaches. These included the performance of the established reaction procedure under altered condition or in the presence of additives such as pre-synthesized oligomers. Given the prerequisite of a suitable aromatic solvent, both oligo( $9,9^{\prime}$-dioctylfluorene) intermediates and poly (9, $9^{\prime}$-dioctylfluorene) were able to align in phases of high order within the forming nanoparticles, as apparent from spectroscopic studies. This was also evidenced by formation of ellipsoidal particles from pre-fabricated polymer or oligomer. To this end, an optimized postpolymerization procedure for the synthesis of non-spherical PF8 nanoparticles was developed as an alternative to the heterophase Suzuki-Miyaura polycondensation. A lower critical oligomer chain length of $D P_{n} \geq c a$. 10 was found to be required for anisotropic particle formation. In order to gain insight into the evolution of polymer chain length and conformation in the course of Suzuki-Miyaura polycondensation, the proceeding reaction was monitored by measurements of molecular weight and fluorescence emission. From these, both the onset of $\beta$-phase generation and the formation of particles was evident at a very early stage of the step-growth polymerization, which proceeded in a rapid manner. Heterophase Suzuki-Miyaura polycondensation was carried out in the presence of multifunctional monomers, proving the presence of linear PF8 to be a prerequisite for the formation of $\beta$-phase regions and of anisotropic particles. Disturbance of ordered chain-packing induced by the presence of crosslinks in the polymer resulted in a loss of non-spherical particle shape. Studies on the behavior of oligoand poly(fluorene) in solution showed significantly greater amounts of $\beta$-phase conformation at increased concentration ( $2 \mathrm{mg} / \mathrm{mL}$ ). For PF8 and oligo(fluorenes) of $D P_{n}>3$ the polymer concentration evidently constituted a decisive factor for the evolution of ordered $\beta$-phase regimes. Concluded from these findings, a mechanism of particle formation was proposed (Scheme 4.2).

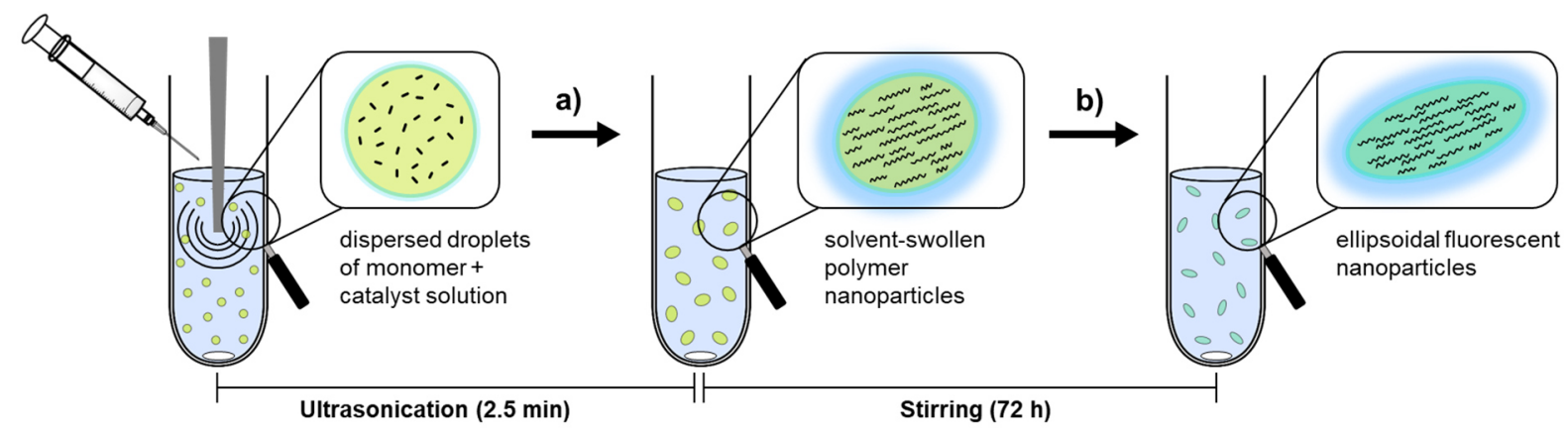

Scheme 4.2: Proposed mechanism of nanoparticle formation including (a) the formation of oligo- and poly(fluorene) chains and their arrangement in ordered phases during the phase of ultrasonication and $(b)$ the evaporation of the organic solvent from the swollen particles, yielding ellipsoidal fluorescent nanoparticles.

The mechanism implies the emulsification of the organic solution of monomers and catalyst in the aqueous phase by high shear force, followed by the rapid formation of oligo- and polymer chains 
immediately after the onset of ultrasonication (a). The growing poly(fluorene) chains form regions of $\beta$ phase conformation already during the first minutes of polymerization by intermolecular interactions, favored by the already high polymer concentration within the emulsified droplets. These are stabilized in the aqueous phase by the present surfactant. With completion of the ultrasonication step (2.5 min), the final $M_{\mathrm{n}}$ of around $40 \mathrm{~kg} / \mathrm{mol}$ is virtually reached. Over the remaining reaction time, the organic solvent slowly evaporates (b), leaving solid particles with high amounts of ordered phases. Overall, the evolution of a non-spherical ellipsoidal particle shape was proven to correlate with the evolution of ordered $\beta$-phase regimes, which shows that polymer nanoparticle shapes can be directed via such chain order. 


\section{POLYDIARYLFLUORENE}

Poly(fluorene) derivatives with bulky aryl substituents have exceptional morphological properties, as they are one of the few conjugated polymers forming regions of $\beta$-phase conformation under suitable conditions..$^{35,39}$ Since they furthermore offer improved stability towards undesirable oxidation reactions at the C-9 position, they are a relevant, yet considerably less investigated alternative to the well-known poly(dialkylfluorenes). ${ }^{15}$ Especially poly(5-octyloxy-9,9'-diphenylfluorene) (PODPF) showed high potential of $\beta$-phase generation, presumably due to the complex interplay of repulsion between bulky phenyl substituents and the octyloxy side chain, inducing attractive van-der-Waals forces. ${ }^{39,55}$ In this chapter, the synthesis of PODPF nanoparticles by different approaches and their shape and size were investigated by various techniques, with the focus on polymer morphology and chain conformation. Initially, the synthesis of the desired monomers bearing either two bromine or boronic acid ester functionalities was aspired. Subsequently, their suitability for heterophase Suzuki-Miyaura polycondensation was evaluated and the reaction conditions were optimized. The resulting particles were analyzed regarding their internal structure, the degree of polymerization and their optical properties.

\subsection{SYNTHESES OF DIARYLFLUORENE MONOMERS}

Unlike alkyl groups, aryl functionalities cannot be attached to fluorene monomers by direct substitution reactions at the C-9 carbon. Instead, alternative pathways had to be developed, which generally include two-step syntheses starting from 9-fluorenone.22 For the system studied, the bromine substituted monomer was synthesized according to a route published by Lin et al. with a Baeyer-Villiger oxidation as one key step (for details cf. Chapter 8.6.1). ${ }^{55}$ The oxidation reaction was followed by introduction of aryl substituents through nucleophilic attack of the corresponding Grignard reagent and ring-closing by Lewis acid-catalyzed condensation (Scheme 5.1). After the efficiently proceeding bromination of 9-fluorenone (a), ${ }^{202}$ the following Bayer-Villiger oxidation (b) required optimization of the reaction conditions in such a way that trifluoroacetic acid and sodium percarbonate as oxidizing species were replaced by $m$-chloroperoxobenzoic acid and sulfuric acid. ${ }^{203}$ Subsequently, the aryl substituents were introduced by reaction of the resulting benzo[c]chromen-6-one with phenylmagnesium bromide as Grignard reagent (c). ${ }^{55}$ The yield of the reaction could be drastically improved by performing the reaction at lower temperatures compared to the literature procedure, which suppressed the formation of biphenyl 
as undesired side product. In contrast, the following alkylation by nucleophilic substitution using 1-bromooctane as reagent under basic conditions (d) proceeded according to literature with satisfying results, as was also the case for the final ring closing reaction by intramolecular Friedel-Crafts alkylation using the complex $\mathrm{BF}_{3} \times \mathrm{Et}_{2} \mathrm{O}$ as catalyzing Lewis acid (e). ${ }^{55}$ The twofold brominated monomer ( $\mathrm{Br}-\mathrm{ODPF}-\mathrm{Br}$ ) was converted to yield the respective boronic acid ester derivative $\left(\mathrm{PrO}_{2} \mathrm{~B}-\mathrm{ODPF}-\mathrm{BO}_{2} \mathrm{Pr}\right)$ by lithiation and borylation on both sides ( $f$ ), thereby completing the set of $A A / B B-t y p e$ monomers. ${ }^{204}$ The corresponding AB-type monomer was obtained in the presence of equimolar amounts of butyl lithium (g), converting only one bromine functionality into the desired bromine acid ester. ${ }^{205}$ Please note, that the respective lithiation did not proceed in a fully regioselective manner. As the octyloxy chain attached to the C-5 position desymmetrisizes the molecule, two different products can be formed. However, the major proportion (approximately $75 \%$ ) of the product mixture was the isomer depicted below, with the boronic acid ester and the alkoxy functionality attached to the same phenyl system. The product was nevertheless considered convenient to be employed in polymerization. The three synthesized monomers as well as the intermediate products were characterized by ${ }^{1} \mathrm{H}$ - and ${ }^{13} \mathrm{C}-\mathrm{NMR}$ spectroscopy (cf. Chapter 9.3.1).
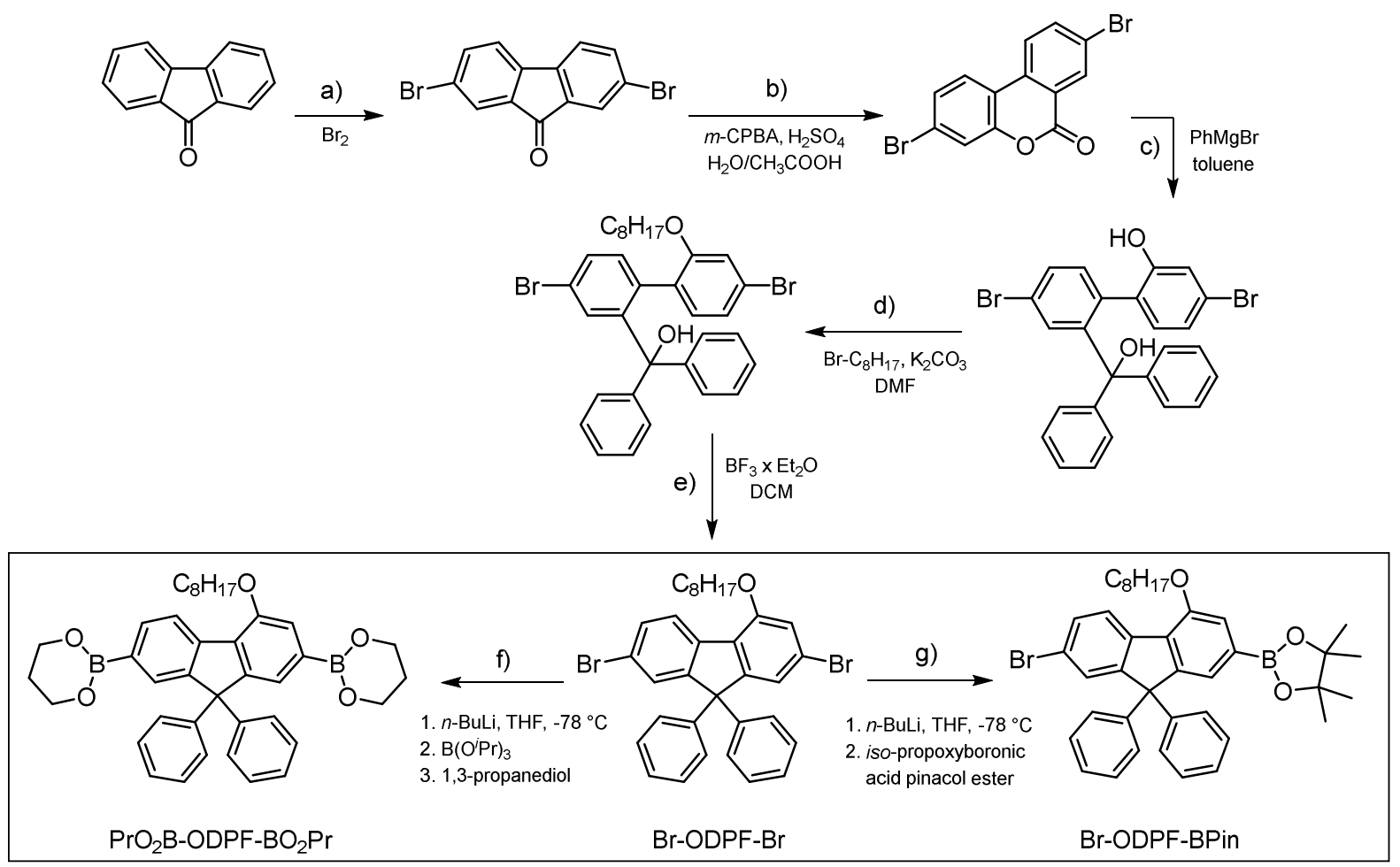

Scheme 5.1: Synthesis route towards 5-octyloxy-9,9'-diphenylfluorene monomers, including (a) bromination of 9fluorenone, (b) Baeyer-Villiger oxidation, (c) introduction of aryl substituents via Grignard reaction, (d) alkylation by nucleophilic substitution, (e) Lewis acid catalyzed condensation and (f, g) borylation. 


\subsection{POLYDIARYLFLUORENE BY EMULSION POLYMERIZATION}

Poly(5-octyloxy-9, $9^{\prime}$-diphenylfluorene) (PODPF) is known for its ability to adapt a $\beta$-phase conformation as one of the few examples apart from poly $\left(9,9^{\prime}\right.$-dioctylfluorene) (PF8). ${ }^{39,55}$ For the latter, the internal arrangement of polymer chains in regions of ordered morphology has been proven to strongly correlate with the external particle dimensions. Therefore, the formation of particles of anisotropic shape and unusual optical properties was anticipated by transferring the emulsion polymerization protocol to the PODPF system. To furthermore extend the scope of heterophase Suzuki-Miyaura polycondensation, the established reaction procedure was performed with the synthesized AA/BB-type 5-octyloxy-9,9'diphenylfluorene monomers described above (for details cf. Chapter 8.6.2). To demonstrate the differences between PF8 and PODPF more clearly, also copolymers were synthesized by using the two monomers sets in different mixing ratios. The resulting materials were compared to the standard poly $\left(9,9^{\prime}\right.$-dioctylfluorene) in view of polymer properties and shape of the resulting particles (Table 5.1). For a 50/50 ratio of the two monomer types (AA/BB-type 9,9'-dioctylfluorene and 5-octyloxy-9,9'diphenylfluorene), the formation of alternating PODPF-PF8 copolymers was expected (entry 2 and 3 ). In contrast, for a reaction mixture of monomers in a 10/90 ratio, the generation of statistical copolymers was assumed (entry 4). With respect to the molecular weight of the polymers and the corresponding distribution, no significant impact of the employed alternative monomer was observed. In order to exclude drastic differences in the reactivity of the PF8 and PODPF monomers, which could adversely affect the polymerization process, the polymerization of a 50/50 mixture was performed twice, with monomers of inverted functionality (entries 2 and 3). More precisely, in addition to the reaction mixture employing $\mathrm{PrO}_{2} \mathrm{~B}-\mathrm{F}_{1}-\mathrm{BO}_{2} \mathrm{Pr}$ and $\mathrm{Br}-\mathrm{ODPF}-\mathrm{Br}$ in equimolar amounts, the procedure was repeated with $\mathrm{PrO}_{2} \mathrm{~B}-\mathrm{ODFP}-\mathrm{BO}_{2} \mathrm{Pr}$ and $\mathrm{Br}-\mathrm{F}_{1}-\mathrm{Br}$, yielding polymers of similar properties.

Table 5.1: Results of heterophase Suzuki-Miyaura polycondensation of AA/BB-type diarylfluorene monomers used in different mixture with the standard $A A / B B$-type dioctylfluorene monomers. ${ }^{a}$

\begin{tabular}{|c|c|c|c|c|c|c|}
\hline \multirow{2}{*}{ entry } & \multicolumn{2}{|c|}{ fractions $^{b}[\%]$} & \multirow{2}{*}{$\begin{array}{c}M_{\mathrm{n}}{ }^{c} \\
{[\mathrm{~kg} / \mathrm{mol}]}\end{array}$} & \multirow{2}{*}{$M_{\mathrm{w}} / M_{\mathrm{n}}{ }^{c}$} & \multirow{2}{*}{$\begin{array}{l}\text { length } \\
{[\mathrm{nm}] / v^{d}}\end{array}$} & \multirow{2}{*}{$\begin{array}{l}\text { aspect } \\
\text { ratio/ } v^{d}\end{array}$} \\
\hline & PODPF & PF8 & & & & \\
\hline $1^{e}$ & 100 & - & 21.9 & 2.5 & - & - \\
\hline 2 & 50 & 50 & 22.5 & 2.9 & $84 / 0.13$ & $1.3 / 0.08$ \\
\hline $3^{f}$ & 50 & 50 & 23.7 & 2.8 & $87 / 0.12$ & $1.5 / 0.13$ \\
\hline 4 & 10 & 90 & 50.1 & 2.5 & $88 / 0.11$ & $1.4 / 0.10$ \\
\hline $5^{g}$ & - & 100 & 42.5 & 2.7 & $111 / 0.11$ & $3.5 / 0.11$ \\
\hline
\end{tabular}

a Conditions: Monomers and $\left[\mathrm{Pd}\left(\mathrm{P}^{t} \mathrm{Bu}_{3}\right)_{2}\right](2.1 \mathrm{mg})$ dissolved in xylene $(1 \mathrm{~mL})$, addition to aqueous Triton $\mathrm{X}-102$ solution $(25 \mathrm{~mL}, 5 \mathrm{wt} \%)$ and $\mathrm{NEt}+\mathrm{OH}(0.16 \mathrm{~g}$ of $20 \%$ aq. solution), simultaneous onset of ultrasonication (2.5 min, $60 \%$ amplitude), $72 \mathrm{~h}$ of stirring. ${ }^{b} \mathrm{As}$ pursued by adjustment of monomer mixture: $\mathrm{PrO}_{2} \mathrm{~B}-\mathrm{F}_{1}-\mathrm{BO}_{2} \operatorname{Pr}(0.1 \mathrm{mmol})$, $\mathrm{Br}-\mathrm{ODPF}-\mathrm{Br}(\mathrm{x} \mathrm{mmol})$ and $\mathrm{Br}-\mathrm{F}_{1}-\mathrm{Br}(0.1-\mathrm{x} \mathrm{mmol})$. 'Determined by GPC with RI detection vs. PS standards. ${ }^{d} \mathrm{Mean}$ values of lengths, aspect ratio and coefficient of variation $v$ in each case. ${ }^{e}$ Monomers: $\mathrm{PrO}_{2} \mathrm{~B}-\mathrm{ODFP}-\mathrm{BO}_{2} \mathrm{Pr}$ $(0.1 \mathrm{mmol})$ and $\mathrm{Br}-\mathrm{ODPF}-\mathrm{Br}(0.1 \mathrm{mmol})$. ${ }^{f}$ Monomers: $\mathrm{PrO}_{2} \mathrm{~B}-\mathrm{ODPF}-\mathrm{BO}_{2} \mathrm{Pr}(0.1 \mathrm{mmol})$ and $\mathrm{Br}-\mathrm{F}_{1}-\mathrm{Br}(0.1 \mathrm{mmol})$. gStandard PF8 particle dispersion for comparison. 
By these findings, a general applicability of the polymerization protocol on diarylfluorene monomers could be evidenced, although the resulting polymers did not reach the molecular weight of the PF8 reference system. Investigations on the dispersions of pure PODPF by microscopy techniques revealed the appearance of fibrous structures as predominant species with negligible quantities of defined particles (Figure 5.1a). For the dispersions of copolymers well distinguishable particles were found which, however, feature a significantly less anisotropic shape as compared to the frequently observed PF8 ellipsoids (b, cf. Figure 9.23). The occurrence of polymer fibers suggested unequal mechanisms of polymer chain arrangement and particle formation as opposed to PF8. These different material properties also became apparent from absorption and emission spectra comparing the homo- and copolymers under investigation (Figure 5.2).
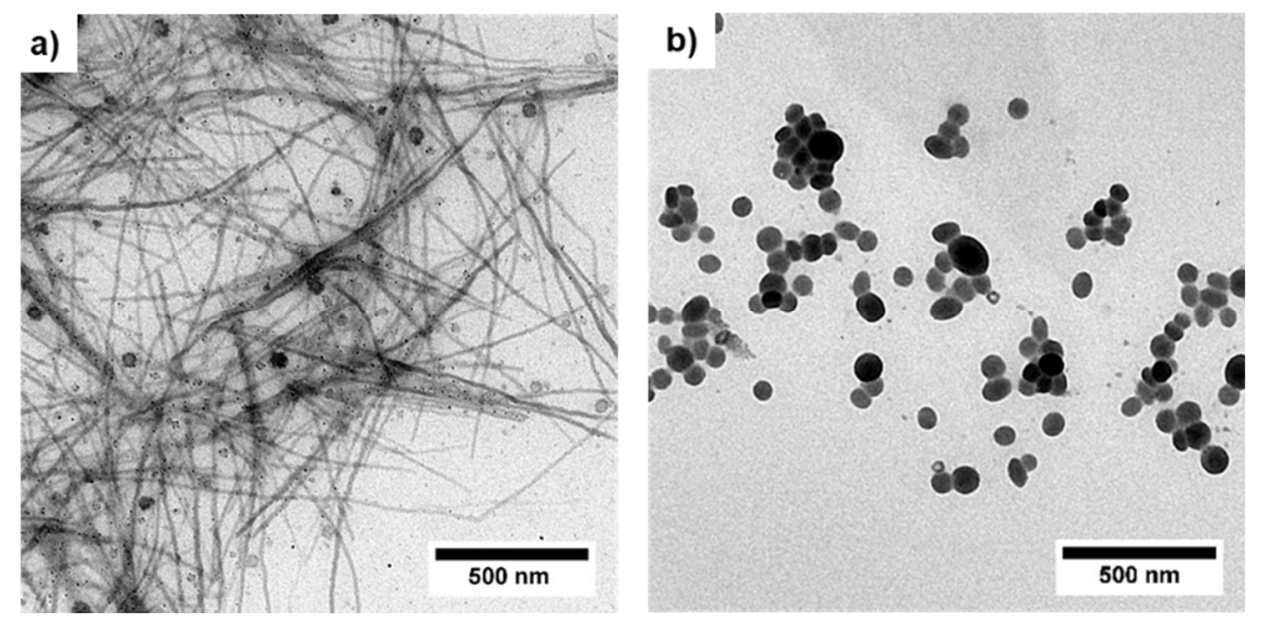

Figure 5.1: TEM images prepared from dispersions of (a) PODPF and (b) alternating poly(5-octyloxy-9,9'diphenylfluorene-9,9'-dioctylfluorene) copolymer synthesized by heterophase Suzuki-Miyaura polycondensation, corresponding to Table 5.1, entry 1 and entry 2.
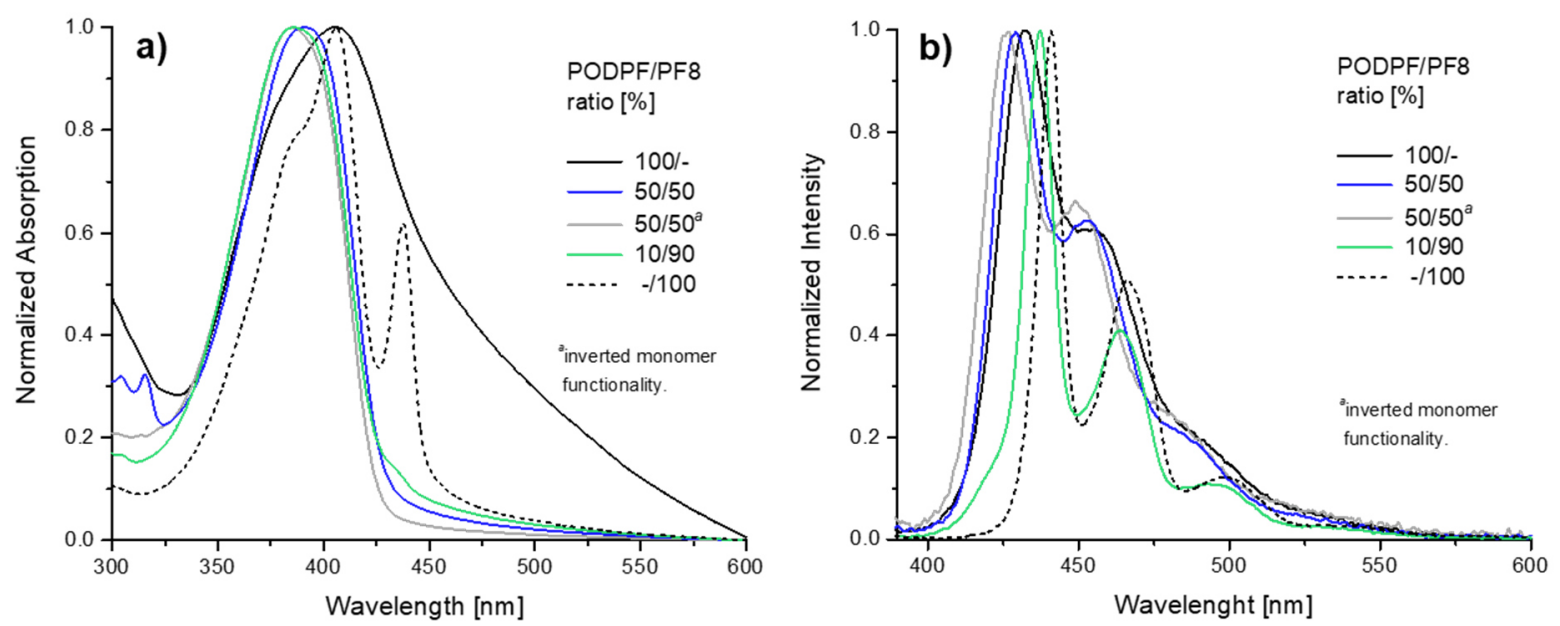

Figure 5.2: (a) UV/Vis absorption and (b) fluorescence emission spectra of dispersions obtained from heterophase Suzuki-Miyaura polycondensation of AA/BB-type 9,9'-dioctylfluorene and 5-octyloxy-9,9'-diphenylfluorene in different ratios.

The sharp peak of $\beta$-phase absorption at $440 \mathrm{~nm}$ diminished significantly in the presence of diarylfluorene monomers. Already at $10 \%$ of diarylfluorene content $\beta$-phase absorption had almost completely vanished, although similar to PF8 an absorption peak in this region was expected to emerge 
upon the occurrence of $\beta$-phase regions in PODPF. Likewise, the corresponding emission peaks at $427 \mathrm{~nm}, 449 \mathrm{~nm}$ and $489 \mathrm{~nm}$ were assignable to amorphous state PODPF by comparison with published literature, while the signals of $\beta$-phase conformation remained absent. ${ }^{39,54,206}$ It can hence be assumed that the polymer backbone planarization required for the formation of $\beta$-phase PODPF in observable quantities involves different requirements than in the case of PF8. Conclusively, under the given reaction conditions, neither the occurrence of $\beta$-phase regions in PODPF nor the formation of defined ellipsoidal nanoparticles could be ensured; an effect which applies to both pure PODPF and for the copolymers studied. Nevertheless, the synthesis of PODPF was provenly possible by emulsion polymerization.

With the aim to find suitable conditions to enable the formation of anisotropic PODPF nanoparticles, the established heterophase polycondensation was adjusted to these demands by variation of several reaction parameters. These included for instance the volume and concentration of the monomer solution employed or the intensity of ultrasonication, in view of the different physical properties such as the reduced solubility of PODPF in most solvents. The dispersions resulting from polymerizations under varying conditions were characterized with regard to their molecular weight, optical properties and the dimensions of the resulting particles (Table 5.2, Figure 9.24 and Figure 9.25). As the synthesis of anisotropic nanoparticles constituted the principal aim of this study, the appearance of all other dispersions was examined by TEM measurements but not further analyzed by calculations of average particle lengths or aspect ratios. The PODPF dispersion synthesized under standard SMPC conditions (cf. Table 5.1) is listed for comparison and as starting point of condition variation (entry 1).

Table 5.2: Heterophase Suzuki-Miyaura polycondensation of AA/BB-type diarylfluorene monomers under varying reaction conditions. ${ }^{a}$

\begin{tabular}{|c|c|c|c|c|c|c|c|c|}
\hline \multirow[b]{2}{*}{ entry } & \multicolumn{4}{|c|}{ organic solution } & \multicolumn{2}{|c|}{ aqueous phase } & \multicolumn{2}{|c|}{ PODPF } \\
\hline & solvent & $\begin{array}{c}\text { volume } \\
{[\mathrm{mL}]}\end{array}$ & $\begin{array}{c}\mathrm{n}_{\text {monomers }}{ }^{b} \\
{[\mathrm{mmol}]}\end{array}$ & $\begin{array}{c}\mathrm{n}_{\mathrm{cat}} \\
{[\mu \mathrm{mol}]}\end{array}$ & $\begin{array}{l}\mathbf{C}_{\text {TritonX }} \\
\text { [wt\%] }\end{array}$ & $\begin{array}{l}\text { US time } \\
\text { [min] }\end{array}$ & $\begin{array}{c}M_{\mathrm{n}}{ }^{c} \\
{[\mathrm{~kg} / \mathrm{mol}]}\end{array}$ & $M_{\mathrm{w}} / M_{\mathrm{n}}{ }^{c}$ \\
\hline 1 & xylene & 1 & 0.2 & 4 & 5 & 2.5 & 21.9 & 2.5 \\
\hline 2 & xylene & 1 & 0.2 & 4 & 5 & 1 & 18.3 & 2.4 \\
\hline 3 & xylene & 1 & 0.2 & 4 & 2.5 & 2.5 & 18.3 & 2.3 \\
\hline 4 & xylene & 1 & 0.2 & 8 & 5 & 2.5 & 16.7 & 2.4 \\
\hline $5^{d}$ & xylene & 1 & 0.2 & 4 & 5 & 2.5 & 2.6 & 1.2 \\
\hline 6 & toluene & 1 & 0.2 & 4 & 5 & 2.5 & 18.0 & 2.5 \\
\hline 7 & xylene & 2 & 0.2 & 4 & 5 & 2.5 & 21.2 & 2.3 \\
\hline 8 & xylene & 2 & 0.1 & 4 & 5 & 2.5 & 15.4 & 2.6 \\
\hline 9 & toluene & 2 & 0.1 & 4 & 5 & 2.5 & 19.5 & 2.4 \\
\hline 10 & toluene & 1 & 0.05 & 4 & 5 & 2.5 & 13.3 & 2.6 \\
\hline 11 & toluene & 1 & 0.05 & 1 & 5 & 2.5 & 14.2 & 2.6 \\
\hline
\end{tabular}

${ }^{a}$ Conditions: $\mathrm{Br}-\mathrm{ODPF}-\mathrm{Br}, \mathrm{PrO}_{2} \mathrm{~B}-\mathrm{ODPF}-\mathrm{BO}_{2} \mathrm{Pr}$ and $\left[\mathrm{Pd}\left(\mathrm{P}^{t} \mathrm{Bu}_{3}\right)_{2}\right]$ dissolved in organic solvent, addition to aqueous phase $(25 \mathrm{~mL})$, containing Triton $\mathrm{X}-102$ and $\mathrm{NEt} \mathrm{t}_{4} \mathrm{OH}(0.16 \mathrm{~g}$ of $20 \%$ aq. solution), simultaneous onset of ultrasonication with $60 \%$ amplitude, $72 \mathrm{~h}$ of stirring. ${ }^{b} \mathrm{Br}-\mathrm{ODPF}-\mathrm{Br}$ and $\mathrm{PrO}_{2} \mathrm{~B}-\mathrm{ODPF}-\mathrm{BO}_{2} \mathrm{Pr}$ used in equimolar amounts in all polymerizations. ${ }^{C}$ Determined by GPC with RI detection vs. PS standards. ${ }^{d}\left[\mathrm{Pd}\left(\mathrm{PPh}_{3}\right)_{4}\right]$ as catalyst. 
As neither the reduction of the ultrasonication time nor the decrease of the surfactant (Triton X-102) concentration showed any advantageous effects concerning the formation of defined particles, the original conditions were assumed to be most suited (entries 2 and 3). The ultrasonication time of 2.5 min appeared to be necessary to ensure sufficient shear force to emulsify the organic solvent in the aqueous phase as stable droplets. The same also applied to the increase of the overall catalyst loading to the double amount or to the replacement of $\left[\mathrm{Pd}\left(\mathrm{P}^{t} \mathrm{Bu}_{3}\right)_{2}\right]$ by the common catalyst precursor $\left[\mathrm{Pd}\left(\mathrm{PPh}_{3}\right)_{4}\right]$ (entries 4 and 5). For the latter, a drastically reduced molecular weight was found, which is in good agreement with results found for the comparison of the two catalyst in the PF8 particle synthesis, revealing a considerably lower reaction rate for $\left[\mathrm{Pd}\left(\mathrm{PPh}_{3}\right)_{4}\right]{ }^{8}$ Especially with regard to the rapid formation of oligomer and polymer chains in early stages of polymerization, as evidenced in abovementioned studies (cf. Chapter 4.3), a sufficient reaction rate provided by the palladium catalyst was considered to be crucial. Compared to the standard PF8 system, both the diarylfluorene monomers and the resulting polymer showed a decreased solubility in xylene. Hence, the usage of ultrasonication was necessary to prepare the organic monomer solution. With regard to this, a series of polymerizations were carried out with reduced monomer concentrations. This was achieved either by increased volumes of the organic solvent, the reduction of monomer amount or by substitution of xylene with toluene, which showed improved solubility behavior for diarylfluorene (entries 6-11). Although ellipsoidal particles were occasionally observable on TEM images of the resulting dispersions, fibrous structures or spherical particles constituted the main component of the samples (Figure 9.24).

Dispersions containing almost exclusively defined, anisotropic nanoparticles could nevertheless be obtained by significant reduction of monomer and catalyst concentration to one quarter of the original amount and the use of toluene as solvent (Figure 5.3, Table 5.2 entry 11). These featured an average length of $92 \mathrm{~nm}(v=0.17)$ and, as a measure of anisotropy, an aspect ratio of $2.5(v=0.15)$.
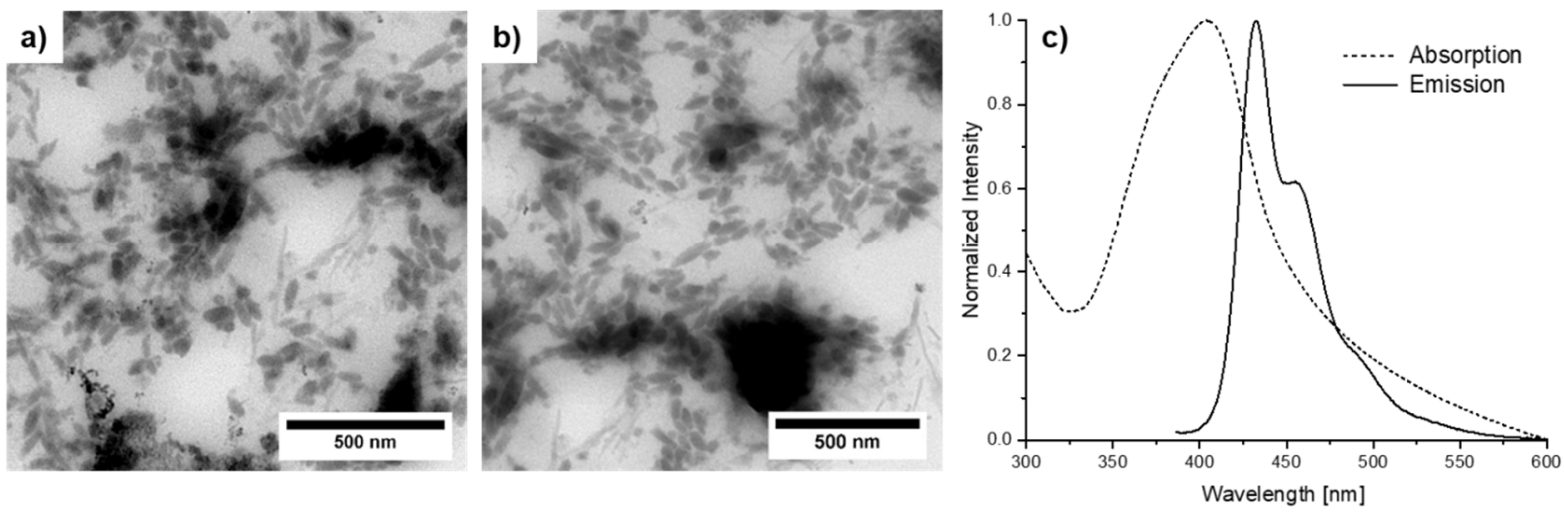

Figure 5.3: $(a, b)$ TEM images of a PODPF dispersion obtained from heterophase Suzuki-Miyaura polycondensation under modified conditions, corresponding to Table 5.2, entry $11(I=92 \mathrm{~nm}(v=0.17), a r=2.5(v=0.15))$ and $(c)$ the respective UVNVis absorption and fluorescence emission spectra.

Although the particles did not reach the quality of standard PF8 dispersions $(I \approx 120 \mathrm{~nm}, \operatorname{ar} \approx 3.5)$ in terms of anisotropy and particle dispersity, these findings proved a general ability of PODPF to form ellipsoidal nanoparticles under carefully adjusted conditions. In contrast to PF8, the formation of particle anisotropy did not obviously correlate with the appearance of $\beta$-phase regions, as indicated by absorption and emission spectra of the dispersion (Figure 5.3c). Also for the case of ellipsoidal particles, 
these showed exclusively signals assignable to polymer in an amorphous state, without apparent presence of $\beta$-phase conformation. ${ }^{54,55,57}$ Considering the molecular weight distributions, exemplarily shown by GPC traces, a bimodal distribution was observed for PODPF synthesized under the initial conditions, which became monomodal for optimized parameters (cf. Figure 9.26). With regard to the general lower solubility of PODPF it was assumed that the polymer chains formed in early stages of the polymerizations precipitated within the emulsified solvent droplets, which impeded further condensation steps. This could be avoided by a general reduction of monomer concentration within the reaction mixture, as enabled by the optimized reaction conditions.

\subsection{CONCENTRATION DEPENDENT PHASE BEHAVIOR IN SOLUTION}

The previous section has shown that poly(5-octyloxy-9,9'-diphenylfluorene) (PODPF) renders the possibility to form anisotropic nanoparticles in a similar fashion as PF8. However, as evidenced from $\beta$ phase absorption and emission of PODPF dispersions, no obvious correlation between the involved particle formation processes and the appearance of regions of high chain order could be ascertained. In order to evaluate the general ability of PODPF to form domains of $\beta$-phase, concentration dependent fluorescence spectra were recorded, analogous to the studies on PF8 outlined in previous sections (cf. Chapter 4.5.1). Polymer solutions of varying concentration in xylene were prepared, using PODPF of high $\left(M_{\mathrm{n}}=22 \mathrm{~kg} / \mathrm{mol}\right)$ and low $\left(M_{\mathrm{n}}=4 \mathrm{~kg} / \mathrm{mol}\right)$ molecular weight. As species of higher $M_{\mathrm{n}}$, PODPF synthesized by emulsion polymerization according to the procedure described above was used (cf. Chapter 8.6.2). The lower $M_{n}$ analogue was obtained from controlled Suzuki-Miyaura coupling polymerization of the respective $A B$-type diarylfluorene monomer. A literature-known procedure established for the synthesis of $P F 8^{72}$ was successfully transferred on the system under investigation (for details cf. Chapter 8.6.3) to yield PODPF $\left(M_{\mathrm{n}}=6.4 \mathrm{~kg} / \mathrm{mol}, M_{w} / M_{\mathrm{n}}=1.5\right)$. The xylene solutions of the two polymer species were investigated by fluorescence emission measurements. Similar to PF8, a distinctly different phase behavior with increasing polymer concentration became apparent (Figure 5.4). For solutions of low concentration, emission peaks at $427 \mathrm{~nm}, 451 \mathrm{~nm}$ and $482 \mathrm{~nm}$ were visible and, in agreement with recent literature, assigned to amorphous state PODPF. ${ }^{39,57}$ With increasing polymer concentration, the peak at $427 \mathrm{~nm}$ diminished while the emission signal at $451 \mathrm{~nm}$ became more enhanced, thereby demonstrating the increasing presence of $\beta$-phase regions and a simultaneous decrease of disordered, amorphous polymer. ${ }^{55,206}$ For the highest concentration investigated $(2 \mathrm{mg} / \mathrm{mL})$, $\beta$-phase polymer constituted the only species captured in the respective emission spectrum. Like in the case of PF8, a definitive 'critical' concentration required for the onset of $\beta$-phase appearance was difficult to determine. Though, a dominance of $\beta$-phase emission was observed for $0.5 \mathrm{mg} / \mathrm{mL}$ and higher concentrations. 

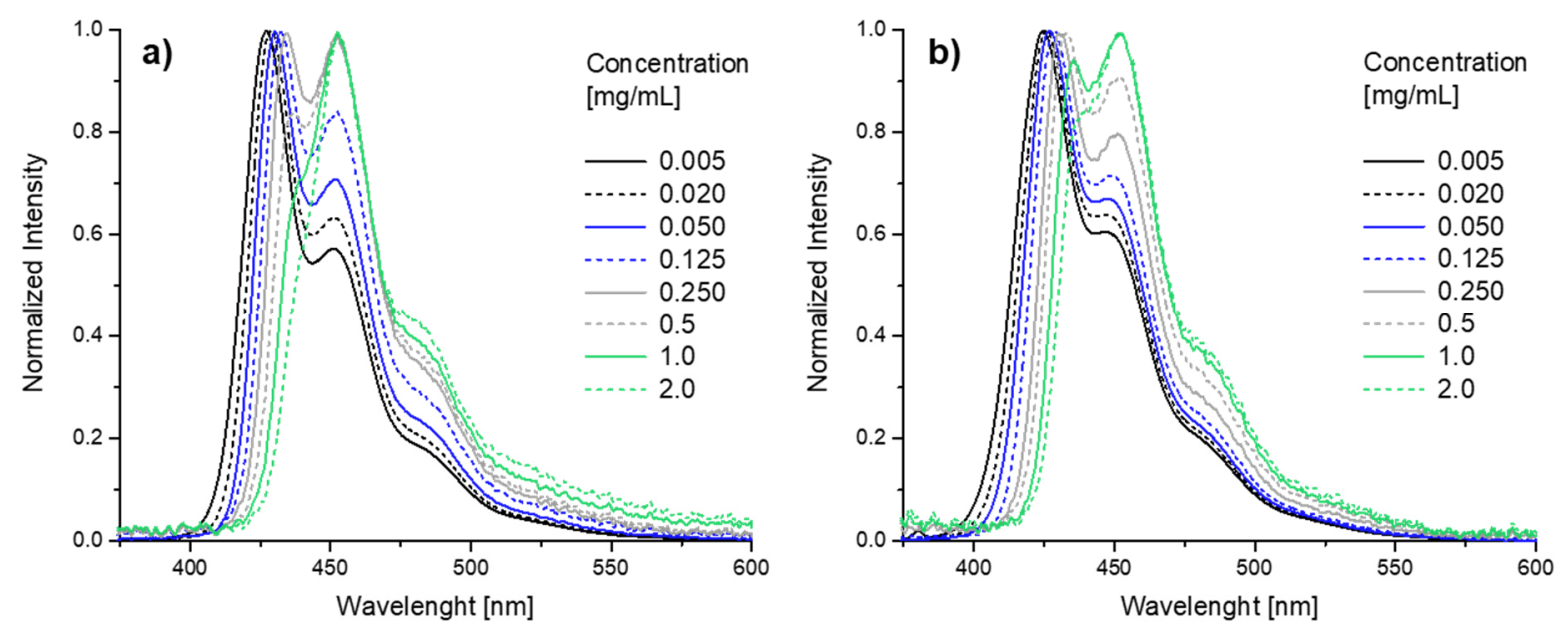

Figure 5.4: Fluorescence emission spectra recorded from a concentration series of PODPF solutions in xylene, prepared from (a) high molecular weight $\left(M_{n}=22 \mathrm{~kg} / \mathrm{mol}\right)$ and (b) low molecular weight $\left(\mathrm{Mn}_{n}=4 \mathrm{~kg} / \mathrm{mol}\right)$ polymer.

For lower molecular weight PODPF, the occurrence of $\beta$-phase regions could similarly be evidenced, but under the prerequisite of higher concentrations. In contrast to PODPF of higher $\mathrm{Mn}$, predominant $\beta$-phase emission was observable above concentrations of $1 \mathrm{mg} / \mathrm{mL}$. Also, emission of amorphous state PODPF was still apparent to a high extent, even at high polymer concentrations. With regard to the PF8 system, which showed an identical phase behavior for polymer chains and oligomer chains of $n \geq 6$, the concentration dependent intermolecular organization to domains of high order in PODPF seemed to be to a higher extent influenced by the polymer chain length. In conclusion, these findings underline the general ability of PODPF to form regions of $\beta$-phase but at the same time emphasizes clear differences in the principles of polymer chain alignment between PODPF and PF8.

\subsection{POST-POLYMERIZATION PARTICLE SYNTHESES}

Similar to the thoroughly studied system of PF8, ellipsoidal nanoparticles have also been found in dispersions of PODPF under carefully adjusted parameters, although the latter have demonstrated to be highly sensitive towards changes of reaction conditions. Additionally, the particle quality did not reach the PF8 dispersions in terms of anisotropy and particle dispersity. For the synthesis of ellipsoidal PF8 nanoparticles, a post-polymerization approach was revealed as valuable alternative to emulsion polymerization (cf. Chapter 4.2). It comprised the emulsification of an organic polymer solution in an aqueous surfactant solution by application of ultrasound. Hence, this method was investigated in view of its applicability on PODPF as starting material and the resulting dispersions were characterized by several techniques, with special focus on the resulting particle shapes. In view of the low solubility of PODPF, various parameters of the post-polymerization protocol were altered in order to provide the best conditions (Table 5.3). 
Table 5.3: Emulsification conditions for the post-polymerization preparation of PODPF particle dispersions. ${ }^{a}$

\begin{tabular}{|c|c|c|c|c|c|c|c|}
\hline \multirow[b]{2}{*}{ entry } & \multirow{2}{*}{$\begin{array}{c}M_{\mathrm{n}}{ }^{b} \\
{[\mathrm{~kg} / \mathrm{mol}]}\end{array}$} & \multirow{2}{*}{$\begin{array}{l}\text { organic } \\
\text { solvent }\end{array}$} & \multirow{2}{*}{$\begin{array}{l}\mathbf{c}_{\text {TritonX }} \\
\text { [wt\%] }\end{array}$} & \multicolumn{2}{|c|}{ ultrasonication } & \multicolumn{2}{|c|}{ TEM data } \\
\hline & & & & $\begin{array}{l}\text { time } \\
\text { [min] }\end{array}$ & $\begin{array}{c}\text { amplitude } \\
\text { [\%] }\end{array}$ & $\begin{array}{l}\text { length } \\
{[\mathrm{nm}] / v^{c}}\end{array}$ & $\begin{array}{l}\text { aspect } \\
\text { ratio/ } / v^{c}\end{array}$ \\
\hline $1^{d}$ & 21.9 & xylene & 5 & 1 & 60 & - & - \\
\hline 2 & 21.2 & xylene & 1 & 1 & 60 & $102(0.13)$ & $2.8(0.14)$ \\
\hline 3 & 21.2 & xylene & 1 & 2 & 30 & - & - \\
\hline 4 & 21.2 & xylene & 5 & 1 & 60 & $108(0.13)$ & $3.3(0.14)$ \\
\hline 5 & 21.2 & toluene & 5 & 1 & 60 & $104(0.11)$ & $3.4(0.12)$ \\
\hline 6 & 21.2 & xylene & 5 & 2 & 30 & - & - \\
\hline 7 & 21.9 & xylene & 5 & 2 & 60 & - & - \\
\hline
\end{tabular}

${ }^{a}$ Conditions: Dissolution of polymer $(3 \mathrm{mg})$ in organic solvent $(1 \mathrm{~mL})$, filtration via $0.45 \mu \mathrm{m}$ PTFE syringe filter, addition to aqueous solution $(15 \mathrm{~mL})$ of Triton X-102 in $50 \mathrm{~mL}$ round bottom flask, simultaneous onset of ultrasonication, stirring for at least $12 \mathrm{~h}$ without cover. ${ }^{b}$ Synthesized by emulsion polymerizations listed in Table 5.2 . ${ }^{c}$ Mean values of lengths, aspect ratio and coefficient of variation $v$ in each case. ${ }^{d}$ Dissolution of 5 mg polymer in $1 \mathrm{~mL}$ of xylene.

As starting material, PODPF previously synthesized by emulsion polymerization and subsequently purified was utilized (for details cf. Chapter 8.6.4). The resulting dispersions were investigated by TEM measurements (Figure 9.27) and, if colloidal systems of defined, anisotropic particles were obtained, the average particle lengths, aspect ratios and the corresponding coefficients of variation $v$ were calculated. For dispersions containing only undefined polymer fragments or fibrous structures, no statistical evaluation was performed, as indicated by a hyphen in the respective table entries. When the conditions approved for PF8 particles were transferred unalteredly to the present system, no evaluable nanoparticles were observable from the TEM images (entry 1 ). With regard to the lower solubility of PODPF, the polymer concentration of $5 \mathrm{mg} / \mathrm{mL}$ was reduced to $3 \mathrm{mg} / \mathrm{mL}$ in the following experiments. Considering the choice of organic solvent, no distinct difference between toluene and xylene became apparent, as for both cases particles of ellipsoidal shape were observed (entries 2, 4 and 5). Likewise, the surfactant concentration of the continuous aqueous phase did not show any influence on the resulting particles. In contrast, the intensity of the shear force applied, adjustable by the duration and amplitude of ultrasonication was revealed as a relevant factor. Extended ultrasonication time resulted in the formation of small, spherical particles or short, fibrous structures instead of defined ellipsoids (entries 3,6 and 7). One possible explanation for this observation is given by the enhanced heat generation within the nascent dispersion by extended ultrasonication, which promotes the evaporation of the organic solvent. As a result, the processes of PODPF chain arrangement within the solvent droplets, already limited by the polymer's solubility, are restricted to a shorter phase of particle formation. Another reason might be the breakage of forming particles into smaller fragments by the persistent application of shear force. 
The best results in terms of particle anisotropy and dispersity were obtained by the emulsification of PODPF, dissolved in toluene, in a $5 \mathrm{wt} \%$ Triton $\mathrm{X}-102$ solution (entry 5 ). Homogenous nanoparticles with an average length of $104 \mathrm{~nm}(v=0.11)$ and an aspect ratio of $3.4(v=0.12)$ were obtained (Figure 5.5a). These nanoparticles were qualitatively almost on par with the well-elaborated PF8 ellipsoids $(I=111 \mathrm{~nm}(v=0.10)$, ar $=3.5(v=0.11))$ and constituted a major improvement in both aspect ratio and particle dispersity as compared to the PODPF particles synthesized via emulsion polymerization $(I=92 \mathrm{~nm}(v=0.17)$, ar $=2.5(v=0.15))$. Similar to the latter studies on emulsion polymerization, no $\beta$-phase absorption or emission was observable in the optical spectra of the PODPF dispersions, which showed exclusively signals assignable to amorphous phase polymer (Figure 5.5b, Figure 9.28).54,57 These became apparent as a broad absorption band at $390 \mathrm{~nm}$ and as emission peaks at $431 \mathrm{~nm}, 457 \mathrm{~nm}$ and $490 \mathrm{~nm}$, respectively.
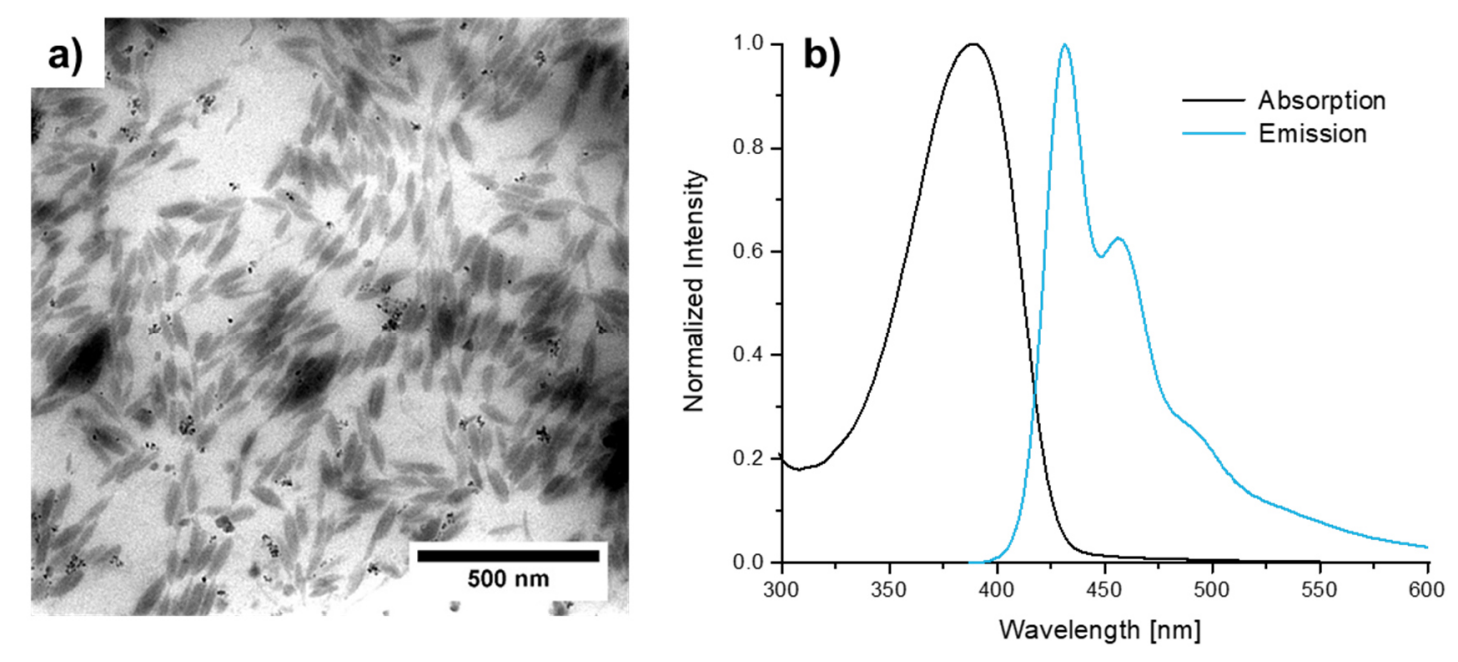

Figure 5.5: (a) TEM images of PODPF particle dispersions prepared by post-polymerization emulsification under conditions corresponding to entry 5 of Table 5.3. (c) Respective UVNis absorption and fluorescence emission spectra.

In conclusion, post-polymerization emulsification under optimized conditions manifested as valuable technique to obtain anisotropic PODPF nanoparticles of high quality, which outperformed particles generated directly in emulsion polymerization in terms of anisotropy and dispersity. In contrast to PF8, the particle shape and the internal polymer chain arrangement into ordered phases were not obviously related, as no presence of $\beta$-phase regions could be spectroscopically evidenced for the ellipsoidal particles. The data showed that also poly(fluorenes) with aryl substituents possess the ability to form ellipsoidal particles, which broadens the scope of accessible conjugated polymer nanoparticles with anisotropic character. 


\subsection{SUMMARY AND CONCLUSION}

Poly(5-octyloxy-9,9'-diphenylfluorene) (PODPF) constitutes a conjugated polymer with a complex interplay of different interactions between the individual polymer chains. These are driven by repulsion between bulky phenyl substituents for one part, competing with attractive van-der-Waals forces induced by octyloxy side chains. ${ }^{35,39,55}$ These internal processes make PODPF a potentially instructive material for the formation of anisotropic nanoparticles and for polymer chain arrangement towards regions of high order, such as $\beta$-phase domains. In this chapter, the synthesis of PODPF and the material's general ability to form nanostructure of anisotropic character were illuminated by various methods. Starting from fluorenone, 5-octyloxy-9,9'-diphenylfluorenes with either two bromine or two boronic acid ester functionalities were synthesized in several steps to yield a set of AA/BB-type monomers. These were employed in the Suzuki-Miyaura polycondensation under heterophase conditions according to the previously established procedure. ${ }^{8}$ As the resulting dispersions contained primarily fibrous, non-defined structures, the protocol was optimized by modification of several conditions, with the monomer concentration in the organic solvent droplets identified as key parameter, to yield exclusively ellipsoidal nanoparticles with an average length of $92 \mathrm{~nm}$ and an aspect ratio of 2.5. In addition to the AA/BB-type monomers, the respective $A B$-type monomer was synthesized and subsequently used in the controlled Suzuki-Miyaura coupling polymerization, which was revealed a suitable alternative to emulsion polymerization, pursuing the synthesis of PODPF. Polymer nanoparticles were also prepared in a postpolymerization approach using pre-synthesized PODPF, which was emulsified in an aqueous surfactant solution. Optimization of the emulsification conditions yielded ellipsoidal nanoparticles $(I=104 \mathrm{~nm}$, ar $=3.4$ ) with improved quality in view of anisotropy and dispersity as compared to particles generated in the course of emulsion polymerization. The synthesized particle dispersions were investigated by acquisition of UV/Vis absorption and fluorescence emission spectra, which revealed the presence of disordered, amorphous phase polymer while the characteristic signals of $\beta$-phase regions remained absent. Nevertheless, the general ability of PODPF to adapt $\beta$-phase conformation was investigated by a series of fluorescence measurements on PODPF solutions of different concentration. Whereas at low concentrations the emission was dominated by amorphous phase signals, the presence of higherordered $\beta$-phase regions was evidenced for higher polymer content. In contrast to PF8, the molecular weight of PODPF seemed to constitute a more crucial factor for $\beta$-phase formation, as the presence of longer polymer chains promote the involved intermolecular processes.

Concluding these findings, poly(5-octyloxy-9,9'-diphenylfluorene) provide the capabilities of both $\beta$-phase evolution and the formation of anisotropic particles. However, unlike in the case of PF8, these two phenomena are not obviously related as far as evident from the experimental spectroscopic observations. Although the internal processes of polymer chain alignment are not necessarily comparable, PODPF and the presented studies broaden the scope of conjugated polymers, which are accessible as nanoparticles with anisotropic shape. 


\section{AMPHPPHLIC BLOCK COPOLYMERS}

One of the most intriguing subjects in nanotechnology and material science is the construction of welldefined, supramolecular structures, which can be approached via noncovalent forces such as hydrophobic and hydrophilic effects or electrostatic interactions. ${ }^{207,208}$ To this end, block copolymers have emerged as important material class, as their tunable composition of covalently linked, different polymer types give opportunity to form precise nanostructures. ${ }^{209}$ Owed to their remarkable optoelectrical features and their rigid backbone character, conjugated polymers, including poly(fluorenes), constitute attractive components in block copolymers. ${ }^{156}$ Upon connection of the hydrophobic, rigid conjugated polymer to a flexible, hydrophilic moiety, rod-coil type block copolymers with an amphiphilic character can be obtained. Given the prerequisite of suitable solvent conditions, these can self-organize, driven by processes of microphase separation. ${ }^{93,142,157}$ As the optoelectrical properties of the conjugated segment can be tuned by its incorporation in amphiphilic systems and the formation of supramolecular aggregates, conjugated rod-coil block copolymers have found application in electronic or luminescent devices. ${ }^{210-212}$ The preparation of conjugated rod-coil block copolymers presents a certain challenge as it requires the linkage of two physically and chemically different block segments. One possible synthetic approach comprises the end-functionalization of the flexible coil segment, which subsequently serves as macroinitiator for the synthesis of the conjugated polymer rod segment, proceeding for instance via transition metal catalyzed polymerization. ${ }^{153,168}$

In addition to poly(fluorenes) serving as material for nanoparticles, as elucidated in the previous chapters, amphiphilic block copolymers with a poly(9,9'-dioctylfluorene) (PF8) segment were studied as reference systems in the following chapter. For this purpose, the preparation of copolymers comprising a PF8-block attached to a hydrophilic poly(ethylene glycol) (PEG) moiety was pursued (Scheme 6.1). Initially, the synthesis of macromolecules consisting of PEGylated bromofluorenes was targeted, followed by the conversion to a catalytically active species by means of an oxidative addition reaction.

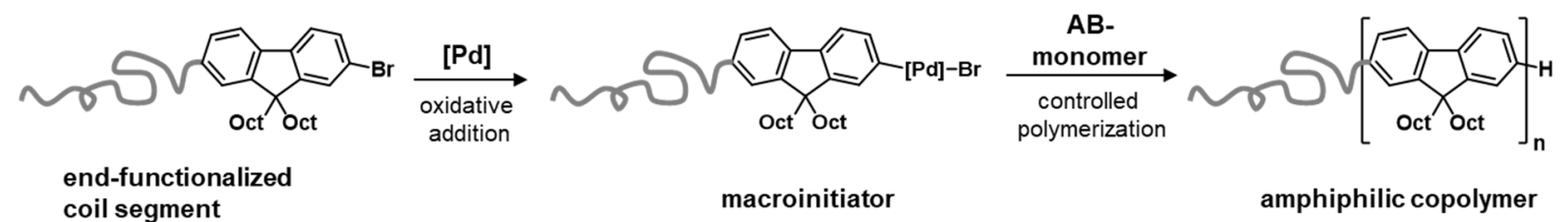

Scheme 6.1: Synthetic approach towards amphiphilic copolymers pursued in this chapter, including the formation of a macroinitiator by oxidative addition of a palladium species to an end-functionalized coil segment (grey line), followed by controlled Suzuki-Miyaura coupling polymerization. 
Starting from the resulting macroinitiators, the synthesis of the conjugated polymer chain via controlled Suzuki-Miyaura coupling polymerization of AB-type monomers was envisaged, thereby evaluating the applicability of the established protocol on the novel system of flexible, hydrophilic macroinitiators. Regarding the characterization of the resulting amphiphilic copolymers, special focus was set on the optical properties and the conformation of the PF8 segment, influenced by the presence of a hydrophilic, covalently linked moiety. In preliminary experiments, the general aggregation behavior of the copolymers in solution and their ability to form hierarchical structures was addressed.

\subsection{SYNTHESES OF MACROINITIATORS}

As counterpart of the hydrophobic conjugated polymers block, poly(ethylene glycol) (PEG), a frequently used moiety in hydrophilic surfactant systems, was chosen as flexible part of the targeted copolymers. To obtain the aryl halide species required for formation of a catalytically active complex, a fluorene monomer was attached to one PEG terminus (Scheme 6.2). The presence of functional groups other than PEG and PF8 with potential unprecedented effects on the self-assembling behavior was thereby avoided; in contrast to other synthetic approaches towards the junction of two segments, which rely for example on click-chemistry reactions and comprise the formation of triazole functionalities. In the synthetic pathway under investigation, the hydroxyl group of a fluorene system was deprotonated under basic conditions. Linkage with an end-functionalized PEG subsequently proceeded via a nucleophilic substitution reaction (for details cf. Chapter 8.7.1). ${ }^{213}$ The PEG-bound bromine atom used in the original protocol was replaced by a tosyl functionality as well-established leaving group to allow for an efficient substitution process. In order to achieve high conversion rates while simultaneously suppressing the occurrence of elimination reactions competing with the substitution, the reaction was carried out at comparably low temperatures $\left(85^{\circ} \mathrm{C}\right)$ over long reaction times.

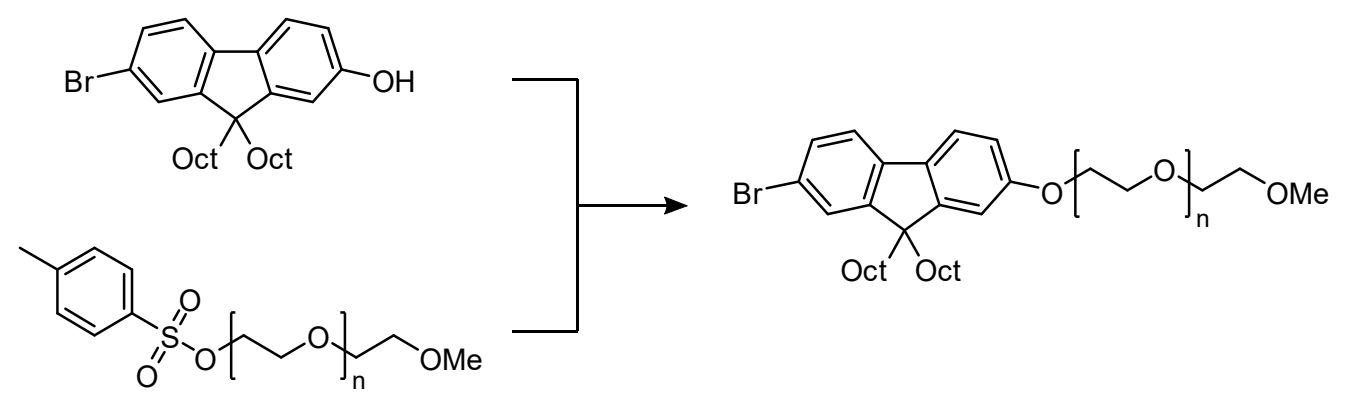

Scheme 6.2: Key synthesis in the route towards fluorene monomers functionalized by poly(ethylene glycol), including a nucleophilic substitution reaction between a tosylated poly(ethylene glycol) and a hydroxyl fluorene.

In this study, commercial PEG monomethyl ethers (mPEG) were used as starting materials, which allowed for a selective tosylation on only one chain terminus, while twofold functionalization was avoided. With the objective to investigate the impact of different degrees of hydrophilicity in the individual copolymers on their properties and aggregation behavior, mPEGs of varying molecular weight were 
applied in the macroinitiator syntheses (for details of synthetic procedures and NMR spectra, cf. Chapters 8.7.1 and 9.4.1). This included the use of mPEG blocks with average molecular weights around $200 \mathrm{~g} / \mathrm{mol}, 500 \mathrm{~g} / \mathrm{mol}$ and $2000 \mathrm{~g} / \mathrm{mol}$, with the $\mathrm{mPEG}-500$ derivatives serving as reference in each case. For the sake of clarity, the average molecular weight of the respective mPEG segment is in the following assigned as subscript in the designation of compounds, for example $\mathrm{Br}_{-} \mathrm{F}_{1}-\mathrm{PEG}_{500}-\mathrm{OMe}_{\text {, }}$ which is based on mPEG-500. Both, the novel nucleophilic substitution products and the tosylated mPEG starting materials, were thoroughly characterized by NMR spectroscopy. The successful formation of the desired target compounds was confirmed by the characteristic ${ }^{1} \mathrm{H}-\mathrm{NMR}$ signals emerging from the ethylene glycol unit directly attached to the aromatic moiety (Figure 9.32). Furthermore, NMR experiments of diffusion-ordered spectroscopy (DOSY) indicated the connection between PEG and aryl to form one molecular species, as both components exhibit the same diffusion behavior (cf. Figure 9.34).

With the PEGylated bromofluorenes in hand, oxidative addition with a palladium source in an in situ type catalyst formation, directly followed by the onset of chain-growth-type polymerization was targeted. In order to initially estimate the general reactivity and applicability of the synthesized macromolecules for the initiator formation, $\mathrm{Br}-\mathrm{F}_{1}-\mathrm{PEG}_{500}-\mathrm{OMe}$ was converted in an oxidative addition step with $\left[\mathrm{Pd}\left(\mathrm{P}^{t} \mathrm{Bu}_{3}\right)_{2}\right]{ }^{70}$ In contrast to the more active in situ type $\mathrm{Pd}-\mathrm{G} 2$ precatalyst used in the following chapter, $\left[\mathrm{Pd}\left(\mathrm{P}^{t} \mathrm{Bu}_{3}\right)_{2}\right]$ was more compatible with the time scales of NMR experiments and was therefore chosen as a model palladium precursor. The progress of the oxidative addition reaction was monitored by ${ }^{31} \mathrm{P}-\mathrm{NMR}$ spectroscopy (Figure 6.1).

a)
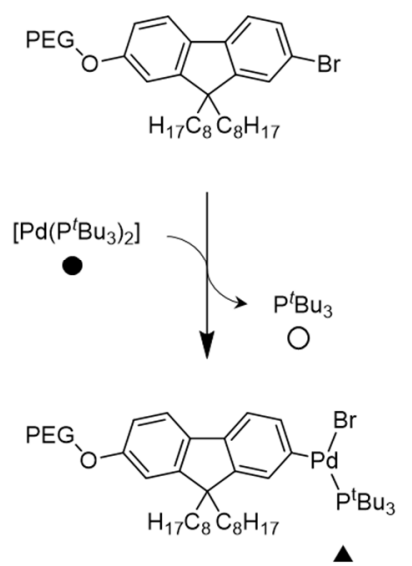

b)

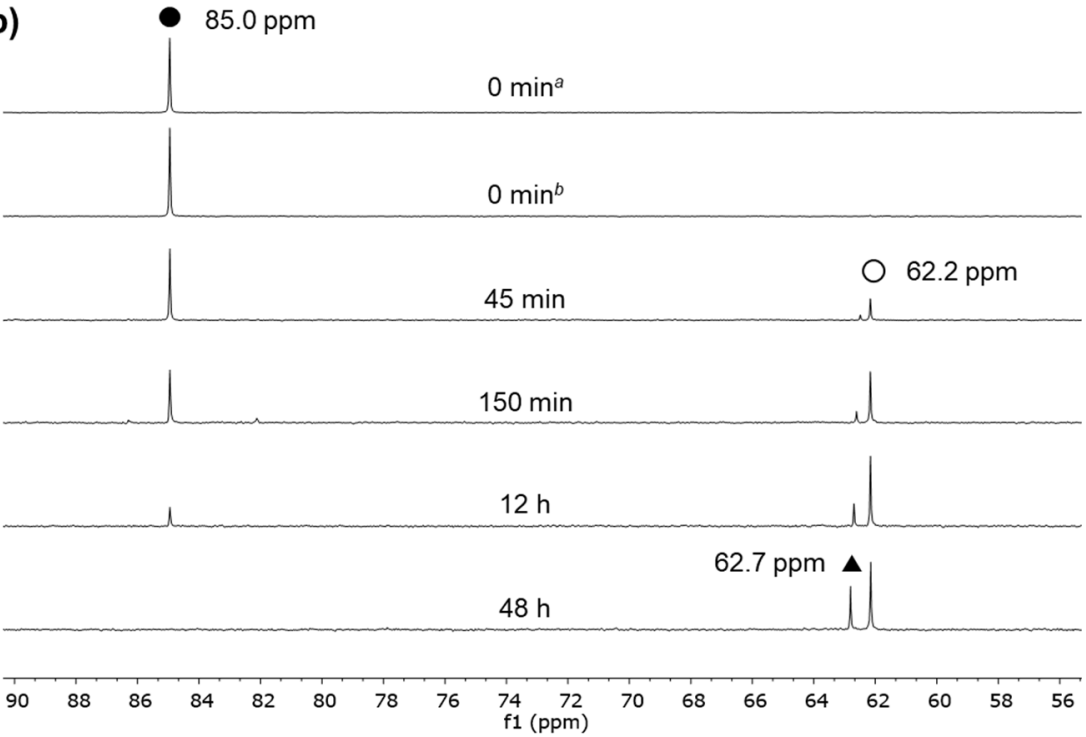

Figure 6.1: (a) Reaction scheme of the oxidative addition of PEGylated bromofluorene to the $\left[P d\left(P^{t} B u_{3}\right)_{2}\right]$ precatalyst and (b) ${ }^{31} \mathrm{P}-\mathrm{NMR}$ spectra $\left(162 \mathrm{~Hz}, C_{6} \mathrm{D}_{6}, 25^{\circ} \mathrm{C}\right)$ after various time intervals. Reaction conditions: $B r-F_{1}-P E G_{500}-O M e\left(60 \mu \mathrm{mol}, 3\right.$ equiv.) and $\left[P d\left(P^{t} B u_{3}\right)_{2}\right]\left(20 \mu \mathrm{mol}, 1\right.$ equiv.) dissolved in $C_{6} D_{6}(1.5 \mathrm{~mL})$, heating to $70{ }^{\circ} \mathrm{C}$. ${ }^{a}\left[\mathrm{Pd}\left(\mathrm{P}^{t} \mathrm{Bu} u_{3}\right)_{2}\right]$ reference. ${ }^{b}\left[\mathrm{Pd}\left(\mathrm{P}^{t} \mathrm{Bu} \mathrm{u}_{3}\right)_{2}\right]$ and $\mathrm{Br}-\mathrm{F}_{1}-\mathrm{PE} \mathrm{G}_{500}-\mathrm{OMe}$.

The signal at $85.0 \mathrm{ppm}$ which diminished over time arose from the precatalyst $\left[\mathrm{Pd}\left(\mathrm{P}^{t} \mathrm{Bu}_{3}\right)_{2}\right]$. In addition to the starting material, two signals of phosphorus species emerged at $62.2 \mathrm{ppm}$ and $62.7 \mathrm{ppm}$ after 45 min of reaction time. The former could be assigned to the free phosphine ligand $\mathrm{P}^{t} \mathrm{Bu}_{3}$, cleaved from the precatalyst in the course of the oxidative addition. The phosphorus species at $62.7 \mathrm{ppm}$ indicated 
the formation of the desired target complex. The corresponding two-dimensional ${ }^{1} \mathrm{H}-{ }^{31} \mathrm{P}-\mathrm{NMR}$ spectra showed clear correlations between a $\mathrm{P}^{t} \mathrm{Bu}_{3}$ species and the aromatic protons of the fluorene moiety, which evidenced the affiliation to the same palladium complex (cf. Figure 9.35).

Conclusively, the results elaborated in the previous section revealed that the linkage of an aromatic moiety with a poly(ethylene glycol) chain by nucleophilic substitution provide a straightforward synthetic approach towards macromolecules with different PEG lengths. Preliminary studies on initiator formation proved the readiness of the PEGylated bromofluorenes to undergo oxidative addition with a suitable palladium source, forming a palladium complex for the synthesis of conjugated copolymers with amphiphilic character.

\subsection{CONTROLLED POLYMERIZATIONS}

The controlled cross-coupling polymerization of AB-type monomers has emerged as a valuable method to access end-group functionalized conjugated polymers by employment of suitable initiators. ${ }^{61,78,214}$ These can either be provided by isolated ${ }^{30,67,75}$ or by in situ formed 77,215 palladium complexes. The former catalyst type entails various disadvantages such as additional synthetic steps of catalyst purification and isolation. These can be omitted by the use of in situ generated complexes. Especially ${ }^{t} \mathrm{Bu} 3 \mathrm{P}^{\mathrm{P}}$-coordinated 2-phenylaniline-based palladacycle complexes have established as both robust and efficient catalyst systems for controlled Suzuki-Miyaura cross-coupling polymerizations (Scheme 6.3). ${ }^{73,74}$

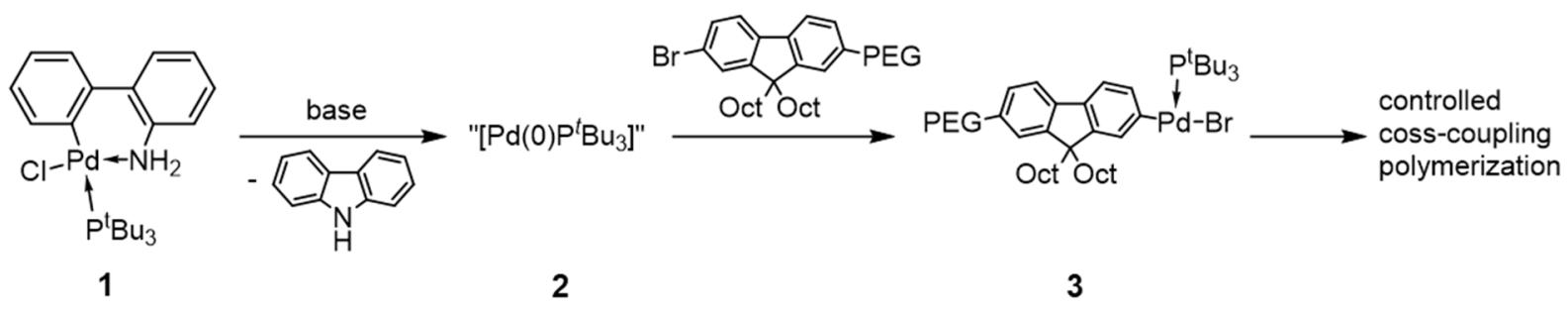

Scheme 6.3: Proposed pathway of the formation of a catalytically active palladium species for controlled SuzukiMiyaura coupling polymerization from a phosphine-coordinated 2-phenylaniline-based palladacycle complex. Adapted with permission from the American Chemical Society (ACS Macro Lett. 2016, 5, 656-660, Copyright 2021).

Such initiators avoid the formation of the less reactive complex $\left[\mathrm{Pd}(0)\left(\mathrm{P}^{t} \mathrm{Bu}_{3}\right)_{2}\right]$, in contrast to initiator systems with excessive use of phosphine ligands. Instead, the phenylaniline moiety is under basic conditions released from the precatalyst (1) as carbazole and a highly reactive 12-electron $\left[\mathrm{Pd}(0)\left(\mathrm{P}^{t} \mathrm{Bu}_{3}\right)\right]$ species (2) is formed. ${ }^{72}$ The latter subsequently reacts with an aryl halide in an oxidative addition to form the catalytically active palladium species (3). In this work, the previously synthesized PEGylated bromofluorene macromolecules constituted the respective aryl halide species, which also provides the end functionality of the forming polymer chain. 
Accordingly, the ${ }^{t} \mathrm{Bu}_{3} \mathrm{P}$-coordinated 2-phenylaniline-based palladacycle complex (Pd-G2) was converted to the catalytic active species by dissolution along with the desired aryl halide in a two-phase mixture of organic solvent and aqueous, basic phase. The initiator mixture containing the active catalyst was added to a solution of the AB-type monomer under investigation to start the polymerization process (for details, cf. Chapter 8.7.3). In reference to the procedure reported in literature, ${ }^{72}$ both the time for the in situ initiator formation and the polymerization time were shortened to 15 minutes in order to maintain a narrow molecular weight distribution and to avoid undesired side reactions such as catalyst decomposition or chain transfer reactions. Upon extended times for initiation and polymerization, a bimodal molecular weight distribution emerged, which was not the case for short time periods (Figure 9.36).

\subsubsection{COPOLYMERS WITH POLYFLUORENE SEGMENT}

In general, the controlled polymerization of AB-type fluorene monomers starting from a PEGylated fluorene moiety was proven to be feasible and amphiphilic copolymers were synthesized. In the following studies, the copolymer synthesized in the presence of 5 equivalents of AB-type monomer, referred to the catalytic palladium species, was chosen as a reference compound for the more detailed characterizations, including one- and two-dimensional NMR spectroscopy (Figure 6.2, cf. Figure 9.37Figure 9.39).

a)
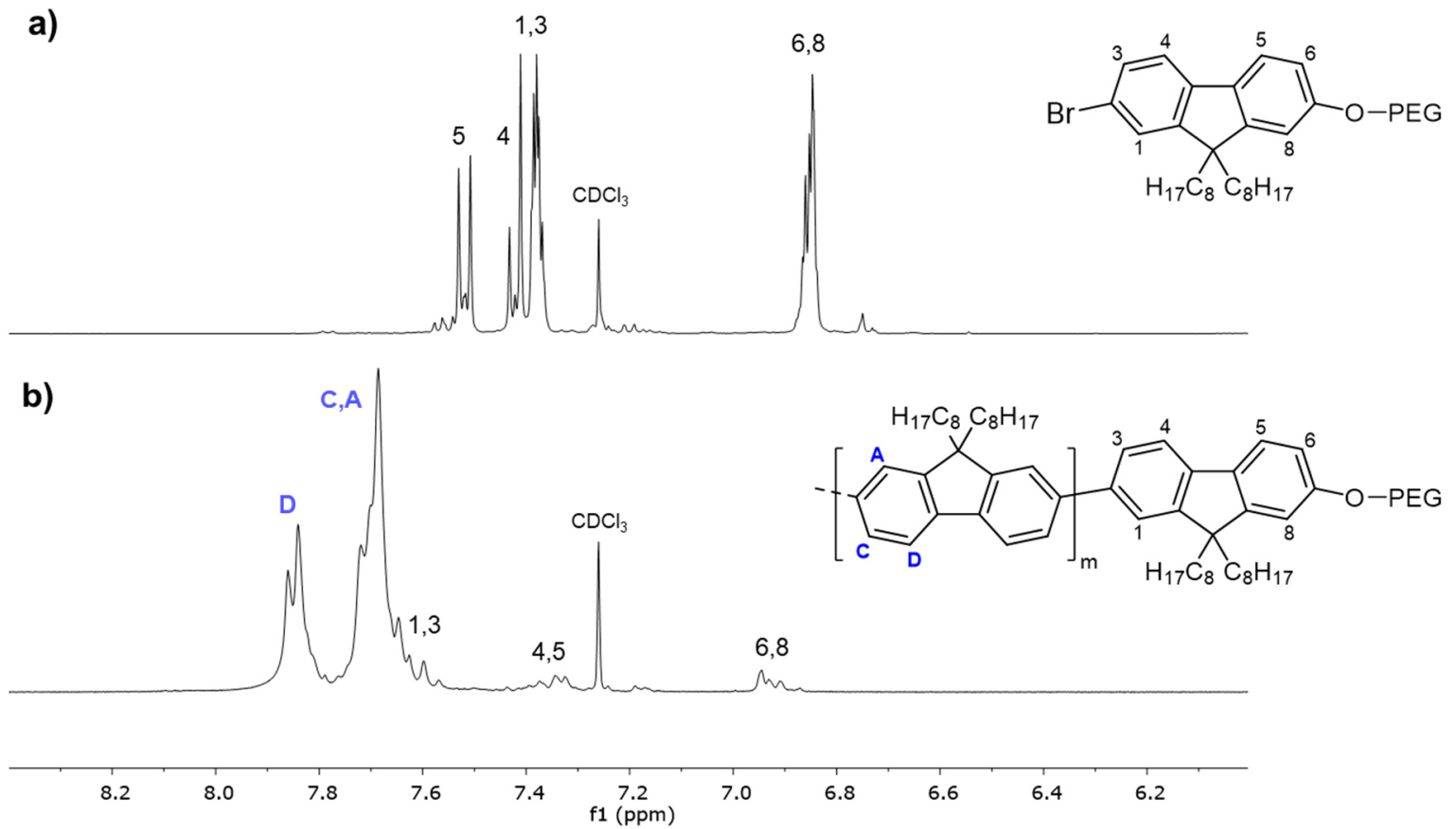

Figure 6.2: Stacked ${ }^{1} \mathrm{H}-\mathrm{NMR}$ spectra $\left(400 \mathrm{MHz}, C D \mathrm{Cl}_{3}, 25{ }^{\circ} \mathrm{C}\right)$ displaying the aromatic regions of (a) Br-F1-PEG500-OMe and (b) amphiphilic PF8-PEG copolymer resulting from controlled Suzuki-Miyaura coupling polymerization, starting from a PEGylated macroinitiator. 
Upon incorporation into the growing poly(fluorene) chain, the ${ }^{1} \mathrm{H}-\mathrm{NMR}$ signals of the PEG-bound fluorene moiety (Figure 6.2a) diminished and the broad signals (,$C$ and $D$ ) of the aromatic poly(fluorene) backbone emerged. The signals assignable to the fluorene monomer in direct vicinity of the PEG chain were observable in strongly decreased intensity as compared to the predominant conjugated polymer backbone, which evidenced the conversion of the starting material and the successful formation of PF8 (cf. also Figure 9.39). Diffusion ordered spectroscopy (DOSY) revealed the same diffusion behavior for the protons associated with both the poly(fluorene) and the poly(ethylene glycol) segment, proving that the two components belong to the same molecular species (cf. Figure 9.40). In addition to NMR spectroscopy, the linkage of the poly(fluorene) and the poly(ethylene glycol) segments was confirmed by matrix-assisted laser desorption/ionization time-of-flight (MALDITOF) mass spectrometry (Figure 6.3).
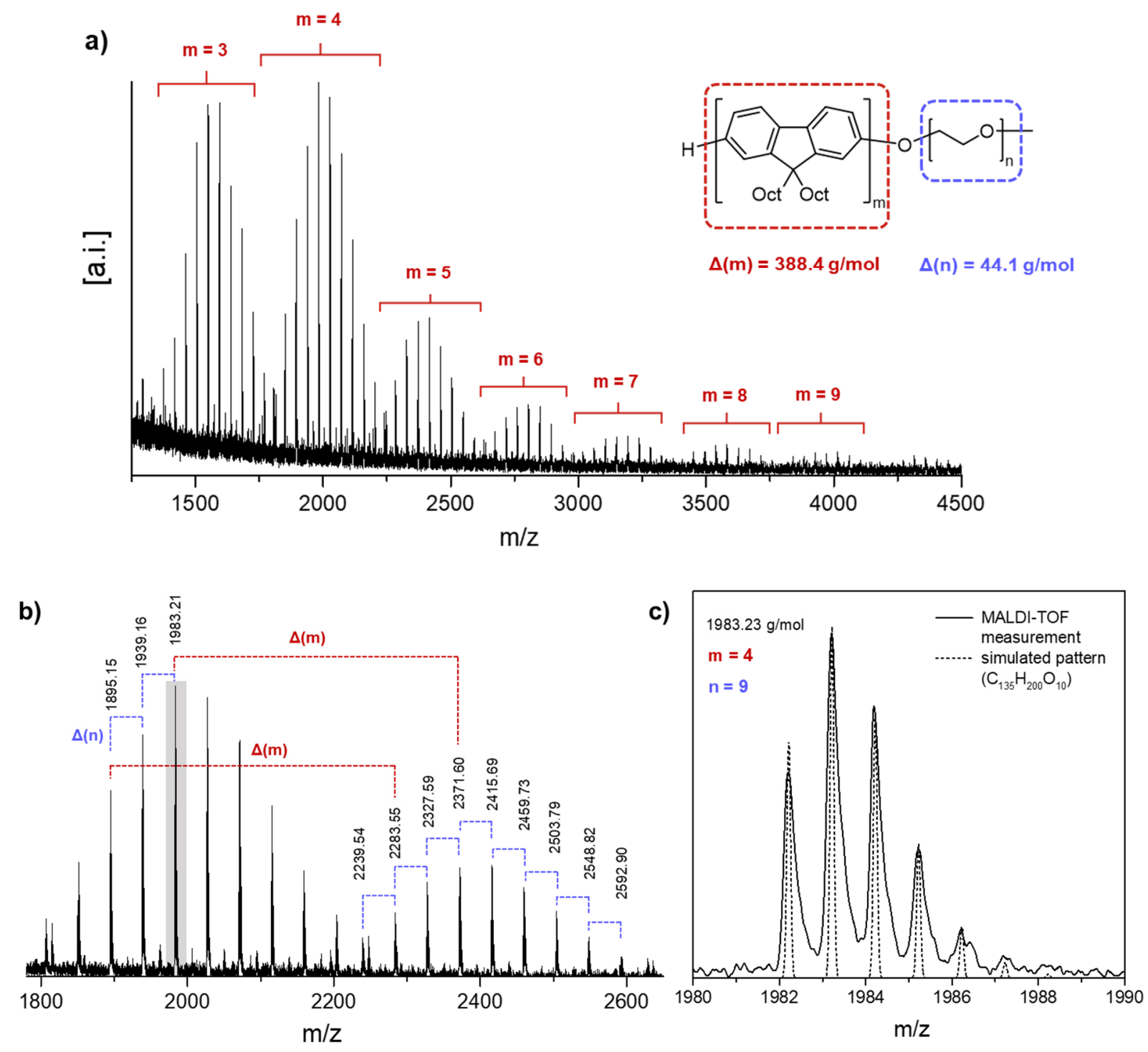

Figure 6.3: (a) MALDI-TOF mass spectrum of an amphiphilic PF8-PEG block copolymer with corresponding molecular structure and assigned regions corresponding to different numbers $m$ of fluorene repeat units. (b) Enlarged section for the region of $m=4$ and $m=5$ with visualized differences for varying numbers of fluorene $(m$, red) and ethylene glycol ( $n$, blue) repeat units. Grey background highlights the most dominant species of the spectrum. (c) Superposition of the highest signal of the mass spectrum and the respective simulated pattern for a species of 4 fluorene and 9 ethylene glycol units. 
For each number of fluorene monomer units $(\mathrm{m})$, an individual peak distribution, resembling a Poissontype distribution, can be observed. This results from the ensemble of different PEG lengths present in the PEGylated fluorene precursor, as furthermore confirmed by the repeating mass difference of $44 \mathrm{~g} / \mathrm{mol}$, which exactly corresponds to the molecular weight of one ethylene glycol. For each 'secondary' distribution, the maximum values were found for PEG segments with 9 or 10 repeat units (n). The species with the most pronounced signal in the mass spectrum is represented by a block copolymer of $m=4$ and $n=9$ (enlarged section $c$ ) and the signal shape agrees excellently with a simulated isotopic pattern. Please note that with varying lengths of each segment, the copolymers feature different properties in view of ionization in the course of the MALDI-TOF measurement and the depicted mixture distribution not necessarily quantitatively reflects the true component mixture present in the obtained polymer.

The self-organizing properties of amphiphilic copolymers in block-selective solvents and the shape of the resulting structures are strongly dominated by the ratio of the two segments. For this reason and to evaluate the scope of the polymerization system studied, conjugated polymer blocks of different chain length were synthesized. This was realized by adjustment of the stoichiometric AB-monomer to initiator ratio and the obtained copolymers were characterized in view of their molecular weight and their optical properties (Table 6.1).

Table 6.1: Block copolymers synthesized by controlled Suzuki-Miyaura coupling polymerization starting from a PEG-based macroinitiator and the corresponding molecular weights and optical properties. ${ }^{a}$

\begin{tabular}{|c|c|c|c|c|c|c|c|}
\hline polymer & $\begin{array}{c}\text { equiv. } \\
\text { monomer }^{b}\end{array}$ & $\begin{array}{c}M_{\mathrm{n}}{ }^{c} \\
{[\mathrm{~kg} / \mathrm{mol}]}\end{array}$ & $M_{\mathrm{w}} / M_{\mathrm{n}}{ }^{c}$ & $\begin{array}{c}\mathrm{DP}_{\mathrm{n}} \\
(\mathrm{PF} 8)^{d}\end{array}$ & $\begin{array}{l}\text { yield } \\
{[\mathrm{mg}]}\end{array}$ & $\begin{array}{c}\lambda_{\mathrm{abs}, \max }{ }^{\mathrm{e}} \\
{[\mathrm{nm}]}\end{array}$ & $\begin{array}{c}\lambda_{\mathrm{em}, \max }{ }^{\mathrm{e}} \\
{[\mathrm{nm}]}\end{array}$ \\
\hline 1 & 1 & 4.9 & 1.34 & 3.6 & 11.5 & 377 & 415 \\
\hline 2 & 2.5 & 7.8 & 1.41 & 7.0 & 39.1 & 382 & 416 \\
\hline 3 & 5 & 10.3 & 1.26 & 10.1 & 57.0 & 388 & 417 \\
\hline 4 & 7.5 & 13.2 & 1.42 & 12.1 & 56.8 & 388 & 417 \\
\hline 5 & 10 & 16.4 & 1.42 & 14.2 & 104.1 & 390 & 417 \\
\hline 6 & 15 & 15.7 & 1.74 & 17.3 & 76.7 & 390 & 417 \\
\hline 7 & 20 & 13.2 & 1.61 & 23.7 & 80.3 & 388 & 416 \\
\hline 8 & 40 & 14.2 & 1.76 & 39.6 & 161.7 & 390 & 417 \\
\hline
\end{tabular}

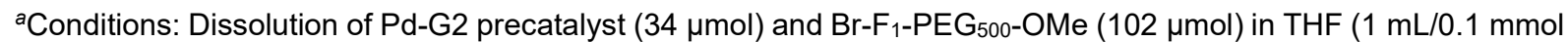
monomer), addition of $2 \mathrm{M}$ aq. $\mathrm{K}_{2} \mathrm{CO}_{3}$ solution (1 mL/0.1 mmol monomer), stirring at $\mathrm{rt}$ for $15 \mathrm{~min}$, addition of mixture to cooled $\left(0{ }^{\circ} \mathrm{C}\right)$ solution of $\mathrm{Br}-\mathrm{F}_{1}$-BPin in THF $(4 \mathrm{~mL} / 0.1 \mathrm{mmol}$ monomer), $15 \mathrm{~min}$ of polymerization while cooling, termination by addition of $2 \mathrm{M}$ aq. $\mathrm{HCl}$ solution $(1 \mathrm{~mL}) .{ }^{b}$ Referred to amount of Pd-G2 catalyst. 'Determined by GPC with RI detection vs. PS standards. ${ }^{d}$ Average number of fluorene repeat units as calculated from ${ }^{1} \mathrm{H}-\mathrm{NMR}$ spectra. ${ }^{e}$ Copolymer solutions in THF (c $\left.\leq 0.01 \mathrm{mg} / \mathrm{mL}\right)$.

For all polymerization experiments employing 1 to 40 equivalents of monomer, PF8-PEG copolymers were yielded and the polymerization system under consideration was revealed to be tolerant towards a variation in monomer amount. Using $\mathrm{Br}-\mathrm{F}_{1}-\mathrm{BP}$ in in a stoichiometry of 1 to 10 equivalents, an increase in molecular weight was evidenced, as it would be expected for a controlled polymerization. The rise in $M_{n}$ 
for higher monomer amounts was accompanied by an increasing dispersity of the copolymers, as indicated by GPC measurements. Please note that, based on their commercial production, all mPEGbased derivatives constituted product mixtures of varying chain lengths and therefore exhibited an intrinsic dispersity. For monomer amounts $>10$ equiv. the increase in molecular weight stagnated, which indicated that the polymer growth process itself is not optimal to obtain copolymers with a higher PF8 proportion. For instance, the comparably short polymerization time of $15 \mathrm{~min}$ or the high concentration within the reaction mixture, leading to premature polymer precipitation, could constitute a limitation in this case, which makes further adjustment of the reaction conditions necessary at this point. This assumption is supported by the yields found for the copolymers. As expected, the absolute mass values increased continuously for higher monomer stoichiometry for the polymers 1-5 but varied widely for polymerizations with $>10$ equivalents of monomer. Challenging post-polymerization work-up for copolymers of higher molecular weight might influence the polymer yields.

Regarding the lengths of the PF8 segments, the number of integrated fluorene monomers as calculated from ${ }^{1} \mathrm{H}-\mathrm{NMR}$ data slightly exceeds the chain length envisaged by the adjusted monomer to initiator ratio. For the exemplary case of polymer 3 , an approximate number of $n \approx 10$ repeat units was calculated by comparative integration of the respective backbone signals, which is higher compared to the stoichiometric ratio of monomer to initiator (5/1). This hints towards an incomplete initiation reaction between PEGylated bromofluorene and Pd-G2 precatalyst, resulting in the formation of less catalytically active palladium species ready to grow a poly(fluorene) chain. As a consequence, a larger amount of monomers is available per initiator molecule. The efficiency of initiation could be increased by extended initiation time, however, this occurred at the expense of a broader molecular weight distribution of the resulting polymer (cf. Figure 9.36).

In conclusion, the polymerization system under investigation constitutes a useful method towards PF8PEG copolymers with variable length of the PF8 segment within a limited range. The obtained copolymers were characterized with regard to their optical properties by means of UV/Vis absorption and fluorescence emission spectroscopy (Figure 6.4).
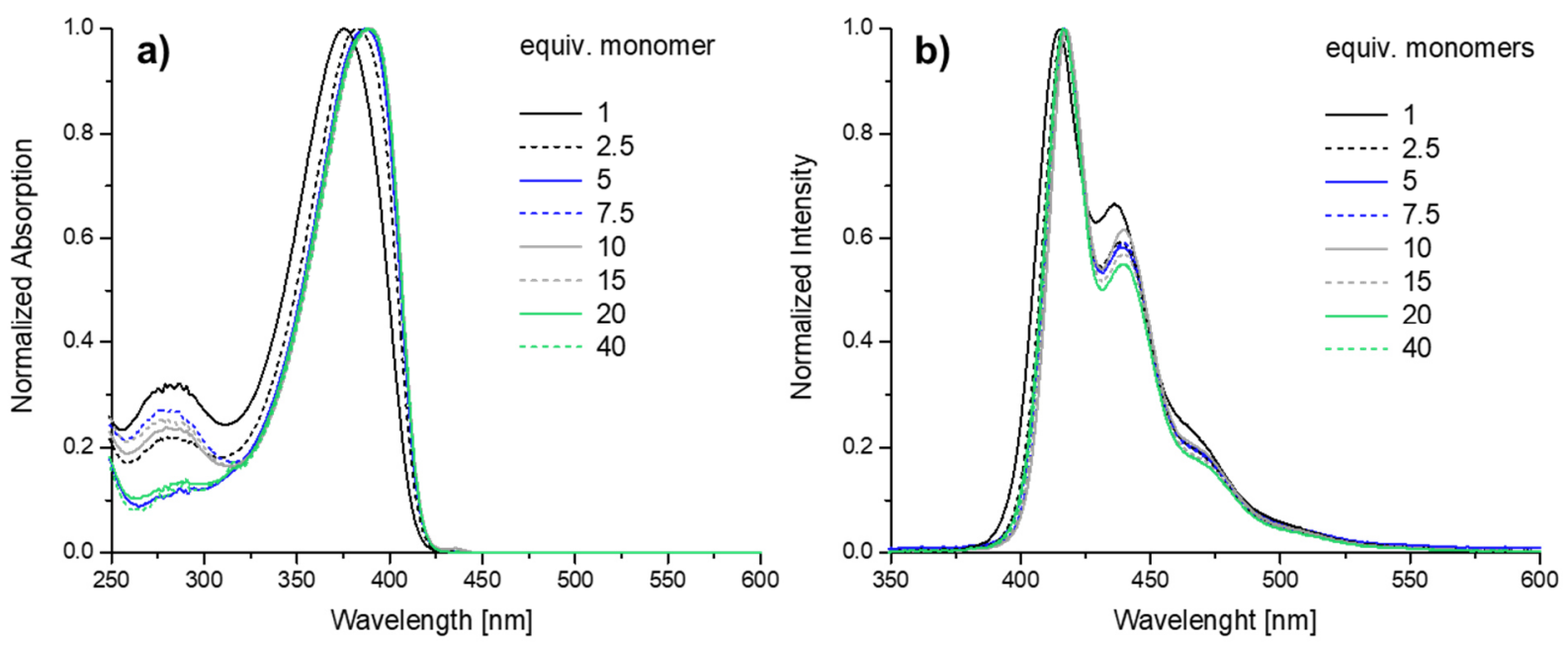

Figure 6.4: (a) UV/Vis absorption and (b) fluorescence emission ( $\lambda_{\text {exc }}=380 \mathrm{~nm}$ ) spectra of amphiphilic block copolymers with increasing length of the poly $\left(9,9^{\prime}\right.$-dioctylfluorene) segment as adjusted by the stoichiometric amount of monomers used relative to the catalytic palladium species. The polymers were dissolved in THF for measurement ( $c \leq 0.01 \mathrm{mg} / \mathrm{mL}$ ). 
The absorption spectra acquired from solutions of the respective copolymers in tetrahydrofuran showed absorption bands around $380 \mathrm{~nm}$, which agrees with the wavelength range expected for amorphous PF8. ${ }^{10}$ With increasing length of the PF8 segment, a slight red-shift in the absorption maxima was observed, caused by the extension of the conjugated system. The shift to higher wavelengths was more pronounced for copolymers with shorter PF8 segments, as the longer homologues approach the optical behavior of regular PF8 homopolymer. From the fluorescence spectra, an emission patters with peaks at $417 \mathrm{~nm}, 437 \mathrm{~nm}$ and $467 \mathrm{~nm}$ became apparent, which also corresponds to amorphous, disordered poly $\left(9,9^{\prime}\right.$-dioctylfluorene) chains, as expectable from the dissolved state of the copolymers.

In order to investigate the concentration dependent phase behavior of the amphiphilic copolymers, a concentration series was prepared, followed by fluorescence emission measurements, in analogy to experiments presented in previous sections (cf. Chapter 4.5). These studies revealed the enhanced presence of ordered $\beta$-phase regions in more concentrated solutions of poly- and oligo(fluorenes). Similarly to regular PF8, the resulting copolymer spectra indicated the presence of $\beta$-phase material upon concentration increase, observable as intensification of the respective fluorescence signal $(440 \mathrm{~nm})$, while the peak assignable to amorphous PF8 diminished (Figure 9.42). Although no exact 'critical' concentration required for the onset of $\beta$-phase formation was ascertained, $\beta$-phase emission first appeared at concentrations $\geq 0.1 \mathrm{mg} / \mathrm{mL}$ and furthermore constituted the exclusive observable contribution to the spectra at $\geq 1 \mathrm{mg} / \mathrm{mL}$. Conclusively, the optical properties observed for the copolymers confirmed the effective formation of a PF8 block with increasing length of the conjugated polymer chain. Furthermore, the ability of the PF8 segments to align via intermolecular processes in concentrated solutions to regions of high chain order was evidenced, without any obvious adverse effect of the attached hydrophilic PEG moieties.

From variation of the copolymers' hydrophilicity by extension or reduction of the flexible PEG segment, significant impacts on the copolymer properties and their self-organizing behavior was expected. In order to investigate these effects, controlled Suzuki-Miyaura polymerization was performed using aryl halide precursors with $\mathrm{MPEG}$ substituents of other lengths. In addition to the reference compound $\mathrm{Br}-\mathrm{F}_{1}-\mathrm{PEG}_{500}-\mathrm{OMe}$, also macromolecules based on mPEG-200 and mPEG-2000 were synthesized as shorter and longer homologue and then applied in polymerizations according to the abovementioned protocol. The resulting block copolymers were characterized in view of material properties, to evaluate the influence of the segment ratio (Table 6.2). 
Table 6.2: Block copolymers synthesized by controlled Suzuki-Miyaura coupling polymerization starting from PEGbased macroinitiators Br-F1-PEG $200-O M e$ (polymers 9 and 10) and Br-F1-PEG $2000-O M e$ (polymers 11 and 12) and the corresponding polymer properties. ${ }^{a}$

\begin{tabular}{c|c|c|c|c|c|c|c} 
polymer & $\begin{array}{c}\text { equiv. } \\
\text { monomer }^{b}\end{array}$ & $\begin{array}{c}\boldsymbol{M}_{\mathbf{n}}{ }^{c} \\
{[\mathbf{k g} / \mathbf{m o l}]}\end{array}$ & $\boldsymbol{M}_{\mathbf{w}} \mathbf{M}_{\mathbf{n}}{ }^{c}$ & $\begin{array}{c}\mathbf{D P}_{\mathbf{n}} \\
\text { (PF8) }^{d}\end{array}$ & $\begin{array}{c}\text { yield } \\
{[\mathbf{m g}]}\end{array}$ & $\begin{array}{c}\boldsymbol{\lambda}_{\mathrm{abs}, \max }{ }^{\mathrm{e}} \\
{[\mathrm{nm}]}\end{array}$ & $\begin{array}{c}\boldsymbol{\lambda}_{\mathrm{em}, \max }{ }^{\mathrm{e}} \\
{[\mathrm{nm}]}\end{array}$ \\
\hline 9 & 2.5 & 6.0 & 1.39 & 6.3 & 32.8 & 381 & 416 \\
10 & 5 & 7.6 & 1.61 & 14.2 & 33.1 & 385 & 417 \\
11 & 2.5 & 10.6 & 1.31 & 1.1 & 208.7 & 381 & 415 \\
12 & 5 & 15.4 & 1.37 & 4.9 & 113.9 & 386 & 416
\end{tabular}

aConditions: Dissolution of Pd-G2 precatalyst $(34 \mu \mathrm{mol})$ and PEG-based aryl halide $(102 \mu \mathrm{mol})$ in THF $\left(1 \mathrm{~mL} / 0.1 \mathrm{mmol}\right.$ monomer), addition of $2 \mathrm{M}$ aq. $\mathrm{K}_{2} \mathrm{CO}_{3}$ solution $(1 \mathrm{~mL} / 0.1 \mathrm{mmol}$ monomer), stirring at $\mathrm{rt}$ for $15 \mathrm{~min}$, addition of mixture to cooled $\left(0^{\circ} \mathrm{C}\right)$ solution of $\mathrm{Br}-\mathrm{F}_{1}$-BPin in THF $(4 \mathrm{~mL} / 0.1 \mathrm{mmol}$ monomer$), 15 \mathrm{~min}$ of polymerization while cooling, termination by addition of $2 \mathrm{M}$ aq. $\mathrm{HCl}$ solution $(1 \mathrm{~mL}) .{ }^{b}$ Referred to amount of Pd-G2 catalyst. 'Determined by GPC with RI detection vs. PS standards. ${ }^{d}$ Average number of fluorene repeat units as calculated from ${ }^{1} \mathrm{H}-\mathrm{NMR}$ spectra. ${ }^{e}$ Copolymer solutions in THF $(\mathrm{c} \leq 0.01 \mathrm{mg} / \mathrm{mL})$.

Starting from macromolecules with longer or shorter PEG segments as compared to the reference system, the elaborated CSMCP protocol successfully yielded amphiphilic copolymers. These, however, featured different solubility properties which became especially apparent for copolymers with a PEG2000-based segment. The respective polymers required adjusted work-up procedures due to their increased hydrophilicity. As was the case for the polymerizations starting from $\mathrm{Br}_{-} \mathrm{F}_{1}-\mathrm{PEG}_{500}-\mathrm{OMe}$, the copolymers exhibited higher molecular weight and longer PF8 segments for the employment of higher monomer amounts. Caused by the varying molecular weight of the starting material, the entire set of copolymers synthesized using $\mathrm{Br}-\mathrm{F}_{1}-\mathrm{PEG}_{200}-\mathrm{OMe}$ or $\mathrm{Br}-\mathrm{F}_{1}-\mathrm{PEG}_{2000}-\mathrm{OMe}$ was shifted to higher or lower molecular weight. Both the UV/Vis absorption and emission spectra recorded for the copolymers showed the expected signals assignable to amorphous phase PF8 (cf. Figure 9.41). Comparing the determined absorption and emission maxima of copolymers with different hydrophilic block lengths, no influence of the PEG segment could be evidenced. This suggested that the optical properties of the copolymers under investigation were, at least in solution, unaffected by their hydrophilicity.

In summary, the established procedure for the synthesis of a PF8 chain starting from a PEGylated initiator was proven to be as well applicable on systems including shorter or longer PEG chains, which opens access to amphiphilic copolymers with variable lengths of both, the hydrophilic coil- and the hydrophobic rod-type segment. 


\subsubsection{COPOLYMERS WITH OTHER CONJUGATED POLYMER SEGMENTS}

In order to vary the electronic and structural nature of individual polymer molecules, the synthetic approach outlined above was performed with alternative monomers (Figure 6.5).

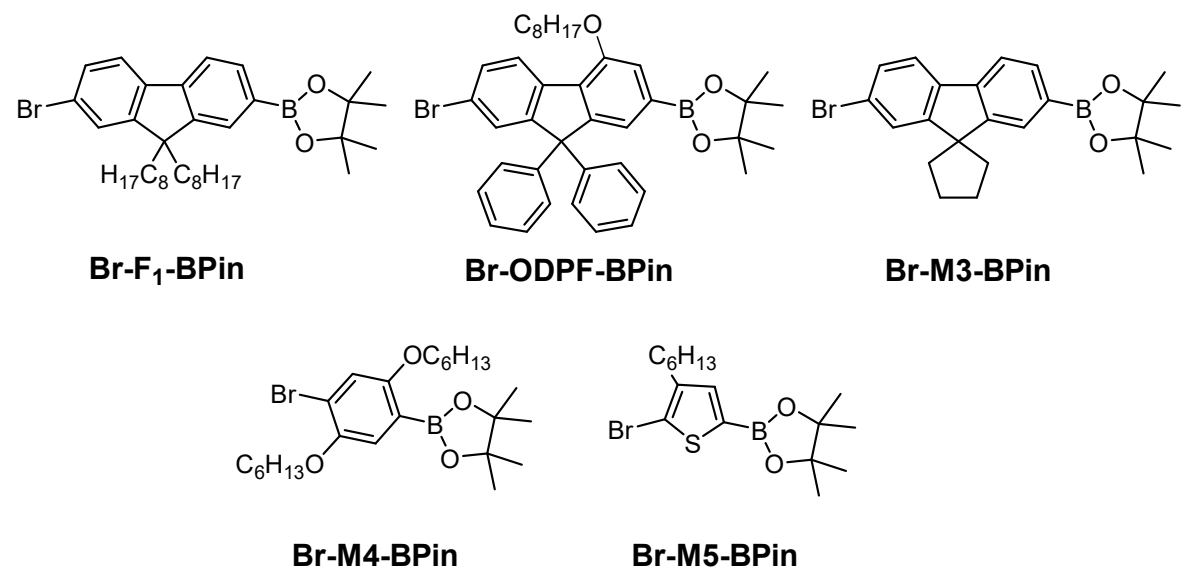

Figure 6.5: Conjugated AB-type monomers employed in controlled Suzuki-Miyaura coupling polymerization towards amphiphilic block copolymers.

In addition to the PF8 monomer used as a reference, the respective AB-type 9,9'-diphenylfluorene monomer (Br-ODPF-BPin, cf. Chapter 8.6.1) was applied in the investigated polymerization. To examine a fluorene-based monomer with a rigid, sterically demanding structure motif, Br-M3-BPin was synthesized by conversion of dibromospiro(cyclopentane-1,9'-fluorene), available in the research group (cf. Chapter 8.7.2). Moreover, the phenyl- and thiophene-based monomers Br-M4-BPin and Br-M5-BPin as starting materials for well-established conjugated polymers were employed. ${ }^{14}$ Especially for the poly(thiophene) based copolymer, drastic changes in optical properties were expected due the strongly different chemical structure of the conjugated system.

For all four alternative monomers, amphiphilic copolymers of varying emission color were obtained by Suzuki-Miyaura coupling polymerization. After the post-polymerization work-up, the copolymers were characterized with regard to molecular weight and optical properties to identify the influence of the different conjugated structure motifs (Table 6.3). As reference system, the data acquired for the copolymer comprising PF8 as conjugated polymer segment are listed (polymer 3 ). In all cases, the polymerizations proceeded unimpaired by the change in conditions and copolymers were obtained in reasonable yields, although their molecular weight was lower in comparison with the reference system. The most pronounced reduction in this regard became apparent for the usage of $\mathrm{Br}$-ODPF-BPin and $\mathrm{Br}$ M3-BPin, which agrees with their higher sterical demand as compared to the standard PF8 monomer, bearing flexible alkyl substituents. With regard to polymer 3 , significant differences in material properties were revealed in studies on the polymers' optical properties investigated by UV/Vis absorption and fluorescence emission spectroscopy (Figure 6.6). 
Table 6.3: Block copolymers synthesized by controlled Suzuki-Miyaura coupling polymerization of varying AB-type monomers, starting from a PEG-based macroinitiator, and the corresponding molecular weights and optical properties. $^{a}$

\begin{tabular}{|c|c|c|c|c|c|c|c|}
\hline polymer & $\begin{array}{l}\text { AB-type } \\
\text { monomer }\end{array}$ & $\begin{array}{c}M_{\mathrm{n}^{c}} \\
{[\mathrm{~kg} / \mathrm{mol}]}\end{array}$ & $M_{\mathrm{w}} / M_{\mathrm{n}}{ }^{c}$ & $\begin{array}{c}\mathrm{DP}_{\mathrm{n}} \\
(\mathrm{PF} 8)^{d}\end{array}$ & $\begin{array}{l}\text { yield } \\
{[\mathrm{mg}]}\end{array}$ & $\begin{array}{c}\lambda_{\text {abs, } \max }{ }^{e} \\
{[\mathrm{~nm}]}\end{array}$ & $\begin{array}{c}\lambda_{e m, \max }{ }^{e} \\
{[n m]}\end{array}$ \\
\hline 3 & $\mathrm{Br}-\mathrm{F}_{1}-\mathrm{BP}$ in & 10.3 & 1.26 & 10.1 & 57.0 & 388 & 417 \\
\hline 13 & Br-ODPF-BPin & 5.8 & 1.50 & 5.4 & 74.8 & 388 & 427 \\
\hline 14 & Br-M3-BPin & 4.5 & 1.31 & 5.4 & 52.7 & 373 & 413 \\
\hline 15 & Br-M4-BPin & 7.0 & 1.28 & 13.0 & 34.8 & 337 & $401^{f}$ \\
\hline 16 & Br-M5-BPin & 7.9 & 1.25 & 14.2 & 50.6 & 437 & 571 \\
\hline
\end{tabular}

${ }^{a}$ Conditions: Dissolution of Pd-G2 precatalyst $(34 \mu \mathrm{mol})$ and Br- $\mathrm{F}_{1}-\mathrm{PEG}{ }_{500}-\mathrm{OMe}(102 \mu \mathrm{mol})$ in THF $(1 \mathrm{~mL} / 0.1 \mathrm{mmol}$ monomer), addition of $2 \mathrm{M}$ aq. $\mathrm{K}_{2} \mathrm{CO}_{3}$ solution (1 mL/0.1 mmol monomer), stirring at $\mathrm{rt}$ for $15 \mathrm{~min}$, addition of mixture to cooled $\left(0^{\circ} \mathrm{C}\right)$ solution of AB-type monomer $(168 \mu \mathrm{mol})$ in THF $(4 \mathrm{~mL} / 0.1 \mathrm{mmol}$ monomer $), 15 \mathrm{~min}$ of polymerization while cooling, termination by addition of $2 \mathrm{M}$ aq. $\mathrm{HCl}$ solution $(1 \mathrm{~mL}) .{ }^{b}$ Referred to amount of $\mathrm{Pd}-\mathrm{G} 2$ catalyst. ${ }^{c}$ Determined by GPC with RI detection vs. PS standards. ${ }^{d}$ Average number of fluorene repeat units as calculated from ${ }^{1} \mathrm{H}-\mathrm{NMR}$ spectra. ${ }^{e}$ Copolymer solutions in THF (c $\leq 0.01 \mathrm{mg} / \mathrm{mL}$ ). ${ }^{f}$ Acquired with an excitation wavelength of $\lambda_{\mathrm{exc}}=350 \mathrm{~nm}$.
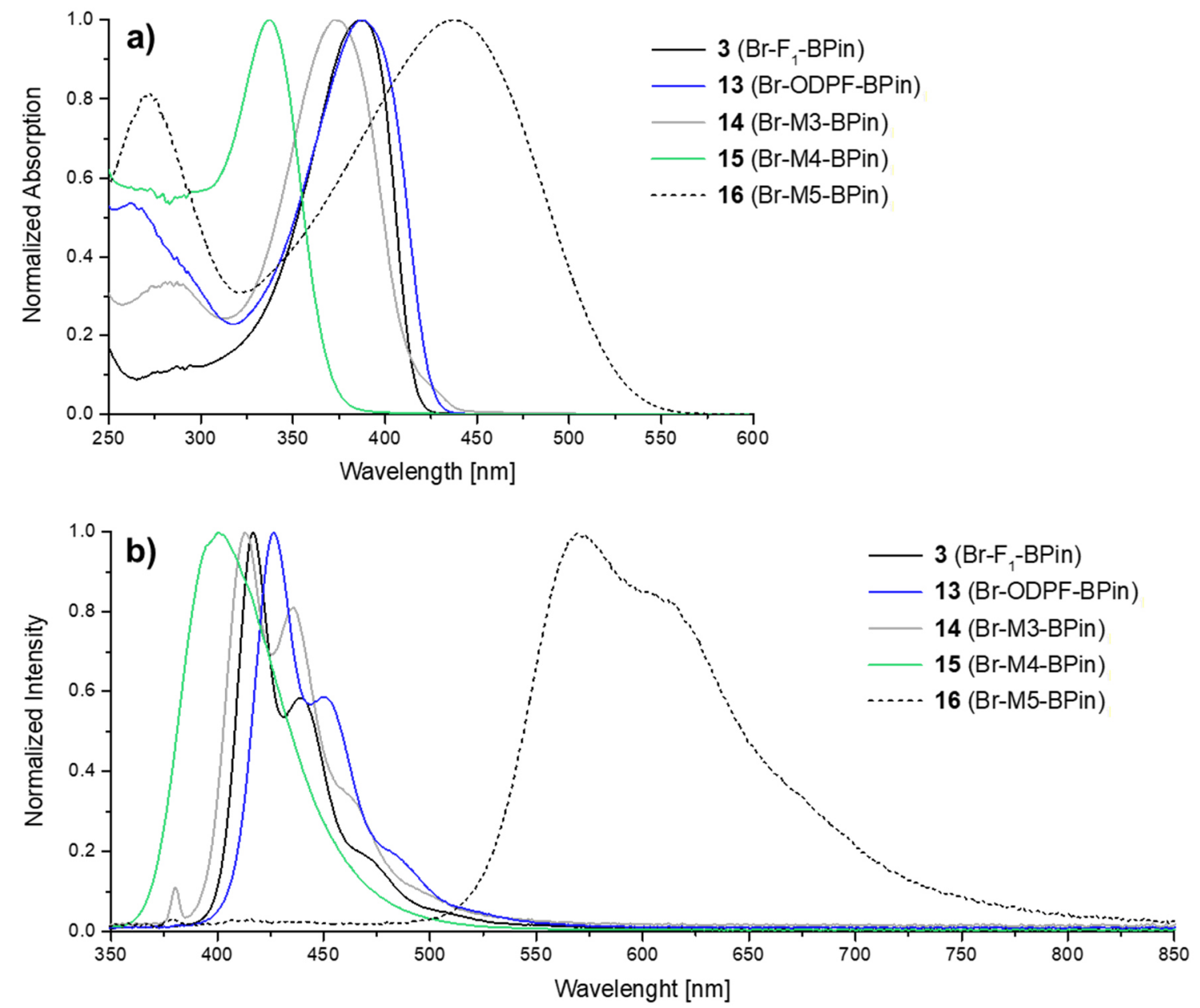

Figure 6.6: (a) UVIVis absorption and (b) fluorescence emission spectra of amphiphilic block copolymers synthesized by use of varying AB-type monomers, corresponding to entries in Table 6.3. Measurements for polymer 15 were conducted with $\lambda_{e x c}=350 \mathrm{~nm}$, all others with $\lambda_{e x c}=380 \mathrm{~nm}$. The polymers were dissolved in THF (c $\leq 0.01 \mathrm{mg} / \mathrm{mL})$. 
Similar to PF8, the structurally related polymer 13 gave rise to an absorption band around $390 \mathrm{~nm}$ and fluorescence emission peaks at $427 \mathrm{~nm}, 450 \mathrm{~nm}$ and $483 \mathrm{~nm}$. Both could be assigned to amorphous phase poly(diarylfluorene), as already elucidated in previous sections (cf. Chapter 5). ${ }^{39}$ Likewise, the fluorene-based polymer 14 featured signals at $412 \mathrm{~nm}, 436 \mathrm{~nm}$ and $462 \mathrm{~nm}$, which represented the characteristic threefold emission peak pattern of the $0-0,0-1$ and $0-2$ intrachain singlet transitions, frequently observed for poly(fluorenes) and related compounds. ${ }^{21}$ This showed the accessibility of copolymers with blocks of poly(fluorenes) other than the well-established PF8. Additionally, the reaction system studied demonstrated tolerance towards structurally completely different motifs of conjugated polymers. Polymer 15 , obtained from polymerization of the corresponding AB-type phenyl monomer, induced a blue-shifted absorption signal around $340 \mathrm{~nm}$ and an emission band at approximately $400 \mathrm{~nm}$, caused by the higher bandgap of poly(para-phenylenes) as compared to poly(fluorene). ${ }^{170}$ As representative for fluorescence emission of lower energy, a poly(thiophen)-poly(ethylene glycol) copolymer was synthesized (polymer 16). In addition to a broad absorption band around $450 \mathrm{~nm}$, emission in the region of red light wavelengths around $600 \mathrm{~nm}$ was observed from fluorescence spectra. This coincided with the optical impression of the copolymer, namely a bright orange-red solution, in contrast to the greenish-blue poly(fluorene) solutions.

In summary, these findings proved that the scope of materials accessible by the established synthetic pathway also extends to amphiphilic copolymers with other conjugated polymer segments, which gives access to materials with tunable emission wavelengths.

\subsection{AGGREGATION BEHAVIOR OF AMPHIPHILIC COPOLYMERS}

Amphiphilic block copolymers with a rod-like, conjugated polymer segments provide promising starting material for the formation of nanoscale superstructures, owed to the differences in polarity and solubility of the different components in appropriate block-selective solvent mixtures. The segments strive to minimize contact to their counterpart and to maximize contact with the more favorable solvent, thereby inducing self-organization processes. ${ }^{158}$ Depending on several factors, such as the relative lengths of hydrophobic and hydrophilic segments, the resulting domains arrange into structures with variable shapes, for example nanofibers with a hydrophobic conjugated polymer core and a flexible, hydrophilic outer shell. ${ }^{144,159,160,216}$

For the amphiphilic PF8-PEG block copolymers under investigation, preliminary aggregation experiments were performed in a tetrahydrofuran/methanol mixture (10/1), chosen as solvent in accordance with published procedures (cf. Chapter 8.7.4). ${ }^{140,162,216}$ Tetrahydrofuran represents a good solvent for both segments, whereas the more polar methanol acts as non-solvent for the conjugated 
polymer and thereby its addition may induce nucleation of, for instance, nanowire-like structures. To this end, the self-organizing behavior and the generation of hierarchical structures upon drying of the abovementioned solutions was investigated. In order to gain a general view on the performance of the synthesized copolymers, mixtures were prepared with specimen of varying segment lengths and different chemical nature of the conjugated segment (cf. Table 9.2). As apparent from visualization of the resulting structures by TEM, several of the samples exhibited regions of lamellar structures (cf. Figure 9.43). Best results regarding the formation of defined superstructures were obtained from solutions of polymer 11, which was synthesized starting from an initiating mPEG-2000 macromolecule. TEM measurements revealed the occurrence of regular, lamellar structures with an average lamella thickness of $9.0 \mathrm{~nm}$ (coefficient of variation $v=0.10$ over 50 measurements) (Figure 6.7). These showed close resemblance to aggregates previously observed for comparable block copolymers, which were assumed to form cylindrical aggregates with a conjugated polymer core and a PEG shell. ${ }^{141,160,217}$
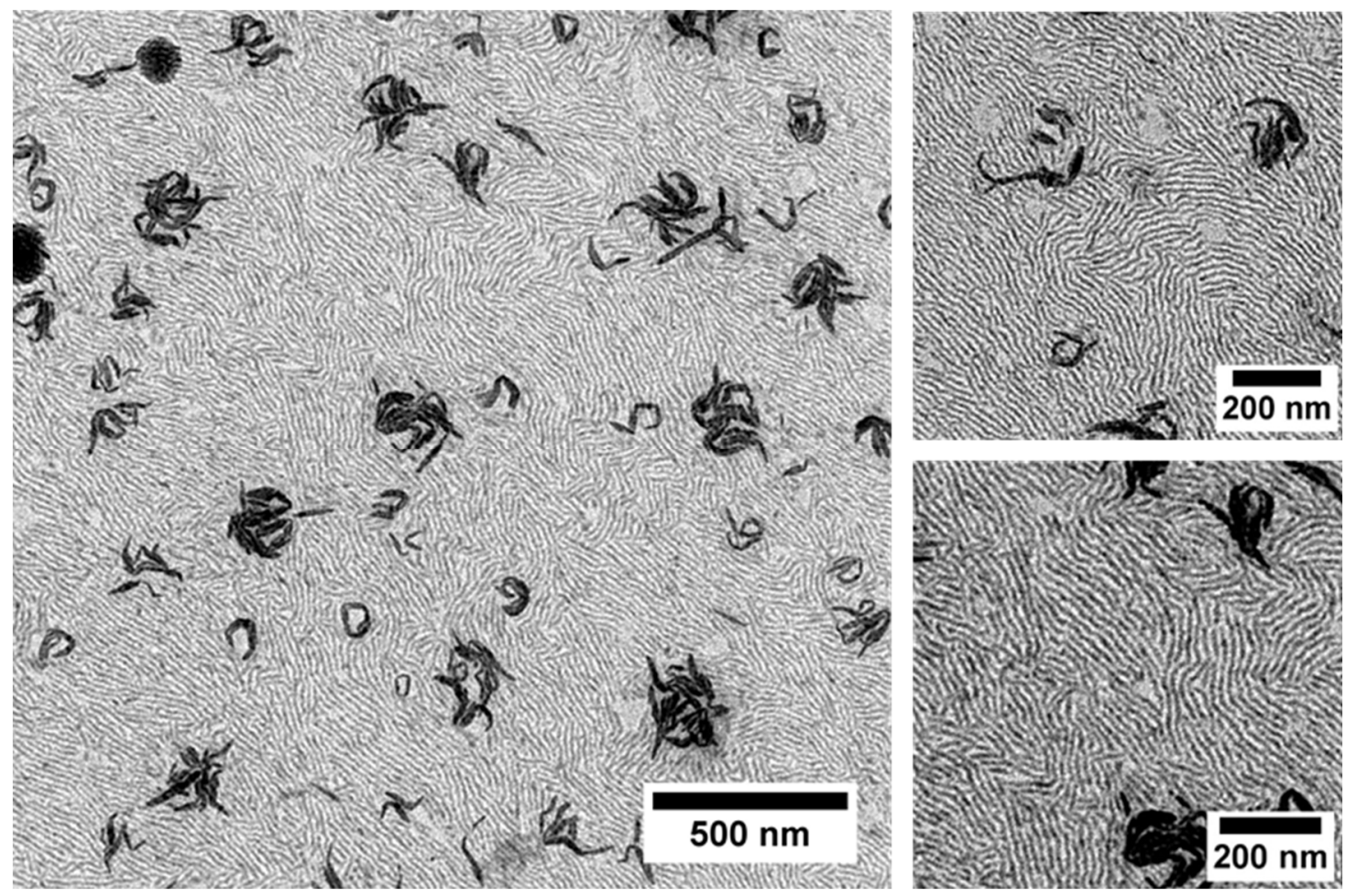

Figure 6.7: TEM images with different magnification of samples prepared from a solution of amphiphilic PF8-PEG polymer 11, dissolved in a tetrahydrofuran/methanol mixture (10/1).

Earlier studies on the fluorescence of films prepared from PF8-PEG block copolymers exhibited an increased occurrence of $\beta$-phase absorption and emission as compared to similar films of PF8 homopolymer. ${ }^{218}$ With regard to the material under investigation, this points to an enhanced structural order of the block copolymers in specific solvent mixtures, enabled by the attached hydrophilic segment and the resulting microphase separation processes.

The general ability of the conjugated polymer segments to exert intermolecular interactions under the given conditions became also apparent from the acquisition of fluorescence emission spectra of the prepared solutions prior to drying. Exemplarily shown for polymer 11, these revealed the emergence of $\beta$-phase conformation in the dissolved material (Figure 6.8). 


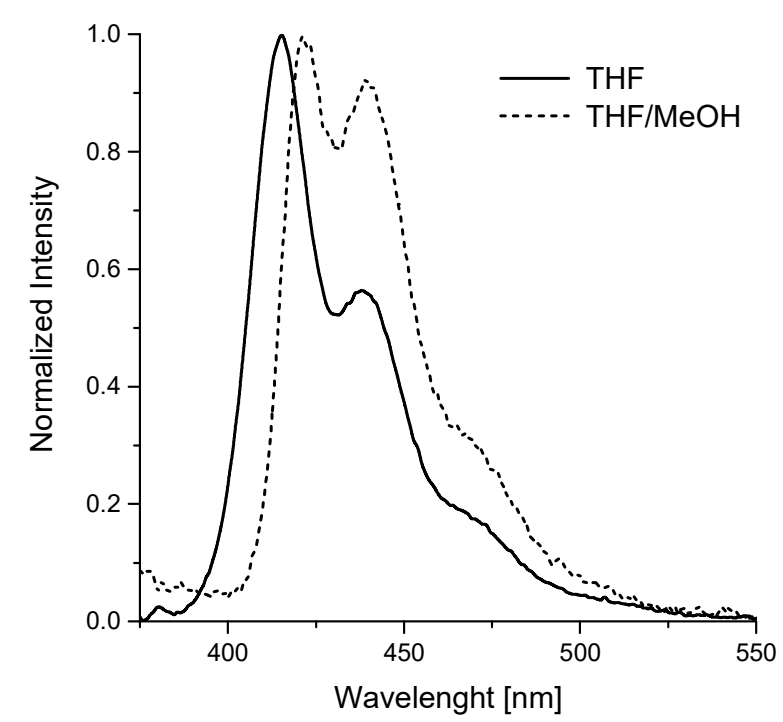

Figure 6.8: Fluorescence emission spectra of amphiphilic copolymer 11, dissolved in tetrahydrofuran (solid lines) and in tetrahydrofuran/methanol mixture (10/1) (dashed lines, polymer concentration c $=0.4 \mathrm{mg} / \mathrm{mL}$ ).

In contrast to diluted solutions of the synthesized amphiphilic copolymers displaying emission signals characteristic for amorphous phase PF8, the less pronounced $\beta$-phase emission peak at approximately $440 \mathrm{~nm}$ gained in intensity for the prepared tetrahydrofuran/methanol mixtures. ${ }^{39}$ The organization of polymer chains to higher ordered phases was presumably favored by both, the comparably high concentration associated with sample preparation (cf. Figure 9.42) and the addition of methanol as nonsolvent for the poly(fluorene) segment. The more pronounced appearance of $\beta$-phase emission for decreasing solvent quality constitutes a literature-known phenomenon observed in studies on PF8 conformation. 48,49

In summary, these initial experiments indicated a general ability of the synthesized amphiphilic copolymers to self-organize in suitable solvents to form ordered, lamellar superstructures. From the general increase of $\beta$-phase regions observable from fluorescence emission it can be assumed that the formation of aggregations to a certain extent coincides with intermolecular interactions between poly(fluorene) segments, which align to phases of higher order under the given conditions. In comparison with the systems of poly(fluorene) as exclusive component addressed in previous chapters, a significant overall impact by the introduction of hydrophilic coils on the formation of supramolecular structures can be concluded. The involved process of phase separation indeed dominates the structure formed by the block copolymers eventually in a dry state. 


\subsection{SUMMARY AND CONCLUSION}

Rod-coil type block copolymers with conjugated polymer segments provide attractive materials for the construction of hierarchical superstructures. Driven by complex processes of microphase separation and electrostatic interactions of the conjugated backbone, such amphiphilic copolymers have the ability to self-assemble under suitable solvent conditions, forming intrinsically stabilized, complex superstructures. Copolymers of two chemically and physically divergent blocks are synthetically accessible by end-functionalization of one segment, which serves subsequently as macroinitiator for the polymerization of the second moiety. In this chapter, poly(9,9'-dioctylfluorene)-poly(ethylene glycol) (PF8-PEG) block copolymers with different lengths of both segments were synthesized and characterized as an alternative nanoscale system based on polyfluorene.

For the synthesis of block copolymers with a flexible, hydrophilic PEG segment and a rigid, hydrophobic conjugated polymer segment, a synthetic pathway was elaborated, based on the controlled SuzukiMiyaura coupling polymerization (CSMCP) procedure. ${ }^{30}$ To this end, PEGylated bromofluorene macromolecules with various PEG lengths were synthesized by linkage of a fluorene moiety with an mPEG chain in a nucleophilic substitution reaction. The new synthesized macromolecules were employed as initiating species in the controlled Suzuki-Miyaura coupling polymerization of AB-type fluorene monomers, to grow a poly $\left(9,9^{\prime}\right.$-dioctylfluorene) chain on the mPEG segment. A variation in the length and the molecular weight of the conjugated polymer block was feasible by the adjustment of the stoichiometric catalyst to monomer ratio. The resulting amphiphilic PF8-PEG block copolymers were investigated by means of several analytic techniques, thereby revealing the connection of the two blocks, which confirmed that the polymerization indeed started from the macroinitiator. Apart from the polymerization of fluorene monomers, serving as a reference system, block copolymers with alternative conjugated polymer segments, including poly(para-phenylene) and poly(thiophene) derivatives, were synthesized. This indicated a high tolerance of the elaborated polymerization system towards different functional groups and broadened the scope of the reaction protocol to copolymers of variable emission color. The synthesized amphiphilic copolymers were investigated with regard to their aggregation behavior. TEM images of the respective samples revealed the occurrence of regions with regular, lamellar structures with average lamella thicknesses around $10 \mathrm{~nm}$.

Conclusively, a valuable synthetic approach towards PF8-PEG block copolymers was elaborated, which gives access to a broad range of amphiphilic, rod-coil-type copolymers with conjugated polymer segments and differing segment lengths. In view of future applications, the copolymers studied bear potential to be employed as surfactants for the stabilization of other nanostructures, for example in the emulsion polymerization of PF8 nanoparticles. Given their general capability to aggregate to regions of higher chain order, a good compatibility with solid-phase PF8 and the ability to align with the internal structure of anisotropic nanoparticles can be assumed, comparable with the situation for oligomers studied in previous chapters, which constitutes a beneficial feature for the implementation as surfactant. 


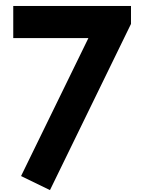

\section{CONCLUSIVE SUMMARY}

The size and shape of nanoscale particles strongly correlate with the internal molecular structure and order. Hence, fundamental understanding of the involved processes and the mechanisms of particle formation gives access to materials with tunable properties. This is especially true for nanoparticles with anisotropic shapes, which represent attractive building blocks for superstructures with unique, directiondependent properties. However, the fabrication of particles with non-spherical shapes is to date primarily a domain of inorganic materials, whereas the induction of anisotropy in polymer nanoparticles directly in the course of their synthesis remains a largely unresolved issue. Only recently, the synthesis of ellipsoidal, fluorescent poly(9,9'-dioctylfluorene) (PF8) nanoparticles via Suzuki-Miyaura polycondensation under heterophase conditions was reported (Figure 7.1). Although the internal arrangement of the polymer chains was suspected to play a role in determining the nanoparticles' anisotropic shape, the origin of the ellipsoidal PF8 particles had remained elusive. PF8 has emerged as exemplary case among conjugated polymers due to its distinct polymorphic behavior. Depending on the polymer backbone conformation, amorphous, nematic, isotropic or ordered phases can be distinguished. The most significant impact on the optoelectrical properties of bulk material arises from the $\beta$-phase, the morphology of highest order. Planarization of the polymer backbone by complex interchain interactions causes an extended conjugation length. A deeper understanding of the internal chain arrangement of conjugated polymers and how it affects the evolution of an anisotropic particle shape is essential to exploit the scope of non-spherical polymer nanoparticles.

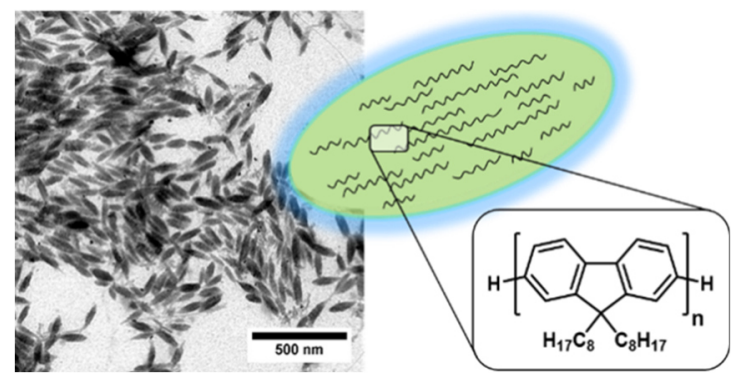

Figure 7.1: TEM image of anisotropic poly(9,9'-dioctylfluorene) nanoparticles with schematic illustration of the internal particle structure and the corresponding molecular structure of the polymer.

With the aim to reveal the internal molecular order of anisotropic CPNs, the conformation of oligo $\left(9,9^{\prime}\right.$ dioctylfluorenes) was monitored by electron paramagnetic resonance (EPR) spectroscopy. Precise oligomers with 1-6 repeat units and TEMPO spin-labels attached to both termini were synthesized either by stepwise synthesis or by controlled Suzuki-Miyaura coupling polymerization and subsequent fractionation via gel permeation chromatography (Figure 7.2a). Especially for the longer oligomers, the latter approach proved to be of advantage due to the reduced synthetic effort. Double electron-electron 
resonance (DEER) experiments were carried out in the Drescher group for each individual oligomer species in solution, to obtain a series of reference data. With increasing chain length, the oligomers displayed a higher flexibility, as revealed by a broadening of the spin-spin distance distributions. A set of characteristic parameters valid for the oligomer system studied, including the contour length $L_{c}$ and the persistence length $L_{p}$, was determined from the acquired data. For the oligomers under investigation, a global persistence length of $L_{p}=14 \mathrm{~nm}$ was elucidated, which corresponds to a semi-flexible character of the oligomer chains.
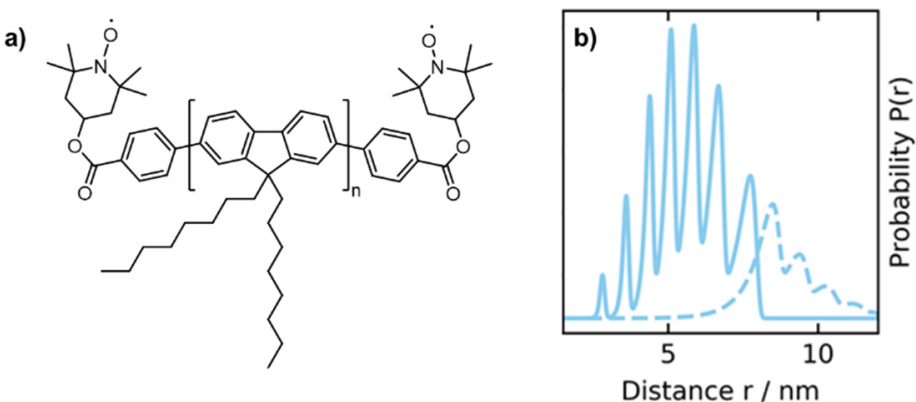

Figure 7.2: (a) Structure of defined, doubly labeled oligo(fluorenes) with $n$ repeat units and (b) distance distributions according to a fitted model following a Poisson distribution for $n=1-7$ (solid line) and for $n>7$ (dashed line) with an expectation value of $\lambda=5.4$ and a fixed global persistence length of $L_{p}=14 \mathrm{~nm}$.

With the obtained reference parameters, DEER studies were performed on spin-labeled oligomer mixtures as obtained from a controlled Suzuki-Miyaura coupling polymerization. A Poisson distribution of reaction products can be expected from this reaction type, which was very well reflected by the determined spin-spin distances and the relative weight fractions of each species (Figure 7.2b). To expand the scope of DEER experiments, the recorded raw data were additionally processed without reference parameters, yielding very consistent results. Apart from studies in solution, the accessibility of oligo(fluorene) conformations within nanoparticles by DEER was evaluated. Due to the nondeuterated, solid environment, the oligomers under investigation were limited to short species (up to 3 repeat units). These were incorporated into spherical PF8 nanoparticles, prepared in an emulsification approach. The oligomer fractions found by DEER measurements were in good agreement with values expected from sample preparation and the chains exhibited unchanged flexibility compared to respective dissolved species. In conclusion, the successful implementation of DEER measurements on mixtures of conjugated oligomers in both solution and in solid material, including the determination of the respective chain flexibility, was demonstrated. For the oligo(fluorenes) studied a semi-flexible, wormlike chain nature, quantifiable by DEER in an isolated state or in mixtures, was found and was furthermore proven to be retained upon incorporation in nanoparticles. However, the DEER method was limited to investigations of oligomers with $n \leq 7$. Especially for measurements in particles, the incorporation of longer oligo- or poly(fluorene) chains was expected to be more conclusive for the internal chain conformation and the mechanisms of particle formation.

To this end, the arrangement of longer oligomers and poly( $9,9^{\prime}$-dioctylfluorene) in particles was addressed by a combination of several preparative and analytic approaches. Using a step-wise synthesis route based on trimers as building blocks, defined oligomers with $n=3,6,9,12$ and 15 repeat units were synthesized and their internal packing in PF8 particles was illuminated. For this purpose, the 
reported Suzuki-Miyaura emulsion-type polycondensation was performed with the pre-synthesized oligomers added to the initial polymerization mixture. As evident from spectroscopic studies, oligo $\left(9,9^{\prime}-\right.$ dioctylfluorenes) of all lengths possessed the ability to align in phases of high order within the forming nanoparticles, while no adverse effect on the particle dimensions was observable from transmission electron microscopy (TEM) images. Similar results were obtained for the alignment of pre-fabricated PF8 in nanoparticles. In this regard, an optimized procedure for the preparation of anisotropic nanoparticles in a post-polymerization emulsification approach was elaborated. The resulting particles were in their particle dimensions and optical features comparable with the reference dispersions obtained from heterophase polycondensation, which made the post-polymerization method a valuable, straightforward alternative pathway towards anisotropic CPNs (Figure 7.3).
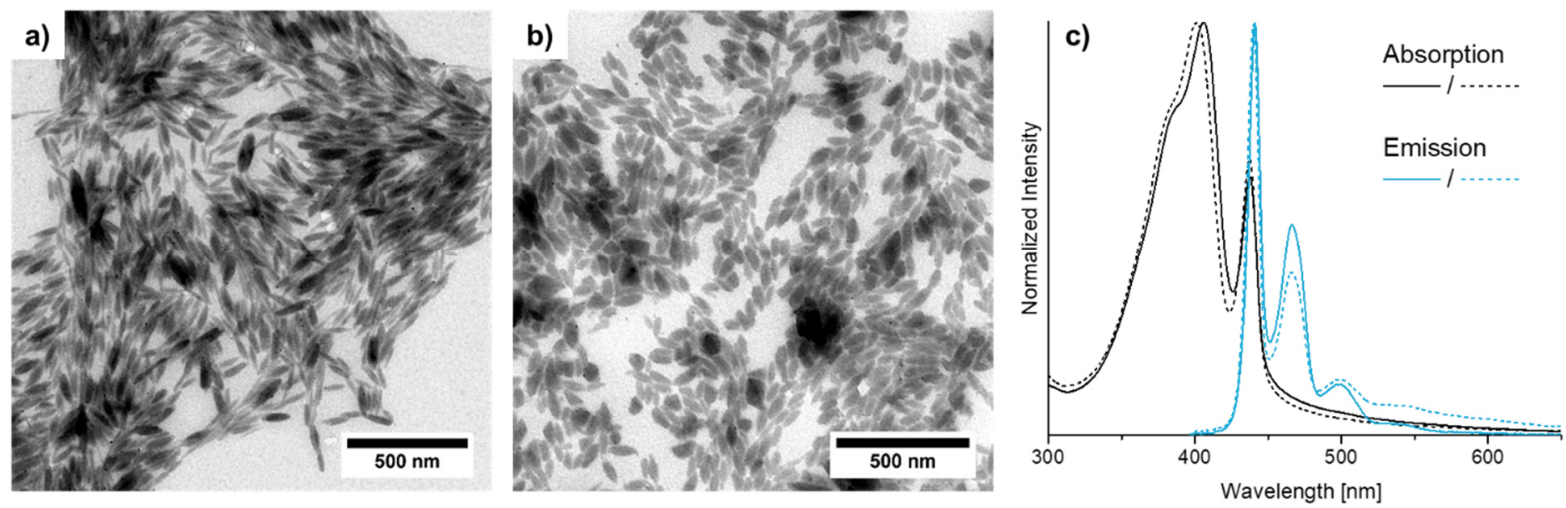

Figure 7.3: TEM images of ellipsoidal PF8 nanoparticles obtained by (a) heterophase Suzuki-Miyaura coupling polymerization ( $(=111 \mathrm{~nm}$, ar $=3.5)$ and (b) post-polymerization particle preparation $(I=100 \mathrm{~nm}, a r=2.9)$. (c) UV/Vis absorption and fluorescence emission spectra of the corresponding aqueous dispersions (solid line: heterophase Suzuki-Miyaura coupling polymerization; dashed line: post-polymerization particle preparation).

These results evidenced that, given the prerequisite of a suitable aromatic solvent, even PF8 polymer chains entail the ability for internal packing into ordered phases and for the formation of ellipsoidal particles. The post-polymerization emulsification protocol was also applied to the abovementioned oligomers as sole starting materials, which revealed a critical oligomer chain length of $n \approx 10$ required for the formation of anisotropic particles. With the aim to illuminate the polymer chain evolution in the course of Suzuki-Miyaura polycondensation, the proceeding reaction was monitored over time by determination of molecular weights. In view of its step-growth character, the polymerization was found to proceed in a very rapid way with the final molecular weight virtually reached after only a few minutes. In parallel with the increase of molecular weight, both the onset of $\beta$-phase emergence by alignment of polymer chains and the formation of particles were evident at a very early stage of the step-growth polymerization. Deliberate disturbance of ordered chain-packing by cross-links introduced during the emulsion polymerization resulted in the formation of increasingly spherical particles of amorphous structure. This underlined the role of chain packing processes of (linear) PF8 segments on the particle shape. Another decisive factor for the evolution of ordered $\beta$-phase regimes was found in the concentration of PF8 in solution, for instance within the emulsified droplets of the heterophase polycondensation. With increasing concentration, the occurrence of $\beta$-phase conformation in a higher proportion was evidenced from fluorescence measurements. 
Based on these findings, a mechanism of particle formation was proposed (Scheme 7.1). Immediately with emulsification of the organic monomer solution in the aqueous phase, oligo- and polymer chains are rapidly formed and align to regions of $\beta$-phase conformation (a). The required intermolecular interactions are the more dominant the higher the polymer concentration in the emulsified droplets. The final molecular weight of the polymer is reached within the first minutes of the reaction and the remaining reaction time is necessary for the organic solvent to evaporate (b). The obtained, fluorescent nanoparticles exhibit a characteristic, ellipsoidal shape and a high portion of ordered phases. Conclusively, the studies outlined provide valuable insight into the evolution of an anisotropic shape of conjugated polymer particles, which was found to strongly correlate with the formation of ordered $\beta$ phase regimes. Hence, the properties of polymer nanoparticles, including their external appearance, can be directed to a high degree via internal chain order.

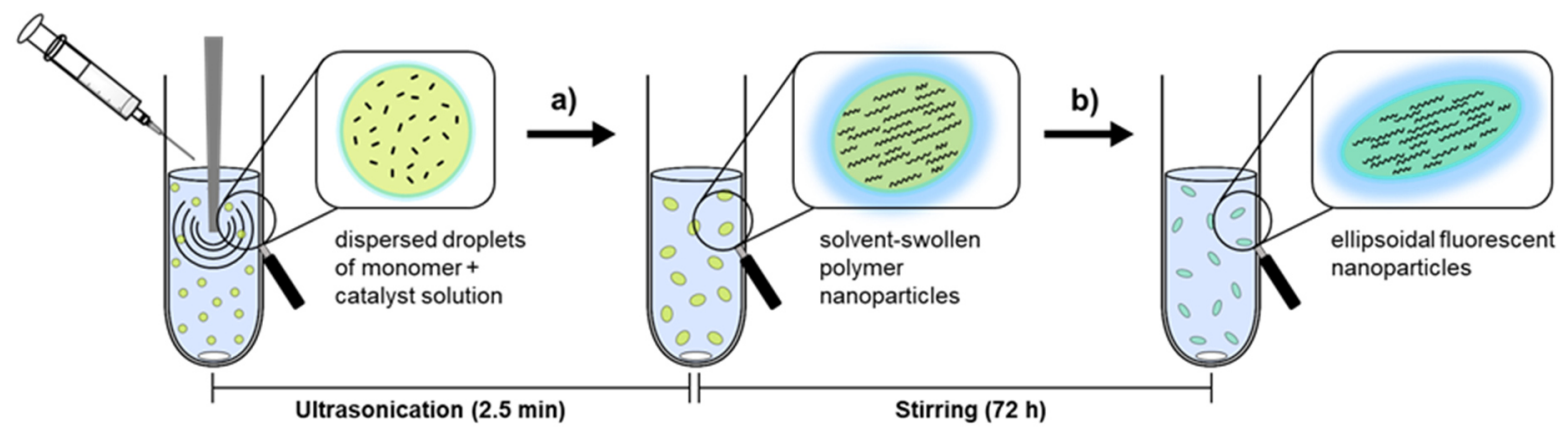

Scheme 7.1: Proposed mechanism of nanoparticle formation including (a) the formation of oligo- and poly(fluorene) chains and their arrangement in ordered phases during ultrasonication and (b) the evaporation of the organic solvent from the swollen particles, yielding ellipsoidal fluorescent nanoparticles.

Apart from poly(9,9'-dioctylfluorene), exceptional morphological properties and the ability of chain arrangement towards regions of high order have also been reported for poly(5-octyloxy-9,9'diphenylfluorene) (PODPF) (Figure 7.4a). The ordered alignment of the polymer backbone is presumably based on the interplay of repulsive and attractive forces induced by the bulky phenyl substituents and the octyloxy side chains.

Therefore, PODPF was studied as a second complementary case to PF8. In the same way as for PF8, the fluorescence emission of PODPF solutions in dependency of the concentration was examined. These studies revealed a change in phase behavior of the material, evident from the increased appearance of $\beta$-phase conformation at higher concentrations. Since PODPF combines the ability of $\beta$ phase formation with high oxidative stability in optoelectrical devices, it was considered an appropriate alternative to PF8. The synthesis of PODPF nanoparticles by different approaches was investigated, focusing in particular on the shape and internal structure of the resulting particles. A set of AA/BB-type monomers was synthesized and employed in the established procedure of heterophase Suzuki-Miyaura polycondensation. By optimization of several reaction parameters, especially the monomer concentration in the initial reaction mixture, the protocol yielded exclusively ellipsoidal nanoparticles $(I=92 \mathrm{~nm}$, ar =2.5). In addition to the AA/BB-type polycondensation, a controlled Suzuki-Miyaura coupling polymerization to PODPF was elaborated, using the respective AB-type monomers. 
With two alternative approaches to PODPF established, nanoparticles were also prepared in a postpolymerization approach, comprising the emulsification of pre-synthesized PODPF in an aqueous surfactant solution. Adjustment of several conditions yielded ellipsoidal nanoparticles $(I=104 \mathrm{~nm}$, ar $=3.4$ ) with improved quality in view of anisotropy and dispersity as compared to particles directly generated in emulsion polymerization (Figure 7.4b). Investigation of the prepared nanoparticle dispersions regarding their optical properties revealed the presence of disordered, amorphous polymer phase, whereas the characteristic signals arising from $\beta$-phase regions remained absent (Figure 7.4c).

a)

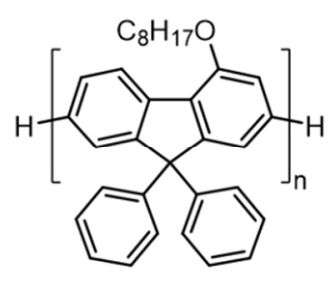

b)

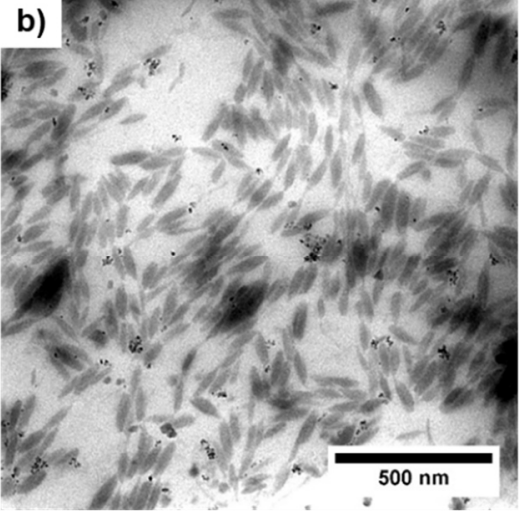

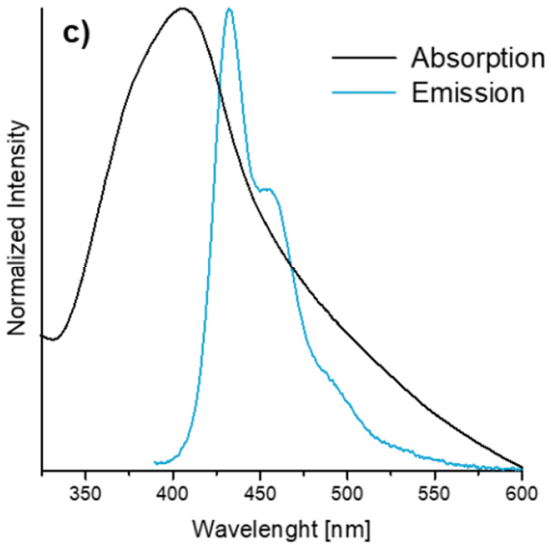

Figure 7.4: (a) Structure of poly(5-octyloxy-9,9'-diphenylfluorene) (PODPF). (b) TEM images of PODPF dispersions prepared via post-polymerization particle preparation. (c) Exemplary UVIVis absorption and fluorescence emission spectra of PODPF dispersions.

Conclusively, poly(5-octyloxy-9, $9^{\prime}$-diphenylfluorene) provided the capabilities of both $\beta$-phase evolution and the formation of anisotropic particle shapes, but unlike in the case of PF8, no obvious correlation of these two phenomena was evidenced from recorded data. Ellipsoidal PODPF nanoparticles of high quality could be obtained in a post-polymerization emulsification approach, which broadens the scope of conjugated polymers which are accessible as anisotropic particles.

Beyond the favorable utilization as basic material for the synthesis of nanoparticles, conjugated polymers, including poly(fluorenes) provide attractive hydrophobic, rigid components in blockcopolymers. ${ }^{140,143,170}$ By attachment of a flexible, hydrophilic moiety, the resulting amphiphilic rod-coiltype copolymers have the ability to organize into complex, nanoscale superstructures, driven by mechanisms of microphase separation. As the close correlation of chain organization on a molecular level and the shape of the resulting nanostructures constituted a major issue of this work, conjugated block copolymers were investigated as an illustrative reference system for comparison. To this end, amphiphilic poly(9,9'-dioctylfluorene)-poly(ethylene glycol) block copolymers were synthesized and examined. Initially, a synthetic approach towards macromolecules consisting of PEGylated fluorene monomers was elaborated. These were converted to a catalytically active palladium species, which initiated the polymerization of $A B$-type monomer via controlled Suzuki-Miyaura coupling polymerization (Figure 7.5a). The established polymerization protocol yielded PF8-PEG block copolymers and the successful connection of the two segments was confirmed. On that basis, a series of copolymers was synthesized, with different block length ratios achieved by either variation of the commercial PEG starting materials or adjustment of the fluorene monomer to catalyst ratio. Examination of the 
copolymers' optical properties evidenced red-shifts in their absorption and emission wavelengths in accordance with extended conjugation lengths for longer PF8 segments, whereas no effect for variation of the hydrophilic moiety was observed. Apart from PF8 as a reference system, block copolymers with other conjugated polymer segments were synthesized via implementation of the reaction procedure.

a)
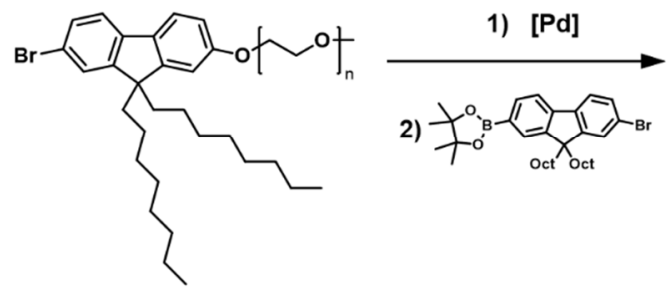

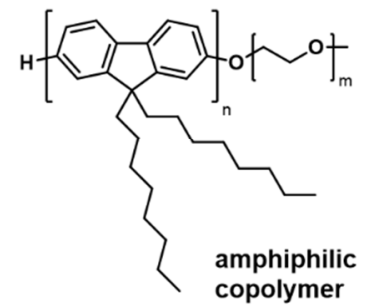

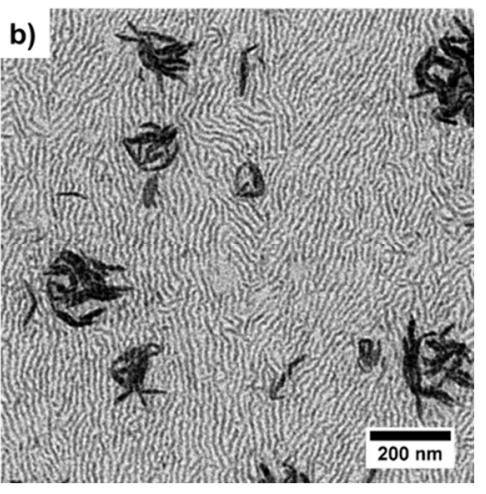

Figure 7.5: (a) Schematic illustration of the synthetic pathway towards amphiphilic block copolymers by SuzukiMiyaura coupling polymerization, starting from PEGylated bromofluorene macromolecules. (b) TEM image of sample prepared from an amphiphilic PF8-PEG copolymer, dissolved in a solvent mixture.

The synthesized PF8-PEG copolymers were studied regarding their aggregation behavior, which revealed the occurrence of regular, lamellar structures under suitable conditions (Figure 7.5b). The formation of these aggregates was presumably dominated by microphase separation processes, enabled by the attached hydrophilic coil segments. In summary, Suzuki-Miyaura coupling polymerization starting from PEGylated bromofluorenes provides an efficient synthetic approach towards amphiphilic block copolymers. The elaborated system gives access to a broad range of copolymers with variable emission color and different segment ratios, which represent promising materials to be applied as stabilizing agents for nanoparticle dispersions.

Overall, the correlation of anisotropic particle shape and internal chain alignment was elucidated for the example of ellipsoidal, fluorescent poly(9,9'-dioctylfluorene) (PF8) nanoparticles, synthesized in a heterophase Suzuki-Miyaura polycondensation. The origin of the unique particle shape was revealed by a combination of synthetic and analytic approaches. EPR experiments performed on spin-labeled oligo(fluorenes) evidenced the semi-flexible character of PF8, which was retained upon incorporation of the chains in solid particles. The alignment behavior of longer species inaccessible by EPR spectroscopy was monitored by several other spectroscopic and microscopic techniques. A general ability of longer oligomers and even of PF8 chains to align within particles to form phases of high order was evidenced. Regarding the processes involved, various decisive factors were outlined, such as the choice of organic solvent or the prerequisite of linear polymer chains. The formation of domains of high chain order was found to be favored by the high polymer concentration, already occurring in early stages of the polymerization. As alternative to emulsion polymerization, a post-polymerization protocol appropriate for the preparation of ellipsoidal PF8 nanoparticles was established. The scope of conjugated polymers accessible as particles of anisotropic shape was extended by poly(fluorene) with alternative substituents. In summary, the evolution of chain order in polymers was found to exceptionally correlate with the formation of non-spherical particle shapes. 


\section{8 experimental SeCtion}

\subsection{MATERIALS AND GENERAL CONSIDERATIONS}

Unless stated otherwise, all reactions of air- and/or water-sensitive compounds were carried out under inert gas atmosphere using standard Schlenk and glovebox techniques.

Solvents were purchased from Sigma-Aldrich, now Merck, or VWR and dried using standard laboratory techniques. Tetrahydrofuran was distilled from sodium/benzophenone ketyl, toluene and diethyl ether were dried and degassed by passing through columns of copper catalyst and $3 \AA$ molecular sieve. $N, N-$ Dimethylformamid was distilled from calcium hydride. Acetonitrile was stored over $3 \AA$ molecular sieve. Xylene was degassed by Freeze-Pump-Thaw cycling prior to use. Solvents of analytical grade were used as purchased. $\mathrm{CDCl}_{3}$ was supplied by Deutero and $\mathrm{C}_{6} \mathrm{D}_{6}$ was supplied by Eurisotop. The deuterated solvents were stored over $3 \AA$ molecular sieves.

Bis(1,5-cyclooctadiene)nickel(0) (98\%), 2-bromofluorene (95\%), 1-bromooctane (98\%), 9-fluorenone $(98 \%)$, tetrakis(triphenylphosphine)palladium(0) (99\%), tetra- $n$-butylammonium bromide (TBAB) $(98 \%)$ and triphenylamine (97\%) were purchased from ABCR. Tetraethylammonium hydroxide ( $20 \%$ solution in water), phenylmagnesium bromide $\left(3 \mathrm{M}\right.$ in $\left.\mathrm{Et}_{2} \mathrm{O}\right)$ and $\mathrm{BF}_{3} \times \mathrm{Et}_{2} \mathrm{O}$ were acquired from Merck. Benzyltriethylammonium chloride (99\%), 2,2'-bipyridine (99\%), 4-bromotoluene (98\%), 1,5-cyclooctadiene $(\geq 99 \%)$, iso-propylboronic acid pinacol ester (98\%), poly(ethylene glycol) 500 monomethylether (mPEG), sodium dodecyl sulfate (SDS) pellets, Triton X-102, $1.6 \mathrm{M}$ and $2.5 \mathrm{M}$ solutions of $n$-butyllithium in hexane, tri-iso-propyl borate (98\%), chloro[(tri-tert-butylphosphine)-2-(amino-biphenyl)] palladium(II) ( $\mathrm{P}(t-\mathrm{Bu})_{3} \mathrm{Pd} \mathrm{G} 2$ ), bromine (reagent grade), $N$-bromosuccinimide (99\%) and 1,3,5tribromobenzene (98\%) were purchased from Sigma-Aldrich, now Merck. $\mathrm{FeCl}_{3} \times 6 \mathrm{H}_{2} \mathrm{O}(\geq 99 \%)$ was purchased from Honeywell. $m$-CPBA (70-75\% in mixture with water) was acquired from acros. 9Fluorene ( $\geq 98 \%$ ), $p$-toluenesulfonyl chloride ( $\geq 89 \%$ ) and 1,3-propanediol ( $98 \%$ ) were supplied by Fluka. Tetraphenylmethane $(\geq 96 \%)$ was supplied by $\mathrm{TCl}$. Bis(tri-tert-butylphosphine) palladium( 0 ) was available in the research group, prepared according to reported procedures. ${ }^{219}$

Column chromatography was performed using silica gel 60M $(0.04-0.063 \mathrm{~mm})$ from Macherey-Nagel. Dialysis was performed with Spectrum Spectra/Por $1 \mathrm{RC}$ Dialysis Membrane Tubings with a diameter of $14.6 \mathrm{~mm}$ and a molecular weight cutoff (MWCO) of 6-8 kD or $3.5 \mathrm{kD}$. 


\subsection{ANALYTICAL METHODS AND TECHNIQUES}

Ultrasonication was applied using a Bandelin GM3200 ultrasonotrode with a KE76 tip, operated at $120 \mathrm{~W}$ with $60 \%$ amplitude. Centrifugation was carried out on a Biofuge Primo centrifuge with a highconic rotor (6000 rpm, 10 minutes). Freeze-drying was carried out with a Christ Alpha 2-4 LDplus freeze-dryer.

${ }^{1} \mathrm{H},{ }^{13} \mathrm{C},{ }^{31} \mathrm{P}$ and the corresponding two-dimensional nuclear magnetic resonance (NMR) spectroscopy was performed on Bruker Avance III 400, Bruker Avance III HD 400, Bruker Avance NEO 800 and Bruker Avance III 600 spectrometers. The chemical shifts in ppm were referenced to the solvent residual proton signals $\left(\mathrm{CDCl}_{3}: 7.26 \mathrm{ppm} ; \mathrm{C}_{6} \mathrm{D}_{6}: 7.16 \mathrm{ppm}\right)$ or to the carbon signals of the deuterated solvent $\left(\mathrm{CDCl}_{3}: 77.16 \mathrm{ppm} ; \mathrm{C}_{6} \mathrm{D}_{6}: 128.06 \mathrm{ppm}\right)$. The multiplicities of the signals were abbreviated as follows: s (singlet), d (doublet), t (triplet), q (quartet), quint (quintet), dd (doublet of doublets), dt (doublet of triplets), $m$ (multiplet) and br (broad signal).

Gel permeation chromatography (GPC) was carried out on a SECcurity2 GPC system from Polymer Standards Service (PSS) with a SDV Linear M $5 \mu \mathrm{m}$ column with RI and UV/Vis detection. The samples were measured in THF at $50{ }^{\circ} \mathrm{C}$ using linear calibration versus poly(styrene) standards. The same device was also used for semi-preparative GPC analysis, using a PSS-SDV column $(300 \times 20 \mathrm{~mm}$, porosity: $1000 \AA$ ) in combination with a SECcurity ${ }^{2}$ fraction collector.

UV/Vis absorption spectra were recorded on a Varian Cary 100 scan spectrometer. Fluorescence emission was measured with a Hamamatsu Absolute PL Quantum Yield Measurement System C9920-02. The default measurement parameters comprise an excitation wavelength of $\lambda_{\mathrm{exc}}=380 \mathrm{~nm}$ with an acquisition time of $50 \mathrm{~ms}$ and averaging over 50 measurement runs. Unless stated otherwise, both absorption and fluorescence measurements were performed on highly diluted solutions or dispersions (c $\leq 0.01 \mathrm{mg} / \mathrm{mL})$.

Matrix-assisted LASER desorption/ionization - time of flight (MALDI-TOF) mass spectra were recorded in THF on a Bruker Microflex mass spectrometer with either $\alpha$-cyano-4-hydroxycinnamic acid (HCCA) or trans-2-[3-(4-tert-butylphenyl)-2-methyl-2-propenylidene]-malononitrile (DCTB) as matrix.

Transmission electron microscopy (TEM) images were captured with a Zeiss Libra 120 instrument with an acceleration voltage of $120 \mathrm{kV}$, using carbon coated copper grids (carbon thickness: $9 \mathrm{~nm}$ ). Samples were not contrasted. Using the ImageJ software, the mean values of particle length and aspect ratio and the corresponding coefficient of variation $v$ were calculated from the measurement of 50 nanoparticles in each case. Outliers were determined and prevented via Grubb's test with a significance level of 0.05 . The coefficient of variation $v$ as a measure for the particle dispersity was defined by the ratio of the standard deviation of either particle length of aspect ratio to the respective mean value.

Dynamic light scattering (DLS) measurements were carried out on diluted particle dispersions using a Malvern Zetasizer Nano-ZS ZEN 3600 instrument $(633 \mathrm{~nm})$ in backscattering mode $\left(173^{\circ}\right)$ at $25^{\circ} \mathrm{C}$ and the acquired data was processed with the Malvern Zetasizer Software 7.12. 
Scanning electron microscopy (SEM) was performed on a Zeiss Auriga CrossBeam with an acceleration voltage of $3 \mathrm{kV}$. SEM samples were prepared on $5 \times 7 \mathrm{~mm}$ silicon wafer chips acquired from Ted Pella Inc. The calculation of the color-indicated particle orientation was performed using the OrientationJ Distribution plugin of the ImageJ software.

Pulsed electron paramagnetic resonance (EPR) measurements were performed in Q-band on a Bruker Elexsys E580 spectrometer, equipped with a $15 \mathrm{~W}$ microwave amplifier and a helium gas flow system (CF935, Oxford Instruments) and a Bruker EN5107D2 resonator, using sample tubes with $1.6 \mathrm{~mm}$ outer diameter. Evaluation of raw data was performed with DEERAnalysis 2019. Continuous wave EPR spectroscopy for spin concentration determination was carried out on a Bruker EMX Nano. 


\title{
8.3 HETEROPHASE SUZUKI-MIYAURA POLYCONDENSATION
}

\subsubsection{SYNTHESES OF MONOMERS}

\section{SYNTHESIS OF 2,7-DIBROMOFLUORENE220}<smiles>Brc1ccc([IH]2=Cc3ccc(Br)cc3C2)cc1</smiles>

\author{
$\mathrm{C}_{13} \mathrm{H}_{8} \mathrm{Br}_{2}$ \\ $\mathrm{M}=324.02 \mathrm{~g} / \mathrm{mol}$
}

A solution of fluorene $\left(40.0 \mathrm{~g}, 241 \mathrm{mmol}, 1\right.$ equiv.) in chloroform $(350 \mathrm{~mL})$ was cooled to $-10{ }^{\circ} \mathrm{C}$ and $\mathrm{FeCl}_{3} \times 6 \mathrm{H}_{2} \mathrm{O}(1.0 \mathrm{~g}, 3.6 \mathrm{mmol}, 0.015$ equiv.) was added. Under exclusion of light, bromine $(3.1 \mathrm{~mL}$, $511 \mathrm{mmol}, 2.1$ equiv.) was added dropwise over a period of $2 \mathrm{~h}$ (caution: bromine is corrosive and toxic) and the solution was stirred over $3 \mathrm{~h}$, while it was allowed to warm to room temperature. The reaction was quenched by addition of aqueous $\mathrm{Na}_{2} \mathrm{~S}_{2} \mathrm{O}_{5}$ and the mixture was stirred for 30 min until the brown color turned into a light yellow. The phases were separated and the aqueous layer was extracted three times with dichloromethane. The combined organic phases were washed with water, dried over $\mathrm{MgSO}_{4}$ and the solvent was then removed under reduced pressure. The crude product was purified by recrystallization from ethanol to yield 2,7-dibromofluorene as white solid $(70.0 \mathrm{~g}, 216 \mathrm{mmol}, 89 \%)$.

${ }^{1} \mathrm{H}-\mathrm{NMR}\left(400 \mathrm{MHz}, \mathrm{CDCl}_{3}, 25^{\circ} \mathrm{C}\right): \delta[p p m]=7.66\left(\mathrm{~d},{ }^{4} \mathrm{JHH}_{\mathrm{HH}}=1.3 \mathrm{~Hz}, 2 \mathrm{H}, \mathrm{H}-3\right), 7.59\left(\mathrm{~d},{ }^{3} \mathrm{JHH}_{\mathrm{HH}}=8.1 \mathrm{~Hz}\right.$, $2 \mathrm{H}, \mathrm{H}-6), 7.50\left(\mathrm{dd},{ }^{3} \mathrm{JHH}_{\mathrm{HH}}=8.1 \mathrm{~Hz},{ }^{4} \mathrm{JHH}_{\mathrm{H}}=1.7 \mathrm{~Hz}, 2 \mathrm{H}, \mathrm{H}-5\right), 3.86(\mathrm{~s}, 2 \mathrm{H}, \mathrm{H}-1)$.

${ }^{13} \mathrm{C}-\mathrm{NMR}\left(101 \mathrm{MHz}, \mathrm{CDCl}_{3}, 25^{\circ} \mathrm{C}\right): \delta[\mathrm{ppm}]=144.9$ (C-2), 139.8 (C-7), 130.3 (C-5), 128.4 (C-3), 121.3 (C-6), 121.1 (C-4), 36.7 (C-1).

\section{SYNTHESIS OF 2,7-DIBROMO-9,9' -DIOCTYLFLUORENE $\left(\mathrm{Br}-\mathrm{F}_{1}-\mathrm{Br}\right)^{221}$}

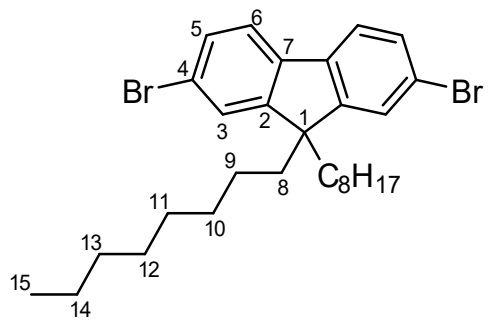

$$
\begin{aligned}
& \mathrm{C}_{29} \mathrm{H}_{40} \mathrm{Br}_{2} \\
& \mathrm{M}=548.45 \mathrm{~g} / \mathrm{mol}
\end{aligned}
$$

A solution of 2,7-dibromofluorene (50.0 g, $154 \mathrm{mmol}, 1$ equiv.), 1-bromooctane (64 mL, $370 \mathrm{mmol}$, 2.4 equiv.) and benzyltriethylammonium chloride $(1.8 \mathrm{~g}, 7.7 \mathrm{mmol}, 0.05$ equiv. $)$ in DMSO (250 $\mathrm{mL})$ was degassed by application of vacuum. A degassed aqueous $50 \mathrm{wt} \% \mathrm{NaOH}$ solution $(70 \mathrm{~mL}, 875 \mathrm{mmol}$, 5.7 equiv.) was added and the mixture was stirred overnight. After addition of diethylether $(500 \mathrm{~mL})$, the organic phase was separated, washed with water, $2 \mathrm{M} \mathrm{HCl}$ and brine and dried over $\mathrm{MgSO}_{4}$. The solvent 
was removed under reduced pressure and the residual brown oil was recrystallized from ethanol, yielding 2,7-dibromo-9,9'-dioctylfluorene $\left(\mathbf{B r}^{\prime}-\mathrm{F}_{1}-\mathbf{B r}\right)$ as white crystals $(70.3 \mathrm{~g}, 128 \mathrm{mmol}, 83 \%)$.

${ }^{1} \mathrm{H}-\mathrm{NMR}\left(400 \mathrm{MHz}, \mathrm{CDCl}_{3}, \mathbf{2 5}^{\circ} \mathrm{C}\right): \delta[\mathrm{ppm}]=7.52\left(\mathrm{~d},{ }^{3} \mathrm{JHH}_{\mathrm{HH}}=8.2 \mathrm{~Hz}, 2 \mathrm{H}, \mathrm{H}-6\right), 7.46-7.44(\mathrm{~m}, 4 \mathrm{H}, \mathrm{H}-3$, $\mathrm{H}-5), 1.93-1.89(\mathrm{~m}, 4 \mathrm{H}, \mathrm{H}-8), 1.26-1.05(\mathrm{~m}, 2 \mathrm{H}, \mathrm{H}-10, \mathrm{H}-11, \mathrm{H}-12, \mathrm{H}-13, \mathrm{H}-14), 0.83\left(\mathrm{t},{ }^{3} \mathrm{JHH}_{\mathrm{HH}}=7.1 \mathrm{~Hz}\right.$, $6 \mathrm{H}, \mathrm{H}-15), 0.61-0.57$ (m, 4H, H-9).

${ }^{13} \mathrm{C}$-NMR (101 MHz, $\mathrm{CDCl}_{3}, 25^{\circ} \mathrm{C}$ ): $\delta$ [ppm] = 152.7 (C-2), 139.2 (C-7), 130.3 (C-3), 126.3 (C-5), 121.6 (C-4), 121.3 (C-6), 55.8 (C-1), 40.3 (C-8), 31.9 (C-13), 30.0 (C-10), 29.3 (C-11), 29.2 (C-12), 23.8 (C-9), 22.7 (C-14), $14.2(\mathrm{C}-15)$.

\section{SYNTHESIS OF 2,2' -(9, $9^{\prime}$-DIOCTYLFLUORENE-2,7-DIYL)BIS-}

\section{(1,3,2-DIOXABORINANE) $\left(\mathrm{PrO}_{2} \mathrm{~B}-\mathrm{F}_{1}-\mathrm{BO}_{2} \mathrm{Pr}\right)^{204}$}

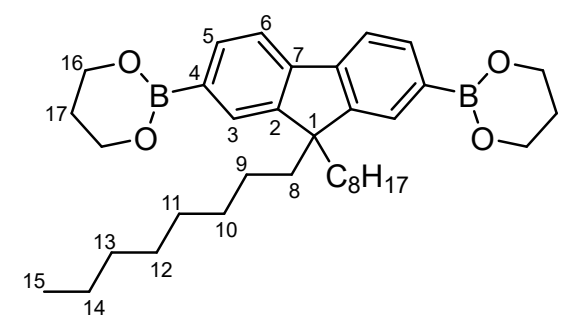

$$
\begin{aligned}
& \mathrm{C}_{35} \mathrm{H}_{52} \mathrm{~B}_{2} \mathrm{O}_{4} \\
& \mathrm{M}=558.42 \mathrm{~g} / \mathrm{mol}
\end{aligned}
$$

A solution of 2,7-dibromo-9,9'-dioctylfluorene $(10.0 \mathrm{~g}, 18.2 \mathrm{mmol}, 1$ equiv.) in dry THF (100 mL) was cooled to $-78{ }^{\circ} \mathrm{C}$ and a $2.5 \mathrm{M}$ solution of $n$-butyllithium in hexane $(25 \mathrm{~mL}, 62.5 \mathrm{mmol}, 3.4$ equiv.) was added dropwise. The solution was stirred for $2 \mathrm{~h}$ at the same temperature and tri-iso-propyl borate (16.5 mL, $71.1 \mathrm{mmol}, 3.9$ equiv.) was added, before it was brought to room temperature and stirred overnight. The reaction mixture was poured into $2 \mathrm{M} \mathrm{HCl}$ containing crushed ice under vigorous stirring and subsequently extracted three times with diethyl ether. The solvent was removed under reduced pressure and the residue was refluxed with 1,3-propanediol ( $4.0 \mathrm{~mL}, 55.2 \mathrm{mmol}, 3$ equiv.) in toluene $(100 \mathrm{~mL})$ overnight. The mixture was cooled to room temperature and diethyl ether $(200 \mathrm{~mL})$ was added. After phase separation the organic layer was washed with brine and water, dried over $\mathrm{MgSO}_{4}$ and the solvent was removed in vacuo. Recrystallization of the crude product from ethanol yielded $2,2^{\prime}-\left(9,9^{\prime}\right.$ dioctylfluorene-2,7-diyl)bis(1,3,2-dioxaborinane) $\left(\mathbf{P r O}_{2} \mathbf{B}-\mathbf{F}_{1}-\mathbf{B O}_{2} \mathbf{P r}\right)$ as white solid $(6.4 \mathrm{~g}, 11.4 \mathrm{mmol}$, $63 \%)$.

${ }^{1} \mathrm{H}-\mathrm{NMR}\left(\mathbf{4 0 0} \mathrm{MHz}, \mathrm{CDCl}_{3}, 25^{\circ} \mathrm{C}\right): \delta[\mathrm{ppm}]=7.75\left(\mathrm{dd},{ }^{3} \mathrm{JHH}_{\mathrm{HH}}=7.4 \mathrm{~Hz},{ }^{4} \mathrm{JHH}_{\mathrm{HH}}=1.0 \mathrm{~Hz}, 2 \mathrm{H}, \mathrm{H}-5\right), 7.72(\mathrm{~s}$, $2 \mathrm{H}, \mathrm{H}-3$ ), 7.69 (dd, $\left.{ }^{3} \mathrm{JHH}=7.6 \mathrm{~Hz},{ }^{5} \mathrm{JHH}_{\mathrm{H}}=0.6 \mathrm{~Hz}, 2 \mathrm{H}, \mathrm{H}-6\right), 4.20$ (t, $\left.{ }^{3} \mathrm{JHH}_{\mathrm{HH}}=5.3 \mathrm{~Hz}, 8 \mathrm{H}, \mathrm{H}-16\right), 2.09$ (quint, $\left.{ }^{3} \mathrm{JHH}_{\mathrm{HH}}=5.3 \mathrm{~Hz}, 4 \mathrm{H}, \mathrm{H}-17\right), 2.00-1.96(\mathrm{~m}, 4 \mathrm{H}, \mathrm{H}-8), 1.25-1.00(\mathrm{~m}, 20 \mathrm{H}, \mathrm{H}-10, \mathrm{H}-11, \mathrm{H}-12, \mathrm{H}-13, \mathrm{H}-14)$, $0.81\left(\mathrm{t},{ }^{3} \mathrm{~J}_{\mathrm{HH}}=7.2 \mathrm{~Hz}, 6 \mathrm{H}, \mathrm{H}-15\right), 0.59-0.51(\mathrm{~m}, 4 \mathrm{H}, \mathrm{H}-9)$.

${ }^{13} \mathrm{C}-\mathrm{NMR}\left(101 \mathrm{MHz}, \mathrm{CDCl}_{3}, 25^{\circ} \mathrm{C}\right): \delta$ [ppm] = 150.5 (C-2), 143.7 (C-7), 132.5 (C-5), 131.7 (C-4), 128.0 (C-3), 119.3 (C-6), 62.2 (C-16), 55.0 (C-1), 40.5 (C-8), 32.0 (C-13), 30.2 (C-10), 29.4 (C-11), 29.3 (C-12), 27.6 (C-17), 23.8 (C-9), 22.7 (C-14), 14.2 (C-15). 


\subsubsection{POLYMERIZATION PROCEDURE}

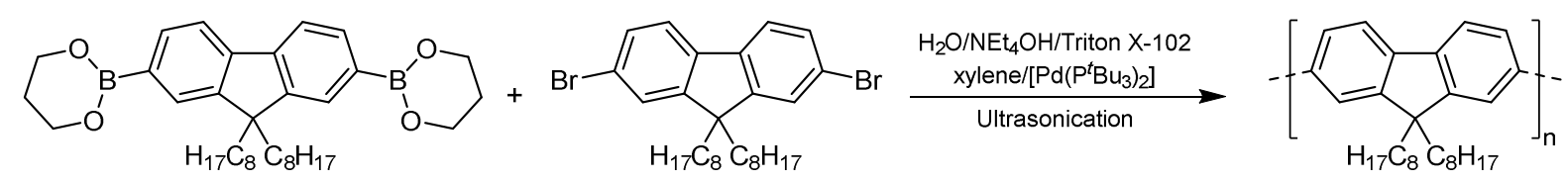

Suzuki-Miyaura polycondensation under heterophase conditions was performed according to the procedure published by Ruiz Perez et al. ${ }^{8}$ In a $100 \mathrm{~mL}$ Schlenk tube, Triton X-102 (1.25 g, $1.75 \mathrm{mmol}$, 17.5 equiv.) was dissolved in deionized water $(25 \mathrm{~mL})$ and $\mathrm{NEt}_{4} \mathrm{OH}$ solution $(20 \%$ in water $)(0.16 \mathrm{~g}$, $0.2 \mathrm{mmol}, 2$ equiv.) was added. The solution was degassed by sparging with $\mathrm{N}_{2}$ for at least $30 \mathrm{~min}$. $\mathrm{PrO}_{2} \mathrm{~B}-\mathrm{F}_{1}-\mathrm{BO}_{2} \mathrm{Pr}$ (55.8 mg, $0.1 \mathrm{mmol}, 1$ equiv.) and $\mathrm{Br}-\mathrm{F}_{1}-\mathrm{Br}(54.8 \mathrm{mg}, 0.1 \mathrm{mmol}, 1$ equiv.) were dissolved in xylene $(1 \mathrm{~mL})$. The solution was degassed by three freeze-pump-thaw cycles and added to bis(tri-tert-butylphosphine) palladium(0) (2.1 mg, $4 \mu \mathrm{mol}, 0.04$ equiv.), followed by three further freezepump-thaw cycles. The monomer/catalyst solution was rapidly injected via syringe into the aqueous phase with simultaneous emulsification by an ultrasonotrode (2.5 min, 60\% amplitude). After ultrasonication, the emulsion was stirred for $72 \mathrm{~h}$ at room temperature to yield a greenish-yellow PF8 dispersion.

For the removal of excessive amounts of surfactant as required for certain analytical methods, the original polymer dispersion $(2 \mathrm{~mL})$ was precipitated by addition of methanol $(6 \mathrm{~mL})$, followed by centrifugation (6000 rpm, $10 \mathrm{~min}$ ). The supernatant, colorless solution was discarded and the solid residue was redispersed in deionized water $(2 \mathrm{~mL}$ ) by ultrasonication ( $1 \mathrm{~min}, 30 \%$ amplitude).<smiles>CCCCCCCCCC1(CC)c2cc(C(C)(C)C)ccc2-c2ccc(C(C)(C)C)cc21</smiles>

${ }^{1} \mathrm{H}-\mathrm{NMR}\left(400 \mathrm{MHz}, \mathrm{CDCl}_{3}, \mathbf{2 5}^{\circ} \mathrm{C}\right): \delta[\mathrm{ppm}]=$ 7.90-7.83 (m, 2H, H-4), 7.77-7.64 (m, 4H, H-1, H-3), 2.232.04 ( $m, 4 \mathrm{H}, \mathrm{H}-8), 1.29-1.13$ (m, 20H, H-10, H-11, H-12, H-13, H-14), 0.93-0.75 (m, 10H, H-9, H-15).

${ }^{13} \mathrm{C}-\mathrm{NMR}\left(101 \mathrm{MHz}, \mathrm{CDCl}_{3}, 25^{\circ} \mathrm{C}\right): \delta[\mathrm{ppm}]=152.0$ (C-6), 140.7 (C-5), 140.2 (C-2), 126.3 (C-1), 121.7 (C-3), 120.1 (C-4), 55.5 (C-7), 40.5 (C-8), 32.0 (C-13), 30.2 (C-10), 29.4 (C-11, C-12), 24.1 (C-9), 22.8 (C-14), 14.2 (C-15). 


\subsection{DOUBLE ELECTRON-ELECTRON RESONANCE MEASUREMENTS ON OLIGOFLUORENES}

\subsubsection{SPIN-LABELED OLIGOMERS BY CONTROLLED SUZUKI-MIYAURA COUPLING POLYMERIZATION}<smiles>CC1(C)OB(c2ccc3c(c2)C([13CH2])([13CH2])c2cc(Br)ccc2-3)OC1(C)C</smiles>

AB-type monomer

Spin-labeled initiator<smiles>CCCCCP1(Cl)(Cl)Nc2ccccc2-c2ccccc21</smiles>

Pd-G2 precatalyst<smiles>CC1(C)CC(OC(=O)c2ccc(B3OC(C)(C)C(C)(C)O3)cc2)CC(C)(C)N1[O]</smiles>

Spin-labeled end-capping agent

Figure 8.1: AB-type fluorene monomer, TEMPO-based spin-labels and catalyst applied in controlled SuzukiMiyaura coupling polymerization.

SYNTHESIS OF 2-(7-BROMO-9,9'-DIOCTYLFLUOREN-2-YL)-4,4,5,5-TETRAMETHYL-

\section{1,2,3-DIOXABOROLANE (Br-F/-BPin) ${ }^{31,205}$}

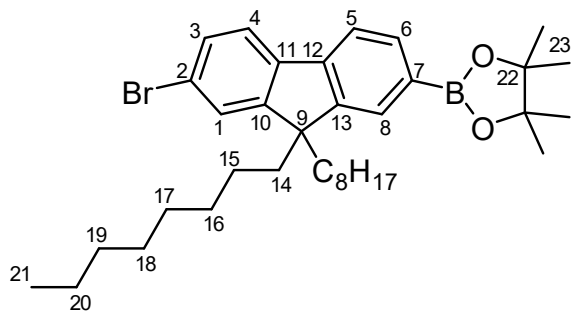

$\mathrm{C}_{35} \mathrm{H}_{52} \mathrm{BBrO}_{2}$

$\mathrm{M}=595.51 \mathrm{~g} / \mathrm{mol}$

A solution of 2,7-dibromo-9,9'-dioctylfluorene $(5.0 \mathrm{~g}, 9.12 \mathrm{mmol}, 1$ equiv. $)$ in dry THF (100 mL) was cooled to $-78{ }^{\circ} \mathrm{C}$ and a $2.5 \mathrm{M}$ solution of $n$-butyllithium in hexane $(4.1 \mathrm{~mL}, 10.02 \mathrm{mmol}, 1.1$ equiv.) was added dropwise. After stirring for $1 \mathrm{~h}$ while cooling, iso-propylboronic acid pinacol ester $(2.2 \mathrm{~mL}$, $10.94 \mathrm{mmol}, 1.2 \mathrm{mmol}$ ) was added and the reaction mixture was allowed to warm up to room temperature and was stirred overnight. For facilitation of phase separation, distilled water and $\mathrm{Et}_{2} \mathrm{O}$ were then added. The organic phase was separated and washed consecutively with brine and distilled water, dried over $\mathrm{MgSO}_{4}$ and the solvents were removed under reduced pressure. The crude product was purified by recrystallization from ethanol, yielding 2-(7-bromo-9,9'-dioctyl-9H-fluoren-2-yl)-4,4,5,5tetramethyl-1,3,2-dioxaborolane (Br-F1-BPin) as a white solid (3.96 g, $6.65 \mathrm{mmol}, 73 \%$ ). 
${ }^{1} \mathrm{H}-\mathrm{NMR}\left(400 \mathrm{MHz}, \mathrm{CDCl}_{3}, 25^{\circ} \mathrm{C}\right): \delta[p p m]=7.80\left(\mathrm{dd},{ }^{3} \mathrm{~J}_{\mathrm{HH}}=7.6 \mathrm{~Hz},{ }^{4} \mathrm{~J}_{\mathrm{HH}}=1.0 \mathrm{~Hz}, 1 \mathrm{H}, \mathrm{H}-6\right), 7.73(\mathrm{~s}$, $1 \mathrm{H}, \mathrm{H}-8), 7.66\left(\mathrm{dd},{ }^{3} \mathrm{JHH}_{\mathrm{HH}}=7.6 \mathrm{~Hz},{ }^{4} \mathrm{~J}_{\mathrm{HH}}=0.7 \mathrm{~Hz}, 1 \mathrm{H}, \mathrm{H}-5\right), 7.57\left(\mathrm{dd},{ }^{3} \mathrm{~J}_{\mathrm{HH}}=7.7 \mathrm{~Hz},{ }^{4} \mathrm{JHH}_{\mathrm{HH}}=0.7 \mathrm{~Hz}, 1 \mathrm{H}\right.$, $\mathrm{H}-4)$, 7.46-7.44 (m, 2H, H-1, H-3), 2.02-1.88 (m, 4H, H-14), $1.39(\mathrm{~s}, 12 \mathrm{H}, \mathrm{H}-23), 1.23-1.02(\mathrm{~m}, 20 \mathrm{H}$, $\mathrm{H}-16, \mathrm{H}-17, \mathrm{H}-18, \mathrm{H}-19, \mathrm{H}-20), 0.82$ (t, $\left.{ }^{3} \mathrm{JHH}_{\mathrm{H}}=7.2 \mathrm{~Hz}, 6 \mathrm{H}, \mathrm{H}-21\right), 0.60-0.54$ (m, 4H, H-15).

${ }^{13} \mathrm{C}-\mathrm{NMR}\left(101 \mathrm{MHz}, \mathrm{CDCl}_{3}, 25^{\circ} \mathrm{C}\right.$ ): $\delta$ [ppm] = 153.7 (C-10), 149.7 (C-13), 143.1 (C-12), 140.1 (C-11), 134.0 (C-6), 130.0 (C-3), 129.0 (C-8), 127.9 (C-7), 126.4 (C-1), 121.7 (C-2), 121.6 (C-4), 119.2 (C-5), 83.9 (C-22), 55.6 (C-9), 40.3 (C-14), 31.9 (C-19), 30.0 (C-16), 29.3 (br, C-17, C-18), 25.1 (C-23), 23.8 (C-15), 22.7 (C-20), 14.2 (C-21).

\section{POLYMERIZATION REACTION}

The following procedure describes the specific synthesis of the mixture of doubly spin-labeled oligo(fluorenes) for DEER measurements via controlled Suzuki-Miyaura coupling polymerization. It was elaborated by J. D. Ruiz Perez upon modification and adjustment of a reported protocol. ${ }^{72}$ By the chosen stoichiometric ratio of precatalyst to monomer, an average polymer chain length of five repeat units was targeted.

The palladium precatalyst $(21.7 \mathrm{mg}, 0.042 \mathrm{mmol}, 1.0$ equiv. $)$ and the spin-labeled initiator $(24.1 \mathrm{mg}$, $0.038 \mathrm{mmol}, 1.6$ equiv.) were dissolved in THF (1 mL). An aqueous $2 \mathrm{M} \mathrm{K}_{2} \mathrm{CO}_{3}$ solution $(1 \mathrm{~mL})$ was added and the mixture was vigorously stirred at room temperature for $40 \mathrm{~min}$ to generate the reactive initiator species in situ. The AB-type monomer ( $126.1 \mathrm{mg}, 0.212 \mathrm{mmol}, 5$ equiv.) was dissolved in THF $(8 \mathrm{~mL})$ and cooled to $0{ }^{\circ} \mathrm{C}$. The initiator solution was quickly injected into the monomer solution and the resulting mixture was stirred for $15 \mathrm{~min}$ at $0{ }^{\circ} \mathrm{C}$. Subsequently, the reaction was quenched by addition of the TEMPO-labeled end-capping agent ( $127.9 \mathrm{mg}, 0.318 \mathrm{mmol}, 7.5$ equiv.) dissolved in THF ( $1 \mathrm{~mL})$. The reaction mixture was stirred overnight at room temperature, before the polymer was extracted three times with dichloromethane and the combined organic layers were washed with brine and dried over $\mathrm{MgSO}_{4}$. After removal of the solvent under reduced pressure, the crude product was dissolved in toluene and precipitated by addition of methanol, followed by centrifugation (6000 rpm, $10 \mathrm{~min}$ ) and decantation of the supernatant solution. This procedure was repeated twice to yield the purified polymer.

\section{ISOLATION OF DEFINED OLIGOFLUORENES BY SEMI-PREPARATIVE GPC}

The purified and dried oligomer mixture was dissolved in THF $(5 \mathrm{mg} / \mathrm{mL})$ and a non-fractionating reference GPC measurement was performed using the semi-preparative column, in order to estimate the respective region of fractionation, with regard to elution time and volume. For each fractionation cycle, $500 \mu \mathrm{L}$ of the prepared solution were injected into the semi-preparative column with a flow rate of $6 \mathrm{~mL} / \mathrm{min}$ and a total elution volume of $7.5 \mathrm{~mL}$ was collected in 15 fractions. The single fractions were characterized by MALDI-TOF measurements and the fractionation was repeated for individual samples, if required. Prior to DEER measurements, the obtained fractions were dried under reduced pressure. 


\subsubsection{PREPARATION OF NANOPARTICLES}

Spherical poly(fluorene) nanoparticles containing oligomers with TEMPO-labels on both chain termini were prepared using an emulsification technique. ${ }^{84,85}$ The poly $\left(9,9^{\prime}\right.$-dioctylfluorene) material used $\left(M_{\mathrm{n}} \approx 30-40 \mathrm{~kg} / \mathrm{mol}\right)$ was obtained from heterophase Suzuki-Miyaura polymerization according to a reported procedure ${ }^{8}$ and purified prior to particle synthesis by three cycles of dissolution in toluene, precipitation in methanol and centrifugation. The polymer $(6 \mathrm{mg})$ was dissolved in THF $(1 \mathrm{~mL})$ and blended with a solution of the defined, labeled oligomers $(n=1,2$ and 3$)$ in toluene-ds. The composition of the oligomer solution was previously adjusted by successive spin concentration measurements and dilution, if required, to yield $20 \mu \mathrm{L}$ of a mixture containing $9 \mu \mathrm{L}$ of the respective 1-mer solution (spin concentration: $0.35 \mathrm{mM}$ ), $5 \mu \mathrm{L}$ of the 2-mer solution (spin concentration: $0.77 \mathrm{mM}$ ) and $6 \mu \mathrm{L}$ of the 3-mer solution (spin concentration: $0.33 \mathrm{mM}$ ). The organic mixture was rapidly injected into an aqueous solution ( $8 \mathrm{~mL}$ ) of sodium dodecyl sulfate $(1.5 \mathrm{wt} \%)$ with simultaneous onset of ultrasonication (2 min, $60 \%$ amplitude). The resulting dispersions were analyzed by DLS and TEM measurement. For DEER measurements, the particles were purified by dialysis in deionized water for three days, using dialysis tubes with a molecular weight cutoff of 6-8 kD, followed by freeze-drying, to yield a light green powder.

\subsubsection{ELECTRON PARAMAGNETIC RESONANCE MEASUREMENTS}

All EPR experiments, including DEER measurements, as well as the processing of the acquired data were performed by D. Bücker from the research group of Prof. Dr. M. Drescher. Pulsed EPR measurements were performed in Q-band on a Bruker Elexsys E580 spectrometer, equipped with a 15 W microwave amplifier and a helium gas flow system (CF935, Oxford Instruments) and a Bruker EN5107D2 resonator, using sample tubes with $1.6 \mathrm{~mm}$ outer diameter. For liquid samples, a sample height of approximately $10 \mathrm{~mm}$ was adjusted, before the samples were flash-frozen in liquid nitrogen and centered in the resonator cavity. All measurements were performed in an over coupled resonator at $40 \mathrm{~K}$. Optimal pulse lengths for the observer channel as well as for the pump channel were determined by nutation experiments. An echo detected field sweep was recorded for all samples and a relaxation experiment (phase memory time) was performed. For all DEER experiments, the observer offset was chosen as approximately $50.4 \mathrm{MHz}$ and the experiments were recorded with 10 shots per point, 8 step nuclear modulation averaging and 8 step phase cycling. Several scans were recorded until a satisfying signal-to-noise level was reached.

Prior to pulsed EPR measurements, the spin concentration of all samples was determined by continuous wave EPR spectroscopy on a Bruker EMX Nano in $50 \mu \mathrm{L}$ Hirschmann ringcaps (diameter $1.02 \mathrm{~mm}$ ). For liquid samples, the resonator height $(125 \mathrm{~mm})$ was completely filled. All continuous wave EPR measurements were carried out at room temperature. 
Evaluation of DEER raw data was performed with DEERAnalysis $2019 .{ }^{189}$ Small alterations were made to the code to allow eight fitting parameters in a model based analysis. In priority, scans were phase corrected individually and added up to a single trace. The cutoff was chosen according to the DEERAnalysis automatic recommendation. DEERNet analysis was used to obtain the background function and to reconstruct the form factor of all DEER traces. ${ }^{190}$ Further analysis was performed either by Tikhonov regularization, ${ }^{222}$ choosing the regularization parameter by the generalized cross validation criterion, ${ }^{223}$ DEERNet (generic) or model based fits.

For the determination of the Gaussian broadening, the model WLC_rigid_gauss provided by DEERAnalysis 2019 was used. It comprises a end-to-end distance distribution of a worm-like chain, broadened by a Gaussian to account for the nitroxide-label flexibility. ${ }^{183,185,224}$ Gaussian broadening was calculated from the doubly spin-labeled fluorene monomer $(n=1)$ by keeping the persistence length $L_{p}$ fixed at a maximum value of $100 \mathrm{~nm}$, resulting in a value of $\sigma=0.06 \mathrm{~nm}$.

Further details on DEER measurement protocols and the following processing of the experimental data can be found in cited literature. ${ }^{18}$ 


\title{
8.5 ORIGIN OF ANISOTROPY
}

\subsubsection{SYNTHESES OF OLIGOFLUORENES}

\section{SYNTHESIS OF 2-BROMO-9,9'-DIOCTYLFLUORENE26}

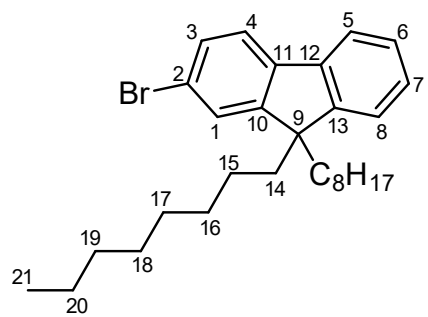

\author{
$\mathrm{C}_{29} \mathrm{H}_{41} \mathrm{Br}$ \\ $\mathrm{M}=469.55 \mathrm{~g} / \mathrm{mol}$
}

2-Bromofluorene (30.0 g, $122 \mathrm{mmol}, 1$ equiv.), 1-bromooctane (71 mL, $409 \mathrm{mmol}, 3.3$ equiv.) and TBAB (2.4 g, $7.3 \mathrm{mmol}, 0.06$ equiv.) were dissolved in DMSO (250 mL) and degassed by application of vacuum. After addition of an aqueous $50 \mathrm{wt} \% \mathrm{NaOH}$ solution $(50 \mathrm{~mL}, 610 \mathrm{mmol}, 5$ equiv.) the solution was stirred for $6 \mathrm{~h}$ at room temperature. The turbid mixture was poured in deionized water $(500 \mathrm{~mL})$ and extracted three times with DCM. The combined organic layers were washed with water, $2 \mathrm{M} \mathrm{HCl}$ and brine, dried over $\mathrm{MgSO}_{4}$ and the solvent was removed under reduced pressure. Remaining 1-bromooctane was removed by distillation and the crude product was purified by column chromatography in $n$-hexane to yield 2-bromo-9,9'-dioctylfluorene as a slightly yellow oil (53.9 g, $115 \mathrm{mmol}, 94 \%)$.

${ }^{1} \mathrm{H}-N M R\left(400 \mathrm{MHz} \mathbf{C D C l}_{3}, \mathbf{2 5}^{\circ} \mathrm{C}\right): \delta[\mathrm{ppm}]=7.69-7.66(\mathrm{~m}, 1 \mathrm{H}, \mathrm{H}-5), 7.56\left(\mathrm{~d},{ }^{3} \mathrm{~J}_{\mathrm{HH}}=7.9 \mathrm{~Hz}, 1 \mathrm{H}, \mathrm{H}-4\right)$, 7.48-7.44 (m, 2H, H-1, H-3), 7.35-7.32 (m, 3H, H-6, H-7, H-8), 2.01-1.89 (m, 4H, H-14), 1.25-1.06 (m, $20 \mathrm{H}, \mathrm{H}-16, \mathrm{H}-17, \mathrm{H}-18, \mathrm{H}-19, \mathrm{H}-20), 0.84\left(\mathrm{t},{ }^{3} \mathrm{~J}_{\mathrm{HH}}=7.2 \mathrm{~Hz}, 6 \mathrm{H}, \mathrm{H}-21\right), 0.66-0.57$ (m, 4H, H-15).

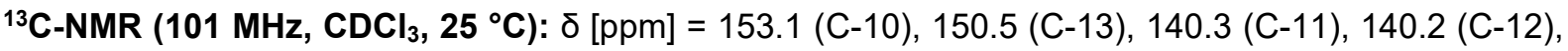
130.0 (C-1), 127.6 (C-6), 127.1 (C-8), 126.3 (C-3), 123.0 (C-7), 121.2 (C-4), 121.1 (C-2), 119.9 (C-5), 55.5 (C-9), 40.4 (C-14), 31.9 (C-19), 30.1 (C-16), 29.4 (C-17), 29.3 (C-18), 23.8 (C-15), 22.7 (C-20), 14.2 (C-21).

\section{SYNTHESIS OF 9,9'-DIOCTYLFLUORENE-2-BORONIC ACID 26}

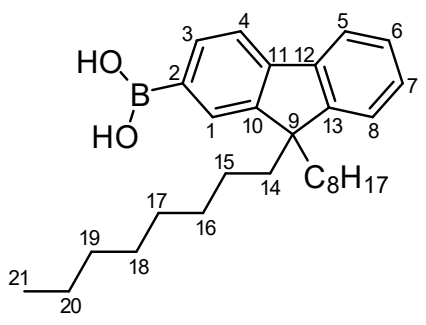

$$
\begin{aligned}
& \mathrm{C}_{29} \mathrm{H}_{43} \mathrm{BO}_{2} \\
& \mathrm{M}=434.47 \mathrm{~g} / \mathrm{mol}
\end{aligned}
$$


A solution of 2-bromo-9,9'-dioctylfluorene (10.0 g, $21.3 \mathrm{mmol}, 1$ equiv.) in dry THF (120 mL) was cooled to $-78{ }^{\circ} \mathrm{C}$ and a $2.5 \mathrm{M}$ solution of $n$-butyllithium in hexane $(10.4 \mathrm{~mL}, 25.6 \mathrm{mmol}, 1.2$ equiv.) was slowly added. After stirring for $1 \mathrm{~h}$ at the same temperature, tri-iso-propyl borate $(9.9 \mathrm{~mL}, 42.6 \mathrm{mmol}, 2$ equiv.) was added and the solution was warmed to room temperature and stirred overnight. $2 \mathrm{M} \mathrm{HCl}(80 \mathrm{~mL})$ was added to the reaction, followed by a further hour of stirring. The phases were separated and the aqueous phase was extracted three times with diethyl ether. The combined organic layers were washed with water and dried over $\mathrm{MgSO}_{4}$. The crude product was purified by column chromatography (gradient from petroleum ether to ethyl acetate) to yield 9,9'-dioctylfluorene-2-boronic acid as a white solid ( $8.5 \mathrm{~g}$, $19.5 \mathrm{mmol}, 92 \%)$.

${ }^{1} \mathrm{H}-\mathrm{NMR}\left(400 \mathrm{MHz}, \mathrm{CDCl}_{3}, \mathbf{2 5}^{\circ} \mathrm{C}\right): \delta[\mathrm{ppm}]=8.33\left(\mathrm{~d},{ }^{3} \mathrm{JHH}_{\mathrm{HH}}=7.5 \mathrm{~Hz}, 1 \mathrm{H}, \mathrm{H}-3\right), 8.24(\mathrm{~s}, 1 \mathrm{H}, \mathrm{H}-1), 7.90$ $\left(\mathrm{d},{ }^{3} \mathrm{~J}_{\mathrm{HH}}=7.5 \mathrm{~Hz}, 1 \mathrm{H}, \mathrm{H}-4\right), 7.84-7.82(\mathrm{~m}, 1 \mathrm{H}, \mathrm{H}-7), 7.44-7.37(\mathrm{~m}, 3 \mathrm{H}, \mathrm{H}-5, \mathrm{H}-6, \mathrm{H}-8), 2.19-2.03(\mathrm{~m}, 4 \mathrm{H}$, $\mathrm{H}-14), 1.24-1.03(\mathrm{~m}, 20 \mathrm{H}, \mathrm{H}-16, \mathrm{H}-17, \mathrm{H}-18, \mathrm{H}-19, \mathrm{H}-20), 0.79\left(\mathrm{t},{ }^{3} \mathrm{JHH}_{\mathrm{HH}}=7.1 \mathrm{~Hz}, 6 \mathrm{H}, \mathrm{H}-21\right), 0.75-0.69$ (m, $4 \mathrm{H}, \mathrm{H}-15)$.

${ }^{13} \mathrm{C}$-NMR (101 MHz, $\left.\mathrm{CDCl}_{3}, 25^{\circ} \mathrm{C}\right): \delta$ [ppm] = 151.8 (C-13), 150.3 (C-10), 145.7 (C-11), 140.9 (C-12), 134.8 (C-3), 129.9 (C-1), 129.1 (C-2), 128.1 (C-5), 127.0 (C-6), 123.2 (C-8), 120.5 (C-7), 119.4 (C-4), 55.2 (C-9), 40.5 (C-14), 32.0 (C-19), 30.2 (C-16), 29.4 (C-17), 29.8 (C-18), 24.0 (C-15), 22.8 (C-20), $14.2(\mathrm{C}-21)$.

\section{SYNTHESIS OF 9,9,9', $9^{\prime}$-TETRAOCTYL-9H, $9^{\prime} H-2,2^{\prime}$-BIFLUORENE $\left(H-F_{2}-H\right)^{10}$}

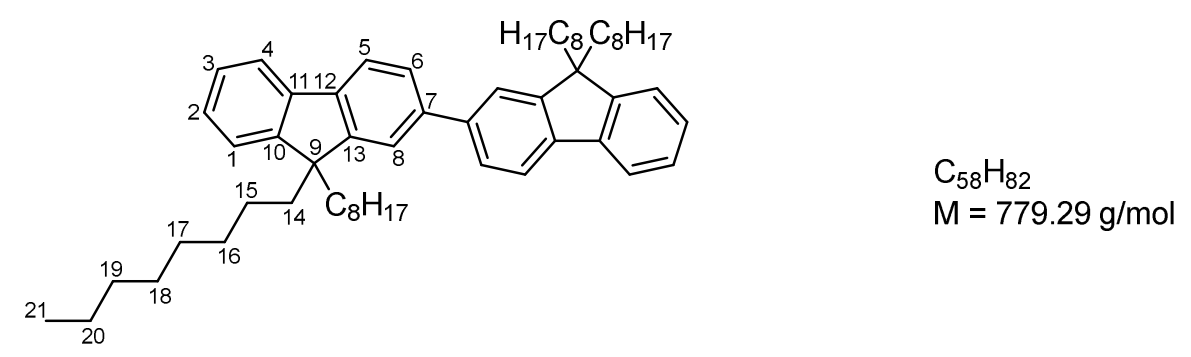

2-Bromo-9,9'-dioctylfluorene (2.75 g, $5.86 \mathrm{mmol}, 1$ equiv.) and 9,9'-dioctylfluorene-2-boronic acid (3.31 g, $7.62 \mathrm{mmol}, 1.3$ equiv.) were dissolved in toluene $(40 \mathrm{~mL}$ ) and the solution was degassed by two freeze-pump-thaw cycles. A $2 \mathrm{M}$ aqueous $\mathrm{K}_{2} \mathrm{CO}_{3}$ solution ( $44 \mathrm{~mL}, 87.9 \mathrm{mmol}, 15$ equiv.) was added, followed by two more freeze-pump-thaw cycles. A solution of tetrakis(triphenylphosphine)palladium $(0)$ ( $0.14 \mathrm{~g}, 0.12 \mathrm{mmol}, 0.02$ equiv.) in toluene $(5 \mathrm{~mL})$ was added and the reaction mixture was heated to $90{ }^{\circ} \mathrm{C}$ overnight. After cooling to ambient temperature, the phases were separated and the organic layer was washed with water and dried over $\mathrm{MgSO}_{4}$. Removal of the solvent under reduced pressure yielded the crude product, which was purified by column chromatography using $n$-hexane as eluent to give the product $\mathbf{H}-\mathbf{F}_{\mathbf{2}} \mathbf{- H}$ as a white solid $(3.69 \mathrm{~g}, 4.73 \mathrm{mmol}, 81 \%)$.

${ }^{1} \mathrm{H}-\mathrm{NMR}\left(\mathbf{4 0 0} \mathrm{MHz}, \mathrm{CDCl}_{3}, \mathbf{2 5}^{\circ} \mathrm{C}\right): \delta[\mathrm{ppm}]=7.78\left(\mathrm{~d},{ }^{3} \mathrm{JHH}_{\mathrm{HH}}=7.9 \mathrm{~Hz}, 2 \mathrm{H}, \mathrm{H}-5\right), 7.74\left(\mathrm{~d},{ }^{3} \mathrm{JHH}_{\mathrm{HH}}=7.2 \mathrm{~Hz}\right.$, $2 \mathrm{H}, \mathrm{H}-4), 7.65\left(\mathrm{dd},{ }^{3} \mathrm{JHH}_{\mathrm{HH}}=7.8 \mathrm{~Hz},{ }^{4} \mathrm{JHH}_{\mathrm{HH}}=1.6 \mathrm{~Hz}, 2 \mathrm{H}, \mathrm{H}-6\right), 7.62\left(\mathrm{~d},{ }^{4} \mathrm{~J}_{\mathrm{HH}}=1.3 \mathrm{~Hz}, 2 \mathrm{H}, \mathrm{H}-8\right), 7.39-7.30$ (m, 6H, H-1, H-2, H-3), 2.08-1.98 (m, 8H, H-14), 1.26-1.09 (m, 40H, H-16, H-17, H-18, H-19, H-20), 0.82 $\left(\mathrm{t},{ }^{3} \mathrm{JHH}_{\mathrm{HH}}=7.2 \mathrm{~Hz}, 12 \mathrm{H}, \mathrm{H}-21\right), 0.78-0.66(\mathrm{~m}, 8 \mathrm{H}, \mathrm{H}-15)$. 
${ }^{13} \mathrm{C}-\mathrm{NMR}\left(\mathbf{1 0 1} \mathrm{MHz}, \mathrm{CDCl}_{3}, 25{ }^{\circ} \mathrm{C}\right): \delta[\mathrm{ppm}]=151.6(\mathrm{C}-13), 151.2(\mathrm{C}-10), 141.0(\mathrm{C}-11), 140.7$ (C-7), 140.4 (C-12), 127.1 (C-2), 126.9 (C-3), 126.2 (C-6), 123.1 (C-1), 121.6 (C-8), 120.0 (C-5), 119.8 (C-4), 55.3 (C-9), 40.5 (C-14), 31.9 (C-19), 30.2 (C-16), 29.4 (C-17, C-18), 24.0 (C-15), 22.7 (C-20), 14.2 (C-21).

\title{
SYNTHESIS OF 7,7'-DIBROMO-9,9,9',9'-TETRAOCTYL-9H,9'H-2,2'-BIFLUORENE
} $\left(\mathrm{Br}-\mathrm{F}_{2}-\mathrm{Br}\right)^{10}$

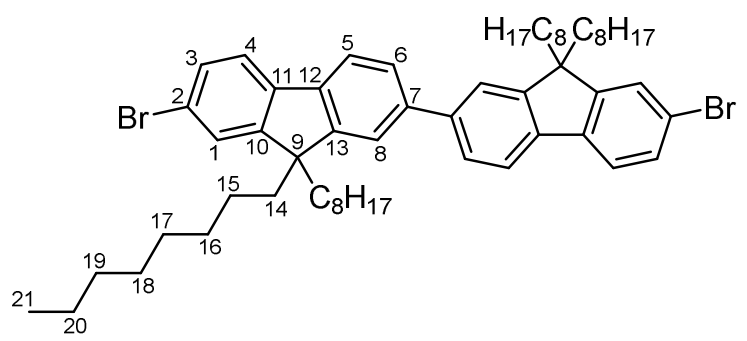

\author{
$\mathrm{C}_{58} \mathrm{H}_{80} \mathrm{Br}$ \\ $\mathrm{M}=937.09 \mathrm{~g} / \mathrm{mol}$
}

A solution of $\mathbf{H}-\mathbf{F}_{2}-\mathbf{H}(6.12 \mathrm{~g}, 7.85 \mathrm{mmol}, 1$ equiv. $)$ and $\mathrm{FeCl}_{3} \times 6 \mathrm{H}_{2} \mathrm{O}(0.04 \mathrm{~g}, 0.16 \mathrm{mmol}, 0.02$ equiv. $)$ in chloroform $(100 \mathrm{~mL})$ was cooled to $-10{ }^{\circ} \mathrm{C}$ and bromine $(0.84 \mathrm{~mL}, 16.48 \mathrm{mmol}, 2.1$ equiv. $)$ was added dropwise. The mixture was brought to room temperature and stirred for $4 \mathrm{~h}$, while the progress of conversion was monitored by TLC. Aqueous $\mathrm{Na}_{2} \mathrm{~S}_{2} \mathrm{O}_{5}$ solution was added and the reaction mixture was stirred vigorously until the brown color disappeared. The organic phase was separated and the aqueous phase was extracted with DCM. The combined organic layers were washed with water, dried over $\mathrm{MgSO}_{4}$ and the solvent was removed in vacuo. The crude product was washed with ethanol, yielding $\mathrm{Br}-\mathrm{F}_{2}-\mathrm{Br}$ as a white solid $(6.52 \mathrm{~g}, 6.96 \mathrm{mmol}, 88 \%)$.

${ }^{1} \mathrm{H}-\mathrm{NMR}\left(400 \mathrm{MHz}, \mathrm{CDCl}_{3}, 25{ }^{\circ} \mathrm{C}\right): \delta[\mathrm{ppm}]=7.74\left(\mathrm{~d},{ }^{3} \mathrm{JHH}_{\mathrm{HH}}=7.8 \mathrm{~Hz}, 2 \mathrm{H}, \mathrm{H}-5\right), 7.62\left(\mathrm{dd},{ }^{3} \mathrm{JHH}_{\mathrm{HH}}=7.8 \mathrm{~Hz}\right.$, $\left.{ }^{4} \mathrm{~J}_{\mathrm{HH}}=1.5 \mathrm{~Hz}, 2 \mathrm{H}, \mathrm{H}-6\right), 7.59\left(\mathrm{~d},{ }^{3} \mathrm{~J}_{\mathrm{HH}}=7.8 \mathrm{~Hz}, 2 \mathrm{H}, \mathrm{H}-4\right), 7.57\left(\mathrm{~d},{ }^{4} \mathrm{JHH}_{\mathrm{HH}}=1.3 \mathrm{~Hz}, 2 \mathrm{H}, \mathrm{H}-8\right), 7.48-7.46(\mathrm{~m}$, $4 \mathrm{H}, \mathrm{H}-1, \mathrm{H}-3), 2.07-1.93(\mathrm{~m}, 8 \mathrm{H}, \mathrm{C}-14), 1.25-1.08(\mathrm{~m}, 40 \mathrm{H}, \mathrm{H}-16, \mathrm{H}-17, \mathrm{H}-18, \mathrm{H}-19, \mathrm{H}-20), 0.81$ (t, $\left.{ }^{3} \mathrm{~J}_{\mathrm{HH}}=7.3 \mathrm{~Hz}, 12 \mathrm{H}, \mathrm{H}-21\right), 0.74-0.64(\mathrm{~m}, 8 \mathrm{H}, \mathrm{H}-15)$.

${ }^{13} \mathrm{C}$-NMR (101 MHz, $\mathrm{CDCl}_{3}, 25{ }^{\circ} \mathrm{C}$ ): $\delta$ [ppm] = 153.4 (C-10), 151.3 (C-13), 140.9 (C-7), 139.9 (C-11), 139.5 (C-12), 130.2 (C-3), 126.5 (C-1), 126.4 (C-6), 121.6 (C-8), 121.3 (C-4), 121.2 (C-2), 120.2 (C-5), 55.7 (C-9), 40.4 (C-14), 31.9 (C-19), 30.1 (C-16), 29.4 (C-17), 29.3 (C-18), 23.9 (C-15), 22.7 (C-20), $14.2(\mathrm{C}-21)$. 


\section{SYNTHESIS OF $9,9,9^{\prime}, 9^{\prime \prime}, 9^{\prime \prime}, 9^{\prime \prime}$-HEXAOCTYL- $9 \mathrm{H}, 9^{\prime} \mathrm{H}, 9^{\prime \prime} \mathrm{H}-2,2^{\prime \prime}: 7^{\prime}, \mathrm{2}^{\prime \prime}$ - \\ TERFLUORENE $\left(\mathrm{H}-\mathrm{F}_{3}-\mathrm{H}\right)^{10}$}

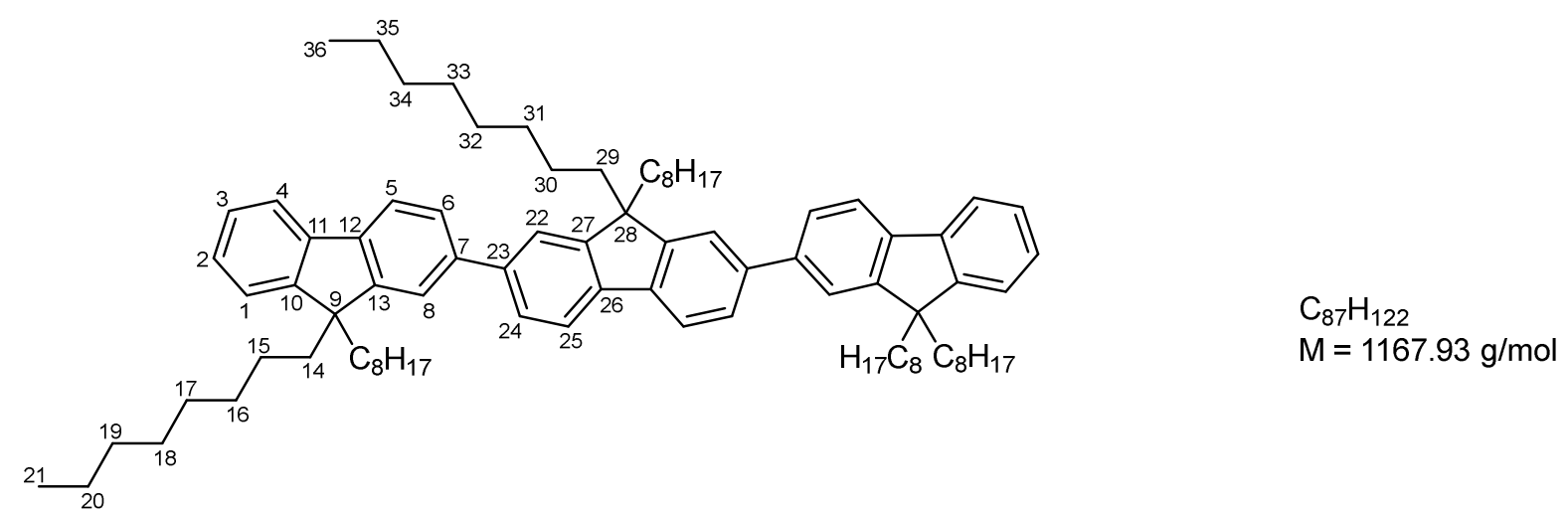

2,7-Dibromo-9,9'-dioctylfluorene (1.98 g, $3.61 \mathrm{mmol}, 1$ equiv.) and 9,9'-dioctylfluorene-2-boronic acid $(3.76 \mathrm{~g}, 8.65 \mathrm{mmol}, 2.4$ equiv.) were dissolved in toluene $(50 \mathrm{~mL})$ and the solution was degassed by two freeze-pump-thaw cycles. A $2 \mathrm{M}$ aqueous $\mathrm{K}_{2} \mathrm{CO}_{3}$ solution $(50 \mathrm{~mL}, 108 \mathrm{mmol}, 30$ equiv.) was added, followed by two more freeze-pump-thaw cycles. Tetrakis(triphenylphosphine)palladium(0) $(0.16 \mathrm{~g}$, $0.14 \mathrm{mmol}, 0.04$ equiv.) was dissolved in toluene $(5 \mathrm{~mL})$, added to the mixture and the reaction was heated to $90{ }^{\circ} \mathrm{C}$ overnight. After cooling to ambient temperature, the phases were separated and the organic layer was washed with water and dried over $\mathrm{MgSO}_{4}$. Removal of the solvent under reduced pressure yielded the crude product, which was purified by column chromatography using $n$-hexane as eluent to give the product $\mathbf{H}-\mathbf{F}_{3}-\mathbf{H}$ as a white solid $(2.82 \mathrm{~g}, 2.41 \mathrm{mmol}, 67 \%)$.

${ }^{1} \mathrm{H}-\mathrm{NMR}\left(400 \mathrm{MHz}, \mathrm{CDCl}_{3}, 25{ }^{\circ} \mathrm{C}\right): \delta[\mathrm{ppm}]=7.82\left(\mathrm{~d},{ }^{3} \mathrm{~J}_{\mathrm{HH}}=7.9 \mathrm{~Hz}, 2 \mathrm{H}, \mathrm{H}-25\right), 7.80\left(\mathrm{~d},{ }^{3} \mathrm{JHH}_{\mathrm{HH}}=7.9 \mathrm{~Hz}\right.$, $2 \mathrm{H}, \mathrm{H}-5), 7.75$ (d, $\left.{ }^{3} \mathrm{~J}_{\mathrm{HH}}=7.1 \mathrm{~Hz}, 2 \mathrm{H}, \mathrm{H}-4\right), 7.69-7.64(\mathrm{~m}, 8 \mathrm{H}, \mathrm{H}-6, \mathrm{H}-8, \mathrm{H}-22, \mathrm{H}-24), 7.39-7.30$ (m, 6H, $\mathrm{H}-1, \mathrm{H}-2, \mathrm{H}-3), 2.13-2.01$ (m, 12H, H-14, H-29), 1.22-1.10 (m, 60H, H-16, H-17, H-18, H-19, H-20, H-31, $\mathrm{H}-32, \mathrm{H}-33, \mathrm{H}-34, \mathrm{H}-35), 0.84-0.79$ (m, 22H, H-21, H-36, H-30), 0.78-0.67 (m, H-15).

${ }^{13} \mathrm{C}-\mathrm{NMR}\left(101 \mathrm{MHz}, \mathrm{CDCl}_{3}, 25^{\circ} \mathrm{C}\right): \delta$ [ppm] = 151.9 (C-27), 151.6 (C-13), 151.2 (C-10), $141.0(\mathrm{C}-11)$, 140.7 (C-7, C-23), 140.5 (C-26), 140.1 (C-12), 127.1 (C-2), 126.9 (C-3), 126.3 (C-24), 126.2 (C-6), 123.1 (C-1), 121.6 (C-22), 121.5 (C-8), 120.1 (C-25), 120.0 (C-5), 119.9 (C-4), 55.5 (C-28), 55.3 (C-9), 40.5 (C-14, C-29), 31.9 (C-19, C-34), 30.2 (C-16, C-31), 29.4 (C-17, C-18, C-32, C-33), 24.1 (C-30), 24.0 (C-15), 22.7 (C-20, C-35), 14.2 (C-21, C-36). 


\section{SYNTHESIS OF 7,7"'-DIBROMO-9,9,9",9",9",9"'-HEXAOCTYL-9H,9'H,9"'H-}

\section{$2,2^{\prime}: 7^{\prime}, 2^{\prime \prime}$-TERFLUORENE $\left(\mathrm{Br}-\mathrm{F}_{3}-\mathrm{Br}\right)^{10}$}

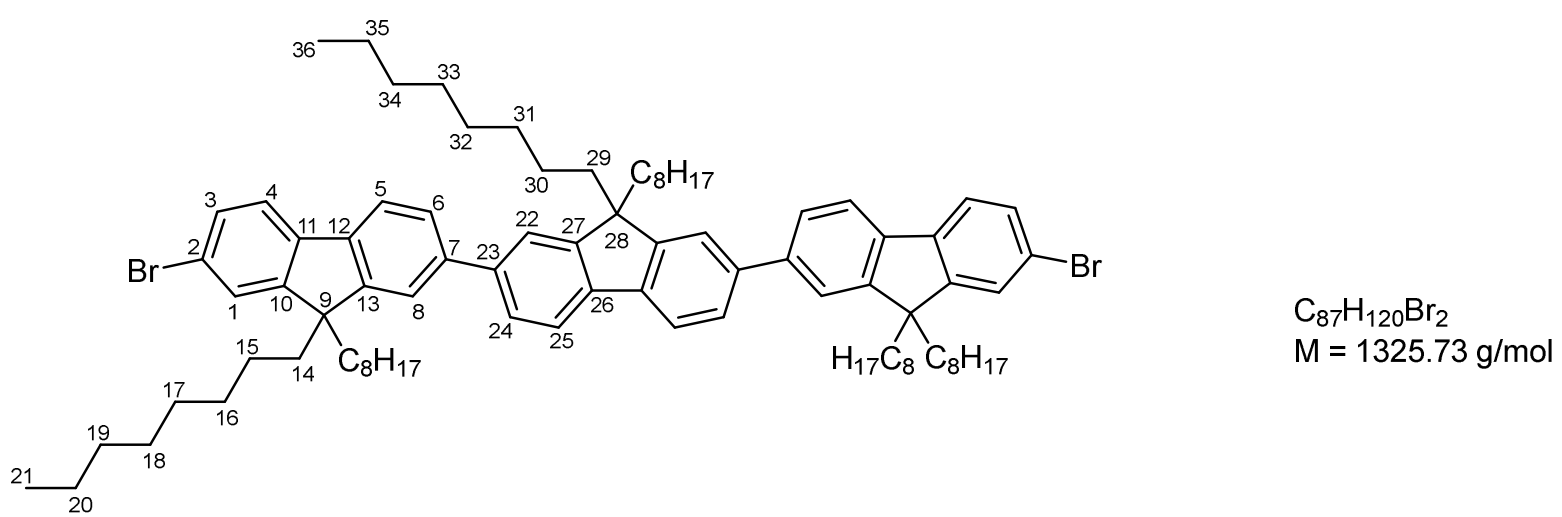

A solution of $\mathbf{H}-\mathbf{F}_{3}-\mathbf{H}\left(7.10 \mathrm{~g}, 6.08 \mathrm{mmol}, 1\right.$ equiv.) and $\mathrm{FeCl}_{3} \times 6 \mathrm{H}_{2} \mathrm{O}(0.03 \mathrm{~g}, 0.12 \mathrm{mmol}, 0.02$ equiv.) in chloroform $(100 \mathrm{~mL})$ was cooled to $-10{ }^{\circ} \mathrm{C}$ and bromine $(0.65 \mathrm{~mL}, 12.76 \mathrm{mmol}, 2.1$ equiv.) was added dropwise. The mixture was brought to room temperature and stirred for $4 \mathrm{~h}$, while the progress of conversion was monitored by TLC. Aqueous $\mathrm{Na}_{2} \mathrm{~S}_{2} \mathrm{O}_{5}$ solution was added and the reaction mixture was stirred vigorously until the brown color disappeared. The organic phase was separated and the aqueous phase was extracted with DCM. The combined organic layers were washed with water, dried over $\mathrm{MgSO}_{4}$ and the solvent was removed in vacuo. The crude product was purified by recrystallization from acetone, yielding $\mathrm{Br}-\mathrm{F}_{3}-\mathrm{Br}$ as a white solid (6.76 g, $5.10 \mathrm{mmol}, 83 \%$ ).

${ }^{1} \mathrm{H}-\mathrm{NMR}\left(400 \mathrm{MHz}, \mathrm{CDCl}_{3}, 25{ }^{\circ} \mathrm{C}\right): \delta[\mathrm{ppm}]=7.82\left(\mathrm{~d},{ }^{3} \mathrm{JHH}_{\mathrm{HH}}=7.8 \mathrm{~Hz}, 2 \mathrm{H}, \mathrm{H}-25\right), 7.75\left(\mathrm{~d},{ }^{3} \mathrm{JHH}=7.9 \mathrm{~Hz}\right.$, $2 \mathrm{H}, \mathrm{H}-5), 7.66\left(\mathrm{~d},{ }^{3} \mathrm{~J}_{\mathrm{HH}}=7.9 \mathrm{~Hz}, 4 \mathrm{H}, \mathrm{H}-6, \mathrm{H}-24\right), 7.63-7.59(\mathrm{~m}, 6 \mathrm{H}, \mathrm{H}-4, \mathrm{H}-8, \mathrm{H}-22), 7.49-7.47(\mathrm{~m}, 4 \mathrm{H}$, $\mathrm{H}-1, \mathrm{H}-3), 2.12-1.95$ (m, 12H, H-14, H-29), 1.26-1.10 (60 H, H-16, H-17, H-18, H-19, H-20, H-31, H-32, $\mathrm{H}-33, \mathrm{H}-34, \mathrm{H}-35), 0.82\left(\mathrm{t},{ }^{3} \mathrm{~J}_{\mathrm{HH}}=7.0 \mathrm{~Hz}, 18 \mathrm{H}, \mathrm{H}-21, \mathrm{H}-36\right.$ ), $0.80\left(\mathrm{t},{ }^{3} \mathrm{~J}_{\mathrm{HH}}=7.1 \mathrm{~Hz}, 4 \mathrm{H}, \mathrm{H}-30\right), 0.75-0.67$ (m, 8H, $\mathrm{H}-15)$.

${ }^{13} \mathrm{C}-\mathrm{NMR}\left(101 \mathrm{MHz}, \mathrm{CDCl}_{3}, 25{ }^{\circ} \mathrm{C}\right): \delta[\mathrm{ppm}]=153.4(\mathrm{C}-10), 152.0(\mathrm{C}-27), 151.3(\mathrm{C}-13), 141.1$ (C-7), 140.5 (C-23), 140.2 (C-26), 140.0 (C-11), 139.4 (C-12), 130.2 (C-3), 126.5 (C-1), 126.4 (C-6), 126.3 (C-24), 121.6 (C-4), 121.5 (C-8), 121.2 (C-22), 121.1 (C-2), 120.2 (C-5, C-25), 55.7 (C-9), 55.5 (C-28), 40.5 (C-29), 40.4 (C-14), 31.9 (C-19, C-34), 30.2 (C-31), 30.1 (C-16), 29.4 (C-17, C-32), 29.3 (C-18, C-33), 24.0 (C-30), 23.9 (C-15), 22.8 (C-20), 22.7 (C-35), 14.2 (C-21), 14.2 (C-36). 


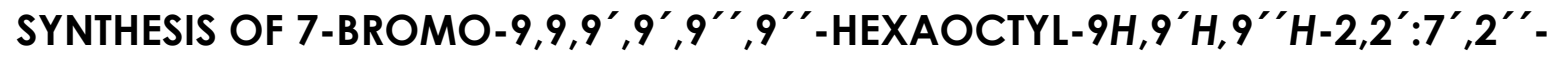 TERFLUORENE $\left(\mathrm{Br}-\mathrm{F}_{3}-\mathrm{H}\right)^{10}$}

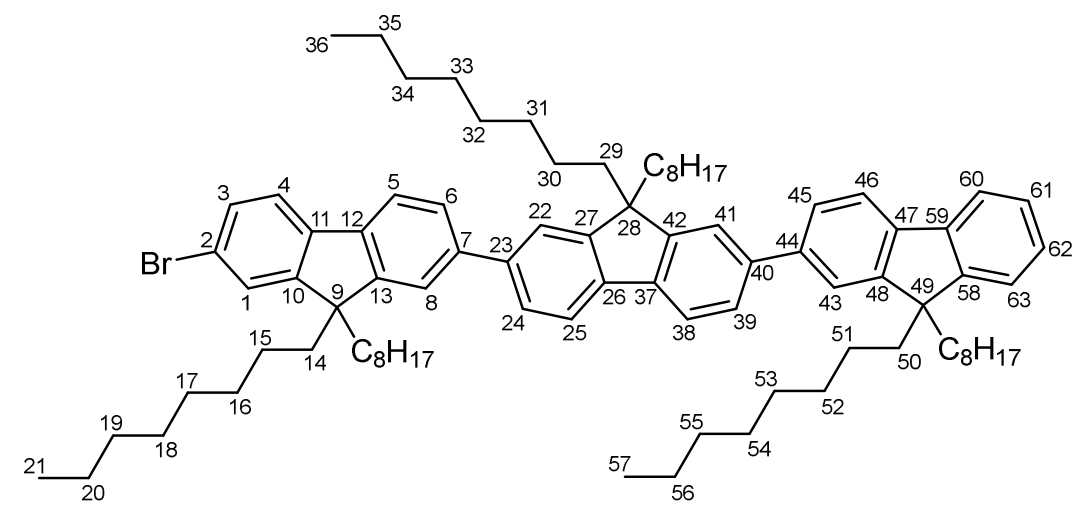

$\mathrm{C}_{87} \mathrm{H}_{121} \mathrm{Br}$ $\mathrm{M}=1246.83 \mathrm{~g} / \mathrm{mol}$

$\mathrm{Br}-\mathrm{F}_{2}-\mathrm{Br}(6.53 \mathrm{~g}, 6.96 \mathrm{mmol}, 1$ equiv.) and 9,9'-dioctylfluorene-2-boronic acid $(3.03 \mathrm{~g}, 6.96 \mathrm{mmol}$, 1 equiv.) were dissolved in toluene $(70 \mathrm{~mL})$ and the solution was degassed via two freeze-pump-thaw cycles. A $2 \mathrm{M}$ aqueous $\mathrm{K}_{2} \mathrm{CO}_{3}$ solution ( $50 \mathrm{~mL}, 100 \mathrm{mmol}$, 15 equiv.) was added, followed by two more freeze-pump-thaw cycles. Tetrakis(triphenylphosphine)palladium( 0$)(0.12 \mathrm{~g}, 0.10 \mathrm{mmol}, 0.015$ equiv.) was dissolved in toluene $(10 \mathrm{~mL})$ and added to the mixture. The reaction was heated to $90^{\circ} \mathrm{C}$ for $48 \mathrm{~h}$, before the phases were separated and the organic layer was washed with water and dried over $\mathrm{MgSO}_{4}$. Removal of the solvent under reduced pressure yielded the crude product, which was purified by column chromatography with $n$-hexane as eluent to give $\mathbf{B r}-\mathbf{F}_{3}-\mathbf{H}$ as white solid $(4.05 \mathrm{~g}, 3.25 \mathrm{mmol}, 47 \%)$.

${ }^{1} \mathrm{H}-\mathrm{NMR}\left(400 \mathrm{MHz}, \mathrm{CDCl}_{3}, 25^{\circ} \mathrm{C}\right): \delta[\mathrm{ppm}]=$ 7.84-7.75 (m, 5H, H-5, H-25, H-38, H-46, H-60), 7.70-7.60 (m, 9H, H-4, H-6, H-8, H-22, H-24, H-39, H-41, H-43, H-45), 7.52-7.48 (m, 2H, H-1, H-3), 7.40-7.31 (m, $3 \mathrm{H}, \mathrm{H}-61, \mathrm{H}-62, \mathrm{H}-63), 2.14-1.96$ (m, 12H, H-14, H-29, H-50), 1.27-1.11 (m, 60H, H-16, H-17, H-18, $\mathrm{H}-19, \mathrm{H}-20, \mathrm{H}-31, \mathrm{H}-32, \mathrm{H}-33, \mathrm{H}-34, \mathrm{H}-35, \mathrm{H}-52, \mathrm{H}-53, \mathrm{H}-54, \mathrm{H}-55, \mathrm{H}-56$ ), 0.86-0.80 (m, 22H, H-21, $\mathrm{H}-30, \mathrm{H}-36, \mathrm{H}-57), 0.79-0.69$ (m, 8H, H-15, H-51).

${ }^{13} \mathrm{C}-\mathrm{NMR}\left(101 \mathrm{MHz}, \mathrm{CDCl}_{3}, 25^{\circ} \mathrm{C}\right.$ ): $\delta$ [ppm] = 153.4 (C-10), 152.0 (C-27), 151.9 (C-42), 151.6 (C-48), 151.3 (C-13), 151.2 (C-58), 141.2 (C-7), 141.0 (C-59), 140.8 (C-40), 140.6 (C-44), 140.5 (C-23), 140.4 (C-37), 140.3 (C-26), 140.1 (C-47), 140.0 (C-11), 139.4 (C-12), 130.2 (C-3), 127.1 (C-62), 126.9 (C-61), 126.4 (C-1), 126.4 (C-6), 126.3 (C-24, C-39), 126.2 (C-45), 123.1 (C-63), 121.6 (C-41), 121.6 (C-4), 121.5 (C-8, C-43), 121.2 (C-22), 121.1 (C-2), 120.1 (C-5), 120.1 (C-25, C-38), 120.0 (C-46), 119.9 (C-60), 55.7 (C-9), 55.5 (C-28), 55.3 (C-49), 40.5 (C-29, C-50), 40.4 (C-14), 31.9 (C-19, C-34, C-55), 30.2 (C-52), 30.2 (C-31), 30.1 (C-16), 29.4 (C-17, C-18, C-32, C-33, C-53, C-54), 24.1 (C-30), 24.0 (C-51), 23.9 (C-15), 22.8 (C-20, C-35, C-56), 14.2 (C-21, C-36, C-57). 


\section{SYNTHESIS OF $\left(9,9,9^{\prime}, 9^{\prime}, 9^{\prime \prime}, 9^{\prime \prime}\right.$-HEXAOCTYL- $9 H, 9^{\prime \prime H}, 9^{\prime \prime} H-\left[2,2^{\prime}: 7^{\prime}, 2^{\prime \prime}\right.$ - TERFLUORENE]-7-YL)BORONIC ACID ((HO) $\left.)_{2} \mathrm{~B}-\mathrm{F}_{3}-\mathrm{H}\right)^{26}$}

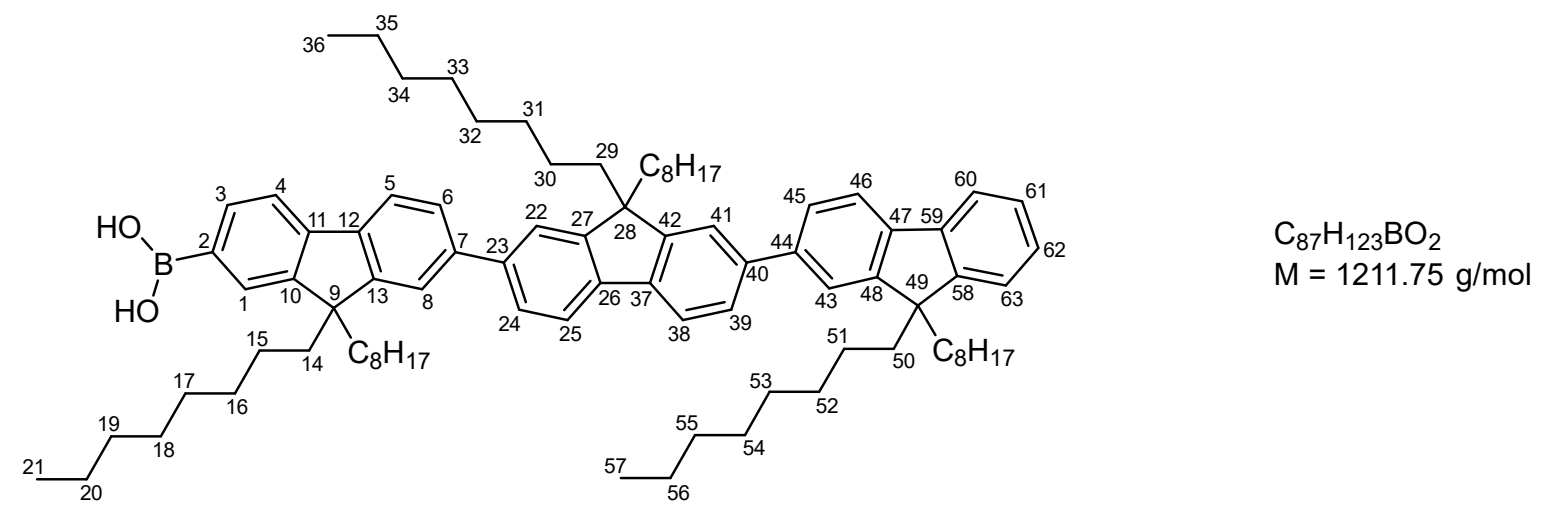

A solution of $\mathbf{B r}-\mathbf{F}_{3}-\mathbf{H}(2.53 \mathrm{~g}, 2.03 \mathrm{mmol}, 1$ equiv. $)$ in dry THF $(50 \mathrm{~mL})$ was cooled to $-78^{\circ} \mathrm{C}$ and a $1.6 \mathrm{M}$ solution of $n$-butyllithium in hexane $(1.5 \mathrm{~mL}, 2.43 \mathrm{mmol}, 1.2$ equiv.) was slowly added. After stirring for $1 \mathrm{~h}$ at the same temperature, tri-iso-propyl borate $(0.94 \mathrm{~mL}, 4.06 \mathrm{mmol}, 2$ equiv.) was added and the solution was warmed to room temperature and stirred overnight. $2 \mathrm{M} \mathrm{HCl}(50 \mathrm{~mL}$ ) was added to the reaction, followed by a further hour of stirring. The phases were separated and the aqueous phase was extracted three times with diethyl ether. The combined organic layers were washed with water and brine and dried over $\mathrm{MgSO}_{4}$. After removal of the solvent under reduced pressure, the crude product was purified by column chromatography (gradient elution from $n$-hexane to ethyl acetate) to yield $(\mathbf{H O})_{2} \mathbf{B}-\mathbf{F}_{3}-\mathbf{H}$ as white solid (1.86 g, $\left.1.53 \mathrm{mmol}, 75 \%\right)$.

${ }^{1} \mathrm{H}-\mathrm{NMR}\left(400 \mathrm{MHz}, \mathrm{CDCl}_{3}, 25^{\circ} \mathrm{C}\right): \delta[\mathrm{ppm}]=8.38\left(\mathrm{~d},{ }^{3} \mathrm{JHH}_{\mathrm{HH}}=7.9 \mathrm{~Hz}, 1 \mathrm{H}, \mathrm{H}-3\right), 8.29(\mathrm{~s}, 1 \mathrm{H}, \mathrm{H}-1), 7.96$ (d, $\left.{ }^{3} \mathrm{JHH}_{\mathrm{HH}}=7.7 \mathrm{~Hz}, 1 \mathrm{H}, \mathrm{H}-4\right), 7.89-7.78(\mathrm{~m}, 4 \mathrm{H}, \mathrm{H}-5, \mathrm{H}-25, \mathrm{H}-38, \mathrm{H}-46), 7.76-7.63(\mathrm{~m}, 9 \mathrm{H}, \mathrm{H}-6, \mathrm{H}-8, \mathrm{H}-22$, $\mathrm{H}-24, \mathrm{H}-39, \mathrm{H}-41, \mathrm{H}-43, \mathrm{H}-45, \mathrm{H}-60)$, 7.39-7.30 (m, 3H, H-61, H-62, H-63), 2.26 (m, 12H, H-14, H-29, $\mathrm{H}-50$ ), 1.24-1.06 (m, 60H, H-16, H-17, H-18, H-19, H-20, H-31, H-32, H-33, H-34, H-35, H-52, H-53, $\mathrm{H}-54, \mathrm{H}-55, \mathrm{H}-56), 0.85-0.67$ (m, 30H, H-15, H-21, H-30, H-36, H-51, H-57).

${ }^{13} \mathrm{C}-\mathrm{NMR}\left(101 \mathrm{MHz}, \mathrm{CDCl}_{3}, 25^{\circ} \mathrm{C}\right): \delta[\mathrm{ppm}]=152.0$ (br, C-13, C-27, C-42), 151.6 (C-48), 151.2 (C-58), 150.6 (br, C-10), 145.3 (br, C-11), 141.6 (br, C-12), 141.0 (C-59), 140.8 (C-23), 140.6 (C-40, C-44), 140.5 (C-26, C-37), 140.3 (C-7), 140.1 (C-47), 134.9 (br, C-3), 129.8 (br, C-1), 129.2 (br, C-2), 127.1 (C-62), 126.9 (C-61), 126.3 (br, C-6, C-24, C-39), 126.2 (C-45), 123.1 (C-63), 121.7 (br, C-8, C-22, C-41), 121.6 (C-43), 120.8 (C-5), 120.1 (br, C-25, C-38), 120.0 (C-46), 119.9 (C-60), 119.5 (C-4), 55.5 (C-28), 55.4 (C-9), 55.3 (C-49), 40.5 (C-14, C-29, C-50), 31.9 (C-19, C-34, C-55), 30.2 (C-16, C-31, C-52), 29.4 (C-17, C-18, C-32, C-33, C-53, C-54), 24.0 (C-15, C-30, C-51), 22.8 (C-20, C-35, C-56), 14.2 (C-21, C-36, C-57). 


\section{SYNTHESIS OF 9,9'-DIOCTYLFLUORENE-HEXAMER (H-F6-H) ${ }^{193}$}

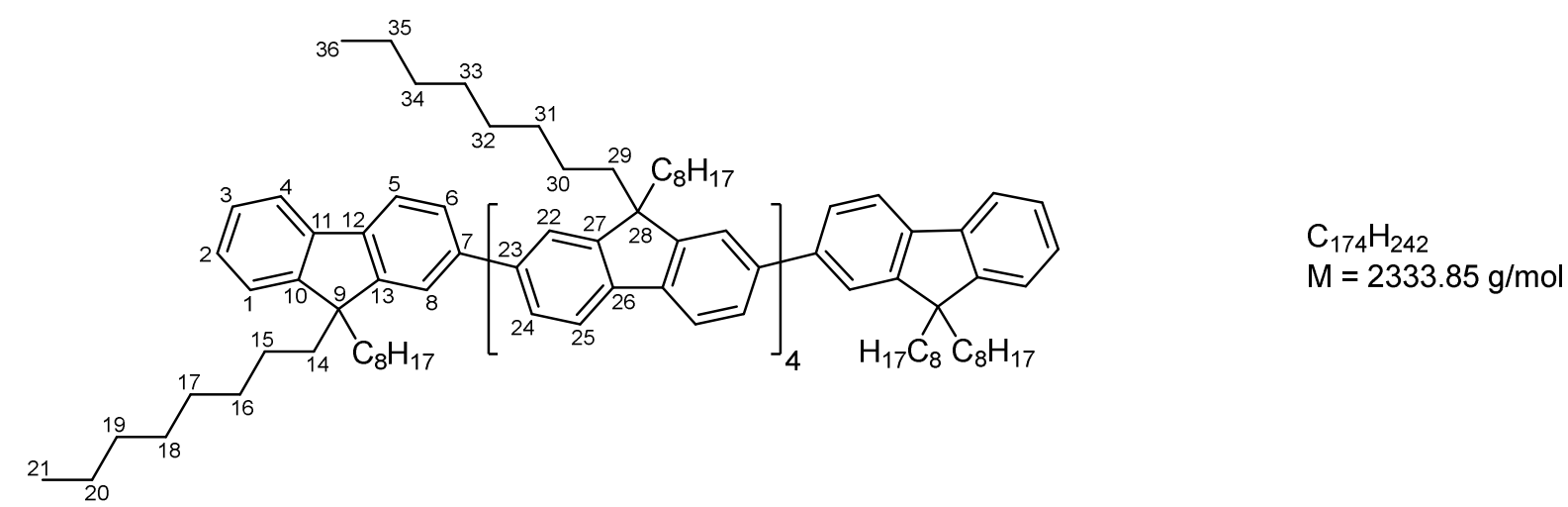

2,2'-Bipyridine (73 mg, $0.47 \mathrm{mmol}, 1.1$ equiv.) and bis(1,5-cyclooctadiene)nickel(0) ( $0.13 \mathrm{~g}, 0.47 \mathrm{mmol}$, 1.1 equiv.) were dissolved in anhydrous DMF $(2 \mathrm{~mL})$. Toluene $(2 \mathrm{~mL})$ and 1,5-cyclooctadiene $(0.06 \mathrm{~mL}$, $0.47 \mathrm{mmol}, 1.1$ equiv.) were added and the mixture was stirred at $80^{\circ} \mathrm{C}$ for $30 \mathrm{~min}$, before a solution of $\mathrm{Br}-\mathrm{F}_{3}-\mathrm{H}$ (0.53 g, $0.43 \mathrm{mmol}, 1$ equiv.) in toluene $(4 \mathrm{~mL})$ was added. The mixture was stirred at $80^{\circ} \mathrm{C}$ for $48 \mathrm{~h}$. After cooling to room temperature, the reaction mixture was poured into a stirred mixture of $\mathrm{CHCl}_{3}$ $(100 \mathrm{~mL})$ and $2 \mathrm{M} \mathrm{HCl}$ solution $(100 \mathrm{~mL})$ and stirred for additional $30 \mathrm{~min}$. The phases were separated, the organic layer was washed with brine, dried over $\mathrm{MgSO}_{4}$ and the solvent was removed under reduced pressure. The crude product was purified by column chromatography using $n$-hexane as eluent to yield $\mathbf{H}-\mathbf{F}_{6}-\mathbf{H}$ as yellowish-green solid $(0.40 \mathrm{~g}, 0.17 \mathrm{mmol}, 79 \%)$.

${ }^{1} \mathrm{H}-\mathrm{NMR}\left(400 \mathrm{MHz}, \mathrm{CDCl}_{3}, 25^{\circ} \mathrm{C}\right): \delta[\mathrm{ppm}]=7.85-7.82(\mathrm{~m}, 8 \mathrm{H}, \mathrm{H}-25), 7.80\left(\mathrm{~d},{ }^{3} \mathrm{JHH}_{\mathrm{HH}}=7.9 \mathrm{~Hz}, 2 \mathrm{H}, \mathrm{H}-5\right)$, $7.75\left(\mathrm{~d},{ }^{3} \mathrm{JHH}_{\mathrm{HH}}=7.0 \mathrm{~Hz}, 2 \mathrm{H}, \mathrm{H}-4\right), 7.71-7.64(\mathrm{~m}, 2 \mathrm{H}, \mathrm{H}-6, \mathrm{H}-8, \mathrm{H}-22, \mathrm{H}-24), 7.39-7.30(\mathrm{~m}, 6 \mathrm{H}, \mathrm{H}-1, \mathrm{H}-2$, $\mathrm{H}-3), 2.17-2.09(\mathrm{~m}, 16 \mathrm{H}, \mathrm{H}-29), 2.08-2.00(\mathrm{~m}, 8 \mathrm{H}, \mathrm{H}-14), 1.26-1.09(\mathrm{~m}, 120 \mathrm{H}, \mathrm{H}-16, \mathrm{H}-17, \mathrm{H}-18, \mathrm{H}-19$, $\mathrm{H}-20, \mathrm{H}-31, \mathrm{H}-32, \mathrm{H}-33, \mathrm{H}-34, \mathrm{H}-35), 0.85-0.79$ (m, 52H, H-21, H-36, H-30), 0.77-0.68 (m, 8H, H-15).

\section{SYNTHESIS OF DIBROMO-(9,9'-DIOCTYLFLUORENE)-HEXAMER (Br-F $-\mathrm{Br})^{10}$}

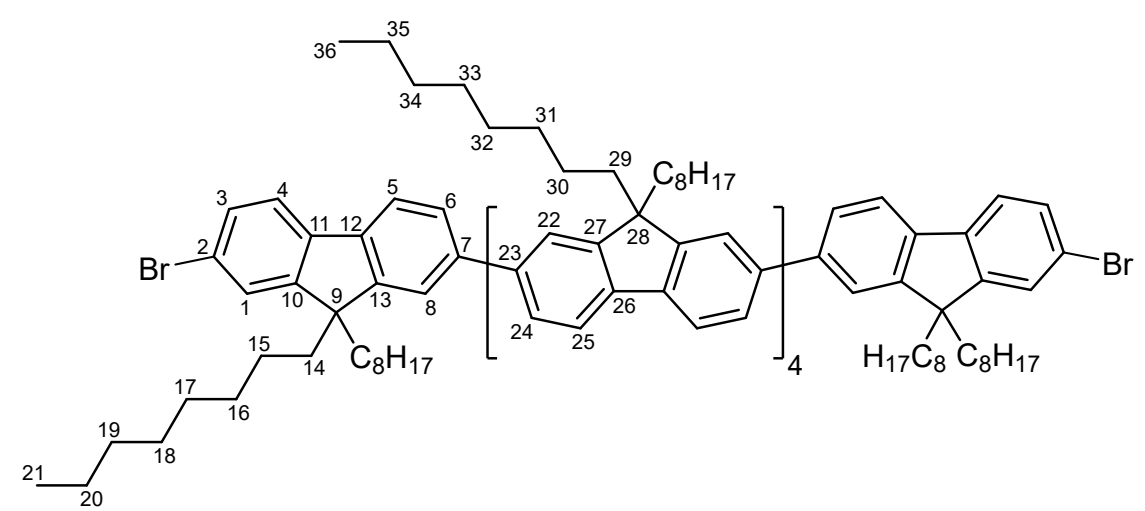

A solution of $\mathbf{H}-\mathbf{F}_{6}-\mathbf{H}\left(0.35 \mathrm{~g}, 0.15 \mathrm{mmol}, 1\right.$ equiv.) and $\mathrm{FeCl}_{3} \times 6 \mathrm{H}_{2} \mathrm{O}(0.8 \mathrm{mg}, 3 \mu \mathrm{mol}, 0.02$ equiv.) was cooled to $-10{ }^{\circ} \mathrm{C}$ and bromine ( $16 \mu \mathrm{L}, 0.31 \mathrm{mmol}, 2.1$ equiv.) was added. The mixture was brought to room temperature and stirred for $4 \mathrm{~h}$. Aqueous $\mathrm{Na}_{2} \mathrm{~S}_{2} \mathrm{O}_{5}$ solution was added and the reaction mixture was stirred vigorously until it showed a light yellow color. The phases were separated and the aqueous 
phase was extracted three times with DCM. The combined organic layers were washed with water, dried over $\mathrm{MgSO}_{4}$ and the solvent was removed in vacuo. The crude product was recrystallized from acetone to yield $\mathbf{B r}-\mathbf{F}_{6}-\mathrm{Br}$ as yellow solid $(0.31 \mathrm{~g}, 0.13 \mathrm{mmol}, 83 \%)$.

${ }^{1} \mathrm{H}-\mathrm{NMR}\left(400 \mathrm{MHz} \mathrm{CDCl}_{3}, \mathbf{2 5}^{\circ} \mathrm{C}\right): \delta[\mathrm{ppm}]=7.84-7.81(\mathrm{~m}, 8 \mathrm{H}, \mathrm{H}-25), 7.75\left(\mathrm{~d},{ }^{3} \mathrm{~J}_{\mathrm{HH}}=7.9 \mathrm{~Hz}, 2 \mathrm{H}, \mathrm{H}-5\right)$, 7.70-7.59 (m, 22H, H-4, H-6, H-8, H-22, H-24), 7.49-7.47 (m, 4H, H-1, H-3), 2.17-2.07 (m, 16H, H-29), 2.06-1.93 (m, 8H, H-14), 1.30-1.09 (m, 120H, H-16, H-17, H-18, H-19, H-20, H-31, H-32, H-33, H-34, $\mathrm{H}-35), 0.90-0.78(\mathrm{~m}, 52 \mathrm{H}, \mathrm{H}-21, \mathrm{H}-36, \mathrm{H}-30), 0.76-0.66(\mathrm{~m}, 8 \mathrm{H}, \mathrm{H}-15)$.

\section{SYNTHESIS OF $9,9^{\prime}$-DIOCTYLFLUORENE-NONAMER $\left(\mathrm{H}-\mathrm{F}_{9}-\mathrm{H}\right)^{10}$}

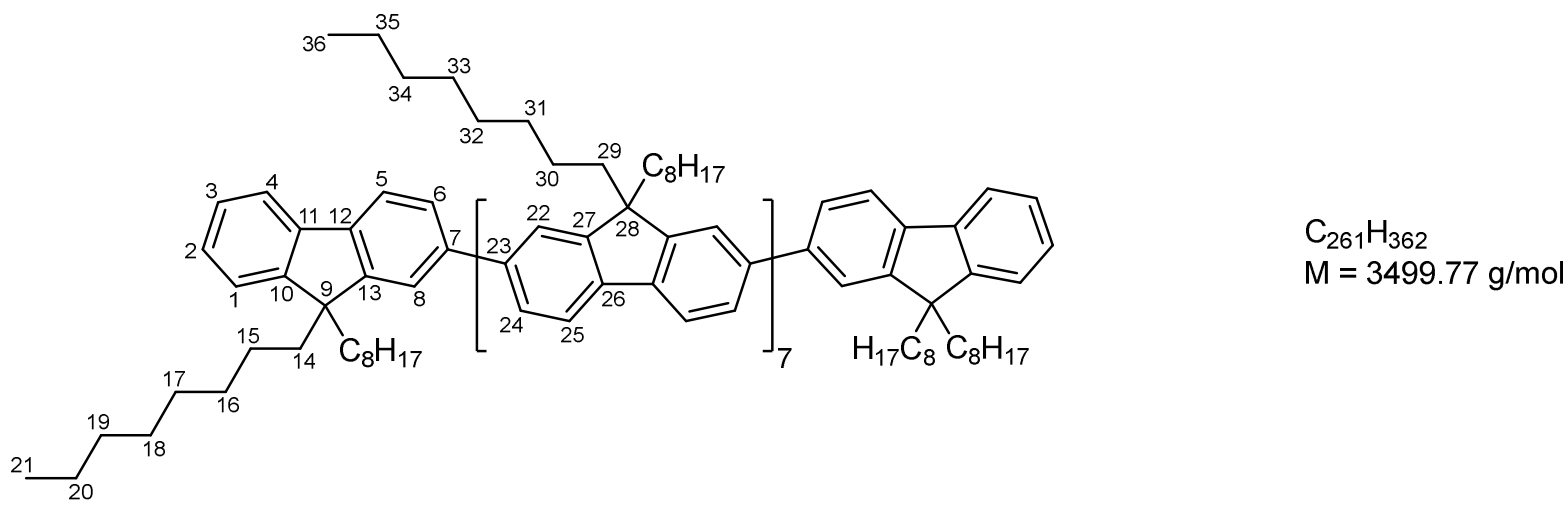

$\mathbf{B r}-\mathbf{F}_{3}-\mathbf{B r}(0.29 \mathrm{~g}, 0.22 \mathrm{mmol}, 1 \text { equiv.) and (HO) })_{2} \mathbf{B}-\mathbf{F}_{3}-\mathbf{H}(0.60 \mathrm{~g}, 0.49 \mathrm{mmol}, 2.2$ equiv.) were dissolved in toluene $(10 \mathrm{~mL})$ and the solution was degassed by two freeze-pump-thaw cycles. A $2 \mathrm{M}$ aqueous $\mathrm{K}_{2} \mathrm{CO}_{3}$ solution $(3.3 \mathrm{~mL}, 6.60 \mathrm{mmol}, 30$ equiv.) was added, followed by two more freeze-pump-thaw cycles. A solution of tetrakis(triphenylphosphine)palladium(0) (10 mg, $9 \mu \mathrm{mol}, 0.04$ equiv.) in toluene $(3 \mathrm{~mL})$ was added and the reaction mixture was heated to $90{ }^{\circ} \mathrm{C}$ overnight. After cooling to ambient temperature, the phases were separated and the organic layer was washed with water and dried over $\mathrm{MgSO}_{4}$. Removal of the solvent under reduced pressure yielded the crude product, which was purified by column chromatography using $n$-hexane as eluent. $\mathbf{H}-\mathbf{F}_{\mathbf{9}}-\mathbf{H}$ was obtained as a greenish-yellow solid (0.46 g, $0.13 \mathrm{mmol}, 59 \%)$.

${ }^{1} \mathrm{H}-\mathrm{NMR}\left(400 \mathrm{MHz} \mathrm{CDCl}_{3}, \mathbf{2 5}^{\circ} \mathrm{C}\right): \delta[\mathrm{ppm}]=7.86-7.82(\mathrm{~m}, 14 \mathrm{H}, \mathrm{H}-25), 7.80\left(\mathrm{~d},{ }^{3} \mathrm{JHH}_{\mathrm{HH}}=7.9 \mathrm{~Hz}, 2 \mathrm{H}, \mathrm{H}-5\right)$, $7.75\left(\mathrm{~d},{ }^{3} \mathrm{~J}_{\mathrm{HH}}=7.3 \mathrm{~Hz}, 2 \mathrm{H}, \mathrm{H}-4\right), 7.71-7.64(\mathrm{~m}, 32 \mathrm{H}, \mathrm{H}-6, \mathrm{H}-8, \mathrm{H}-22, \mathrm{H}-24), 7.39-7.30(\mathrm{~m}, 6 \mathrm{H}, \mathrm{H}-1, \mathrm{H}-2$, $\mathrm{H}-3)$, 2.19-2.09 (m, 28H, H-29), 2.08-1.99 (m, 8H, H-14), 1.26-1.10 (m, 180H, H-16, H-17, H-18, H-19, $\mathrm{H}-20, \mathrm{H}-31, \mathrm{H}-32, \mathrm{H}-33, \mathrm{H}-34, \mathrm{H}-35), 0.88-0.79$ (m, 82H, H-21, H-36, H-30), 0.77-0.67 (m, 8H, H-15). 


\section{SYNTHESIS OF DIBROMO-(9,9'-DIOCTYLFLUORENE)-NONAMER (Br-F9-Br) ${ }^{10}$}

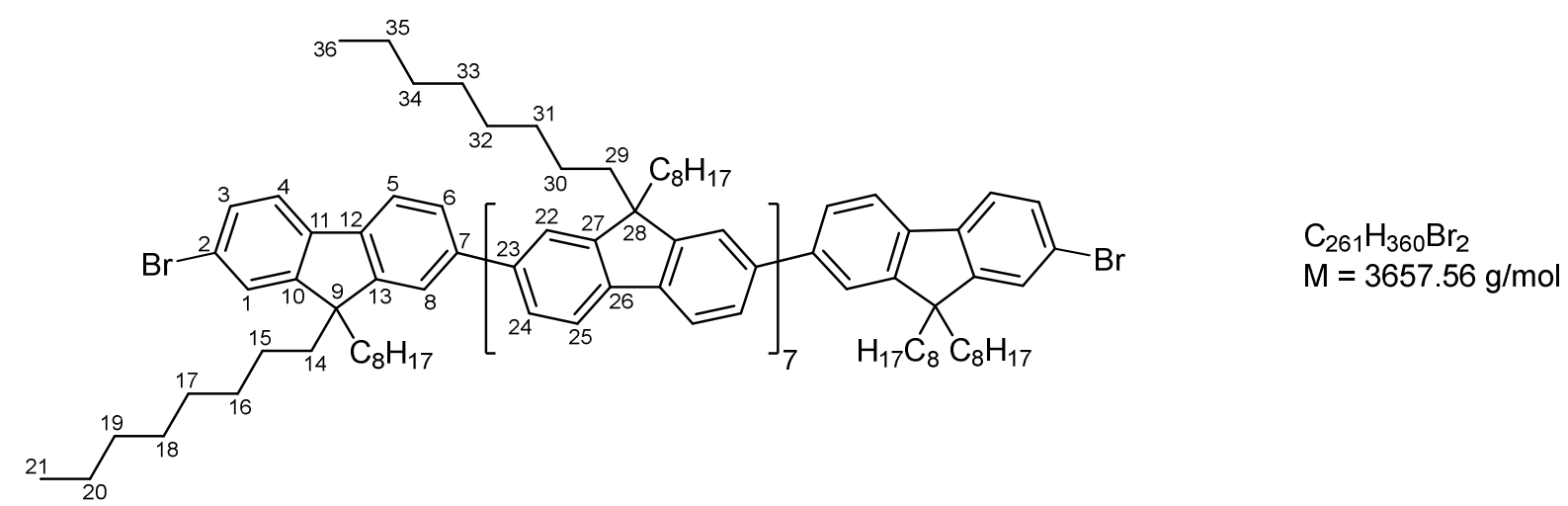

A solution of $\mathbf{H}-\mathbf{F}_{\mathbf{9}}-\mathbf{H}\left(0.43 \mathrm{~g}, 0.12 \mathrm{mmol}, 1\right.$ equiv.) and $\mathrm{FeCl}_{3} \times 6 \mathrm{H}_{2} \mathrm{O}(0.6 \mathrm{mg}, 2 \mu \mathrm{mol}, 0.02$ equiv.) was cooled to $-10{ }^{\circ} \mathrm{C}$ and bromine ( $13 \mu \mathrm{L}, 0.26 \mathrm{mmol}, 2.1$ equiv.) was added. The mixture was brought to room temperature and stirred for $4 \mathrm{~h}$. Aqueous $\mathrm{Na}_{2} \mathrm{~S}_{2} \mathrm{O}_{5}$ solution was added and the reaction mixture was stirred vigorously until it showed a light yellow color. The phases were separated and the aqueous phase was extracted three times with DCM. The combined organic layers were washed with water, dried over $\mathrm{MgSO}_{4}$ and the solvent was removed in vacuo. The crude product was recrystallized from acetone to yield $\mathbf{B r}-\mathrm{F}_{9}-\mathrm{Br}$ as yellow solid $(0.41 \mathrm{~g}, 0.11 \mathrm{mmol}, 92 \%)$.

${ }^{1} \mathrm{H}-\mathrm{NMR}\left(400 \mathrm{MHz}, \mathrm{CDCl}_{3}, 25^{\circ} \mathrm{C}\right): \delta[\mathrm{ppm}]=7.85-7.82(\mathrm{~m}, 14 \mathrm{H}, \mathrm{H}-25), 7.76\left(\mathrm{~d},{ }^{3} \mathrm{JHH}_{\mathrm{HH}}=7.8 \mathrm{~Hz}, 2 \mathrm{H}, \mathrm{H}-5\right)$, 7.71-7.60 (m, 34H, H-4, H-6, H-8, H-22, H-24), 7.49-7.47 (m, 4H, H-1, H-3), 2.16-1.96 (m, 36H, H-14, $\mathrm{H}-29$ ), 1.31-1.10 (m, 180H, H-16, H-17, H-18, H-19, H-20, H-31, H-32, H-33, H-34, H-35), 0.88 (t, $\left.{ }^{3} \mathrm{JHH}_{\mathrm{HH}}=7.1 \mathrm{~Hz}, 12 \mathrm{H}, \mathrm{H}-21\right), 0.86-0.79(\mathrm{~m}, 70 \mathrm{H}, \mathrm{H}-36, \mathrm{H}-30), 0.74-0.68(\mathrm{~m}, 8 \mathrm{H}, \mathrm{H}-15)$.

\section{SYNTHESIS OF $9,9^{\prime}$-DIOCTYLFLUORENE-DODECAMER $\left(H-F_{12}-H\right)^{10}$}

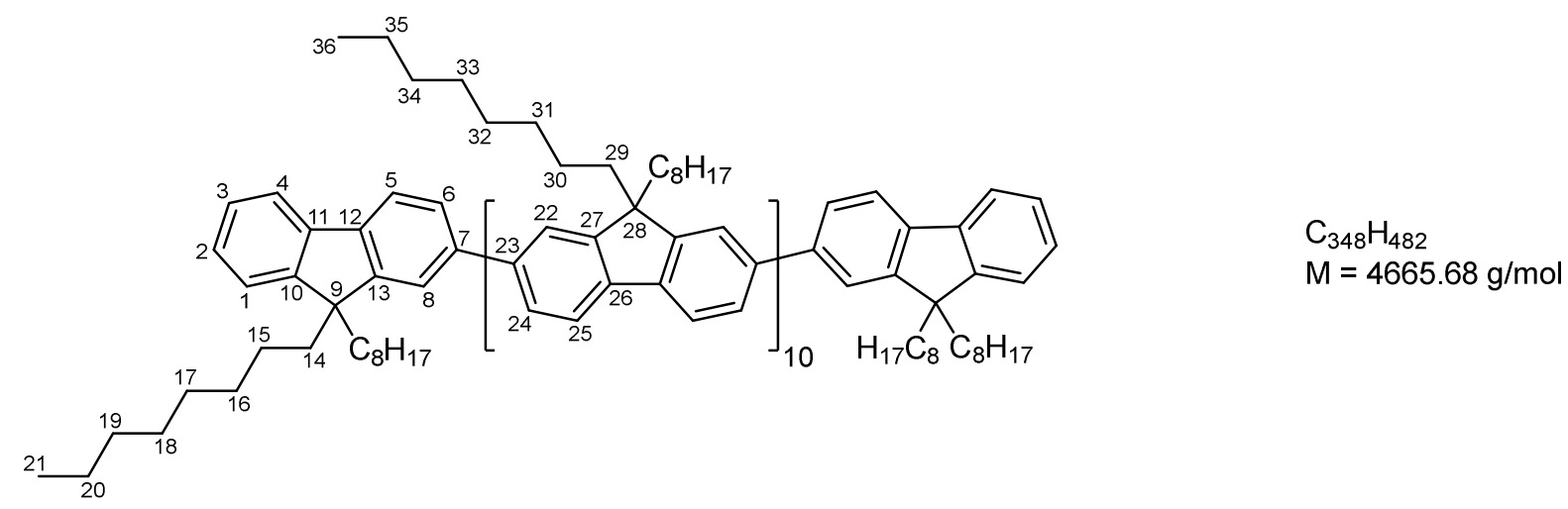

A solution of $\mathbf{B r}-\mathbf{F}_{6}-\mathrm{Br}\left(0.30 \mathrm{~g}, 0.12 \mathrm{mmol}, 1\right.$ equiv.) and $(\mathbf{H O})_{2} \mathbf{B}-\mathbf{F}_{3}-\mathbf{H}(0.32 \mathrm{~g}, 0.27 \mathrm{mmol}, 2.2$ equiv. $)$ in toluene $(15 \mathrm{~mL})$ was degassed by two freeze-pump-thaw cycles. A $2 \mathrm{M}$ aqueous $\mathrm{K}_{2} \mathrm{CO}_{3}$ solution $(1.8 \mathrm{~mL}, 3.60 \mathrm{mmol}, 30$ equiv.) was added, followed by two more freeze-pump-thaw cycles. A solution of tetrakis(triphenylphosphine)palladium( 0 ) $(6 \mathrm{mg}, 5 \mu \mathrm{mol}, 0.04$ equiv.) in toluene $(3 \mathrm{~mL})$ was added and the reaction mixture was heated to $90{ }^{\circ} \mathrm{C}$ overnight. After cooling to ambient temperature, the phases were separated and the organic layer was washed with water and dried over $\mathrm{MgSO}_{4}$. Removal of the 
solvent under reduced pressure yielded the crude product, which was purified by column chromatography (gradient elution from $n$-hexane to ethyl acetate). $\mathbf{H}-\mathbf{F}_{\mathbf{1 2}} \mathbf{- H}$ was obtained as a greenishyellow solid (0.30 g, $0.06 \mathrm{mmol}, 53 \%)$.

${ }^{1} \mathrm{H}-\mathrm{NMR}\left(400 \mathrm{MHz}, \mathrm{CDCl}_{3}, 2{ }^{\circ} \mathrm{C}\right): \delta[p p m]=7.89-7.77(\mathrm{~m}, 22 \mathrm{H}, \mathrm{H}-5, \mathrm{H}-25), 7.76-7.58(\mathrm{~m}, 46 \mathrm{H}, \mathrm{H}-4$, $\mathrm{H}-6, \mathrm{H}-8, \mathrm{H}-22, \mathrm{H}-24), 7.40-7.29$ (m, 6H, H-1, H-2, H-3), 2.23-1.97 (m, 48H, H-14, H-29), 1.29-1.03 (m, $240 \mathrm{H}, \mathrm{H}-16, \mathrm{H}-17, \mathrm{H}-18, \mathrm{H}-19, \mathrm{H}-20, \mathrm{H}-31, \mathrm{H}-32, \mathrm{H}-33, \mathrm{H}-34, \mathrm{H}-35), 0.90-0.67$ (m, 120H, H-15, H-21, $\mathrm{H}-36, \mathrm{H}-30)$.

\section{SYNTHESIS OF 9,9'-DIOCTYLFLUORENE-PENTADECAMER $\left(\mathrm{H}-\mathrm{F}_{15}-\mathrm{H}\right)^{10}$}

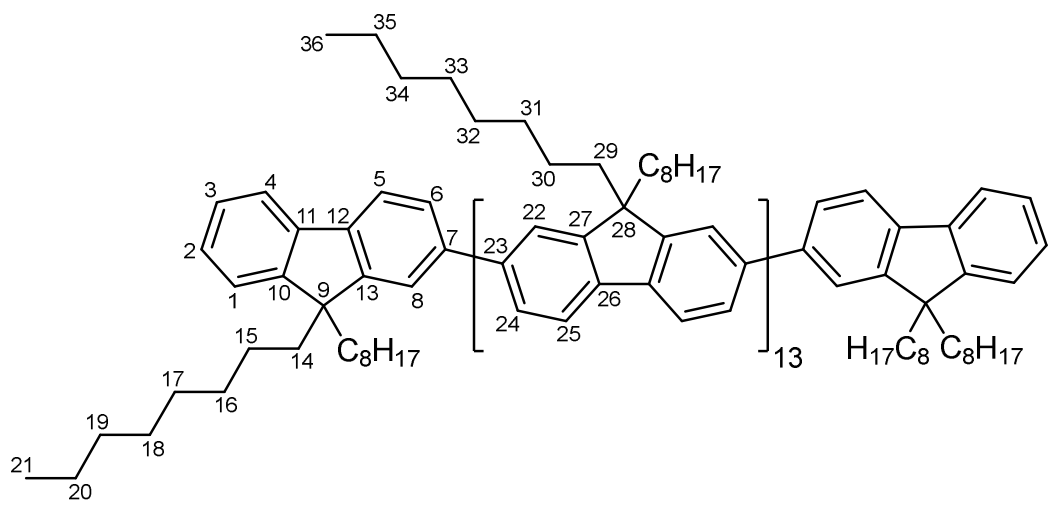

$$
\begin{aligned}
& \mathrm{C}_{435} \mathrm{H}_{602} \\
& \mathrm{M}=5831.60 \mathrm{~g} / \mathrm{mol}
\end{aligned}
$$

A solution of $\mathbf{B r}-\mathbf{F}_{9}-\mathbf{B r}(0.40 \mathrm{~g}, 0.11 \mathrm{mmol}, 1$ equiv. $)$ and $(\mathbf{H O})_{2} \mathbf{B}-\mathbf{F}_{3}-\mathbf{H}(0.29 \mathrm{~g}, 0.24 \mathrm{mmol}, 2.2$ equiv. $)$ in toluene $(25 \mathrm{~mL})$ was degassed by two freeze-pump-thaw cycles. A $2 \mathrm{M}$ aqueous $\mathrm{K}_{2} \mathrm{CO}_{3}$ solution (1.6 $\mathrm{mL}, 3.30 \mathrm{mmol}, 30$ equiv.) was added, followed by two more freeze-pump-thaw cycles. A solution of tetrakis(triphenylphosphine)palladium(0) $(5 \mathrm{mg}, 4 \mu \mathrm{mol}, 0.04$ equiv.) in toluene $(3 \mathrm{~mL})$ was added and the reaction mixture was heated to $90^{\circ} \mathrm{C}$ overnight. After cooling to ambient temperature, the phases were separated and the organic layer was washed with water and dried over $\mathrm{MgSO}_{4}$. Removal of the solvent under reduced pressure yielded the crude product, which was purified by column chromatography (gradient elution from $n$-hexane to ethyl acetate). $\mathbf{H}-\mathbf{F}_{15}-\mathbf{H}$ was obtained as a greenishyellow solid (91 mg, $16 \mu \mathrm{mol}, 14 \%)$.

${ }^{1} \mathrm{H}-\mathrm{NMR}\left(400 \mathrm{MHz}, \mathrm{CDCl}_{3}, 2{ }^{\circ} \mathrm{C}\right): \delta[\mathrm{ppm}]=7.87-7.77(\mathrm{~m}, 28 \mathrm{H}, \mathrm{H}-5, \mathrm{H}-25), 7.75-7.62(\mathrm{~m}, 58 \mathrm{H}, \mathrm{H}-4$, $\mathrm{H}-6, \mathrm{H}-8, \mathrm{H}-22, \mathrm{H}-24), 7.39-7.30$ (m, 6H, H-1, H-2, H-3), 2.20-1.99 (m, 60H, H-14, H-29), 1.27-1.04 (m, $300 \mathrm{H}, \mathrm{H}-16, \mathrm{H}-17, \mathrm{H}-18, \mathrm{H}-19, \mathrm{H}-20, \mathrm{H}-31, \mathrm{H}-32, \mathrm{H}-33, \mathrm{H}-34, \mathrm{H}-35), 0.89-0.66$ (m, 150H, H-15, H-21, $\mathrm{H}-36, \mathrm{H}-30$ ). 


\subsubsection{SUZUKI-MIYAURA POLYCONDENSATION WITH ADDED OLIGOMERS}

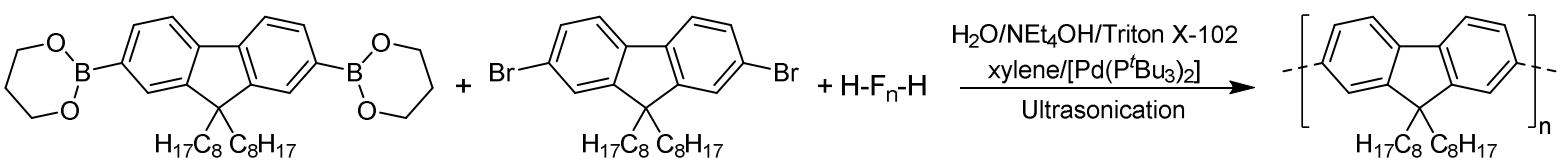

The polymerization was performed according to the general procedure for heterophase Suzuki-Miyaura polycondensation described in Chapter 8.3.2. The respective oligo(fluorene) $\mathrm{H}-\mathrm{F}_{\mathrm{n}}-\mathrm{H}(11.1 \mathrm{mg}$ ) was dissolved in xylene $(1 \mathrm{~mL})$ along with the monomers $\mathrm{PrO}_{2} \mathrm{~B}-\mathrm{F}_{1}-\mathrm{BO}_{2} \operatorname{Pr}(55.8 \mathrm{mg}, 0.1 \mathrm{mmol}, 1$ equiv.) and $\mathrm{Br}-\mathrm{F}_{1}-\mathrm{Br}(54.8 \mathrm{mg}, 0.1 \mathrm{mmol}, 1$ equiv.). In all cases ( $\mathrm{n}=3,6,9,12$ and 15$)$, the oligomers were applied in amounts of $10 \mathrm{wt} \%$, referred to the total mass of both monomers $(111 \mathrm{mg})$.

\subsubsection{POST-POLYMERIZATION PARTICLE SYNTHESIS}

\section{POST-POLYMERIZATION EMULSIFICATION OF POLYFLUORENE}

A solution of the respective poly $\left(9,9^{\prime}\right.$-dioctylfluorene $)(5 \mathrm{mg})$ in xylene $(1 \mathrm{~mL})$ was filtered via a $0.45 \mu \mathrm{m}$ PTFE syringe filter. The solution was added to an aqueous solution (10 mL) of Triton X-102 (5 wt\%) with ultrasonication starting simultaneously ( $1 \mathrm{~min}, 60 \%$ amplitude). The resulting dispersion was stirred at $300 \mathrm{rpm}$ for at least $24 \mathrm{~h}$.

Prior to use in the above post-polymerization procedure, poly(fluorene) synthesized by heterophase Suzuki-Miyaura polycondensation was purified. The polymer was precipitated by addition of methanol to the polymer dispersion, followed by centrifugation $(6000 \mathrm{rpm}, 10 \mathrm{~min})$ and decantation of the supernatant solution. The residual solid was dissolved in hot toluene, and the precipitation procedure was repeated twice. The resulting polymer was dried under reduced pressure.

Commercial poly(fluorene) purchased from Sigma-Aldrich was used without further purification.

\section{POST-POLYMERIZATION EMULSIFICATION OF OLIGOFLUORENES}

A solution of the respective oligo(fluorene) $(5 \mathrm{mg})$ in xylene $(1 \mathrm{mg})$ was filtered via a $0.45 \mu \mathrm{m}$ PTFE syringe filter. The solution was added to an aqueous solution $(10 \mathrm{~mL})$ of Triton X-102 (5 wt\%) with ultrasonication starting simultaneously ( $1 \mathrm{~min}, 60 \%$ amplitude). The resulting dispersion was stirred at $300 \mathrm{rpm}$ for at least $24 \mathrm{~h}$. 


\subsubsection{EVOLUTION OF MOLECULAR WEIGHT IN THE COURSE OF POLYCONDENSATION}

\section{SAMPLE PREPARATION FOR GPC MEASUREMENTS}

For molecular weight measurement of the monomer/catalyst mixture before emulsification in the aqueous phase, a solution of the two AA/BB-type monomers and the Pd-catalyst in xylene was prepared according to the SMPC procedure described in detail in Chapter 8.3.2. After degasification of the monomer/catalyst solution by three freeze-pump-thaw cycles, the solvent was removed under reduced pressure and the remaining solid was dissolved in THF for GPC and fluorescence measurements.

For monitoring the molecular weight during the period of ultrasonication as first phase of polymerization, reaction mixtures were precipitated completely by addition of methanol in order to avoid any distorting effects of the sample extraction on the further polymerization process. Four different reaction mixtures were prepared according to the standard SMPC procedure (vide supra). After the onset of ultrasonication, the polymerization process was stopped by pouring the reaction mixture into a large excess of methanol $(50 \mathrm{~mL}$ ) after $0.5 \mathrm{~min}, 1 \mathrm{~min}, 2 \mathrm{~min}$ and $2.5 \mathrm{~min}$ of ultrasonication. The ultrasonotrode was immediately removed and the precipitated mixture was rapidly stirred for $30 \mathrm{~min}$. The polymer was collected by centrifugation (6000 rpm, $10 \mathrm{~min}$ ) and dried under reduced pressure. Samples for GPC and fluorescence measurements were prepared including purification of the required polymer amount by two cycles of dissolution in hot toluene, precipitation by addition of methanol and centrifugation. The resulting solid was dried in vacuo and dissolved in THF for analytics.

For the aliquots drawn between $1 \mathrm{~h}$ and $72 \mathrm{~h}$ of reaction time, $0.1 \mathrm{~mL}$ of reaction mixture was removed from the reaction flask via syringe under inert gas atmosphere and immediately added to $8 \mathrm{~mL}$ of methanol. After centrifugation ( $6000 \mathrm{rpm}, 10 \mathrm{~min}$ ) and removal of the supernatant solution, the remaining precipitate was purified by two cycles of dissolution in hot toluene $(0.5-1 \mathrm{~mL})$, precipitation by addition of methanol $(6 \mathrm{~mL})$ and centrifugation. The resulting solid was dried in vacuo.

\section{SAMPLE PREPARATION FOR FLUORESCENCE AND MICROSCOPY MEASUREMENTS}

The Suzuki-Miyaura polycondensation was performed according to the standard procedure described above (Chapter 8.3.2). After $0.5 \mathrm{~min}, 1 \mathrm{~min}, 2 \mathrm{~min}$ and $2.5 \mathrm{~min}$ of ultrasonication samples were drawn, as well as after $1 \mathrm{~h}, 2 \mathrm{~h}, 4 \mathrm{~h}, 6 \mathrm{~h}, 24 \mathrm{~h}, 48 \mathrm{~h}$ and $72 \mathrm{~h}$ of stirring. The dispersions $(0.5 \mathrm{~mL})$ were diluted in deionized water $(10 \mathrm{~mL})$, which was acidified by addition of small amounts of $\mathrm{HCl}$ prior to use, and the samples were immediately examined by fluorescence emission measurements.

In addition, TEM samples were prepared from the aliquots drawn after $1 \mathrm{~min}$ and $2.5 \mathrm{~min}$ of ultrasonication as well as after $1 \mathrm{~h}, 2 \mathrm{~h}, 4 \mathrm{~h}, 6 \mathrm{~h}, 24 \mathrm{~h}, 48 \mathrm{~h}$ and $72 \mathrm{~h}$ of stirring. Note that although the sample preparation was conducted immediately after the withdrawal of the respective aliquot, the dispersion was allowed to dry on the TEM grid for several hours before the measurement. 


\subsubsection{SYNTHESIS OF CROSS-LINKED POLYFLUORENE}

\section{SYNTHESIS OF TRIS(4-BROMOPHENYL)AMINE225}<smiles>Brc1ccc(N(c2ccc(Br)cc2)c2ccc(Br)cc2)cc1</smiles>

Triphenylamine $(0.25 \mathrm{~g}, 1.05 \mathrm{mmol}, 1$ equiv. ) and silica gel $(1 \mathrm{~g})$ were added to a solution of NBS $(0.56 \mathrm{~g}$, $3.15 \mathrm{mmol}, 3$ equiv. $)$ in DCM $(200 \mathrm{~mL})$ and the suspension was stirred overnight at room temperature under exclusion of light. The silica gel was removed by filtration and the organic phase was washed thoroughly with water and brine followed by drying over $\mathrm{MgSO}_{4}$. Removal of the solvent under reduced pressure yielded tris(4-bromophenyl)amine as a white solid $(0.45 \mathrm{~g}, 0.93 \mathrm{mmol}, 89 \%)$.

${ }^{1} \mathrm{H}-\mathrm{NMR}\left(400 \mathrm{MHz}, \mathrm{CDCl}_{3}, 25{ }^{\circ} \mathrm{C}\right): \delta[\mathrm{ppm}]=7.36\left(\mathrm{~d},{ }^{3} \mathrm{JHH}_{\mathrm{HH}}=8.8 \mathrm{~Hz}, 6 \mathrm{H}, \mathrm{H}-3\right), 6.92\left(\mathrm{~d},{ }^{3} \mathrm{~J}_{\mathrm{HH}}=8.8 \mathrm{~Hz}\right.$, $6 \mathrm{H}, \mathrm{H}-2)$.

${ }^{13} \mathrm{C}-\mathrm{NMR}\left(101 \mathrm{MHz}, \mathrm{CDCl}_{3}, 25^{\circ} \mathrm{C}\right): \delta[\mathrm{ppm}]=146.2(\mathrm{C}-1), 132.7(\mathrm{C}-3), 125.8$ (C-2), $116.2(\mathrm{C}-4)$.

\section{SYNTHESIS OF TETRAKIS(4-BROMOPHENYL)METHANE226}<smiles>Brc1ccc(C(c2ccc(Br)cc2)(c2ccc(Br)cc2)c2ccc(Br)cc2)cc1</smiles>

$$
\begin{aligned}
& \mathrm{C}_{25} \mathrm{H}_{16} \mathrm{Br}_{4} \\
& \mathrm{M}=636.02 \mathrm{~g} / \mathrm{mol}
\end{aligned}
$$

Bromine ( $0.44 \mathrm{~mL}, 17.2 \mathrm{mmol}, 11$ equiv. $)$ was added dropwise to tetraphenylmethane $(0.5 \mathrm{~g}, 1.56 \mathrm{mmol}$, 1 equiv.) and the resulting slurry was stirred for $5 \mathrm{~h}$ at room temperature. Ethanol (50 mL), cooled to $-78^{\circ} \mathrm{C}$ by liquid nitrogen, was added and the resulting precipitate was filtered and washed with saturated aqueous $\mathrm{Na}_{2} \mathrm{~S}_{2} \mathrm{O}_{5}$ solution and water. After drying, the solid was dissolved in warm toluene and the solution was filtered. Removal of the solvent from the filtrate under reduced pressure yielded tetrakis(4-bromophenyl)methane as yellow solid $(0.79 \mathrm{~g}, 1.24 \mathrm{mmol}, 80 \%)$.

${ }^{1} \mathrm{H}-\mathrm{NMR}\left(400 \mathrm{MHz}, \mathrm{CDCl}_{3}, 25^{\circ} \mathrm{C}\right): \delta[p p m]=7.39\left(\mathrm{~d},{ }^{3} \mathrm{JHH}=8.7 \mathrm{~Hz}, 8 \mathrm{H}, \mathrm{H}-4\right), 7.01\left(\mathrm{~d},{ }^{3} \mathrm{JHH}=8.8 \mathrm{~Hz}\right.$, $8 \mathrm{H}, \mathrm{H}-3)$.

${ }^{13} \mathrm{C}-\mathrm{NMR}\left(101 \mathrm{MHz}, \mathrm{CDCl}_{3}, 25^{\circ} \mathrm{C}\right): \delta[\mathrm{ppm}]=144.6$ (C-2), 132.5 (C-4), $131.3(\mathrm{C}-3), 121.0$ (C-5), 63.8 (C-1). 


\section{POLYMERIZATION PROCEDURE}<smiles>CCCC1(CCC)c2cc(Br)ccc2-c2ccc(B3OC[CH+]CO3)cc2C1(CCC)c1ccc(Br)cc1</smiles><smiles>Brc1ccc(N(c2ccc(Br)cc2)c2ccc(Br)cc2)cc1</smiles><smiles>Brc1ccc(C(c2ccc(Br)cc2)(c2ccc(Br)cc2)c2ccc(Br)cc2)cc1</smiles>

$\mathrm{H}_{2} \mathrm{O} / \mathrm{NEt}_{4} \mathrm{OH} /$ Triton $\mathrm{X}-102$ xylene/[Pd(Pt $\mathrm{P}^{\mathrm{B}} \mathrm{H}_{3} / \mathrm{d}$

Ultrasonication

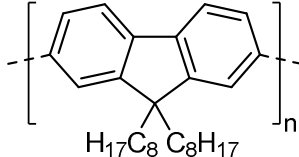

cross-linked PF8

The syntheses of cross-linked poly(fluorenes) were performed according to the general Suzuki-Miyaura polycondensation procedure outlined above in Chapter 8.3.2, with the addition of either tris(4bromophenyl)amine or tetrakis(4-bromophenyl)methane as cross-linker. The respective cross-linking agent ( $x$ equiv.) was dissolved in xylene $(1 \mathrm{~mL})$ along with $\mathrm{PrO}_{2} \mathrm{~B}-\mathrm{F}_{1}-\mathrm{BO}_{2} \operatorname{Pr}(55.8 \mathrm{mg}, 0.1 \mathrm{mmol}, 1$ equiv.) and $\mathrm{Br}-\mathrm{F}_{1}-\mathrm{Br}$ (1-x equiv.). Polymerizations were conducted using $0.5 \mathrm{~mol} \%, 1.25 \mathrm{~mol} \%, 1.5 \mathrm{~mol} \%$ and $5 \mathrm{~mol} \%$ of cross-linker, referred to the total amount of both monomers $(0.2 \mathrm{mmol})$.

Table 8.1: Composition of reaction mixtures regarding amounts of 2,7-dibromo-9,9'-dioctylfluorene $\left(\mathrm{Br}-\mathrm{F}_{1}-\mathrm{Br}\right)$ and cross-linking additives for synthesis of cross-linking poly(fluorene). ${ }^{a}$

\begin{tabular}{c|c|c|c|c|c}
\multicolumn{4}{c|}{ multi-functional monomer } & \multicolumn{2}{c}{$\begin{array}{c}\text { 2,7-dibromo-9,9'- } \\
\text { dioctylfluorene }\end{array}$} \\
\hline type & $\begin{array}{c}\text { amount }^{b} \\
{[\mathrm{~mol} \%]}\end{array}$ & $\begin{array}{c}\mathbf{n} \\
{[\mu \mathrm{mol}]}\end{array}$ & $\begin{array}{c}\text { mass } \\
{[\mathrm{mg}]}\end{array}$ & $\begin{array}{c}\mathbf{n} \\
{[\mu \mathrm{mol}]}\end{array}$ & $\begin{array}{c}\text { mass } \\
{[\mathrm{mg}]}\end{array}$ \\
\hline \multirow{3}{*}{$\mathbf{N}\left(\mathrm{C}_{6} \mathrm{H}_{4} \mathrm{Br}\right)_{3}$} & 0.5 & 1.0 & 0.5 & 99.0 & 54.3 \\
& 1.25 & 2.5 & 1.2 & 97.5 & 53.5 \\
& 2.5 & 5.0 & 2.4 & 95.0 & 52.1 \\
& 5 & 10.0 & 4.8 & 90.0 & 49.4 \\
\hline & 0.5 & 1.0 & 0.6 & 99.0 & 54.3 \\
$\mathbf{C}\left(\mathbf{C}_{6} \mathrm{H}_{4} \mathrm{Br}\right)_{4}$ & 1.25 & 2.5 & 1.6 & 97.5 & 53.5 \\
& 2.5 & 5.0 & 3.2 & 95.0 & 52.1 \\
& 5 & 10.0 & 6.4 & 90.0 & 49.4
\end{tabular}

${ }^{a}$ Conditions: Diboronic acid ester $(0.1 \mathrm{mmol})$ and dibromo-substituted fluorene monomers $(0.1-\mathrm{x} \mathrm{mmol})$, multifunctional monomer $(x \mathrm{mmol})$ and $\left[\mathrm{Pd}\left(\mathrm{P}^{t} \mathrm{Bu}_{3}\right)_{2}\right](4 \mu \mathrm{mol})$ dissolved in xylene $(1 \mathrm{~mL})$, addition to $5 \mathrm{wt} \%$ aqueous Triton $\mathrm{X}-102$ solution $(25 \mathrm{~mL})$, simultaneous ultrasonication ( $2.5 \mathrm{~min}, 60 \%$ amplitude), $72 \mathrm{~h}$ of stirring. ${ }^{b}$ Referred to the total amount of both AA/BB-type monomers $(0.2 \mathrm{mmol})$. 


\subsubsection{PHASE BEHAVIOR IN SOLUTION}

\section{SAMPLE PREPARATION}

Polymer solutions with a concentration of $2 \mathrm{mg} / \mathrm{mL}$ were prepared by dissolution of commercial PF8 (Sigma-Aldrich) or the oligo(fluorene) of choice in the respective organic solvent (at least $3 \mathrm{~mL}$ ) by application of heat, if required. After cooling, the homogenized solutions were filtered via $0.45 \mu \mathrm{m}$ PTFE syringe filters. In the course of the measurements, the solutions were gradually diluted to achieve the desired concentration.

\section{MEASUREMENT PROCEDURE}

Fluorescence emission was measured with a Hamamatsu Absolute PL Quantum Yield Measurement System C9920-02 with an excitation wavelength of $\lambda_{\mathrm{exc}}=380 \mathrm{~nm}$. The default measurement conditions (50 ms of exposure time, averaging over 50 measurements) were adjusted for the samples of high concentration to ensure a sufficient signal to noise ratio $(20 \mathrm{~ms}$ of exposure time, averaging over 500 measurements).

\subsubsection{VERTICAL DEPOSITION}

Prior to vertical deposition experiments, the $5 \times 7 \mathrm{~mm}$ silicon wafers were hydrophilized. To this end, a $5 / 1$ mixture of concentrated sulphuric acid and hydrogen peroxide $(30 \%)$ was heated to $105^{\circ} \mathrm{C}$ and the wafers were immersed. After $10 \mathrm{~min}$ the wafers were removed, rinsed with deionized water and immediately used for particle deposition experiments.

The PF8 particle dispersion $(2 \mathrm{~mL})$ as obtained from heterophase Suzuki-Miyaura polycondensation was purified by precipitation in methanol $(6 \mathrm{~mL})$, centrifugation $(6000 \mathrm{rpm}, 10 \mathrm{~min})$ and redispersion of the precipitate in deionized water $(2 \mathrm{~mL}$ ) by ultrasonication ( $1 \mathrm{~min}, 30 \%$ amplitude).

The silicon wafer was placed diagonally in an $8 \times 50 \mathrm{~mm}$ glass tube and $160 \mu \mathrm{L}$ of particle dispersion was added, followed by drying over $5 \mathrm{~d}$ at room temperature. 


\title{
8.6 POLYDIARYLFLUORENE
}

\subsubsection{SYNTHESES OF DIARYLFLUORENE MONOMERS}

\section{SYNTHESIS OF 2,7-DIBROMO-9-FLUORENONE202}<smiles>O=C1c2cc(Br)ccc2-c2ccc(Br)cc21</smiles>

$\mathrm{C}_{13} \mathrm{H}_{6} \mathrm{Br}_{2} \mathrm{O}$

$\mathrm{M}=338.00 \mathrm{~g} / \mathrm{mol}$

9-Fluorenone (10.0 g, $55.5 \mathrm{mmol}$, 1 equiv.) was suspended in deionized water (140 $\mathrm{mL}$ ) and cooled to $0{ }^{\circ} \mathrm{C}$. Bromine $(14.3 \mathrm{~mL}, 277.5 \mathrm{mmol}, 5$ equiv.) was added dropwise over a period of $10 \mathrm{~min}$ and the suspension was heated to $80^{\circ} \mathrm{C}$ for $10 \mathrm{~h}$ while stirring. After cooling down to ambient temperature, aqueous $\mathrm{Na}_{2} \mathrm{~S}_{2} \mathrm{O}_{5}$ solution was added until the brown color of the mixture disappeared. The yellow precipitate was filtered, washed with water and dried in vacuo to yield 2,7-dibromo-9-fluorenone (18.4 g, $54.6 \mathrm{mmol}, 98 \%)$.

${ }^{1} \mathrm{H}-\mathrm{NMR}\left(400 \mathrm{MHz}, \mathrm{CDCl}_{3}, 25^{\circ} \mathrm{C}\right): \delta[\mathrm{ppm}]=7.76\left(\mathrm{~d},{ }^{4} \mathrm{JHH}_{\mathrm{HH}}=1.8 \mathrm{~Hz}, 2 \mathrm{H}, \mathrm{H}-1\right), 7.62\left(\mathrm{dd},{ }^{3} \mathrm{JHH}_{\mathrm{HH}}=7.9 \mathrm{~Hz}\right.$, $\left.{ }^{4} \mathrm{~J}_{\mathrm{HH}}=1.8 \mathrm{~Hz}, 2 \mathrm{H}, \mathrm{H}-3\right), 7.38\left(\mathrm{~d},{ }^{3} \mathrm{~J}_{\mathrm{HH}}=7.9 \mathrm{~Hz}, 2 \mathrm{H}, \mathrm{H}-4\right)$.

${ }^{13} \mathrm{C}-\mathrm{NMR}\left(101 \mathrm{MHz}, \mathrm{CDCl}_{3}, 2 \mathbf{2}^{\circ} \mathrm{C}\right.$ ): $\delta$ [ppm] = 191.1 (C-7), 142.4 (C-2), 137.6 (C-3), 135.5 (C-6), 128.0 (C-1), 123.5 (C-5), 122.0 (C-4).

\section{SYNTHESIS OF 3,8-DIBROMO-6H-BENZO[C]CHROMEN-6-ONE203}<smiles>O=c1oc2cc(Br)ccc2c2ccc(I)cc12</smiles>

\author{
$\mathrm{C}_{13} \mathrm{H}_{6} \mathrm{Br}_{2} \mathrm{O}_{2}$ \\ $\mathrm{M}=354.00 \mathrm{~g} / \mathrm{mol}$
}

2,7-Dibromo-9-fluorenone (2.0 g, $5.64 \mathrm{mmol}, 1$ equiv.) and 70\% $\mathrm{m}$-CPBA in water ( $2.13 \mathrm{~g}, 8.66 \mathrm{mmol}$, 1.5 equiv.) were dissolved in glacial acetic acid $(24 \mathrm{~mL})$ and concentrated $\mathrm{H}_{2} \mathrm{SO}_{4}(4 \mathrm{~mL})$ was added. The mixture was heated to $80^{\circ} \mathrm{C}$ for $24 \mathrm{~h}$, before additional amounts of $m$-CPBA $(0.4 \mathrm{~g}, 1.62 \mathrm{mmol}$, 0.3 equiv.) and concentrated $\mathrm{H}_{2} \mathrm{SO}_{4}(1 \mathrm{~mL})$ were added and the reaction was stirred at $80^{\circ} \mathrm{C}$ for further $72 \mathrm{~h}$. After quenching by addition of saturated aqueous $\mathrm{Na}_{2} \mathrm{SO}_{3}$ solution, the mixture was extracted three times with dichloromethane. The combined organic layers were washed with aqueous $\mathrm{Na}_{2} \mathrm{CO}_{3}$ solution and water, dried over $\mathrm{MgSO}_{4}$ and the solvent was removed under reduced pressure. The crude product was purified by column chromatography using an eluent mixture of toluene and petroleum ether in a 1:1 ratio, yielding 3,8-dibromo-6H-benzo[c]chromen-6-one as white solid (1.23 g, $3.49 \mathrm{mmol}, 62 \%)$. 
${ }^{1} \mathrm{H}-\mathrm{NMR}\left(400 \mathrm{MHz}, \mathrm{CDCl}_{3}, \mathbf{2 5}{ }^{\circ} \mathrm{C}\right): \delta[\mathrm{ppm}]=8.51\left(\mathrm{dd},{ }^{4} \mathrm{~J}_{\mathrm{HH}}=1.8,{ }^{5} \mathrm{JHH}_{\mathrm{HH}}=0.8 \mathrm{~Hz}, 1 \mathrm{H}, \mathrm{H}-1\right), 7.96-7.92(\mathrm{~m}$, $2 \mathrm{H}, \mathrm{H}-3, \mathrm{H}-4), 7.87\left(\mathrm{~d},{ }^{3} \mathrm{~J}_{\mathrm{HH}}=8.6 \mathrm{~Hz}, 1 \mathrm{H}, \mathrm{H}-5\right), 7.54\left(\mathrm{~d},{ }^{4} \mathrm{~J}_{\mathrm{HH}}=1.9 \mathrm{~Hz}, 1 \mathrm{H}, \mathrm{H}-8\right), 7.48\left(\mathrm{dd},{ }^{3} \mathrm{JHH}_{\mathrm{HH}}=8.6\right.$, $\left.{ }^{4} \mathrm{JHH}_{\mathrm{HH}}=1.9 \mathrm{~Hz}, 1 \mathrm{H}, \mathrm{H}-6\right)$.

${ }^{13} \mathrm{C}-\mathrm{NMR}\left(101 \mathrm{MHz}, \mathrm{CDCl}_{3}, 25^{\circ} \mathrm{C}\right): \delta[\mathrm{ppm}]=159.4(\mathrm{C}-9), 151.5(\mathrm{C}-13), 138.3(\mathrm{C}-3), 133.5(\mathrm{C}-1), 133.0$ (C-11), 128.3 (C-6), 124.4 (C-7), 124.0 (C-5), 123.6 (C-4), 123.4 (C-2), 122.6 (C-10), 121.2 (C-8), 116.6 (C-12).

\section{SYNTHESIS OF 4,4'-DIBROMO-2'-(HYDROXYDIPHENYLMETHYL)BIPHENYL-2-OL55}<smiles>Oc1cc(Br)ccc1-c1ccc(Br)cc1C(O)(c1ccccc1)c1ccccc1</smiles>

$\mathrm{C}_{25} \mathrm{H}_{18} \mathrm{Br}_{2} \mathrm{O}_{2}$

$\mathrm{M}=510.23 \mathrm{~g} / \mathrm{mol}$

A solution of 3,8-dibromo-6H-benz[c]ochromen-6-one ( $3.71 \mathrm{~g}, 10.5 \mathrm{mmol}, 1$ equiv.) in toluene $(80 \mathrm{~mL})$ was degassed by three freeze-pump-thaw cycles and cooled to $0{ }^{\circ} \mathrm{C}$. A $3 \mathrm{M}$ solution of $\mathrm{PhMgBr}$ in diethyl ether (17.5 mL, $52.4 \mathrm{mmol}, 5$ equiv.) was added dropwise; the reaction was allowed to warm to room temperature and stirred overnight. After quenching by addition of aqueous $\mathrm{NH}_{4} \mathrm{Cl}$ solution, the phases were separated and the aqueous layer was extracted two times with dichloromethane. The combined organic phases were dried over $\mathrm{MgSO}_{4}$ and the solvent was removed under reduced pressure. The crude product was purified by column chromatography (dichloromethane/petroleum ether in a 1/1 ratio) to yield $4,4^{\prime}$-dibromo-2'-(hydroxydiphenylmethyl)biphenyl-2-ol as a white solid $(3.87 \mathrm{~g}, 7.58 \mathrm{mmol}$, $72 \%)$.

${ }^{1} \mathrm{H}-\mathrm{NMR}\left(400 \mathrm{MHz}, \mathrm{CDCl}_{3}, 25^{\circ} \mathrm{C}\right): \delta[\mathrm{ppm}]=7.49\left(\mathrm{dd},{ }^{3} \mathrm{JHH}_{\mathrm{HH}}=8.1 \mathrm{~Hz},{ }^{4} \mathrm{JHH}_{\mathrm{HH}}=2.1 \mathrm{~Hz}, 1 \mathrm{H}, \mathrm{H}-3\right), 7.36-7.32$ $(\mathrm{m}, 3 \mathrm{H}, \mathrm{Ph}-\mathrm{H}), 7.24-7.21(\mathrm{~m}, 5 \mathrm{H}, \mathrm{Ph}-\mathrm{H}), 7.05\left(\mathrm{~d},{ }^{4} \mathrm{JHH}_{\mathrm{HH}}=2.1 \mathrm{~Hz}, 1 \mathrm{H}, \mathrm{H}-1\right), 7.02\left(\mathrm{~d},{ }^{4} \mathrm{JHH}_{\mathrm{HH}}=2.1 \mathrm{~Hz}, 1 \mathrm{H}\right.$, $\mathrm{H}-6), 7.01-6.98(\mathrm{~m}, 2 \mathrm{H}, \mathrm{Ph}-\mathrm{H}), 6.95\left(\mathrm{~d},{ }^{3} \mathrm{JHH}_{\mathrm{HH}}=8.1 \mathrm{~Hz}, 1 \mathrm{H}, \mathrm{H}-4\right), 6.65\left(\mathrm{dd},{ }^{3} \mathrm{JHH}_{\mathrm{HH}}=8.1 \mathrm{~Hz},{ }^{4} \mathrm{JHH}_{\mathrm{HH}}=1.9 \mathrm{~Hz}\right.$, $1 \mathrm{H}, \mathrm{H}-8), 6.03\left(\mathrm{~d},{ }^{3} \mathrm{JHH}_{\mathrm{HH}}=8.1 \mathrm{~Hz}, 1 \mathrm{H}, \mathrm{H}-13\right), 5.54(\mathrm{~s}, 1 \mathrm{H}, \mathrm{H}-15), 3.11(\mathrm{~s}, 1 \mathrm{H}, \mathrm{H}-14)$.

${ }^{13} \mathrm{C}-\mathrm{NMR}\left(101 \mathrm{MHz}, \mathrm{CDCl}_{3}, 25{ }^{\circ} \mathrm{C}\right): \delta[\mathrm{ppm}]=153.8$ (C-5), $148.7(\mathrm{C}-10), 145.5(\mathrm{Ph}), 145.1(\mathrm{Ph}), 135.0$ (C-4), 134.8 (C-11), 133.1 (C-1), 131.8 (C-13), 131.4 (C-3), 128.5 (Ph), 128.4 (C-12), $128.3(\mathrm{Ph}), 128.2$ (Ph), $128.1(\mathrm{Ph}), 127.6(\mathrm{Ph}), 127.3(\mathrm{Ph}), 123.2(\mathrm{C}-8), 122.3$ (C-7), 122.0 (C-2), 119.8 (C-6), 83.1 (C-9). 


\section{SYNTHESIS OF (4,4'-DIBROMO-2'-OCTYLOXYBIPHENYL-2-YL)- DIPHENYLMETHANOL55}

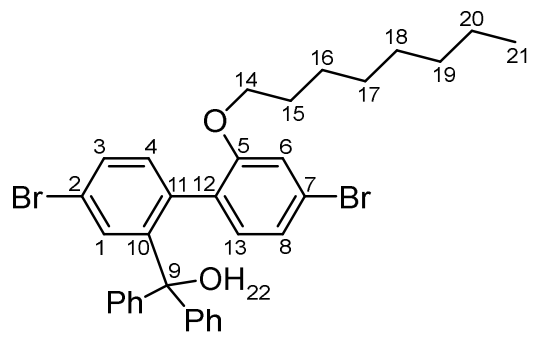

4,4' 'Dibromo-2' -(hydroxydiphenylmethyl)biphenyl-2-ol (3.80 g, $7.45 \mathrm{mmol}, 1$ equiv.) and $\mathrm{K}_{2} \mathrm{CO}_{3}(3.60 \mathrm{~g}$, $26.08 \mathrm{mmol}, 3.5$ equiv.) were dissolved in dry DMF $(20 \mathrm{~mL})$ and degassed 1-bromooctane $(1.56 \mathrm{~mL}$, $8.94 \mathrm{mmol}, 1.2$ equiv.) was added dropwise. The mixture was heated to $70^{\circ} \mathrm{C}$ and stirred for $24 \mathrm{~h}$ under exclusion of light. Solid residues were filtered off and the solution was diluted with $1 \mathrm{M} \mathrm{HCl}$. The aqueous phase was extracted three times with DCM and the combined organic layers were washed with $1 \mathrm{M} \mathrm{HCl}$ and water and dried over $\mathrm{MgSO}_{4}$. After removing the solvent under reduced pressure, the crude product was purified by column chromatography (petroleum ether/DCM in a 3:1 ratio), yielding (4,4'dibromo-2'octyloxybiphenyl-2-yl)-diphenylmethanol as a white solid (3.32 g, $5.33 \mathrm{mmol}, 72 \%)$.

${ }^{1} \mathrm{H}-\mathrm{NMR}\left(400 \mathrm{MHz}, \mathrm{CDCl}_{3}, 25^{\circ} \mathrm{C}\right): \delta[p p m]=7.42\left(\mathrm{dd},{ }^{3} \mathrm{~J}_{\mathrm{HH}}=8.1 \mathrm{~Hz},{ }^{4} \mathrm{~J}_{\mathrm{HH}}=2.1 \mathrm{~Hz}, 1 \mathrm{H}, \mathrm{H}-3\right), 7.29-7.24$ (m, 5H, Ph-H), 7.17-7.15 (m, 3H, Ph-H), 7.10-7.08 (m, 2H, Ph-H), $7.06\left(\mathrm{~d},{ }^{4} J_{\mathrm{HH}}=2.1 \mathrm{~Hz}, 1 \mathrm{H}, \mathrm{H}-1\right), 6.91$ $\left(\mathrm{d},{ }^{4} \mathrm{~J}_{\mathrm{HH}}=1.8 \mathrm{~Hz}, 1 \mathrm{H}, \mathrm{H}-6\right), 6.87\left(\mathrm{~d},{ }^{3} \mathrm{~J}_{\mathrm{HH}}=8.1 \mathrm{~Hz}, 1 \mathrm{H}, \mathrm{H}-4\right), 6.66\left(\mathrm{dd},{ }^{3} \mathrm{~J}_{\mathrm{HH}}=8.1 \mathrm{~Hz},{ }^{4} J_{\mathrm{HH}}=1.8 \mathrm{~Hz}, 1 \mathrm{H}\right.$, $\mathrm{H}-8), 6.14\left(\mathrm{~d},{ }^{3} \mathrm{~J}_{\mathrm{HH}}=8.1 \mathrm{~Hz}, 1 \mathrm{H}, \mathrm{H}-13\right), 3.98(\mathrm{~s}, 1 \mathrm{H}, \mathrm{H}-22), 3.94-3.81(\mathrm{~m}, 2 \mathrm{H}, \mathrm{H}-14), 1.68-1.61(\mathrm{~m}, 2 \mathrm{H}$, $\mathrm{H}-15), 1.32-1.25(\mathrm{~m}, 10 \mathrm{H}, \mathrm{H}-16, \mathrm{H}-17, \mathrm{H}-18, \mathrm{H}-19, \mathrm{H}-20), 0.89\left(\mathrm{t},{ }^{3} \mathrm{JHH}_{\mathrm{HH}}=7.0 \mathrm{~Hz}, 3 \mathrm{H}, \mathrm{H}-21\right)$.

${ }^{13} \mathrm{C}-\mathrm{NMR}\left(101 \mathrm{MHz} \mathrm{CDCl}_{3}, \mathbf{2 5}^{\circ} \mathrm{C}\right.$ ): $\delta$ [ppm] = $155.9(\mathrm{C}-5), 148.4(\mathrm{C}-10), 146.5(\mathrm{Ph}), 146.1(\mathrm{Ph}), 136.1$ (C-11), 134.4 (C-4), 133.0 (C-1), 132.3 (C-13), 130.4 (C-3), 129.8 (C-12), 128.3 (Ph), 128.0 (Ph), 127.7 (Ph), $127.5(\mathrm{Ph}), 127.4(\mathrm{Ph}), 127.0(\mathrm{Ph}), 123.1$ (C-8), 121.9 (C-7), 121.3 (C-2), 115.3 (C-6), $82.2(\mathrm{C}-9)$, 69.2 (C-14), 31.9 (C-19), 29.4 (C-17), 29.3 (C-18), 29.0 (C-15), 26.0 (C-16), 22.8 (C-20), 14.3 (C-21).

\section{SYNTHESIS OF 2,7-DIBROMO-5-OCTYLOXY-9,9'-DIPHENYLFLUORENE $(\mathrm{Br}-\mathrm{ODPF}-\mathrm{Br})^{55}$}

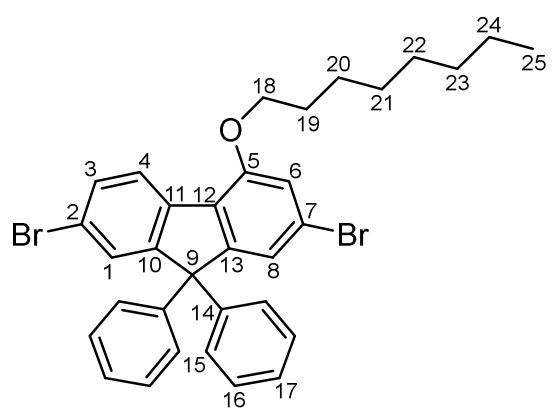

$$
\begin{aligned}
& \mathrm{C}_{33} \mathrm{H}_{32} \mathrm{Br}_{2} \mathrm{O} \\
& M=604.43 \mathrm{~g} / \mathrm{mol}
\end{aligned}
$$


$\mathrm{BF}_{3} \times \mathrm{Et}_{2} \mathrm{O}$ (0.98 mL, $7.72 \mathrm{mmol}, 1.2$ equiv.) was dissolved in dry $\mathrm{DCM}(40 \mathrm{~mL})$ and added dropwise to a solution of (4,4'dibromo-2'-(octyloxy)biphenyl-2-yl)-diphenylmethanol (4.00 g, $6.43 \mathrm{mmol}, 1$ equiv.) in DCM $(40 \mathrm{~mL})$. After stirring for $24 \mathrm{~h}$ at room temperature, the reaction was quenched by addition of water and ethanol. The phases were separated and the aqueous layer was extracted three times with DCM. The combined organic phases were washed with water, dried over $\mathrm{MgSO}_{4}$ and the solvent was removed in vacuo. Column chromatography (petroleum ether/DCM in a 3:1 ratio) of the crude product yielded 2,7-dibromo-5-octyloxy-9,9'-diphenylfluorene (Br-ODPF-Br) as a white solid (3.62 g, $5.99 \mathrm{mmol}$, $93 \%)$.

${ }^{1} \mathrm{H}-\mathrm{NMR}\left(400 \mathrm{MHz} \mathrm{CDCl}_{3}, \mathbf{2 5}^{\circ} \mathrm{C}\right): \delta[\mathrm{ppm}]=7.98\left(\mathrm{dd},{ }^{3} \mathrm{~J}_{\mathrm{HH}}=5.4 \mathrm{~Hz}, 1 \mathrm{H}, \mathrm{H}-4\right), 7.50-7.47(\mathrm{~m}, 2 \mathrm{H}, \mathrm{H}-1$, $\mathrm{H}-3), 7.29-7.25(\mathrm{~m}, 6 \mathrm{H}, \mathrm{H}-16, \mathrm{H}-17), 7.19-7.16(\mathrm{~m}, 4 \mathrm{H}, \mathrm{H}-15), 7.12\left(\mathrm{~d},{ }^{4} \mathrm{~J}_{\mathrm{HH}}=1.5 \mathrm{~Hz}, 1 \mathrm{H}, \mathrm{H}-8\right), 7.01(\mathrm{~d}$, $\left.{ }^{4} J_{\mathrm{HH}}=1.5 \mathrm{~Hz}, 1 \mathrm{H}, \mathrm{H}-6\right), 4.14\left(\mathrm{t},{ }^{3} \mathrm{~J}_{\mathrm{HH}}=6.5 \mathrm{~Hz}, 2 \mathrm{H}, \mathrm{H}-18\right), 1.97$ (quint, $\left.{ }^{3} \mathrm{~J}_{\mathrm{HH}}=6.6 \mathrm{~Hz}, 2 \mathrm{H}, \mathrm{H}-19\right), 1.63-1.55$ $(\mathrm{m}, 2 \mathrm{H}, \mathrm{H}-20), 1.48-1.33(\mathrm{~m}, 8 \mathrm{H}, \mathrm{H}-21, \mathrm{H}-22, \mathrm{H}-23, \mathrm{H}-24), 0.93\left(\mathrm{t}, 3^{3} J_{\mathrm{HH}}=6.9 \mathrm{~Hz}, 3 \mathrm{H}, \mathrm{H}-25\right)$.

${ }^{13} \mathrm{C}-N M R\left(101 \mathrm{MHz} \mathbf{C D C l}_{3}, 25{ }^{\circ} \mathrm{C}\right): \delta[\mathrm{ppm}]=155.7$ (C-5), 154.2 (C-13), $152.6(\mathrm{C}-10), 144.7$ (C-14), 137.8 (C-11), 131.0 (C-3), 128.9 (C-1), 128.6 (C-15), 128.2 (C-16), 127.2 (C-17), 126.4 (C-12), 125.4 (C-4), 122.2 (C-7), 121.5 (C-8), 121.0 (C-2), 114.0 (C-6), 68.8 (C-18), 66.1 (C-9), 31.9 (C-23), 29.5 (C-19), 29.4 (C-21, C-22), 26.3 (C-20), 22.8 (C-24), 14.3 (C-25).

\section{SYNTHESIS OF 2,2' -(5-OCTYLOXY-9,9' -DIPHENYLFLUORENE-2,7-DIYL)BIS(1,3,2- DIOXABORINANE) (PrO $\left.{ }_{2} \mathrm{~B}-\mathrm{ODPF}-\mathrm{BO}_{2} \mathrm{Pr}\right)^{204}$}

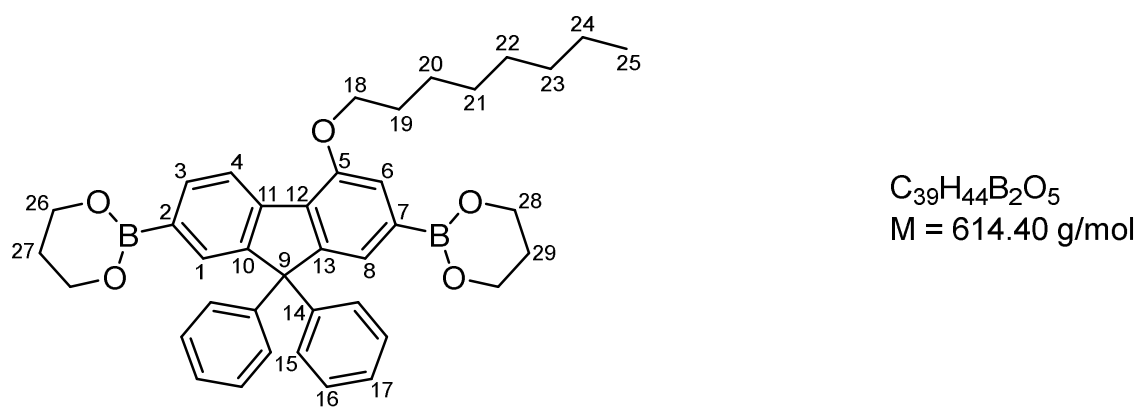

A solution of 2,7-dibromo-5-octyloxy-9,9'-diphenylfluorene (1.00 g, $1.65 \mathrm{mmol}, 1$ equiv.) in anhydrous THF was cooled to $-78{ }^{\circ} \mathrm{C}$ and a $1.6 \mathrm{M} \mathrm{n}$-butyllithium solution in hexane (3.54 mL, $5.66 \mathrm{mmol}$. 3.4 equiv.) was added dropwise. After stirring for $2 \mathrm{~h}$ at constant cooling, tri-iso-propyl borate $(1.49 \mathrm{~mL}, 6.45 \mathrm{mmol}$, 3.9 equiv.) was added. The reaction mixture was brought to room temperature, stirred overnight and poured into $2 \mathrm{M} \mathrm{HCl}$ containing crushed ice. After threefold extraction with diethyl ether, the combined organic phases were washed with water and brine, dried over $\mathrm{MgSO}_{4}$ and the solvent was removed under reduced pressure. The residue was dissolved in toluene $(10 \mathrm{~mL})$ and 1,3-propanediol $(0.36 \mathrm{~mL}$, 5.02 mmol, 3 equiv.) was added. The mixture was refluxed overnight and diluted with diethyl ether. The organic phase was washed with water and brine, dried over $\mathrm{MgSO}_{4}$ and the solvent was removed in vacuo. Recrystallization of the crude product from ethanol yielded 2,2'-(5-octyloxy-9,9'diphenylfluorene-2,7-diyl)bis(1,3,2-dioxaborinane) $\left(\mathbf{P r O}_{2} \mathbf{B}-\mathbf{O D P F}-\mathbf{B O}_{2} \mathbf{P r}\right)$ as a white solid (0.78 $\mathrm{g}$, $1.25 \mathrm{mmol}, 75 \%)$. 
${ }^{1} \mathrm{H}-\mathrm{NMR}\left(400 \mathrm{MHz}, \mathrm{CDCl}_{3}, 2{ }^{\circ} \mathrm{C}\right): \delta[\mathrm{ppm}]=8.16\left(\mathrm{~d},{ }^{3} \mathrm{JHH}_{\mathrm{HH}}=7.3 \mathrm{~Hz}, 1 \mathrm{H}, \mathrm{H}-4\right), 7.78-7.76(\mathrm{~s}, 2 \mathrm{H}, \mathrm{H}-1$, $\mathrm{H}-3$ ), 7.41 (s, 1H, H-8), 7.25 (s, 1H, H-6), 7.21-7.15 (m, 10H, H-15, H-16, H-17), 4.20 (t, ${ }^{3} J_{\mathrm{HH}}=6.4 \mathrm{~Hz}$, $2 \mathrm{H}, \mathrm{H}-18$ ), $4.11\left(\mathrm{t},{ }^{3} \mathrm{~J}_{\mathrm{HH}}=5.5 \mathrm{~Hz}, 8 \mathrm{H}, \mathrm{H}-26, \mathrm{H}-28\right), 2.04-1.92(\mathrm{~m}, 6 \mathrm{H}, \mathrm{H}-19, \mathrm{H}-27, \mathrm{H}-29), 1.60$ (quint, $\left.{ }^{3} \mathrm{~J}_{\mathrm{HH}} \mathrm{p}=7.5 \mathrm{~Hz}, 2 \mathrm{H}, \mathrm{H}-20\right), 1.45-1.29(\mathrm{~m}, 8 \mathrm{H}, \mathrm{H}-21, \mathrm{H}-22, \mathrm{H}-23, \mathrm{H}-24), 0.91\left(\mathrm{t},{ }^{3} \mathrm{~J}_{\mathrm{HH}}=7.8 \mathrm{~Hz}, 3 \mathrm{H}, \mathrm{H}-25\right)$.

${ }^{13} \mathrm{C}$-NMR (101 MHz, $\left.\mathbf{C D C l}_{3}, 25{ }^{\circ} \mathrm{C}\right): \delta[\mathrm{ppm}]=155.2$ (C-5), 153.0 (C-13), 150.5 (C-10), 146.3 (C-14), 142.0 (C-11), 133.2 (C-3), 130.9 (C-1), 130.6 (C-7), 128.6, (C-15), 128.5 (C-2), 128.2 (C-12), 128.1 (C-16), 126.4 (C-17), 123.8 (C-8), 123.6 (C-4), 114.8 (C-6), 68.2 (C-18), 65.6 (C-9), 62.0 (C-26, C-28), 32.0 (C-23), 29.7 (C-19), 29.6 (C-21), 29.4 (C-22), 27.5 (C-27, C-29), 26.5 (C-20), 22.8 (C-24), 14.27 (C-25).

\section{SYNTHESIS OF 2-(7-BROMO-5-OCTYLOXY-9,9'-DIPHENYLFLUOREN-2-YL)-4,4,5,5- TETRAMETHYL-1,3,2-DIOXABOROLANE (Br-ODPF-BPin) ${ }^{205}$}

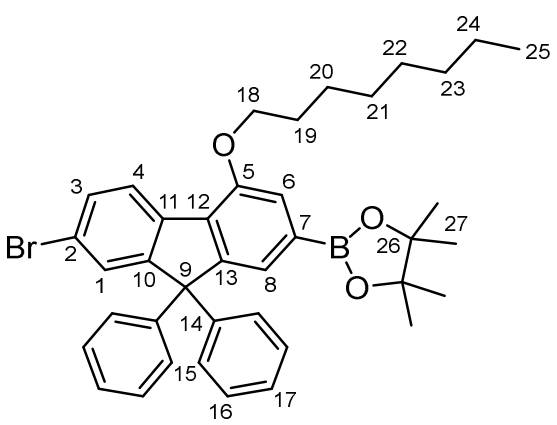

$$
\begin{aligned}
& \mathrm{C}_{39} \mathrm{H}_{44} \mathrm{BBrO}_{3} \\
& \mathrm{M}=651.49 \mathrm{~g} / \mathrm{mol}
\end{aligned}
$$

2,7-Dibromo-5-octyloxy-9,9'-diphenylfluorene (Br-ODPF-Br) (2.00 g, $3.31 \mathrm{mmol}, 1$ equiv.) was dissolved in dry THF $(50 \mathrm{~mL})$ and the solution was cooled to $-78^{\circ} \mathrm{C}$. A $2.5 \mathrm{M}$ solution of $n$-butyllithium in hexane (1.45 mL, $3.64 \mathrm{mmol}, 1.1$ equiv.) was added dropwise and the reaction mixture was stirred for $1 \mathrm{~h}$ under cooling. Iso-propoxylboronic acid pinacol ester $(0.81 \mathrm{~mL}, 3.97 \mathrm{mmol}, 1.2$ equiv.) was added, the mixture was allowed to come to room temperature and was stirred overnight. For facilitation of phase separation, distilled water and dichloromethane were then added. The organic layer was washed consecutively with brine and distilled water, dried over $\mathrm{MgSO}_{4}$ and the solvents were removed under reduced pressure. The crude product was purified by recrystallization from a mixture of methanol and ethanol in a 10/1 ratio, yielding 2-(7-bromo-5-octyloxy-9,9'-diphenylfluoren-2-yl)-4,4,5,5tetramethyl-1,3,2-dioxaborolane (Br-ODPF-BPin) as a white powder (1.45 g, $2.22 \mathrm{mmol}, 67 \%$ ).

Please note, that the abovementioned lithiation did not proceed in a fully regioselective manner and, consequently, two different products were formed. However, as the major proportion (approximately $75 \%$ ) of the product mixture was constituted by the isomer depicted above, the following NMR characterization refers exclusively to this compound.

${ }^{1} \mathrm{H}-\mathrm{NMR}\left(400 \mathrm{MHz} \mathrm{CDCl}_{3}, 25^{\circ} \mathrm{C}\right): \delta[\mathrm{ppm}]=8.07\left(\mathrm{~d},{ }^{3} \mathrm{JHH}_{\mathrm{HH}}=8.0 \mathrm{~Hz}, 1 \mathrm{H}, \mathrm{H}-4\right), 7.49-7.46(\mathrm{~m}, 2 \mathrm{H}, \mathrm{H}-1$, $\mathrm{H}-3), 7.43(\mathrm{~s}, 1 \mathrm{H}, \mathrm{H}-8), 7.29(\mathrm{~s}, 1 \mathrm{H}, \mathrm{H}-6), 7.25-7.17(\mathrm{~m}, 10 \mathrm{H}, \mathrm{H}-15, \mathrm{H}-16, \mathrm{H}-17), 4.22\left(\mathrm{t},{ }^{3} \mathrm{JHH}_{\mathrm{HH}}=6.4 \mathrm{~Hz}\right.$, $2 \mathrm{H}, \mathrm{H}-18), 2.00-1.93(\mathrm{~m}, 2 \mathrm{H}, \mathrm{H}-19), 1.65-1.55(\mathrm{~m}, 2 \mathrm{H}, \mathrm{H}-20), 1.48-1.32(\mathrm{~m}, 2 \mathrm{H}, \mathrm{H}-21, \mathrm{H}-22, \mathrm{H}-23$, $\mathrm{H}-24, \mathrm{H}-27), 0.92\left(\mathrm{t},{ }^{3} \mathrm{~J}_{\mathrm{HH}}=6.5 \mathrm{~Hz}, 3 \mathrm{H}, \mathrm{H}-25\right)$. 
${ }^{13} \mathrm{C}-\mathrm{NMR}\left(101 \mathrm{MHz}, \mathrm{CDCl}_{3}, 25{ }^{\circ} \mathrm{C}\right): \delta[\mathrm{ppm}]=155.0(\mathrm{C}-5), 153.6(\mathrm{C}-10), 152.3(\mathrm{C}-13), 145.3(\mathrm{C}-14)$, 138.5 (C-11), 130.8 (C-3), 130.1 (C-12), 129.0 (C-1), 128.5 (C-15), 128.4 (C-16), 126.9 (C-17), 126.0 (C-4), 124.6 (C-8), 121.1 (C-2), 116.1 (C-6), 83.9 (C-26), 68.4 (C-18), 65.8 (C-9), 32.0 (C-23), 29.6 (C-19), 29.5 (C-22), 29.4 (C-21), 26.4 (C-20), 25.0 (C-27), 22.8 (C-24), 14.3 (C-25), C-7 not found.

\subsubsection{POLYDIARYLFLUORENE BY EMULSION POLYMERIZATION}

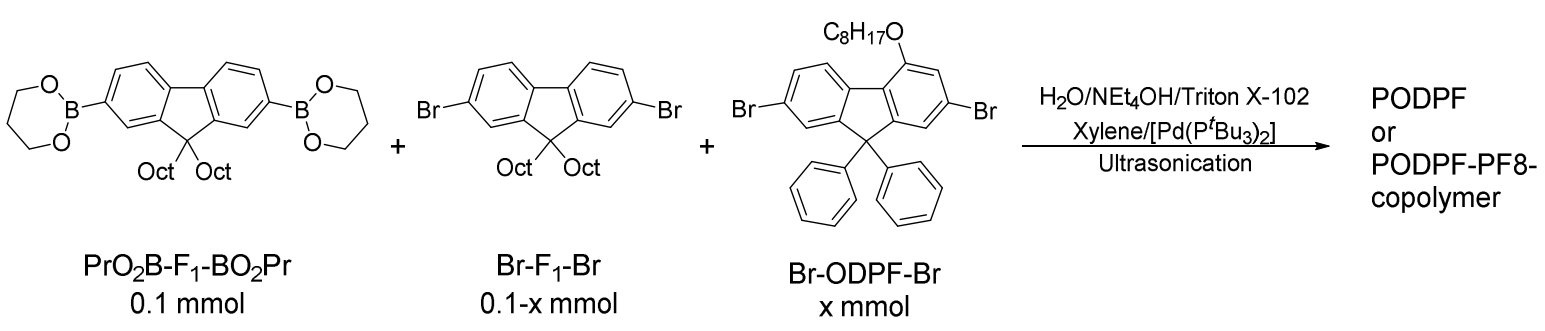

Suzuki-Miyaura polycondensation under heterophase conditions was performed according to the procedure published by Ruiz Perez et al. ${ }^{8}$ In a $100 \mathrm{~mL}$ Schlenk tube, Triton X-102 (1.25 g, $1.75 \mathrm{mmol}$, 17.5 equiv.) was dissolved in deionized water $(25 \mathrm{~mL})$ and $\mathrm{NEt}_{4} \mathrm{OH}$ solution ( $20 \%$ in water) $(0.16 \mathrm{~g}$, $0.2 \mathrm{mmol}, 2$ equiv.) was added. The solution was degassed by sparging with $\mathrm{N}_{2}$ for at least $30 \mathrm{~min}$. For the synthesis of PODPF as homopolymer, $\mathrm{PrO}_{2} \mathrm{~B}-\mathrm{ODPF}-\mathrm{BO}_{2} \operatorname{Pr}(61.4 \mathrm{mg}, 0.1 \mathrm{mmol}, 1$ equiv.) and Br-ODPF-Br (60.4 mg, $0.1 \mathrm{mmol}, 1$ equiv.) were dissolved in xylene (1 mL). For the synthesis of PF8PODPF copolymers, monomer mixtures were prepared according to the compositions indicated in Table 5.1. The respective monomer solution was degassed by three freeze-pump-thaw cycles and added to bis(tri-tert-butylphosphine) palladium(0) $(2.1 \mathrm{mg}, 4 \mu \mathrm{mol}, 0.04$ equiv.), followed by three further freeze-pump-thaw cycles. The monomer/catalyst solution was rapidly injected via syringe into the aqueous phase with simultaneous emulsification by an ultrasonotrode ( $2.5 \mathrm{~min}, 60 \%$ amplitude). After ultrasonication, the emulsion was stirred for $72 \mathrm{~h}$ at room temperature to yield a greenish-yellow polymer dispersion.

As detailed in Table 5.2, the above-mentioned procedure was optimized concerning particle shape and uniformity. To this end, only AA/BB-type diarylfluorene monomers were used $\left(\mathrm{PrO}_{2} \mathrm{~B}-\mathrm{ODPF}-\mathrm{BO}_{2} \mathrm{Pr}\right.$ and $\mathrm{Br}-\mathrm{ODPF}-\mathrm{Br}$ ) in equimolar amounts.

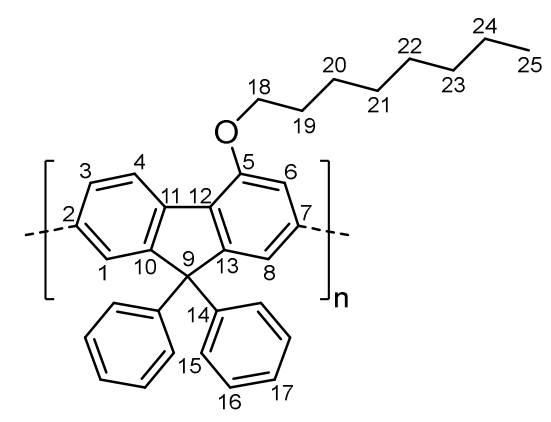


${ }^{1} \mathrm{H}-\mathrm{NMR}\left(400 \mathrm{MHz}, \mathrm{CDCl}_{3}, \mathbf{2 5}^{\circ} \mathrm{C}\right): \delta[\mathrm{ppm}]=8.16-8.12(\mathrm{~m}, 1 \mathrm{H}, \mathrm{H}-4), 7.56-7.47(\mathrm{~m}, 2 \mathrm{H}, \mathrm{H}-1, \mathrm{H}-3), 7.25-$ 7.17 (m, 10H, H-15, H-16, H-17), 7.16-7.07 (m, 1H, H-8), 7.00-6.94 (m, 1H, H-6), 4.19-4.15 (m, 2H, $\mathrm{H}-18), 1.99-1.94(\mathrm{~m}, 2 \mathrm{H}, \mathrm{H}-19), 1.63-1.57$ (m, 2H, H-20), 1.46-1.29 (m, 8H, H-21, H-22, H-23, H-24), $0.89\left(\mathrm{t},{ }^{3} \mathrm{JHH}_{\mathrm{HH}}=6.5 \mathrm{~Hz}, 1 \mathrm{H}, \mathrm{H}-25\right)$.

${ }^{13} \mathrm{C}$-NMR (101 MHz, $\left.\mathbf{C D C l}_{3}, 25{ }^{\circ} \mathrm{C}\right): \delta[\mathrm{ppm}]=155.5$ (C-5), 153.5 (C-13), 151.4 (C-10), 146.1 (C-14), 142.6 (C-7), 140.0 (C-2), 138.4 (C-11), 128.4 (br, C-15, C-16), 127.3 (C-12), 126.9 (C-1), 126.7 (C-17), 124.3 (C-3), 124.2 (C-4), 117.4 (C-8), 109.6 (C-6), 68.3 (C-18), 66.0 (C-9), 32.0 (C-23), 29.6 (C-19), 29.5 (C-21), 29.4 (C-22), 26.4 (C-20), 22.9 (C-24), 14.3 (C-25).

\subsubsection{POLYDIARYLFLUORENE BY CONTROLLED SUZUKI-MIYAURA COUPLING POLYMERIZATION}

The controlled polymerization of AB-type diarylfluorene monomer was carried out according to a literature-known procedure established for the synthesis of PF8. ${ }^{72}$ Chloro[(tri-tert-butylphosphine)-2-(2aminobiphenyl)] palladium(II) (12 mg, $23 \mu \mathrm{mol}, 1$ equiv.) and 4-bromotoluene ( $8 \mathrm{mg}, 46 \mu \mathrm{mol}, 2$ equiv.) were dissolved in THF $(1 \mathrm{~mL}) .2 \mathrm{M}$ aqueous $\mathrm{K}_{2} \mathrm{CO}_{3}$ solution was added $(1 \mathrm{~mL})$ and the resulting initiator mixture was vigorously stirred at room temperature for $40 \mathrm{~min}$. Br-ODPF-BPin $(0.15 \mathrm{~g}, 0.23 \mathrm{mmol}$, 10 equiv.) was dissolved in THF ( $5 \mathrm{~mL})$ and the solution was cooled to $0{ }^{\circ} \mathrm{C}$. The initiator solution was rapidly added and the mixture was stirred under continuous cooling for $30 \mathrm{~min}$, before the polymerization was terminated by the addition of $2 \mathrm{M}$ aqueous $\mathrm{HCl}$ solution $(1 \mathrm{~mL})$. After stirring overnight at room temperature, the phases were separated, the aqueous phase was extracted with dichloromethane and the combined organic layers were washed with water and brine. The solution was dried over $\mathrm{MgSO}_{4}$ and the solvent was removed under reduced pressure. The crude product was purified by dissolution in hot toluene and precipitation in methanol, followed by centrifugation. The supernatant solution was discarded and the precipitate was dried under reduced pressure to yield PODPF as green-yellow solid (63 mg, $0.14 \mathrm{mmol}, 62 \%$ ).

\subsubsection{POST-POLYMERIZATION SYNTHESIS OF POLYDIARYLFLUORENE PARTICLES}

The following procedure represents the optimized conditions found for the post-polymerization preparation of anisotropic PODPF nanoparticles, corresponding to Table 5.3, entry 5.

Prior to use in post-polymerization particle preparation, PODPF synthesized by heterophase SuzukiMiyaura polycondensation was purified. The polymer was precipitated by addition of methanol to the polymer dispersion, followed by centrifugation (6000 rpm, $10 \mathrm{~min}$ ), and decantation of the supernatant 
solution. The residual solid was dissolved in hot toluene and the precipitation procedure was repeated twice. The resulting polymer was dried under reduced pressure.

A solution of PODPF ( $3 \mathrm{mg}$ ) in toluene $(1 \mathrm{~mL})$ was filtered via a $0.45 \mu \mathrm{m}$ PTFE syringe filter. The solution was added to an aqueous solution $(15 \mathrm{~mL})$ of Triton X-102 (5 wt\%) in a $50 \mathrm{~mL}$ round bottom flask with ultrasonication starting simultaneously ( $1 \mathrm{~min}, 60 \%$ amplitude). The resulting dispersion was stirred at $300 \mathrm{rpm}$ for at least $24 \mathrm{~h}$. 


\subsection{AMPHIPHILIC BLOCK COPOLYMERS}

\subsubsection{SYNTHESES OF MACROINITIATORS}

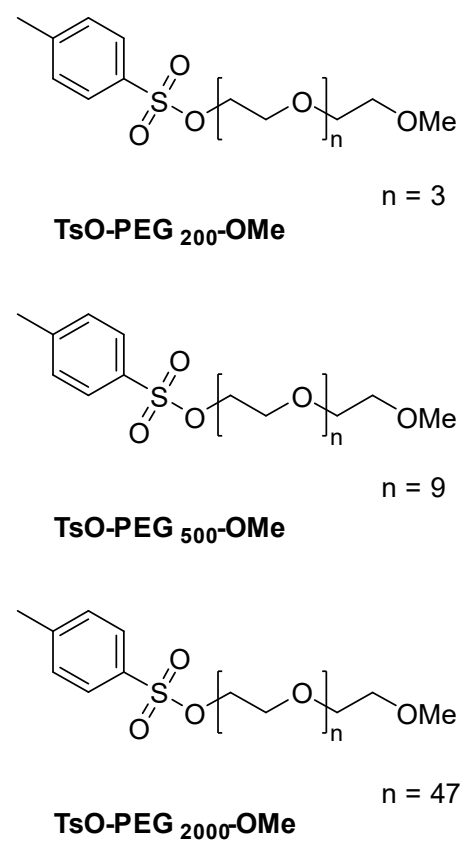<smiles>COCCCOc1ccc2c(c1)C(Cl)(Cl)c1cc(Br)ccc1-2</smiles>

$\mathrm{Br}-\mathrm{F}_{1}-\mathrm{PEG}_{200} \mathrm{OMe}$<smiles>COCCCOc1ccc2c(c1)C(Cl)(Cl)c1cc(Br)ccc1-2</smiles>

$\mathrm{Br}-\mathrm{F}_{1}-\mathrm{PEG}_{500}-\mathrm{OMe}$<smiles>COCCC(C)OCCOc1ccc2c(c1)C(Cl)(OCl)c1cc(Br)ccc1-2</smiles>

$\mathrm{Br}-\mathrm{F}_{1}-\mathrm{PEG}_{2000}-\mathrm{OMe}$

Figure 8.2: Functionalized poly(ethylene glycols) and macroinitiators applied in Chapter 6 with the respective abbreviations used in the following and the average number of poly(ethylene glycol) repeat units calculated from ${ }^{1} \mathrm{H}-\mathrm{NMR}$ spectra.

\section{SYNTHESIS OF 7-BROMO-9,9'-DIOCTYLFLUORENE-2-BORONIC ACID218}

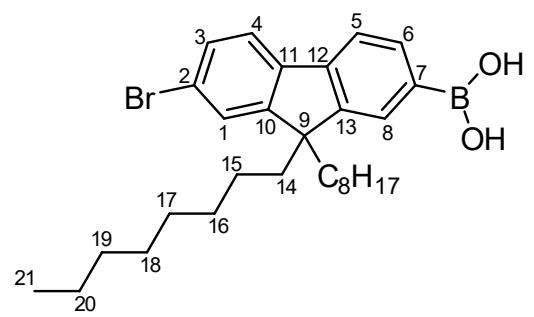

$$
\begin{aligned}
& \mathrm{C}_{29} \mathrm{H}_{42} \mathrm{BBrO}_{2} \\
& \mathrm{M}=513.37 \mathrm{~g} / \mathrm{mol}
\end{aligned}
$$

A solution of 2,7-dibromo-9,9'-dioctylfluorene $\left(\mathbf{B r}^{-} \mathbf{F}_{\mathbf{1}}-\mathbf{B r}\right)(10.0 \mathrm{~g}, 18.3 \mathrm{mmol}, 1$ equiv.) in dry THF $(120 \mathrm{~mL})$ was cooled to $-78{ }^{\circ} \mathrm{C}$ and a $1.6 \mathrm{M}$ solution of $n$-butyllithium in hexane $(12.5 \mathrm{~mL}, 20.1 \mathrm{mmol}$, 1.1 equiv.) was slowly added. After stirring for $1 \mathrm{~h}$ at constant temperature, tri-iso-propyl borate $(4.7 \mathrm{~mL}$, $20.1 \mathrm{mmol}, 1.1$ equiv.) was added and the reaction temperature was kept at $-78{ }^{\circ} \mathrm{C}$ for another hour before the mixture was allowed to warm to room temperature and stirred overnight. Water $(25 \mathrm{~mL})$ and $1 \mathrm{M} \mathrm{HCl}(25 \mathrm{~mL})$ were added and the phases were separated. The organic layer was washed with water, dried over $\mathrm{MgSO}_{4}$ and the solvent was removed in vacuo. The crude product was purified by column chromatography (gradient from petroleum ether to ethyl acetate) to yield 7-bromo-9,9'-dioctylfluorene2-boronic acid as a white solid (6.5 g, $12.6 \mathrm{mmol}, 69 \%)$. 
${ }^{1} \mathrm{H}-\mathrm{NMR}\left(\mathbf{4 0 0} \mathrm{MHz}, \mathrm{CDCl}_{3}, \mathbf{2 5}^{\circ} \mathrm{C}\right): \delta[\mathrm{ppm}]=8.30\left(\mathrm{~d},{ }^{3} \mathrm{JHH}_{\mathrm{HH}}=7.5 \mathrm{~Hz}, 1 \mathrm{H}, \mathrm{H}-6\right), 8.19(\mathrm{~s}, 1 \mathrm{H}, \mathrm{H}-8), 7.86$ $\left(\mathrm{d},{ }^{3} \mathrm{~J}_{\mathrm{HH}}=7.6 \mathrm{~Hz}, 1 \mathrm{H}, \mathrm{H}-5\right), 7.68\left(\mathrm{~d},{ }^{3} \mathrm{~J}_{\mathrm{HH}}=8.0 \mathrm{~Hz}, 1 \mathrm{H}, \mathrm{H}-4\right), 7.54\left(\mathrm{~d},{ }^{4} \mathrm{~J}_{\mathrm{HH}}=1.5 \mathrm{~Hz}, 1 \mathrm{H}, \mathrm{H}-1\right), 7.52(\mathrm{dd}$, $\left.{ }^{3} \mathrm{JHH}_{\mathrm{HH}}=7.9 \mathrm{~Hz},{ }^{4} \mathrm{~J}_{\mathrm{HH}}=1.7 \mathrm{~Hz}, 1 \mathrm{H}, \mathrm{H}-3\right), 2.17-1.99(\mathrm{~m}, 4 \mathrm{H}, \mathrm{H}-14), 1.23-1.04(\mathrm{~m}, 2 \mathrm{H}, \mathrm{H}-16, \mathrm{H}-17, \mathrm{H}-18$, $\mathrm{H}-19, \mathrm{H}-20), 0.79\left(\mathrm{t},{ }^{3} \mathrm{JHH}_{\mathrm{HH}}=7.1 \mathrm{~Hz}, 6 \mathrm{H}, \mathrm{H}-21\right), 0.71-0.63(\mathrm{~m}, 4 \mathrm{H}, \mathrm{H}-15)$.

${ }^{13} \mathrm{C}-\mathrm{NMR}\left(101 \mathrm{MHz}, \mathrm{CDCl}_{3}, 25^{\circ} \mathrm{C}\right.$ ): $\delta$ [ppm] = 154.0 (C-10), 149.9 (C-13), 144.7 (C-12), 139.9 (C-11), 134.9 (C-6), 130.3 (C-3), 129.8 (C-8), 129.5 (br, C-7), 126.5 (C-1), 122.2 (C-2), 121.9 (C-4), 119.5 (C-5), 55.6 (C-9), 40.4 (C-14), 31.9 (C-19), 30.1 (C-16), 29.4 (C-17), 29.3 (C-18), 23.9 (C-15), 22.7 (C-20), $14.2(\mathrm{C}-21)$.

\title{
SYNTHESIS OF 7-BROMO-9,9'-DIOCTYLFLUORENE-2-OL227
}

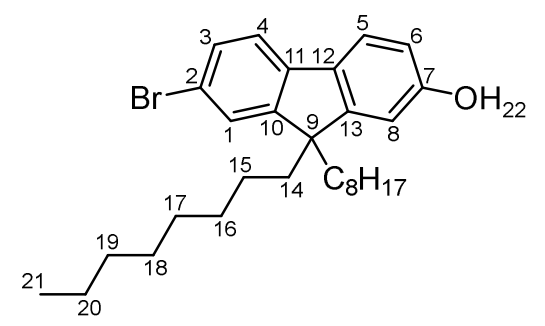

\author{
$\mathrm{C}_{29} \mathrm{H}_{41} \mathrm{BrO}$ \\ $\mathrm{M}=485.55 \mathrm{~g} / \mathrm{mol}$
}

7-Bromo-9,9'-dioctylfluorene-2-boronic acid (6.5 g, $12.5 \mathrm{mmol}, 1$ equiv.) was dissolved in a mixture of dimethyl carbonate $(70 \mathrm{~mL})$ and diethyl ether $(30 \mathrm{~mL})$ and $30 \%$ aqueous $\mathrm{H}_{2} \mathrm{O}_{2}$ solution $(10.8 \mathrm{~mL}$, $106.3 \mathrm{mmol}, 8.5$ equiv.) was added. The reaction was stirred overnight at room temperature, monitoring the progress of conversion via TLC. The reaction mixture was diluted with deionized water $(30 \mathrm{~mL})$ and ethyl acetate $(30 \mathrm{~mL})$ to facilitate phase separation. The organic layer was washed with deionized water and brine, dried over $\mathrm{MgSO}_{4}$ and the solvent was removed under reduced pressure, yielding 7-bromo9,9'-dioctylfluorene-2-ol as a white solid $(4.82 \mathrm{~g}, 11.8 \mathrm{mmol}, 94 \%)$.

${ }^{1} \mathrm{H}-\mathrm{NMR}\left(400 \mathrm{MHz}, \mathrm{CDCl}_{3}, \mathbf{2 5}^{\circ} \mathrm{C}\right): \delta[\mathrm{ppm}]=7.51\left(\mathrm{dd},{ }^{3} \mathrm{JHH}_{\mathrm{HH}}=6.7,{ }^{4} \mathrm{JHH}=1.8 \mathrm{~Hz}, 1 \mathrm{H}, \mathrm{H}-6\right), 7.45-7.39(\mathrm{~m}$, $3 \mathrm{H}, \mathrm{H}-1, \mathrm{H}-3, \mathrm{H}-4), 6.80-6.78$ (m, 2H, H-5, H-8), 4.99 (s, 1H, H-22), 1.91-1.87 (m, 4H, H-14), 1.25-1.05 $(\mathrm{m}, 20 \mathrm{H}, \mathrm{H}-16, \mathrm{H}-17, \mathrm{H}-18, \mathrm{H}-19, \mathrm{H}-20), 0.83\left(\mathrm{t},{ }^{3} \mathrm{JHH}=7.0 \mathrm{~Hz}, 6 \mathrm{H}, \mathrm{H}-21\right), 0.63-0.58(\mathrm{~m}, 4 \mathrm{H}, \mathrm{H}-15)$.

${ }^{13} \mathrm{C}-\mathrm{NMR}\left(101 \mathrm{MHz}, \mathrm{CDCl}_{3}, 25{ }^{\circ} \mathrm{C}\right): \delta[\mathrm{ppm}]=155.8$ (C-7), 152.9 (C-13), 152.6 (C-10), 140.2 (C-11), 133.4 (C-12), 130.0 (C-4), 126.1 (C-3), 120.9 (C-6), 120.3 (C-1), 119.9 (C-2), 114.3 (C-5), 110.3 (C-8), 55.5 (C-9), 40.6 (C-14), 31.9 (C-19), 30.1 (C-16), 29.4 (C-17, C-18), 23.8 (C-15), 22.7 (C-20), 14.2 $(\mathrm{C}-21)$. 


\section{SYNTHESIS OF TOSYLATED POLY(ETHYLENE GLYCOL) MONOMETHYLETHERS153}

Please note that for the following reaction products containing poly(ethylene glycol) chains, the average number of ethylene glycol repeat units and the corresponding molecular weight were determined from ${ }^{1} \mathrm{H}-N M R$ spectra. All quantitative calculations for starting materials as well as for the resulting products are based on these average values. In consequence of the work-up procedure during synthesis, the average number of PEG repeat units for the products can be altered in comparison to the starting material and are not necessarily identical. The tosylated low molecular weight PEG TsO-PEG ${ }_{200}-\mathrm{OMe}$ was already available in the research group.

\section{TsO-PEG ${ }_{500}-\mathrm{OMe}$}

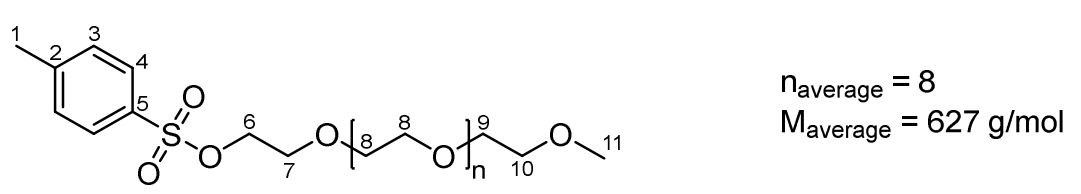

Poly(ethylene glycol) monomethyl ether $500(2.0 \mathrm{~g}, 4.0 \mathrm{mmol}, 1$ equiv.) was dissolved in a solution of sodium hydroxide ( $4.8 \mathrm{~g}, 120.0 \mathrm{mmol}, 30$ equiv.) in deionized water $(40 \mathrm{~mL})$ and the reaction mixture was cooled to $0{ }^{\circ} \mathrm{C}$. $p$-Toluenesulfonyl chloride $(7.6 \mathrm{~g}, 40.0 \mathrm{mmol}, 10$ equiv.) dissolved in THF (40 mL) was added dropwise, followed by $3 \mathrm{~h}$ of stirring at $0{ }^{\circ} \mathrm{C}$. The reaction was allowed to come to room temperature and stirred overnight. The phases were separated and the aqueous phase was extracted with chloroform. The combined organic layers were washed with brine, dried over $\mathrm{MgSO}_{4}$ and the solvent was removed under reduced pressure to yield TsO-PEG ${ }_{500}-\mathbf{O M e}$ as a colorless oil $(2.5 \mathrm{~g}$, $4.0 \mathrm{mmol}$, quantitative yield).

${ }^{1} \mathrm{H}-\mathrm{NMR}\left(800 \mathrm{MHz}, \mathrm{CDCl}_{3} ; 25^{\circ} \mathrm{C}\right): \delta[\mathrm{ppm}]=7.72\left(\mathrm{~d},{ }^{3} \mathrm{JHH}_{\mathrm{HH}}=8.3 \mathrm{~Hz}, 2 \mathrm{H}, \mathrm{H}-4\right), 7.28\left(\mathrm{~d},{ }^{3} \mathrm{JHH}_{\mathrm{HH}}=8.3 \mathrm{~Hz}\right.$, $2 \mathrm{H}, \mathrm{H}-3), 4.08\left(\mathrm{t},{ }^{3} \mathrm{JHH}_{\mathrm{HH}}=4.8 \mathrm{~Hz}, 2 \mathrm{H}, \mathrm{H}-6\right), 3.61\left(\mathrm{t},{ }^{3} \mathrm{~J}_{\mathrm{HH}}=4.8 \mathrm{~Hz}, 2 \mathrm{H}, \mathrm{H}-7\right), 3.59-3.51(\mathrm{~m}, 34 \mathrm{H}, \mathrm{H}-8, \mathrm{H}-9)$, $3.47\left(\mathrm{t},{ }^{3} \mathrm{~J}_{\mathrm{HH}}=4.7 \mathrm{~Hz}, 2 \mathrm{H}, \mathrm{H}-10\right), 3.30(\mathrm{~s}, 3 \mathrm{H}, \mathrm{H}-11), 2.38(\mathrm{~s}, 3 \mathrm{H}, \mathrm{H}-1)$.

${ }^{13} \mathrm{C}$-NMR (200 MHz, $\mathrm{CDCl}_{3}, 25{ }^{\circ} \mathrm{C}$ ): $\delta$ [ppm] = 144.7 (C-2), 132.9 (C-5), 129.8 (C-3), 127.9 (C-4), 71.8 (C-10), 70.6 (C-9), 70.5 (br, C-8), 69.2 (C-6), 68.6 (C-7), 58.9 (C-11), 21.6 (C-1).

\section{TsO-PEG $2000-O M e$}

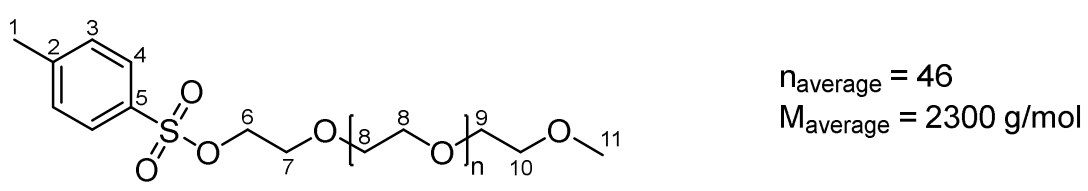

Poly(ethylene glycol) monomethyl ether 2000 (5.0 g, $2.5 \mathrm{mmol}, 1$ equiv.) was dissolved in a solution of sodium hydroxide $(2.0 \mathrm{~g}, 50.0 \mathrm{mmol}, 20$ equiv. $)$ in deionized water $(25 \mathrm{~mL})$ and the reaction mixture was cooled to $0{ }^{\circ} \mathrm{C}$. $p$-Toluenesulfonyl chloride $(9.5 \mathrm{~g}, 50.0 \mathrm{mmol}, 20$ equiv.) dissolved in THF $(25 \mathrm{~mL})$ was added dropwise, followed by $3 \mathrm{~h}$ of stirring at $0{ }^{\circ} \mathrm{C}$. The reaction was allowed to come to room 
temperature and stirred overnight. The phases were separated and the aqueous phase was extracted with dichloromethane. The combined organic layers were washed with brine, dried over $\mathrm{MgSO}_{4}$ and the solvent was removed under reduced pressure to yield TsO-PEG ${ }_{2000}-\mathbf{O M e}$ as a white solid $(4.4 \mathrm{~g}$, $1.9 \mathrm{mmol}, 76 \%)$.

${ }^{1} \mathrm{H}-\mathrm{NMR}\left(800 \mathrm{MHz}, \mathrm{CDCl}_{3} ; \mathbf{2 5}^{\circ} \mathrm{C}\right): \delta[\mathrm{ppm}]=7.74\left(\mathrm{~d},{ }^{3} \mathrm{JHH}_{\mathrm{HH}}=8.0 \mathrm{~Hz}, 2 \mathrm{H}, \mathrm{H}-4\right), 7.30\left(\mathrm{~d},{ }^{3} \mathrm{JHH}_{\mathrm{HH}}=8.0 \mathrm{~Hz}\right.$, $2 \mathrm{H}, \mathrm{H}-3), 4.11\left(\mathrm{t},{ }^{3} \mathrm{~J}_{\mathrm{HH}}=4.7 \mathrm{~Hz}, 2 \mathrm{H}, \mathrm{H}-6\right), 3.74-3.44(\mathrm{~m}, 192 \mathrm{H}, \mathrm{H}-7, \mathrm{H}-8, \mathrm{H}-9, \mathrm{H}-10), 3.33(\mathrm{~s}, 3 \mathrm{H}, \mathrm{H}-11)$, $2.40(\mathrm{~s}, 3 \mathrm{H}, \mathrm{H}-1)$.

${ }^{13} \mathrm{C}$-NMR (200 MHz, $\mathbf{C D C l}_{3}, 25^{\circ} \mathrm{C}$ ): $\delta$ [ppm] = 144.8 (C-2), 133.1 (C-5), 129.8 (C-3), 128.0 (C-4), 72.5, 71.9 (C-10), 70.7 (C-9), 70.6 (br, C-8), 70.4, 69.2 (C-6), 68.7 (C-7), 61.7, 59.0 (C-11), 56.2, 21.6 (C-1).

\section{SYNTHESIS OF Br-F - PEG $_{500}-\mathrm{OMe}$}

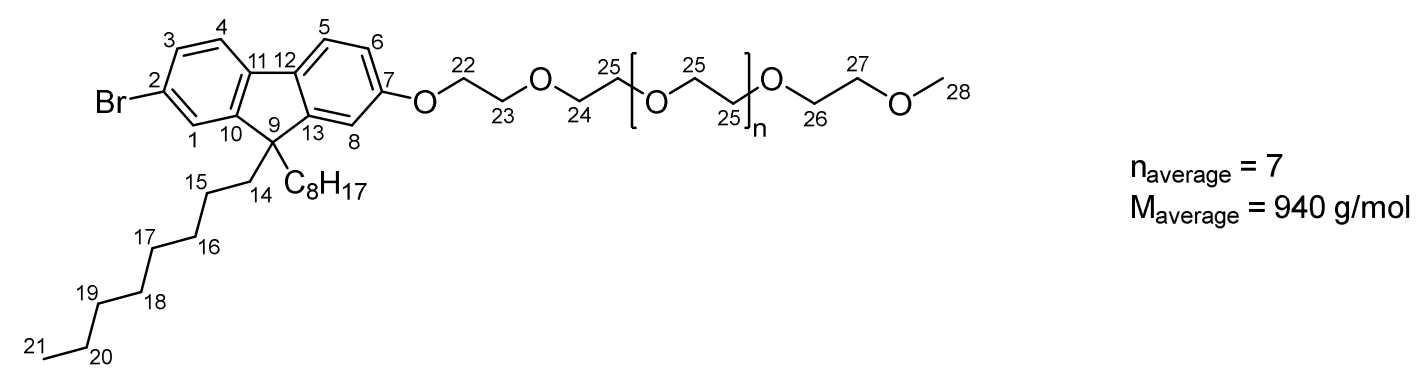

7-Bromo-9,9'-dioctylfluorene-2-ol $(1.00 \mathrm{~g}, 2.06 \mathrm{mmol}, 2$ equiv. $)$ and TsO-PEG $_{500}-\mathbf{O M e}(0.67 \mathrm{~g}$, $1.03 \mathrm{mmol}, 1$ equiv.) were dissolved in dry acetonitrile $(20 \mathrm{~mL})$ and $\mathrm{Na}_{2} \mathrm{CO}_{3}(0.27 \mathrm{~g}, 2.58 \mathrm{mmol}$, 2.5 equiv.) was added. The mixture was stirred at $85^{\circ} \mathrm{C}$ for 7 days, before it was allowed to cool down to room temperature. After filtration, the solvent was removed in vacuo and the crude product was purified by column chromatography using toluene as eluent to remove unreacted 7-bromo-9,9'dioctylfluorene-2-ol. The product was subsequently eluted from the column with a mixture of dichloromethane and methanol in a $10: 1$ ratio to yield $\mathbf{B r}-\mathbf{F}_{1}-\mathbf{P E G}_{500}-\mathbf{O M e}$ as yellow oil $(0.91 \mathrm{~g}$, $0.97 \mathrm{mmol}, 94 \%)$.

${ }^{1} \mathrm{H}-\mathrm{NMR}\left(400 \mathrm{MHz}, \mathrm{CDCl}_{3}, 25{ }^{\circ} \mathrm{C}\right): \delta[\mathrm{ppm}]=7.52\left(\mathrm{~d},{ }^{3} \mathrm{JHH}_{\mathrm{HH}}=8.9 \mathrm{~Hz}, 1 \mathrm{H}, \mathrm{H}-5\right), 7.43-7.41(\mathrm{~m}, 1 \mathrm{H}, \mathrm{H}-4)$, 7.39-7.37 (m, 2H, H-1, H-3), 6.87-6.84 (m, 2H, H-6, H-8), 4.17 (t, $\left.{ }^{3} \mathrm{JHH}_{\mathrm{HH}}=4.7 \mathrm{~Hz}, 2 \mathrm{H}, \mathrm{H}-22\right), 3.88$ (t, $\left.{ }^{3} \mathrm{JHH}_{\mathrm{HH}}=4.7 \mathrm{~Hz}, 2 \mathrm{H}, \mathrm{H}-23\right), 3.75-3.73(\mathrm{~m}, 2 \mathrm{H}, \mathrm{H}-26), 3.70-3.62(\mathrm{~m}, 34 \mathrm{H}, \mathrm{H}-24, \mathrm{H}-25), 3.54-3.52(\mathrm{~m}, 2 \mathrm{H}$, $\mathrm{H}-27$ ), 3.36 (s, 3H, H-28), 1.89-1.84 (m, 4H, H-14), 1.22-1.00 (m, 20H, H-16, H-17, H-18, H-19, H-20), $0.81\left(\mathrm{t},{ }^{3} \mathrm{JHH}=7.1 \mathrm{~Hz}, 6 \mathrm{H}, \mathrm{H}-21\right), 0.62-0.53(\mathrm{~m}, 4 \mathrm{H}, \mathrm{H}-15)$.

${ }^{13} \mathrm{C}-\mathrm{NMR}\left(101 \mathrm{MHz}, \mathrm{CDCl}_{3}, 25{ }^{\circ} \mathrm{C}\right): \delta[\mathrm{ppm}]=159.2(\mathrm{C}-7), 152.6(\mathrm{C}-10), 152.4(\mathrm{C}-13), 140.2(\mathrm{C}-11)$, 133.3 (C-12), 129.9 (C-1), 126.0 (C-3), 120.5 (C-5), 120.3 (C-4), 119.8 (C-2), 113.1 (C-8), 109.7 (C-6), 72.0 (C-27), 71.0 (C-24), 70.7 (br, C-25), 70.6 (C-26), 69.3 (C-23), 67.7 (C-22), 59.1 (C-28), 55.4 (C-9), 40.5 (C-14), 31.8 (C-19), 30.0 (C-16), 29.3 (br, C-17, C-18), 23.7 (C-15), 22.7 (C-20), 14.2 (C-21). 


\section{SYNTHESIS OF Br-F1-PEG $200-O M e$}

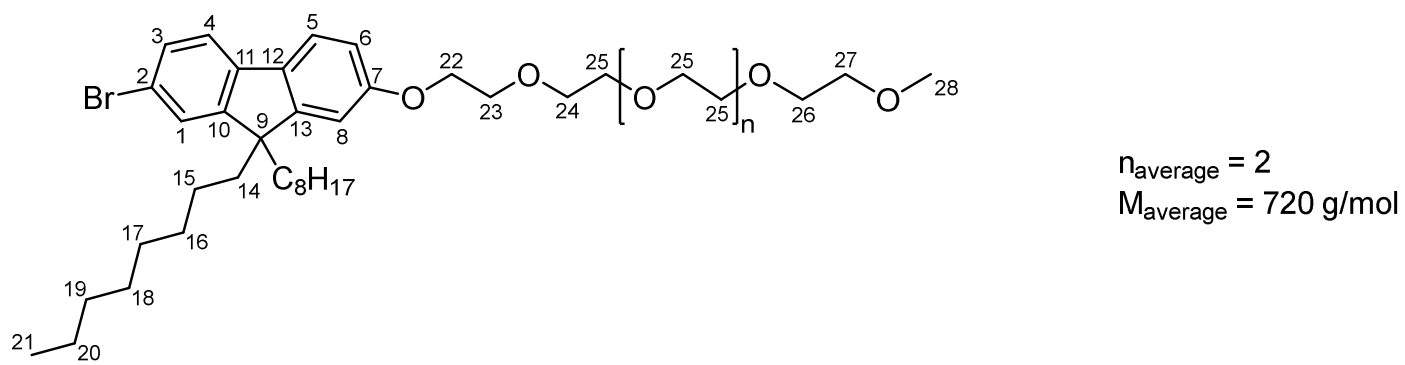

7-Bromo-9,9'-dioctylfluorene-2-ol $(3.35 \mathrm{~g}, 6.89 \mathrm{mmol}, 2$ equiv. $)$ and TsO-PEG ${ }_{200}-\mathbf{O M e}^{\prime}(1.25 \mathrm{~g}$, $3.45 \mathrm{mmol}$, 1 equiv.) were dissolved in dry acetonitrile $(40 \mathrm{~mL})$ and $\mathrm{Na}_{2} \mathrm{CO}_{3}(0.91 \mathrm{~g}, 8.63 \mathrm{mmol}$, 2.5 equiv.) was added. The mixture was stirred at $85^{\circ} \mathrm{C}$ for 7 days, before it was allowed to cool down to room temperature. After filtration, the solvent was removed in vacuo and the crude product was purified by column chromatography using toluene as eluent to remove unreacted 7-bromo-9,9'dioctylfluorene-2-ol. The product was subsequently eluted from the column with a mixture of dichloromethane and methanol in a $10: 1$ ratio to yield $\mathbf{B r}-\mathbf{F}_{1}-\mathbf{P E G}_{\mathbf{2 0 0}}-\mathbf{O M e}$ as brown oil $(1.34 \mathrm{~g}$, $1.86 \mathrm{mmol}, 54 \%)$.

${ }^{1} \mathrm{H}-\mathrm{NMR}\left(400 \mathrm{MHz}, \mathrm{CDCl}_{3}, 25^{\circ} \mathrm{C}\right): \delta[\mathrm{ppm}]=7.52\left(\mathrm{~d},{ }^{3} \mathrm{JHH}_{\mathrm{HH}}=9.3 \mathrm{~Hz}, 1 \mathrm{H}, \mathrm{H}-5\right), 7.42\left(\mathrm{~d},{ }^{3} \mathrm{JHH}_{\mathrm{HH}}=8.5 \mathrm{~Hz}\right.$, $1 \mathrm{H}, \mathrm{H}-4), 7.39-7.37$ (m, 2H, H-1, H-3), 6.87-6.85 (m, 2H, H-6, H-8), 4.17 (t, ${ }^{3} \mathrm{~J}_{\mathrm{HH}}=4.8 \mathrm{~Hz}, 2 \mathrm{H}, \mathrm{H}-22$ ), $3.89\left(\mathrm{t},{ }^{3} \mathrm{JHH}=4.8 \mathrm{~Hz}, 2 \mathrm{H}, \mathrm{H}-23\right), 3.76-3.74(\mathrm{~m}, 2 \mathrm{H}, \mathrm{H}-26), 3.71-3.63(\mathrm{~m}, 12 \mathrm{H}, \mathrm{H}-24, \mathrm{H}-25), 3.55-3.53$ (m, 2H, H-27), 3.37 (s, 3H, H-28), 1.90-1.84 (m, 4H, H-14), 1.22-1.01 (m, 20H, H-16, H-17, H-18, H-19, $\mathrm{H}-20), 0.81\left(\mathrm{t},{ }^{3} \mathrm{~J}_{\mathrm{HH}}=7.3 \mathrm{~Hz}, 6 \mathrm{H}, \mathrm{H}-21\right), 0.61-0.54(\mathrm{~m}, 4 \mathrm{H}, \mathrm{H}-15)$.

${ }^{13} \mathrm{C}-\mathrm{NMR}\left(101 \mathrm{MHz}, \mathrm{CDCl}_{3}, 2{ }^{\circ} \mathrm{C}\right): \delta[\mathrm{ppm}]=159.2$ (C-7), 152.6 (C-10), 152.4 (C-13), 140.2 (C-11), 133.3 (C-12), 129.9 (C-1), 126.0 (C-3), 120.5 (C-5), 120.3 (C-4), 119.8 (C-2), 113.1 (C-8), 109.7 (C-6), 72.0 (C-27), 71.0 (C-24), 70.8-70.6 (br, C-25, C-26), 69.9 (C-23), 67.7 (C-22), 59.1 (C-28), 55.4 (C-9), 40.5 (C-14), 31.9 (C-19), 30.1 (C-16), 29.3 (br, C-17, C-18), 23.7 (C-15), 22.7 (C-20), 14.2 (C-21).

\section{SYNTHESIS OF Br-F - PEG $_{2000}-\mathrm{OMe}$}

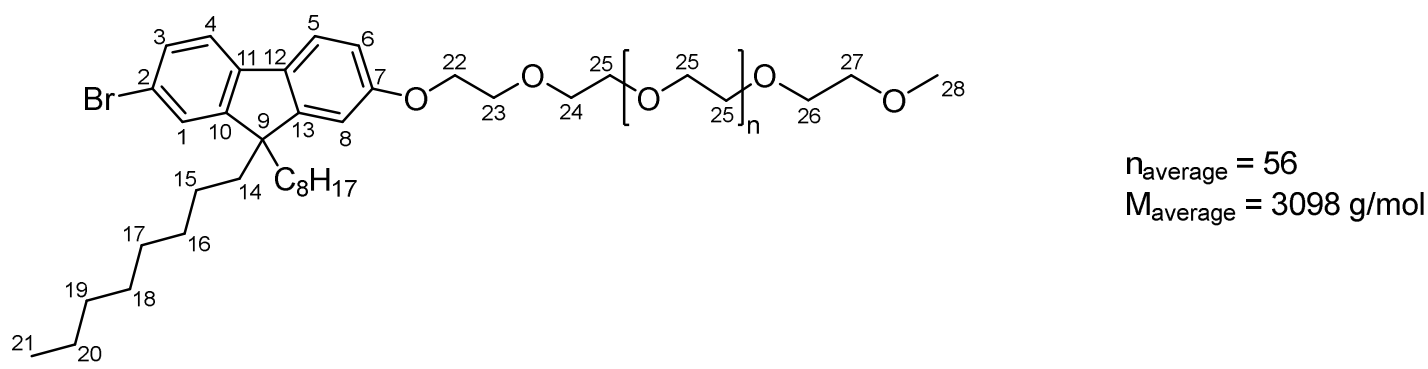

7-Bromo-9,9'-dioctylfluorene-2-ol (1.81 g, $3.72 \mathrm{mmol}, 2$ equiv.) and TsO-PEG ${ }_{2000}-\mathbf{O M e}^{\prime}(4.00 \mathrm{~g}$, $1.86 \mathrm{mmol}, 1$ equiv.) were dissolved in dry acetonitrile $(40 \mathrm{~mL})$ and $\mathrm{Na}_{2} \mathrm{CO}_{3}(0.49 \mathrm{~g}, 4.65 \mathrm{mmol}$, 2.5 equiv.) was added. The mixture was stirred at $85^{\circ} \mathrm{C}$ for 7 days, before it was allowed to cool down to room temperature. After filtration, the solvent was removed in vacuo and the crude product was 
purified by column chromatography using toluene as eluent to remove unreacted 7-bromo-9,9'dioctylfluorene-2-ol. The product was subsequently eluted from the column with a mixture of dichloromethane and methanol in a 10:1 ratio to yield $\mathbf{B r}-\mathbf{F}_{\mathbf{1}}-\mathbf{P E G}_{\mathbf{2 0 0 0}} \mathbf{- O M e}$ as slightly yellow solid (2.87 g, $0.93 \mathrm{mmol}, 50 \%)$.

${ }^{1} \mathrm{H}-\mathrm{NMR}\left(400 \mathrm{MHz}, \mathrm{CDCl}_{3}, 25^{\circ} \mathrm{C}\right): \delta[\mathrm{ppm}]=7.51\left(\mathrm{~d},{ }^{3} \mathrm{JHH}_{\mathrm{HH}}=9.0 \mathrm{~Hz}, 1 \mathrm{H}, \mathrm{H}-5\right), 7.41\left(\mathrm{~d},{ }^{3} \mathrm{JHH}_{\mathrm{HH}}=8.7 \mathrm{~Hz}\right.$, $1 \mathrm{H}, \mathrm{H}-4), 7.38-7.36(\mathrm{~m}, 2 \mathrm{H}, \mathrm{H}-1, \mathrm{H}-3), 6.85-6.83(\mathrm{~m}, 2 \mathrm{H}, \mathrm{H}-6, \mathrm{H}-8), 4.15$ (t, $\left.{ }^{3} \mathrm{~J}_{\mathrm{HH}}=4.7 \mathrm{~Hz}, 2 \mathrm{H}, \mathrm{H}-22\right)$, $3.87\left(\mathrm{t},{ }^{3} \mathrm{JHH}_{\mathrm{HH}}=4.7 \mathrm{~Hz}, 2 \mathrm{H}, \mathrm{H}-23\right), 3.76-3.55(\mathrm{~m}, 230 \mathrm{H}, \mathrm{H}-24, \mathrm{H}-25, \mathrm{H}-26), 3.53-3.51(\mathrm{~m}, 2 \mathrm{H}, \mathrm{H}-27), 3.35$ (s, 3H, H-28), 1.88-1.83 (m, 4H, H-14), 1.20-0.99 (m, 20H, H-16, H-17, H-18, H-19, H-20), 0.79 (t, $\left.{ }^{3} \mathrm{~J}_{\mathrm{HH}}=7.2 \mathrm{~Hz}, 6 \mathrm{H}, \mathrm{H}-21\right), 0.58-0.52(\mathrm{~m}, 4 \mathrm{H}, \mathrm{H}-15)$.

${ }^{13} \mathrm{C}-\mathrm{NMR}\left(101 \mathrm{MHz}, \mathrm{CDCl}_{3}, 25{ }^{\circ} \mathrm{C}\right): \delta[\mathrm{ppm}]=159.1(\mathrm{C}-7), 152.6(\mathrm{C}-10), 152.4(\mathrm{C}-13), 140.2(\mathrm{C}-11)$, 133.3 (C-12), 129.9 (C-1), 126.0 (C-3), 120.5 (C-5), 120.3 (C-4), 119.8 (C-2), 113.0 (C-8), 109.7 (C-6), 72.1 (C-27), 70.9 (C-24), 70.6 (br, C-25, C-26), 69.9 (C-23), 67.7 (C-22), 59.1 (C-28), 55.4 (C-9), 40.5 (C-14), 31.8 (C-19), 30.0 (C-16), 29.3 (br, C-17, C-18), 23.7 (C-15), 22.6 (C-20), 14.1 (C-21).

\subsubsection{SYNTHESES OF AB-TYPE MONOMERS}

The synthesis of 2-(7-bromo-9,9'-dioctyl-9H-fluoren-2-yl)-4,4,5,5-tetramethyl-1,3,2-dioxaborolane (Br-F $\left.F_{1}-B P i n\right)$, used as a standard monomer in this chapter, is outlined in detail in a previous section (cf. Chapter 8.4.1) as it is also the case for 2-(7-bromo-5-octyloxy-9,9'-diphenylfluoren-2-yl)-4,4,5,5tetramethyl-1,3,2-dioxaborolane (Br-ODPF-BPin) (cf. Chapter 8.6.1). The starting material for the synthesis of 2-(2'-bromospiro(cyclopentane-1,9'-fluoren)-7'-yl)-4,4,5,5-tetramethyl-1,3,2-dioxaborolane (Br-M3-Pin) described below was available in the research group. As well, the monomers 2-(4-bromo2,5-bis(hexyloxy)phenyl)-4,4,5,5-tetramethyl-1,3,2-dioxaborolane (Br-M4-BPin) and 2-(5-bromo-4hexylthiophen-2-yl)-4,4,5,5-tetramethyl-1,3,2-dioxaborolane (Br-M5-BPin) were already available.

\section{SYNTHESIS OF 2-(2'-BROMOSPIRO(CYCLOPENTANE-1, 9'-FLUOREN)-7'-YL)-}

\section{4,4,5,5-TETRAMETHYL-1,2,3-DIOXABOROLANE (Br-M3-BPin) ${ }^{31,205}$}

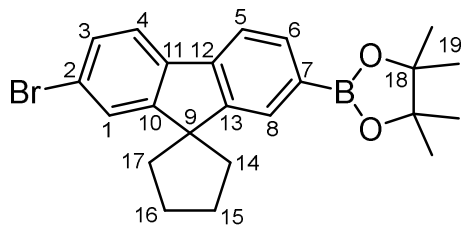

$$
\begin{aligned}
& \mathrm{C}_{23} \mathrm{H}_{26} \mathrm{BBrO}_{2} \\
& \mathrm{M}=425.17 \mathrm{~g} / \mathrm{mol}
\end{aligned}
$$

A solution of 2',7'-dibromospiro(cyclopentane-1,9'-fluorene) (2.00 g, $5.29 \mathrm{mmol}, 1$ equiv.) in dry THF ( $25 \mathrm{~mL}$ ) was cooled to $-78^{\circ} \mathrm{C}$ and a $2.5 \mathrm{M}$ solution of $n$-BuLi in hexane $(2.33 \mathrm{~mL}, 5.82 \mathrm{mmol}, 1.1$ equiv.) was added dropwise. The solution was stirred for $1 \mathrm{~h}$ at $-78^{\circ} \mathrm{C}$, before iso-propylboronic acid pinacol 
ester (1.29 mL, $6.35 \mathrm{mmol}, 1.2$ equiv.) was slowly added and the mixture was allowed to come to room temperature. After stirring overnight, deionized water $(5 \mathrm{~mL})$ was added, the mixture was stirred for further $30 \mathrm{~min}$ and the phases were separated. The aqueous phase was extracted with dichloromethane and the combined organic layers were washed with water and brine and dried over $\mathrm{MgSO}_{4}$. The solvent was removed and the remaining solid was suspended in ethanol by use of ultrasonication. After centrifugation (6000 rpm), the supernatant solution was discarded and the remaining precipitate was dried in vacuo to yield Br-M3-BPin as white solid (0.82 g, $1.92 \mathrm{mmol}, 36 \%$ ).

${ }^{1} \mathrm{H}-\mathrm{NMR}\left(400 \mathrm{MHz} \mathrm{CDCl}_{3}, 25^{\circ} \mathrm{C}\right): \delta[\mathrm{ppm}]=7.84(\mathrm{br}, 1 \mathrm{H}, \mathrm{H}-8), 7.80\left(\mathrm{dd},{ }^{3} \mathrm{~J}_{\mathrm{HH}}=7.7 \mathrm{~Hz},{ }^{4} \mathrm{~J}_{\mathrm{HH}}=0.8 \mathrm{~Hz}\right.$, $1 \mathrm{H}, \mathrm{H}-6), 7.66\left(\mathrm{dd},{ }^{3} \mathrm{~J}_{\mathrm{HH}}=7.5 \mathrm{~Hz},{ }^{5} \mathrm{~J}_{\mathrm{HH}}=0.6 \mathrm{~Hz}, 1 \mathrm{H}, \mathrm{H}-5\right), 7.58\left(\mathrm{~d},{ }^{3} \mathrm{~J}_{\mathrm{HH}}=8.2 \mathrm{~Hz}, 1 \mathrm{H}, \mathrm{H}-4\right), 7.56(\mathrm{~d}$, $\left.{ }^{4} J_{\mathrm{HH}}=1.7 \mathrm{~Hz}, 1 \mathrm{H}, \mathrm{H}-1\right), 7.45\left(\mathrm{dd},{ }^{3} \mathrm{~J}_{\mathrm{HH}}=8.2 \mathrm{~Hz},{ }^{4} J_{\mathrm{HH}}=1.7 \mathrm{~Hz}, 1 \mathrm{H}, \mathrm{H}-3\right), 2.20-2.00(\mathrm{~m}, 8 \mathrm{H}, \mathrm{H}-14, \mathrm{H}-15$, $\mathrm{H}-16, \mathrm{H}-17), 1.37$ (s, 12H, H-19).

${ }^{13} \mathrm{C}-N M R\left(101 \mathrm{MHz} \mathbf{C D C l}_{3}, 25^{\circ} \mathrm{C}\right): \delta$ [ppm] = 157.4 (C-10), 153.1 (C-13), 141.6 (C-12), 138.4 (C-11), 133.9 (C-6), 129.9 (C-3), 129.1 (C-8), 126.6 (C-1), 121.8 (C-2), 121.5 (C-4), 119.1 (C-5), 84.0 (C-18), 57.9 (C-9), 39.7 (br, C-14, C-17), 27.2 (br, C-15, C-16), 25.0 (C-19).

\subsubsection{CONTROLLED SUZUKI-MIYAURA COUPLING POLYMERIZATION}

\section{GENERAL PROCEDURE}

The controlled polymerization of AB-type diarylfluorene monomers was carried out according to a literature-known procedure established for the synthesis of PF8 ${ }^{72}$ which was optimized for the system under investigation by shorter time periods for initiation and polymerization. The following procedure refers to the polymerization using $\mathbf{B r}_{-} \mathbf{F}_{\mathbf{1}}-\mathbf{P E G}_{\mathbf{5 0 0}}-\mathbf{O M e}$ as aryl halide species.

Chloro[(tri-tert-butylphosphine)-2-(2-aminobiphenyl)] palladium(II) (17 mg, $34 \mu \mathrm{mol}, 1$ equiv.) and Br-F - PEG $_{500}-$ OMe (98 mg, $102 \mu \mathrm{mol}, 3$ equiv.) were dissolved in THF (1 mL per $0.1 \mathrm{mmol}$ monomer). $2 \mathrm{M}$ aqueous $\mathrm{K}_{2} \mathrm{CO}_{3}$ solution was added ( $1 \mathrm{~mL}$ per $0.1 \mathrm{mmol}$ monomer) and the resulting initiator mixture was vigorously stirred at room temperature for $15 \mathrm{~min}$. The desired AB-type monomer (for instance Br-F1-BPin, $100 \mathrm{mg}, 168 \mu \mathrm{g}, 5$ equiv.) was dissolved in THF (4 mL per $0.1 \mathrm{mmol}$ monomer) and the solution was cooled to $0{ }^{\circ} \mathrm{C}$ using an ice bath. The initiator solution was rapidly added and the mixture was stirred under continuous cooling for $15 \mathrm{~min}$, before the polymerization was terminated by the addition of $2 \mathrm{M}$ aqueous $\mathrm{HCl}$ solution $(1 \mathrm{~mL})$. After stirring overnight at room temperature, the phases were separated, the aqueous phase was extracted with chloroform and the combined organic layers were washed with brine. The solution was dried over $\mathrm{MgSO}_{4}$ and the solvent was removed under reduced pressure. The crude product was purified by dissolution in hot toluene and precipitation in methanol, followed by centrifugation. The supernatant solution was discarded and the precipitate was dried under reduced pressure to yield the respective amphiphilic copolymer. 
For the synthesis of copolymers with different lengths of the hydrophilic PEG segment, the same procedure was carried out using $\mathbf{B r}_{-} \mathbf{F}_{\mathbf{1}}-\mathbf{P E G}_{\mathbf{2 0 0}}-\mathbf{O M e}\left(69 \mathrm{mg}, 102 \mu \mathrm{mol}, 3\right.$ equiv.) or $\mathbf{B r}-\mathbf{F}_{\mathbf{1}}-\mathbf{P E G}_{\mathbf{2 0 0 0}}-\mathbf{O M e}_{\mathbf{M}}$ (251 mg, $102 \mu \mathrm{mol}, 3$ equiv.), respectively. In cases of copolymers with longer conjugated copolymer segments ( $\geq 10$ equiv. of $A B$-type monomer), the chloroform used in the work-up was replaced by toluene in order to increase the solubility.

\subsubsection{AGGREGATION BEHAVIOR OF AMPHIPHILIC COPOLYMERS}

For the experiments addressing the aggregation behavior of the amphiphilic copolymers studied, the respective copolymer $(1 \mathrm{mg})$ was dissolved in tetrahydrofuran $(2.5 \mathrm{~mL})$ and the solution was filtered via a $0.45 \mu \mathrm{m}$ PTFE syringe filter. In a $8 \mathrm{~mL}$ vial, methanol $(0.25 \mathrm{~mL})$ was added dropwise to the polymer solution. The mixture was manually shaken for $10 \mathrm{~s}$ and aged for $24 \mathrm{~h}$ before TEM samples were prepared.

For the acquisition of fluorescence emission spectra from the resulting solutions, the default measurement settings were adjusted by shortage of the exposure time to $20 \mathrm{~ms}$ and the signal to noise ratio was improved by averaging over 200 measurement runs. 


\section{APPENDIX}

\subsection{STUDIES ON OLIGOFLUORENE CONFORMATION BY DOUBLE ELECTRON- ELECTRON RESONANCE}

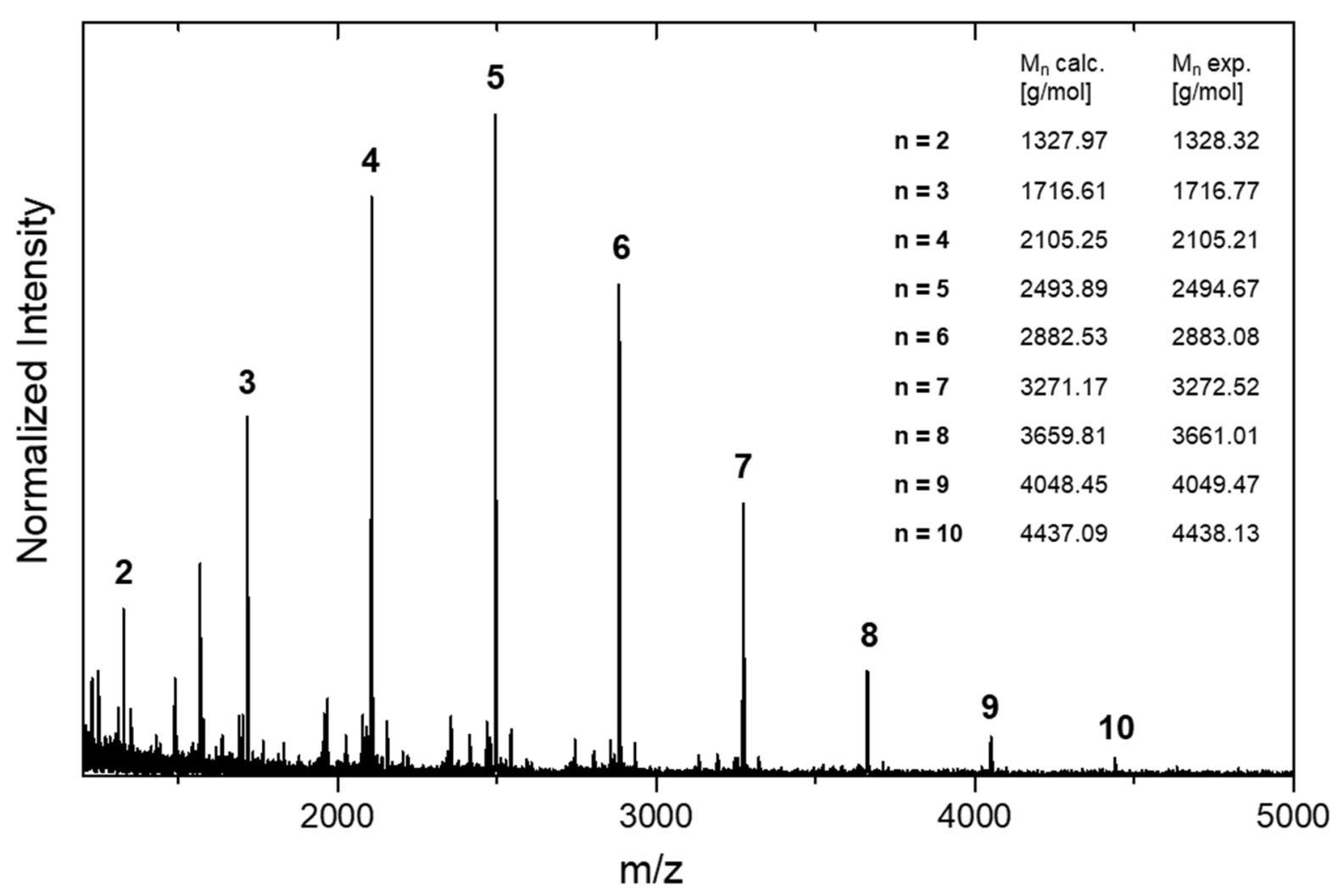

Figure 9.1: MALDI-TOF mass spectrum of a mixture of doubly spin-labeled oligo(fluorenes), obtained from controlled Suzuki-Miyaura coupling polymerization. The peaks are labeled with the respective number $n$ of repeat units. The calculated and experimentally found values for $M_{n}$ are listed. 

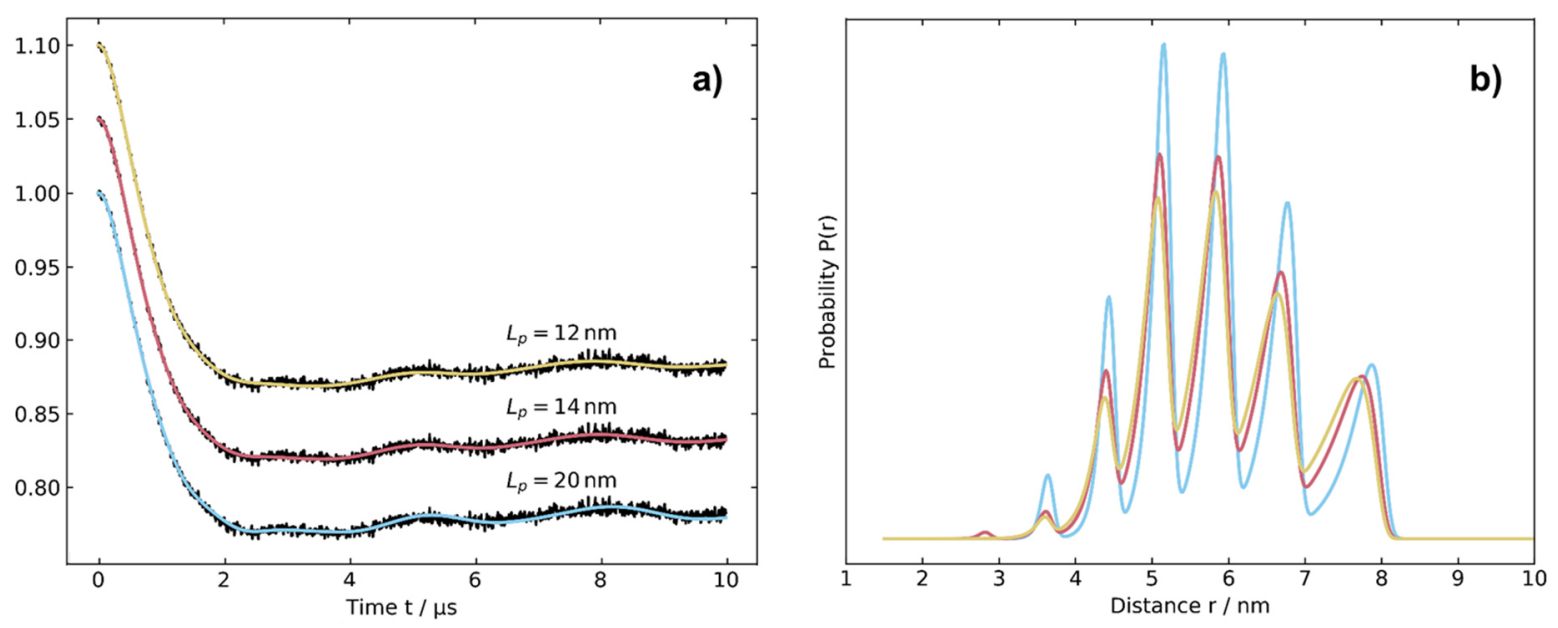

Figure 9.2: Performance of the fit for the mixture of spin-labeled oligomers obtained from controlled Suzuki-Miyaura coupling polymerization with differently set persistence lengths but identical contour lengths. (a) Comparison of form factors with fit and (b) resulting distance distributions. Each fit was obtained by weighting of seven WLCs with fixed Gaussian broadening, contour lengths and persistence length ( $L_{p}=12 \mathrm{~nm}, 14 \mathrm{~nm}$ or $\left.20 \mathrm{~nm}\right)$.
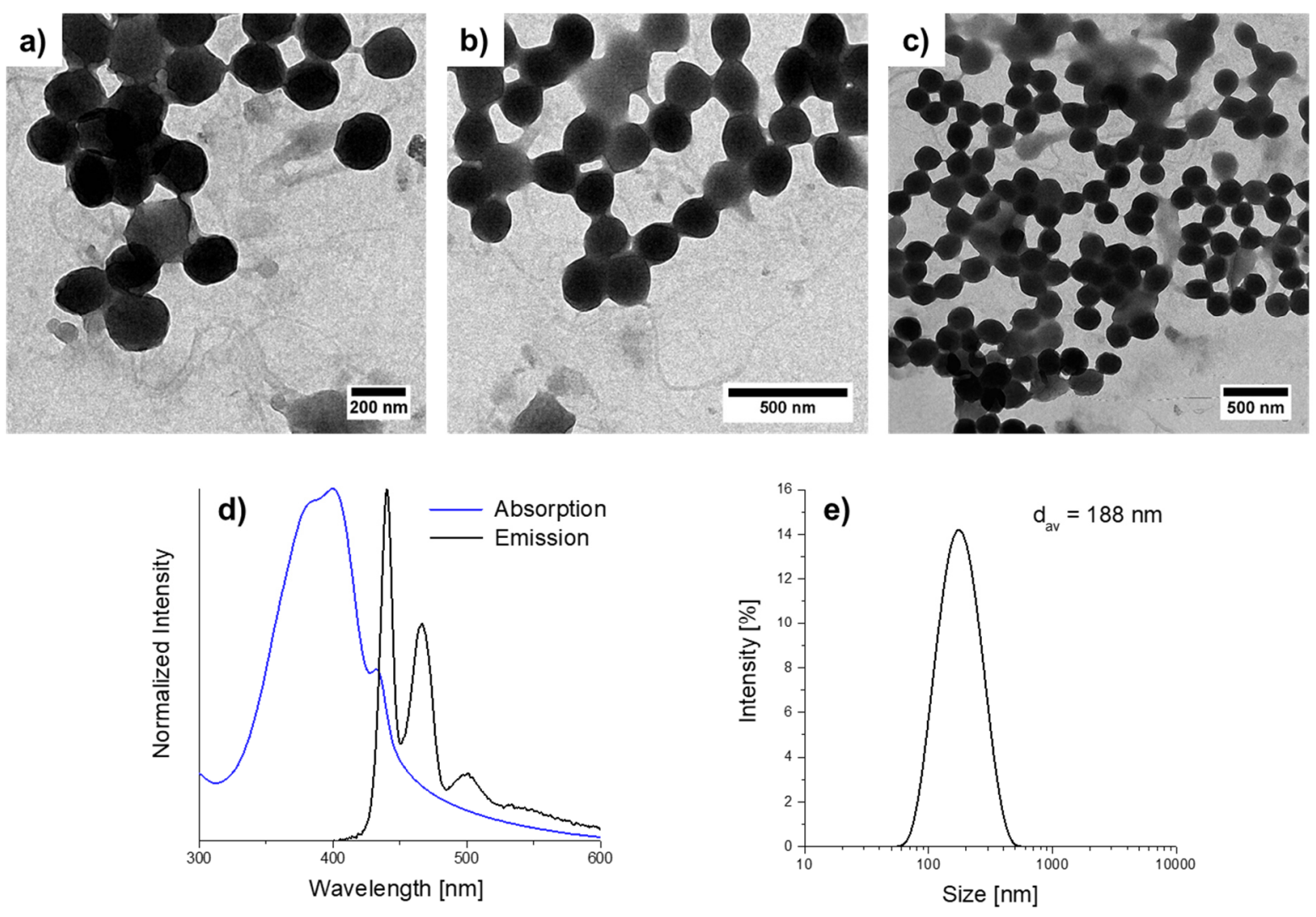

Figure 9.3: (a-c) Additional TEM images of PF8 particles containing spin-labeled oligomers, prepared in an emulsification approach, (d) corresponding UV/Vis absorption and fluorescence emission spectra of the dispersions and (e) particle size distribution determined by DLS. 


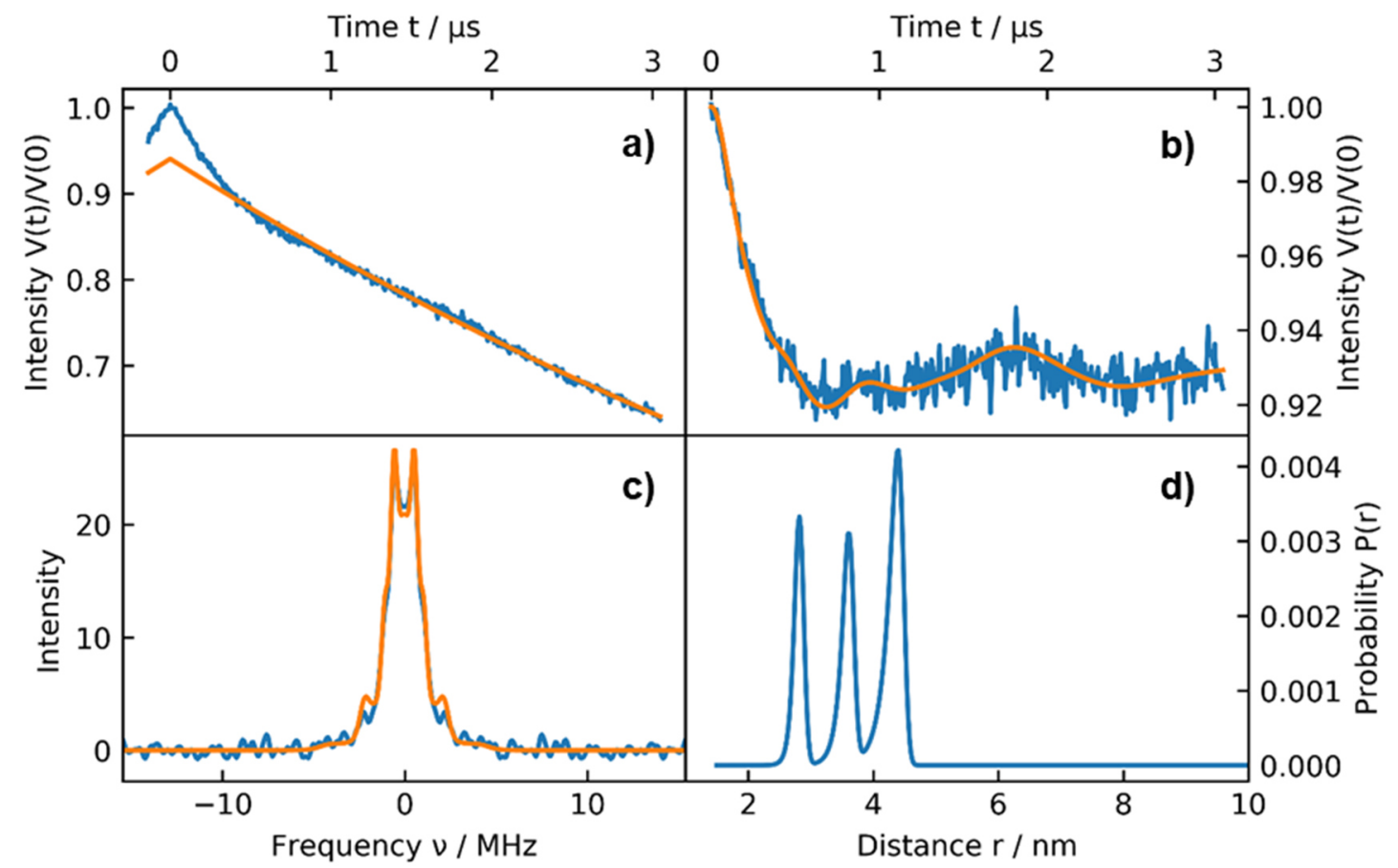

Figure 9.4: DEER measurements on PF8 particles containing spin-labeled oligomers, performed with $20 \mathrm{~ns}$ pump pulse length and 44 ns observer $\pi$-pulse length with 300 averages. Dipolar evolution time was set to $t=3.2 \mu \mathrm{s}$ without cutoff. (a) DEER trace with background from DEERNet. (b) Form factor with model based fit (3 WLCs). (c) Dipolar spectrum with model based fit. (d) Distance distribution by model based fit.

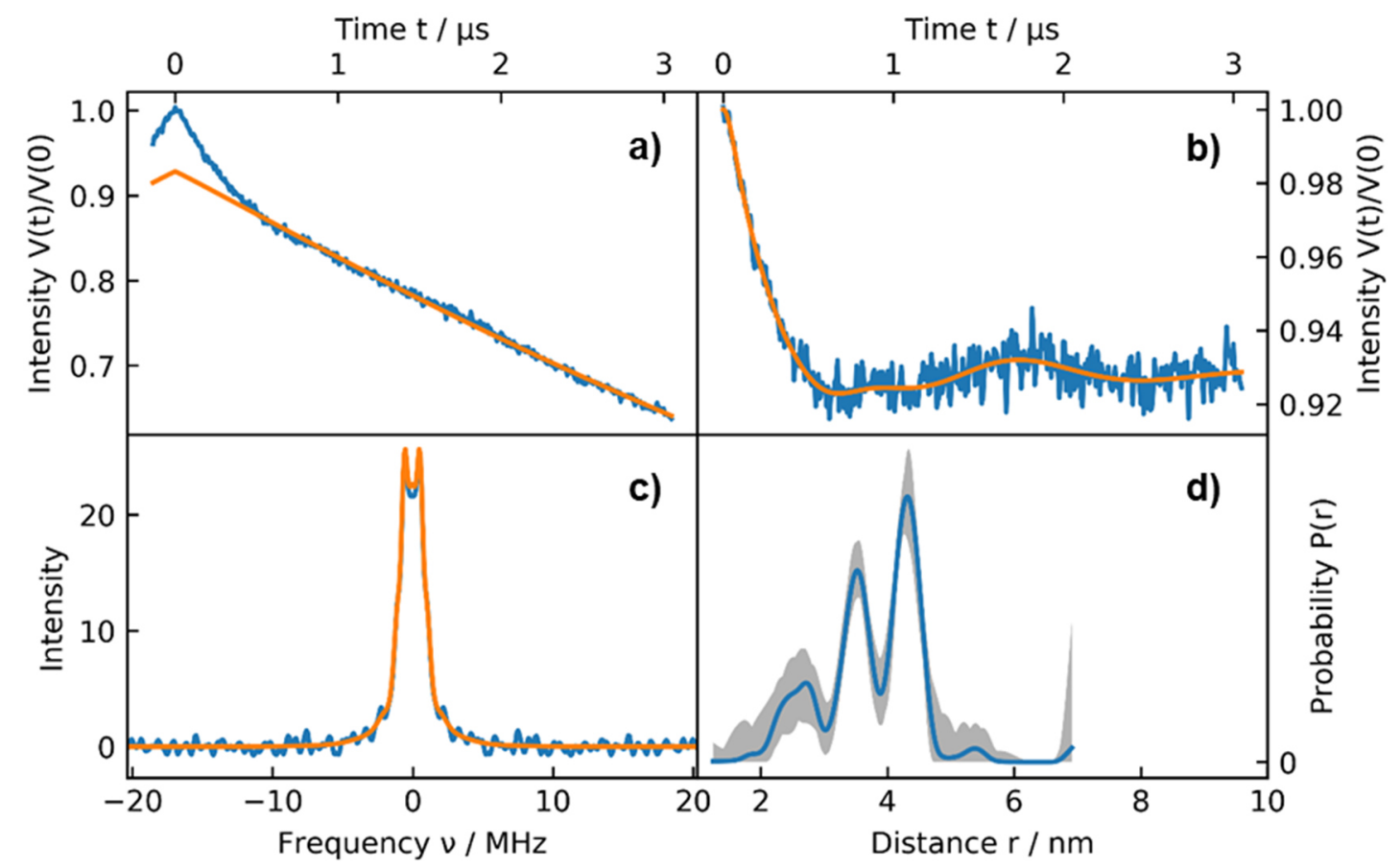

Figure 9.5: DEER measurements on PF8 particles containing spin-labeled oligomers, performed with 20 ns pump pulse length and 44 ns observer m-pulse length with 300 averages. Dipolar evolution time was set to $t=3.2 \mu s$ without cutoff. (a) DEER trace with background from DEERNet. (b) Form factor with model free fit. (c) Dipolar spectrum with model free fit. (d) Distance distribution obtained by Tikhonov regularization, using the GCV criterion. The grey area indicates the uncertainty estimate resulting from DEERNet background correction. 


\subsection{ORIGIN OF ANISOTROPY}

\subsubsection{SYNTHESES OF OLIGOFLUORENES}
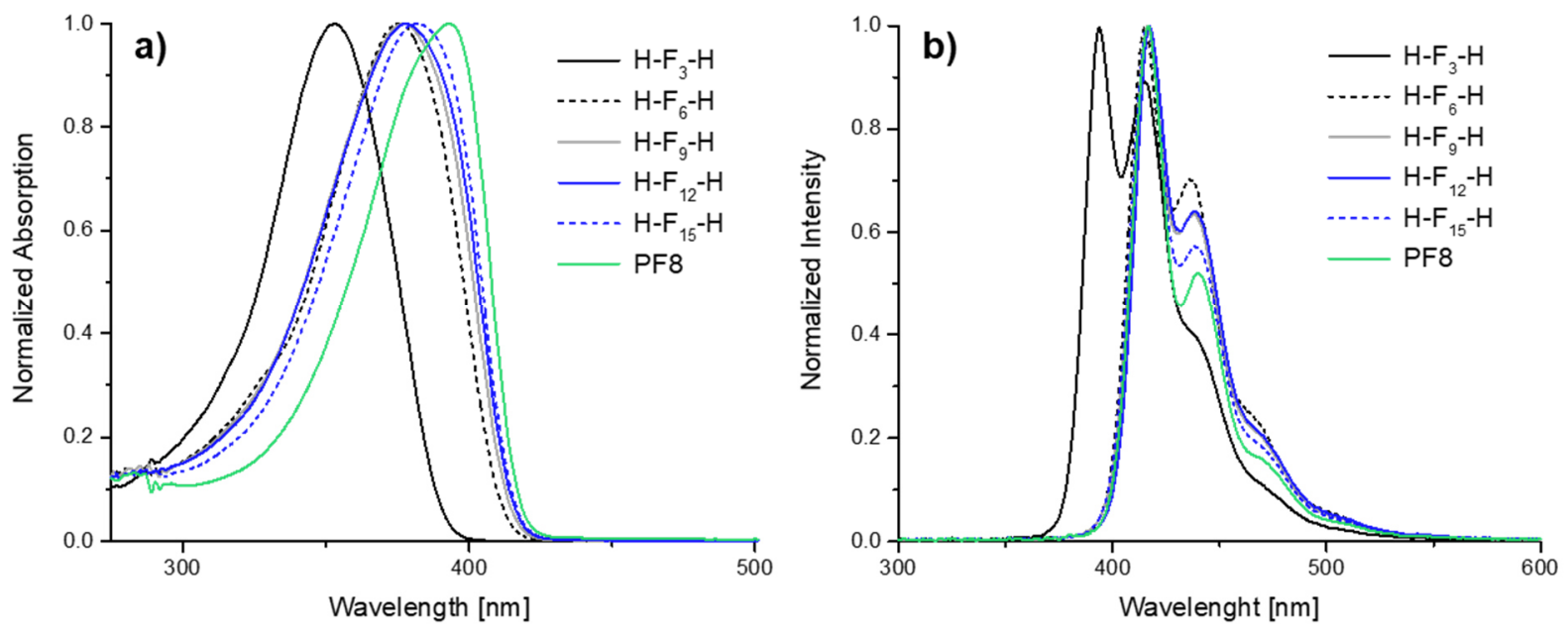

Figure 9.6: (a) UV/Vis absorption spectra and (b) fluorescence emission spectra $\left(\lambda_{\text {exc }}=380 \mathrm{~nm}\right)$ of $\mathrm{H}-F_{n}-\mathrm{H}$ oligomers dissolved in THF (c $\leq 0.01 \mathrm{mg} / \mathrm{mL})$.

\subsubsection{HETEROPHASE SUZUKI-MIYAURA POLYCONDENSATION WITH} ALTERNATIVE SOLVENTS
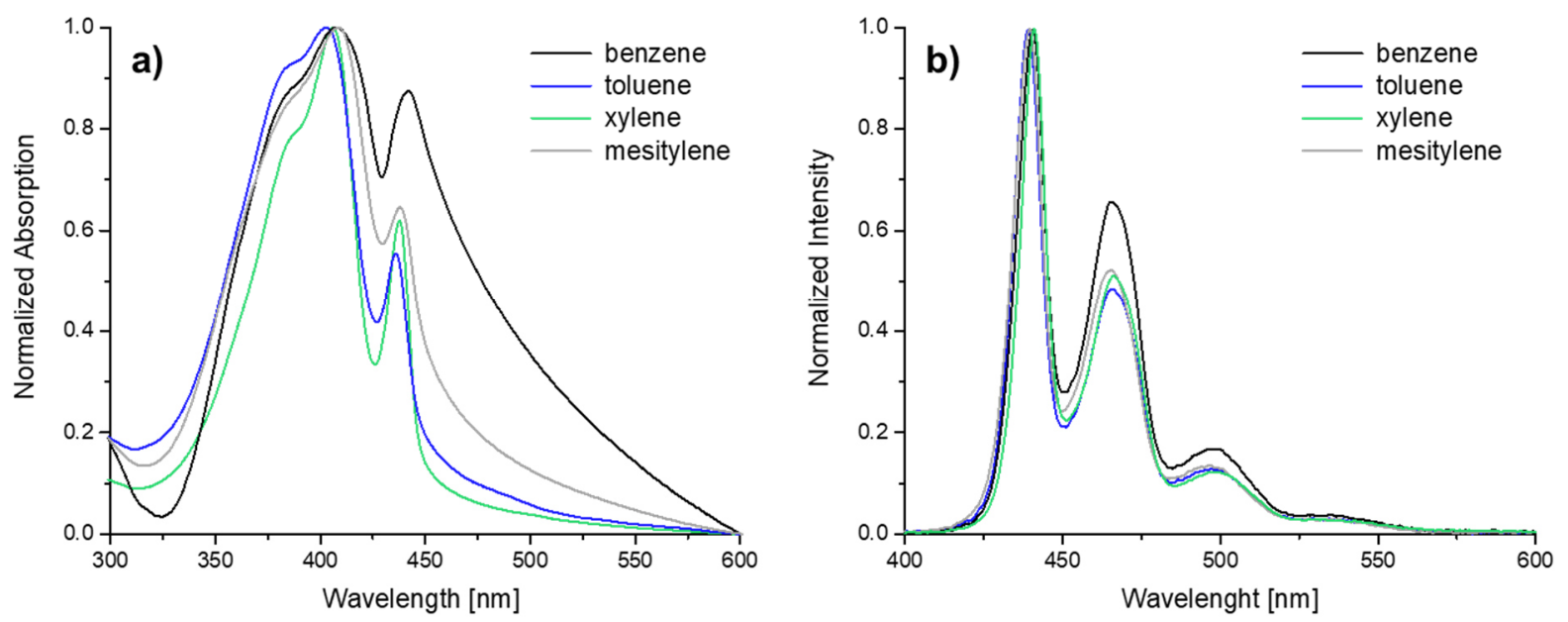

Figure 9.7: (a) UV/Vis absorption spectra and (b) fluorescence emission spectra ( $\lambda_{\text {exc }}=380 \mathrm{~nm}$ ) of poly(fluorene) dispersions prepared by Suzuki-Miyaura polycondensation with alternative aromatic solvents. 


\subsubsection{POST-POLYMERIZATION PARTICLE SYNTHESES}
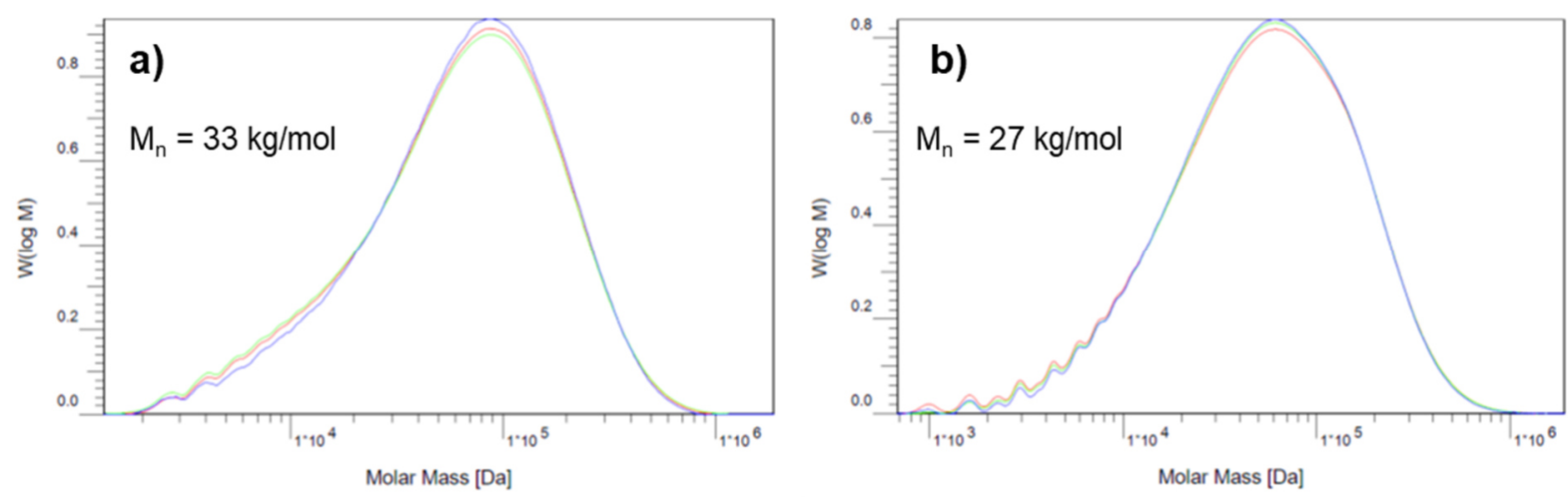

Figure 9.8: GPC traces of poly(fluorenes) applied in post-polymerization particle synthesis, (a) previously synthesized by Suzuki-Miyaura polycondensation and (b) commercially acquired from Sigma-Aldrich. Indicated $M_{n}$ values were determined from RI detection vs. PS standards (blue line).

Table 9.1: Preparation of particle dispersions by post-polymerization emulsification under varied conditions. Entry 1 represents the procedure described as reference system in previous sections (cf. Chapter 8.5.3). ${ }^{a}$

\begin{tabular}{c|c|c|c} 
entry & organic solvent & $\begin{array}{c}\text { ultrasonication } \\
\text { time [min] }\end{array}$ & surfactant \\
\hline 1 & xylene & 1 & Triton X-102 \\
2 & benzene & 1 & Triton X-102 \\
3 & toluene & 1 & Triton X-102 \\
4 & mesitylene & 1 & Triton X-102 \\
5 & xylene & 2.5 & Triton X-102 \\
6 & xylene & 1 & Triton X-405
\end{tabular}

aFurther conditions: Dissolution of poly(fluorene) $(5 \mathrm{mg})$, synthesized by heterophase Suzuki-Miyaura polycondensation and purified prior to usage, in the organic solvent of choice $(1 \mathrm{~mL})$, rapid injection into the aqueous solution $(10 \mathrm{~mL})$ of surfactant $(5 \mathrm{wt} \%)$, simultaneous onset of ultrasonication (60\% amplitude), stirring for at least $24 \mathrm{~h}$. 

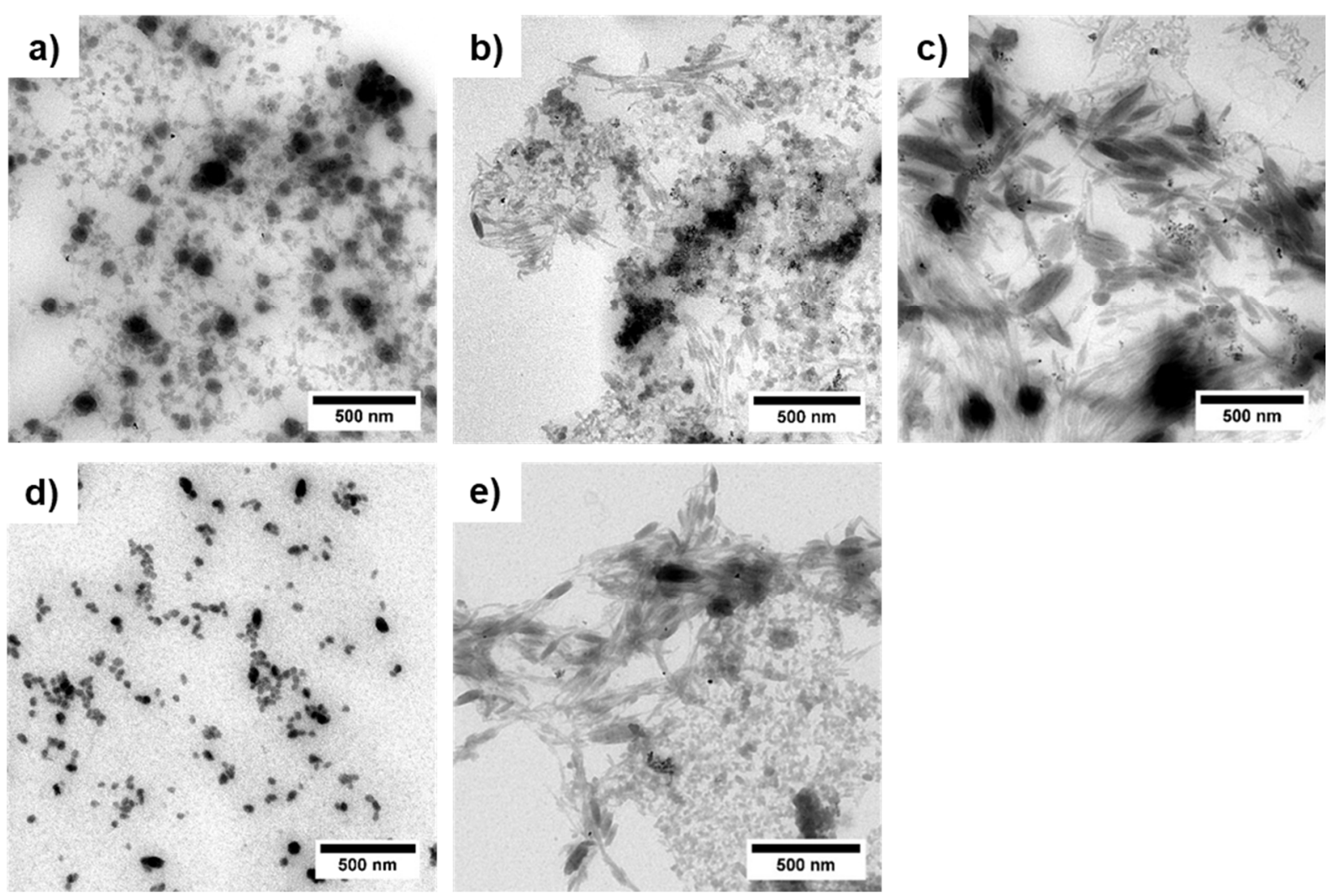

Figure 9.9: TEM images of dispersions prepared by post-polymerization emulsification under varied conditions, corresponding to entries 2 (a), 3 (b), 4 (c), 5 (d) and 6 (e) of Table 9.1.

\subsubsection{EVOLUTION OF MOLECULAR WEIGHT IN THE COURSE OF POLYCONDENSATION}

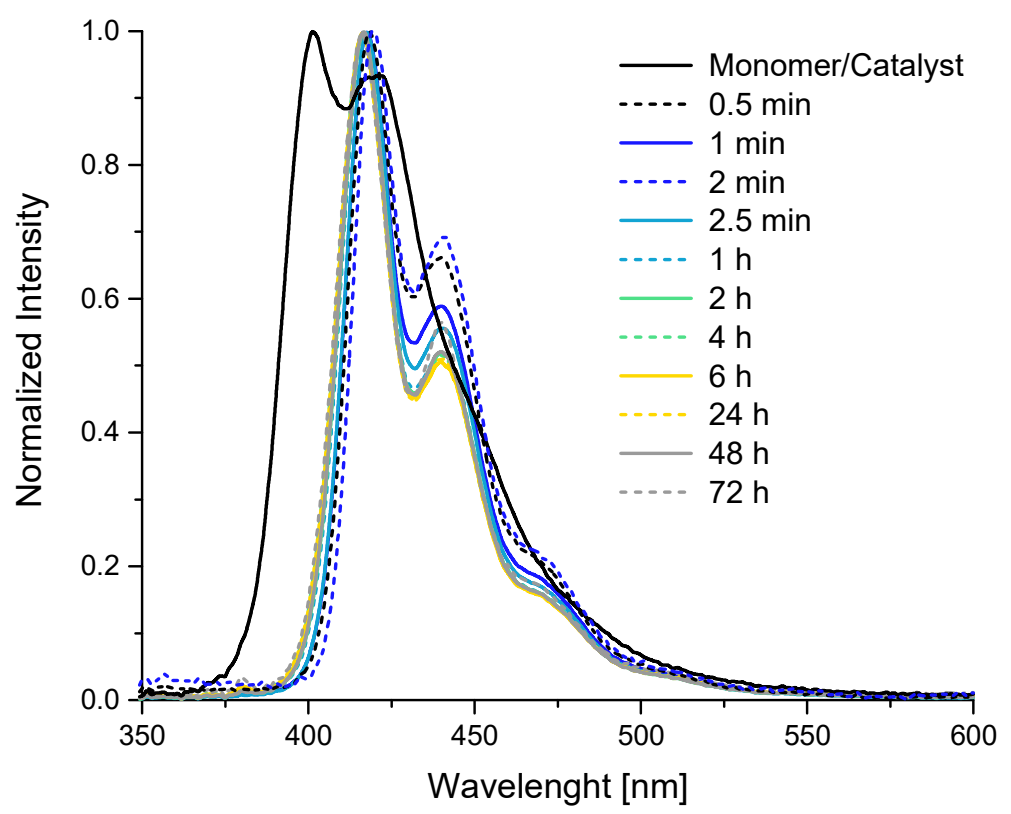

Figure 9.10: Fluorescence emission spectra $\left(\lambda_{e x c}=380 \mathrm{~nm}\right)$ of samples drawn from the reaction mixture and precipitated at various stages of the polycondensation. Measurement of polymer solutions in THF (c $\leq 0.01 \mathrm{mg} / \mathrm{mL})$. 


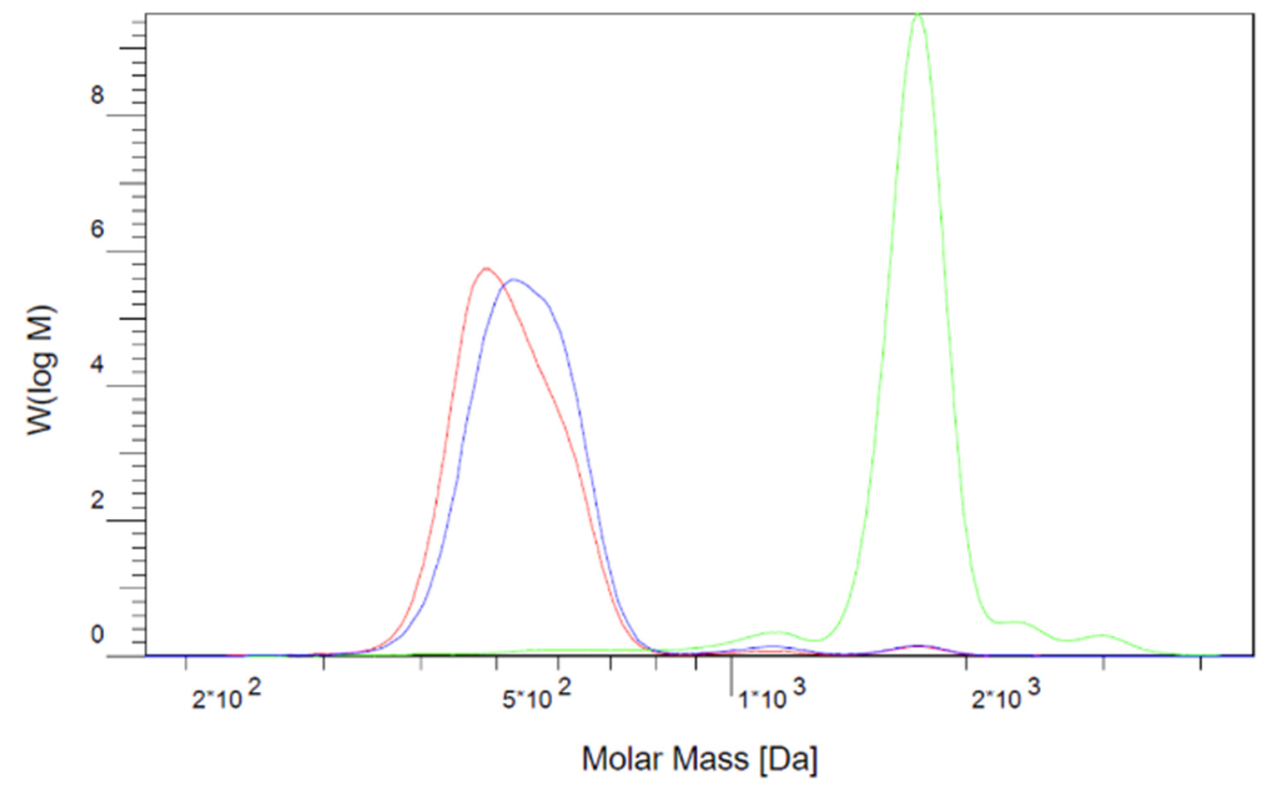

Figure 9.11: GPC trace of the monomer/catalyst mixture acquired by UVIVis detection (red and green line) and RI detection (blue line) in THF vs. PS standards.
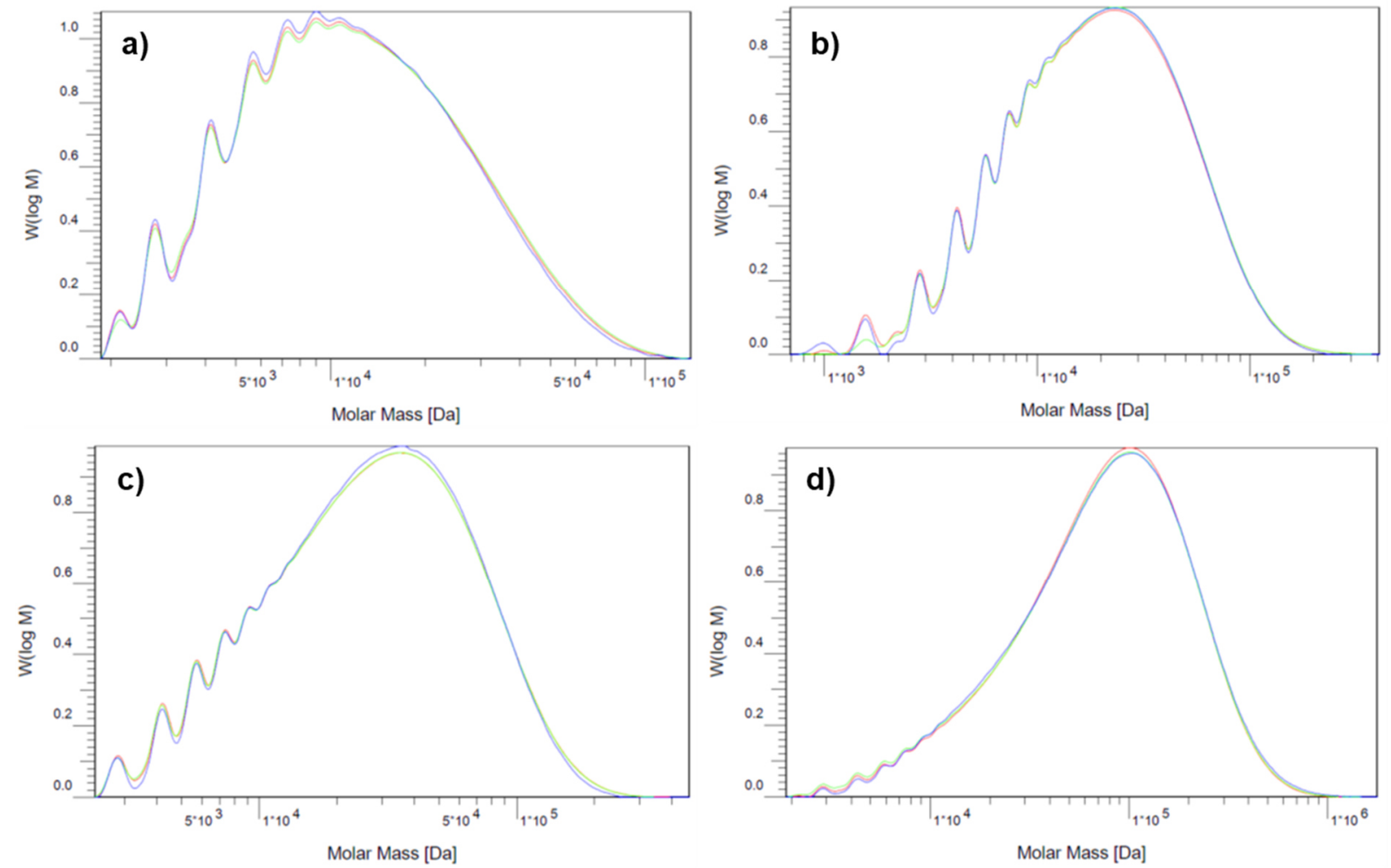

Figure 9.12: GPC traces of poly(fluorene) obtained from polycondensation reactions stopped after (a) $0.5 \mathrm{~min}$, (b) $1 \mathrm{~min}$, (c) $2 \mathrm{~min}$, (d) $2.5 \mathrm{~min}$ of ultrasonication, acquired by UV detection (red and green line) and RI detection (blue line) in THF vs. PS standards. 

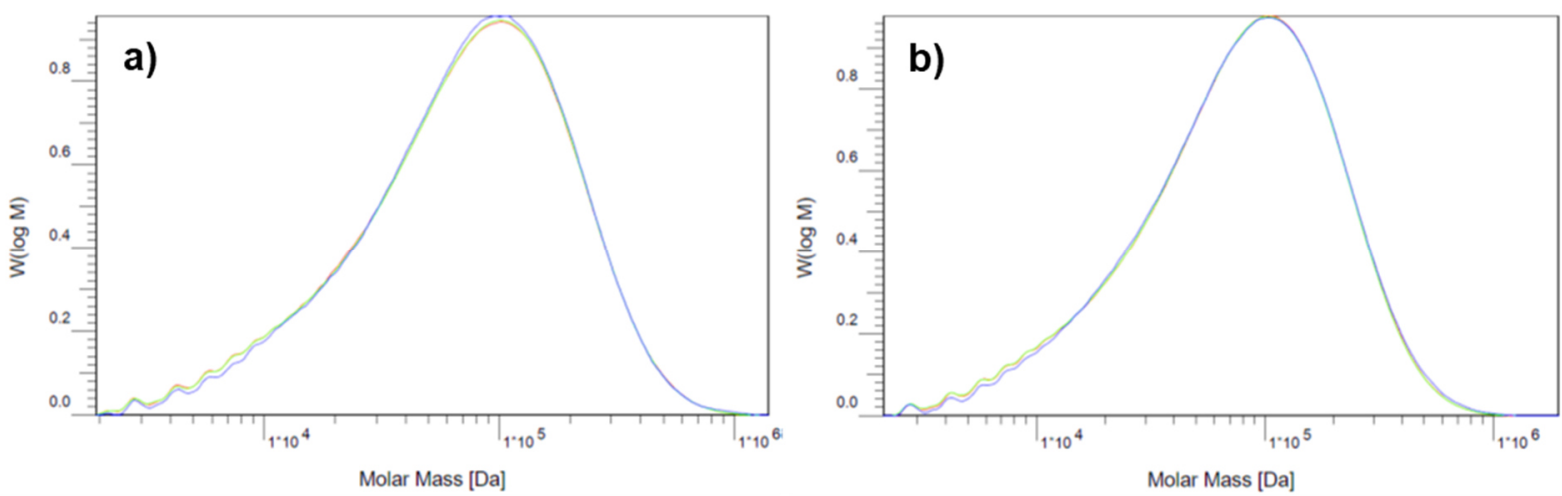

Figure 9.13: GPC traces of poly(fluorene) samples drawn from polycondensation reactions after (a) $1 h$ and (b) $72 h$ of reaction time, acquired by UVNis detection (red and green line) and RI detection (blue line) in THF vs. PS standards.
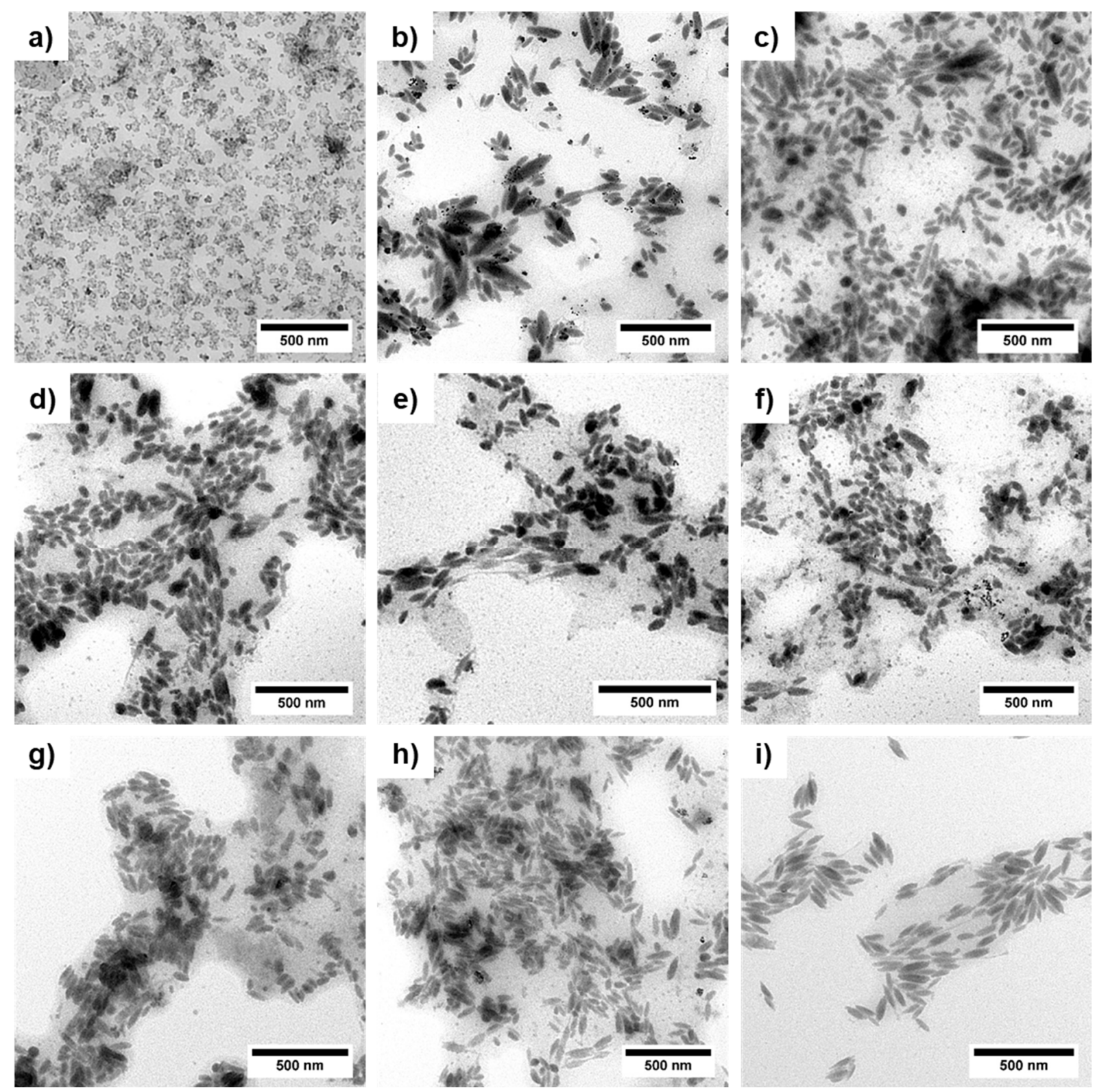

Figure 9.14: TEM images of aqueous dispersion samples drawn after (a) $1 \mathrm{~min}$, (b) $2.5 \mathrm{~min}$, (c) $1 \mathrm{~h}$, (d) $2 \mathrm{~h}$, (e) $4 \mathrm{~h}$, (f) $6 h$, (g) $24 h$, (h) $48 h$ and (i) $72 h$ of the heterophase Suzuki-Miyaura polycondensation reaction, followed by immediate sample preparation. 


\subsubsection{SYNTHESIS OF CROSS-LINKED POLYFLUORENE}
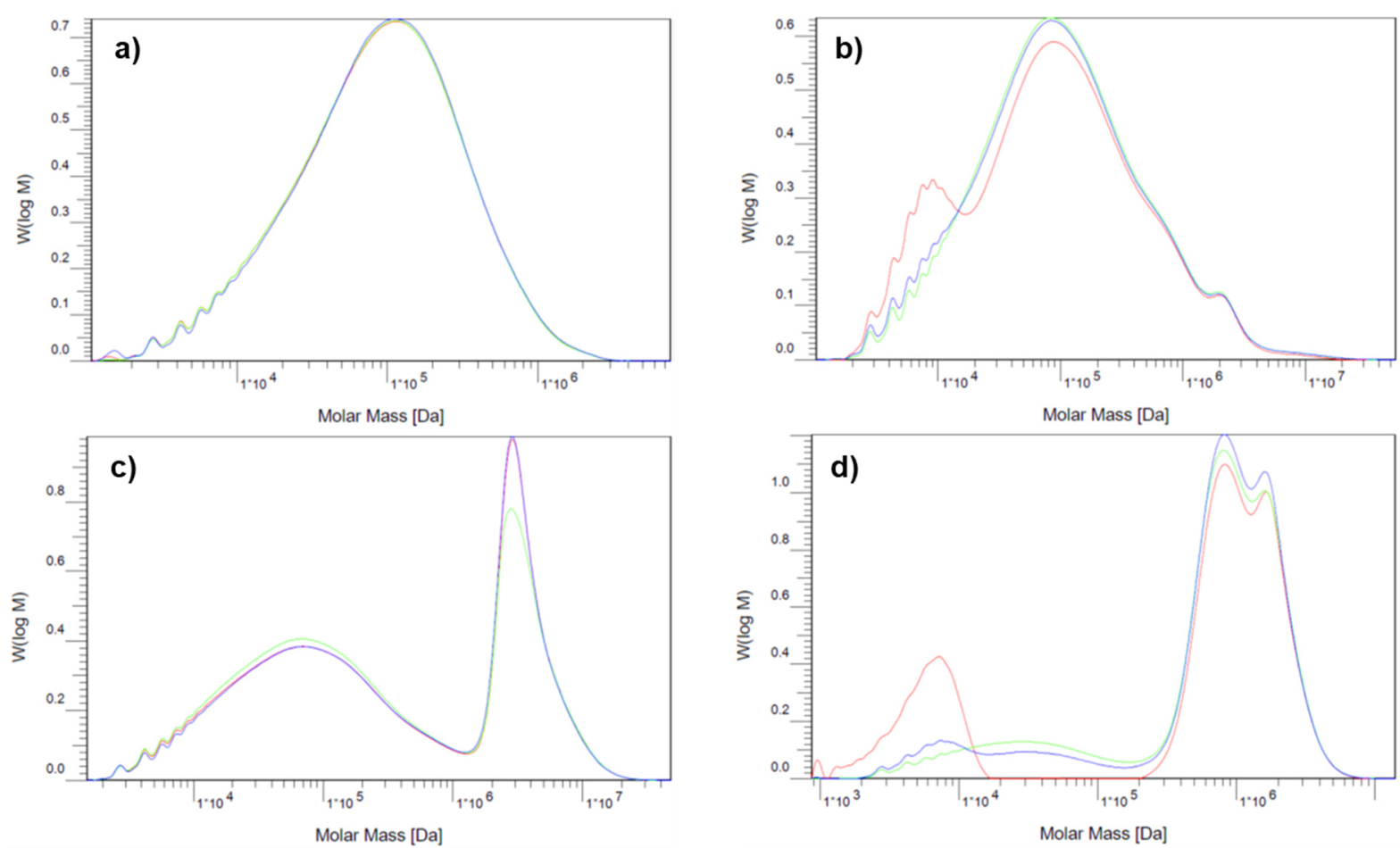

Figure 9.15: GPC traces of cross-linked poly(fluorene) samples synthesized with (a) $0.5 \mathrm{~mol} \%$, (b) $1.25 \mathrm{~mol} \%$, (c) $2.5 \mathrm{~mol} \%$ and (d) $5 \mathrm{~mol} \%$ of tris(4-bromophenyl)amine as cross-linking additive, acquired by UVNis detection (red and green line) and RI detection (blue line) in THF vs. PS standards.
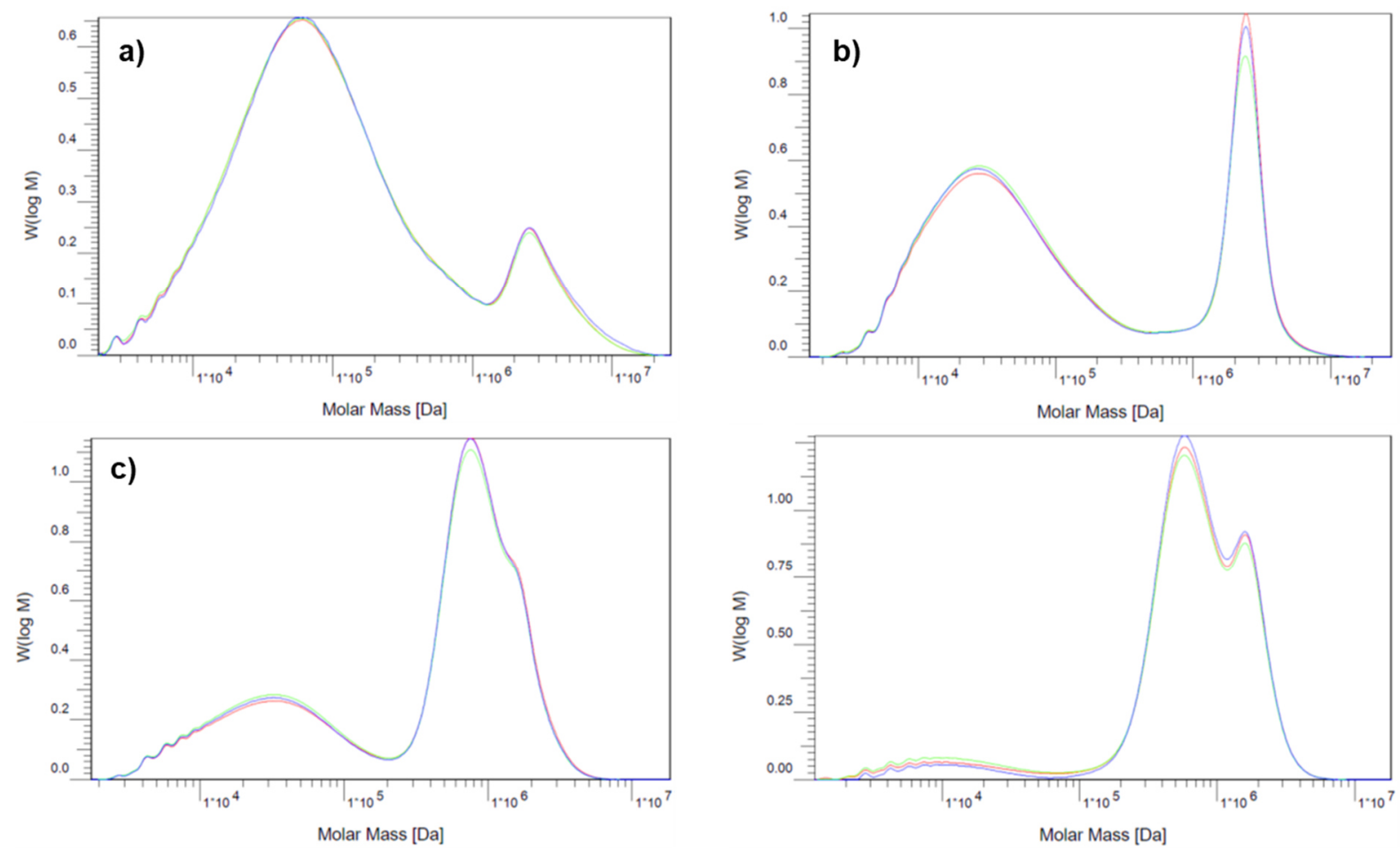

Figure 9.16: GPC traces of cross-linked poly(fluorene) samples synthesized with (a) $0.5 \mathrm{~mol} \%$, (b) $1.25 \mathrm{~mol} \%$, (c) $2.5 \mathrm{~mol} \%$ and (d) $5 \mathrm{~mol} \%$ of tetrakis(4-bromophenyl)methane as cross-linking additive, acquired by UV detection (red and green line) and RI detection (blue line) in THF vs. PS standards. 


\subsection{POLYDIARYLFLUORENE}

\subsubsection{NMR-SPECTRA OF DIARYLFLUORENE MONOMERS}

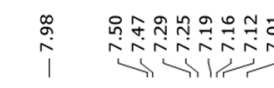

$\stackrel{+}{\vec{f}}$

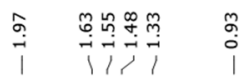

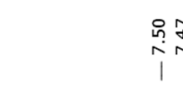

突先
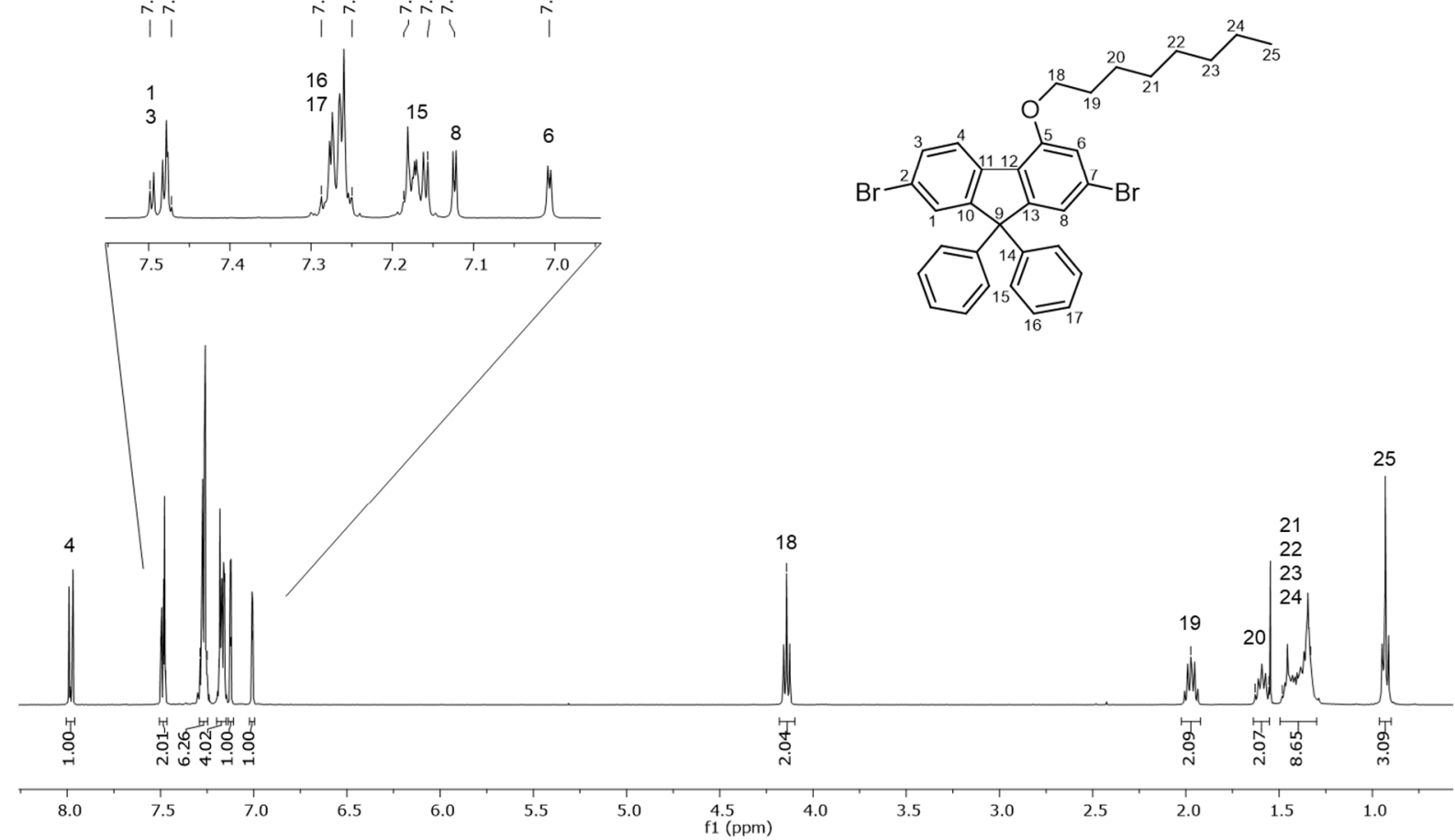

Figure 9.17: ${ }^{1} \mathrm{H}-\mathrm{NMR}$ spectrum $\left(400 \mathrm{MHz}, \mathrm{CDCl}_{3}, 25^{\circ} \mathrm{C}\right)$ of $\mathrm{Br}-\mathrm{ODPF}-\mathrm{Br}$ with assigned signals. 


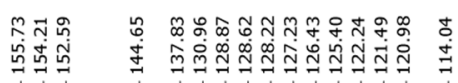

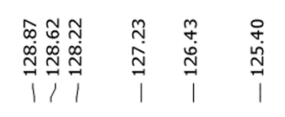

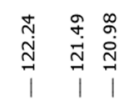
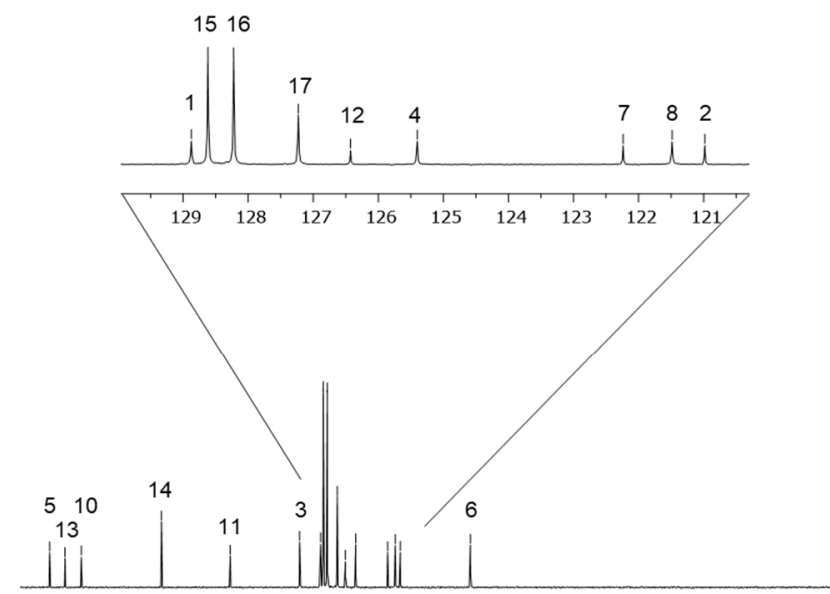

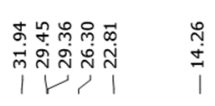

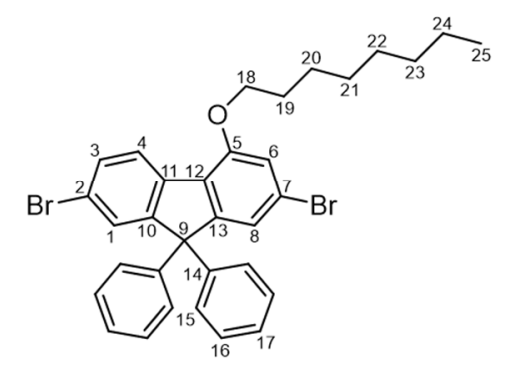

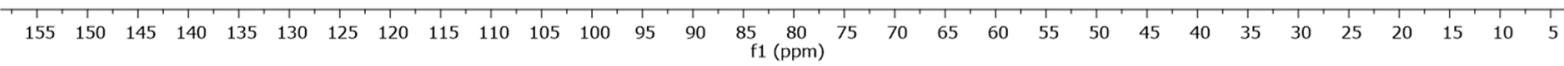

Figure 9.18: ${ }^{13} \mathrm{C}-\mathrm{NMR}$ spectrum $\left(101 \mathrm{MHz}, \mathrm{CDCl}_{3}, 25^{\circ} \mathrm{C}\right)$ of $\mathrm{Br}-\mathrm{ODPF}-\mathrm{Br}$ with assigned signals. 


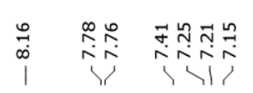

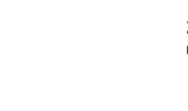

$\underset{i}{\vec{j}}$

$\stackrel{\stackrel{n}{i}}{i} \stackrel{\vec{n}}{i} \stackrel{\stackrel{n}{*}}{i}$

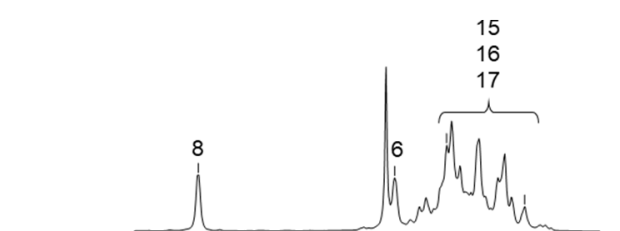

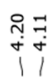

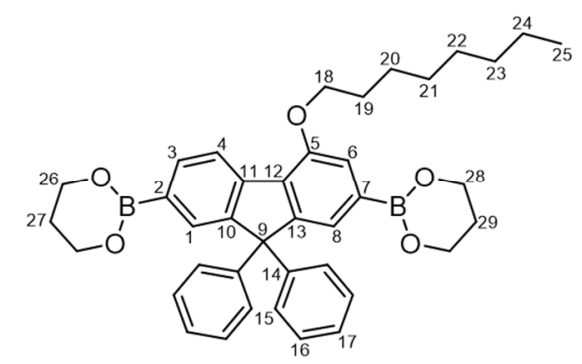

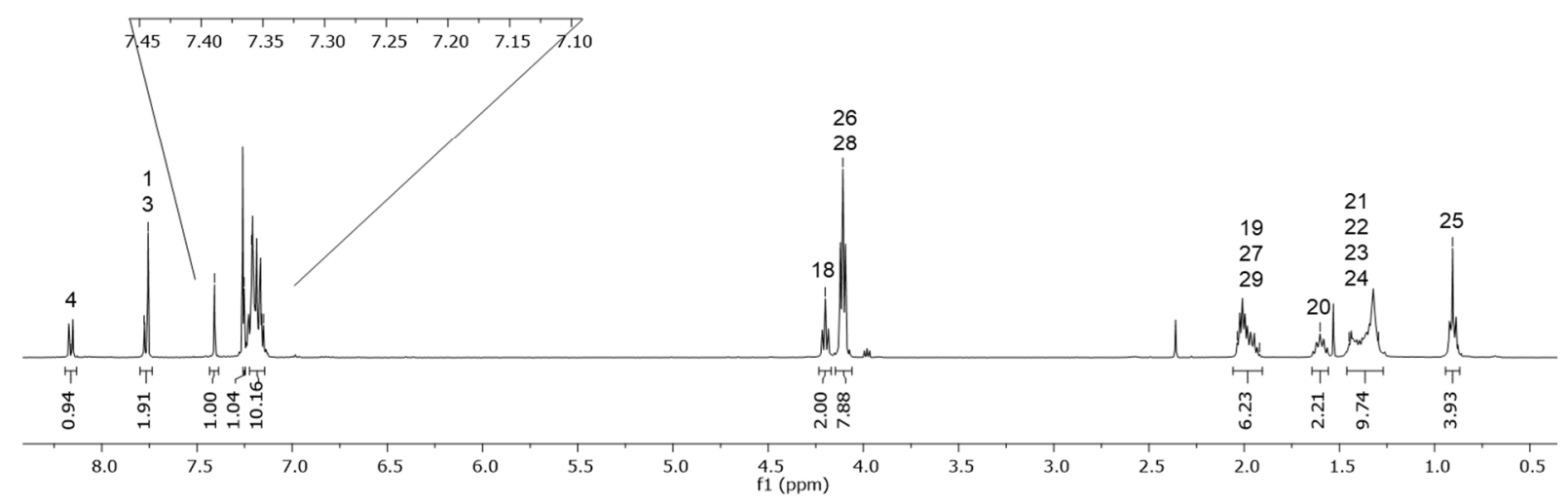

Figure 9.19: ${ }^{1} \mathrm{H}-\mathrm{NMR}$ spectrum $\left(400 \mathrm{MHz}, \mathrm{CDCl}_{3}, 25^{\circ} \mathrm{C}\right)$ of $\mathrm{PrO}_{2} \mathrm{~B}-\mathrm{ODPF}-\mathrm{BO}{ }_{2} \mathrm{Pr}$ with assigned signals.

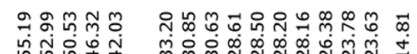

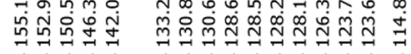

농ํำ

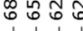

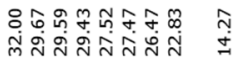

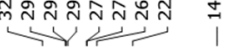

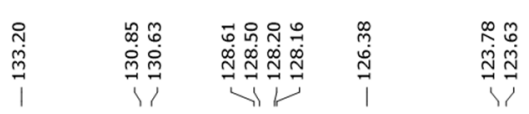<smiles>CCCCCCCCOc1cc(B2OCCCO2)cc2c1[C@@H]1c3ccc(B4OCCCO4)cc3C(C)(C)C21</smiles>

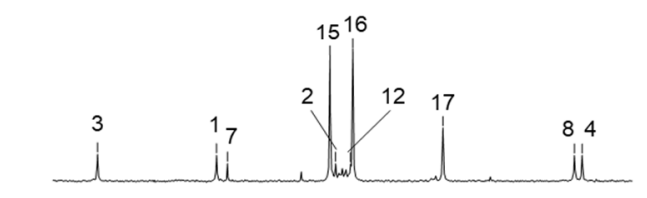

$\begin{array}{lllllllllllllllll}134 & 133 & 132 & 131 & 130 & 129 & 128 & 1 & 127 & 1 & 124 & 1 / 25\end{array}$<smiles>C1=CC(=CC2=CC=[Te]C=C2)C=C1</smiles>

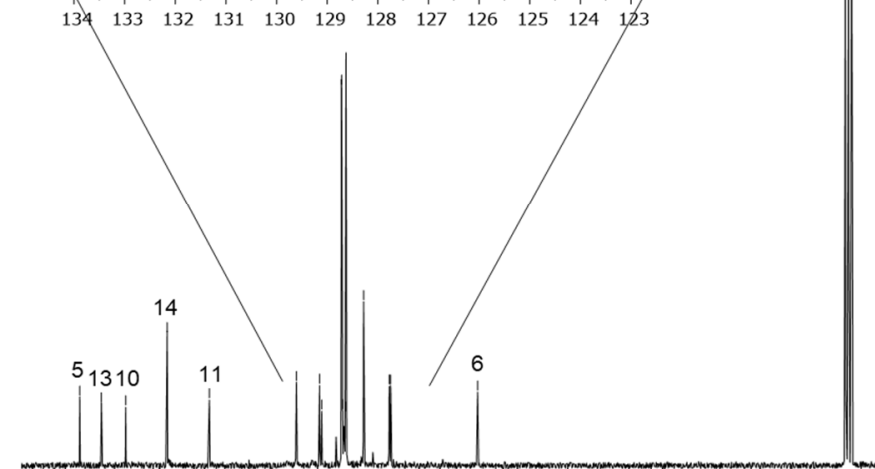

ปูi
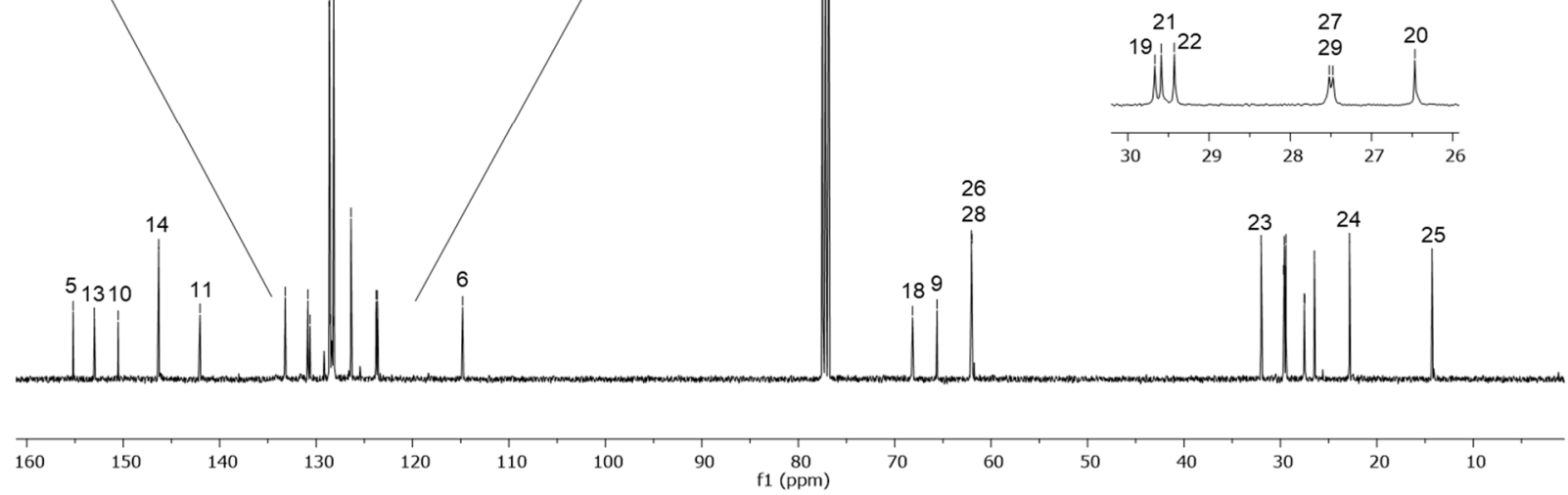

Figure 9.20: ${ }^{13} \mathrm{C}-\mathrm{NMR}$ spectrum $\left(101 \mathrm{MHz}, \mathrm{CDCl}_{3}, 25^{\circ} \mathrm{C}\right)$ of $\mathrm{PrO}_{2} \mathrm{~B}-\mathrm{ODPF}-\mathrm{BO}_{2} \mathrm{Pr}$ with assigned signals. 


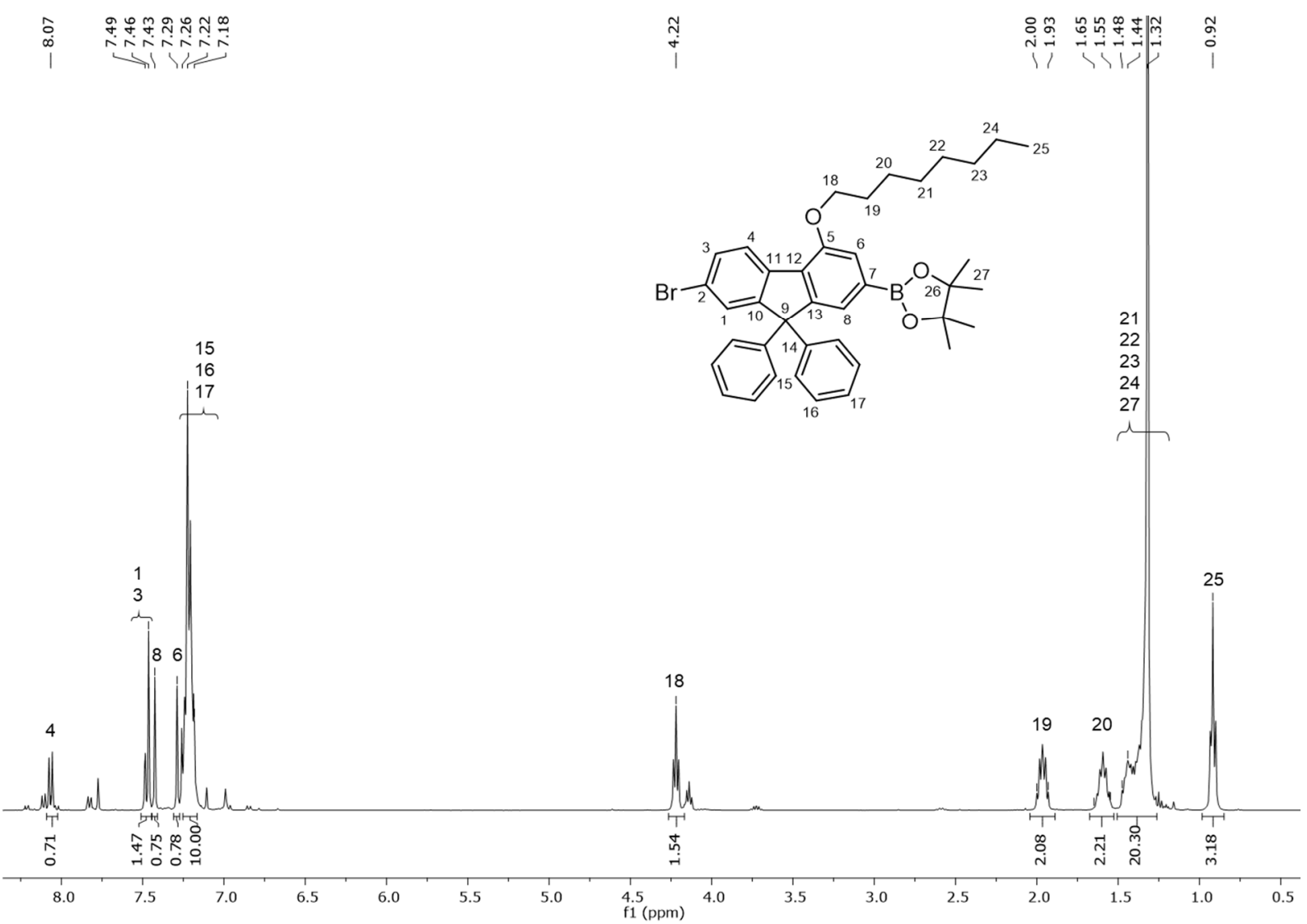

Figure 9.21: ${ }^{1} \mathrm{H}-\mathrm{NMR}$ spectrum $\left(400 \mathrm{MHz}, \mathrm{CDCl}_{3}, 25^{\circ} \mathrm{C}\right)$ of $\mathrm{Br}-\mathrm{ODPF}-\mathrm{BP}$ in with assigned signals.

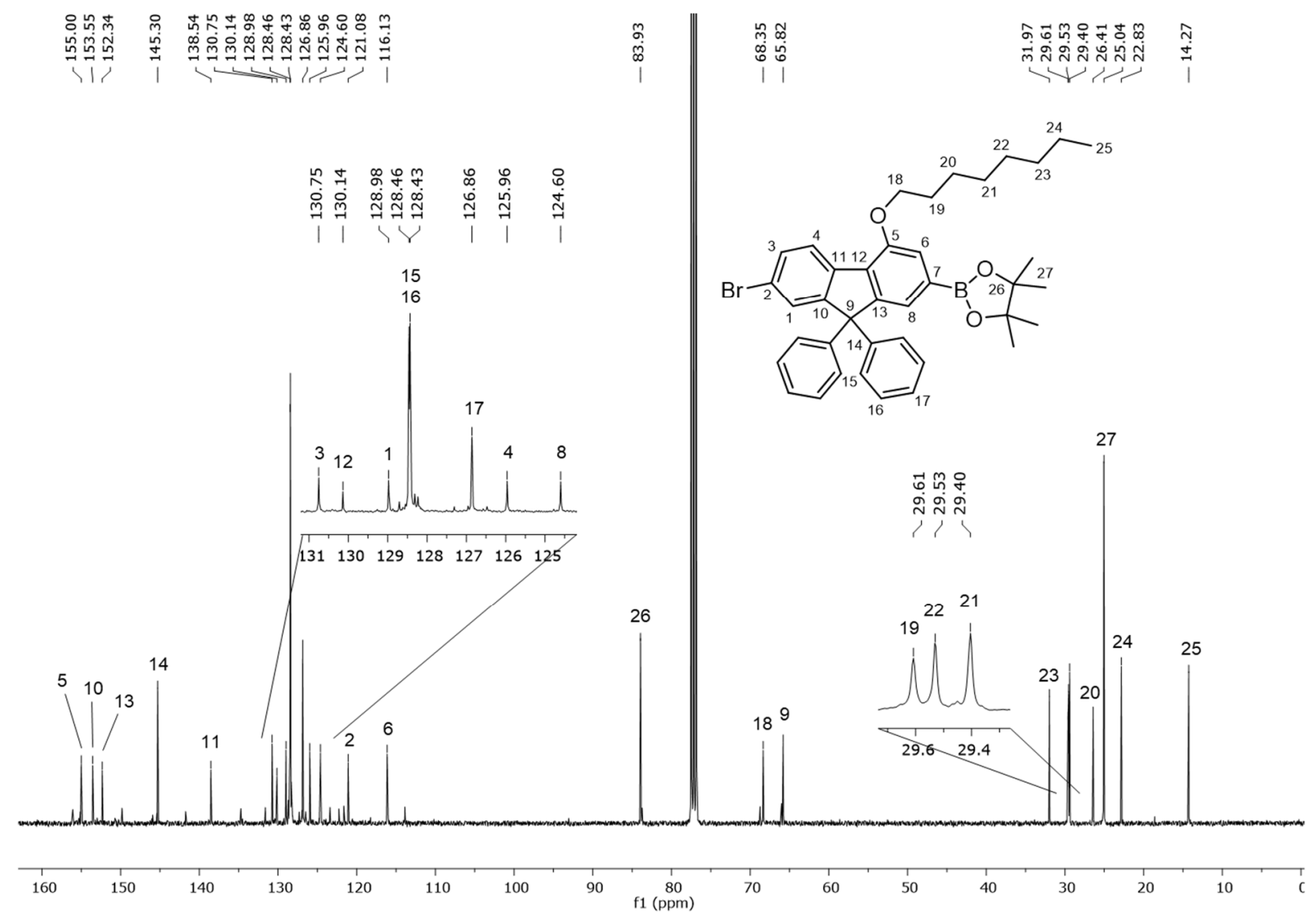

Figure 9.22: ${ }^{13} \mathrm{C}-\mathrm{NMR}$ spectrum $\left(101 \mathrm{MHz}, \mathrm{CDCl}_{3}, 25^{\circ} \mathrm{C}\right)$ of $\mathrm{Br}-\mathrm{ODPF}-\mathrm{BP}$ in with assigned signals. 


\subsubsection{POLYDIARYLFLUORENE BY EMULSION POLYMERIZATION}
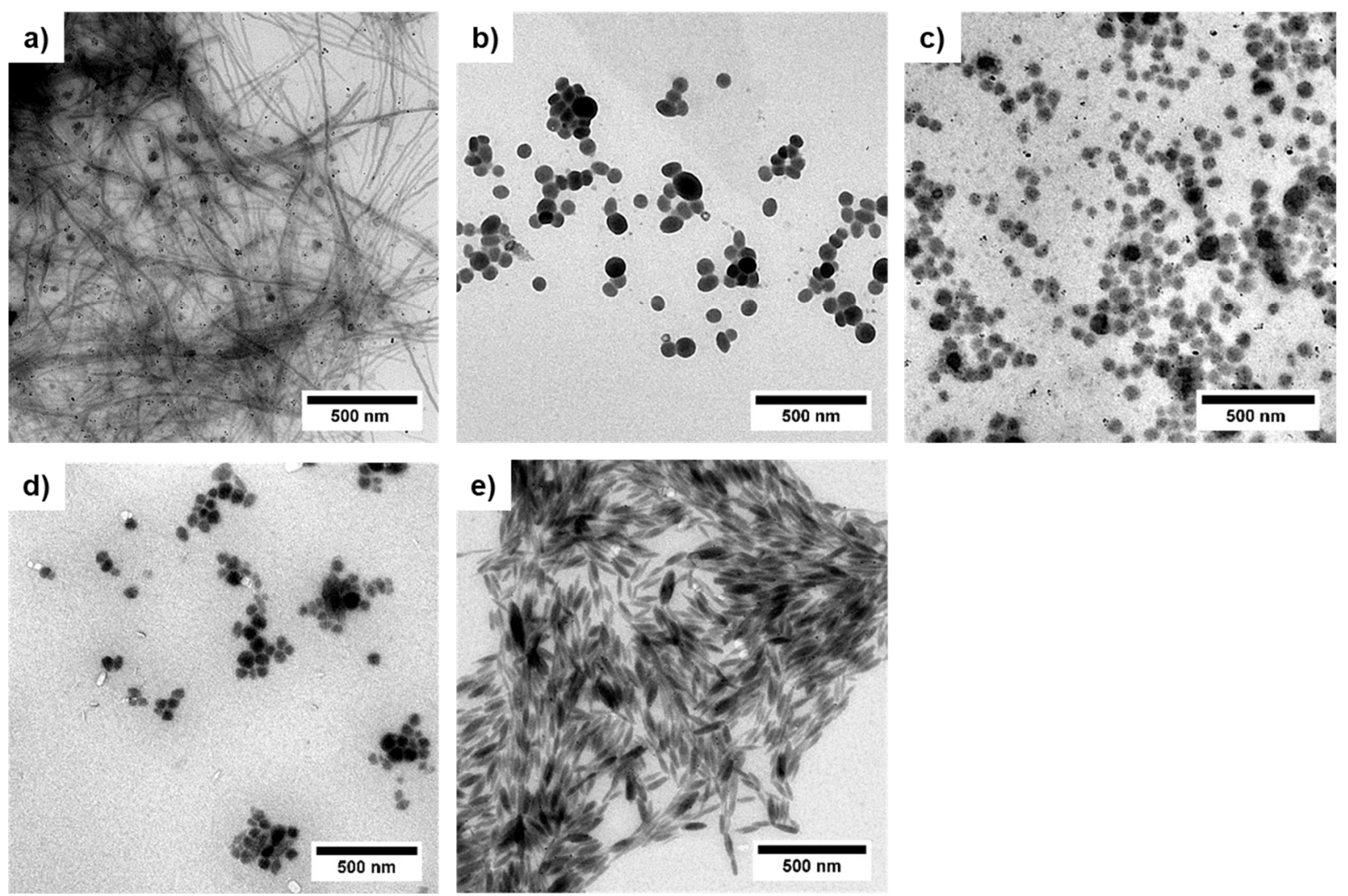

Figure 9.23: TEM images prepared from dispersions of PODPF, PODPF-PF8 copolymers and PF8 synthesized by heterophase Suzuki-Miyaura polycondensation, corresponding to Table 5.1, (a) entry 1, (b) entry 2, (c) entry 3, (d) entry 4 and (e) entry 5. 
a)

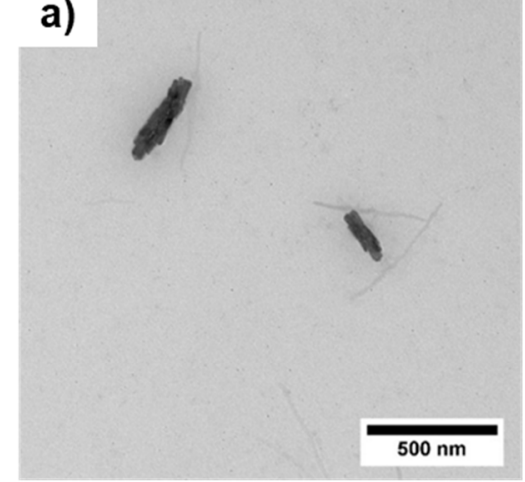

\section{d)}

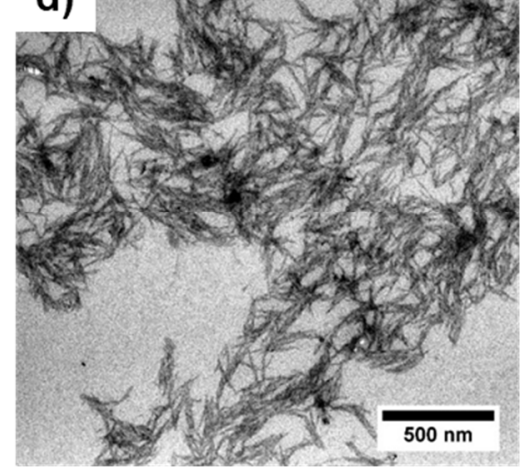

\section{g)}

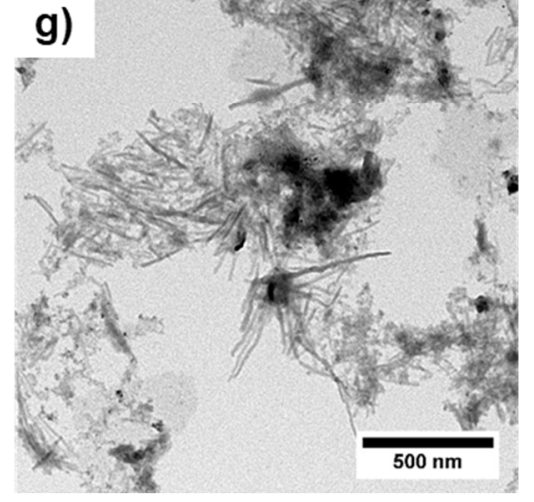

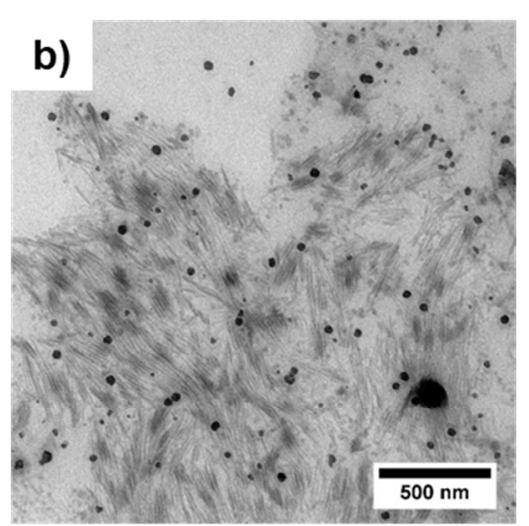

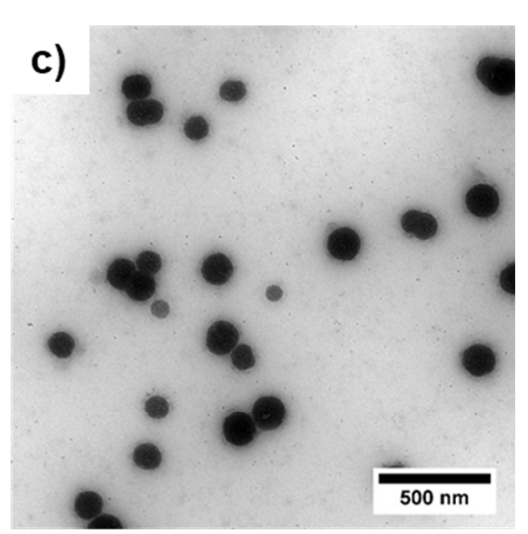

\section{e)}
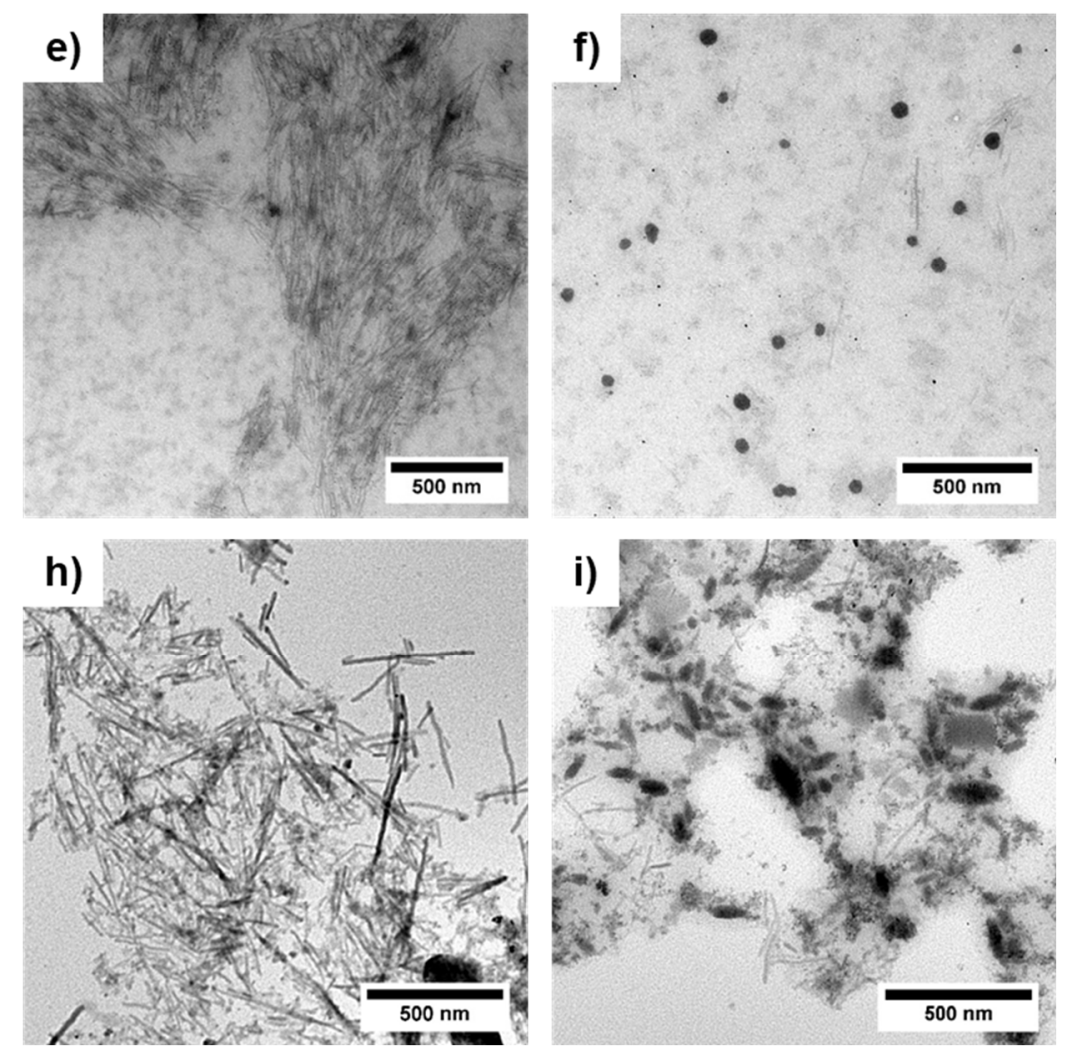

Figure 9.24: TEM images of dispersions obtained from heterophase Suzuki-Miyaura polycondensation of AA/BBtype diarylfluorene monomers under varying reaction conditions, corresponding to the entries of Table 5.2: (a) entry 2, (b) entry 3, (c) entry 4, (d) entry 5, (e) entry 6, (f) entry 7, (g) entry 8, (h) entry 9 and (i) entry 10. 

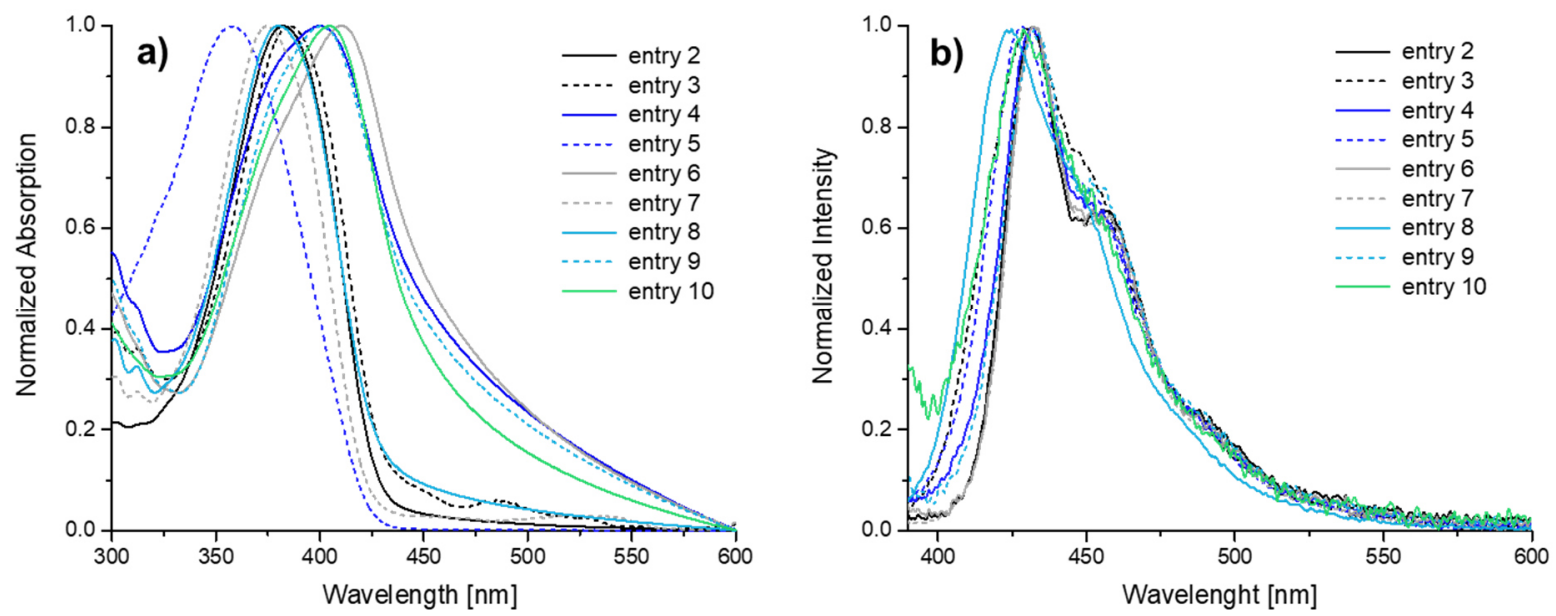

Figure 9.25: (a) UV/Vis absorption and (b) fluorescence emission spectra of dispersions obtained from heterophase Suzuki-Miyaura polycondensation of $A A / B B$-type diarylfluorene monomers under varying reaction conditions, corresponding to the entries 2-10 of Table 5.2.
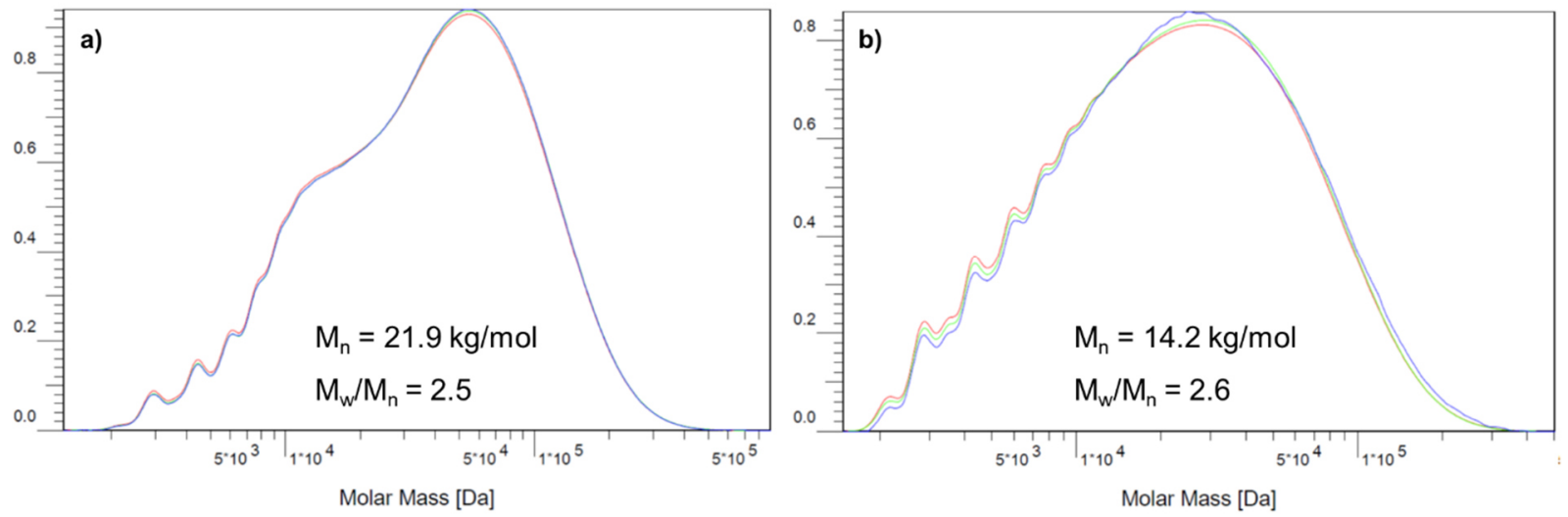

Figure 9.26: Exemplary GPC traces of PODPF prepared under the reaction conditions given in Table 5.2: (a) entry 1 and (b) entry 11, acquired by UV detection (red and green line) and RI detection (blue line) in THF vs. PS standards. 


\subsubsection{POST-POLYMERIZATION PARTICLE SYNTHESES}
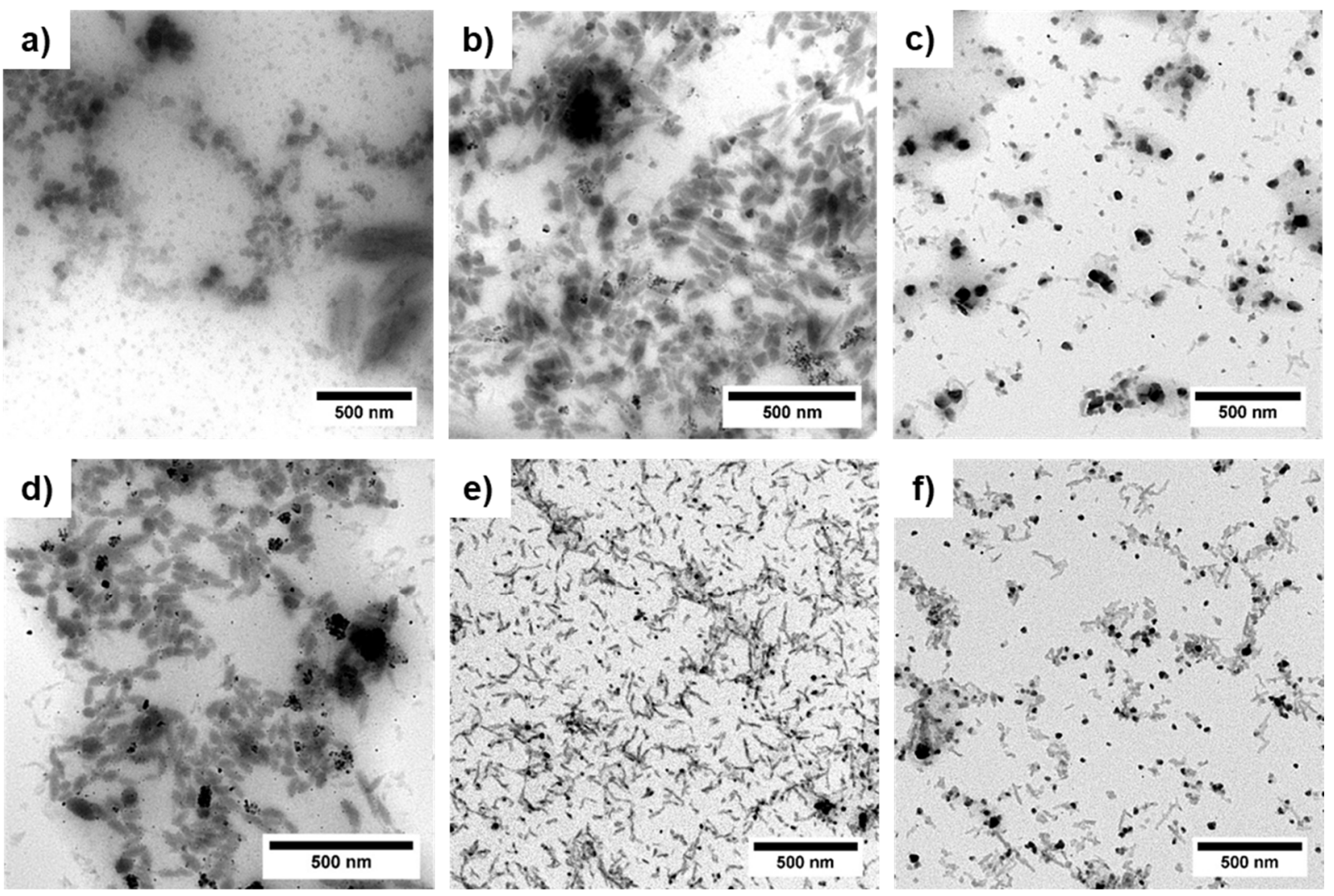

Figure 9.27: TEM images of PODPF dispersions obtained from post-polymerization particle syntheses under varying reaction conditions listed in Table 5.3: (a) entry 1, (b) entry 2, (c) entry 3, (d) entry 4, (e) entry 6, (f) entry 7.
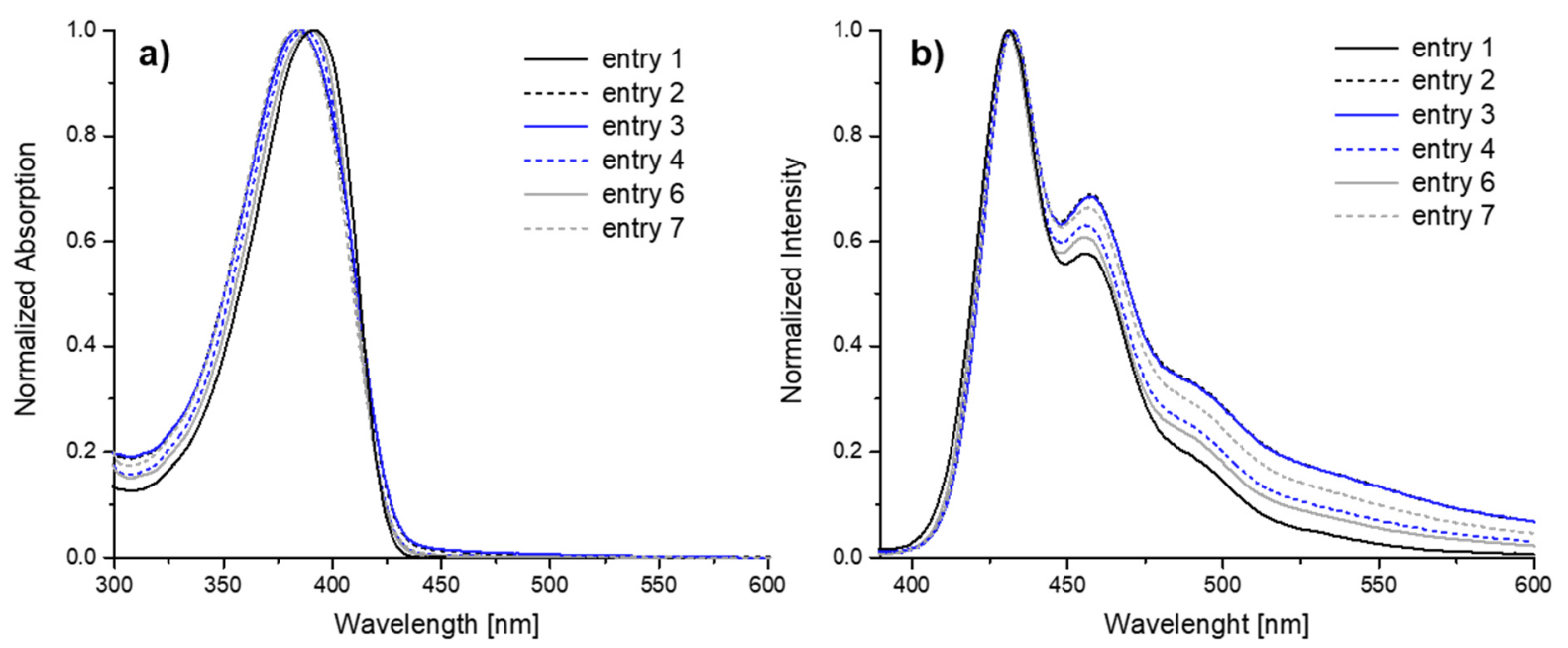

Figure 9.28: (a) UVNis absorption and (b) fluorescence emission spectra of PODPF dispersions obtained from post-polymerization particle syntheses under varying reaction conditions corresponding to the entries in Table 5.3. 


\subsection{AMPHIPHILIC BLOCK COPOLYMERS}

\subsubsection{SYNTHESES OF MACROINITIATORS}

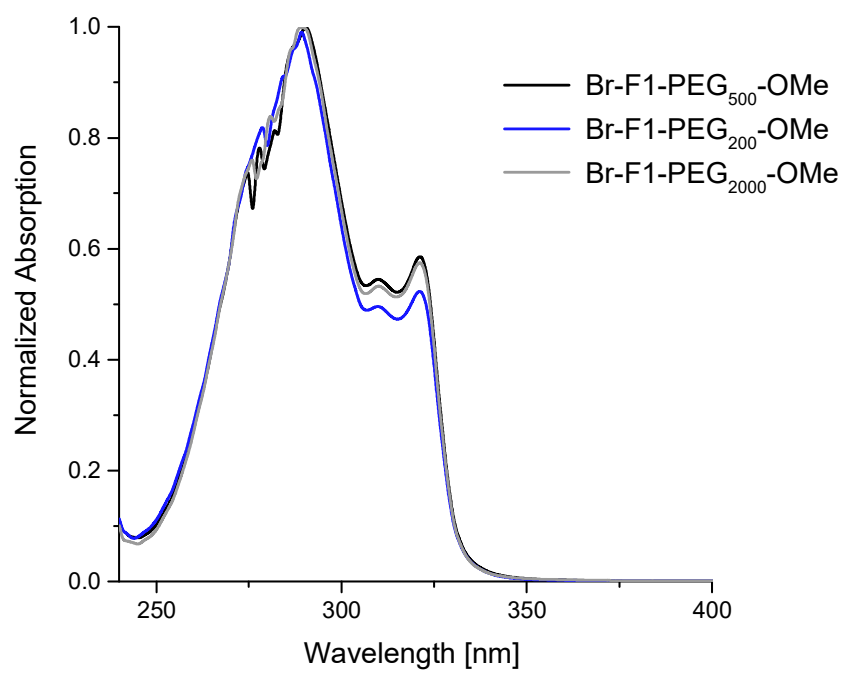

Figure 9.29: UV/Vis absorption spectra of PEGylated bromofluorene macromolecules dissolved in THF (c $\leq 0.01 \mathrm{mg} / \mathrm{mL})$.
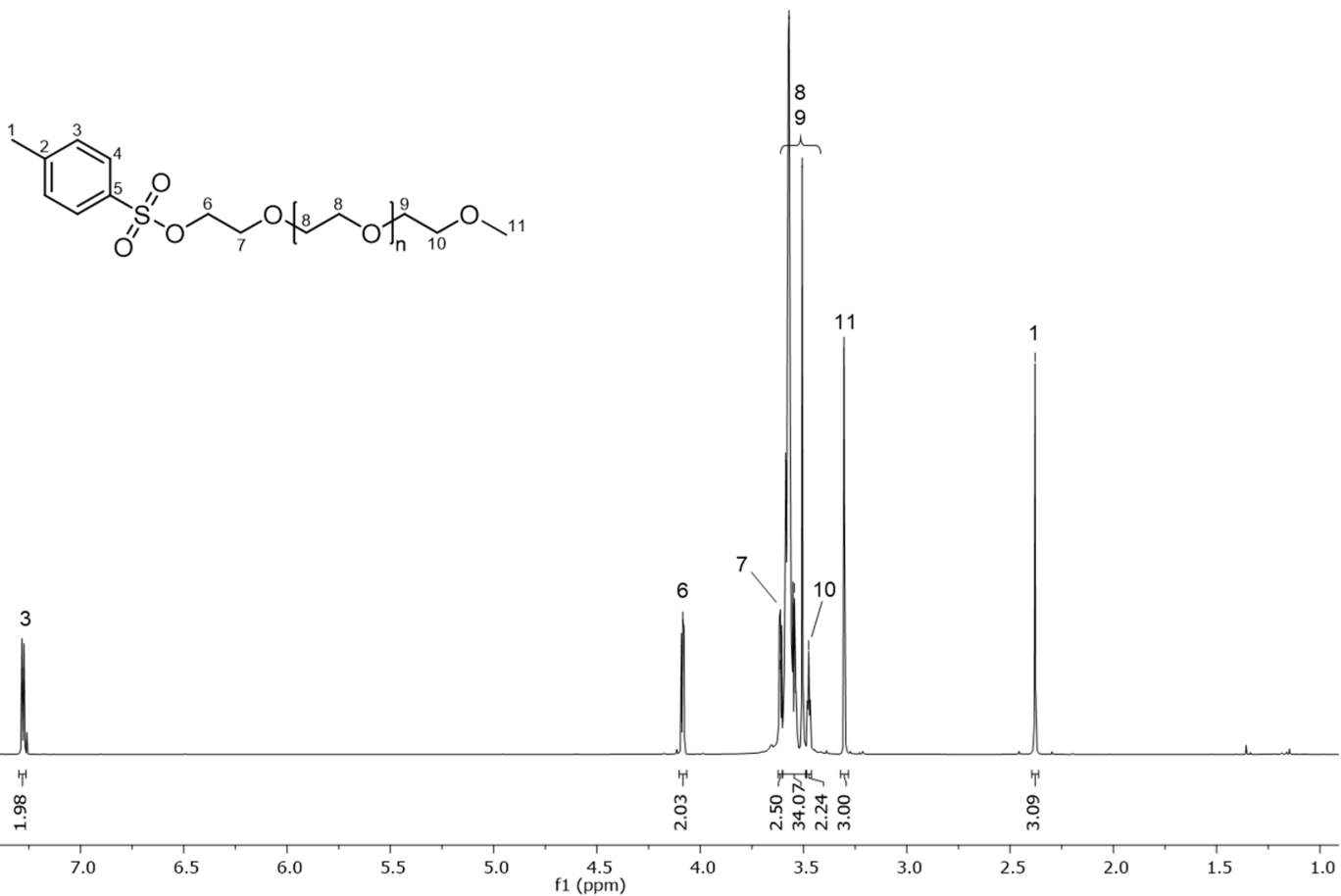

Figure 9.30: ${ }^{1} \mathrm{H}-\mathrm{NMR}$ spectrum $\left(400 \mathrm{MHz}, \mathrm{CDCl}_{3}, 25^{\circ} \mathrm{C}\right)$ of tosylated poly(ethylene glycol) monomethyl ether, using the example of TsO-PEG $500-\mathrm{OMe}$, with assigned signals. 
APPENDIX

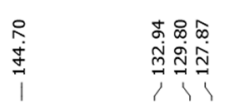

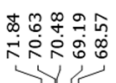

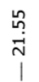
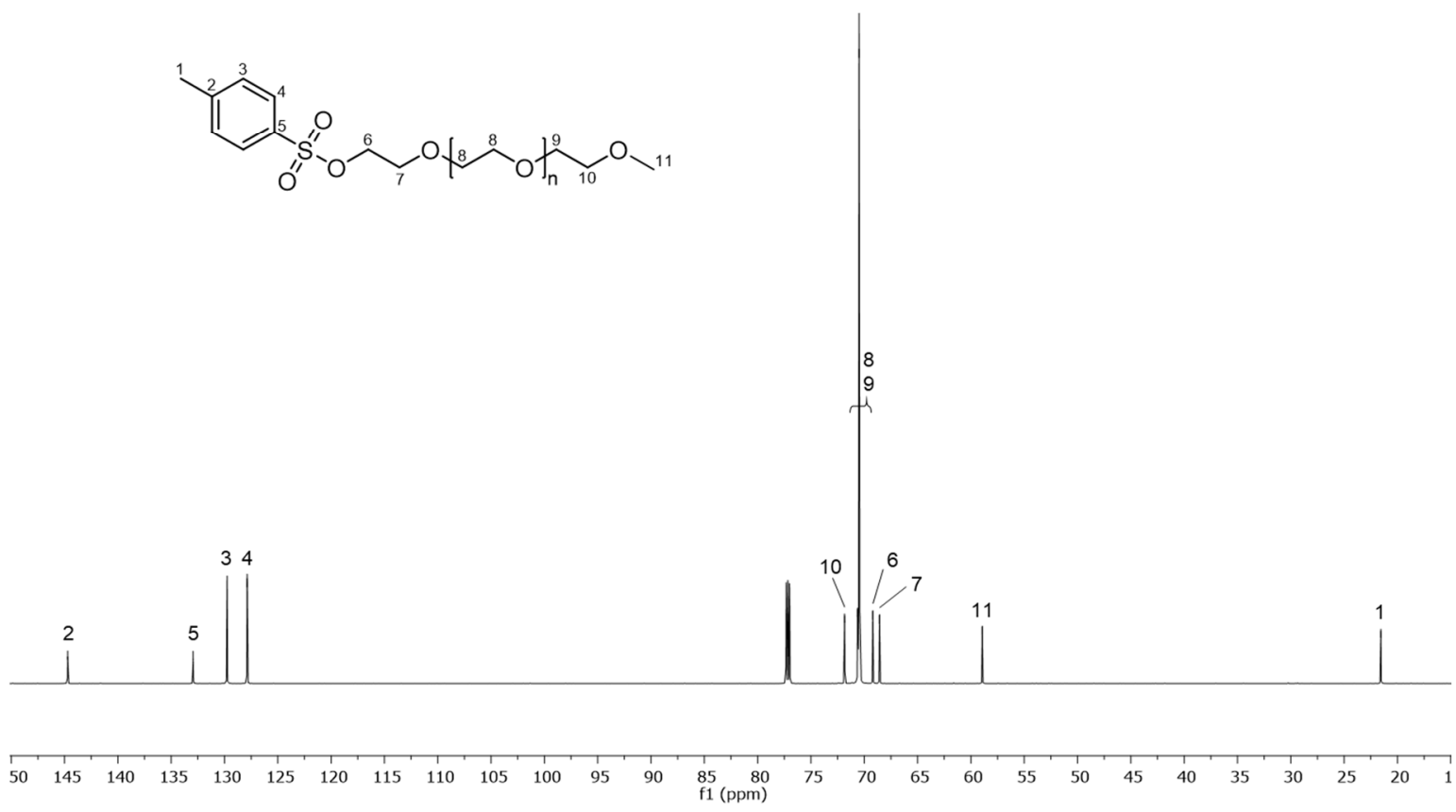

Figure 9.31: ${ }^{13} \mathrm{C}-\mathrm{NMR}$ spectrum $\left(101 \mathrm{MHz}, \mathrm{CDCl}_{3}, 25^{\circ} \mathrm{C}\right)$ of tosylated polyethylene glycol), using the example of TSO-PEG500-OMe with assigned signals.

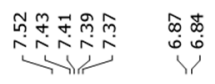
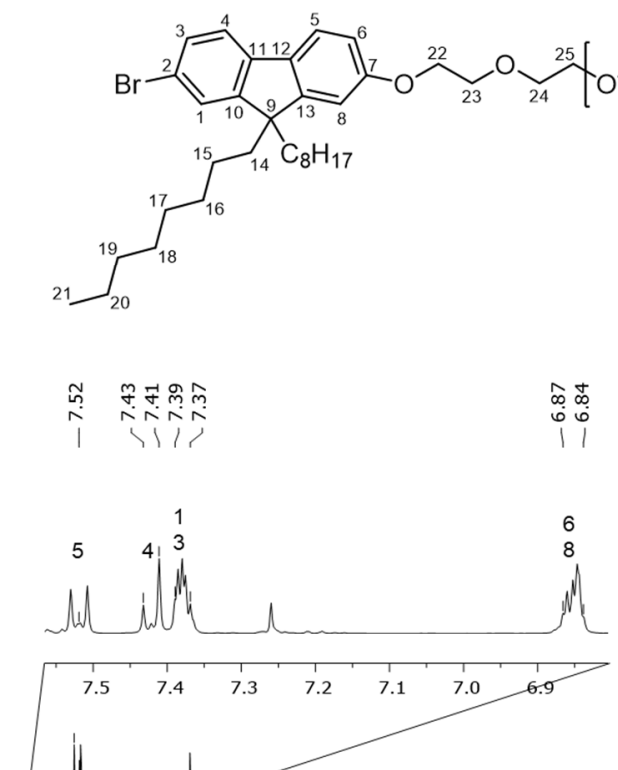


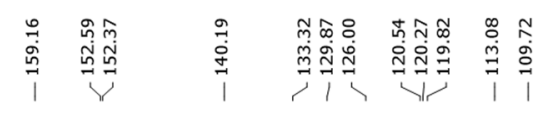

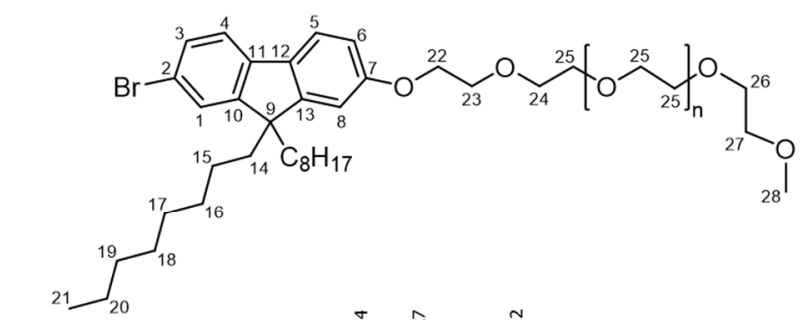

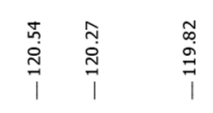
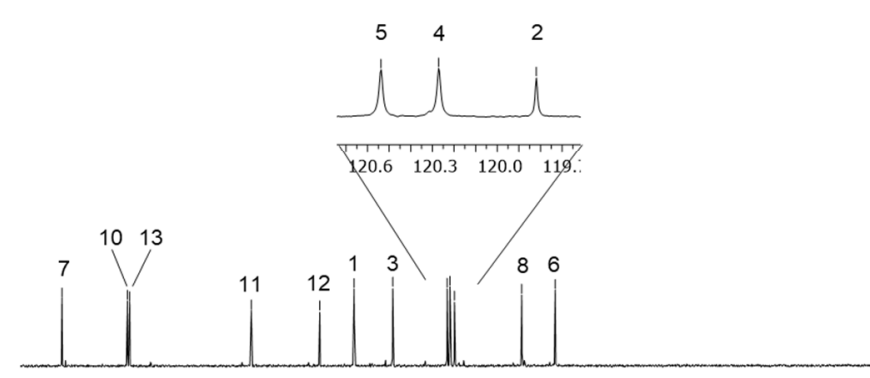

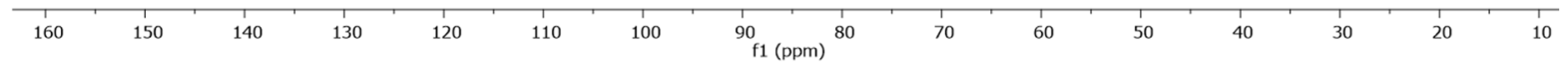

Figure 9.33: ${ }^{13} \mathrm{C}-\mathrm{NMR}$ spectrum $\left(101 \mathrm{MHz}, \mathrm{CDCl} 3,25^{\circ} \mathrm{C}\right)$ of poly(ethylene glycol) monomethyl ether, endfunctionalized with one fluorene moiety, shown on the example of $B r-F_{1}-P E G_{500}-O M e$, with assigned signals.

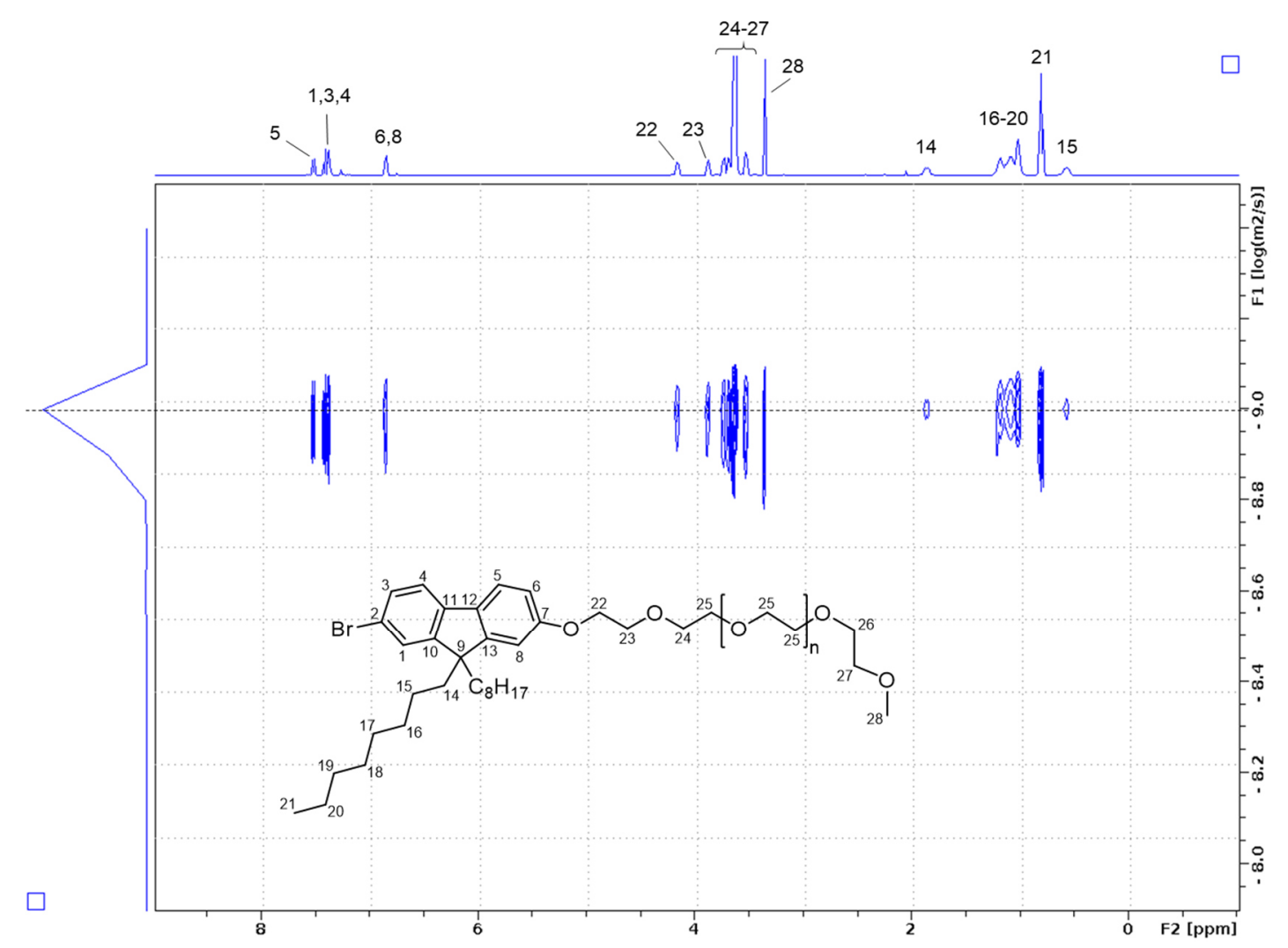

Figure 9.34: Exemplary DOSY-NMR spectrum (400 MHz, CDCl $\left.3,25^{\circ} \mathrm{C}\right)$ of poly(ethylene glycol) monomethyl ether, end-functionalized with one fluorene moiety $\left(B r-F_{1}-P E G_{500}-O M e\right)$. The dashed line serves as a visual guide to show that all signals caused by the macromolecule have the same diffusion behavior. 


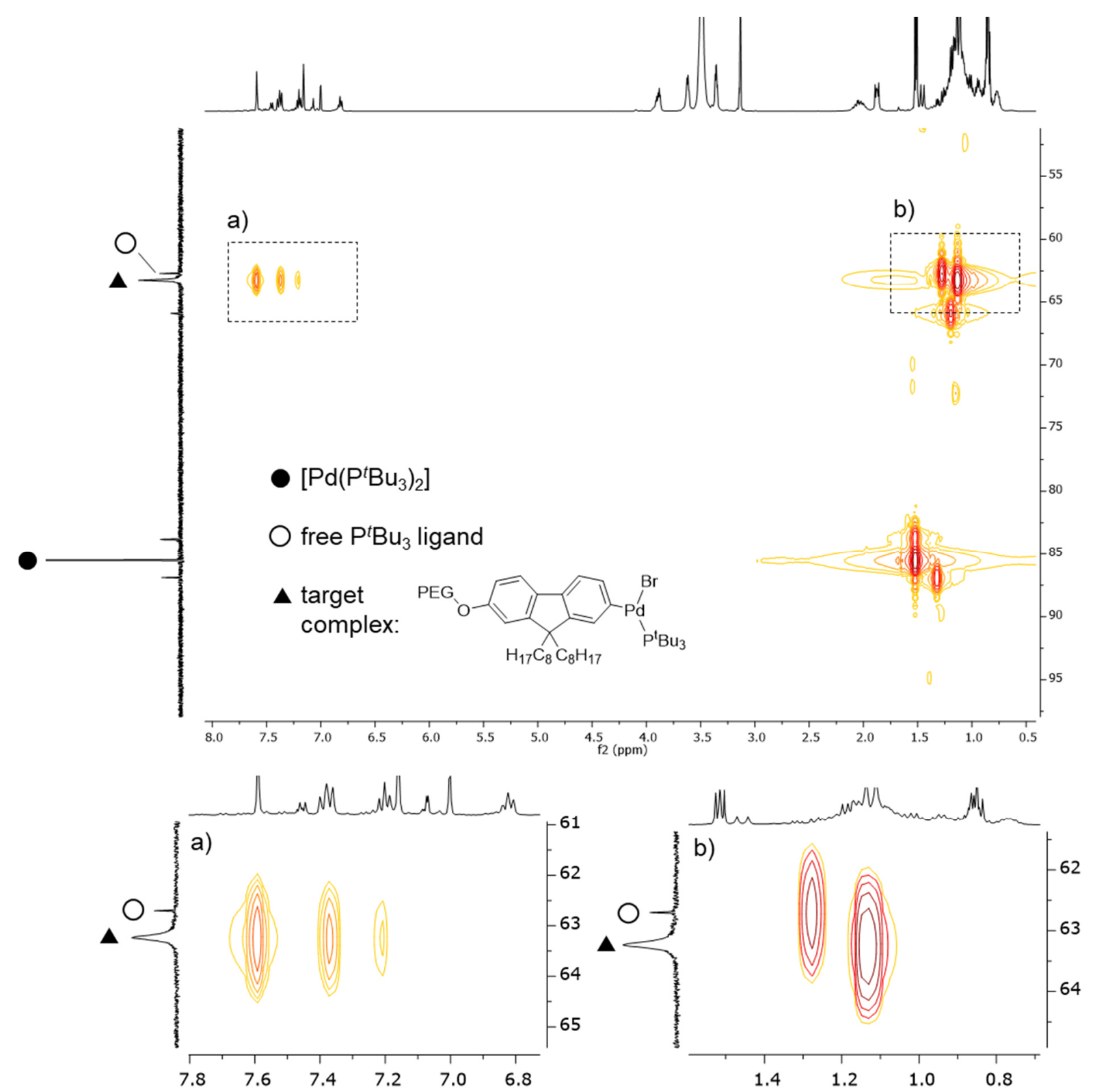

Figure 9.35: ${ }^{1} \mathrm{H},{ }^{31} \mathrm{P}-\mathrm{HMBC}$ NMR spectrum acquired for the reaction mixture of preliminary studies on the oxidative addition between $\mathrm{Br}-\mathrm{F}_{1}-\mathrm{PEG} \mathrm{G}_{500}-\mathrm{OH}$ and $\left[\mathrm{Pd}\left(\mathrm{P}^{t} \mathrm{Bu}_{3}\right)_{2}\right]$ with enlarged sections of $(\mathrm{a})$ the aromatic and $(\mathrm{b})$ the aliphatic region, with assignment of the different phosphorus species. Reaction conditions: $\left[\mathrm{Pd}\left(\mathrm{P}^{t} \mathrm{Bu} \mathrm{u}_{3}\right)_{2}\right](40 \mu \mathrm{mol}, 1$ equiv.) and $\mathrm{Br}_{-} \mathrm{F}_{1}-\mathrm{PEG} \mathrm{G}_{500}-\mathrm{OH}\left(80 \mu \mathrm{mol}, 2\right.$ equiv.) in $\mathrm{C}_{6} \mathrm{D}_{6}(2 \mathrm{~mL}), 70^{\circ} \mathrm{C}, 4 \mathrm{~h}$. 


\subsubsection{CONTROLLED SUZUKI-MIYAURA COUPLING POLYMERIZATION}
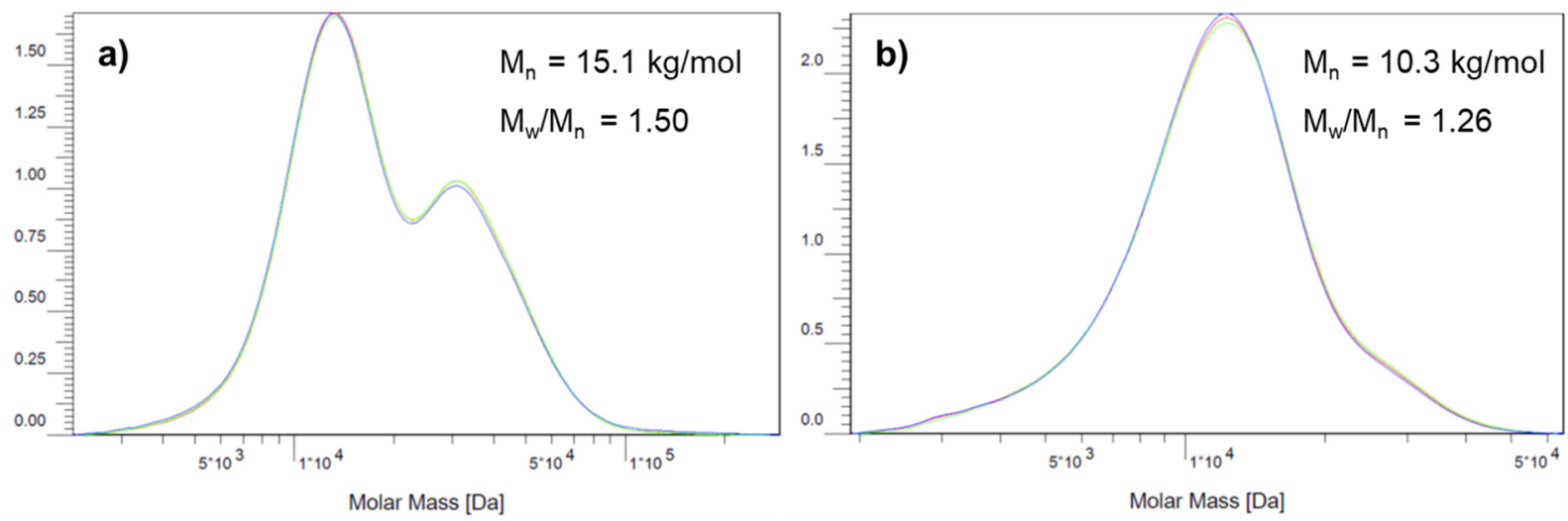

Figure 9.36: Exemplary GPC traces of amphiphilic PEG-PF8 block copolymers (a) prepared according to a published procedure ${ }^{72}$ and (b) prepared under optimized reaction conditions including initiation time and reaction time of 15 min each. Data are acquired by UV detection (red and green line) and RI detection (blue line) in THF vs. PS standards.

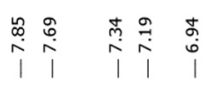

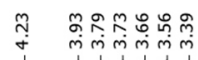

$\stackrel{\sim}{i}$

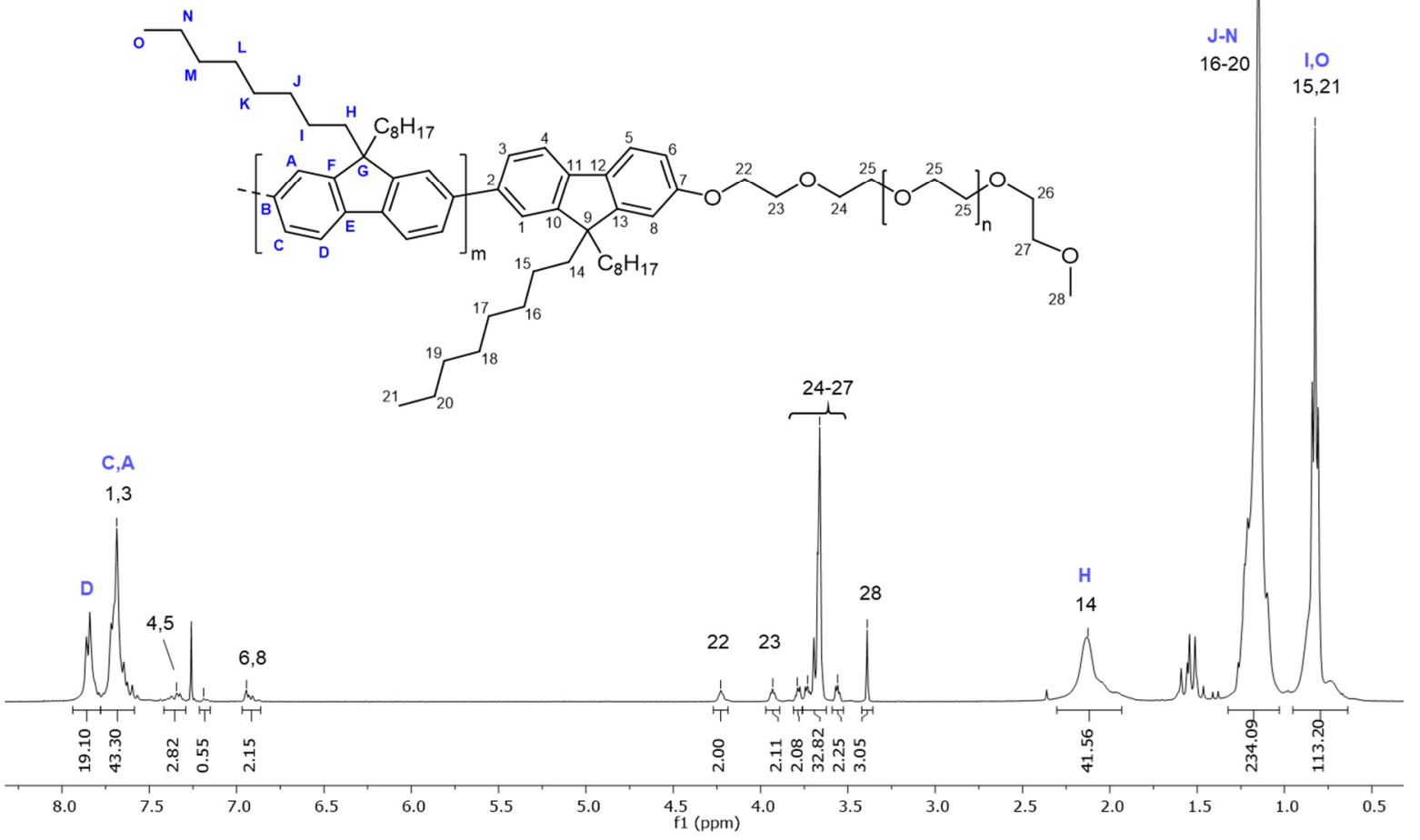
1) 1 1 1

Figure 9.37: ${ }^{1} \mathrm{H}-\mathrm{NMR}$ spectrum $\left(400 \mathrm{MHz}, \mathrm{CDCl} 3,25^{\circ} \mathrm{C}\right)$ of amphiphilic PF8-PEG block-copolymer obtained from controlled Suzuki-Miyaura coupling polymerization, corresponding to Table 6.1, polymer 3, with assigned signals. 


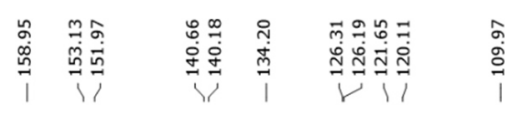
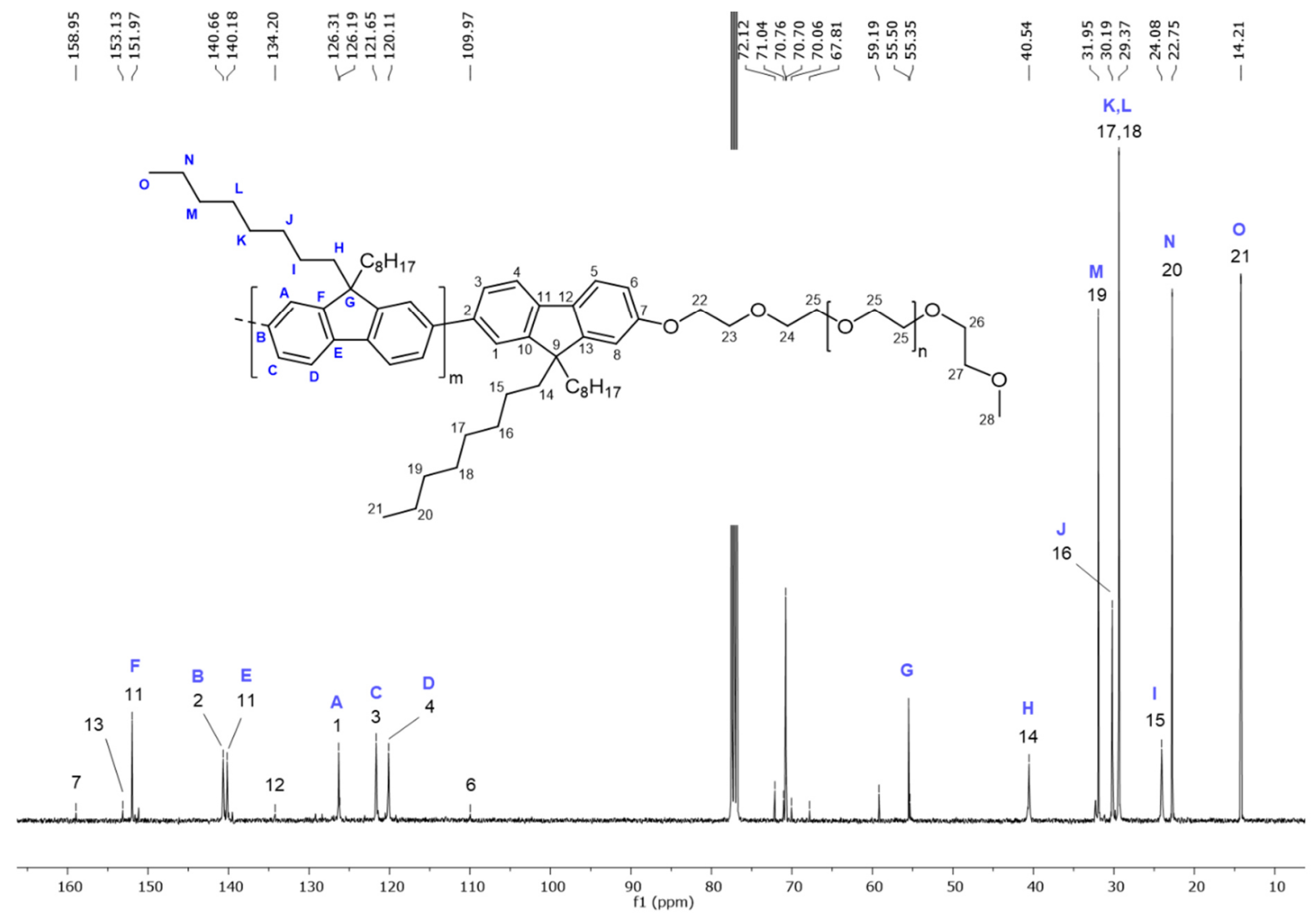

Figure 9.38: ${ }^{13} \mathrm{C}-\mathrm{NMR}$ spectrum $\left(101 \mathrm{MHz}, \mathrm{CDCl}_{3}, 25^{\circ} \mathrm{C}\right)$ of amphiphilic PF8-PEG block-copolymer obtained from controlled Suzuki-Miyaura coupling polymerization, corresponding to Table 6.1, polymer 3, with assigned signals.

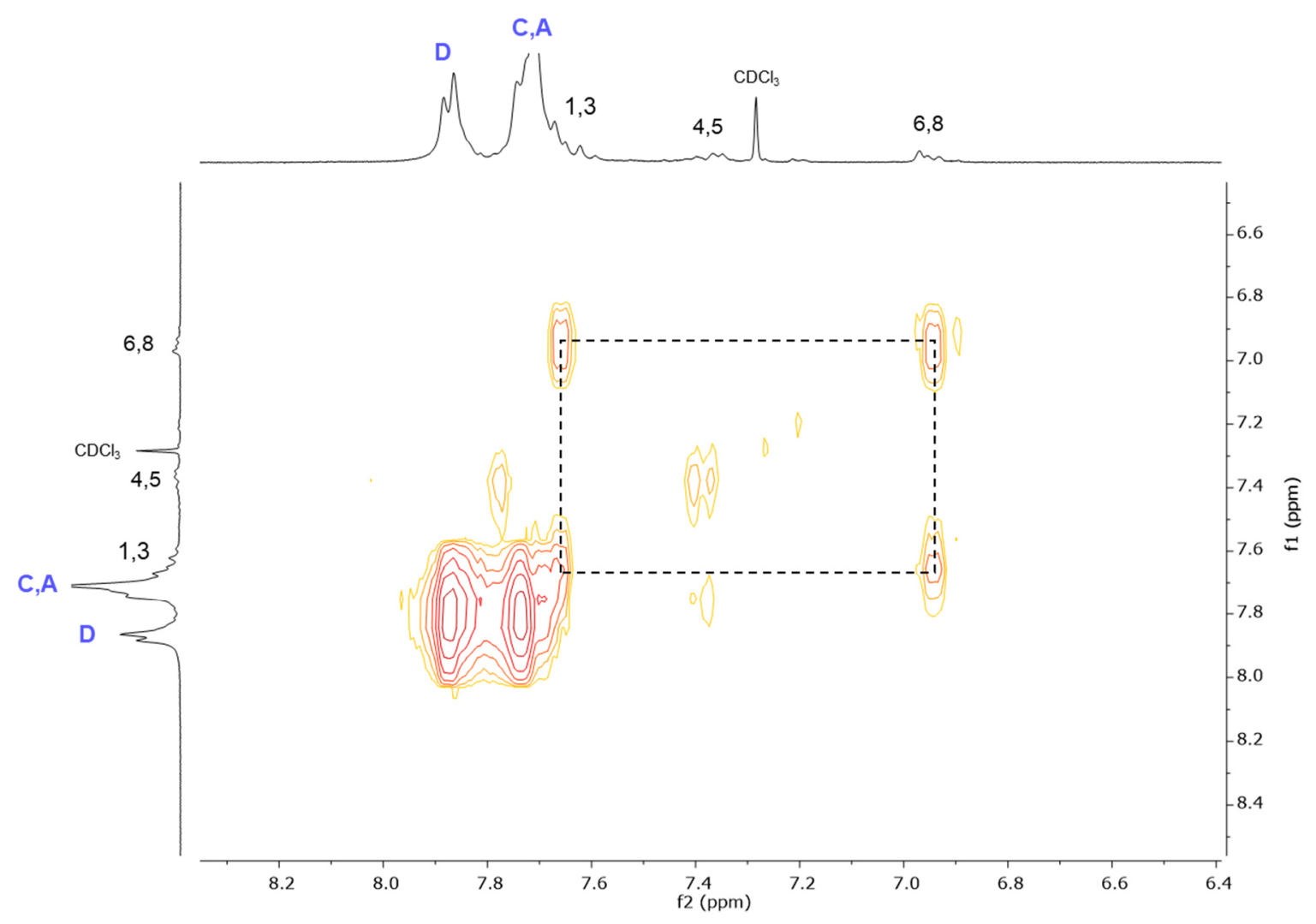

Figure 9.39: Enlarged section of spectrum recorded by ${ }^{1} \mathrm{H}-{ }^{1} \mathrm{H}$ correlated spectroscopy (COSY) measurements on amphiphilic PF8-PEG block-copolymer. Correlation of interest is marked by dashed lines. 


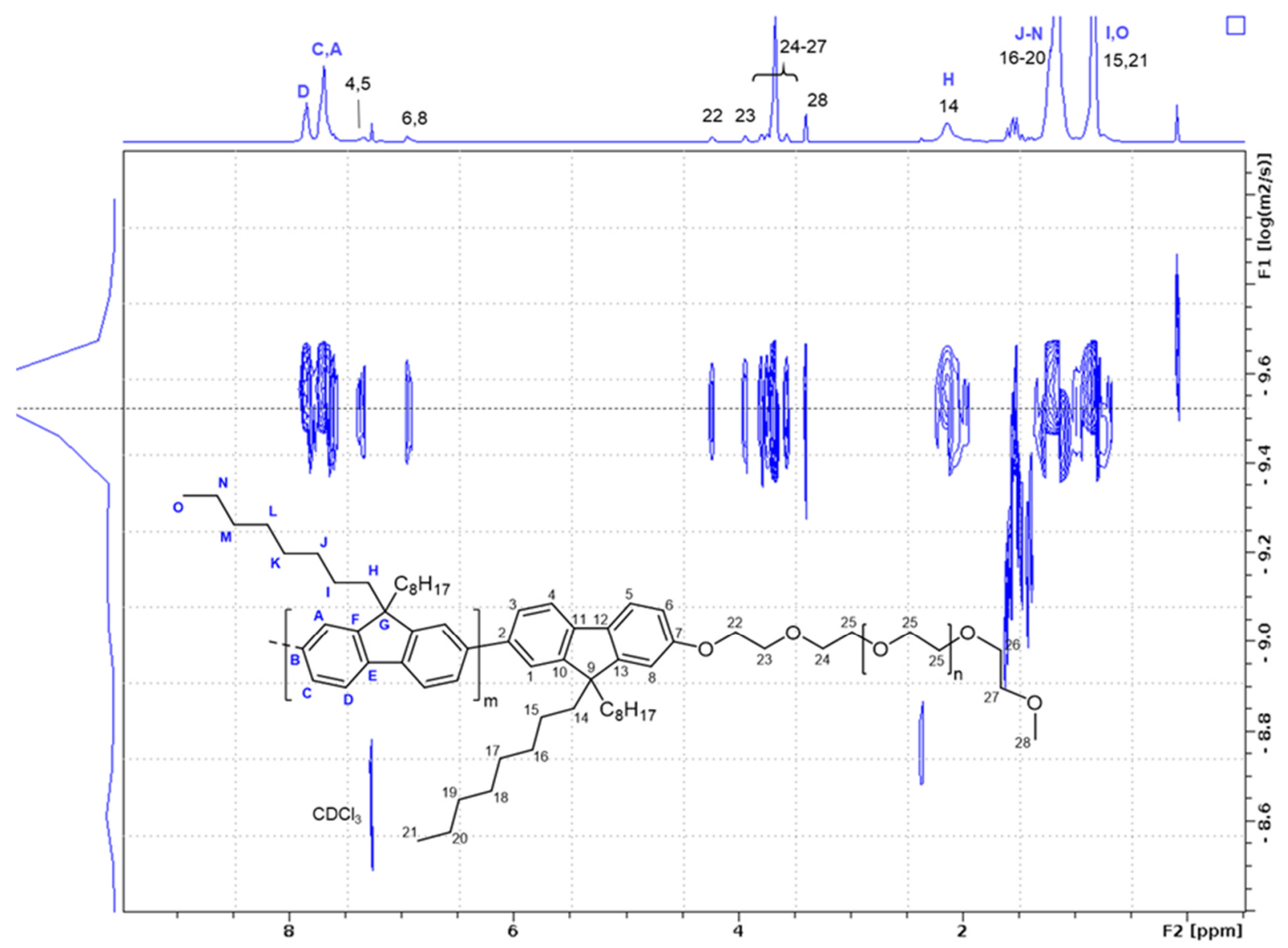

Figure 9.40: Exemplary DOSY-NMR spectrum (400 MHz, CDCl $\left.3,25^{\circ} \mathrm{C}\right)$ of amphiphilic PF8-PEG block-copolymer obtained from controlled Suzuki-Miyaura coupling polymerization, corresponding to Table 6.1, polymer 3. The dashed line serves as a visual guide to show that all signals caused by the macromolecule have the same diffusion behavior.
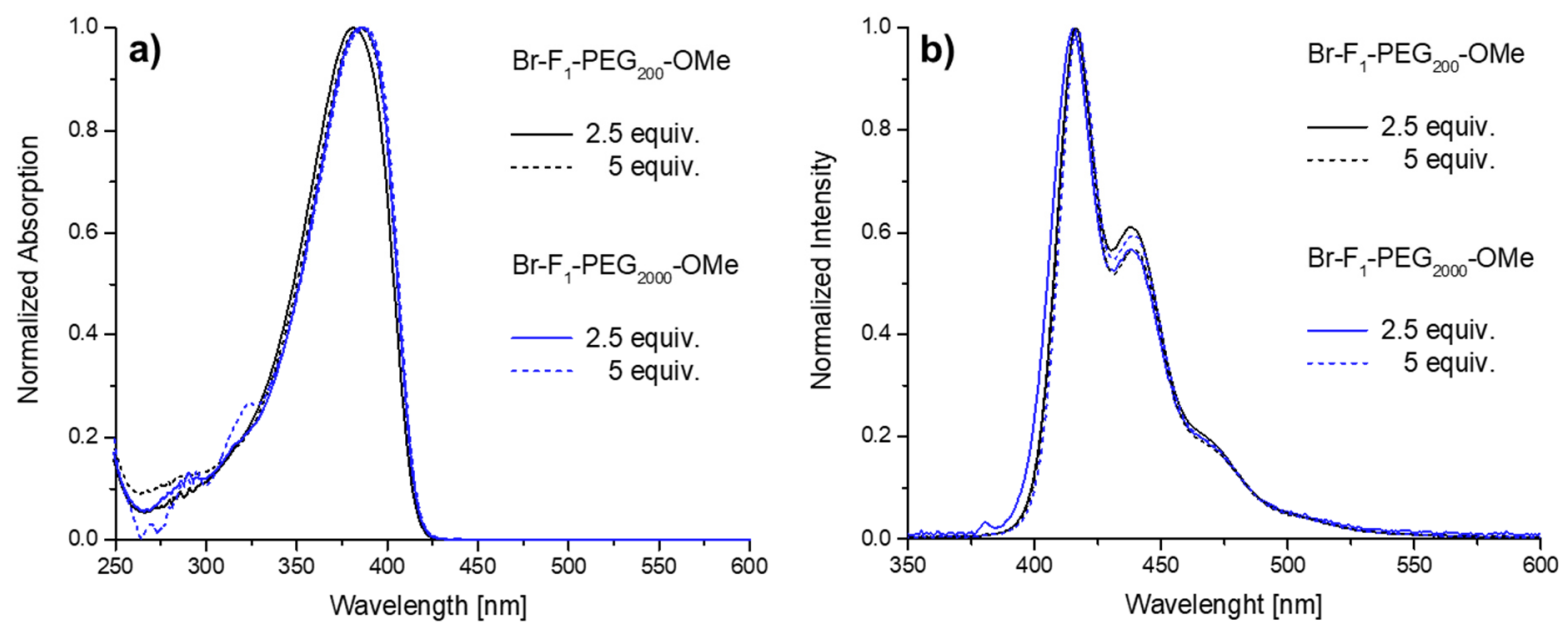

Figure 9.41: (a) UVNis absorption and (b) fluorescence emission spectra ( $\lambda_{e x c}=380 \mathrm{~nm}$ ) of amphiphilic PF8-PEG block-copolymers synthesized via controlled Suzuki-Miyaura coupling polymerization using Br-F $-P \mathrm{EG}_{200-O M e}$ and $B r-F_{1}-P E G_{2000}-O M e$ as initiating macromolecules, corresponding with Table 6.2. Copolymers were dissolved in THF for measurements (c $\leq 0.01 \mathrm{mg} / \mathrm{mL}$ ). 


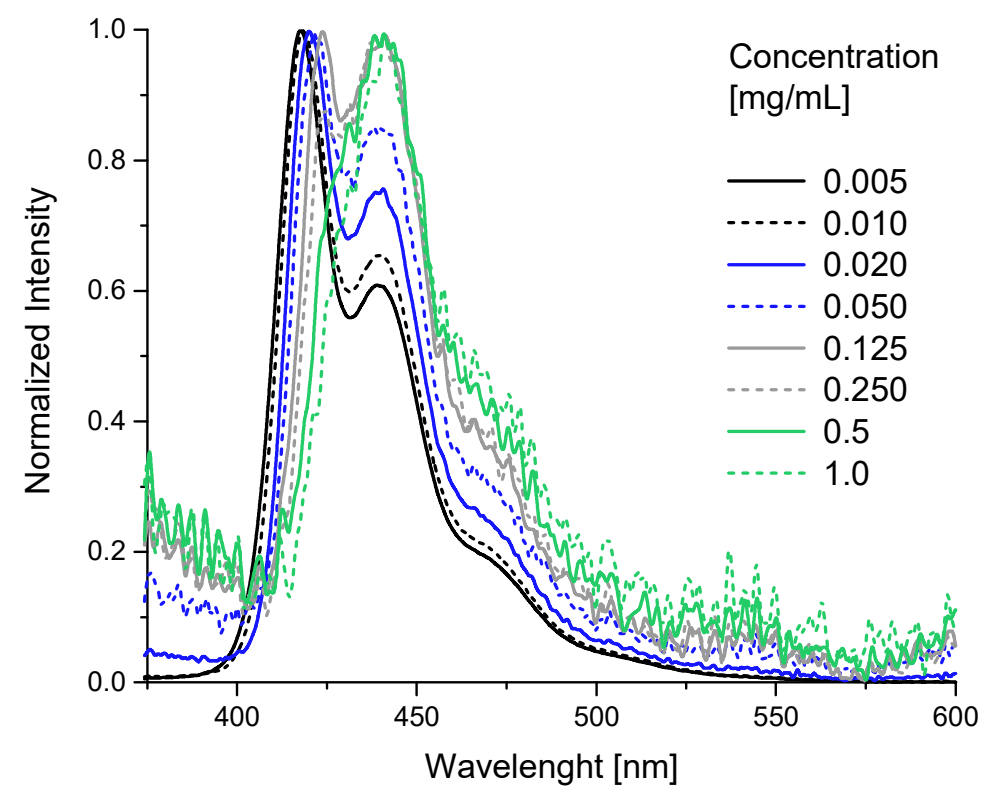

Figure 9.42: Fluorescence emission spectra recorded from a concentration series of amphiphilic PF8-PEG copolymer 3 (Table 6.1), dissolved in tetrahydrofuran. Spectra were recorded with an excitation wavelength of $\lambda_{\text {exc }}=380 \mathrm{~nm}$, an exposure time of $50 \mathrm{~ms}$ and averaging over 50 measurement runs.

\subsubsection{AGGREGATION BEHAVIOR OF AMPHIPHILIC COPOLYMERS}

Table 9.2: Experiments on aggregation behavior of several amphiphilic copolymers in solution. ${ }^{\text {a }}$ If possible, the average lamella thickness was determined by TEM. Polymers corresponding with entries in Table 6.1, Table 6.2 and Table 6.3.

\begin{tabular}{|c|c|c|c|c|}
\hline $\begin{array}{l}\text { polymer } \\
\text { number }\end{array}$ & aryl halide & $\begin{array}{l}\text { AB-type } \\
\text { monomer }\end{array}$ & $\begin{array}{c}M_{\mathrm{n}}{ }^{b} \\
{[\mathrm{~kg} / \mathrm{mol}]}\end{array}$ & $\begin{array}{l}\text { average lamella } \\
\text { thickness [nm]/ } v^{c}\end{array}$ \\
\hline 1 & $\mathrm{Br}-\mathrm{F}_{1}-\mathrm{PEG} \mathrm{G}_{500}-\mathrm{OMe}$ & $\mathrm{Br}-\mathrm{F}_{1}-\mathrm{BPin}$ & 4.9 & - \\
\hline 3 & $\mathrm{Br}-\mathrm{F}_{1}-\mathrm{PEG} \mathrm{G}_{500}-\mathrm{OMe}$ & $\mathrm{Br}-\mathrm{F}_{1}$-BPin & 10.3 & $11.8 / 0.15$ \\
\hline 5 & $\mathrm{Br}-\mathrm{F}_{1}-\mathrm{PEG} \mathrm{G}_{500}-\mathrm{OMe}$ & $\mathrm{Br}-\mathrm{F}_{1}$-BPin & 16.4 & - \\
\hline 9 & $\mathrm{Br}-\mathrm{F}_{1}-\mathrm{PEG} \mathrm{G}_{200}-\mathrm{OMe}$ & $\mathrm{Br}-\mathrm{F}_{1}$-BPin & 6.0 & - \\
\hline 11 & $\mathrm{Br}-\mathrm{F}_{1}-\mathrm{PEG}_{2000}-\mathrm{OMe}$ & $\mathrm{Br}-\mathrm{F}_{1}$-BPin & 10.6 & $9.0 / 0.10$ \\
\hline 13 & $\mathrm{Br}-\mathrm{F}_{1}-\mathrm{PEG} \mathrm{G}_{500}-\mathrm{OMe}$ & Br-ODPF-BPin & 5.8 & $9.6 / 0.14$ \\
\hline 14 & $\mathrm{Br}-\mathrm{F}_{1}-\mathrm{PEG} \mathrm{G}_{500}-\mathrm{OMe}$ & Br-M3-BPin & 4.5 & - \\
\hline 15 & $\mathrm{Br}-\mathrm{F}_{1}-\mathrm{PEG} \mathrm{G}_{500}-\mathrm{OMe}$ & Br-M4-BPin & 7.0 & $9.1 / 0.14$ \\
\hline 16 & $\mathrm{Br}-\mathrm{F}_{1}-\mathrm{PEG} \mathrm{G}_{500}-\mathrm{OMe}$ & Br-M5-BPin & 7.9 & $5.5 / 0.13$ \\
\hline
\end{tabular}

${ }^{a}$ Conditions: Dissolution of respective copolymer $(1 \mathrm{mg})$ in THF $(2.5 \mathrm{~mL})$, filtration via $0.45 \mu \mathrm{m}$ syringe filter, dropwise addition of $\mathrm{MeOH}(0.25 \mathrm{~mL})$, shaking for $10 \mathrm{~s}$, aging for $24 \mathrm{~h}$ at rt. ${ }^{b}$ Determined by GPC with RI detection vs. PS standards. "Mean values of lengths and coefficient of variation $v$ as calculated from 50 measurements. 

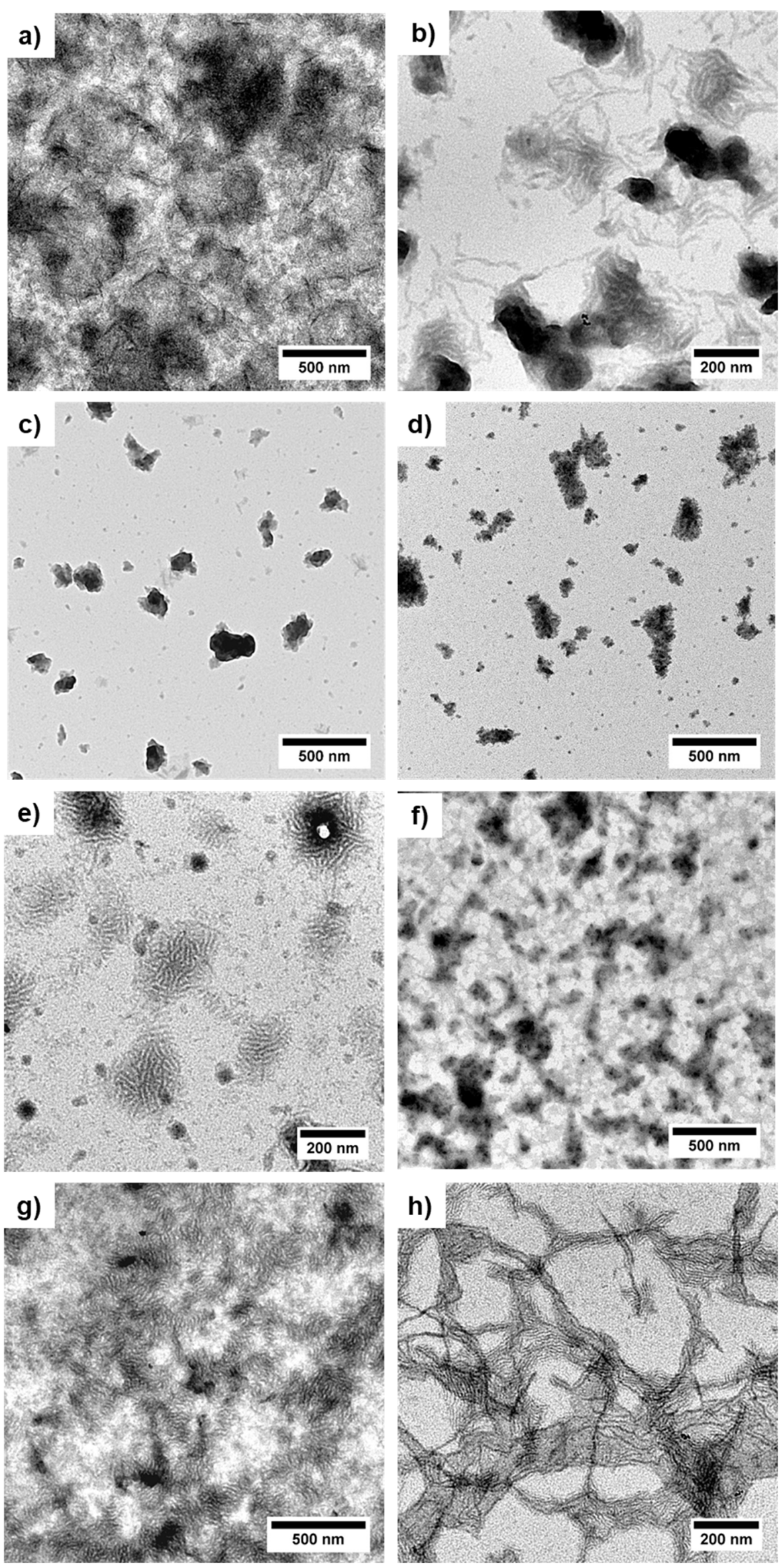

Figure 9.43: TEM images of samples corresponding to entries of Table 9.2: (a) polymer 1, (b) polymer 3, (c) polymer 5, (d) polymer 9, (e) polymer 13, (f) polymer 14, (g) polymer 15 and (h) polymer 16. 


\section{$1 \mathbf{0}_{\text {References }}$}

(1) Burroughes, J. H.; Bradley, D. D. C.; Brown, A. R.; Marks, R. N.; Mackay, K.; Friend, R. H.; Burns, P. L.; Holmes, A. B. Light-Emitting Diodes Based on Conjugated Polymers. Nature 1990, 347, 539-541.

(2) Friend, R. H.; Gymer, R. W.; Holmes, A. B.; Burroughes, J. H.; Marks, R. N.; Taliani, C.; Bradley, D. D. C.; Dos Santos, D. A.; Bredas, J. L.; Lögdlund, M. Electroluminescence in Conjugated Polymers. Nature 1999, 397, 121.

(3) Pecher, J.; Mecking, S. Nanoparticles of Conjugated Polymers. Chem. Rev. 2010, 110, 6260-6279.

(4) Edwards, J.; Fisher, R.; Vincent, B. Polyacetylene Latices: Preparation and Characterisation. Makromol. Chem. Rapid Comm. 1983, 4, 393-397.

(5) Sun, Y.; Xia, Y. Shape-Controlled Synthesis of Gold and Silver Nanoparticles. Science 2002, 298, 21762179.

(6) Thorkelsson, K.; Bai, P.; Xu, T. Self-Assembly and Applications of Anisotropic Nanomaterials: A Review. Nano Today 2015, 10, 48-66.

(7) Kuehne, A. J.; Gather, M. C.; Sprakel, J. Monodisperse Conjugated Polymer Particles by Suzuki-Miyaura Dispersion Polymerization. Nat. Commun. 2012, 3, 3:1088.

(8) Ruiz Perez, J. D.; Mecking, S. Anisotropic Polymer Nanoparticles with Tunable Emission Wavelengths by Intersegmental Chain Packing. Angew. Chem. Int. Ed. 2017, 56, 6147-6151.

(9) Da Como, E.; Scheler, E.; Strohriegl, P.; Lupton, J. M.; Feldmann, J. Single Molecule Spectroscopy of Oligofluorenes: How Molecular Length Influences Polymorphism. Appl. Phys. A 2009, 95, 61-66.

(10) Shiraki, T.; Shindome, S.; Toshimitsu, F.; Fujigaya, T.; Nakashima, N. Strong Main-Chain LengthDependence for the $\beta$-Phase Formation of Oligofluorenes. Polym. Chem. 2015, 6, 5103-5109.

(11) Feast, W. J.; Tsibouklis, J.; Pouwer, K. L.; Groenendaal, L.; Meijer, E. W. Synthesis, Processing and Material Properties of Conjugated Polymers. Polymer 1996, 37, 5017-5047.

(12) Shirakawa, H.; Louis, E. J.; MacDiarmid, A. G.; Chiang, C. K.; Heeger, A. J. Synthesis of Electrically Conducting Organic Polymers: Halogen Derivatives of Polyacetylene, $(\mathrm{CH})_{x}$. J. Chem. Soc. 1977, 578580.

(13) Friend, R. H.; Bradley, D. D. C.; Townsend, P. D. Photo-Excitation in Conjugated Polymers. J. Phys. D: Appl. Phys. 2000, 20, 1367-1384.

(14) Kraft, A.; Grimsdale, A. C.; Holmes, A. B. Electroluminescent Conjugated Polymers - Seeing Polymers in a New Light. Angew. Chem. Int. Ed. 1998, 37, 402-428.

(15) Leclerc, M.; Morin, J.-F. Design and Synthesis of Conjugated Polymers; John Wiley \& Sons: Weinheim, 2010.

(16) Burroughes, J. H.; Friend, R. H. The Semiconductor Device Physics of Polyacetylene. In Conjugated Polymers: The Novel Science and Technology of Highly Conducting and Nonlinear Optically Active Materials; Brédas, J. L., Silbey, R., Eds.; Springer Netherlands: Dordrecht, 1991; pp 555-622.

(17) Noriega, R.; Rivnay, J.; Vandewal, K.; Koch, F. P. V.; Stingelin, N.; Smith, P.; Toney, M. F.; Salleo, A. A General Relationship Between Disorder, Aggregation and Charge Transport in Conjugated Polymers. Nat. Mater. 2013, 12, 1038-1044.

(18) Bücker, D.; Sickinger, A.; Ruiz Perez, J. D.; Oestringer, M.; Mecking, S.; Drescher, M. Direct Observation of Chain Lengths and Conformations in Oligofluorene Distributions from Controlled Polymerization by Double Electron-Electron Resonance. J. Am. Chem. Soc. 2020, 142, 1952-1956. 
(19) Jiao, X.; Wang, C.; McNeill, C. R. Detecting the Onset of Molecular Reorganization in Conjugated Polymer Thin Films Using an Easily Accessible Optical Method. Macromolecules 2019, 52, 4646-4654.

(20) Peet, J.; Heeger, A. J.; Bazan, G. C. "Plastic" Solar Cells: Self-Assembly of Bulk Heterojunction Nanomaterials by Spontaneous Phase Separation. Acc. Chem. Res. 2009, 42, 1700-1708.

(21) Neher, D. Polyfluorene Homopolymers: Conjugated Liquid-Crystalline Polymers for Bright Blue Emission and Polarized Electroluminescence. Macromol. Rapid Commun. 2001, 22, 1365-1385.

(22) Scherf, U.; Neher, D.; Becker, K. Polyfluorenes; Advances in Polymer Science 212; Springer: Berlin, Heidelberg, 2008.

(23) Leclerc, M. Polyfluorenes: Twenty Years of Progress. J. Polym. Sci., Part A: Polym. Chem. 2001, 39, 2867-2873.

(24) Abbel, R.; Schenning, A. P. H. J.; Meijer, E. W. Fluorene-Based Materials and their Supramolecular Properties. J. Polym. Sci., Part A: Polym. Chem. 2009, 47, 4215-4233.

(25) Fukuda, M.; Sawada, K.; Yoshino, K. Fusible Conducting Poly(9-alkylfluorene) and Poly(9,9-dialkylfluorene) and Their Characteristics. Jpn. J. Appl. Phys. 1989, 28, L1433-L1435.

(26) Ding, J.; Day, M.; Robertson, G.; Roovers, J. Synthesis and Characterization of Alternating Copolymers of Fluorene and Oxadiazole. Macromolecules 2002, 35, 3474-3483.

(27) Liu, B.; Yu, W.-L.; Lai, Y.-H.; Huang, W. Blue-Light-Emitting Fluorene-Based Polymers with Tunable Electronic Properties. Chem. Mater. 2001, 13, 1984-1991.

(28) Rusu, R.-D.; Schlüter, A. D. Progress in the Suzuki Polycondensation of Fluorene Monomers. RSC Adv. 2014, 4, 57026-57034.

(29) Verheyen, L.; Janssens, K.; Marinelli, M.; Salatelli, E.; Koeckelberghs, G. Rational Design of Poly(fluorene)-b-poly(thiophene) Block Copolymers to Obtain a Unique Aggregation Behavior. Macromolecules 2019, 52, 6578-6584.

(30) Yokoyama, A.; Suzuki, H.; Kubota, Y.; Ohuchi, K.; Higashimura, H.; Yokozawa, T. Chain-Growth Polymerization for the Synthesis of Polyfluorene via Suzuki-Miyaura Coupling Reaction from an Externally Added Initiator Unit. J. Am. Chem. Soc. 2007, 129, 7236-7237.

(31) Zhang, X.; Tian, H.; Liu, Q.; Wang, L.; Geng, Y.; Wang, F. Synthesis of Fluorene-Based Oligomeric Organoboron Reagents via Kumada, Heck, and Stille Cross-Coupling Reactions. J. Org. Chem. 2006, $71,4332-4335$.

(32) Grell, M.; Bradley, D. D. C.; Inbasekaran, M.; Woo, E. P. A Glass-Forming Conjugated Main-Chain Liquid Crystal Polymer for Polarized Electroluminescence Applications. Adv. Mater. 1997, 9, 798-802.

(33) Grell, M.; Bradley, D. D. C.; Ungar, G.; Hill, J.; Whitehead, K. S. Interplay of Physical Structure and Photophysics for a Liquid Crystalline Polyfluorene. Macromolecules 1999, 32, 5810-5817.

(34) Chen, S. H.; Chou, H. L.; Su, A. C.; Chen, S. A. Molecular Packing in Crystalline Poly(9,9-di-n-octyl-2,7fluorene). Macromolecules 2004, 37, 6833-6838.

(35) Surin, M.; Hennebicq, E.; Ego, C.; Marsitzky, D.; Grimsdale, A. C.; Müllen, K.; Brédas, J.-L.; Lazzaroni, R.; Leclère, P. Correlation between the Microscopic Morphology and the Solid-State Photoluminescence Properties in Fluorene-Based Polymers and Copolymers. Chem. Mater. 2004, 16, 994-1001.

(36) Chunwaschirasiri, W.; Tanto, B.; Huber, D. L.; Winokur, M. J. Chain Conformations and Photoluminescence of Poly(di-n-octylfluorene). Phys. Rev. Lett. 2005, 94, 107402.

(37) Arif, M.; Volz, C.; Guha, S. Chain Morphologies in Semicrystalline Polyfluorene: Evidence from Raman Scattering. Phys. Rev. Lett. 2006, 96, 25503.

(38) Tsoi, W. C.; Charas, A.; Cadby, A. J.; Khalii, G.; Adawi, A. M.; Iraqi, A.; Hunt, B.; Morgado, J.; Lidzey, D. G. Observation of the $\beta$-Phase in Two Short-Chain Oligofluorenes. Adv. Funct. Mater. 2008, 18, 600606. 
(39) Yu, M.-N.; Soleimaninejad, H.; Lin, J.-Y.; Zuo, Z.-Y.; Liu, B.; Bo, Y.-F.; Bai, L.-B.; Han, Y.-M.; Smith, T. A.; Xu, M. Photophysical and Fluorescence Anisotropic Behavior of Polyfluorene $\beta$-Conformation Films. J. Phys. Chem. Lett. 2018, 9, 364-372.

(40) Liu, C.; Wang, Q.; Tian, H.; Liu, J.; Geng, Y.; Yan, D. Morphology and Structure of the $\beta$-Phase Crystals of Monodisperse Polyfluorenes. Macromolecules 2013, 46, 3025-3030.

(41) Chen, S. H.; Su, A. C.; Chen, S. A. Noncrystalline Phases in Poly(9,9-di-n-octyl-2,7-fluorene). J. Phys. Chem. B 2005, 109, 10067-10072.

(42) Knaapila, M.; Dias, F. B.; Garamus, V. M.; Almásy, L.; Torkkeli, M.; Leppänen, K.; Galbrecht, F.; Preis, E.; Burrows, H. D.; Scherf, U.; et al. Influence of Side Chain Length on the Self-Assembly of Hairy-Rod Poly(9,9-dialkylfluorene)s in the Poor Solvent Methylcyclohexane. Macromolecules 2007, 40, 93989405.

(43) Wu, C.; McNeill, J. Swelling-Controlled Polymer Phase and Fluorescence Properties of Polyfluorene Nanoparticles. Langmuir 2008, 24, 5855-5861.

(44) Dias, F. B.; Morgado, J.; Maçanita, A. L.; da Costa, F. P.; Burrows, H. D.; Monkman, A. P. Kinetics and Thermodynamics of Poly(9,9-dioctylfluorene) $\beta$-Phase Formation in Dilute Solution. Macromolecules 2006, 39, 5854-5864.

(45) Bright, D. W.; Dias, F. B.; Galbrecht, F.; Scherf, U.; Monkman, A. P. The Influence of Alkyl-Chain Length on Beta-Phase Formation in Polyfluorenes. Adv. Funct. Mater. 2009, 19, 67-73.

(46) Becker, K.; Lupton, J. M. Dual Species Emission from Single Polyfluorene Molecules: Signatures of Stress-Induced Planarization of Single Polymer Chains. J. Am. Chem. Soc. 2005, 127, 7306-7307.

(47) Rahman, M. H.; Chen, C.-Y.; Liao, S.-C.; Chen, H.-L.; Tsao, C.-S.; Chen, J.-H.; Liao, J.-L.; Ivanov, V. A.; Chen, S.-A. Segmental Alignment in the Aggregate Domains of Poly(9,9-dioctylfluorene) in Semidilute Solution. Macromolecules 2007, 40, 6572-6578.

(48) Knaapila, M.; Garamus, V. M.; Dias, F. B.; Almásy, L.; Galbrecht, F.; Charas, A.; Morgado, J.; Burrows, H. D.; Scherf, U.; Monkman, A. P. Influence of Solvent Quality on the Self-Organization of Archetypical Hairy Rods - Branched and Linear Side Chain Polyfluorenes: Rodlike Chains versus "Beta-Sheets" in Solution. Macromolecules 2006, 39, 6505-6512.

(49) Liu, C.; Wang, Q.; Tian, H.; Liu, J.; Geng, Y.; Yan, D. Control of Crystal Morphology in Monodisperse Polyfluorenes by Solvent and Molecular Weight. J. Phys. Chem. B 2013, 117, 8880-8886.

(50) Cone, C. W.; Cheng, R. R.; Makarov, D. E.; Vanden Bout, D. A. Molecular Weight Effect on the Formation of $\beta$-Phase Poly(9,9'-dioctylfluorene) in Dilute Solutions. J. Phys. Chem. B 2011, 115, 12380-12385.

(51) Chen, J.-H.; Chang, C.-S.; Chang, Y.-X.; Chen, C.-Y.; Chen, H.-L.; Chen, S.-A. Gelation and Its Effect on the Photophysical Behavior of Poly(9,9-dioctylfluorene-2,7-diyl) in Toluene. Macromolecules 2009, 42, 1306-1314.

(52) Rosencrantz, R. R.; Rahimi, K.; Kuehne, A. J. C. Morphology Control in Poly(9,9-di-n-octyl-2,7-fluorene) Spherulite Particles Prepared via Dispersion Polymerization. J. Phys. Chem. B 2014, 118, 6324-6328.

(53) Gaal, M.; List, E. J. W.; Scherf, U. Excimers or Emissive On-Chain Defects? Macromolecules 2003, 36, 4236-4237.

(54) Li, T.; Zhang, H.; Liu, B.; Ma, T.; Lin, J.; Xie, L.; Lu, D. Effect of Solvent on the Solution State of Conjugated Polymer P7DPF Including Single-Chain to Aggregated State Structure Formation, Dynamic Evolution, and Related Mechanisms. Macromolecules 2020, 53, 4264-4273.

(55) Lin, J.-Y.; Zhu, W.-S.; Liu, F.; Xie, L.-H.; Zhang, L.; Xia, R.; Xing, G.-C.; Huang, W. A Rational Molecular Design of $\beta$-Phase Polydiarylfluorenes: Synthesis, Morphology, and Organic Lasers. Macromolecules 2014, 47, 1001-1007.

(56) Trimpin, S.; Grimsdale, A. C.; Räder, H. J.; Müllen, K. Characterization of an Insoluble Poly(9,9-diphenyl2,7-fluorene) by Solvent-Free Sample Preparation for MALDI-TOF Mass Spectrometry. Anal. Chem. 2002, 74, 3777-3782. 
(57) Liu, B.; Lin, J.; Liu, F.; Yu, M.; Zhang, X.; Xia, R.; Yang, T.; Fang, Y.; Xie, L.; Huang, W. A Highly Crystalline and Wide-Bandgap Polydiarylfluorene with $\beta$-Phase Conformation Toward Stable Electroluminescence and Dual Amplified Spontaneous Emission. ACS Appl. Mater. Interfaces 2016, 8 , 21648-21655.

(58) Zhu, W.; Wei, C.; An, X.; Weng, J.; Liu, B.; Wang, N.; Sun, N.; Sun, L.; Yu, M.; Lin, J.; et al. Polydiarylfluorene Molecular Weight Effects on $\beta$-Conformation Formation for Amplified Spontaneous Emission for Optoelectronic Applications. ACS Appl. Polym. Mater. 2019, 1, 2352-2359.

(59) Schlüter, A.-D.; Wegner, G. Palladium and Nickel Catalyzed Polycondensation - The Key to Structurally Defined Polyarylenes and Other Aromatic Polymers. Acta Polym. 1993, 44, 59-69.

(60) Yamamoto, T. ח-Conjugated Polymers with Electronic and Optical Functionalities: Preparation by Organometallic Polycondensation, Properties, and Applications. Macromol. Rapid Commun. 2002, 23, 583.

(61) Verheyen, L.; Leysen, P.; van den Eede, M.-P.; Ceunen, W.; Hardeman, T.; Koeckelberghs, G. Advances in the Controlled Polymerization of Conjugated Polymers. Polymer 2017, 108, 521-546.

(62) Yamamoto, T.; Hayashi, Y.; Yamamoto, A. A Novel Type of Polycondensation Utilizing Transition MetalCatalyzed C-C Coupling. I. Preparation of Thermostable Polyphenylene Type Polymers. Bull. Chem. Soc. Jpn. 1978, 51, 2091-2097.

(63) Miyaura, N.; Yamada, K.; Suzuki, A. A New Stereospecific Cross-coupling by the Palladium-catalyzed Reaction of 1-Alkenylboranes with 1-Alkenyl or 1-Alkynyl Halides. Tetrahedron Lett. 1979, 20, 34373440.

(64) Miyaura, N.; Suzuki, A. Stereoselective Synthesis of Arylated (E)-Alkenes by the Reaction of Alk-1enylboranes with Aryl Halides in the Presence of Palladium Catalyst. J. Chem. Soc., Chem. Commun. $1979,866$.

(65) Zhang, K.; Tkachov, R.; Ditte, K.; Kiriy, N.; Kiriy, A.; Voit, B. AB- Versus AA+BB-Suzuki Polycondensation: A Palladium/Tris(tert-butyl)phosphine Catalyst Can Outperform Conventional Catalysts. Macromol. Rapid Commun. 2019, 1900521, 1-6.

(66) Miyaura, N.; Suzuki, A. Palladium-Catalyzed Cross-Coupling Reactions of Organoboron Compounds. Chem. Rev. 1995, 95, 2457-2483.

(67) Elmalem, E.; Kiriy, A.; Huck, W. T. S. Chain-Growth Suzuki Polymerization of n-Type Fluorene Copolymers. Macromolecules 2011, 44, 9057-9061.

(68) de Roo, T.; Haase, J.; Keller, J.; Hinz, C.; Schmid, M.; Seletskiy, D. V.; Cölfen, H.; Leitenstorfer, A.; Mecking, S. A Direct Approach to Organic/Inorganic Semiconductor Hybrid Particles via Functionalized Polyfluorene Ligands. Adv. Funct. Mater. 2014, 24, 2714-2719.

(69) Elmalem, E.; Biedermann, F.; Johnson, K.; Friend, R. H.; Huck, W. T. S. Synthesis and Photophysics of Fully $\pi$-Conjugated Heterobis-Functionalized Polymeric Molecular Wires via Suzuki Chain-Growth Polymerization. J. Am. Chem. Soc. 2012, 134, 17769-17777.

(70) Fischer, C. S.; Baier, M. C.; Mecking, S. Enhanced Brightness Emission-Tuned Nanoparticles from Heterodifunctional Polyfluorene Building Blocks. J. Am. Chem. Soc. 2013, 135, 1148-1154.

(71) Yokozawa, T.; Ohta, Y. Transformation of Step-Growth Polymerization into Living Chain-Growth Polymerization. Chem. Rev. 2016, 116, 1950-1968.

(72) Zhang, H.-H.; Peng, W.; Dong, J.; Hu, Q.-S. t-Bu3P-Coordinated 2-Phenylaniline-Based Palladacycle Complex/ArBr as Robust Initiators for Controlled $\mathrm{Pd}(0) / t-\mathrm{Bu}_{3} \mathrm{P}$-Catalyzed Suzuki Cross-Coupling Polymerization of AB-Type Monomers. ACS Macro Lett. 2016, 5, 656-660.

(73) Biscoe, M. R.; Fors, B. P.; Buchwald, S. L. A New Class of Easily Activated Palladium Precatalysts for Facile C-N Cross-Coupling Reactions and the Low Temperature Oxidative Addition of Aryl Chlorides. J. Am. Chem. Soc. 2008, 130, 6686-6687. 
(74) Kinzel, T.; Zhang, Y.; Buchwald, S. L. A New Palladium Precatalyst Allows for the Fast Suzuki-Miyaura Coupling Reactions of Unstable Polyfluorophenyl and 2-Heteroaryl Boronic Acids. J. Am. Chem. Soc. 2010, 132, 14073-14075.

(75) Yokozawa, T.; Kohno, H.; Ohta, Y.; Yokoyama, A. Catalyst-Transfer Suzuki-Miyaura Coupling Polymerization for Precision Synthesis of Poly ( $p$-phenylene). Macromolecules 2010, 43, 7095-7100.

(76) Yokozawa, T.; Suzuki, R.; Nojima, M.; Ohta, Y.; Yokoyama, A. Precision Synthesis of Poly(3-hexylthiophene) from Catalyst-Transfer Suzuki-Miyaura Coupling Polymerization. Macromol. Rapid Commun. 2011, 32, 801-806.

(77) Zhang, H.-H.; Xing, C.-H.; Hu, Q.-S. Controlled Pd(0)/t-Bu33P-Catalyzed Suzuki Cross-Coupling Polymerization of $\mathrm{AB}-\mathrm{Type}$ Monomers with $\mathrm{PhPd}\left(t-\mathrm{Bu} \mathrm{u}_{3} \mathrm{P}\right) \mathrm{l}$ or $\mathrm{Pd}_{2}\left(\mathrm{dba}_{3} / t-\mathrm{Bu}_{3} \mathrm{P} / \mathrm{Arl}\right.$ as the Initiator. J. Am. Chem. Soc. 2012, 134, 13156-13159.

(78) Kosaka, K.; Uchida, T.; Mikami, K.; Ohta, Y.; Yokozawa, T. AmPhos Pd-Catalyzed Suzuki-Miyaura Catalyst-Transfer Condensation Polymerization: Narrower Dispersity by Mixing the Catalyst and Base Prior to Polymerization. Macromolecules 2018, 51, 364-369.

(79) Huber, J.; Mecking, S. Processing of Polyacetylene from Aqueous Nanoparticle Dispersions. Angew. Chem. Int. Ed. 2006, 45, 6314-6317.

(80) Baier, M. C.; Huber, J.; Mecking, S. Fluorescent Conjugated Polymer Nanoparticles by Polymerization in Miniemulsion. J. Am. Chem. Soc. 2009, 131, 14267-14273.

(81) Behrendt, J. M.; Esquivel Guzman, J. A.; Purdie, L.; Willcock, H.; Morrison, J. J.; Foster, A. B.; O’Reilly, R. K.; McCairn, M. C.; Turner, M. L. Scalable Synthesis of Multicolour Conjugated Polymer Nanoparticles via Suzuki-Miyaura Polymerisation in a Miniemulsion and Application in Bioimaging. React. Funct. Polym. 2016, 107, 69-77.

(82) Pecher, J.; Huber, J.; Winterhalder, M.; Zumbusch, A.; Mecking, S. Tailor-Made Conjugated Polymer Nanoparticles for Multicolor and Multiphoton Cell Imaging. Biomacromolecules 2010, 11, 2776-2780.

(83) Tuncel, D.; Demir, H. V. Conjugated Polymer Nanoparticles. Nanoscale 2010, 2, 484-494.

(84) Landfester, K.; Montenegro, R.; Scherf, U.; Güntner, R.; Asawapirom, U.; Patil, S.; Neher, D.; Kietzke, T. Semiconducting Polymer Nanospheres in Aqueous Dispersion Prepared by a Miniemulsion Process. Adv. Mater. 2002, 14, 651-655.

(85) Kietzke, T.; Neher, D.; Kumke, M.; Montenegro, R.; Landfester, K.; Scherf, U. A Nanoparticle Approach to Control the Phase Separation in Polyfluorene Photovoltaic Devices. Macromolecules 2004, 37, 48824890.

(86) Piok, T.; Gamerith, S.; Gadermaier, C.; Plank, H.; Wenzl, F. P.; Patil, S.; Montenegro, R.; Kietzke, T.; Neher, D.; Scherf, U.; et al. Organic Light-Emitting Devices Fabricated from Semiconducting Nanospheres. Adv. Mater. 2003, 15, 800-804.

(87) Bao, B.; Ma, M.; Zai, H.; Zhang, L.; Fu, N.; Huang, W.; Wang, L. Conjugated Polymer Nanoparticles for Label-Free and Bioconjugate-Recognized DNA Sensing in Serum. Adv. Sci. 2015, 2, 1400009.

(88) Singh, A.; Bezuidenhout, M.; Walsh, N.; Beirne, J.; Felletti, R.; Wang, S.; Fitzgerald, K. T.; Gallagher, W. M.; Kiely, P.; Redmond, G. Functionalization of Emissive Conjugated Polymer Nanoparticles by Coprecipitation: Consequences for Particle Photophysics and Colloidal Properties. Nanotechnology 2016, 27, 305603.

(89) Sickinger, A. Anisotropic Conjugated Polymer Nanoparticles with Charged Surfaces. Master Thesis, University of Konstanz, Konstanz, 2017.

(90) Wu, C.; Szymanski, C.; McNeill, J. Preparation and Encapsulation of Highly Fluorescent Conjugated Polymer Nanoparticles. Langmuir 2006, 22, 2956-2960.

(91) Szymanski, C.; Wu, C.; Hooper, J.; Salazar, M. A.; Perdomo, A.; Dukes, A.; McNeill, J. Single Molecule Nanoparticles of the Conjugated Polymer MEH-PPV, Preparation and Characterization by Near-Field Scanning Optical Microscopy. J. Phys. Chem. B 2005, 109, 8543-8546. 
(92) Grey, J. K.; Kim, D. Y.; Norris, B. C.; Miller, W. L.; Barbara, P. F. Size-Dependent Spectroscopic Properties of Conjugated Polymer Nanoparticles. J. Phys. Chem. B 2006, 110, 25568-25572.

(93) Kaeser, A.; Schenning, A. P. H. J. Fluorescent Nanoparticles Based on Self-Assembled $\pi-C o n j u g a t e d$ Systems. Adv. Mater. 2010, 22, 2985-2997.

(94) Schütze, F.; Krumova, M.; Mecking, S. Size Control of Spherical and Anisotropic Fluorescent Polymer Nanoparticles via Precise Rigid Molecules. Macromolecules 2015, 48, 3900-3906.

(95) Yang, Z.; Huck, W. T. S.; Clarke, S. M.; Tajbakhsh, A. R.; Terentjev, E. M. Shape-Memory Nanoparticles from Inherently Non-Spherical Polymer Colloids. Nat. Mater. 2005, 4, 486-490.

(96) Piwoński, H.; Michinobu, T.; Habuchi, S. Controlling Photophysical Properties of Ultrasmall Conjugated Polymer Nanoparticles Through Polymer Chain Packing. Nat. Commun. 2017, 8, 15256.

(97) Pecher, J.; Mecking, S. Nanoparticles from Step-Growth Coordination Polymerization. Macromolecules 2007, 40, 7733-7735.

(98) Schork, F. J.; Luo, Y.; Smulders, W.; Russum, J. P.; Butté, A.; Fontenot, K. Miniemulsion Polymerization. In Polymer Particles; Ōkubo, M., Butté, A., Eds.; Advances in Polymer Science 175; Springer: Berlin, Heidelberg, 2005; pp 129-255.

(99) Landfester, K. Synthesis of Colloidal Particles in Miniemulsions. Annu. Rev. Mater. Res. 2006, 36, $231-$ 279.

(100) Landfester, K.; Bechthold, N.; Tiarks, F.; Antonietti, M. Miniemulsion Polymerization with Cationic and Nonionic Surfactants: A Very Efficient Use of Surfactants for Heterophase Polymerization. Macromolecules 1999, 32, 2679-2683.

(101) Ciftci, S.; Kuehne, A. J. C. Monodisperse Conjugated Polymer Particles via Heck Coupling - A Kinetic Study to Unravel Particle Formation in Step-Growth Dispersion Polymerization. Macromolecules 2015, 48, 8389-8393.

(102) Landfester, K. Miniemulsion Polymerization and the Structure of Polymer and Hybrid Nanoparticles. Angew. Chem. Int. Ed. 2009, 48, 4488-4507.

(103) Negele, C.; Haase, J.; Leitenstorfer, A.; Mecking, S. Polyfluorene Nanoparticles and Quantum Dot Hybrids via Miniemulsion Polymerization. ACS Macro Lett. 2012, 1, 1343-1346.

(104) McClements, D. J. Nanoemulsions versus Microemulsions: Terminology, Differences, and Similarities. Soft Matter 2012, 8, 1719-1729.

(105) Anton, N.; Vandamme, T. F. Nano-Emulsions and Micro-Emulsions: Clarifications of the Critical Differences. Pharm. Res. 2011, 28, 978-985.

(106) Li, H.; Wu, X.; Xu, B.; Tong, H.; Wang, L. Solution-Processible Hyperbranched Conjugated Polymer Nanoparticles with Tunable Particle Sizes by Suzuki Polymerization in Miniemulsion. RSC Adv. 2013, 3, 8645.

(107) Chandler, D. Interfaces and the Driving Force of Hydrophobic Assembly. Nature 2005, 437, 640-647.

(108) Anjali, T. G.; Basavaraj, M. G. Shape Anisotropic Colloids at Interfaces. Langmuir 2019, 35, 3-20.

(109) Murphy, C. J.; Sau, T. K.; Gole, A. M.; Orendorff, C. J.; Gao, J.; Gou, L.; Hunyadi, S. E.; Li, T. Anisotropic Metal Nanoparticles: Synthesis, Assembly, and Optical Applications. J. Phys. Chem. B 2005, 109, 13857-13870.

(110) Burda, C.; Chen, X.; Narayanan, R.; El-Sayed, M. A. Chemistry and Properties of Nanocrystals of Different Shapes. Chem. Rev. 2005, 105, 1025-1102.

(111) Halas, N. J. Plasmonics: An Emerging Field Fostered by Nano Letters. Nano Lett. 2010, 10, 38163822.

(112) Lee, E.; Xia, Y.; Ferrier, R. C.; Kim, H.-N.; Gharbi, M. A.; Stebe, K. J.; Kamien, R. D.; Composto, R. J.; Yang, S. Fine Golden Rings: Tunable Surface Plasmon Resonance from Assembled Nanorods in Topological Defects of Liquid Crystals. Adv. Mater. 2016, 28, 2731-2736. 
(113) Xia, Y.; Halas, N. J. Shape-Controlled Synthesis and Surface Plasmonic Properties of Metallic Nanostructures. MRS Bull. 2005, 30, 338-348.

(114) Lee, K. J.; Yoon, J.; Lahann, J. Recent Advances with Anisotropic Particles. Curr. Opin. Colloid Interface Sci. 2011, 16, 195-202.

(115) Mittal, M.; Furst, E. M. Electric Field-Directed Convective Assembly of Ellipsoidal Colloidal Particles to Create Optically and Mechanically Anisotropic Thin Films. Adv. Funct. Mater. 2009, 19, 3271-3278.

(116) Glotzer, S. C.; Solomon, M. J. Anisotropy of Building Blocks and their Assembly into Complex Structures. Nat. Mater. 2007, 6, 557-562.

(117) Glotzer, S. C.; Solomon, M. J.; Kotov, N. A. Self-Assembly: From Nanoscale to Microscale Colloids. AIChE J. 2004, 50, 2978-2985.

(118) Grzelczak, M.; Vermant, J.; Furst, E. M.; Liz-Marzán, L. M. Directed Self-Assembly of Nanoparticles. ACS Nano 2010, 4, 3591-3605.

(119) Whitesides, G. M.; Grzybowski, B. Self-Assembly at All Scales. Science 2002, 295, 2418.

(120) Bhardwaj, R.; Fang, X.; Somasundaran, P.; Attinger, D. Self-Assembly of Colloidal Particles from Evaporating Droplets: Role of DLVO Interactions and Proposition of a Phase Diagram. Langmuir 2010, 26, 7833-7842.

(121) Ganesan, M.; Solomon, M. J. High-Density Equilibrium Phases of Colloidal Ellipsoids by Application of Optically Enhanced, Direct Current Electric Fields. Soft Matter 2017, 13, 3768-3776.

(122) Singh, J. P.; Lele, P. P.; Nettesheim, F.; Wagner, N. J.; Furst, E. M. One- and Two-Dimensional Assembly of Colloidal Ellipsoids in AC Electric Fields. Phys. Rev. E 2009, 79, 50401.

(123) Takashima, S.; Schwan, H. P. Alignment of Microscopic Particles in Electric Fields and its Biological Implications. Biophys. J. 1985, 47, 513-518.

(124) Zhang, S.; Pelligra, C. I.; Feng, X.; Osuji, C. O. Directed Assembly of Hybrid Nanomaterials and Nanocomposites. Adv. Mater. 2018, 30, 1705794.

(125) Tao, H.; Galati, E.; Kumacheva, E. Temperature-Responsive Self-Assembly of Nanoparticles Grafted with UCST Polymer Ligands. Macromolecules 2018, 51, 6021-6027.

(126) Verwey, E. J. Theory of the Stability of Lyophobic Colloids. J. Phys. Chem. 1947, 51, 631-636.

(127) Derjaguin, B. V.; Landau, L. Theory of the Stability of Strongly Charged Lyophobic Sols and of the Adhesion of Strongly Charged Particles in Solution of Electrolytes. Acta Physicochim. U.R.S.S. 1941, 14, 633-662.

(128) Davies, R.; Schurr, G. A.; Meenan, P.; Nelson, R. D.; Bergna, H. E.; Brevett, C. A. S.; Goldbaum, R. H. Engineered Particle Surfaces. Adv. Mater. 1998, 10, 1264-1270.

(129) Ethirajan, A.; Ziener, U.; Landfester, K. Surface-Functionalized Polymeric Nanoparticles as Templates for Biomimetic Mineralization of Hydroxyapatite. Chem. Mater. 2009, 21, 2218-2225.

(130) Rey, M.; Yu, T.; Bley, K.; Landfester, K.; Buzza, D. M. A.; Vogel, N. Amphiphile-Induced Anisotropic Colloidal Self-Assembly. Langmuir 2018, 34, 9990-10000.

(131) Yablonovitch; Gmitter; Leung. Photonic Band Structure: The Face-Centered-Cubic Case Employing Nonspherical Atoms. Phys. Rev. Lett. 1991, 67, 2295-2298.

(132) Yu, B.; Cong, H.; Peng, Q.; Gu, C.; Tang, Q.; Xu, X.; Tian, C.; Zhai, F. Current Status and Future Developments in Preparation and Application of Nonspherical Polymer Particles. Adv. Colloid Interface Sci. 2018, 256, 126-151.

(133) Kim, J.-W.; Larsen, R. J.; Weitz, D. A. Synthesis of Nonspherical Colloidal Particles with Anisotropic Properties. J. Am. Chem. Soc. 2006, 128, 14374-14377.

(134) Tang, C.; Zhang, C.; Sun, Y.; Liang, F.; Wang, Q.; Li, J.; Qu, X.; Yang, Z. Janus Anisotropic Hybrid Particles with Tunable Size from Patchy Composite Spheres. Macromolecules 2013, 46, 188-193. 
(135) Rolland, J. P.; Maynor, B. W.; Euliss, L. E.; Exner, A. E.; Denison, G. M.; DeSimone, J. M. Direct Fabrication and Harvesting of Monodisperse, Shape-Specific Nanobiomaterials. J. Am. Chem. Soc. 2005, 127, 10096-10100.

(136) Herrmann, C.; Turshatov, A.; Crespy, D. Fabrication of Polymer Ellipsoids by the Electrospinning of Swollen Nanoparticles. ACS Macro Lett. 2012, 1, 907-909.

(137) Lo, Y.-C.; Tseng, H.-F.; Chiu, Y.-J.; Wu, B.-H.; Li, J.-W.; Chen, J.-T. Solvent-Induced Shape Recovery of Anisotropic Polymer Particles Prepared by a Modified Thermal Stretching Method. Langmuir 2018, 34, 8326-8332.

(138) Lele, P. P.; Furst, E. M. Assemble-and-Stretch Method for Creating Two- and Three-Dimensional Structures of Anisotropic Particles. Langmuir 2009, 25, 8875-8878.

(139) Champion, J. A.; Katare, Y. K.; Mitragotri, S. Making Polymeric Micro- and Nanoparticles of Complex Shapes. Proc. Natl. Acad. Sci. U.S.A. 2007, 104, 11901-11904.

(140) Jin, X.-H.; Price, M. B.; Finnegan, J. R.; Boott, C. E.; Richter, J. M.; Rao, A.; Menke, S. M.; Friend, R. H.; Whittell, G. R.; Manners, I. Long-Range Exciton Transport in Conjugated Polymer Nanofibers Prepared by Seeded Growth. Science 2018, 360, 897-900.

(141) Park, S.-J.; Kang, S.-G.; Fryd, M.; Saven, J. G.; Park, S.-J. Highly Tunable Photoluminescent Properties of Amphiphilic Conjugated Block Copolymers. J. Am. Chem. Soc. 2010, 132, 9931-9933.

(142) Zhang, J.; Chen, X.-F.; Wei, H.-B.; Wan, X.-H. Tunable Assembly of Amphiphilic Rod-Coil Block Copolymers in Solution. Chem. Soc. Rev. 2013, 42, 9127-9154.

(143) MacFarlane, L. R.; Shaikh, H.; Garcia-Hernandez, J. D.; Vespa, M.; Fukui, T.; Manners, I. Functional Nanoparticles through m-Conjugated Polymer Self-Assembly. Nat. Rev. Mater. 2021, 6, 7-26.

(144) Schmalz, H.; Schmelz, J.; Drechsler, M.; Yuan, J.; Walther, A.; Schweimer, K.; Mihut, A. M. ThermoReversible Formation of Wormlike Micelles with a Microphase-Separated Corona from a Semicrystalline Triblock Terpolymer. Macromolecules 2008, 41, 3235-3242.

(145) Dai, X.; Qiang, X.; Hils, C.; Schmalz, H.; Gröschel, A. H. Frustrated Microparticle Morphologies of a Semicrystalline Triblock Terpolymer in 3D Soft Confinement. ACS Nano 2021, 15, 1111-1120.

(146) He, T.; Adams, D. J.; Butler, M. F.; Yeoh, C. T.; Cooper, A. I.; Rannard, S. P. Direct Synthesis of Anisotropic Polymer Nanoparticles. Angew. Chem. 2007, 119, 9403-9407.

(147) Lesov, I.; Valkova, Z.; Vassileva, E.; Georgiev, G. S.; Ruseva, K.; Simeonov, M.; Tcholakova, S.; Denkov, N. D.; Smoukov, S. K. Bottom-Up Synthesis of Polymeric Micro- and Nanoparticles with Regular Anisotropic Shapes. Macromolecules 2018, 51, 7456-7462.

(148) Osichow, A.; Rabe, C.; Vogtt, K.; Narayanan, T.; Harnau, L.; Drechsler, M.; Ballauff, M.; Mecking, S. Ideal Polyethylene Nanocrystals. J. Am. Chem. Soc. 2013, 135, 11645-11650.

(149) Schnitte, M.; Staiger, A.; Casper, L. A.; Mecking, S. Uniform Shape Monodisperse Single Chain Nanocrystals by Living Aqueous Catalytic Polymerization. Nat. Commun. 2019, 10, 1-6.

(150) Schnitte, M.; Lipinski, S.; Schiebel, E.; Mecking, S. Pentafluorophenyl Groups as Remote Substituents in $\mathrm{Ni}(\mathrm{II})$ Polymerization Catalysis. Organometallics 2020, 39, 13-17.

(151) Muenmart, D.; Foster, A. B.; Harvey, A.; Chen, M.-T.; Navarro, O.; Promarak, V.; McCairn, M. C.; Behrendt, J. M.; Turner, M. L. Conjugated Polymer Nanoparticles by Suzuki-Miyaura Cross-Coupling Reactions in an Emulsion at Room Temperature. Macromolecules 2014, 47, 6531-6539.

(152) Xi, Y.; Li, D. S.; Newbloom, G. M.; Tatum, W. K.; O’Donnell, M.; Luscombe, C. K.; Pozzo, L. D. Sonocrystallization of Conjugated Polymers with Ultrasound Fields. Soft Matter 2018, 14, 4963-4976.

(153) Choi, H.-N.; Yang, H.-S.; Chae, J.-H.; Choi, T.-L.; Lee, I.-H. Synthesis of Conjugated Rod-Coil Block Copolymers by RuPhos Pd-Catalyzed Suzuki-Miyaura Catalyst-Transfer Polycondensation: Initiation from Coil-Type Polymers. Macromolecules 2020, 53, 5497-5503. 
(154) Liu, C.-L.; Lin, C.-H.; Kuo, C.-C.; Lin, S.-T.; Chen, W.-C. Conjugated Rod-Coil Block Copolymers: Synthesis, Morphology, Photophysical Properties, and Stimuli-Responsive Applications. Prog. Polym. Sci. 2011, 36, 603-637.

(155) Klok, H.-A.; Lecommandoux, S. Supramolecular Materials via Block Copolymer Self-Assembly. Adv. Mater. 2001, 13, 1217-1229.

(156) Surin, M.; Marsitzky, D.; Grimsdale, A. C.; Müllen, K.; Lazzaroni, R.; Leclère, P. Microscopic Morphology of Polyfluorene-Poly(ethylene oxide) Block Copolymers: Influence of the Block Ratio. Adv. Funct. Mater. 2004, 14, 708-715.

(157) Lee, M.; Cho, B. K.; Zin, W. C. Supramolecular Structures from Rod-Coil Block Copolymers. Chem. Rev. 2001, 101, 3869-3892.

(158) Qian, J.; Zhang, M.; Manners, I.; Winnik, M. A. Nanofiber Micelles from the Self-Assembly of Block Copolymers. Trends Biotechnol. 2010, 28, 84-92.

(159) Wang, H.; Wang, H. H.; Urban, V. S.; Littrell, K. C.; Thiyagarajan, P.; Yu, L. Syntheses of Amphiphilic Diblock Copolymers Containing a Conjugated Block and Their Self-Assembling Properties. J. Am. Chem. Soc. 2000, 122, 6855-6861.

(160) Wang, H.; You, W.; Jiang, P.; Yu, L.; Wang, H. H. Supramolecular Self-Assembly of Conjugated Diblock Copolymers. Chem. Eur. J. 2004, 10, 986-993.

(161) Chochos, C. L.; Tsolakis, P. K.; Gregoriou, V. G.; Kallitsis, J. K. Influence of the Coil Block on the Properties of Rod-Coil Diblock Copolymers with Oligofluorene as the Rigid Segment. Macromolecules 2004, 37, 2502-2510.

(162) Gwyther, J.; Gilroy, J. B.; Rupar, P. A.; Lunn, D. J.; Kynaston, E.; Patra, S. K.; Whittell, G. R.; Winnik, M. A.; Manners, I. Dimensional Control of Block Copolymer Nanofibers with a $\pi$-Conjugated Core: Crystallization-Driven Solution Self-Assembly of Amphiphilic Poly(3-hexylthiophene)-b-poly(2vinylpyridine). Chem. Eur. J. 2013, 19, 9186-9197.

(163) Liu, J.; Sheina, E.; Kowalewski, T.; McCullough, R. D. Tuning the Electrical Conductivity and SelfAssembly of Regioregular Polythiophene by Block Copolymerization: Nanowire Morphologies in New Di-and Triblock Copolymers. Angew. Chem. 2002, 114, 339-342.

(164) Qian, J.; Li, X.; Lunn, D. J.; Gwyther, J.; Hudson, Z. M.; Kynaston, E.; Rupar, P. A.; Winnik, M. A.; Manners, I. Uniform, High Aspect Ratio Fiber-Like Micelles and Block Co-Micelles with a Crystalline $\pi-$ Conjugated Polythiophene Core by Self-Seeding. J. Am. Chem. Soc. 2014, 136, 4121-4124.

(165) Patra, S. K.; Ahmed, R.; Whittell, G. R.; Lunn, D. J.; Dunphy, E. L.; Winnik, M. A.; Manners, I. Cylindrical Micelles of Controlled Length with a $\pi$-Conjugated Polythiophene Core via Crystallization-Driven SelfAssembly. J. Am. Chem. Soc. 2011, 133, 8842-8845.

(166) Urien, M.; Erothu, H.; Cloutet, E.; Hiorns, R. C.; Vignau, L.; Cramail, H. Poly(3-hexylthiophene) Based Block Copolymers Prepared by "Click” Chemistry. Macromolecules 2008, 41, 7033-7040.

(167) Marsitzky, D.; Brand, T.; Geerts, Y.; Klapper, M.; Müllen, K. Synthesis of Rod-Coil Block Copolymers via End-Functionalized Poly(p-phenylene)s. Macromol. Rapid Commun. 1998, 19, 385-389.

(168) Kobayashi, S.; Fujiwara, K.; Jiang, D.-H.; Yamamoto, T.; Tajima, K.; Yamamoto, Y.; Isono, T.; Satoh, T. Suzuki-Miyaura Catalyst-transfer Polycondensation of Triolborate-type Fluorene Monomer: Toward Rapid Access to Polyfluorene-containing Block and Graft Copolymers from Various Macroinitiators. Polym. Chem. 2020, 11, 6832-6839.

(169) Hintze, C.; Schütze, F.; Drescher, M.; Mecking, S. Probing of Chain Conformations in Conjugated Polymer Nanoparticles by Electron Spin Resonance Spectroscopy. Phys. Chem. Chem. Phys. 2015, 17, 32289-32296.

(170) Cuendias, A. de; Hiorns, R. C.; Cloutet, E.; Vignau, L.; Cramail, H. Conjugated Rod-Coil Block Copolymers and Optoelectronic Applications. Polym. Int. 2010, 59, 1452-1476. 
(171) Williams, D. R. M.; Fredrickson, G. H. Cylindrical Micelles in Rigid-Flexible Diblock Copolymers. Macromolecules 1992, 25, 3561-3568.

(172) Schweiger, A.; Jeschke, G. Principles of Pulse Electron Paramagnetic Resonance; Oxford University Press: New York, 2001.

(173) Al'tshuler, S. A.; Kozyrev, B. M., Eds. Electron Paramagnetic Resonance; Academic Press: New York, London, 1964.

(174) Weil, J. A.; Bolton, J. R. Electron Paramagnetic Resonance: Elementary Theory and Practical Applications; John Wiley \& Sons: Hoboken, New Jersey, 2007.

(175) Likhtenshtein, G.; Yamauchi, J.; Nakatsuji, S.; Smirnov, A. I.; Tamura, R. Nitroxides: Applications in Chemistry, Biomedicine, and Materials Science; Wiley-VCH: Weinheim, 2008.

(176) Jeschke, G.; Polyhach, Y. Distance Measurements on Spin-Labelled Biomacromolecules by Pulsed Electron Paramagnetic Resonance. Phys. Chem. Chem. Phys. 2007, 9, 1895-1910.

(177) Jeschke, G. DEER Distance Measurements on Proteins. Annu. Rev. Phys. Chem. 2012, 63, 419-446.

(178) Ward, R.; Bowman, A.; Sozudogru, E.; El-Mkami, H.; Owen-Hughes, T.; Norman, D. G. EPR Distance Measurements in Deuterated Proteins. J. Magn. Reson. 2010, 207, 164-167.

(179) Hubbell, W. L.; Altenbach, C. Investigation of Structure and Dynamics in Membrane Proteins Using Site-Directed Spin Labeling. Curr. Opin. Struc. Biol. 1994, 4, 566-573.

(180) Pannier, M.; Veit, S.; Godt, A.; Jeschke, G.; Spiess, H. W. Dead-Time Free Measurement of DipoleDipole Interactions Between Electron Spins. J. Magn. Reson. 2000, 142, 331-340.

(181) Jeschke, G.; Sajid, M.; Schulte, M.; Ramezanian, N.; Volkov, A.; Zimmermann, H.; Godt, A. Flexibility of Shape-Persistent Molecular Building Blocks Composed of $p$-Phenylene and Ethynylene Units. J. Am. Chem. Soc. 2010, 132, 10107-10117.

(182) Bode, B. E.; Margraf, D.; Plackmeyer, J.; Dürner, G.; Prisner, T. F.; Schiemann, O. Counting the Monomers in Nanometer-Sized Oligomers by Pulsed Electron-Electron Double Resonance. J. Am. Chem. Soc. 2007, 129, 6736-6745.

(183) Wilhelm, J.; Frey, E. Radial Distribution Function of Semiflexible Polymers. Phys. Rev. Lett. 1996, 77, 2581-2584.

(184) Doose, S.; Barsch, H.; Sauer, M. Polymer Properties of Polythymine as Revealed by Translational Diffusion. Biophys. J. 2007, 93, 1224-1234.

(185) Godt, A.; Schulte, M.; Zimmermann, H.; Jeschke, G. How Flexible Are Poly(para-phenylene ethynylene)s? Angew. Chem. Int. Ed. 2006, 45, 7560-7564.

(186) Ruiz Perez, J. D. Anisotropic Conjugated Polymer Nanoparticles: Anisotropic Conjugated Polymer Nanoparticles. Doctoral Thesis, University of Konstanz, Konstanz, 2019.

(187) Li, Y.; Ding, J.; Day, M.; Tao, Y.; Lu, J.; D'iorio, M. Synthesis and Properties of Random and Alternating Fluorene/Carbazole Copolymers for Use in Blue Light-Emitting Devices. Chem. Mater. 2004, 16, 21652173.

(188) Lawrence, J.; Goto, E.; Ren, J. M.; McDearmon, B.; Kim, D. S.; Ochiai, Y.; Clark, P. G.; Laitar, D.; Higashihara, T.; Hawker, C. J. A Versatile and Efficient Strategy to Discrete Conjugated Oligomers. J. Am. Chem. Soc. 2017, 139, 13735-13739.

(189) Jeschke, G.; Chechik, V.; Ionita, P.; Godt, A.; Zimmermann, H.; Banham, J.; Timmel, C. R.; Hilger, D.; Jung, H. DeerAnalysis2006 - A Comprehensive Software Package for Analyzing Pulsed ELDOR Data. Appl. Magn. Reson. 2006, 30, 473-498.

(190) Worswick, S. G.; Spencer, J. A.; Jeschke, G.; Kuprov, I. Deep Neural Network Processing of DEER Data. Sci. Adv. 2018, 4, eaat5218.

(191) Fytas, G.; Nothofer, H. G.; Scherf, U.; Vlassopoulos, D.; Meier, G. Structure and Dynamics of Nondilute Polyfluorene Solutions. Macromolecules 2002, 35, 481-488. 
(192) Wollmann, J.; Kahle, F.-J.; Bauer, I.; Köhler, A.; Strohriegl, P. Versatile Approach to Well-Defined Oligofluorenes and Polyfluorenes with Low Dispersity. Macromolecules 2020, 53, 10137-10146.

(193) Geng, Y.; Trajkovska, A.; Katsis, D.; Ou, J. J.; Culligan, S. W.; Chen, S. H. Synthesis, Characterization, and Optical Properties of Monodisperse Chiral Oligofluorenes. J. Am. Chem. Soc. 2002, 124, 83378347.

(194) Schwartz, B. J. Conjugated Polymers as Molecular Materials: How Chain Conformation and Film Morphology Influence Energy Transfer and Interchain Interactions. Annu. Rev. Phys. Chem. 2003, 54, 141-172.

(195) Tanaka, S.; Takeuchi, K.; Asai, M.; Iso, T.; Ueda, M. Preparation of Hyperbranched Copolymers Constituted of Triphenylamine and Phenylene Units. Synth. Met. 2001, 119, 139-140.

(196) Gao, C.; Yan, D. Hyperbranched Polymers: From Synthesis to Applications. Prog. Polym. Sci. 2004, 29, 183-275.

(197) Sun, M.; Fu, Y.; Li, J.; Bo, Z. Star Polyfluorenes with a Triphenylamine-Based Core. Macromol. Rapid Commun. 2005, 26, 1064-1069.

(198) Dugyala, V. R.; Basavaraj, M. G. Self-Assembly of Nano-Ellipsoids into Ordered Structures via Vertical Deposition. RSC Adv. 2015, 5, 60079-60084.

(199) Jiang, P.; Bertone, J. F.; Hwang, K. S.; Colvin, V. L. Single-Crystal Colloidal Multilayers of Controlled Thickness. Chem. Mater. 1999, 11, 2132-2140.

(200) Cuesta, J. A.; Frenkel, D. Monte Carlo Simulation of Two-Dimensional Hard Ellipses. Phys. Rev. A 1990, 42, 2126-2136.

(201) Odriozola, G. Revisiting the Phase Diagram of Hard Ellipsoids. J. Chem. Phys. 2012, 136, 134505.

(202) Freeman, D. M. E.; Musser, A. J.; Frost, J. M.; Stern, H. L.; Forster, A. K.; Fallon, K. J.; Rapidis, A. G.; Cacialli, F.; McCulloch, I.; Clarke, T. M. Synthesis and Exciton Dynamics of Donor-Orthogonal Acceptor Conjugated Polymers: Reducing the Singlet-Triplet Energy Gap. J. Am. Chem. Soc. 2017, 139, $11073-$ 11080.

(203) Ruhland, K.; Brück, A.; Herdtweck, E. Investigation of the Thermal Decomposition of [biphen(QPiPr)Pt(alk)2]: An Entry to C-C Single Bond Activation? Eur. J. Inorg. Chem. 2007, 2007, 944-964.

(204) Padalkar, V. S.; Tsutsui, Y.; Sakurai, T.; Sakamaki, D.; Tohnai, N.; Kato, K.; Takata, M.; Akutagawa, T.; Sakai, K.; Seki, S. Optical and Structural Properties of ESIPT Inspired HBT-Fluorene Molecular Aggregates and Liquid Crystals. J. Phys. Chem. B 2017, 121, 10407-10416.

(205) Roo, T. de; Huber, S.; Mecking, S. CdSe/CdS—Conjugated Polymer Core-Shell Hybrid Nanoparticles by a Grafting-From Approach. ACS Macro Lett. 2016, 5, 786-789.

(206) Liu, B.; Lin, J.; Yu, M.; Li, B.; Xie, L.; Ou, C.; Liu, F.; Li, T.; Lu, D.; Huang, W. Hereditary Character of Alkyl-Chain Length Effect on $\beta$-Phase Conformation from Polydialkylfluorenes to Bulky Polydiarylfluorenes. J. Phys. Chem. C 2017, 121, 19087-19096.

(207) Whitesides, G. M.; Mathias, J. P.; Seto, C. T. Molecular Self-Assembly and Nanochemistry: A Chemical Strategy for the Synthesis of Nanostructures. Science 1991, 254, 1312-1319.

(208) Muthukumar, M. Competing Interactions and Levels of Ordering in Self-Organizing Polymeric Materials. Science 1997, 277, 1225-1232.

(209) Förster, S.; Antonietti, M. Amphiphilic Block Copolymers in Structure-Controlled Nanomaterial Hybrids. Adv. Mater. 1998, 10, 195-217.

(210) Malliaras, G. G.; Hadziioannou, G.; Herrema, J. K.; Wildeman, J.; Wieringa, R. H.; Gill, R. E.; Lampoura, S. S. Tuning of the Photo- and Electroluminescence in Multi-Block Copolymers of Poly[(silanylene)thiophene]s via Exciton Confinement. Adv. Mater. 1993, 5, 721-723.

(211) Javier, A. E.; Patel, S. N.; Hallinan, D. T.; Srinivasan, V.; Balsara, N. P. Simultaneous Electronic and Ionic Conduction in a Block Copolymer: Application in Lithium Battery Electrodes. Angew. Chem. Int. Ed. 2011, 50, 9848-9851. 
(212) Sary, N.; Richard, F.; Brochon, C.; Leclerc, N.; Lévêque, P.; Audinot, J.-N.; Berson, S.; Heiser, T.; Hadziioannou, G.; Mezzenga, R. A New Supramolecular Route for Using Rod-Coil Block Copolymers in Photovoltaic Applications. Adv. Mater. 2010, 22, 763-768.

(213) Rauwald, U.; Scherman, O. A. Supramolecular Block Copolymers with Cucurbit[8]uril in Water. Angew. Chem. Int. Ed. 2008, 47, 3950-3953.

(214) Sui, A.; Shi, X.; Tian, H.; Geng, Y.; Wang, F. Suzuki-Miyaura Catalyst-Transfer Polycondensation with $\mathrm{Pd}(\operatorname{IPr})(\mathrm{OAc})_{2}$ as the Catalyst for the Controlled Synthesis of Polyfluorenes and Polythiophenes. Polym. Chem. 2014, 5, 7072-7080.

(215) Grisorio, R.; Suranna, G. P. Impact of Precatalyst Activation on Suzuki-Miyaura Catalyst-Transfer Polymerizations: New Mechanistic Scenarios for Pre-transmetalation Events. ACS Macro Lett. 2017, $6,1251-1256$.

(216) Shaikh, H.; Jin, X.-H.; Harniman, R. L.; Richardson, R. M.; Whittell, G. R.; Manners, I. Solid-State DonorAcceptor Coaxial Heterojunction Nanowires via Living Crystallization-Driven Self-Assembly. J. Am. Chem. Soc. 2020, 142, 13469-13480.

(217) Mori, T.; Watanabe, T.; Minagawa, K.; Tanaka, M. Self-Assembly of Oligo( $p$-phenylenevinylene)-blockPoly(ethylene oxide) in Polar Media and Solubilization of an Oligo( $p$-phenylenevinylene) Homooligomer inside the Assembly. J. Polym. Sci., Part A: Polym. Chem. 2005, 43, 1569-1578.

(218) Marsitzky, D.; Klapper, M.; Müllen, K. End-Functionalization of Poly(2,7-fluorene): A Key Step toward Novel Luminescent Rod-Coil Block Copolymers. Macromolecules 1999, 32, 8685-8688.

(219) Dai, C.; Fu, G. C. The First General Method for Palladium-Catalyzed Negishi Cross-Coupling of Aryl and Vinyl Chlorides: Use of Commercially Available $\mathrm{Pd}\left(\mathrm{P}(t-\mathrm{Bu})_{3}\right)_{2}$ as a Catalyst. J. Am. Chem. Soc. 2001, 123, 2719-2724.

(220) Price, D. W.; Tour, J. M. Biphenyl- and Fluorenyl-Based Potential Molecular Electronic Devices. Tetrahedron 2003, 59, 3131-3156.

(221) Keshtov, M. L.; Mal'tsev, E. I.; Lopatin, A. M.; Nikitin, L. N.; Blagodatskikh, I. A.; Buzin, M. N.; Pozin, S. M.; Khokhlov, A. R. New Electyroluminescent Phenyl-Subsituted Polyfluorenes Synthesized in Supercritical Carbon Dioxide. Dokl. Chem. 2010, 432.

(222) Edwards, T. H.; Stoll, S. Optimal Tikhonov Regularization for DEER Spectroscopy. J. Magn. Reson. 2018, 288, 58-68.

(223) Farquharson, C. G.; Oldenburg, D. W. Automatic Estimation of the Trade-off Parameter in Nonlinear Inverse Problems Using the GCV and L-curve Criteria. SEG Technical Program Expanded Abstracts; Society of Exploration Geophysicists 2000, 265-268.

(224) Kratky, O.; Porod, G. Röntgenuntersuchung Gelöster Fadenmoleküle. Recl. Trav. Chim. Pays-Bas 1949, 68, 1106-1122.

(225) Lee, H. J.; Sohn, J.; Hwang, J.; Park, S. Y.; Choi, H.; Cha, M. Triphenylamine-Cored Bifunctional Organic Molecules for Two-Photon Absorption and Photorefraction. Chem. Mater. 2004, 16, 456-465.

(226) Zhu, L.; Sheng, D.; Xu, C.; Dai, X.; Silver, M. A.; Li, J.; Li, P.; Wang, Y.; Wang, Y.; Chen, L.; et al. Identifying the Recognition Site for Selective Trapping of ${ }^{99} \mathrm{TcO}^{-}$in a Hydrolytically Stable and Radiation Resistant Cationic Metal-Organic Framework. J. Am. Chem. Soc. 2017, 139, 14873-14876.

(227) Wagh, R. B.; Nagarkar, J. M. Facile and Effective Approach for Oxidation of Boronic Acids. Tetrahedron Lett. 2017, 58, 4572-4575. 